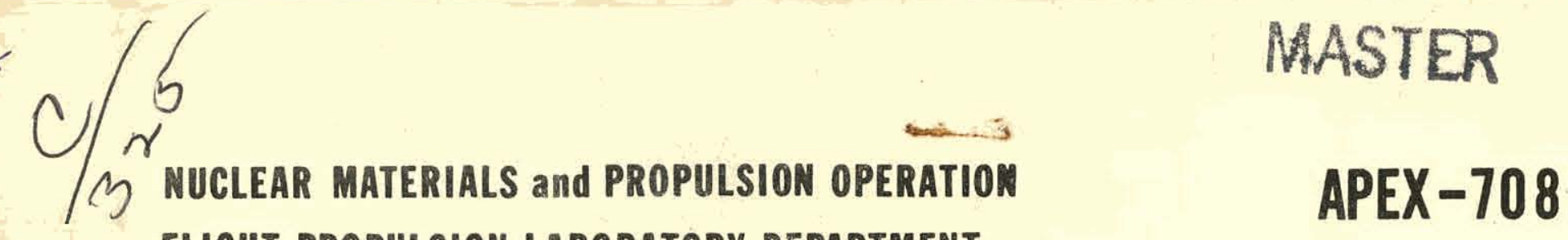
FLIGHT PROPULSION LABORATORY DEPARTMENT
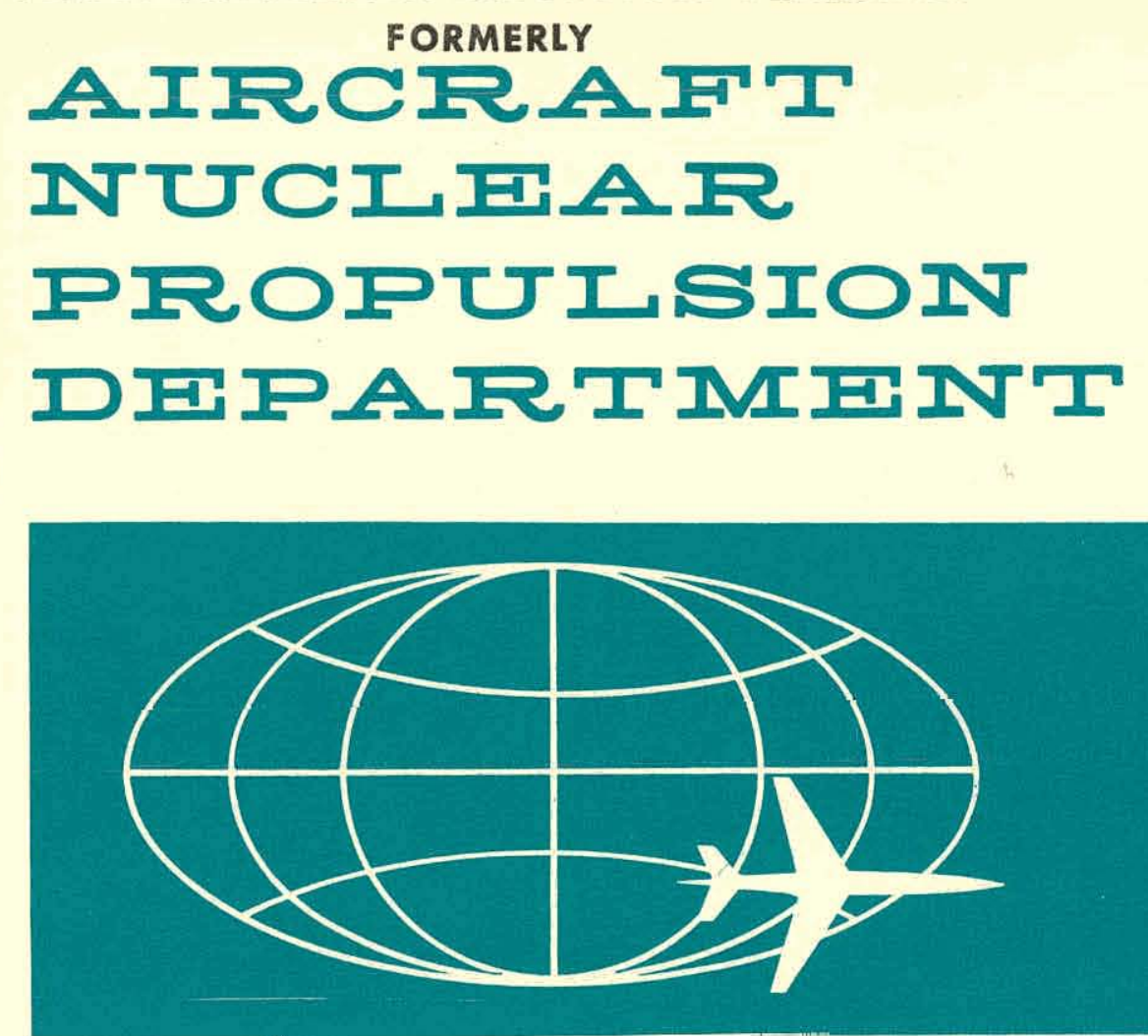

\title{
IBM-7090 Programs to Compile and Modify A Nuclear Data Tape
}

R. G. Herrmann

T. A. Hoffman

F. D.Wenstrup

A. Wilcox 


\section{DISCLAIMER}

This report was prepared as an account of work sponsored by an agency of the United States Government. Neither the United States Government nor any agency Thereof, nor any of their employees, makes any warranty, express or implied, or assumes any legal liability or responsibility for the accuracy, completeness, or usefulness of any information, apparatus, product, or process disclosed, or represents that its use would not infringe privately owned rights. Reference herein to any specific commercial product, process, or service by trade name, trademark, manufacturer, or otherwise does not necessarily constitute or imply its endorsement, recommendation, or favoring by the United States Government or any agency thereof. The views and opinions of authors expressed herein do not necessarily state or reflect those of the United States Government or any agency thereof. 


\section{DISCLAIMER}

Portions of this document may be illegible in electronic image products. Images are produced from the best available original document. 


\section{LEGAL NOTICE}

This report was prepared as an account of Government sponsored work. Neither the United States, nor the Commission, nor the Air Force, nor any person acting on behalf of the Cornmission or the Air Force:

A. Makes any warranty or representation, express or implied, with respect to the accuracy, completeness, or usefulness of the information contained in this report, or that the use of any information, apparatus, method, or prosess disclosed in this report may not infringe privately owned rights; or

B. Assumes any liabilities with respect to the use of, or for damages resulting from the use of any information, apparatus, method, or process disclosed in this report.

As used in the above "person acting on behalf of the Commission or Air Force" includes any employee or contractor of the Commission or Air Force to the extent that such employee or contractor prepares, handles, or distributes, or provides access to, any information pursuant to his employment or contract with the Commission or Air Force.

Printed in USA. Price \$4.00. Available from the

Office of Technical Services

U.S. Department of Commerce Washington 25, D.C. 
IBM-7090 PROGRAMS TO COMPILE AND

MODIFY A NUCLEAR DATA TAPE

\author{
R. G。 Herrmann \\ T. A. Hoffman \\ F. D。 Wenstrup \\ A. Wilcox
}

May 31, 1961

\title{
GENERAL (ge? ELCTRIC \\ NUCLEAR MATERIALS AND PROPULSION OPERATION (Formerly Aircraft Nuclear Propulsion Department) \\ Cincinnati 15, Ohio
}

Published

August 1961

UNCLASSIFIED 
3 Aberdeen Proving Ground

1 Aerolet-General Corporation

1 Aerojet-General Nucleonice

1 AFPR, Boeing, Seattle

3 AFPR, Lockheed, Marletta

2 Alr Force Special Weapons Center

2 ANP Project Office, Convalr, Fort Worth

1 Alco Products, Inc.

1 Alli-Chalmers Manufacturing Company

1 Alls-Chalmers Manufacturing Company, Washington

1 Argonne Cancer Research Hospltal

10 Argonne National Laboratory

1 Army Ballistic Missile Agency

4 Army Chemlcal Center

1 Army Signal Research and Development Laboratory

1 Atomic Bomb Casualty Commissiod

1 AEC Scientific Representative, France

1 AEC Scientific Representative, Japan

3 Atomic Energy Commisgion, Washington

4 Atomic Energy of Canada Limited

4 Atomice International

2 Baboock and willcox Company

2 Battelle Memorial Institute

4 Brookhaven National Laboratory

1 Bruhh Beryllium Company

1 Bureau of Shipe (Code 1500)

1 Bureau of Yards and Docks

1 Carnegle Institute of Technology

1 Chicago Operation Office

1 Chicago Patent Group

1 Columbla Univeraity (Havens)

1 Columbla Unlversity (\$OO-187)

1 Combustion Englneering, Inc.

1 Combustion Engineering, Inc. (NRD)

1 Convalr-General Dynamics Corporation, San Dlego

2 Defence Research Member

Defense Atomic Support Agency, Washington

Department of the Army, G-2

duPont Company, Alken

1 duPont Company, Wilmington

Edgerton, Germeshausen and Grier, Inc., Las Vegas

Frankford Arsenal

Franklen Institute of Pennsylvania

Genoral Atomic Division

General Electris Company (ANPD)

General Electric Company, Richland

1 General Electric Company, St. Peteraburg

General Nuclear Engineering Corporation

Gibbs and Cox, inc.

Glasstone, Samuel

Goodyoor Atnmls finrmration

Grand Junction Operations Offlce

Hughes Alrcraft Company

Iown State Univeraity

Jet Propul sion Laboratory

Knolls Atomic Power Laboratory

Los Alamos Sclentlfic Laboratory

LO8 Alamos Sclentific Laboratory (Sesonske)

Lovelace Foundation

Maritime Administration

Martin Company

Massachusetts Instltute of Technology (Profio)

Massaciusetts Institute of Technology (Thompson)

Midwestern Unlversitles Research Assoclation

Mound Laboratory

NASA Lewis Research Center

National Bureau of Standand

National Bureau of Standards (Library)

1 National Lead Company of Ohto
Naval Medical Research Institute

Naval Research Laboratory

New Brunswick Area Office

New York Operations Offlce

New York Unlversity (Figher)

New York Univeraity (Richtmyer)

Northern Research and Engineering Corporation

Nuclear Development Corporation of America

Nuclear Materlals and Equlpment Corporation

Nuclear Metalo, inc.

Oak Ridge Institute of Nuclear Studies

Offlce of Naval Research

Offlce of Naval Research (Code 422)

Orfice of Ordnance Research

Offlce of the Chief of Naval Operations

Ordnance Materlala Research Office

Ordnance Tank-Automotive Command

Patent Branch, Washington

Pennaylvania State Unlveraity (Blanchard)

Phillipo Petroleum Company (NRTS)

Power Reactor Development Company

Pratt and Whitney Aircraft Division

Princeton University (White)

Public Health Service

Public Health Service, Cincinnat

Public Health Service, Las Vegae

Public Health Service, Montgomery

Renaselaer Polytechnic Inatitute

Qusrtermaster Research and Development Command

Sandia Corporation, Albuquerque

States Marine LInes, Inc.

Steveng Institute of Technology

Strategic Air Command

Sylvania Electric Products, Inc.

Technical Research Group

Tennessee Valley Authority

Texas Nuclear Corporation

The Surgeon Genera

Union Carbide Nuclear Company (ORGDP)

Union Carbide Nuclear Company (ORNL)

Unlon Carbide Nuclear Company (Paducah Plant)

USAF Project RAND

U. S. Geological Survey, Denver

U. S. Geological Survey, Menlo Park

U. S. Geological Survey, Naval Gun Factory

U. S. Geological Survey, Washington

U. S. Naval Ordnance Laboratory

U. S. Naval Postgraduate School

U. S. Naval Radiological Dafense Laboratory

U. S. Patent Office

Univeroity of Callfornia at Los Angeles

Undversity of Californla, Berkeley

Unlverefty of Callfornia, Lvermore

Univeraity of Callfornda, San Francisco

University of Puerto Rico

University of Rochester

University of Rochester (Marahak)

Univeraity of Washington (Geballe)

Univerulty of Washington (Robde)

Walter Reed Army Medical Center

Watertown Arsenal

Westinghouse Bettio Atomic Power Laboratory

Westinghouse Electric Corporation

Wright Ar Development Division

Yale Univeralty (Brelt)

Yale University (Schultz)

Yankee Atomic Electric Company

Office of Technical information Extension

Office of Technical Bervices, Washingtion 
SPECIAL DISTRIBUTION

External

Atomic Power Equipment Department, San Jose, Calif.

Attn: R. M. Cohen

J. Weil

General Atomic, San Diego, Califormia

Attn: C. Cyl-Champlin

J. M. Krase

Hanford Atomic Products Operation, Richland, Wash.

Attn: F. G. Dawson

W. B. Henderson

The Internuclear Company, Clayton, Missouri

Attn: E. E. Mason

Knolls Atomic Power Laboratory, Schenectady, N.Y. Attn: D. S. Selengut

Lawrence Radiation Laboratory, Livermore, Calif. Attn: R. N. Stuart

Missile and Space Vehicle Department, Philadelphia, Pennsylvania

Attn: J. F. Heyda

The Martin Company, Nuclear Division, Baltimore,

Maryland

Attn: J. E. Long

F. D. Wenstrup

Technical Military Planning Operation, Defense

Systems Department, Santa Barbara, Calif.

Attn: P. G. Fischer

R. G. Herrmann

Westinghouse, Atomic Products Division, Pittsburgh, Pennsylvania

Attn: F. L. Langford

Internal

J. R. Beeler

V. P. Calkins

C. L. Chase (2)

E. B. Delson

M. B. Goldstein

G. G. Leeth

W. H. Long

J. E. MacDonald

H. F. Matthiesen

F. W. Mezger

J. W. Morfitt (ITS)
R. E. Motsinger

W. E. Niemuth

C. S. Robertson

A. J. Rothstein

J. D. Selby

J. D. Simpson

G. Thornton

J. W. Zwick

Library (ITS)

Reports Library (12)

(54323) 


\section{ABSTRACT}

The Reactor Nuclear Data Tape Programs, as described in this report, were deslgned to compile and/or modify a nuclear data library tape from basic nuclear cross-section input. It is intended that this crosssection library tape $w 11 l$ be utilized to provide up-to-date nuclear data for various reactor codes.

Program RNDP was designed to complie an Initial tape from certain minimal imput data,or to add or delete complete blocks of data from an existing tape.

Program RNDM was designed to complle and add to an existing tape macroscopic cross section data consisting of mixtures of microscopic cross sections available on the tape.

Program RNDC was designed to modify and copy an existing tape, or to compile a new tape from sets of binary cards punched by Program RNDP.

These programs were written using the FORIRAN-II system of automatic coding for the IBM-7090.

\section{ACKMOWLEDGMENT}

The original specifications for Programs RNDP, RNDM, and RNDC were prepared by P. G. Fischer and J. R. Cooper. 
Page

1.0 Introduction.

2.0 Reactor Nuclear Data Preparation Program - . . - . . 15

2.1 General Information. . . . . . . . . . . 15

2.2 Data Requirements . . . . . . . . . . 17

2.3 Equations . . . . . . . . . . . . . 20

2.4 Subroutine Descriptions. . . . . . . . . . 25

2.5 Program Flow Charts. . . . . . . . . . . 31

2.6 Program Diagnost1cs. . . . . . . . . . . 31

2.7 Program Input Forms. . . . . . . . . . . 35

2.8 Program Source Deck Listing. . . . . . . . . 80

2.9 Program Mnemonic Glossary . . . . . . . . . . . . . .

3.0 Reector Nuclear Data Macroscopic Program - . . . . .148

3.1 General Information. . . . . . . . . . . . . . . .

3.2 Equations and Data Requirements. : : : : . .148

3.3 Description of Subroutines Unique to this Program . . . .149

3.4 Program Flow Chart . . . . . . . . . . . 150

3.5 Program Diagnost1cs. . . . . . . . . . . . . . . . .

3.6 Program Input Forms. . . . . . . . . . .154

3.7 Program Source Deck Llsting. . . . . . . . .157

3.8 Program Mnemonic Glossary . . . . • • • . .197

4.0 Reactor Nuclear Data Copy and Correct Program. - . . . 201

4.1 General Information. . . . . . . . . . . . . . .

4.2 Description of Subroutines Unique to this Program . . .201

4.3 Program Flow Chart . . . . . . . . . . .205

4.4 Program Dlagnost1cs. . . . . . . . . . .206

4.5 Program Input Forms. . . . . . . . . . .209

4.6 Program Source Deck Ifsting. . . . . . . . .228

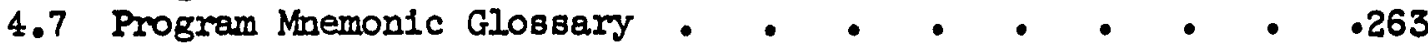

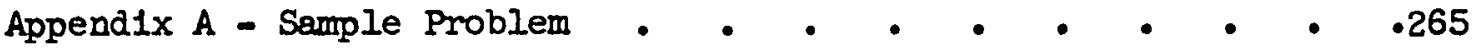

Al - Sample Problem Input . • . . . . . . . . . . . 65

A2 - Sample Problem Output . • . . . . • • • .277

Appendix B - DIP Input Subroutine • • • • • • • • 302 
Fig. 1.1 - Reactor Nuclear Data Tape Format

F1g. 1.2 - Reactor Nuclear Data Tape Index Record

Fig. 1.3 - Reactor Nuclear Data Tape Second Record

F1g. 1.4 - Reactor Nuclear Data Tape Material Data Block

Fig. 1.5 - Materlal Data Block Record I

Fig. 1.6 - Material Data Block Record 2

FIg. 1.7 - Material Data Block Record 3

F1g. 1.8 - Materlal Data Block Record 4

12

Fig. 1.9 - Materlal Data Block Record 5

13

F1g. 1.10- Reactor Nuclear Data Tape Spectral Data Block

13

Fig. 1.11- Reactor Nuclear Data Tape Final Record

14

Fig. 2.1 - Logic for Calculation of

Fig. 2.2 - Logic for Calcutiation of

$$
\sigma_{s_{j}}, \sigma_{A_{i}},\left(\nu \sigma_{f}\right)_{i} \text { lor } \Sigma_{s_{i}}, \Sigma_{A_{i}},\left(\nu \Sigma_{f}\right)_{i} \text { ] }
$$

22

$\left(\varepsilon \sigma_{\mathrm{s}}\right)_{\mathrm{i}}, \sigma_{\mathrm{TR}_{\mathrm{i}}},\left(\varepsilon_{1} \sigma_{\mathrm{sl}}\right)_{\mathrm{i}}$ [or $\left(\xi \Sigma_{\mathrm{s}}\right)_{\mathrm{i}}, \Sigma_{\mathrm{TR}},\left(\xi_{1} \Sigma_{\mathrm{s} 1}\right)_{\mathrm{i}}$ ]

23

F1g. 2.3 - Logic for Calctiation of Density Factor (DF)

24

F1g. 2.4 - Flow Chart of Control Routine

32

F1g. 2.5 - Flow Chart of DATACT Control Routine

33

F1g. 2.6 - Flow Chart of INPIDB Subroutine

34

F1g. 3.1 - Program Gross Flow Chart

Fig. 4.1 - Flow Chart of Control Routine

F1g. 4.2 - Macroscopic-Canstituent Index (Final Record) 


\subsection{INTRODUCTION}

The Reactor Nuclear Data Tape programs complle and/or modify a nuclear data library tape to be used by reactor analysis codes as an input source of cross section data. The data tape energy lattice is flexible, allowing up to a maximu of thirty-two epithermal levels and ten thermal temperature levels. Thermal scattering-transfer data coupling the thermal and near-thermal levels, are limited to a maximum five-by-five matrix. Resonance data may be glven at two-hundred energy points maximum.

The format of the overall Reactor Nuclear Data Tape (RNDT) is 1llustrated in Figure 1.1. The first logicalt record of the tape can be considered a table of contents for the whole tape. Fach data block is described in this first record, Figure 1.2, by four words: two words of binary-coded Hollerith information giving the deslgnation, one word giving the material code number in floating point, and one integer word giving the logical record number on the tape of the first record of the data block.

The energy and lethargy lattlces and a set of $\mathrm{I} / \mathrm{v}$ factors constitute the second record on the tape as shown in Figure 1.3 .

- The "Data Blocks" shown in Flgure 1.1 may contaln two different types of data. The first type, Figure 1.4, is material cross-section data having a data-block code number less than 500.0, and a maximm of five records within the block. The first record, Figure 1.5, will always be present for each of these types of blocks. The second, third, fourth, and fifth records, Flgures 1.6 through 1.9, will appear only for those materials for which such data areavailable or necessary.

The second type of data block, Figure 1.10, baving a data-block code number equal to or greater than 500.0 , requires only one record on the tape.

The final record on the tape, illustrated in Figure 1.11, contains a table listing the microscopic constituents of each macroscopic crosssection data block present on the tape. The final record always appears in full, one thousand words, as the last record on the tape, even though no macroscopic cross-section data are avaliable from the tape. In that case, the final record would contain all zeros. It should be noted that the fixed length of the final record limits the number of macroscopic material data blocks and/or the number of microscopic constituents. After the final record, a single end-of-file is written to indicate the end of all data.

These programs were written in the FORTRAN-II system of automatic coding and assembled on the IBM-7090. Binary tapes, such as the Reactor Nuclear Data Tape, complled and written in FORTRAN language should generally be used only in confunction with other programs written in the same syotem, because of the tape structure in terms of 128 or less words per physical record.

Fuless specifically stated, all references to tape record imply logical rather than physical tape records. 


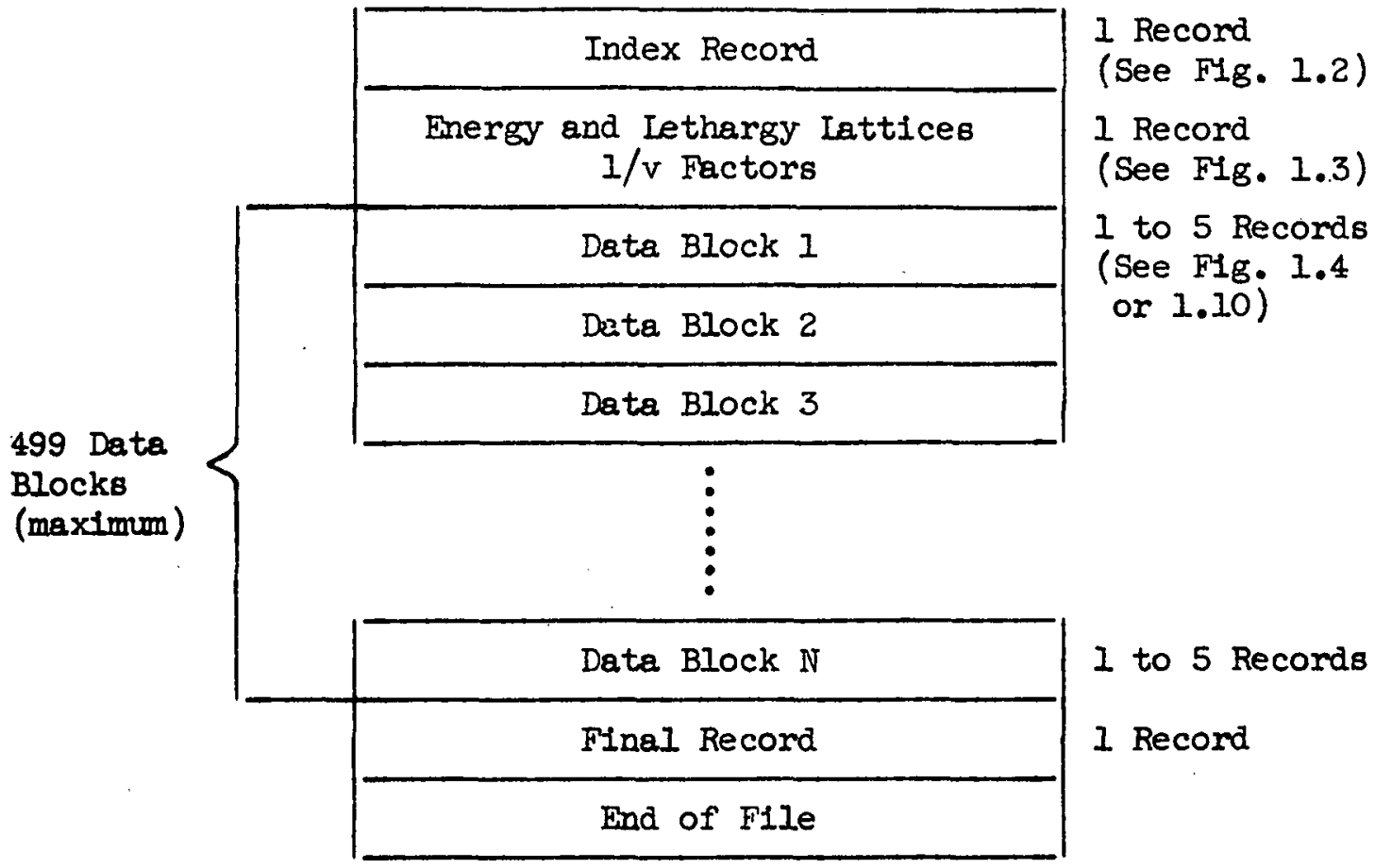

F1g. 1.1 - Reactor Nuclear Data Tape Format

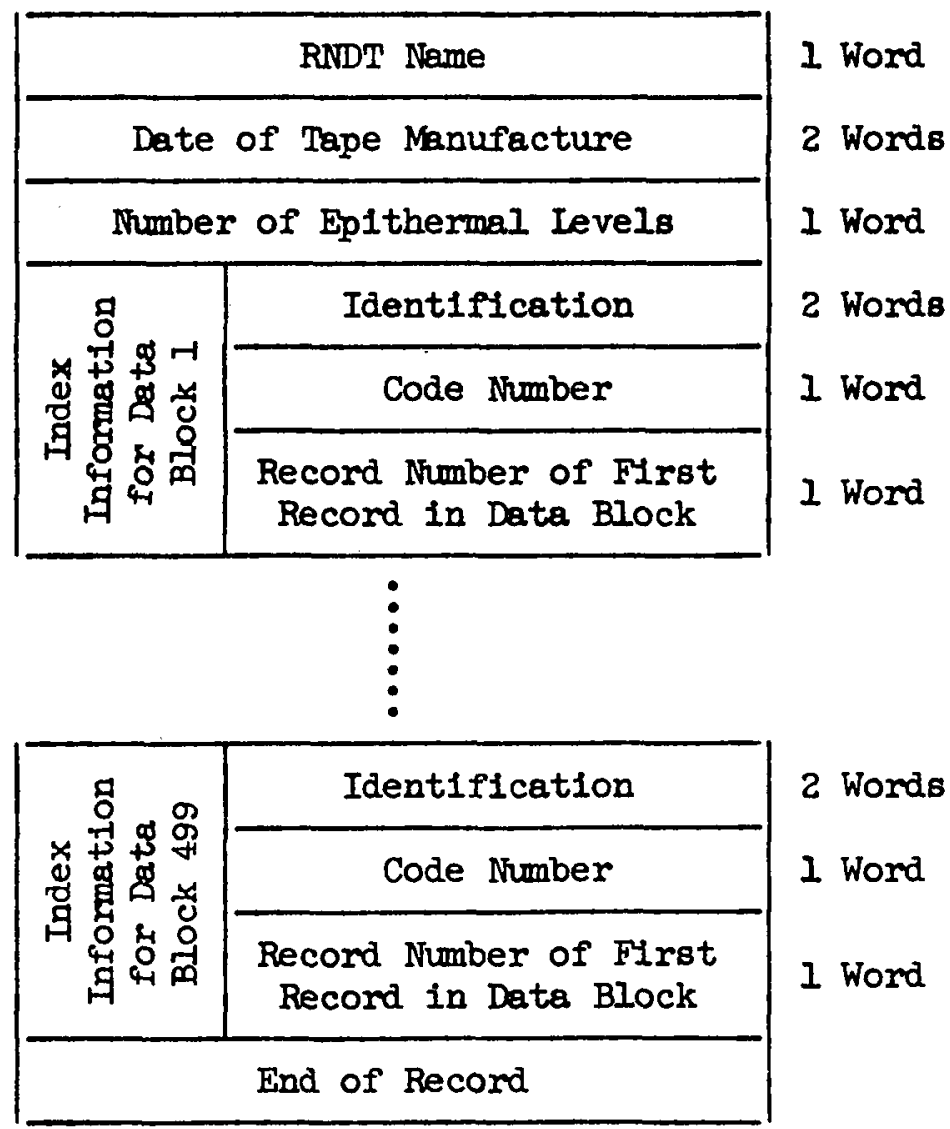

F1g. 1.2 - Reactor Nuclear Data Tape Index Record NOTE: Index information for nonexistent data blocks w1ll contain zeros. 


\begin{tabular}{|c|}
\hline Record Number \\
\hline $\begin{array}{c}\text { Energy (E) } \\
\text { Level Structure }\end{array}$ \\
\hline $\begin{array}{c}\text { Lethargy (U) } \\
\text { Level Structure }\end{array}$ \\
\hline $\begin{array}{c}\text { Energy (E) } \\
\text { Group Structure }\end{array}$ \\
\hline $\begin{array}{c}\text { Lethargy (U) } \\
\text { Group Structure }\end{array}$ \\
\hline I/v Factors \\
\hline Fnd of Record
\end{tabular}

1 Word

42 Words Maximum

(Includes the thermal levels)

42 Words Maximum

(Includes the thermal levels)

33 Words Maximum

33 Words Maximum

32 Words Maximum

FIg. 1.3 - Reactor Nuclear Data Tape Second Record

NOTE: Group $m$ extends from $E_{m-1}$ and $U_{m-1}$ on the high-energy end to $E_{m}$ and $U_{m}$ on the low-energy end. 


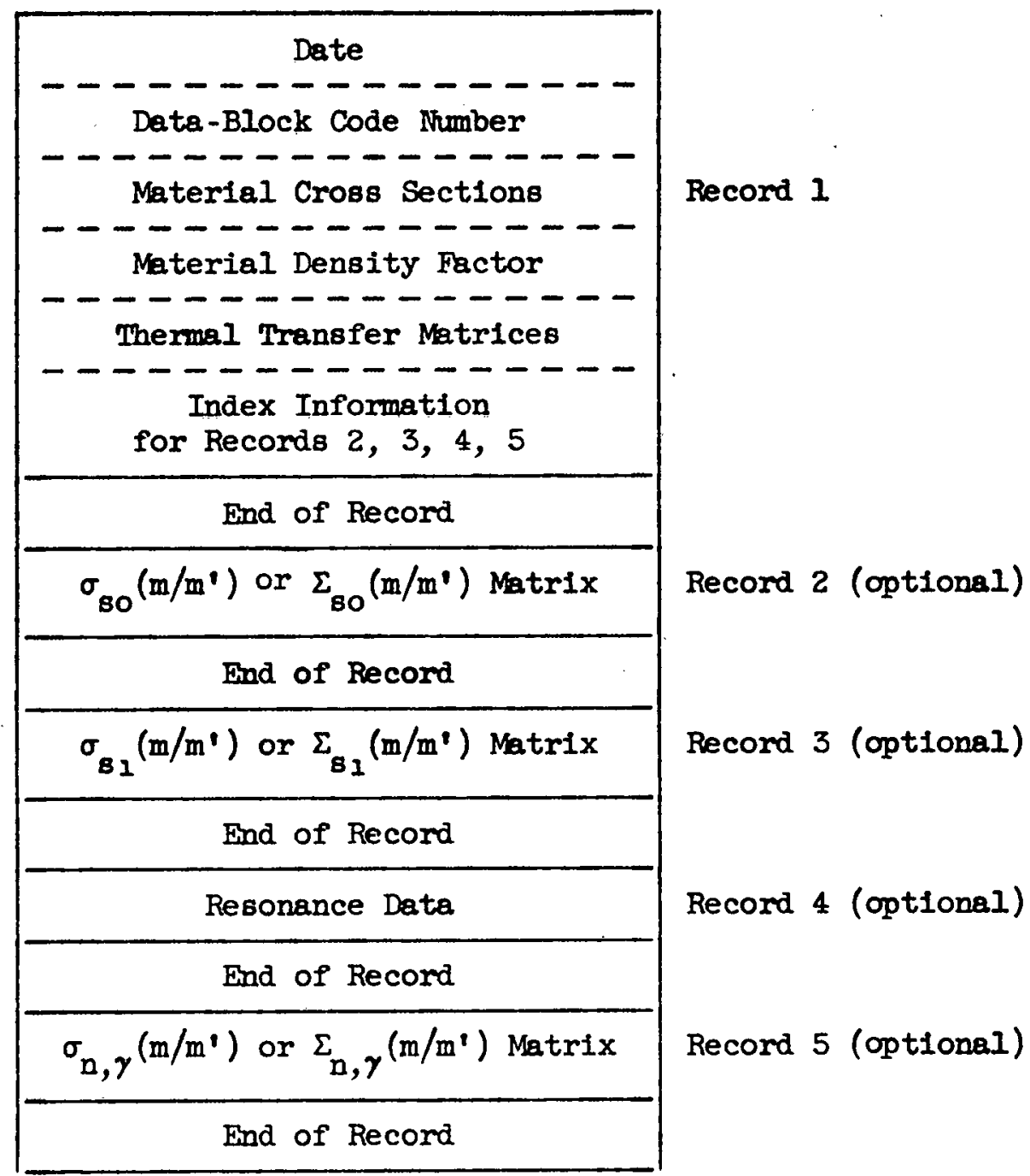

FIg. 1.4 - Reactor Muclear Data Tape Material Data Block 


\begin{tabular}{|c|c|}
\hline Record Number & 1 Word \\
\hline Date & 2 Words \\
\hline Code Number & 1 Word \\
\hline$\sigma_{s}$ or $\Sigma_{s}$ & 42 Words Meximm \\
\hline$\xi \sigma_{\mathrm{s}}$ or $\xi \Sigma_{\mathrm{s}}$ & 42 Words Maximum \\
\hline$\sigma_{t r}$ or $\Sigma_{t r}$ & 42 Words Maximum \\
\hline$\sigma_{a}$ or $\Sigma_{a}$ & 42 Words Meximum \\
\hline$\xi_{2} \sigma_{81}$ or $\xi_{1} \Sigma_{82}$ & 42 Words Maximum \\
\hline$v \sigma_{f}$ or $v \Sigma_{f}$ & 42 Words Maximum \\
\hline Dena1ty Factor $\left(A / N_{0}\right.$ or $\left.\rho\right)$ & 1 Word \\
\hline $\begin{aligned} \sigma\left(k / k^{\prime}\right) & \text { or } \Sigma\left(k / k^{\prime}\right) \\
k & =1,6 \\
k^{\prime} & =1,5\end{aligned}$ & 300 Words Meximum \\
\hline $\begin{array}{l}\text { MOMIN } \\
\text { MO }_{\text {MAX }} \\
M^{\prime} O_{\text {MAX }}\end{array}$ & 3 Words \\
\hline $\begin{array}{l}M_{\text {MII }} \\
M_{\text {MAX }} \\
M^{\prime} I_{\text {MAX }}\end{array}$ & 3 Words \\
\hline Number of Resonances (NRES) & 1 Word \\
\hline $\begin{array}{l}\mathrm{M} \Gamma_{\text {MIN }} \\
M \Gamma_{\text {MAX }} \\
M^{\prime} \Gamma_{\text {MAX }}\end{array}$ & 3 Words \\
\hline $\begin{array}{l}\text { Total Number of Words in Data } \\
\text { Block Following First Record }\end{array}$ & 1 Word \\
\hline End of Record & \\
\hline
\end{tabular}

Fig. 1.5 - Material Data Block Record 1

NOTE: A value of zero for $M_{M I N}, M_{M I N}$, NRES, or MIMIN indicates that records $2,3,4$, or 5 respectively do not exist on the tape. 


\begin{tabular}{c}
\hline Record Number \\
\hline$\sigma_{s o}\left(m / m^{\prime}\right)$ or $\Sigma_{s o}\left(m / m^{\prime}\right)$ \\
$m=M_{M I N}, M_{M A X}$ \\
$m^{\prime}=M_{M I N}, M^{\prime} O_{M A X}$ \\
\hline End of Record
\end{tabular}

1 Word

925 Words Maximum

F1g. 1.6 - Material Data Block Record 2

\begin{tabular}{|c|}
\hline Record Number \\
\hline$\sigma_{s 1}\left(m / m^{\prime}\right)$ or $\Sigma_{s I}\left(m / m^{\prime}\right)$ \\
$m=M_{M I N}, M_{M A X}$ \\
$m^{\prime}=M_{M I N}, M^{\prime} I_{M A X}$ \\
\hline End of Record
\end{tabular}

1 Word

925 Words Maximum

F1g. 1.7 - Material Data Block Record 3

\begin{tabular}{c}
\hline Record Number \\
\hline Resonance Energy $E_{o}$ \\
\hline Resonance Integral $I_{0}$ \\
\hline Peek Cross Section $\sigma_{0}$ \\
\hline$\eta=\frac{v \Gamma_{f}}{\Gamma_{\gamma}+\Gamma_{f}}$ \\
End of Record
\end{tabular}

1 Word

200 Words Maximum

200 Words Maximum

200 Words Maximum

200 Words Maximum

200 Words Maximum

Fig. 1.8 - Material Data Block Record 4 


\begin{tabular}{|c|}
\hline Record Number \\
\hline$\sigma_{n, \gamma}\left(\mathrm{m} / \mathrm{m}^{\prime}\right)$ or $\Sigma_{\mathrm{n}, \boldsymbol{\gamma}}\left(\mathrm{m} / \mathrm{m}^{\prime}\right)$ \\
$m=\mathrm{M} \Gamma_{\mathrm{MIN}}, \mathrm{M} \Gamma_{\mathrm{MAX}}$ \\
$\mathrm{m}^{\prime}=\mathrm{M} \Gamma_{\mathrm{MIN}}, \mathrm{M}^{\prime} \Gamma_{\mathrm{MAX}}$ \\
\hline End of Record
\end{tabular}

1 Word

Flg. 1.9 - Materlal Data Block Record 5

\begin{tabular}{|c|}
\hline Record Number \\
\hline Date \\
\hline Data-Block Code Number \\
\hline Source Spectrum $S$ \\
\hline Delay Fraction $\beta$ \\
\hline Mean Iffe $t_{m}$ \\
\hline End of Record
\end{tabular}

1 Word

2 Words

1 Word

42 Words Maximum

1 Word

1 Word

F1g. 1.10 - Reactor Nuclear Data Tape Spectral Data Block 


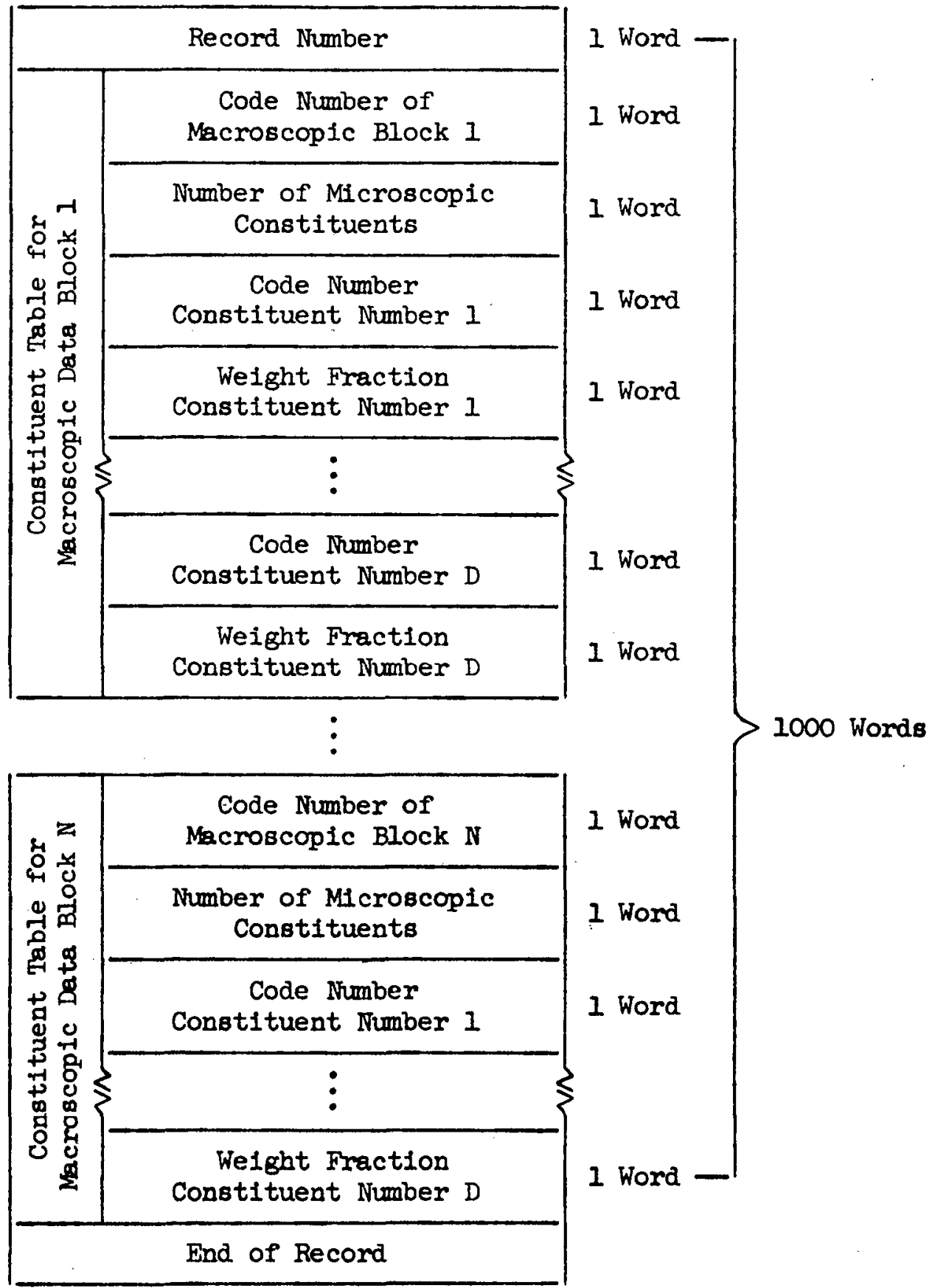

FIg. 1.11 - Reactor Nuclear Data Tape Final Record 


\subsection{REACTOR NUCLFAR DATA PREPARATION PROGRAM}

\subsection{General Information}

Program RNDP has two main functions which are the initial compilation of a nuclear data tape from decimal card data, and the revision of an existing data tape by the addition, deletion, or relocation of complete data blocks. In addition, the program has a restart capability for confining an initial compllation or revision of a tape if it is interrupted by a DIPt-type data error while processing the decimal data. Such a feature is a necessity when large quantities of decimal data are to be processed. Printing and punching is automatic for some records; for others it will occur if code numbers are specified.

For both tape revision and initial compilation of a tape, an attempt is made to manufacture two new tapes, denoted by the mnemonics MTAPE and NTAPE. In case one of these fails to process, the compilation of the other will continue. If both fail, the program is stopped.

All card imput to program RNDP, except the column binary cards punched by subroutine DIPERR ${ }^{4}$ for restart purposes, is read in by subroutine DIP; hence, the input format must conform to DIP conventions.

\subsubsection{Initial Tape Manufacture}

The imput requirements and equations needed for the initial compilation of a tape are given in the next section. A new tape must be manufactured whenever any changes not indicated in section 2.1 .2 are made.

\subsubsection{Revision of an Existing Tape}

An existing data tape can be revised by program RNDP in any of the three ways indicated above. In all such cases, the level structure of added data blocks must conform to that of the existing tape. The equations in section 2.3 are still applicable for calculations involving the index record and data blocks.

In tape revision, the index record is first corrected to reflect any additions, deletions, or relocations. Once this record is updated, it serves as a guide for the remaining tape structure. It is possible to modify a data block (so long as the level structure is not changed) without changing its code number or designation code by simultaneous deletion and addition. Its code number must be included in the NBDEL list and the revised data block must be supplied as card data. The final record is the last to be updated. The constituent tables are constructed on the basis of the final tape structure as indicated by the updated index record.

\footnotetext{
FIP is a flexible format, decimal imput, FORTRAN library routine. For convenience, the subroutine writeup is included as Appendix B. HIndividual program subroutines are discussed in greater detail in section 2.4 .
} 
In addition to both MTAPE and NTAPE, tape revision requires an existing tape (ITAPE). If relocation of data blocks is requested, an intermediate tape (KTAPE) is needed for the temporary storage of these blocks.

\subsubsection{Restart after a DIP Error}

If a DIP error is encountered in the input-specified index record data or in the data for the second record, no attempt is made to preserve the machine setup and memory status. However, if a DIP error occurs while reading-in data-block information, a transfer is made to subroutine DIPERR to punch out all the information in core which is needed to continue the compilation of the tape on a subsequent run. Th1s punched card output is labeled "RERUN", and on the next attempt, should be inserted in place of all the data preceding the first record of the data block in which the error occurred. At the time of an interruption due to such an error, an on-line comment is printed, requesting the machine operator to save all tapes which are being used in the compilation; that is, MTAPE and NTAPE, ITAPE in case of tape revision, and KIAPE in case of relocation.

\subsubsection{Printing and Punching}

The following mules govern printing:

(1) The index record and final record are printed each time a tape is made, whether an initial compilation or a revision.

(2) All data blocks added to the tape are printed, whether specified or not.

(3) The second record and all other data blocks are printed if their code number is given in the PC $\phi D E$ 11st. (The code number for the second record is $2 \times 10^{-6}$.)

Thus, in the case of an initial compilation, all records are printed automatically except the second; for tape revision, the only records printed automatically are the index record, final record, and added data blocks.

If e1ther MTAPE or NTAPE (or both) falls to process, a suitable comment is printed. Additionally, in the case of a DIP-type error in a data block, its code number is printed. Mention 18 also made of the deletion or modification of any constituents of macroscopic data blocks.

The following rules govern punching:

(1) Except for two decimal cards punched in DIPERR for a rerun case, all punching is in absolute-column binary-card format.

(2) The index record is always punched, followed by a transfer card.

(3) The second record, all data blocks, and the final record are punched if their code numbers are given in the PUNCOD list $\left(2 \times 10^{-6}\right.$ for second record, 0 for final record $)$. The second and final record, if punched, are followed by a transfer card. Each complete data block is followed by a one transfer card, regardless of how many records it contains. 
(4) All punched data, except for the two decimal cards for a rerun, are acceptable to subroutine ACBIP. All punched data, except rerun data, are also acceptable to program RNDC.

\subsection{Data Reguirements}

\subsubsection{Index Record}

Tape Name

Date of Manufacture

Number of Levels

Index Groups

a - 2-Word Identification

b - Data-Block Code Irmber

c - Physical Record Number or Flrst Record in Data Block.
- oupplied as imput

- supplied as Imput

- supplied as imput

- supplied as imput with crosssection or spectral data block

- supplied as input with crosssection or spectral data block

- calculated from block number (NB) supplied as Input with crosssection or spectral data block. See Eq. (2.1).

\section{2 .2 Record $\underline{2}$}

Record Number

$\mathrm{EL}_{1}$<smiles>[14CH3]</smiles>

EG

$\mathrm{UG}_{\mathrm{m}}$

FV
- always equal to 2

- supplied as imput

- supplied as Imput

- supplied as Input

- supplied as input

- supplied as 1mput

2.2.3 Location of Data Blocks on Tape

The position of each Data Block will be determined by the block number (NB) which is supplied as imput.

\subsubsection{Cross-Section Data Blocks}

\section{$\underline{\text { Record } 1}$}

The logic for calculating $\sigma_{8}, \sigma_{\Omega}, v \sigma_{f}, \xi \sigma_{8}, \xi \sigma_{81}, \sigma_{t r}$, and DF appears in Flgures $2.1,2.2$, and 2.3. In general, provision has been made for internally generating as much of the data as possible. Thus, for example, $\xi$, the average logarithmic energy decrement is required as input only where the total scattering is not simply isotropic elastic scattering.

Record Number (NIR)
- calculated with Eq. (2.1) with $j=$ block number (NB) 


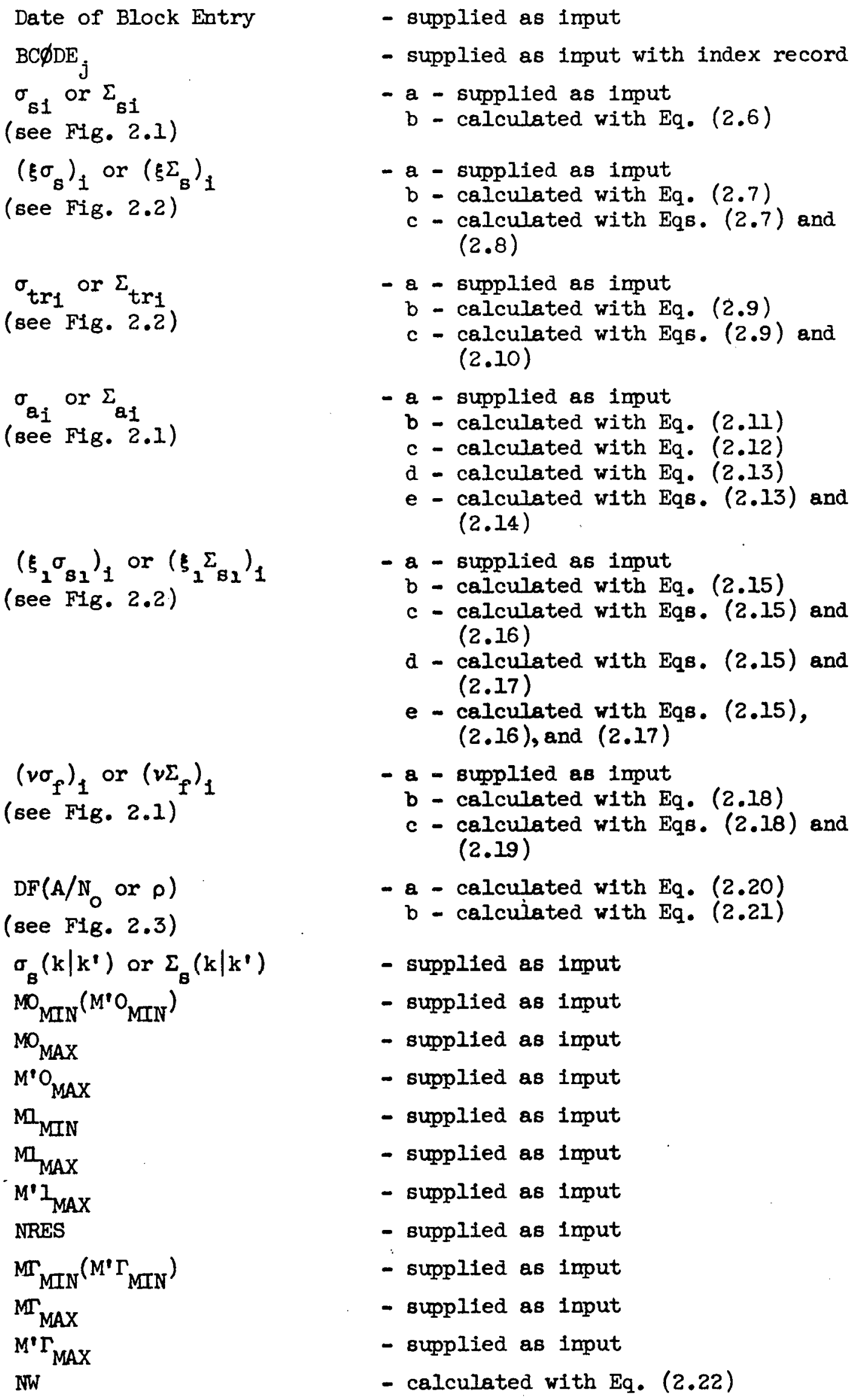


$\underline{\text { Record } 2}$

Record Number (N2R)

$\sigma_{s o}\left(m / m^{\prime}\right)$ or $\Sigma_{s o}\left(m / m^{\prime}\right)$

Record Number (N3R)

$\sigma_{61}\left(\mathrm{~m} / \mathrm{m}^{\prime}\right)$ or $\Sigma_{81}\left(\mathrm{~m} / \mathrm{m}^{\prime}\right)$

Record Number (N4R)

Eol

Iol

$\sigma_{\circ \ell}$

$R_{\ell}$

$\eta_{\ell}$

Record Number (N5R)

$\sigma_{n, \gamma}\left(\mathrm{m} / \mathrm{m}^{\prime}\right)$
- calculated with Eq. (2.2) with $j=N B$

- supplied as input

Record 3

- calculated with Eq. (2.3) with $J=\mathrm{NB}$

- supplied as imput

\section{Record 4}

- calculated with Eq. (2.4) with $j=\mathrm{NB}$

- supplied as input

- a - supplied as imput b - calculated with Eqs. (2.23), $(2.24),(2.25)$, and $(2.26)$

- a - supplied as imput

b - calculated with Eq. (2.27)

- a - supplied as imput

b - calculated with Eq. (2.28)

- a - supplied as input

b - calculated w1th Eq. (2.29)

Record 5

- calculated with Eq. (2.5) with $\mathfrak{j}=\mathrm{NB}$

- supplied es Input

\section{2 .5 Spectral Data Blocks}

Record Number (NIR)

Date of Block Entry

$S_{1}$

$\beta$

$t_{m}$

- calculated with Eq. (2.1) with $j=$ block number (NB)

- supplied as imput

- supplied as input

- supplied as lmput

- supplied as input

- calculated with Eq. (2.1) with $\mathrm{j}=\mathrm{J}+\mathrm{I}$

- supplied as imput

Code Number of Macroscoplc Block D 
Number of Microscopic

Constituents

Weight Fraction of

Constituent
- supplied as Imput

- supplied as imput

\subsection{Equations}

The following equations are used in the calculation of crosssection data, as indicated in section 1.2 .

$$
\begin{aligned}
& N I R=N I R_{j-1}+5-\left(\delta_{M O}, 0+\delta_{M I N}, 0+\delta_{N R E S, 0}+\delta_{M}, 0,0\right)_{j-1} \\
& \left(N 2 R_{O}=2\right) \\
& N 2 R_{j}=N 2 R_{j}+1 \\
& N 3 R_{j}=N R_{j}+2-\left(8_{M O}, 0\right)_{j} \\
& N 4 R_{j}=N I R_{j}=3-\left(8_{M_{M I N}}, 0+8_{M M_{M I N}}, 0\right)_{j} \\
& \mathrm{NSR}_{j}=\mathrm{NIR}_{\mathrm{J}}+4-\left(8_{\mathrm{MO}}, \mathrm{MIN}, 0+\delta_{\mathrm{MR}_{\mathrm{MIN}}, 0}+8_{\mathrm{NRES}, 0}\right)_{\mathrm{J}} \\
& \sigma_{\sigma_{i}}=\sigma_{t_{i}}-\sigma_{\theta_{i}} \\
& \left(\xi \sigma_{B}\right)_{1}=\xi_{1} \times \sigma_{s_{1}} \\
& \xi_{1}=1+\frac{(A / A N-I)^{2}}{2(A / A N)} \ln [(A / A N-I) /(A / A N+I)] \\
& \sigma_{t r_{1}}=B_{1} \times \sigma_{B_{1}} \\
& \mathrm{~B}_{1}=1-2 / 3(\mathrm{~A} / \mathrm{AN}) \\
& \sigma_{a_{1}}=\sigma_{t_{1}}-\sigma_{s_{i}} \\
& \sigma_{a_{1}}=F V_{1} \times \sigma_{Q}(2200 \mathrm{~m} / \mathrm{s}) \\
& \sigma_{a_{1}}=\left(v \sigma_{f}\right)_{1} / \eta_{1} \\
& \eta_{1}=A_{2}+\left(B_{2}+C_{2} \times\left(E L_{1}\right)^{D_{2}} \text { en } E L_{1}\right. \\
& \left(\xi_{1} \sigma_{s_{1}}\right)_{1}=\xi_{11} \times \sigma_{s_{11}}
\end{aligned}
$$




$$
\begin{aligned}
& \sigma_{B 11}=\sigma_{B 1}-\sigma_{t r_{1}} \\
& \xi_{11}=\frac{5}{3}-(\mathrm{A} / \mathrm{AN})^{2}+\frac{(\mathrm{A} / \mathrm{AN}+2)(\mathrm{A} / \mathrm{AN}-1)^{2}}{2} \times \\
& \ln [(A / A N+I) /(A / A N-1)]^{2} \\
& \left(v \sigma_{f}\right)_{1}=v_{1} \times \sigma_{f_{1}} \\
& v_{1}=A_{1}+B_{1} E+C_{1} E^{D_{1}} \\
& D F_{j}=\rho_{j} \\
& D_{j}=A_{j} / N_{0} \\
& \mathrm{NW}_{\mathrm{J}}=\left[\left(\mathrm{MO}_{\mathrm{MAX}}-\mathrm{MO}_{\mathrm{MIN}}+1\right) \times\left(\mathrm{M}^{\prime} \mathrm{O}_{\mathrm{MAX}}-\mathrm{M}^{\prime} \mathrm{O}_{\mathrm{MIN}}+1\right)\right]_{\mathrm{J}} \\
& +\left[\left(M_{M A X}-M_{M I N}+1\right) \times\left(M^{\prime} I_{\text {MAX }}-M^{\prime} I_{M I N}+1\right)\right]_{J} \\
& +\left[5 \times \mathrm{NRES}_{j}+\left[\left(\mathrm{M} \Gamma_{\mathrm{MAX}}-\mathrm{M} \Gamma_{\mathrm{MIN}}+1\right) \times\left(\mathrm{M}^{\prime} \Gamma_{\mathrm{MAX}}-\mathrm{M}^{\prime} \Gamma_{\mathrm{MIN}}+1\right)\right]_{j}\right.
\end{aligned}
$$

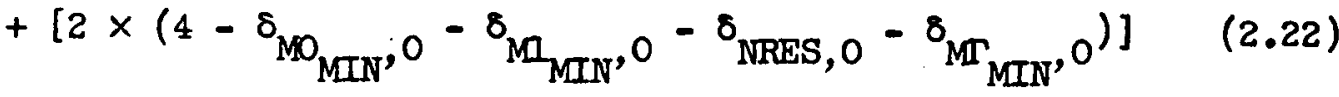

$$
\begin{aligned}
& I_{0}=\frac{Q^{2} 2 \pi^{2} \pi_{0}^{2} g}{E_{0}}\left(\Gamma_{\gamma}+\Gamma_{f}\right) \Gamma_{n} \\
& Q=(A+A N) / A \\
& \hbar_{0}=4.5517359 \times 10^{-10} / \sqrt{E_{0}} \\
& \Gamma=\Gamma_{n}+\Gamma_{\gamma}+\Gamma_{f} \\
& \sigma_{0}=\frac{2 E_{0} I_{0}}{\pi \Gamma} \\
& R=\frac{4 \cdot A N \cdot Q \cdot E_{O} \cdot k}{A \Gamma^{2}} \\
& \eta=\frac{v \Gamma_{f}}{\Gamma_{y}+\Gamma_{f}} \quad \text { NOTE: Use Eq. (2.19) to evaluate } v
\end{aligned}
$$




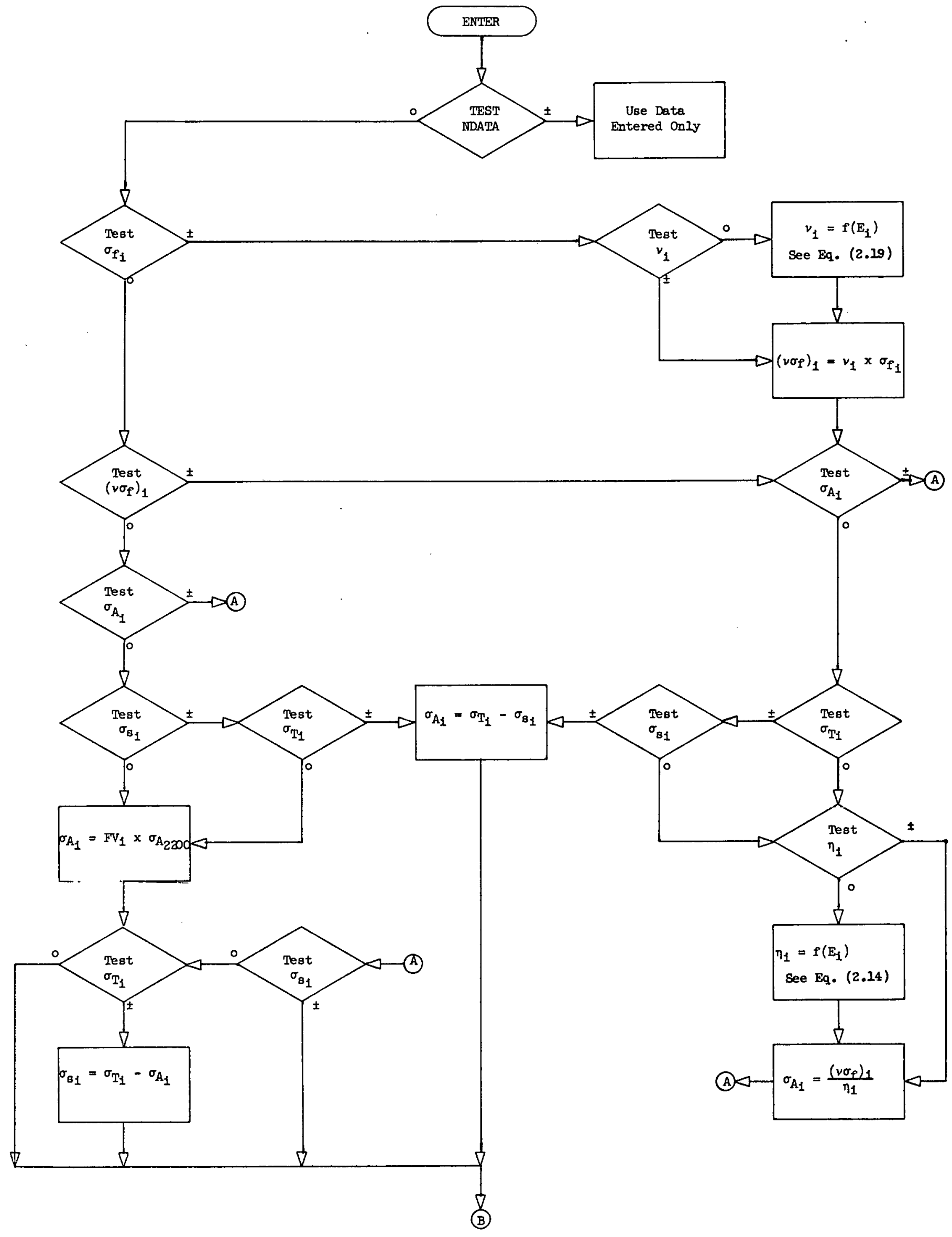

FIGURE 2.1

IOGIC FOR CALCULATION OF $\sigma_{8_{1}}, \sigma_{\mathrm{A}_{1}},\left(v \sigma_{f}\right)_{1}$ [or $\Sigma_{8_{1}}, \Sigma_{\mathrm{A}_{1}},\left(v \Sigma_{f}\right)_{1}$ ] 


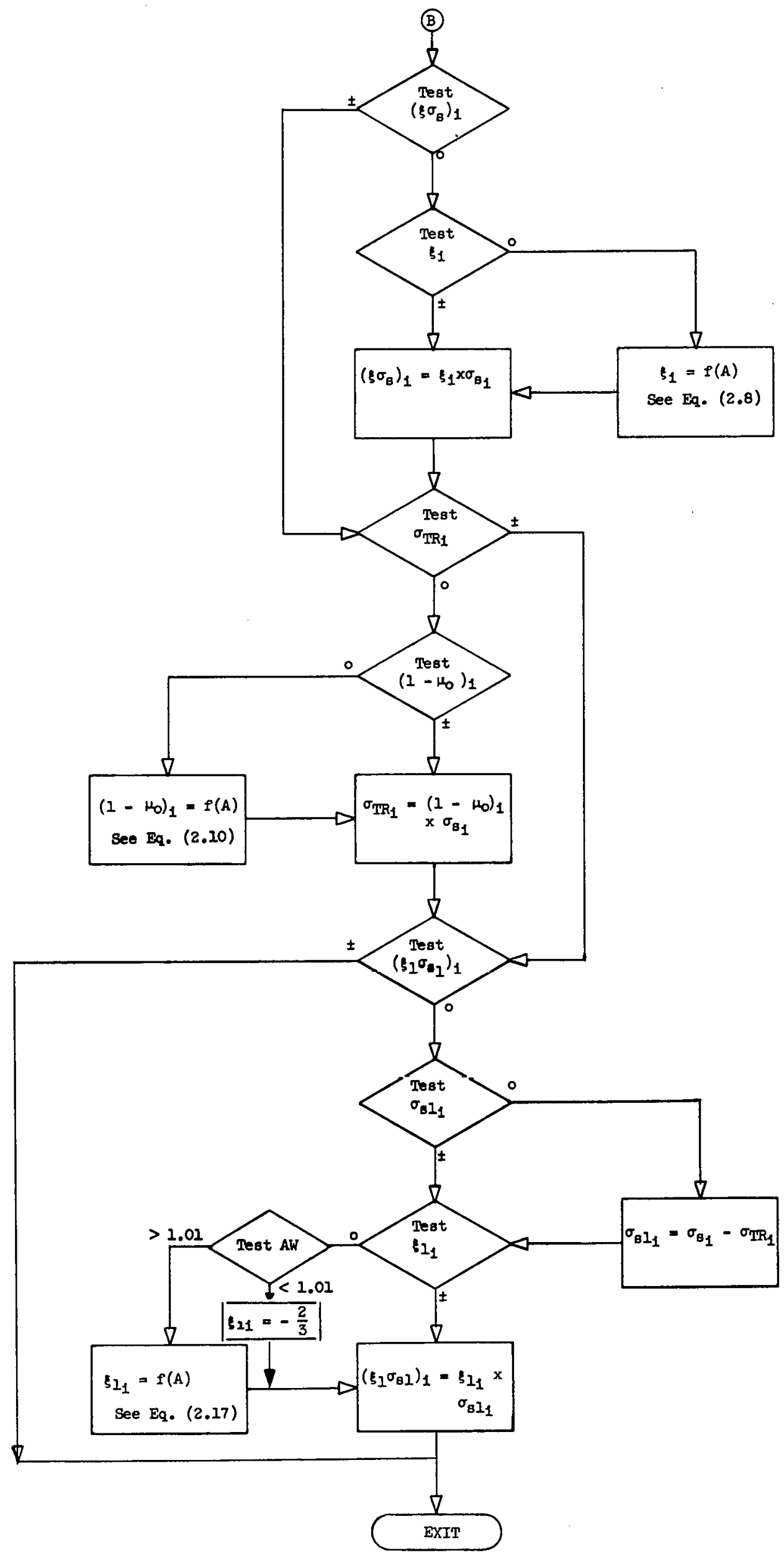

FIGURE 2.2

LOGIC FOR CALCULATION OF $\left(\xi \sigma_{8}\right)_{1}, \sigma_{\text {IR }_{1}},\left(\xi \sigma_{E_{1}}\right)_{1}$ [or $\left(\xi \Sigma_{\varepsilon}\right)_{1}, \Sigma_{\text {TP }},\left(\xi, \Sigma_{E]}\right)_{1}$ 


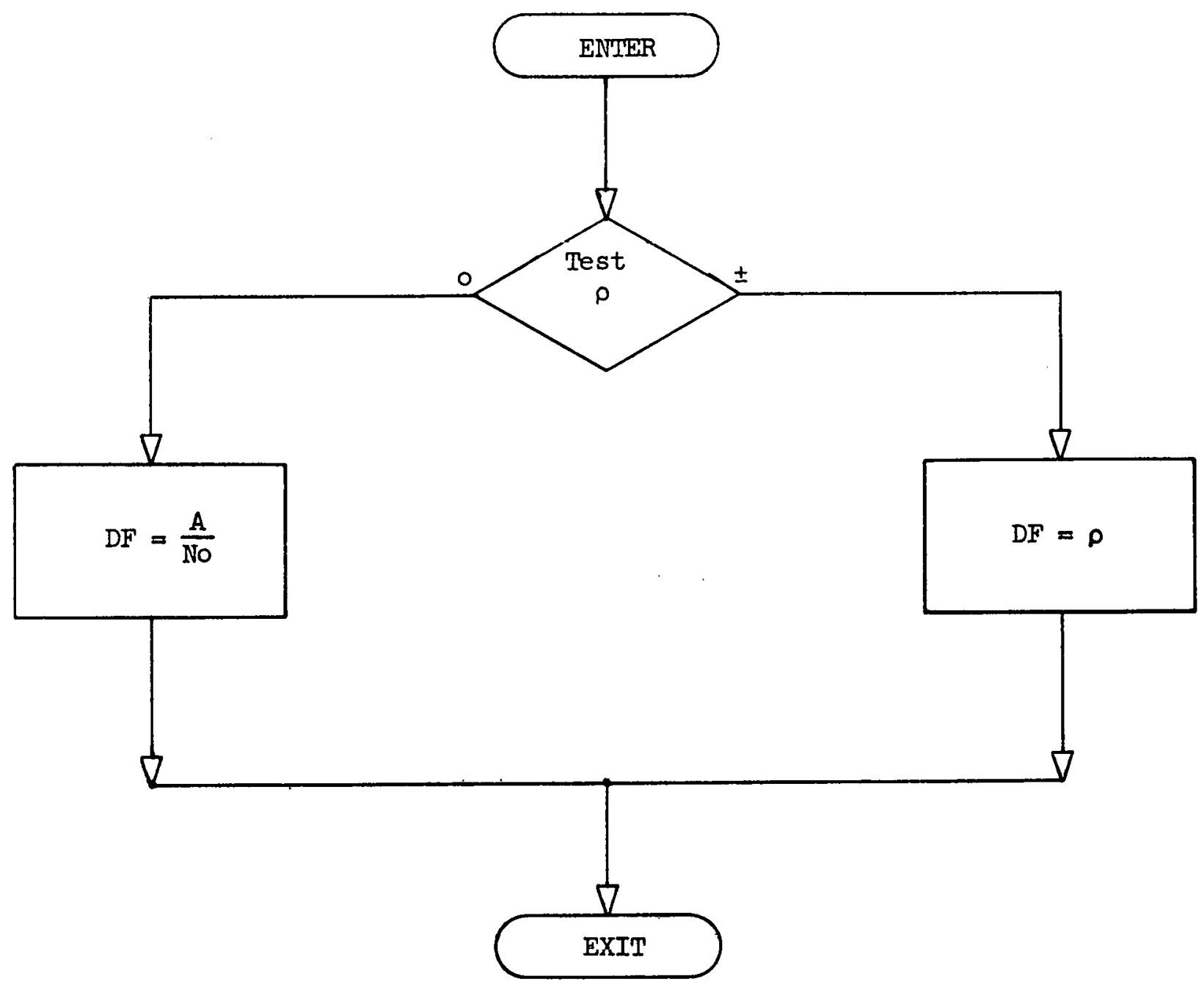

FIGURE 2.3

LOGIC FOR CALCULATION OF DENSITY FACTOR (DF) 


\subsection{Subroutine Descriptions}

\subsubsection{Control Routine}

This routine directs the overall flow of the program beginning with the initialization subroutine INIT. For a rerun case, control is passed to subroutine TRY $\phi V R$ to restore machine setup and status as it existed at the time of the DIP error interruption, followed by a transfer to the subsidiary control routine DATACT. In a normal case, control is passed to subroutine INDXNT to complle the index record for a new tape or to INDXRV to update this record from the old tape. This 1s followed by a transfer to NMIPRC to write this record on the new tapes, to WRINDX for printing output, to REC2 for compiling or copying the second record, and then to DATACT. From DATACT, control is to FINREC for compling or updating the final record.

\subsubsection{Subroutine ER\%R(N)}

This subroutine contains all the anticlpated error stops in program RNDP. The error type 18 indicated by the argument $N$ and is readily obtained from the program listing. A list of the absolute locations of these stops is given in a later section of the report.

ER $\phi R$ is used by the following subroutines: TAPECK, INIT, INDXRV, REC2, MMIPRC, KTAPRC, and DIPERR.

\section{4 .3 Subroutine TAPECK (IфC, NWфRD, NфTAPE)}

TAPECK is used for reading a single record of NW\$RD words from tape N $\varnothing \mathrm{TAPE}$ Into storage beginning at location I $\phi \mathrm{C}$. If the tape cannot be read, sense light 2 is turned on in subroutine (TSB), and the condition is diagnosed by the interrogation of this sense light upon return. FINREC.

TAPECK is used by INDXRV, REC2, DATACT, KTAPRC, TRNSDB, and

\section{4 .4 Subroutine INIT}

INIT has four functions: (1) It assigns values to those program parameters which, although they cannot be regarded as absolute constants, are expected to be changed too infrequently to warrant inclusion as input. Included are tape assignments, thermal levels, and control parameters related to dimension specifications. (2) It reads in the first record of data. (3) Table sizes are calculated on the basis of preassigned parameters and input data. Included in this category are the calculation of the number of words for the second record and the number of words in the first record of a material data block. (4) The input is checked to see if any dimensions are exceeded or if there is an inconsistency between the imput and the format of the existing tape.

INIT is reached only from the control routine. 


\section{4 .5 Subroutine INDXNT}

INDXNT is reached only from the control routine and then only if NEWTAP = 1. It complles the index record on the basis of the information given in the first record of card input.

\subsubsection{Subroutine INDXRV}

This subroutine revises the index record to reflect any deletions, additions, or relocations indicated in the first record of card input. It is instructive to consider an example at this point.

Suppose that the existing tape contalns the seven data blocks A, B, C, $D, E, F, G$ and the revised tape is to contain the nine data blocks $A$, H, G, I, D, J, F, K, B in the indicated order. The tape formats are then as follows:

Existing Tape

\begin{tabular}{|l|}
\hline Index Record \\
\hline Record 2 \\
\hline Data Block A \\
\hline Data Block B \\
\hline Data Block C \\
\hline Data Block D \\
\hline Data Block E \\
\hline Data Block F \\
\hline Data Block G \\
\hline Flnal Record \\
\hline
\end{tabular}

Revised Tape

\begin{tabular}{|l|}
\hline Index Record \\
\hline Record 2 \\
\hline Data Block A \\
\hline Data Block H \\
\hline Data Block G \\
\hline Data Block I \\
\hline Data Block D \\
\hline Data Block J \\
\hline Data Block F \\
\hline Data Block K \\
\hline Data Block B \\
\hline Flnal Record \\
\hline
\end{tabular}

The required revision may be effected as follows:

(1) Delete blocks $\mathrm{C}$ and $\mathrm{E}$ to give the data-block sequence $\mathrm{ABDFG}$.

(2) Add block $\mathrm{H}$ at position 2 to yield the sequence ABBDFG. Add block I at position 3 to yield the sequence AHIBDFG. Add block $J$ at position 6 to yleld the sequence AHIBDJFG. Add block $\mathrm{K}$ at position 8 to yleld the sequence AHIBDJFKG.

(3) Relocate block G to position 3 to give the sequence AHGIBDJFK. Relocate block B to position 9 to give the sequence AHGIDJFKB. 
The important point to remember is that the sequence of operations when revising the index record is always deletion, addition, and relocation. Additions are made on the basis of the index record structure after all deletions have been made; relocations are made on the basis of the structure after both deletions and additions have occurred.

The exact sequence of operation within INDXRV is as follows:

(1) The index record is read from the old tape into array $\mathrm{NBl}$.

(2) A check is made for the attempted deletion of a non-existent data block-also for the attempted deletion and relocation of the same data block. If either of these Inconsistencles is present, a transfer is made to ERdR.

(3) Blocks to be relocated, if any, are placed on the intermediate (K). tape in the same sequence in which they appear on the old tape. A list is concurrently compiled in array IRRDB of the physical-record number on this tape of the initial record of such data blocks. The corresponding code numbers are stored in array RLQCRC. These lists will be used in the subsidiary control routine DATACT for tape positioning.

(4) The index record, as it appeared on the old tape; 1s transferred to array NB2 if elther deletion or addition is to occur.

(5) Deletion is effected by simply falling to copy the Indexrecord Information for the deleted blocks into the new index record. The record numbers of subsequent data blocks are updated to reflect this change, the resultant index record being stored in array $\mathrm{NBl}$.

(6) If deletion has occurred, the index record is transferred from array NBI to NB2. Addition is effected by inserting the data-block information at the appropriate position with an updating of subsequent record numbers.

(7) Relocation occurs in four steps:

(a) For each data block, the record number is replaced by the actual number of records in that data block.

(b) The information for data blocks to be relocated is placed in the lower region of array NB6, and for data blocks which are not to be relocated into the upper region of NB6, the order in both cases being as they occurred on the index record after deletions and additions.

(c) The Index data for those blocks to be relocated are inserted at the appropriate position among those not to be relocated.

(d) The record numbers are calculated.

INDXRV is reached only from the control routine.

\subsubsection{Subroutine REC2}

If NEWTAP $=0$, the second record is simply copied from the existing tape. If NEWTAP $=1$, it is compiled from the second record of input data. In the former case, the energy levels and $1 / v$ factors are copied into blocks EL and FV for future use.

This subroutine is reached only from the control routine. 


\section{4 .8 Subroutine DATACT}

DATACT is a secondary-level control routine which directs the flow of data blocks from both the existing and intermediate tapes and card imput to the new tapes. A copy of the index record (in array NB6) is examined to ascertain the medium from which each data block can be obtained, that is, ITAPE, KTAPE, or card input. The following sequence of operations is applied to each data block represented in the Index record:

(1) If NEWTAP $=0$, the block is immediately read in as card imput; otherwise, the data block will be read in as card input if its code number is contained in the list of blocks added. This is done by means of subroutine INPTDB.

(2) If the data block is not added as input, the list of relocated blocks is scanned to see if it is on the intermediate tape. If so, this tape is positioned by means of the information in arrays NRRDB and RL $\phi C R C$ and the data block transferred to the new tape by means of TRNSDB.

(3) If the data block is neither one being added nor one being relocated, it must be present on the old tape. Succeeding date blocks are thus checked on this tape until the one in question is found. It is then transferred to the new tape by means of IRNSDB.

DATACT is reached only from the control routine.

\subsubsection{Subroutine INPTDB}

This subroutine processes each new data block, recelving control only from DATACT. Its functions are as follows:

(1) Check to see if this is a spectral or materlal data block. If it is a spectral block, the spectral data is read in and the record set up, written on the new tapes, and printed. Punching will be done if requested.

(2) For a material data block, the data is read in and processed according to the special flow charts (Flgs. 2.1, 2.2, 2.3). The remainder of the first record for the data block is complied from information in storage, using subroutine DIMEN to calculate table sizes. This record is then written on the new tapes and printed and is punched if desired.

(3) The SIGSO and SIGSI records, if present, are read in, written on the new tapes, printed, and punched if requested.

(4) The resonance record, if present, is read in, tables completed. and the record written on the new tapes, printed, and punched if requested.

(5) The SIGNG record is processed as were the SIGSO and SIGSI records.

\subsubsection{Subroutine NMIPRC (NW)}

This subroutine controls the writing of a record of length NW words on the new tepes, MTAPE and NTAPE. In case elther tape should fa1l, its mnemonic is set to zero and a comment to this effect printed. Thus, the mnemonics MEAPE and NTAPE are used both as tape designations 
and as code words. If only one tape falls, the other continues to be written; if both fall, control is transferred to ERdR after a printed comment.

NMIPRC is used by the control routine as well as the subroutines NMIPRC, INPIDB, TRNBDB, and FINREC.

\subsubsection{Subroutine KTAPRC (NW)}

KTAPRC tranefers a record of NW words from the existing tape to the intermediate tape using array $\mathrm{NBZ}$ for temporary storage. It is used only in the subroutine INDXRV.

\section{4 .12 Subroutine DIMEN (IDC)}

This routine calculates the number of words in the SIGSO, SIGSI, resonance, and SIGNG records, denoting these by $\mathbb{N}, N 2, N 3, N 4$. The Indices are stored sequentially in array MATDIM. IdC is the location of these Indices when calling the routine.

DIMEN 18 used by INDXRV, DATACT, INPIDB, and TRNSDB.

\section{4 .13 Subroutine TRNSDB (ITAPI)}

TRNSDB 18 used to transfer a complete data block from ITAPI (either ITAPE or KTAPE) to the new tapes and w1ll initlate the printing and/or punching of the data block if requested. Thls routine is used only by the routine DATACT.

\subsubsection{Subroutine WRINDX}

WRINDX is used to print and punch the Index record and transfer the index record from array NBI to NB6 for future reference. It 18 entered only from the control routine.

\subsubsection{Subroutine WRSECR}

WRSECR prints the second record if the code number 0.000002 is listed in the PC $\phi D E$ table and punches this record if the PUNC $\phi D$ table contains the same number. WRSECR is reached only through subroutine REC2.

\subsubsection{Subroutine WRSPDB (NG)}

This subroutine is used to print and punch a spectral data block. When WRSPDB 18 entered from INPIDB, the argument NG 18 set to 1 so that printing is automatic. If NG $=0$, as when entered from TRNSDB, a check is made for the presence of the code number in the PCDDE Iist. In e1ther case, a check is made of the PUNC $\phi D$ list for punching. No other subroutines use WRSPDB.

\subsubsection{Subrout1ne WRRECl (NG, PTCфDE, PNCфDE)}

WRRECl is used to print the first record of a material data block. NG is used in exactly the same fashion as in WRSPDB. PTC $\phi D E$ is 
set to 1 if this date block is printed and to 0 if not--simliarly for PNCQDE with regard to punching. These two code words will be used in WRREC2, WRREC3, WRREC4, WRREC5 (denoted by II3 and II4) to avold another check for printing and punching.

This routine is used in INPTDB and TRNSDB.

\subsubsection{Subroutine WRREC2 (II3, II4)}

WRREC2 prints the SIGSO record of a material data block if II3 = 1, using subroutine RC235. Th1s record 18 punched if II4 $=1$. WRREC2 1s called by INPTDB and TRNSDB.

\subsubsection{Subrout1ne WRREC3 (II3, II4)}

WRREC3 prints the SIGSI record of a materlal data block if II3 = 1, using subroutine RC235. Th1s record is punched if II4 $=1$. RREC3 is called by INPTDB and TRNSDB.

\subsubsection{Subrout1ne WRREC4 (II3, II4)}

WRREC4 prints the resonance record of a material data block if II3 $=1$ and punches if II4 $=1$. Th1s subroutine is called by INPTDB and TRNSDB.

\subsubsection{Subroutine WRREC5 (II3,II4)}

WRREC5 prints the SIGNG record of a material data block if II3 = 1 , using subroutine RC235. Th1s record is punched if II4 $=1$. WRREC5 is called by INPTDB and TRNSDB.

\subsubsection{Subroutine RC235 (Mn, M2, NW, NG)}

RC235 is used by subroutines WRREC2, WRREC3, and WRREC5 to set up the output format and perform the printing. The formats are 1dentical for each of the three routines, only the page beadings being different. The arguments have the following meanings, indicated in order, for these three routines: (1) $\mathrm{MI}=\mathrm{MO}_{\mathrm{MIN}}, \mathrm{MI}_{\mathrm{MIN}}, \mathrm{M} \Gamma_{\mathrm{MIN}}$, (2) $\mathrm{N2}=\mathrm{MO}_{\mathrm{MAX}}$, $\mathrm{M}_{\mathrm{MAX}}, \mathrm{M}_{\mathrm{MAX}},(3) \mathrm{NW}=\mathrm{NI}, \mathrm{N2}, \mathrm{N4},(1) \mathrm{NG}=0$, 1, 2. The quant1 tes $N I$, N2, and N4 are the number of words in the SIGSO, SIGSI, and SIGNG records as calculated in DIMEN.

\subsubsection{Subrout1ne FINREC}

FINREC complles the final record, assigning a record number and Inftializing the remainder of the record to zero in the case of a new tape. For a revised tape, this subroutine reads in the final record from the old tape, corrects the record number, and updates the record information to reflect any deletions or relocations of macroscopic blocks. If there are no macroscopic data blocks on the revised tape, a comment is printed to that effect; otherwise, for each macroscopic block, the constituents are listed together with their welght fractions. Also, if the constituent of any macroscopic block has been deleted, a comment to this effect is printed. This record is then punched if 
requested, an end of flle written, all tapes rewound, and a return 18 executed to the control routine.

It should be noted that if a macroscoplc constituent is not present on the revised tape, the designation code is not avaliable.

\subsubsection{Subrout1ne DIPERR}

If a DIP-type error is encountered in a given record of datablock imput, a provision has been made to permit continuing the compllation of the new data tepes in a subsequent restart case. This is accomplyshed by means of DIPHRR which punches on binary cards the necessary restart information and prints on-line the comment to save all tapes involved in the present run. Tape compllation can then be continued in a subsequent run by remounting the same tapes. The punched cards labeled "remun", replace all the declmal-card data preceding the input for the data block in wh1ch the DIP error occurred. ERQR.

This routine is entered only from INPIDB, and Its only exit is to

\subsubsection{Subroutine TRYQVR}

This subroutine 18 a companion to DIPERR in the sense that, while DIPERR is used to preserve all necessary data for rerunning a case in wh1ch compilation was interrupted by a DIP error within a data block, TRY $\varnothing$ VR is used to read in thesedata and reposition all tapes to their setting when intermuted. Subroutine TRY $\oint$ VR is entered from the control routine.

\subsection{Program Flow Charts}

The program flow charts are Flgs. 2.4, 2.5, and 2.6. They are shown on pages 32,33 , and 34 .

\subsection{Program Diagnostics}

External Formula Numbert

Error

2022

DIP error in first record of laput data

2023

DIP error in data for record 2

2024

DIP error in data for spectral-data block

2025

DIP error in data for record 1 of a materialdata block

2026

DIP error in $\sigma_{B O}$ record of a material-data block

2027

DIP error in $\sigma_{B 1}$ record of a materlal-data block

\footnotetext{
F
}

When an error occurs, a printed corment giving the external (source program) formula number of the statement at which the library subroutine ERRod was entered. 


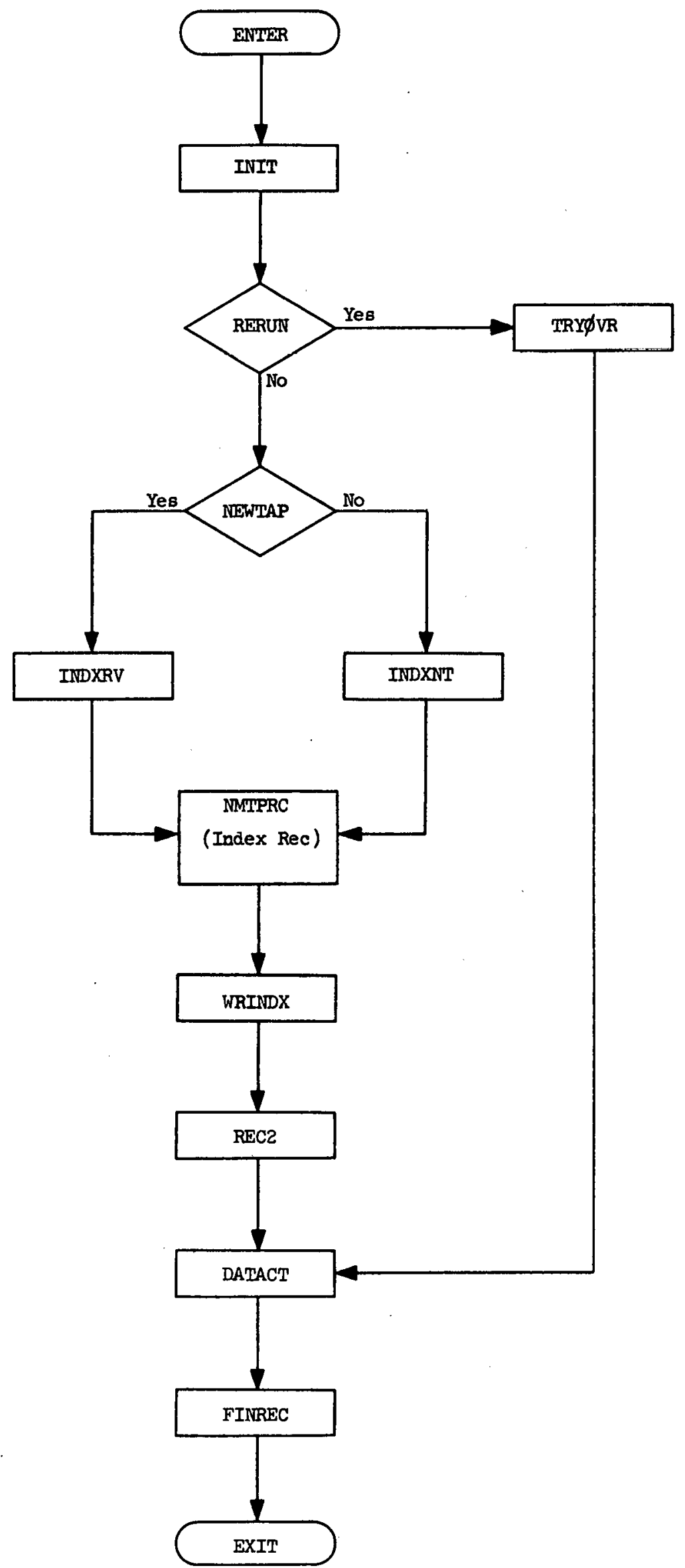

FTG. 24 - FLOW CHART OT CONTROL ROUTINE 


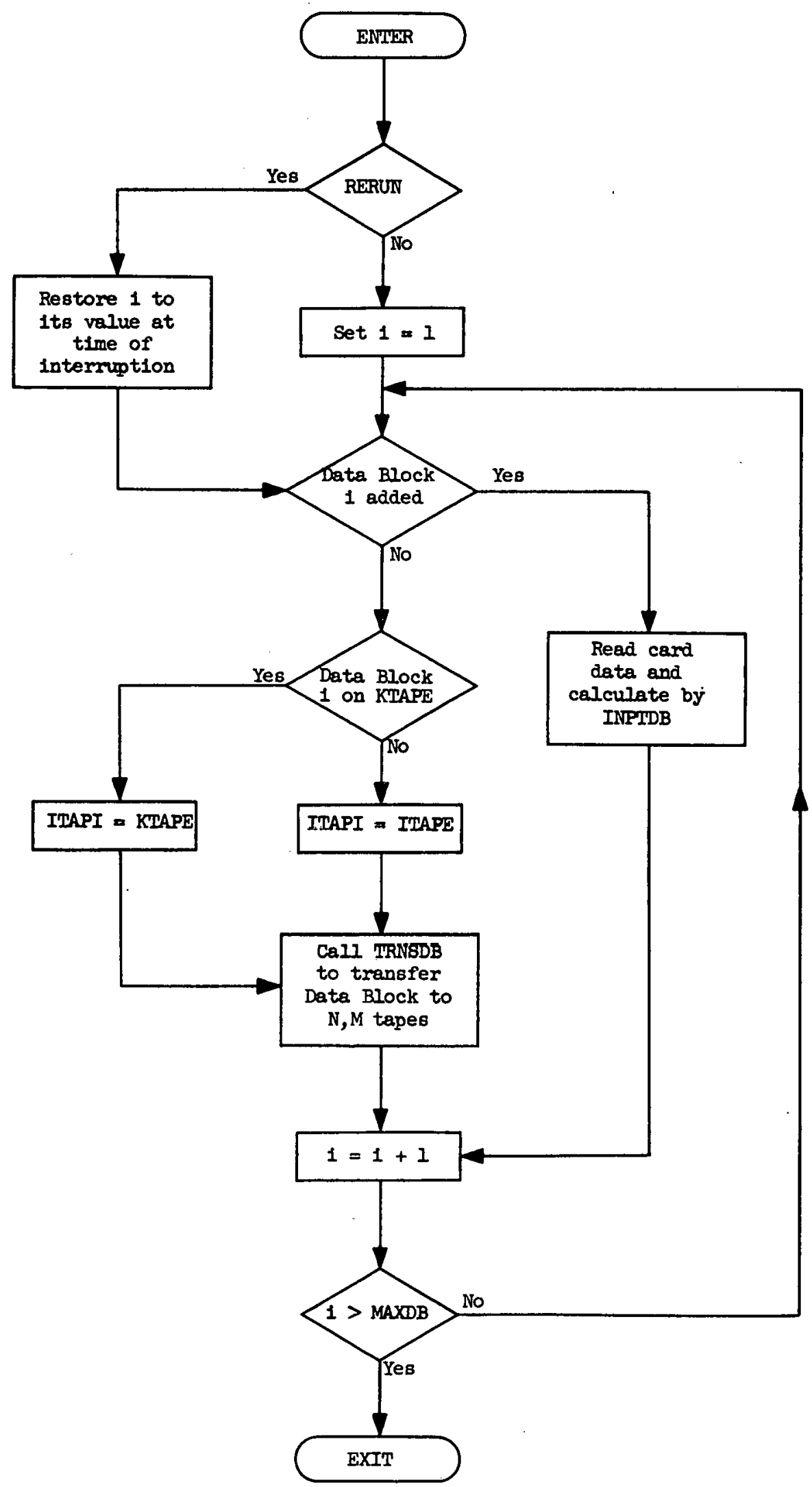

FIG. 2.5 - FLOW CHART OF DATACT CONTROL ROUTINE 


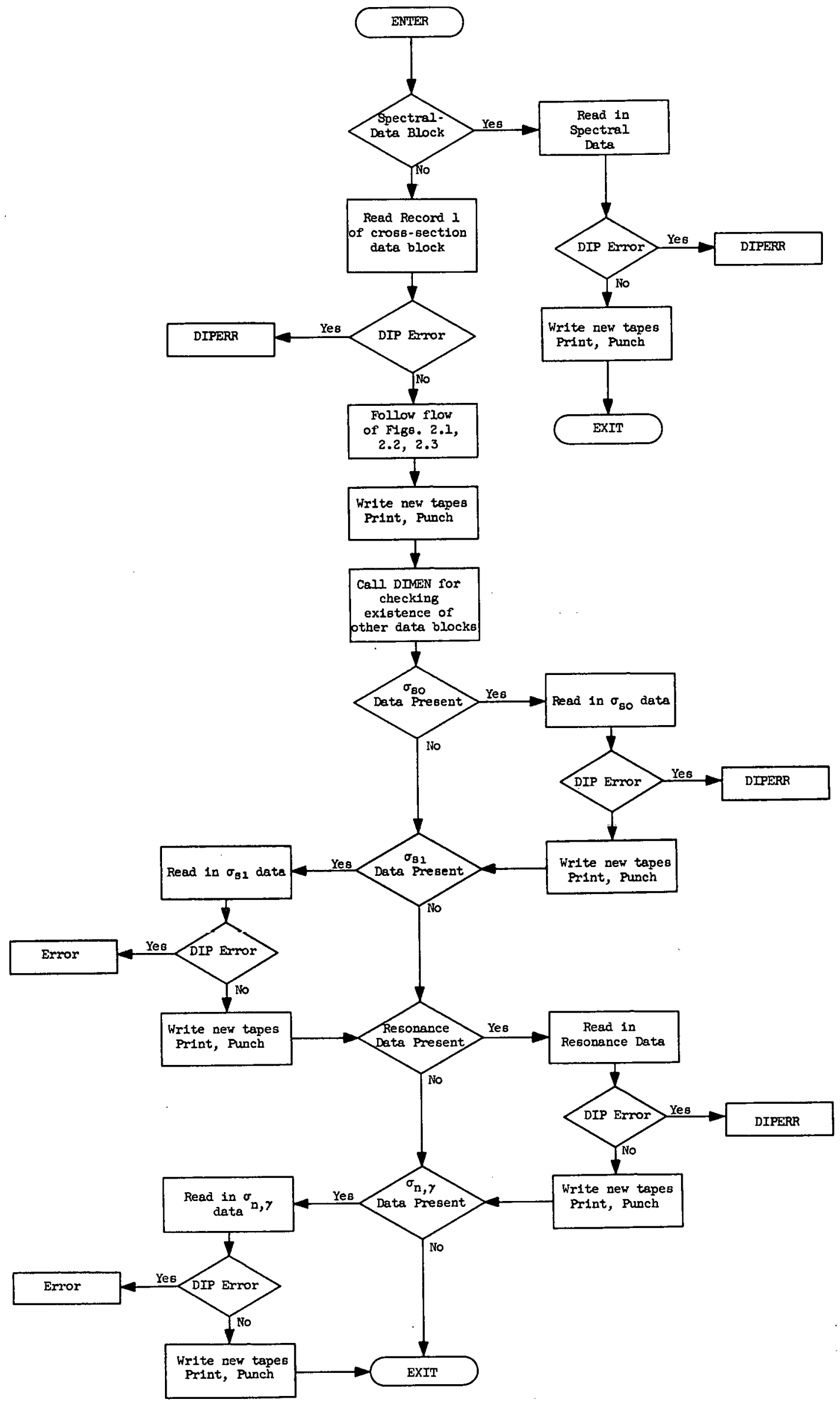

FIG. 2.6 - FLOW CHART OF INPTDB SUBROUTINE 
External Formula Number

$\begin{array}{ll}2028 & \begin{array}{l}\text { DIP error in resonance record of a material } \\ \text { data block }\end{array} \\ 2029 & \text { DIP error in } \sigma_{n, \gamma} \text { record of a material data } \\ \text { block } & \text { ITAPE could not be read } \\ 2030 & \text { KTAPE could not be read } \\ 2031 & \text { Both MTAPE and NTAPE failed to complle } \\ 2032 & \text { KTAPE failed to complle } \\ 2033 & \text { Dimensions in NOMIN table are too large } \\ 2034 & \text { Too many levels } \\ 2035 & \text { Attempt made to delete non-existent data block } \\ 2036 & \text { Attempt made to relocate deleted data block } \\ 2037 & \text { Attempt made to relocate non-existent data } \\ 2038 & \text { block }\end{array}$

\subsection{Program Input Forms}

This section contains the program input forms. They are shown on the following peges. 
REACTOR NUCLEAR DATA PROGRAM

Index Data

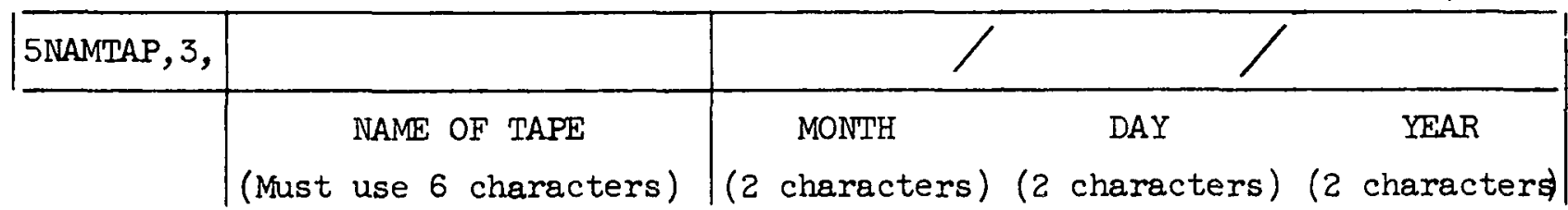

\begin{tabular}{|l|l|}
\hline $4 N L E V$, & \\
\hline $\begin{array}{l}\text { Number of } \\
\text { Epithermal } \\
\text { Levels }\end{array}$ \\
\hline
\end{tabular}

\begin{tabular}{|c|c|c|c|c|c|}
\hline 4NEWTAP, & , & NBA, & , & NBD, & , \\
\hline & $\begin{array}{l}0 \rightarrow \text { Revising old tape } \\
1 \rightarrow \text { Making new tape }\end{array}$ & & $\begin{array}{c}\text { Number Data Blocks } \\
\text { to be added }\end{array}$ & & $\begin{array}{c}\text { Number Data Blocks } \\
\text { to be deleted }\end{array}$ \\
\hline
\end{tabular}

\begin{tabular}{|l|r|r|r|r|r|}
\hline 3NBDEL, &, &, &, &, \\
\hline & Code numbers of Data Blocks to be deleted. \\
(Enter only if $\mathrm{NBD}>0)$ &
\end{tabular}

\begin{tabular}{|c|c|}
\hline 4NPSN, \\
\hline \\
$\begin{array}{c}\text { Number Blocks } \\
\text { to be } \\
\text { Relocated }\end{array}$ \\
\hline
\end{tabular}


Use This Page Orly If NBA $>0$ or NPSN $>0$

\section{Index Data}

(Enter blocks to be added followed by blocks to be relocated)

\begin{tabular}{|c|c|} 
& $\begin{array}{c}\text { Data Block Designation } \\
\text { (12 characters) }\end{array}$ \\
\hline 5BNAM, 2, & \\
\hline$N_{1}^{*}$ & \\
\hline E28406,2, & \\
\hline$N_{2}$ & \\
\hline E28402,2, & \\
\hline$N_{3}$ & \\
\hline E28398,2, & \\
\hline$N_{4}$ & \\
\hline$E 28394,2$, & \\
\hline$N_{5}$ & \\
\hline$E 28390,2$, & \\
\hline$N_{6}$ & \\
\hline$E 28386,2$ & \\
\hline$N_{7}$ & \\
\hline$E 28382,2$, & \\
\hline
\end{tabular}

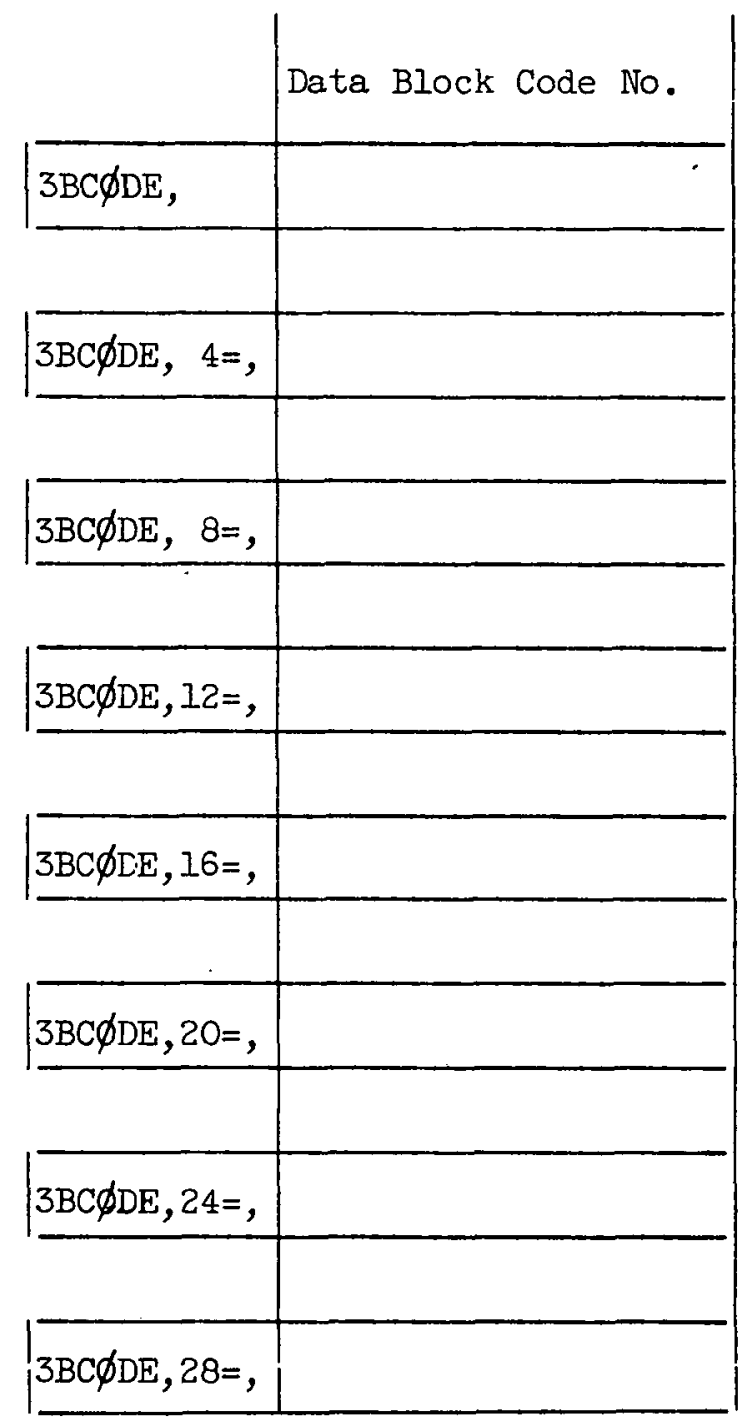

\begin{tabular}{|l|l|} 
& Data Block Position \\
\hline 4NBPSN, & \\
\hline & \\
\hline 4NBPSN, $4=$, & \\
\hline & \\
\hline 4NBPSN, $8=$, & \\
\hline & \\
\hline $4 \mathrm{NBPSN}, \quad 12=$, & \\
\hline & \\
\hline $4 \mathrm{NBPSN}, \quad 16=$, & \\
\hline & \\
\hline $4 \mathrm{NBPSN}, \quad 20=$, & \\
\hline & \\
\hline $4 \mathrm{NBPSN}, \quad 24=$, & \\
\hline
\end{tabular}


Us $\equiv$ This Page only If NBA $>0$

Index Data

\begin{tabular}{|c|c|c|c|c|c|c|c|c|c|c|}
\hline & $\mid \begin{array}{c}\text { Minimum } \\
M \text { in } \\
\sigma_{\text {se }}\left(M / M^{*}\right)\end{array}$ & $\mid \begin{array}{c}\text { Maximum } \\
M \text { in } \\
\sigma_{\mathrm{so}}\left(\mathrm{M} / \mathrm{M}^{\prime}\right) \\
\end{array}$ & $\mid \begin{array}{r}\text { Maximun } \\
\mathrm{M}^{1} \text { in } \\
\sigma_{\mathrm{s}}\left(\mathrm{M} / \mathrm{M}^{\prime}\right)\end{array}$ & $\begin{array}{c}\text { Minimum } \\
M \text { in } \\
\sigma_{S I}\left(M^{\prime} / M^{1}\right)\end{array}$ & $\mid \begin{array}{c}\text { Maximum } \\
\mathrm{M} \mathrm{in} \\
\sigma_{5]}\left(\mathrm{M} / \mathrm{M}^{\circ}\right)\end{array}$ & $\mid \begin{array}{c}\operatorname{Maximum} \\
\mathrm{M}^{+} \text {in } \\
\left.\sigma_{s} \mathrm{M}^{\prime} / \mathrm{M}^{\mathrm{I}}\right)\end{array}$ & $\begin{array}{r}\text { Number of } \\
\text { Resonances }\end{array}$ & $\begin{array}{c}\underset{M i n i m u m}{M} \\
\mathrm{M} \text { in } \\
\sigma_{n, \gamma\left(\mathrm{M} / \mathrm{M}^{\prime}\right)}\end{array}$ & $\mid \begin{array}{c}\text { Maximum } \\
M \text { in } \\
\sigma_{n}, \gamma\left(M / M^{\prime}\right)\end{array}$ & $\mid \begin{array}{c}\text { Maximum } \\
M^{\prime} \text { in } \\
\sigma_{n, \gamma\left(M / M^{\prime}\right)}\end{array}$ \\
\hline 4MOMIN, & , & , & , & , & , & , & , & , & & , \\
\hline 1 & | & 1 & 1 & | & I & I & 1 & 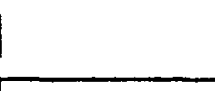 & 1 & 1 \\
\hline 4 MOMIN, $10=$ & , & , & , & , & , & , & , & & 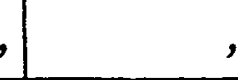 & 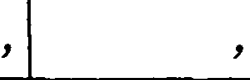 \\
\hline 1 & | & 1 & 1 & 1 & I & 1 & 1 & & 1 & 1 \\
\hline $4 \mathrm{MOMIN}, 20=$ & , & , & , & , & 1 & , &. & & , & 1 \\
\hline 1 & | & 1 & 1 & 1 & $!$ & 1 & 1 & I & 1 & 1 \\
\hline $4 \mathrm{MOMIN}, 30=$ & ' & 1 & $\dot{\prime}$ & , & , & , & , & & 1 & , \\
\hline 1 & 1 & I & I & 1 & 1 & | & I & 1 & 1 & 1 \\
\hline $4 \mathrm{MOMIN}, 40=$ & , & I & , & , & 1 & , & , & & 1 & , \\
\hline 1 & | & I & I & 1 & 1 & 1 & 1 & 1 & 1 & 1 \\
\hline 4 MOMIN, $50=$ & , & $\perp$ & 1 & , & $\perp$ & , &, & & 1 & 1 \\
\hline & I & I & I & 1 & 1 & 1 & 1 & 1 & 1 & 1 \\
\hline 4 MOMIN, $60=$ & , & $\perp$ & 1 & , & $\perp$ & 1 & , & &, 1 & 1 \\
\hline 1 & 1 & 1 & 1 & 1 & 1 & 1 & 1 & 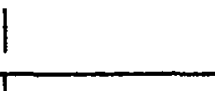 & 1 & 1 \\
\hline 4 MOMIN, $70=$ & , & 1 & , & , & 1 & , & , & & , & , \\
\hline
\end{tabular}


RNDP

\begin{tabular}{|c|c|c|c|}
\hline 4NסPRNT, & , & NфPUN, & , \\
\hline & $\begin{array}{l}\text { Number of blocks to be } \\
\text { printed out. }\end{array}$ & & $\begin{array}{l}\text { Number of blocks to be } \\
\text { punched out. }\end{array}$ \\
\hline
\end{tabular}

NOTE: The program will always print and punch the first record and print the final record.

Code Numbers of Blocks To Be Printed

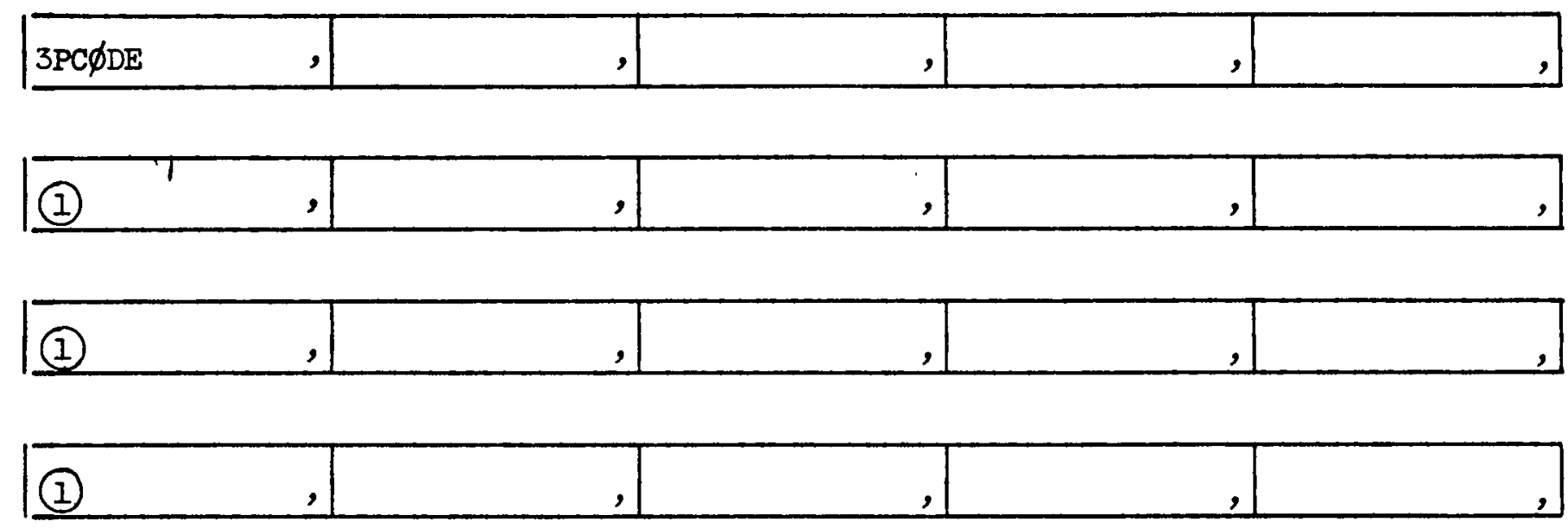

Code Numbers of Blocks To Be Punched

$$
\left[\begin{array}{rl}
2 \times 10^{-6} & =\text { Record } 2 \\
0 & =\text { Final Record }
\end{array}\right]
$$

\begin{tabular}{|l|l|l|l|l|}
\hline 3PUNC $\phi \mathrm{D}$ &, &, &, & \\
\hline
\end{tabular}

\begin{tabular}{|l|l|r|r|r|}
\hline (1) &, &, & & \\
\hline
\end{tabular}
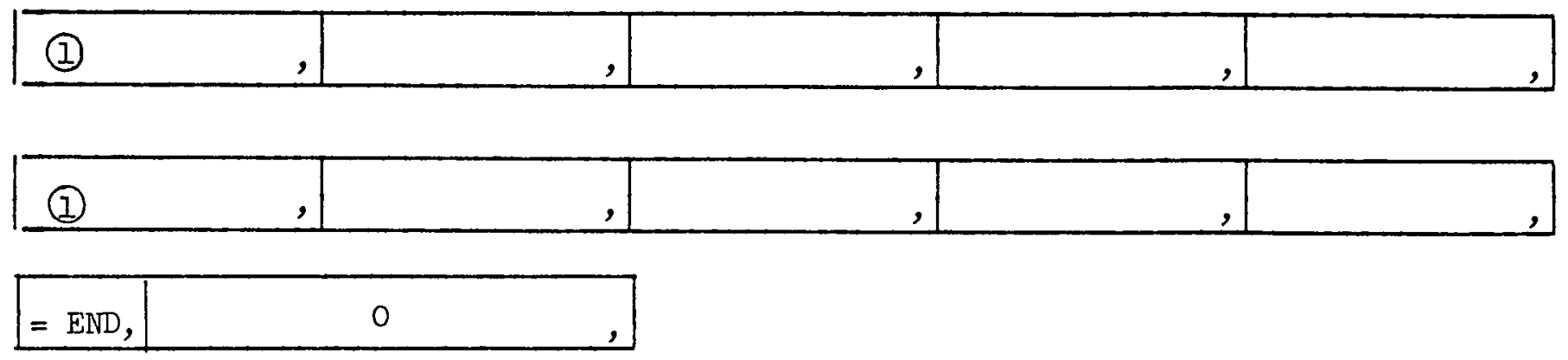

This Must Be Last Card of Index Data 
Use This Page Only If NEWTAP $=1$

Record 2 Input

Level Energies

\begin{tabular}{|c|c|c|c|c|c|c|}
\hline & & Level 1 & 2 & 3 & 4 & 5 \\
\hline 3EL &, & , & , & , & , & \\
\hline & & 6 & 7 & 8 & 9 & 10 \\
\hline $3 \mathrm{EL}, 5=$ & , & , & , & , & , & \\
\hline & & 11 & 12 & 13 & 14 & 15 \\
\hline $3 E L, 10=$ & , & , & , & , & , & \\
\hline & & 16 & 17 & 28 & 19 & 20 \\
\hline $3 E L, \quad 15=$ & , & , & , & , & , & \\
\hline & & 21 & 22 & 23 & 24 & 25 \\
\hline $3 E L, 20=$ & , & , & , & , & , & \\
\hline & & 26 & 27 & 28 & 29 & 30 \\
\hline $3 E L, 25=$ & , & , & , & , & , & \\
\hline & & 31 & 32 & 33 & 34 & \\
\hline $3 E L, \quad 30=$ & , & , & , & , & , & \\
\hline
\end{tabular}


Use This Page Only If NEWTAP = 1

Record 2 Input

\section{Level Lethargies}

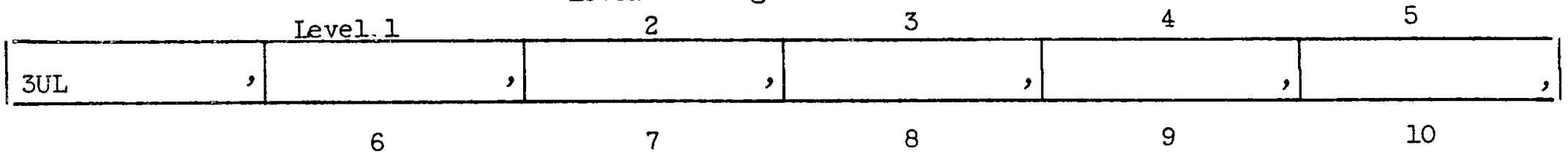

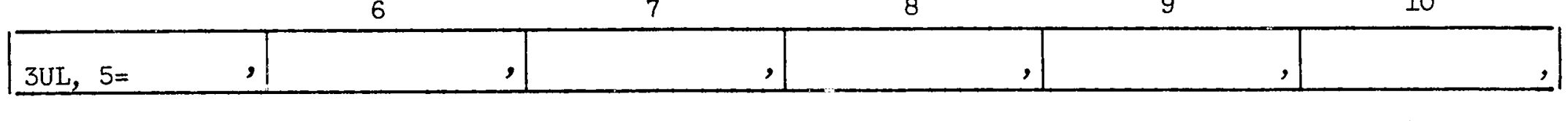

\begin{tabular}{|l|l|l|l|l|l|}
\hline 11 & 12 & 13 & 14 \\
\hline $3 U L, 10=$ & & & 15 & \\
\hline
\end{tabular}

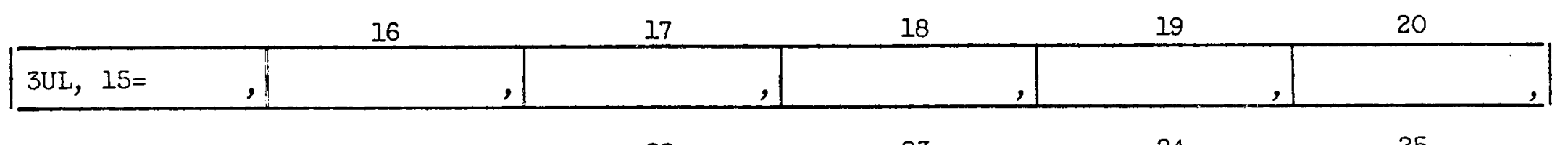

\begin{tabular}{|c|c|c|c|c|c|c|}
\hline & & 21 & 22 & 23 & 24 & 25 \\
\hline 3UL, $20=$ & , & , & , & , & , & ， \\
\hline & & 26 & 27 & 28 & 29 & 30 \\
\hline
\end{tabular}

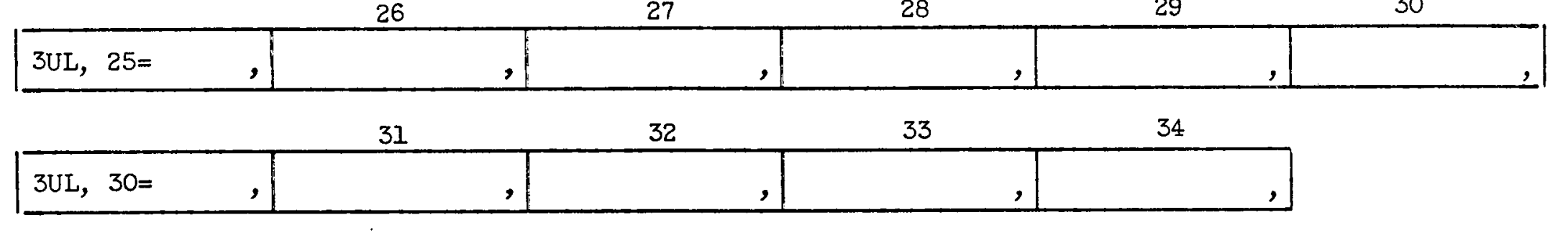


Use This Page Only If NEWTAP $=1$

Record 2 Input

Froup Boundary Energies

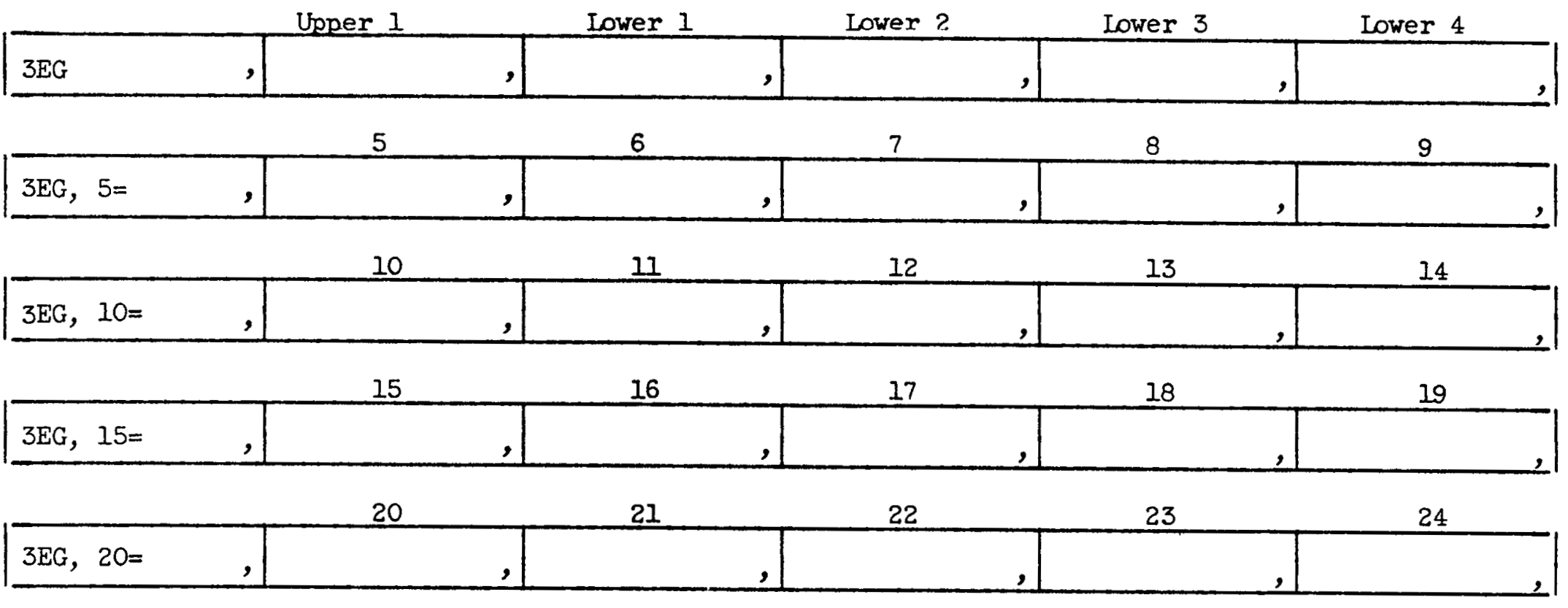

Group Boundary Lethargles

\begin{tabular}{|l|l|l|l|l|l|l|l|}
\hline 3UG & Lower 1 & Upper 1 & Upper 2 & Upper 3 & Upper 4 \\
\hline 3UG, 5= & & & & & \\
\hline
\end{tabular}


Use This Page Only If NEWTAP = I

Record 2 Input

Group 1/v Factors

(= 1.0 at $2200 \mathrm{~m} / \mathrm{sec}$ )

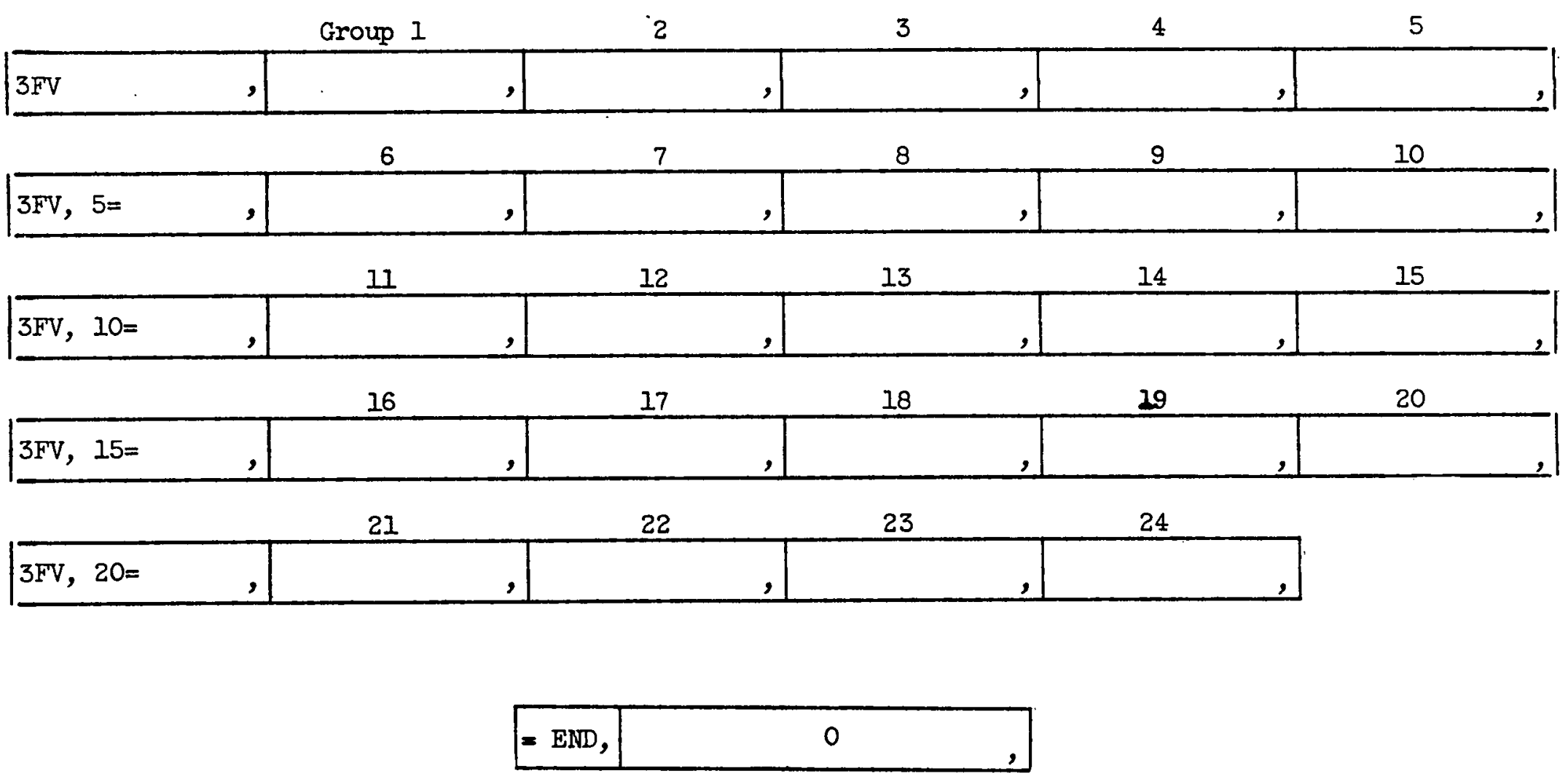


Cross-Section Data-Block Imput

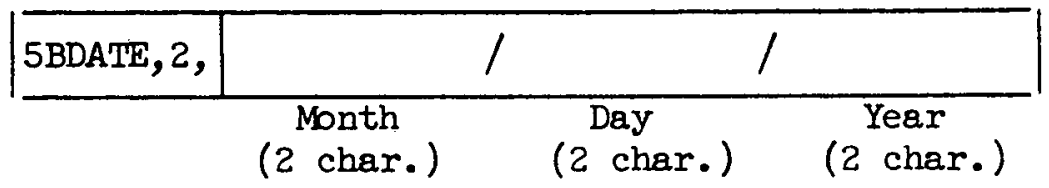

Atomic Weight

\begin{tabular}{|l|l|}
\hline 3AW, & , \\
\hline
\end{tabular}

Density $\left(\mathrm{gm} / \mathrm{cm}^{3}\right)$

\begin{tabular}{|l|l|}
\hline 3DEN, \\
\hline
\end{tabular}

$\sigma_{\mathrm{A}}(2200 \mathrm{~m} / \mathrm{sec})$

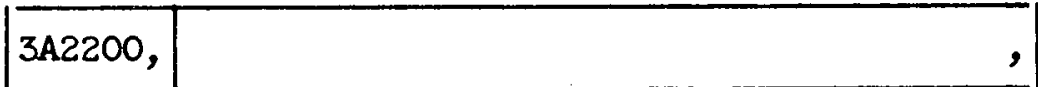

\begin{tabular}{|l|l|}
\hline 4NDATA, & \\
\hline$=1 \rightarrow$ Use data entered only & data
\end{tabular} 


\section{Cross Section Input}

Coefficients For Calculating $v$

$$
v_{i}=A I+B I E L_{i}+C L E L_{i}^{D 1}
$$

\begin{tabular}{|l|l|l|l|l|l|l|}
\hline $3 \mathrm{Al}$, &, $\mathrm{Bl}$, &, & $\mathrm{Cl}$, &, & $\mathrm{Dl}$, & \\
\hline
\end{tabular}

Coefficients For Calculating $\eta$

$\eta=A 2+\left(B 2+C 2 E L_{i}^{D 2}\right) \operatorname{lnEL} L_{i}$

\begin{tabular}{|l|l|l|l|l|l|l|}
\hline $3 \mathrm{~A} 2$, &, $\mathrm{B} 2$, &, $\mathrm{C} 2$, &, $\mathrm{D} 2$, &, \\
\hline
\end{tabular}




\section{Cross Section Input}

$$
\sigma_{s_{1}}
$$

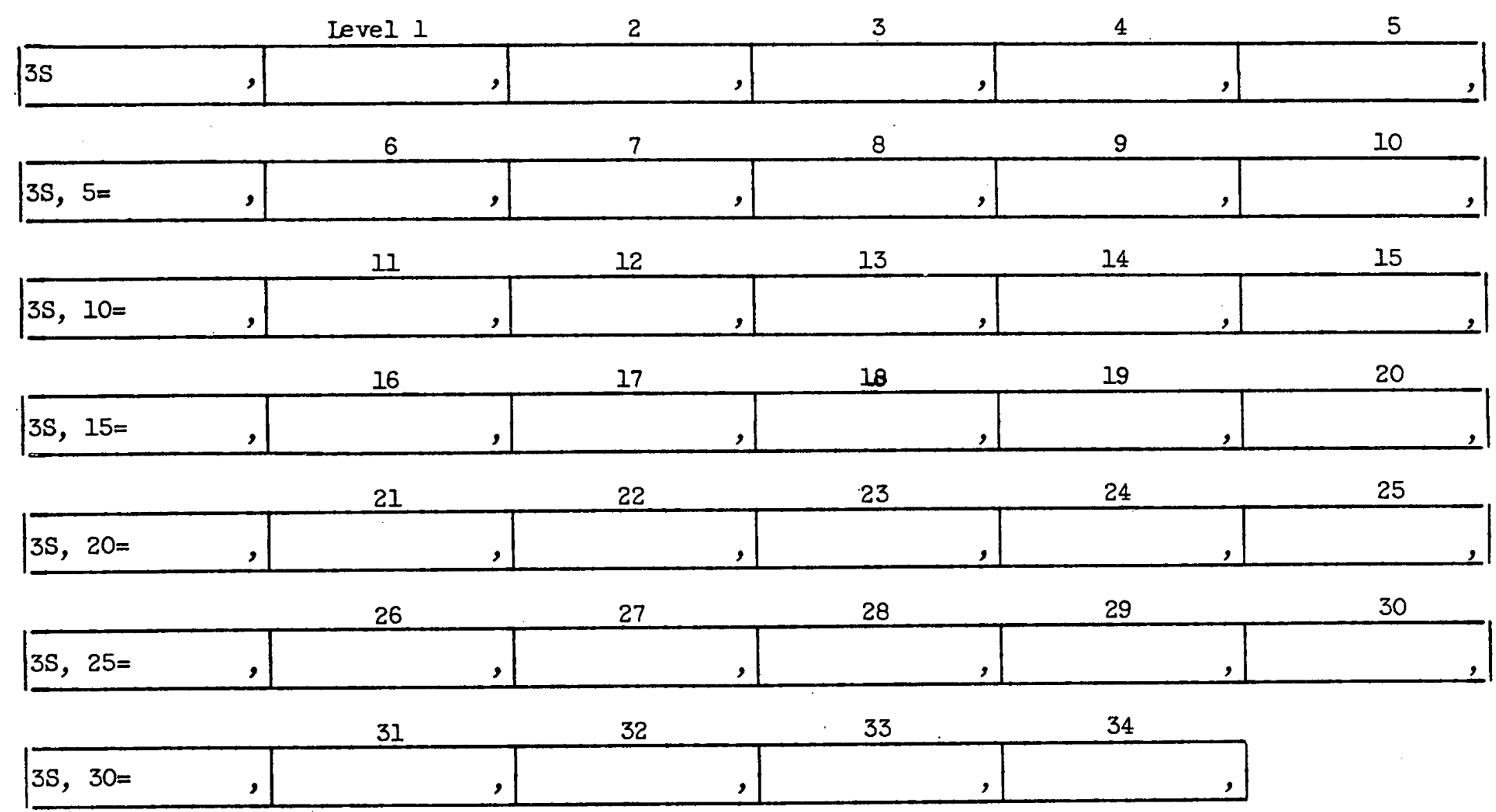


Cross Section Input

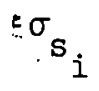

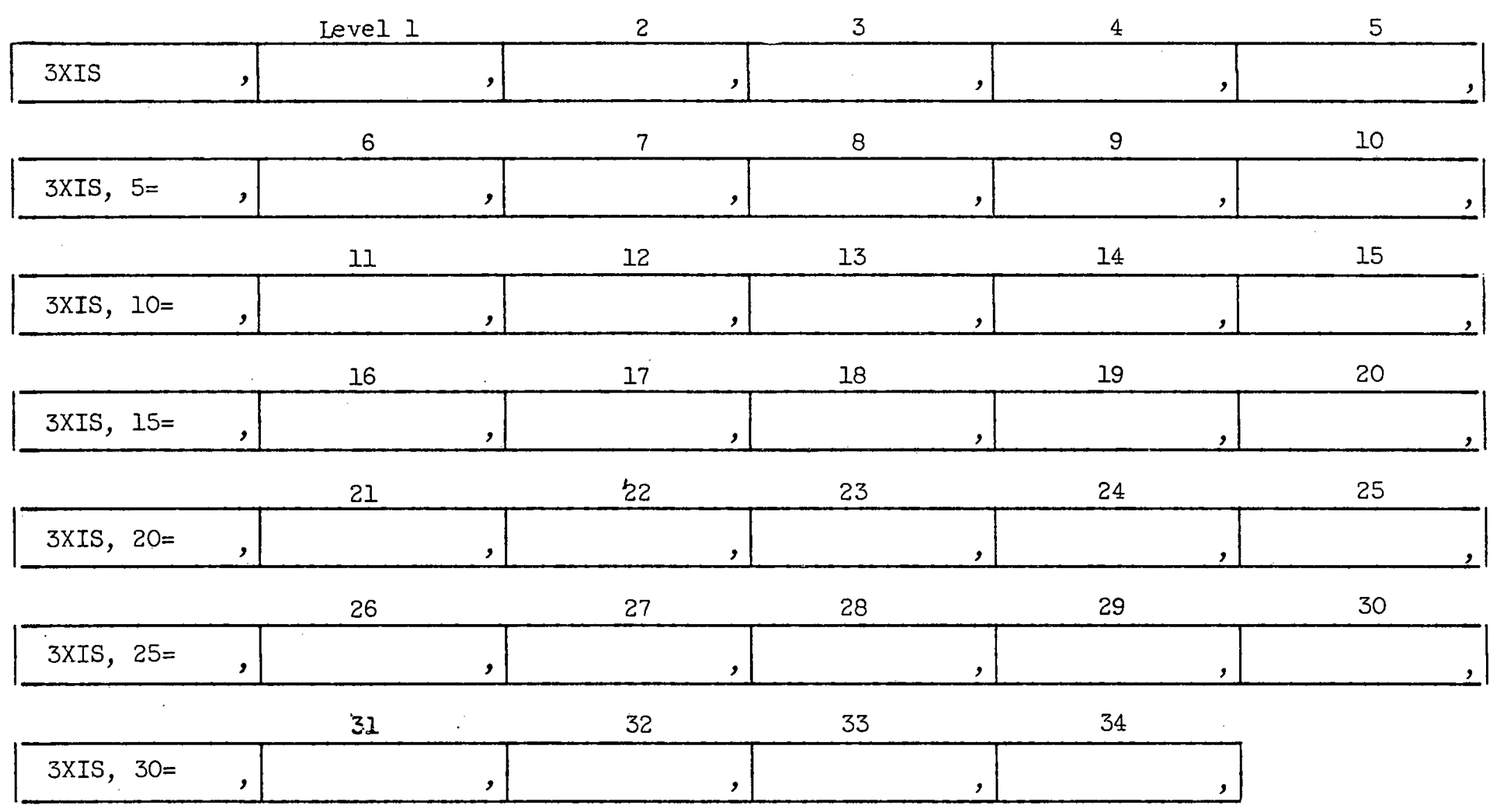


Cross Section Input

$$
\sigma_{\mathrm{TR}_{1}}
$$

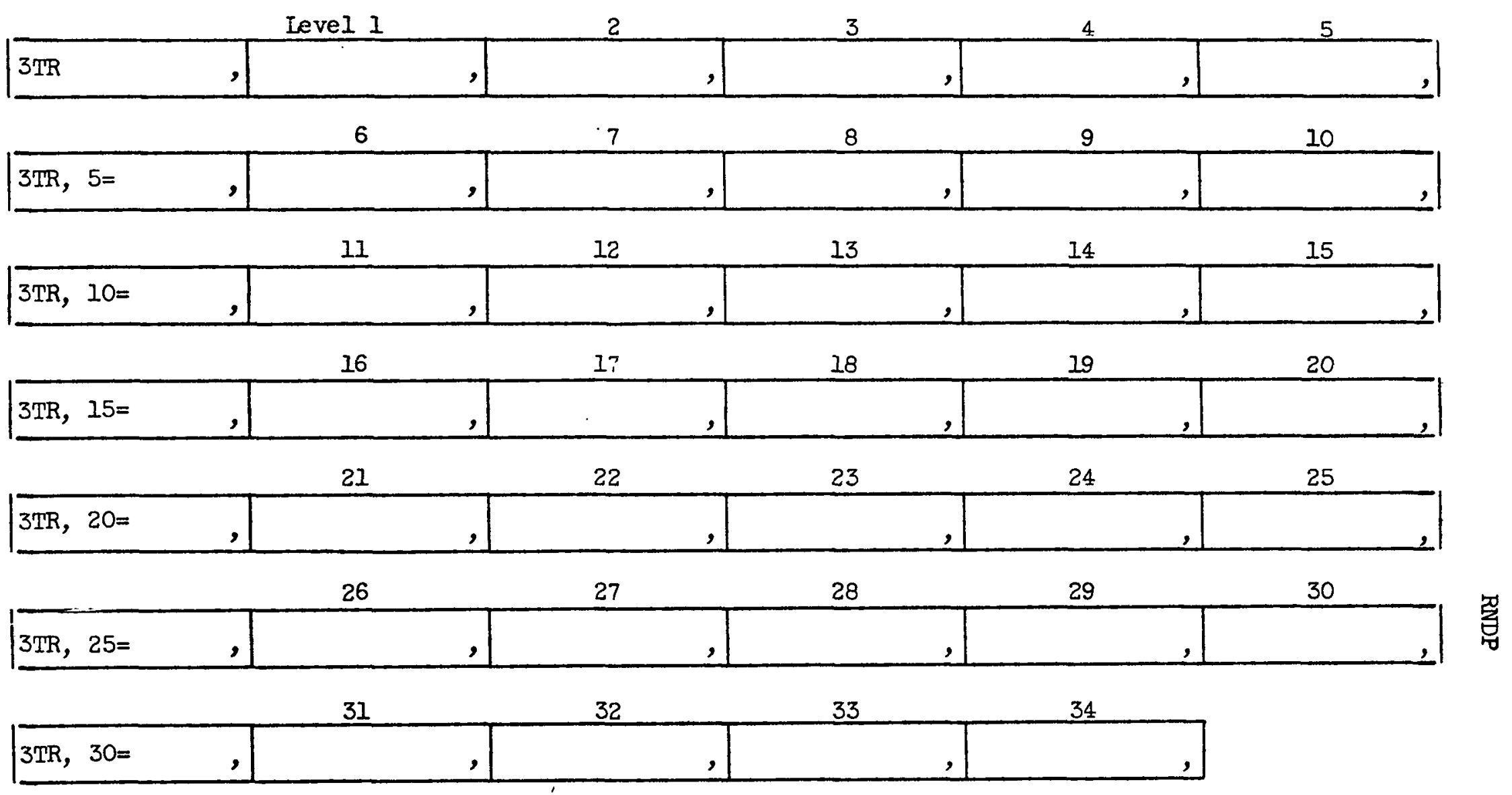


Cross Section Input

$$
\sigma_{A_{1}}
$$

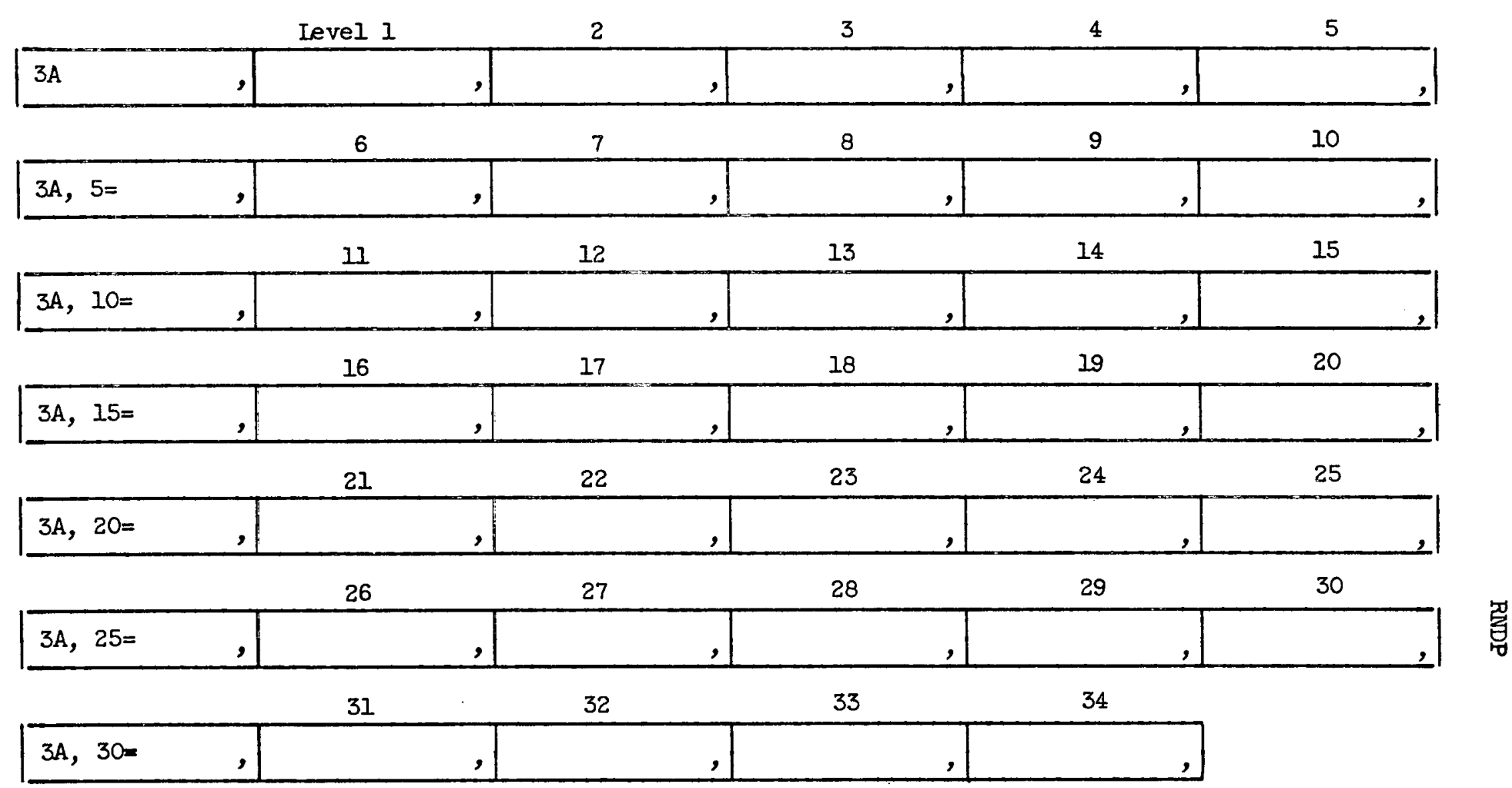


Cross Section Input

$$
\left(\varepsilon_{1} \sigma_{s_{1}}\right)_{1}
$$

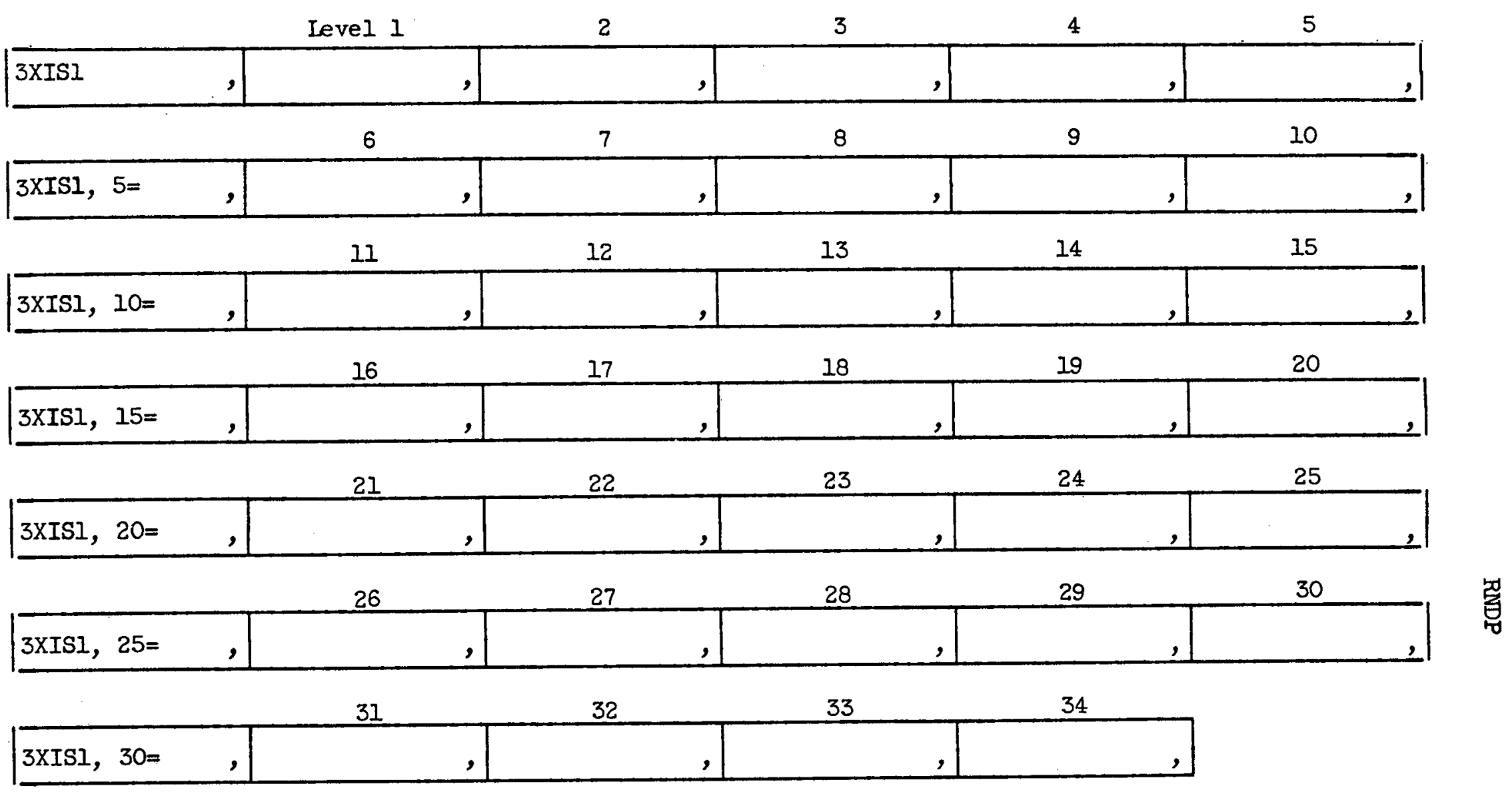


Cross Section Input

$v \sigma_{F_{1}}$

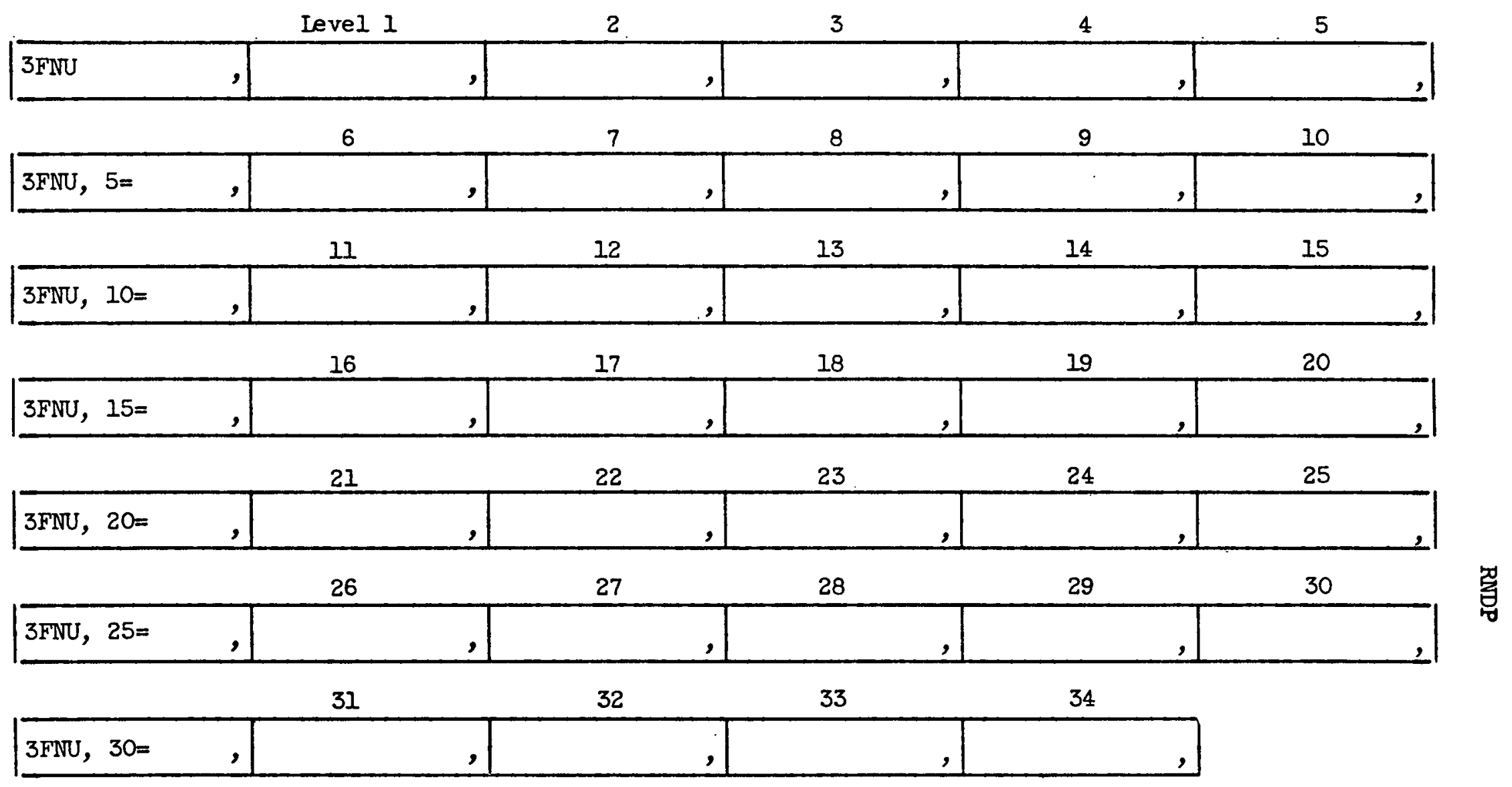


Cross Section Input

$\sigma_{F_{i}}$

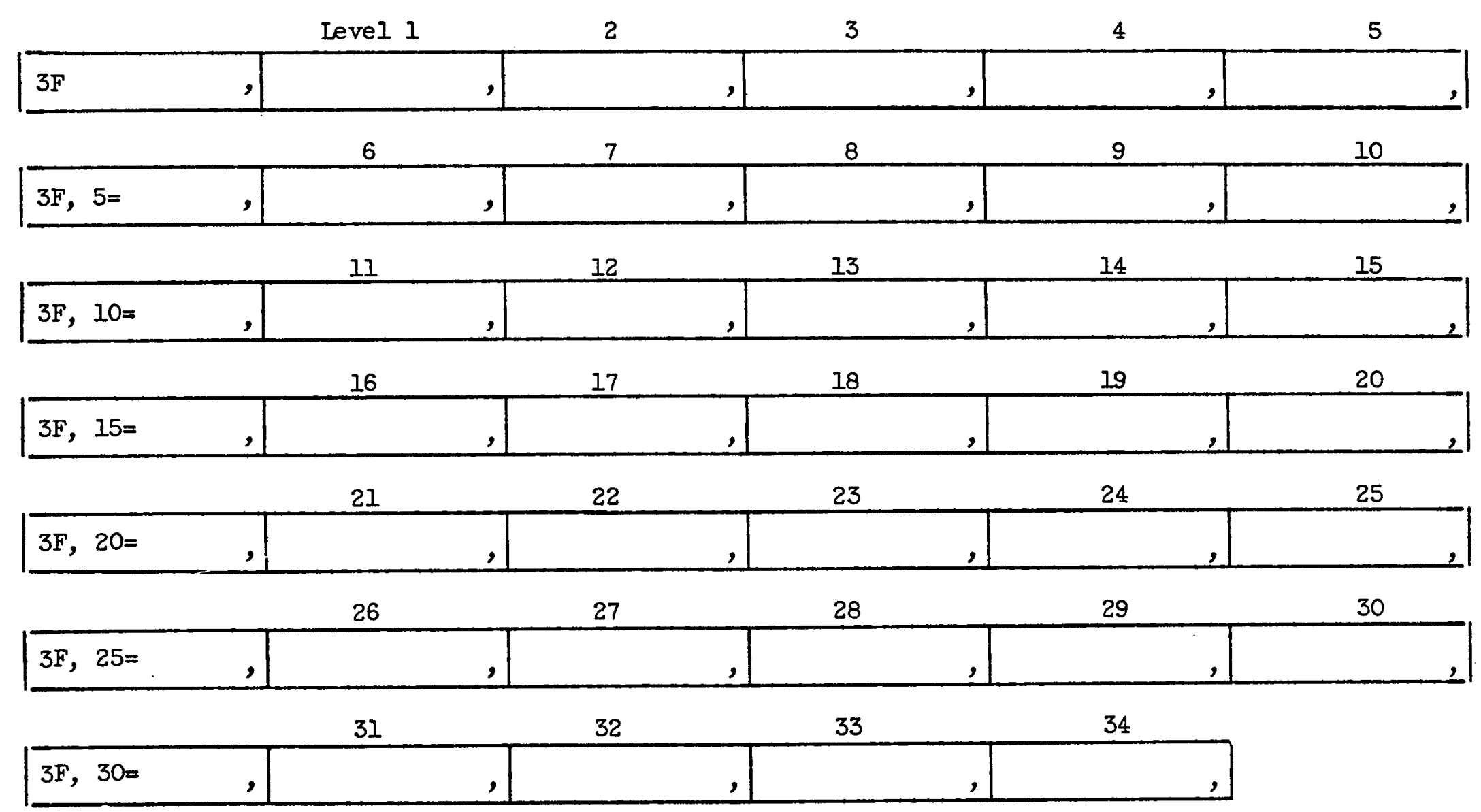




\section{Cross Section Input}

$$
\left(\sigma_{81}\right)_{1}
$$

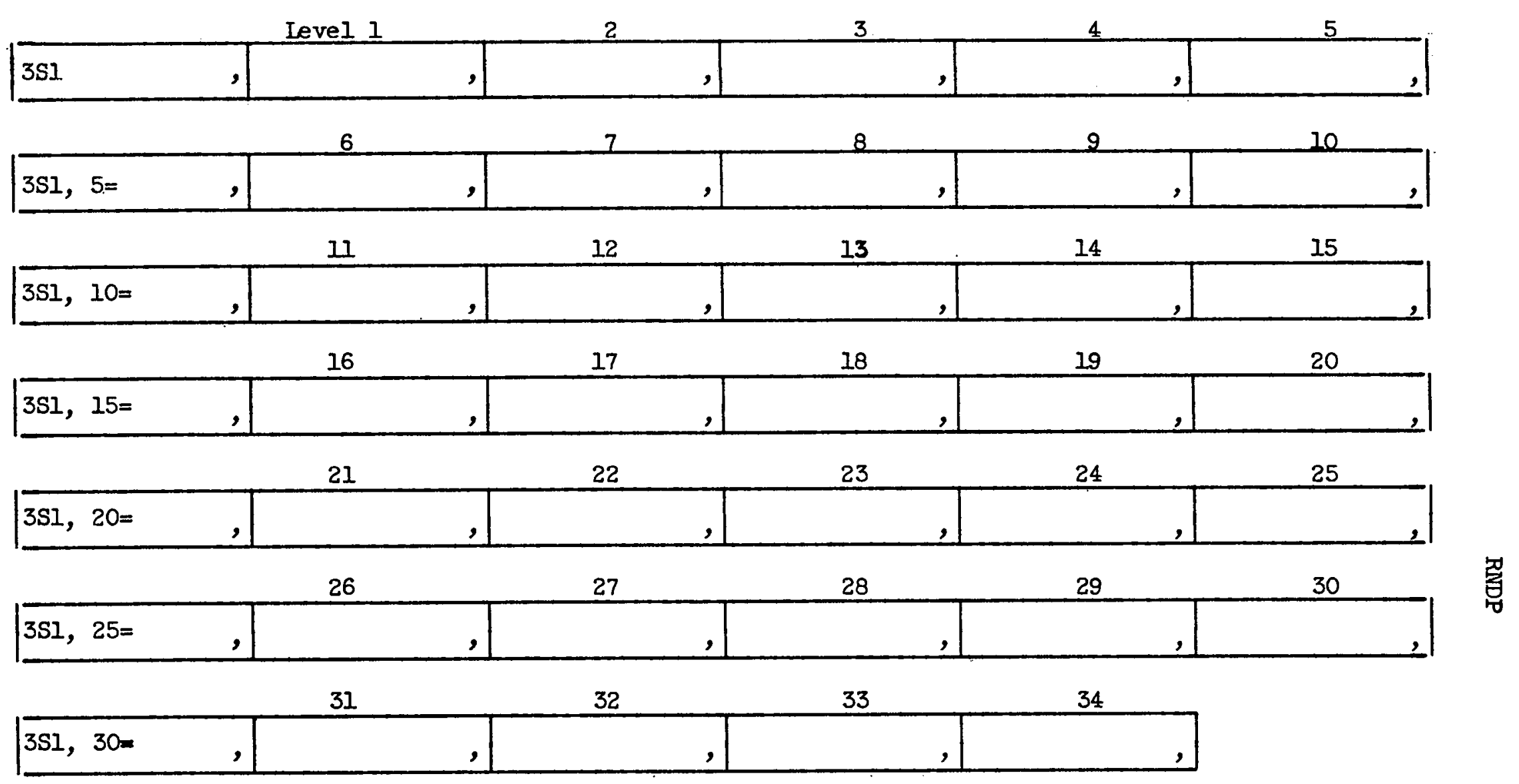


Cross Section Input

$\sigma_{\mathrm{T}_{1}}$

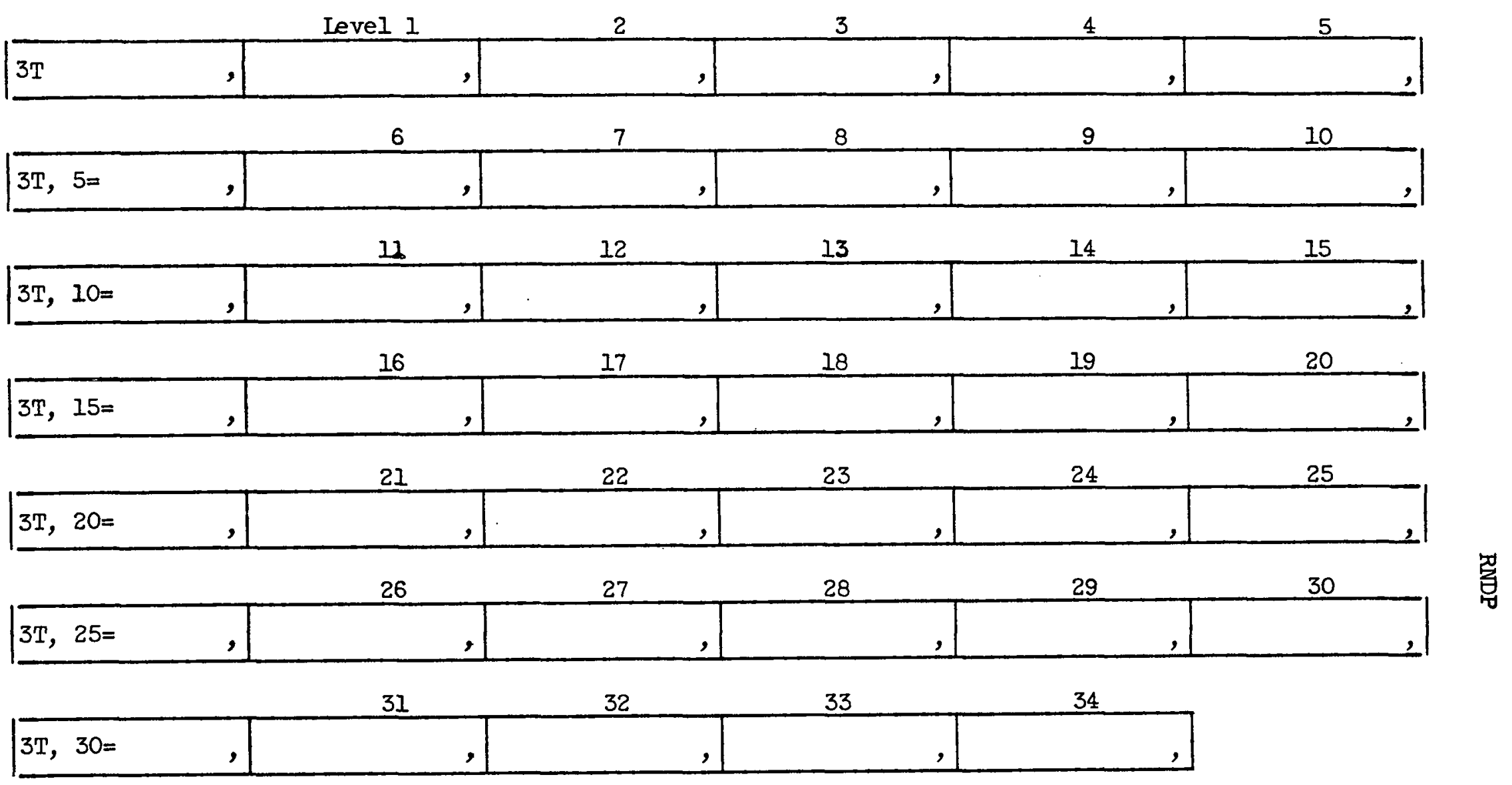


Cross Section Input

$\xi_{i}$

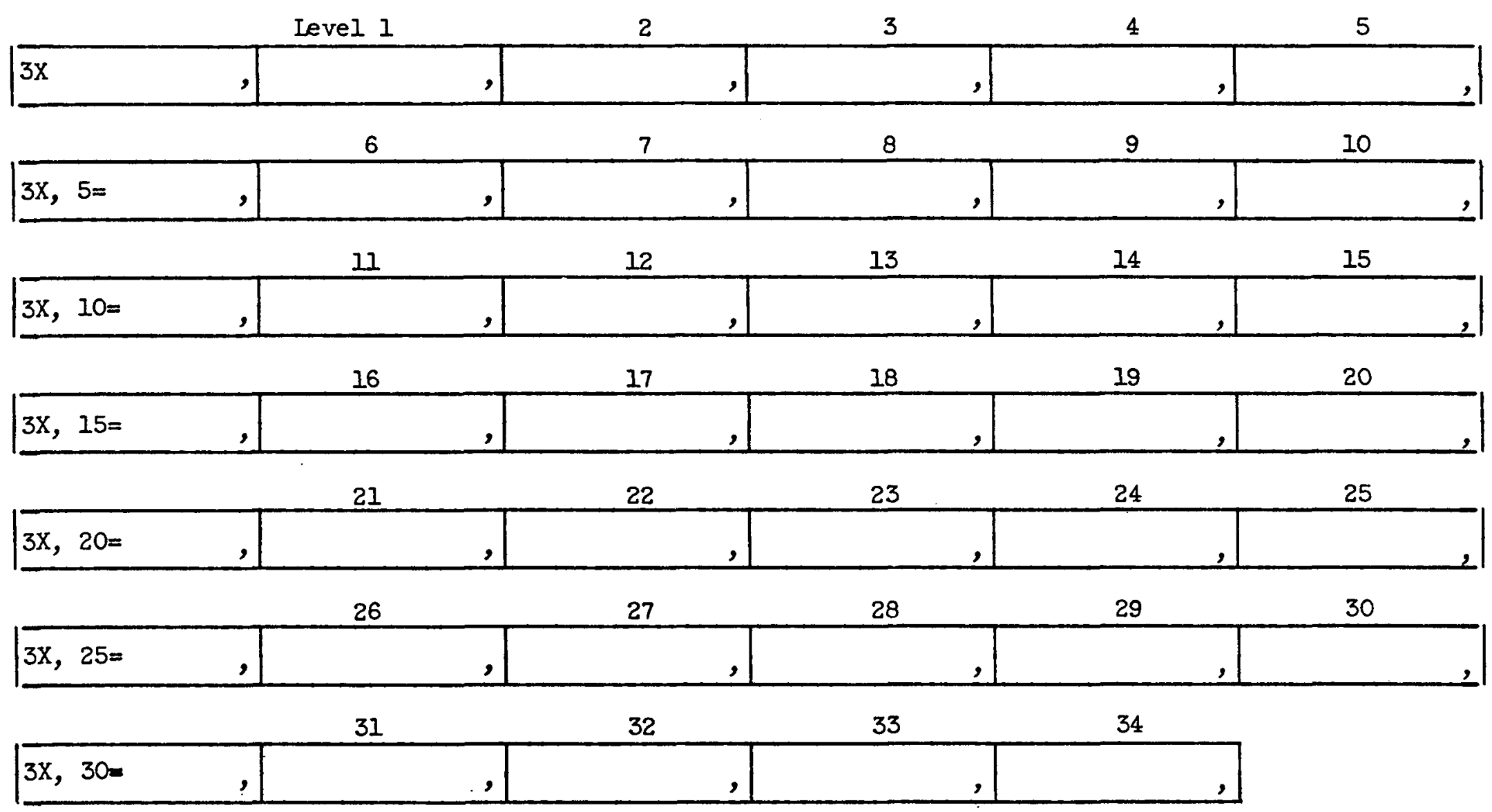


Cross Section Imput

${ }^{\xi_{1}}{ }_{1}$

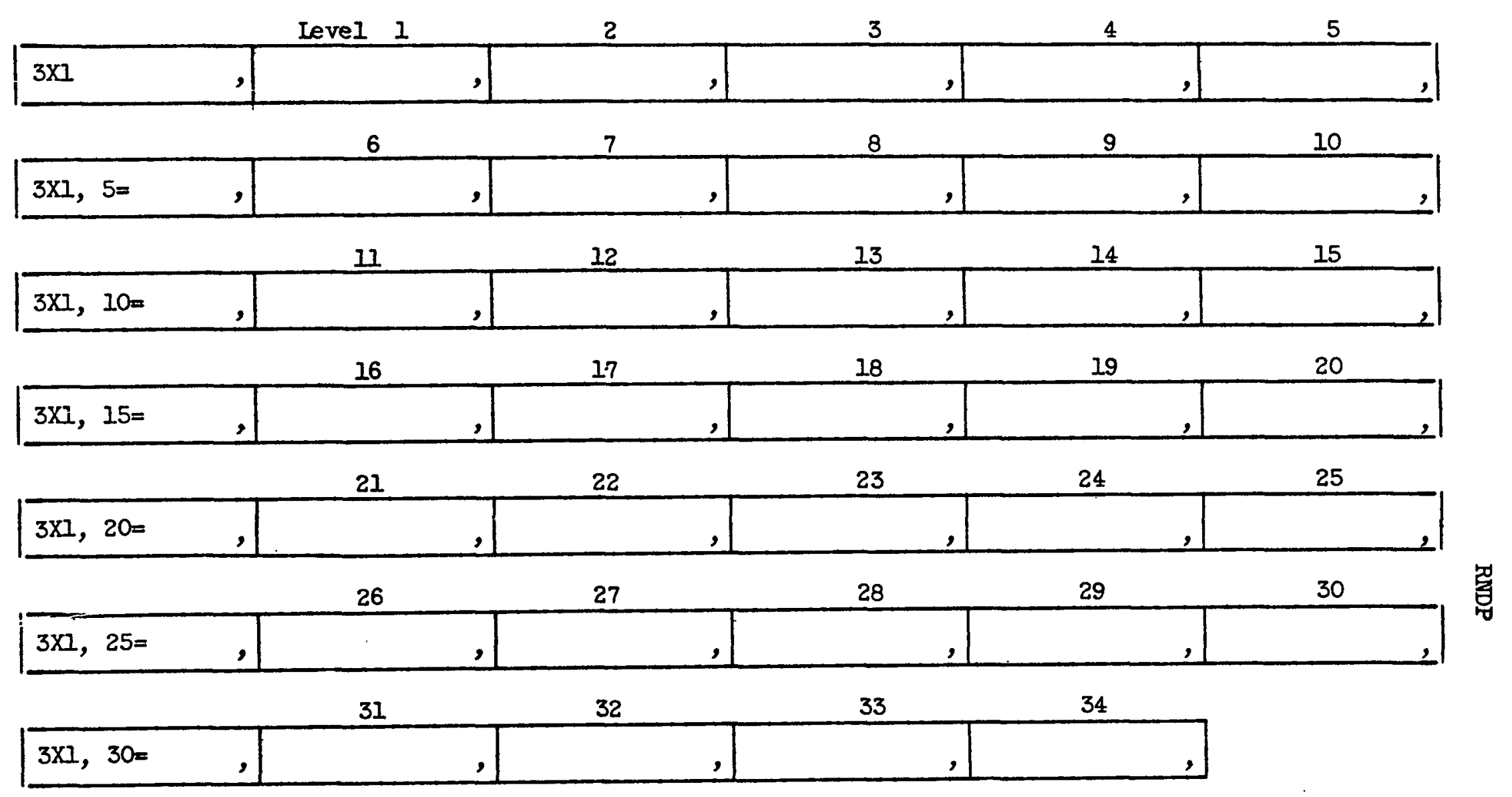


Cross Section Input

$$
B_{1}=\left(1-\bar{\mu}_{0}\right)_{1}
$$

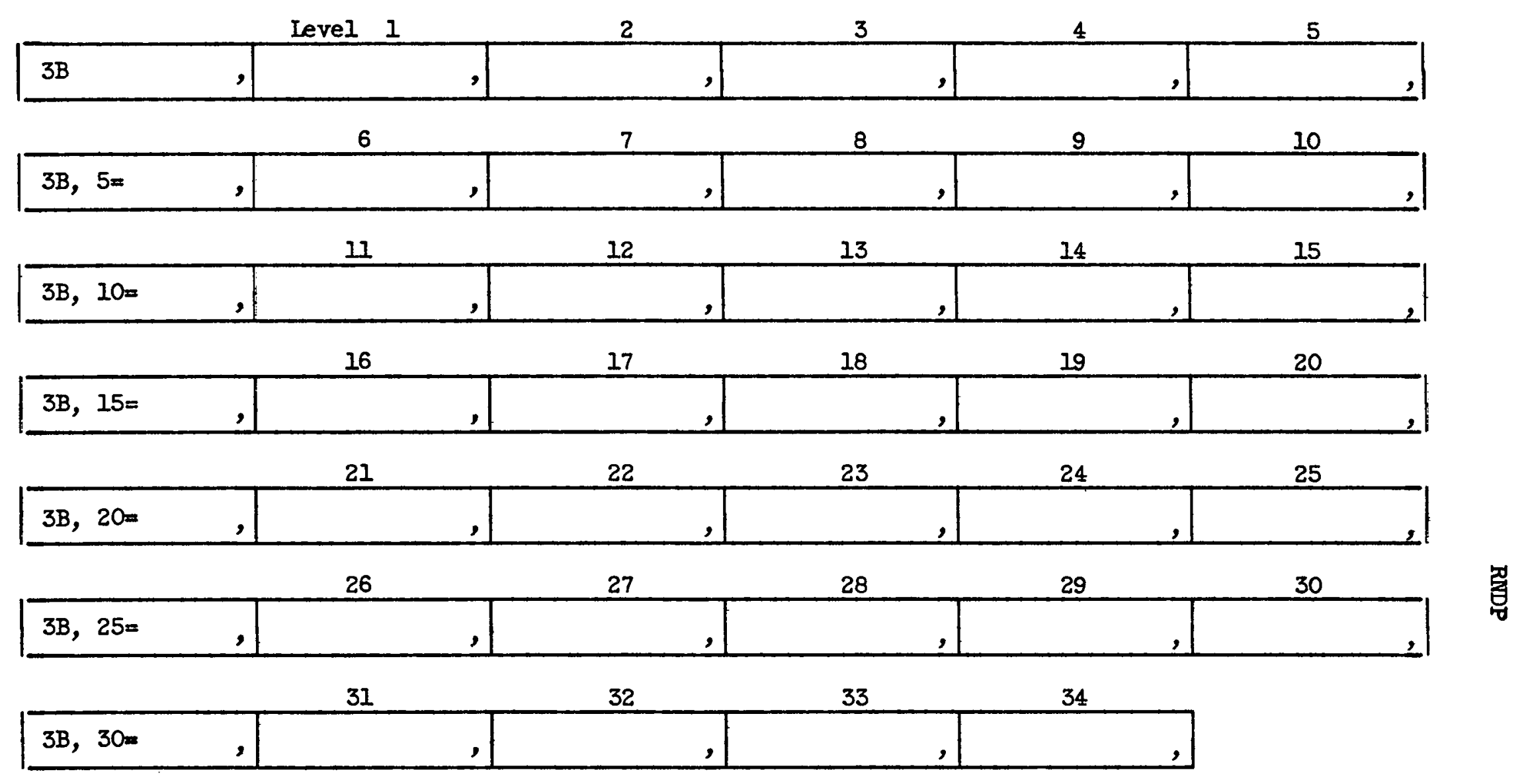


Cross Section Input

$$
v_{1}
$$

\begin{tabular}{|c|c|c|c|c|c|c|}
\hline & & Ievel I & 2 & 3 & 4 & 5 \\
\hline \multirow[t]{2}{*}{ 3PNU } &, 1 &, 1 & , &, &, 1 & \\
\hline & & 6 & 7 & 8 & 9 & 10 \\
\hline \multirow[t]{2}{*}{$3 \mathrm{PNU}, 5=$} & , &, 1 & , &, &, & \\
\hline & & 11 & 12 & 13 & 14 & 15 \\
\hline \multirow[t]{2}{*}{$3 \mathrm{PNU}, 10=$} & , & , &, & , & , & \\
\hline & & 16 & 17 & 18 & 19 & 20 \\
\hline \multirow[t]{2}{*}{ 3PNU, $15=$} & , & , &, 1 & , & & \\
\hline & & 21 & 22 & 23 & 24 & 25 \\
\hline \multirow[t]{2}{*}{ 3PNU, $20=$} & , &, 1 & , & 2 &, 1 & \\
\hline & & 26 & 27 & 28 & 29 & 30 \\
\hline \multirow[t]{2}{*}{$3 \mathrm{PNU}, 25=$} &, &, & , &, 1 & , & \\
\hline & & 31 & 32 & 33 & 34 & \\
\hline 3PNU, $30=$ &, &, 1 &, 1 &, & , & \\
\hline
\end{tabular}




\section{Cross Section Imput}

$\eta_{1}$

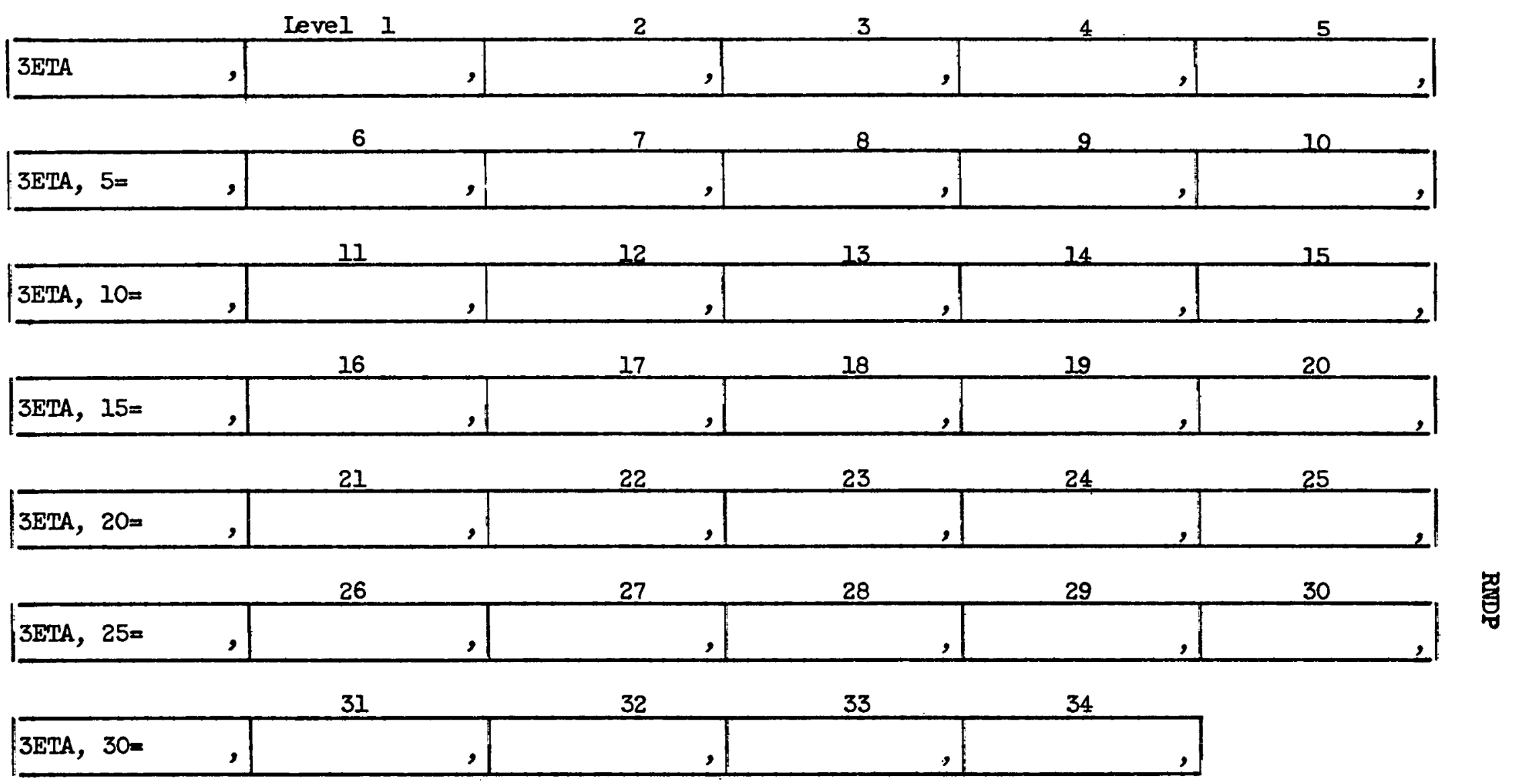


Cross Section Imput

Thermal Transfer Matrix $4500^{\circ} \mathrm{F}$

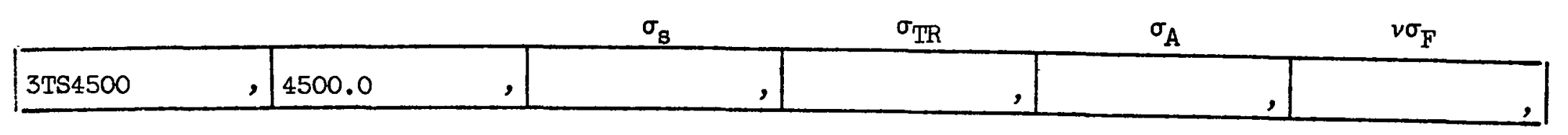

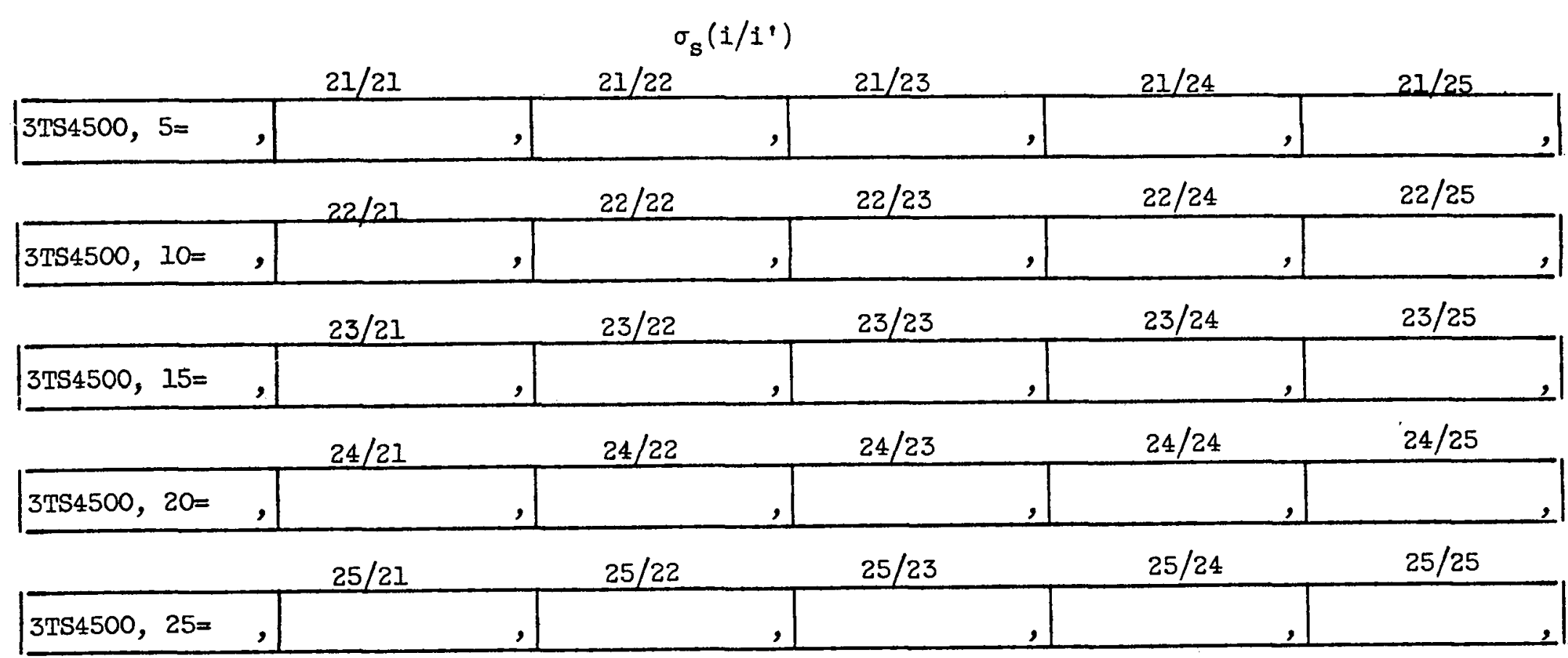


Cross Section Imput

Thermal Transfer Matrix

$4000^{\circ} \mathrm{F}$

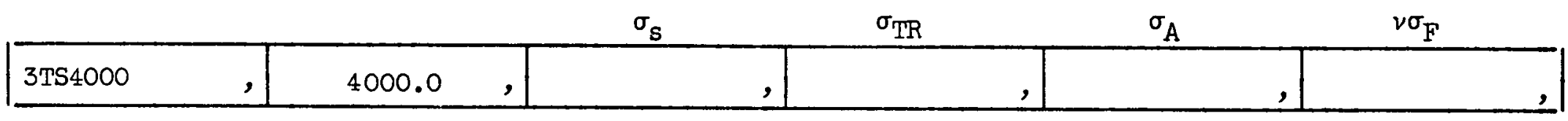

$\sigma_{s}\left(i / i^{\prime}\right)$

\begin{tabular}{|c|c|c|c|c|c|c|}
\hline & & $21 / 21$ & $21 / 22$ & $21 / 23$ & $21 / 24$ & $21 / 26$ \\
\hline $3 \mathrm{TS} 4000,5=$ & , &, &, & , & , & , \\
\hline & & $22 / 21$ & $22 / 22$ & $22 / 23$ & $22 / 24$ & $22 / 26$ \\
\hline $3 \mathrm{TS} 4000,10=$ & , & , & , & , & , & , \\
\hline & & $23 / 21$ & $23 / 22$ & $23 / 23$ & $23 / 24$ & $23 / 26$ \\
\hline $3 T S 4000,15=$ &, & , & , & , & , & \\
\hline & & $24 / 21$ & $24 / 22$ & $24 / 23$ & $24 / 24$ & $24 / 26$ \\
\hline $3 T S 4000,20=$ & , & , & , & , &, & \\
\hline & & $26 / 21$ & $26 / 22$ & $26 / 23$ & $26 / 24$ & $26 / 26$ \\
\hline $3 T S 4000,25=$ &, & , & , & , & , & \\
\hline
\end{tabular}


Cross Section Input

Thermal Transfer Matrix

$3500^{\circ} \mathrm{F}$

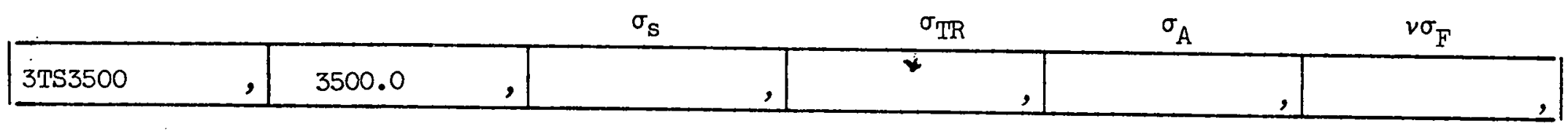

$$
\sigma_{s}\left(i / i^{\prime}\right)
$$

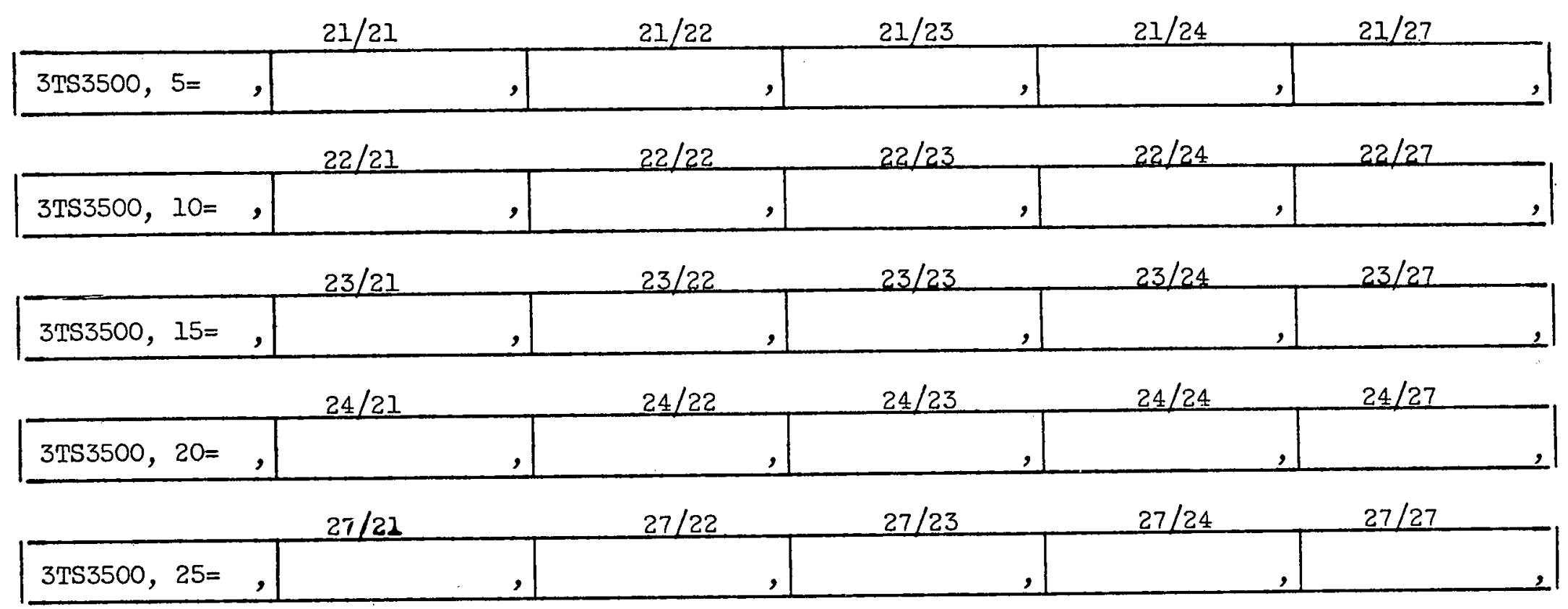




\section{Cross Section Input}

Thermal Transfer Matrix

$3000^{\circ} \mathrm{F}$

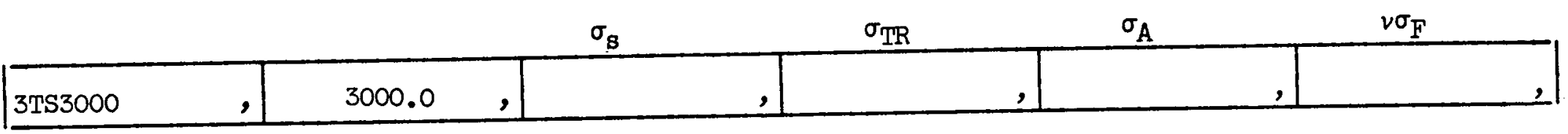

$$
\sigma_{s}\left(1 / i^{\prime}\right)
$$

\begin{tabular}{|c|c|c|c|c|c|c|}
\hline & & $21 / 21$ & $21 / 22$ & $21 / 23$ & $21 / 24$ & $21 / 28$ \\
\hline \multirow{2}{*}{$3 T S 3000,5=$} &, &, &, &, &, 1 & \\
\hline & & $22 / 21$ & $22 / 22$ & $22 / 23$ & $22 / 24$ & $22 / 28$ \\
\hline \multirow[t]{2}{*}{$3 \mathrm{TS} 3000,10=$} & , & , & , & , & , & \\
\hline & & $23 / 21$ & $23 / 22$ & $23 / 23$ & $23 / 24$ & $23 / 28$ \\
\hline \multirow[t]{2}{*}{$3 T S 3000,15=$} &, & , & , &, &, & \\
\hline & & $24 / 21$ & $24 / 22$ & $24 / 23$ & $24 / 24$ & $24 / 28$ \\
\hline \multirow[t]{2}{*}{$3 T S 3000,20=$} & , & , & , &, &, & \\
\hline & & $28 / 21$ & $28 / 22$ & $28 / 23$ & $28 / 24$ & $28 / 28$ \\
\hline $3 T S 3000,25$ & , & , & , & , &, & \\
\hline
\end{tabular}


Cross Section Input

Thermal Transfer Matrix

$2500^{\circ} \mathrm{F}$

$\sigma_{5}$

$\sigma_{\text {TR }}$

$\sigma_{\mathrm{A}}$

$v \sigma_{F}$

\begin{tabular}{|l|l|l|l|l|l|l|}
\hline TS2500 & 2500.0 &, &, &, &, \\
\hline
\end{tabular}

\begin{tabular}{|c|c|c|c|c|c|c|}
\hline & \multirow{2}{*}{\multicolumn{2}{|c|}{$21 / 21$}} & \multicolumn{2}{|c|}{$\sigma_{s}\left(1 / i^{\prime}\right)$} & \multirow[b]{2}{*}{$21 / 24$} & \multirow[b]{2}{*}{$21 / 29$} \\
\hline & & & $21 / 22$ & $21 / 23$ & & \\
\hline \multirow[t]{2}{*}{$3 \operatorname{Ts} 2500,5=$} & , & , &, &, & , & , \\
\hline & & $22 / 21$ & $22 / .22$ & $22 / 23$ & $22 / 24$ & $22 / 29$ \\
\hline \multirow[t]{2}{*}{$3 \mathrm{TS} 2500,10=$} & , &, & , & , & , & , \\
\hline & & $23 / 21$ & $23 / 22$ & $23 / 23$ & $23 / 24$ & $23 / 29$ \\
\hline \multirow[t]{2}{*}{$3 T S 2500,15=$} & , &, &, & , & , & , \\
\hline & & $24 / 21$ & $24 / 22$ & $24 / 23$ & $24 / 24$ & $24 / 29$ \\
\hline \multirow[t]{2}{*}{$3 \mathrm{TS} 2500,20=$} & , &, & , & , & , & \\
\hline & & $29 / 21$ & $29 / 22$ & $29 / 23$ & $29 / 24$ & $29 / 29$ \\
\hline $3 T S 2500,25=$ & , & , & , & , & , & \\
\hline
\end{tabular}


Cross Section Input

Thermal Transfer Matrix

$2000^{\circ} \mathrm{F}$

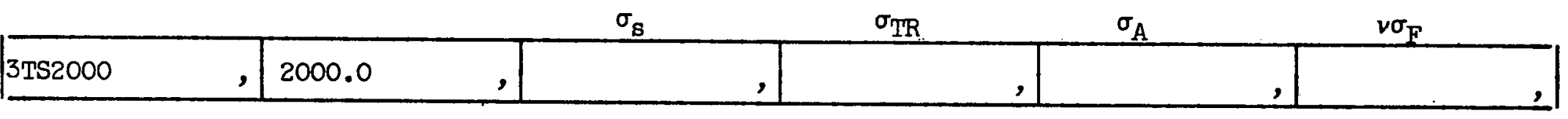

$\sigma_{s}\left(i / i^{\prime}\right)$

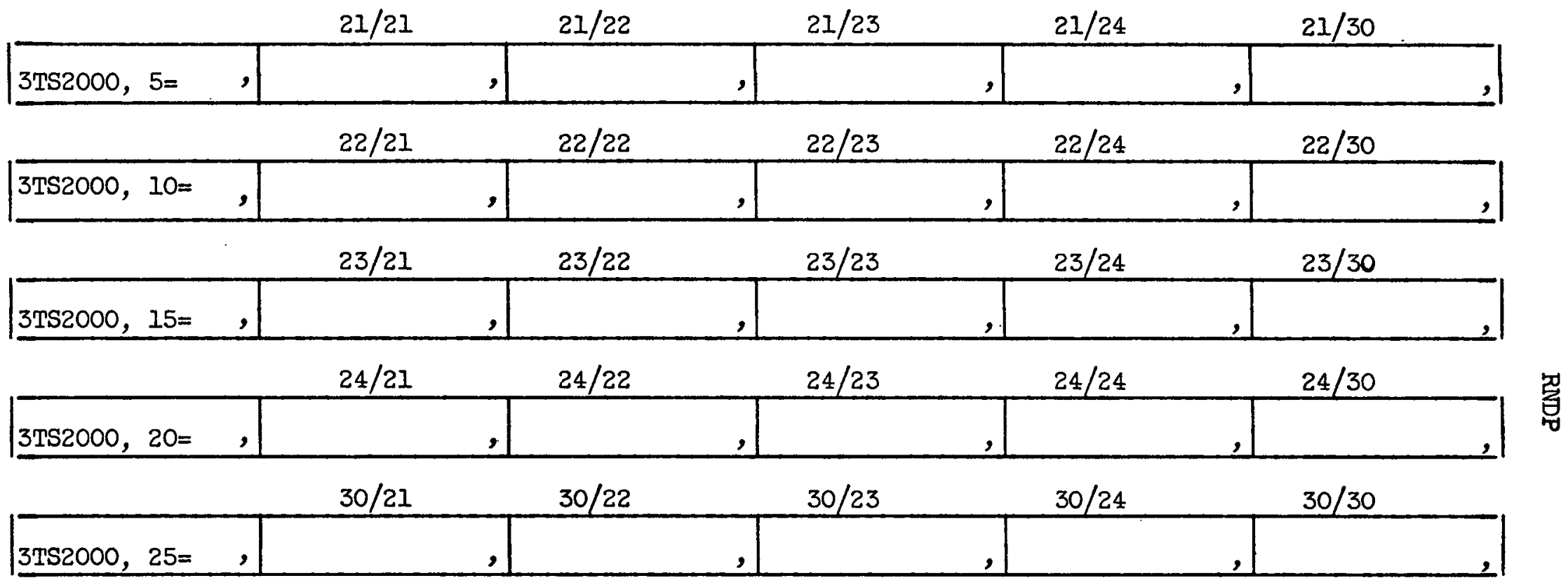


Cross Section Input

Thermal Transfer Matrix $1500^{\circ} \mathrm{F}$

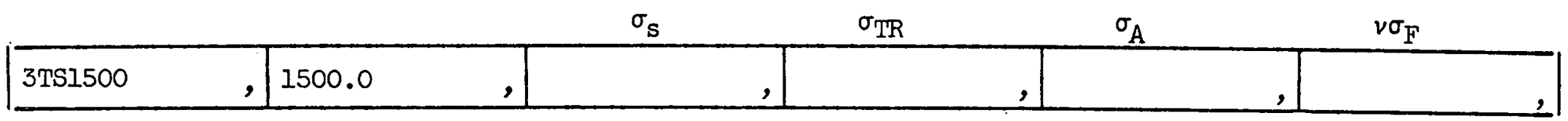

$$
\sigma_{s}\left(1 / 1^{\prime}\right)
$$

\begin{tabular}{|c|c|c|c|c|c|c|}
\hline & & $21 / 21$ & $21 / 22$ & $21 / 23$ & $21 / 24$ & $21 / 31$ \\
\hline \multirow[t]{2}{*}{$3 \mathrm{TSl} 500,5=$} & , & , & $\therefore$ & , & , & , \\
\hline & & $22 / 21$ & $22 / 22$ & $22 / 23$ & $22 / 24$ & $22 / 31$ \\
\hline \multirow[t]{2}{*}{$3 T S 1500,10=$} & , & , & , & , & , & , \\
\hline & & $23 / 21$ & $23 / 22$ & $23 / 23$ & $23 / 24$ & $23 / 31$ \\
\hline \multirow[t]{2}{*}{$3 m 51500,15=$} & , & , & , & , & , & \\
\hline & & $24 / 21$ & $24 / 22$ & $24 / 23$ & $24 / 24$ & $24 / 31$ \\
\hline \multirow[t]{2}{*}{$3 T S 1500,20=$} & , & , & , & , &, & \\
\hline & & $31 / 21$ & $31 / 22$ & $31 / 23$ & $31 / 24$ & $31 / 31$ \\
\hline $3 \mathrm{TS} 1500,25=$ & , & , & , & , & , & \\
\hline
\end{tabular}




\section{Cross Section Imput}

Thermal Transfer Matrix

\begin{tabular}{|c|c|c|c|c|c|c|c|}
\hline & & & & & & & \\
\hline & & & & $\sigma_{\mathrm{s}}$ & $\sigma_{\mathrm{TR}}$ & $\sigma_{A}$ & $\nu \sigma_{\mathrm{F}}$ \\
\hline 3TS1000 & , & 1000.0 & , & & & & \\
\hline
\end{tabular}

$$
\sigma_{s}\left(i / 1^{\prime}\right)
$$

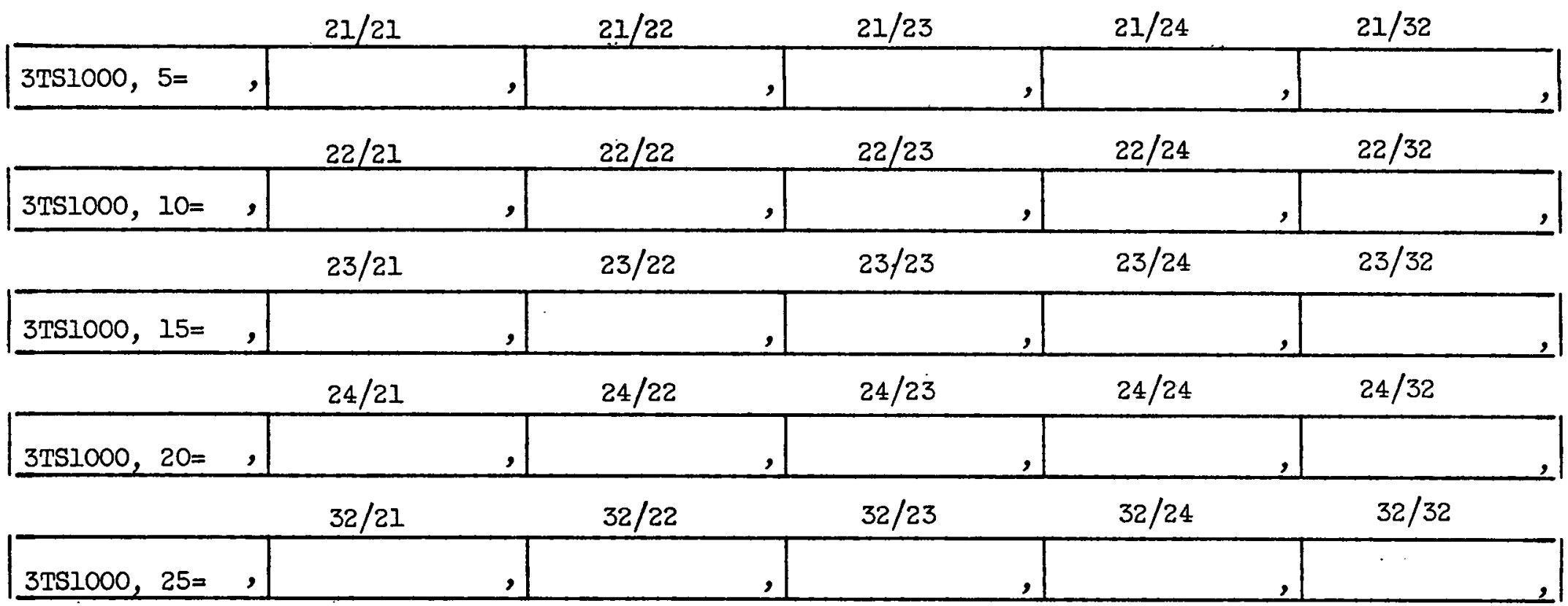


Cross Section Input

Thermal Transfer Matrix

$$
500^{\circ} \mathrm{F}
$$

$\sigma_{\mathrm{s}}$

$$
\sigma_{\mathrm{TR}}
$$

$\sigma_{A}$

$v \sigma_{F}$

\begin{tabular}{|l|l|l|l|l|l|}
\hline $3 \operatorname{TS} 500$ & 500.0 & &, &, & \\
\hline
\end{tabular}

$$
\sigma_{s}\left(i / 1^{\prime}\right)
$$

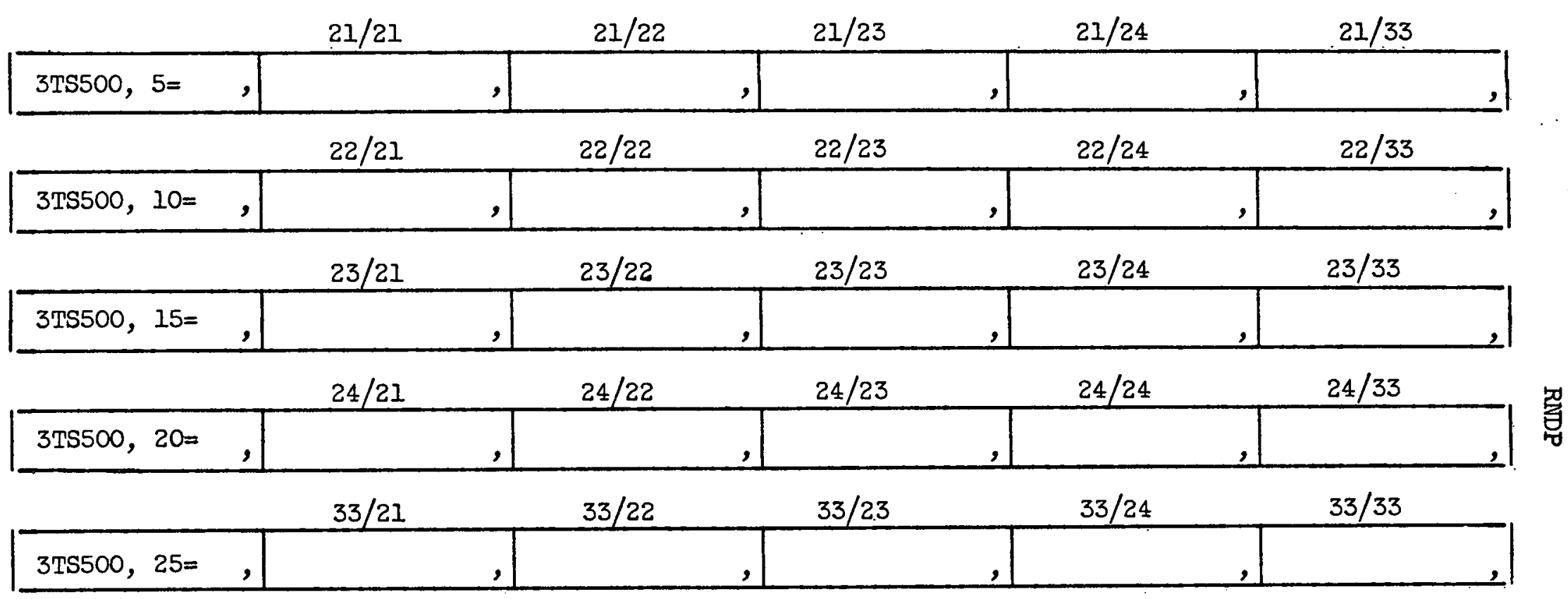


Cross Section Imput

Thermal Transfer Matrix

$68^{\circ} \mathrm{F}$

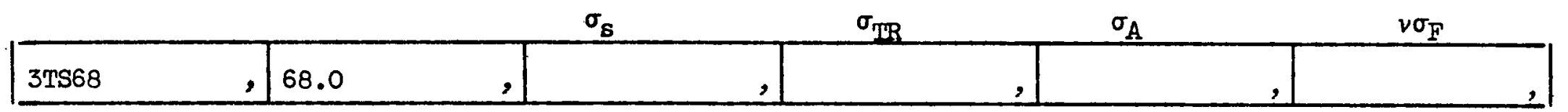

$$
\sigma_{s}\left(1 / 1^{\prime}\right)
$$

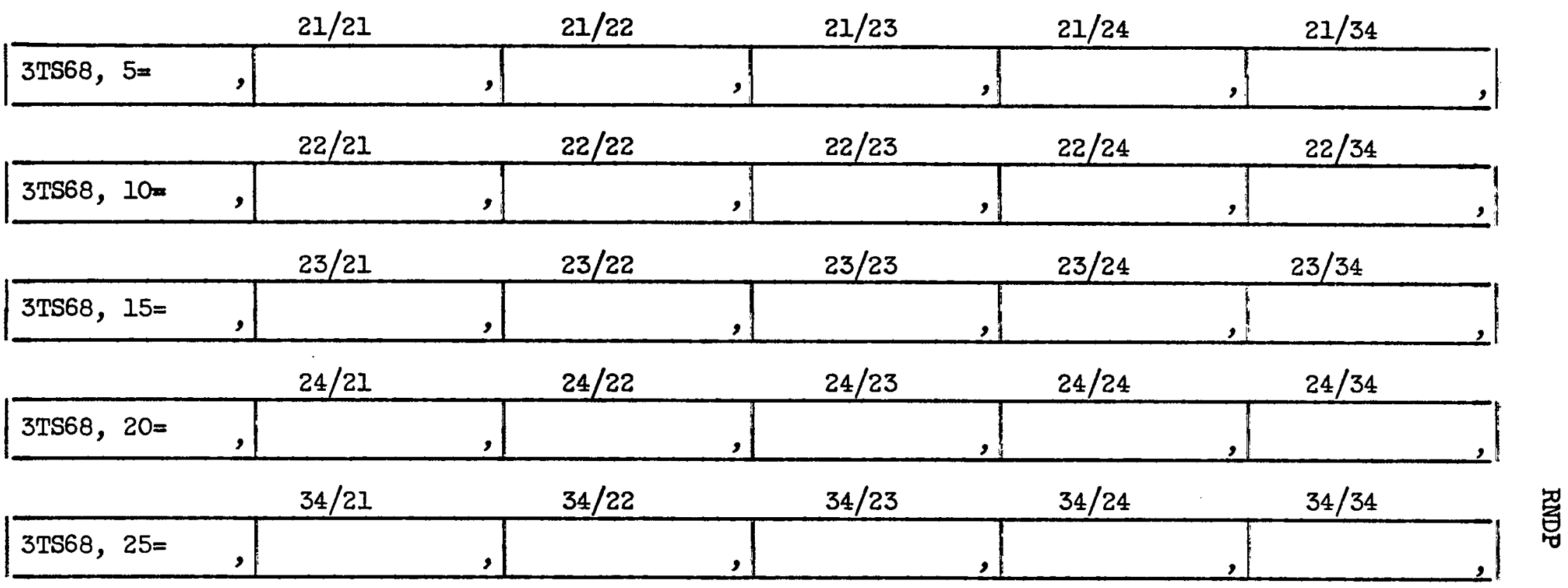

\begin{tabular}{|l|l|}
\hline$=\operatorname{END}$, & 0 \\
\hline
\end{tabular}


Cross Section Input

Scattering Transfer Matrix

$$
\sigma_{\mathrm{BO}}\left(\mathrm{m} / \mathrm{m}^{\prime}\right)
$$

The data should be ordered on the cards as shown below.

$$
\begin{aligned}
& \sigma_{\mathrm{BO}}\left(\mathrm{MO}_{\mathrm{MIN}} / \mathrm{M}^{\prime} \mathrm{O}_{\mathrm{MIN}}\right), \sigma_{\mathrm{SO}}\left(\mathrm{MO}_{\mathrm{MIN}} / \mathrm{M}^{\prime} \mathrm{O}_{\mathrm{MIN}}+1\right), \cdots \sigma_{\mathrm{BO}}\left(\mathrm{MO}_{\mathrm{MIN}} / \mathrm{M}^{\prime} \mathrm{O}_{\mathrm{MAX}}\right) \text {, } \\
& \sigma_{\text {SO }}\left(M_{M I N}+1 / M^{\prime} O_{M I N}\right), \sigma_{S O}\left(M_{M I N}+1 / M^{\prime} O_{M I N}+1\right) \cdots \sigma_{s O}\left(M_{M I N}+1 / M^{\prime} O_{M A X}\right) \text {, } \\
& \sigma_{\mathrm{BO}}\left(\mathrm{MO}_{\mathrm{MAX}} / \mathrm{M}^{\prime} \mathrm{O} \mathrm{MIN}\right), \sigma_{\mathrm{SO}}\left(\mathrm{MO}_{\mathrm{MAX}} / \mathrm{M}^{\prime} \mathrm{O}_{\mathrm{MIN}}+1\right), \cdots \cdots \sigma_{\mathrm{SO}}\left(\mathrm{MO}_{\mathrm{MAX}} / \mathrm{M}^{\prime} \mathrm{O}_{\mathrm{MAX}}\right)
\end{aligned}
$$

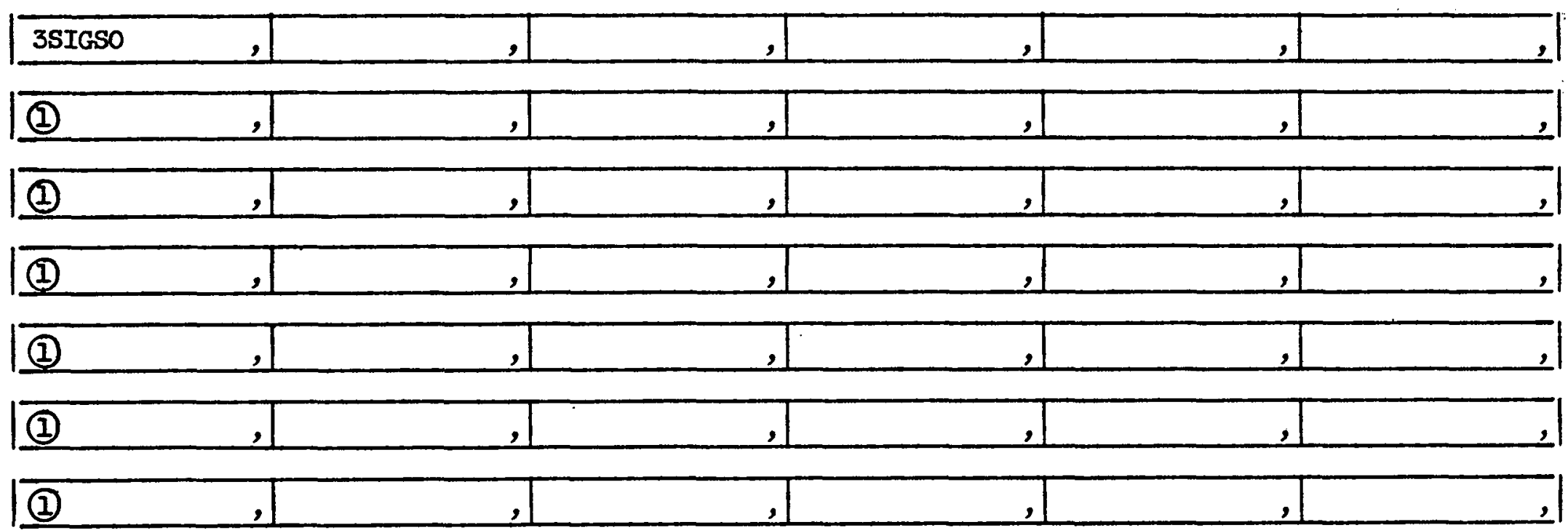

The table above is limited to 625 entries.

$=\operatorname{END}$,




\section{Cross Section Imput}

Scattering Trarsfer Matrix

$$
\sigma_{s l}\left(m / m^{\prime}\right)
$$

The data should be ordered as shown below.

$$
\begin{aligned}
& \sigma_{\mathrm{BI}}\left(\mathrm{MI}_{\mathrm{MIN}} / \mathrm{M}^{\prime} \mathrm{I}_{\mathrm{MIN}}\right), \sigma_{\mathrm{SI}}\left(\mathrm{MI}_{\mathrm{MIN}} / \mathrm{M}^{\prime} \mathrm{I}_{\mathrm{MIN}}+1\right), \cdots \cdots \sigma_{\mathrm{SI}}\left(\mathrm{MI}_{\mathrm{MIN}} / \mathrm{M}^{\prime} \mathrm{I}_{\mathrm{MAX}}\right) \text {, } \\
& \sigma_{\mathrm{sI}}\left(\mathrm{MI}_{\mathrm{MIN}}+1 / \mathrm{M}^{\prime} \mathrm{I}_{\mathrm{MIN}}\right), \sigma_{\mathrm{s} 1}\left(\mathrm{MI}_{\mathrm{MIN}}+1 / \mathrm{M}^{\prime} \mathrm{I}_{\mathrm{MIN}}+1\right), \cdots \sigma_{\mathrm{sI}}\left(\mathrm{MI}_{\mathrm{MIN}}+1 / \mathrm{M}^{\prime} \mathrm{M}_{\mathrm{MAX}}\right) \text {, } \\
& \sigma_{s 1}\left(M_{M A X} / M^{\prime} 1_{M I N}\right), \sigma_{s 1}\left(M_{M A X} / M^{\prime} l_{M I N}+1\right), \cdots \cdots \sigma_{s 1}\left(M_{M A X} / M^{\prime} l_{M A X}\right)
\end{aligned}
$$

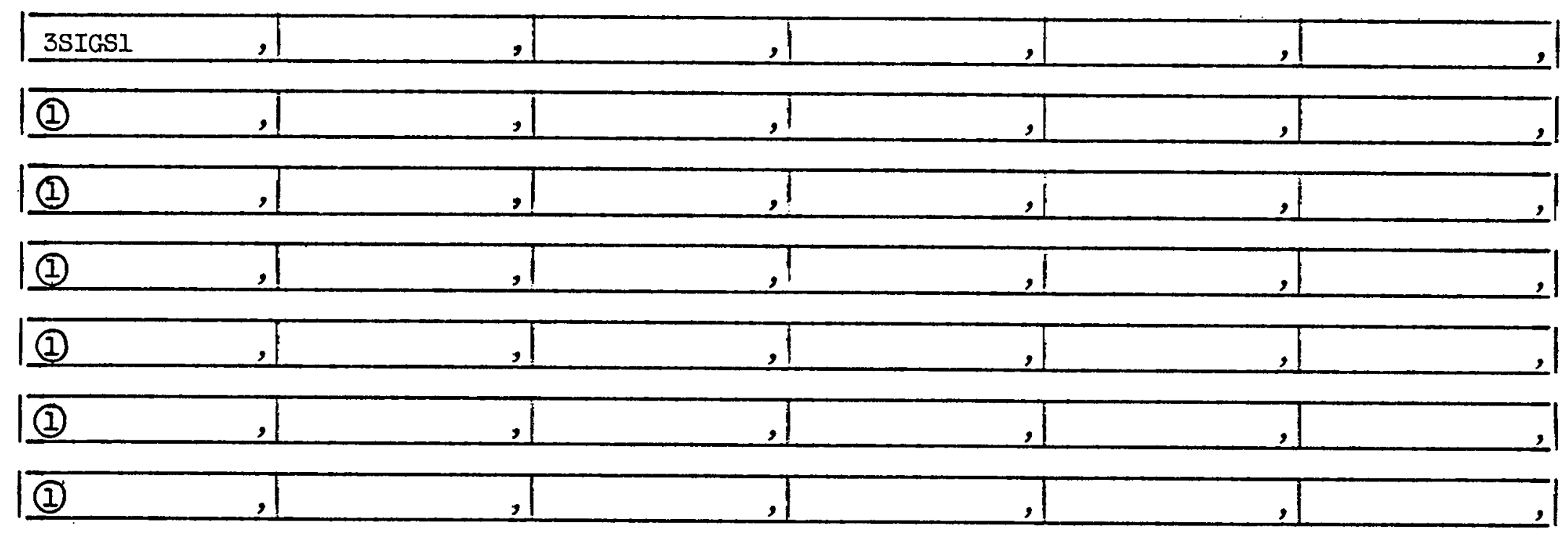

The table above is limited to 625 entries

\begin{tabular}{|l|l|}
\hline$=E N D$, & 0 \\
\hline
\end{tabular}




\section{Cross Section Input}

Resonance Data

Resonance Energies

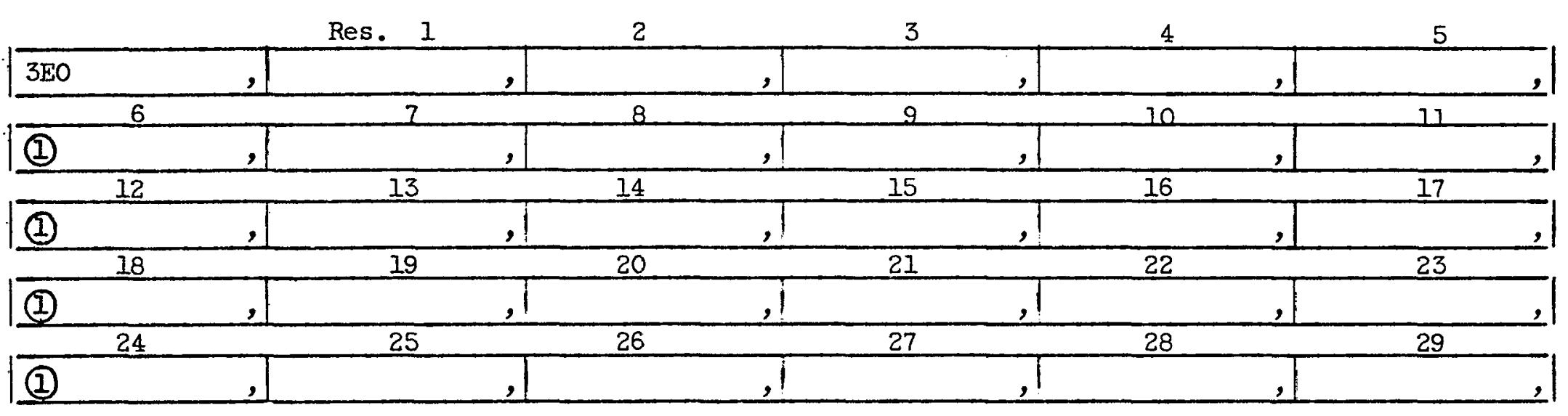

The table above is limited to 200 entries

Resonance Integrals

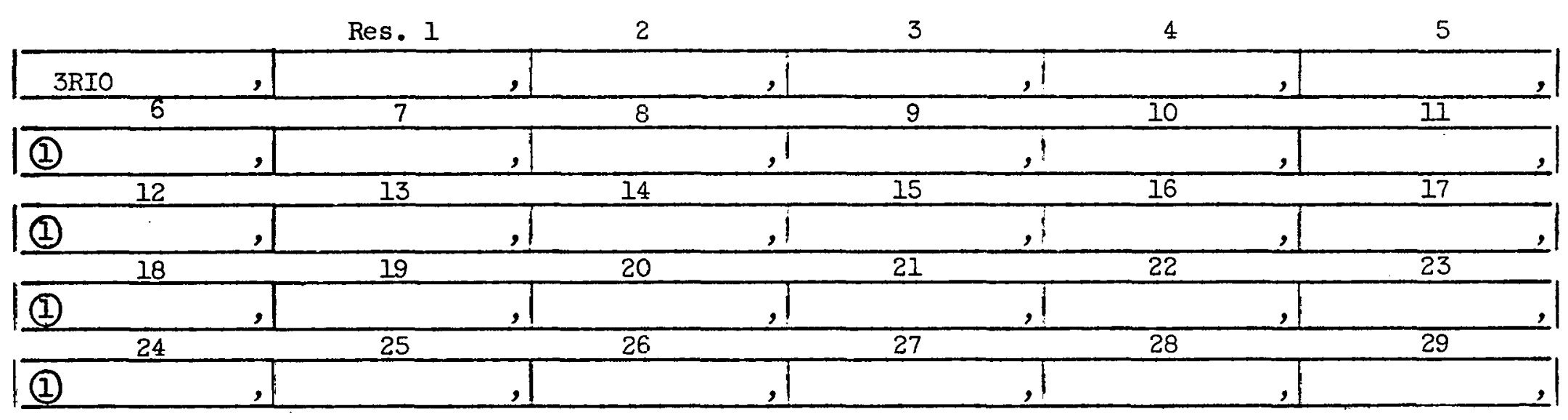

The table above is limited to 200 entries 


\section{Cross Section Input}

Resonance Data

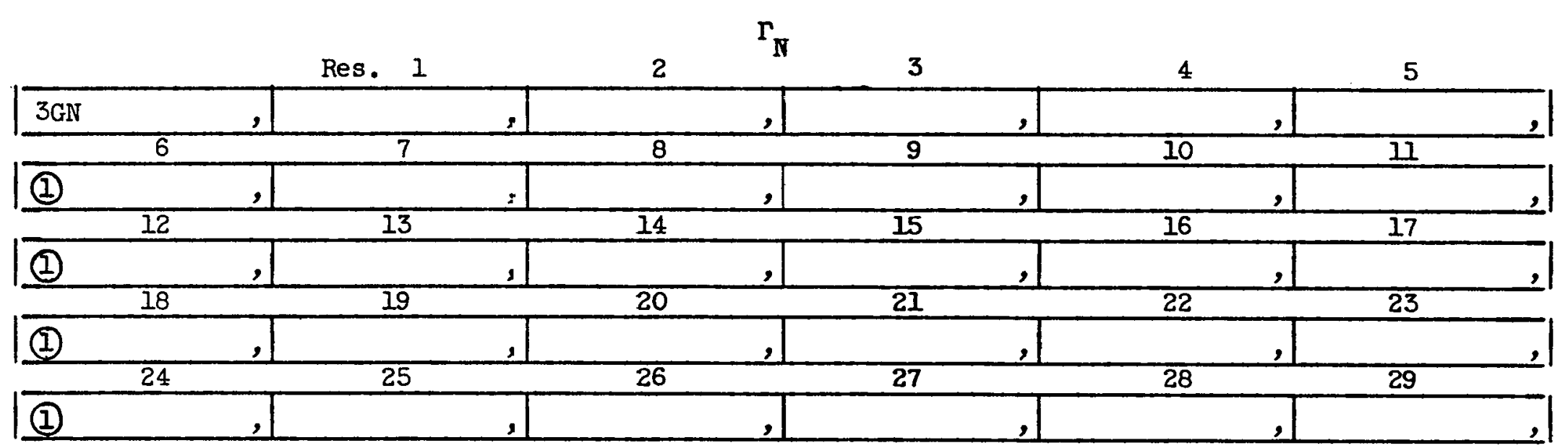

The table above is limited to 200 entries

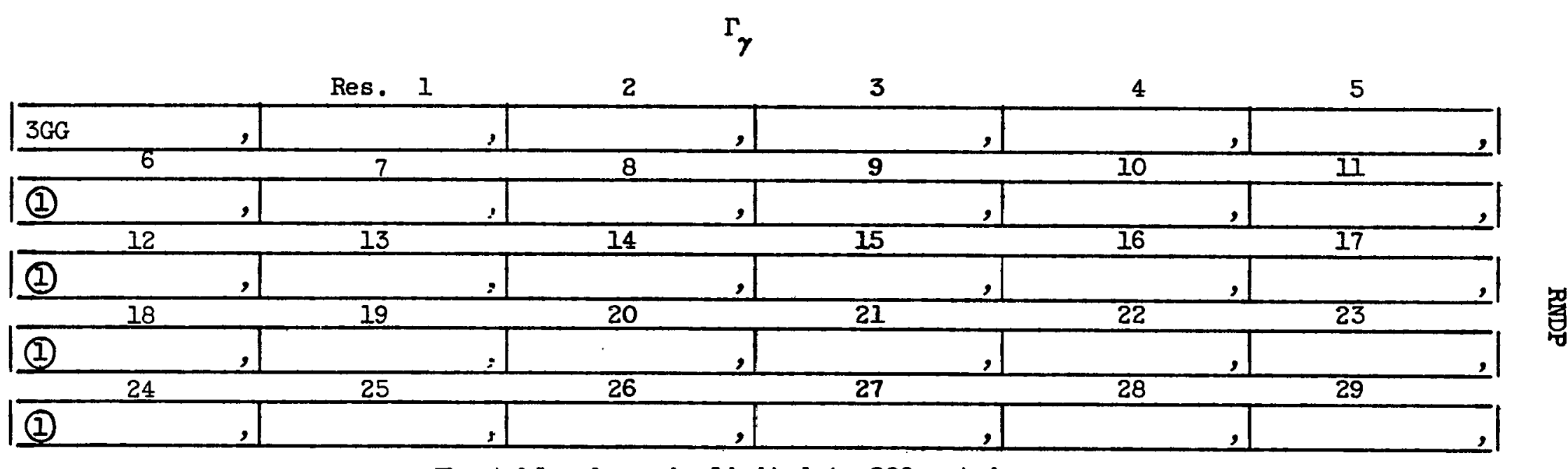

The table above is limited to 200 entries 


\section{Cross Section Input}

Resonance Data

$$
\Gamma_{\mathrm{F}}
$$

\begin{tabular}{|c|c|c|c|c|c|}
\hline & Res. I & 2 & 3 & 4 & 5 \\
\hline $3 \mathrm{GF}$ & & & & & \\
\hline 6 & 7 & 8 & 9 & 10 & II \\
\hline (1) & & & & & \\
\hline 12 & 13 & 14 & 15 & 16 & 17 \\
\hline (1) & & & & & \\
\hline 18 & 19 & 20 & 21 & 22 & 23 \\
\hline (1) & & & & & \\
\hline 24 & 25 & 26 & 27 & 28 & 29 \\
\hline (1) & & & & & \\
\hline
\end{tabular}

The table above is limited to 200 entries

Statistical Welghting Factor, g,

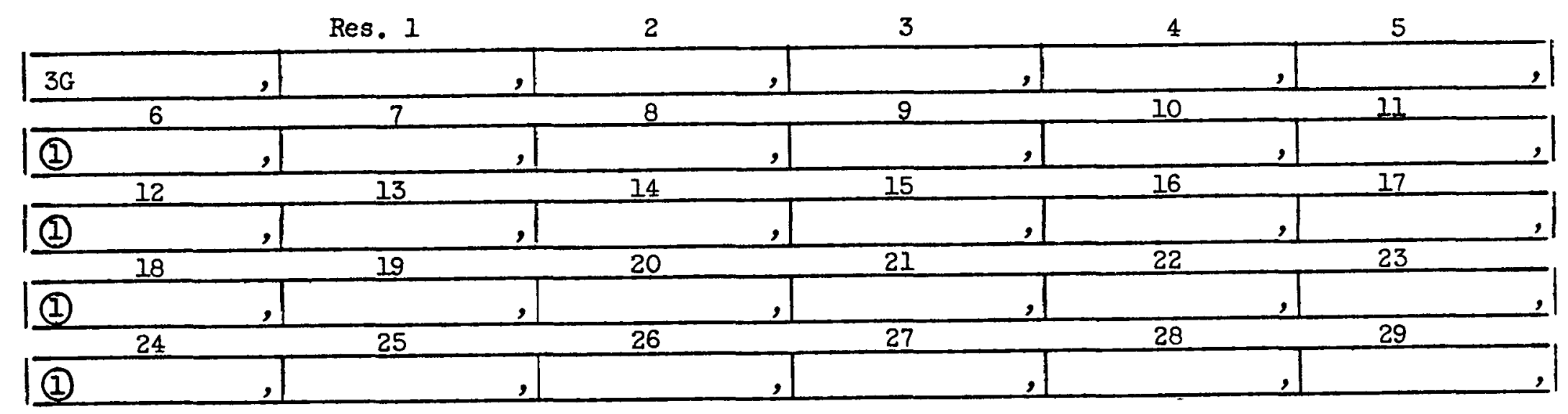




\section{Cross Section Input}

\section{Resonance Data}

Peak Cross Sections

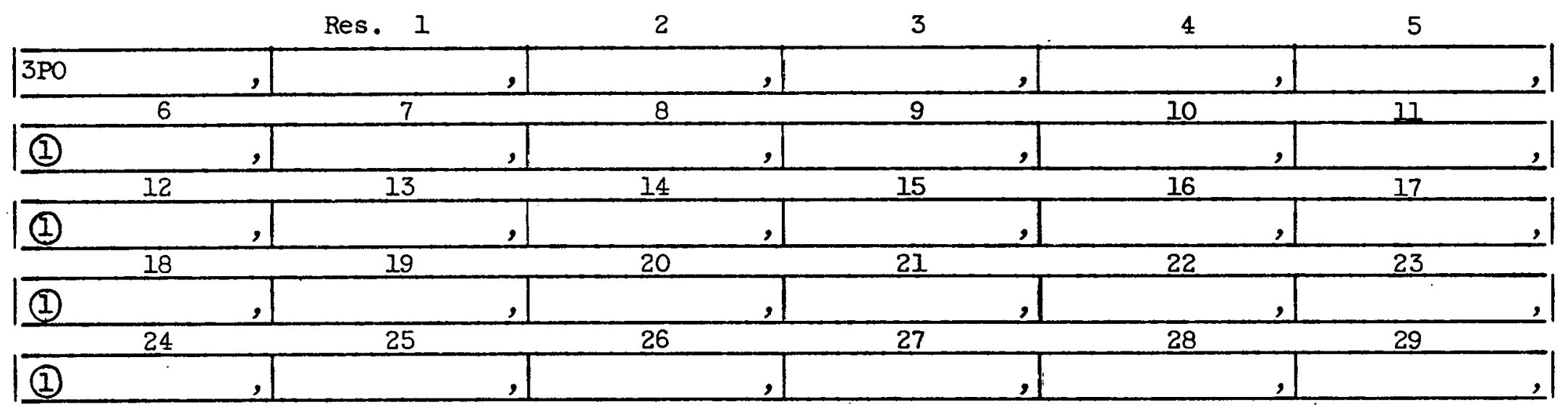

The table above is limited to 200 entries

Doppler Temperature Coefficients

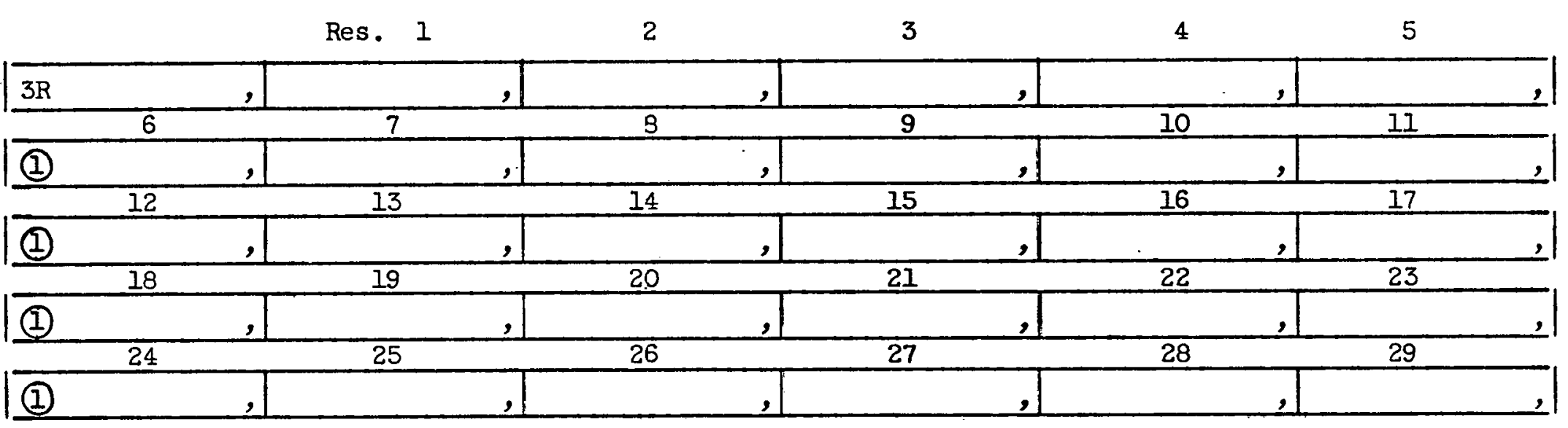


Cross Section Input

$$
\eta=\frac{\Gamma_{F}}{\Gamma_{\gamma}+\Gamma_{F}}
$$

\begin{tabular}{|c|c|c|c|c|c|}
\hline & Res. I & 2 & 3 & 4 & 5 \\
\hline 3ETAR & & & & & \\
\hline 6 & 7 & 8 & 9 & 10 & 11 \\
\hline (1) & & & & & \\
\hline 12 & 13 & 14 & 15 & 16 & 17 \\
\hline (1) & & & & & \\
\hline 18 & 19 & 20 & 21 & 22 & 23 \\
\hline (1) & & & & & \\
\hline 24 & 25 & 26 & 27 & 28 & 29 \\
\hline (1) & & & & & \\
\hline
\end{tabular}

The table above is limited to 200 entries

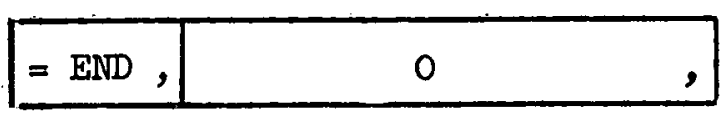


Cross Section Imput

n,y Transfer Matrix

$$
\sigma_{n, \gamma}\left(m / m^{2}\right)
$$

The data should be ordered as shown below

$$
\begin{gathered}
\sigma_{n, \gamma}\left(M_{M I N} / M^{\prime} G_{M I N}\right), \sigma_{n, \gamma}\left(M_{M I N} / M^{\prime} G_{M I N}+1\right), \cdots \cdot \sigma_{n, \gamma}\left(M_{M I N} / M^{\prime} G_{M A X}\right), \\
\sigma_{n, \gamma}\left(M G_{M I N}+1 / M^{\prime} G_{M I N}\right), \sigma_{n, \gamma}\left(M_{M I N}+1 / M^{\prime} G_{M I N}+1\right), \cdots \sigma_{n, \gamma}\left(M_{M I N}+1 / M^{\prime} G_{M A X}\right), \\
\sigma_{n, \gamma}\left(M_{M A X} / M^{\prime} G_{M I N}\right), \sigma_{n, \gamma}\left(M_{M A X} / M^{\prime} G_{M I N}+1\right), \cdots \sigma_{n, \gamma}\left(M_{M A X} / M^{\prime} G_{M A X}\right)
\end{gathered}
$$

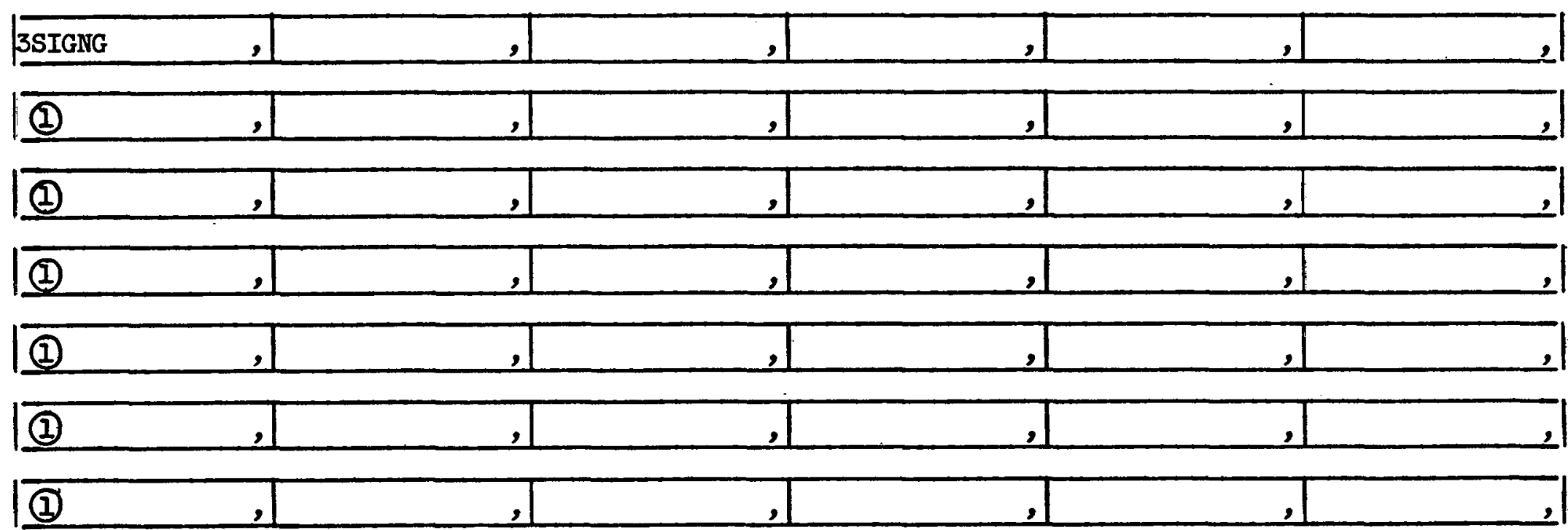

The table above is limited to 680 entries

\begin{tabular}{|l|l|}
\hline$=$ END, & 0 \\
\hline
\end{tabular}


RIDP

Spectral Data Block Input

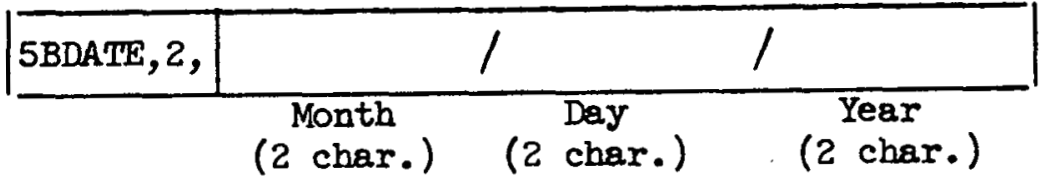

$B$

(delay fraction)

$t_{M}=I / \lambda$

3BETA,

(mean Iife)

$\mathrm{TM}$,

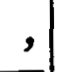


Spectrel Date Block Imput

Ievel Spectral Values

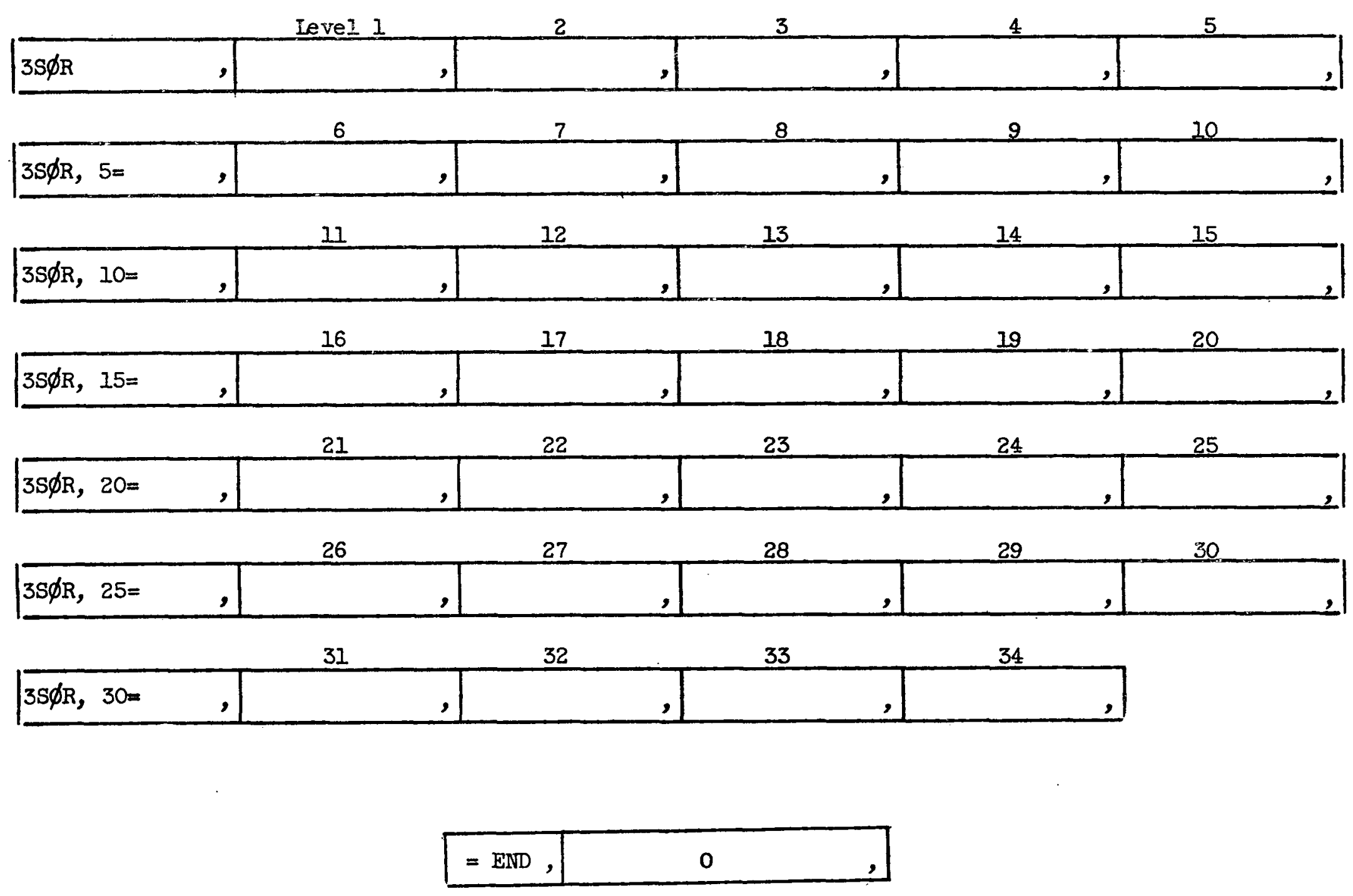




\subsection{Program Source Deck Iisting}

C CONTROL BLOCK

C

COMMON NBI, NB2, NB6, NBPSN,MOMIN,PCODE, PUNCOD, NBDEL, NRRDB, RLOCRC, 1 THERM, MATDIN, NAMTAP, NLEV , NEWTAP, NBA, NBD, NPSH, NOPRNT , NOPUN, NRES,. $2 N T H G R P, N L E V I, N T L E V, N W R$, NWINOX, NWRC2,NWDBRI, N1, N2, N3, N4, NSPECT, 3NWFR, ITAPE, KTAPE, LTAPE, MTAPE, NTAPE, I, MAXIB2, 4MAXDB1, MAXDB, NAXDBM, IDHE11, IDMB I2, IDMB21, IOMB22, AN, RERUN

C

EQUIVALENCE (NBI,FBI), (NBI $(1006), E L, E N E R G Y),(N B 1(1051), F V),(N B 1(1$ 10961 , UL, BDATE), (NBI (1141),EG), (NB1(1186),UG), (NBI(1098),AW), (NB1) (1 $2099), D E N),(N B 1(1100), A 2200)$, (NB1(1101), NDATA), (NBI(1102),A1), (NB1) $31103), B 1),(N B 1(1104), C 1),(N B 1(1105), D 1),(N B 1(1106), A 2),(N B 1(1107)$, $4 B 2),(N B 1(1108), C 2),(N B I(1109), 02),(N B)(I I 10), S, S I G S O, S I G S I, E O, S I G N$ $5 G, 3 E T A),(N B I(1111), T M),(N B 1(1112), S O R),(N B I(1155), X I S),(N B 1(1200)$, $6 T R),(N B I(1245), A),(N B 1(1290), X I S 1),(N B 1(1335), F N U),(N B 1(1380), F), 1$ $7 N B 1(1425), S 1),(N B 1(1470), T),(N B 1(1515), X),(N B 1(1560), X 1),(N B 1(1605$ $8), 3),(N B I(1650)$, PNU), (NBI (1695), ETA)

C

EQUIVALENCE (NBI(1740),TS4500), (NBI(1770),TS4000), (NRI(1800), TS35 $100)$, (NE1 (1830), TS3000), (NB] (1860),TS2500), (N31(1890), TS2000), (NB1) $21920)$, TS1500), (NBI(1950), TS1000), (NB1(1980),TS500), (NB1(2010), TS68 $3),(N B I(1310), R I 0),(N B I(1510), G N)$, (NEI (1710), GG), (NB2(1006), GF), (NB $42(1206), G),(N 32(1406), P 0),(N Q 2(1606), R),(N B 2(1806), E T A R),(N B 2, F B 2)$ $5,(N B P S N(2), B C D D E),(N B P S N(3), B N A N),(N S 6, F B 6)$

C

DIMENSION $\quad$ IIBI $(2100), F B 1(2100), N B 2(2050), F B 2(2050)$, NB6 $(2010)$, IFP6 (2010), NEPSN!(1992), FCODE (1991), BNAN(1990), MOMIN(4980), PCODE(500 2), PUNCOD(500), NBDEL (499), NRRDS(499), RLOCRC(499), THERM(15), MATDIM(1 $30), E L(45), F V(45), U L(45), E G(45), U G(45)$, RDATE(2),S(45), SIGSO(925), SI 4GSI (0.25), EO (200), SIGNG $(025)$, SOR $(45), X I S(45)$, TR $(45), A(45), X I S I(45)$, 5FNU(45), F(45), SI (45), T(45), $,(45), X 1(45), B(45)$, PNU(45), ETA(45), TS 45 $600(30), T 54000(30), T S 3500(30), T 53000(30)$, TS2500(30), TS2000(30), TS15 $700(30)$, TS1000(30), TSEDO(30), TSS8(30), RIO(200), GN(200), GG(200), GF(2 8.00), G(200), PO(200), R (200), ETAR(200), ENERGY (45), NAMTAP(3)

C

1 CALL INIT IF (RERUN) $11,12,11$

11 CALL TRYOVR GO TO 5

12. IF (NEWTAP) $2,3,2$

C SET UP INDEX RECORD FOR NEN TAPE

2 CALL INDXNT GO TO 4 REVISE OLD INDEX RECORD

3 CALL INDXRV WRITE INDEX RECORD ON MTAPE, NTAPE

4 CALL NMTPRC (NWINDX)

C WRITE AND PUNCH INDEX RECORD CALL WRINDX

C COMPILE RECORD 2

CALL REC2 
C DATA BLOCK CONTROL ROUTINE

C 5 CALL DATACT

CALL FINREC

CALL EXIT

END $(0,0,0)$ 
SUBROUTINE EROR (N)

THIS ROUTINE IS INTENDED TO PROVIDE AN ABSOLUTE LOCATION OF

C ISTOPS RESULTING FROM DIFFERENT TYPES OF. ERRORS INDICATED BELOW.

C 2 AS THE REASSEMBLY OF THE CONTROL ROUTINE IS NOT ANTICIPATED. THE

C 3ORJECT DECK FOR THIS ROUTINE MAY EE PLACED IMMEDIATELY BEHIND

C 4THE DECK FOR THE CONTROL ROUTINE TO GIVE THE DESIRED ABSOLUTE

C. 5 LIST OF STOPS. AS OF THIS ASSEMBLY, THE FOLLONING STOPS ARE

C GOBTAINAELE - THE RENIAINDER MAY BE OBTAINED BY USING

C TTHE APPROPRIATE ARGUMENT.

C

2000 If $(\mathrm{N}-1) \quad 2021,2022,2001$

$200 I$ IF $(\mathrm{N}-3) \quad 2023,2024,2002$

2002 IF $(N-5) \ldots 2025,2026,2003$

2003 IF $(N-7) \quad 2027,2028,2004$

2004 IF $(N-9) \quad 2029,2030,2005$

2005 IF $(N-11) \quad 2031,2032,2006$

2006 IF $(\mathrm{N}-13) \quad 2033,2034,2007$

2007 If $(\mathrm{N}-15) \quad 2035,2036,2008$

2008 IF $(\mathrm{N}-17), 2037,2038 \cdot 2009$

2009 IF $(N-19) \quad 2039,2040,2040$

2021 CALL EXIT

$C$ DIP ERROR FIRST RECORD (INIT ROUTINE)

2022 CALL ERRORA

CALL EXIT

C ... DIP ERROR IN DATA FOR RECORD. 2. (REC2 ROUTINE)

2023 CALL ERRORA

C DIP ERROR IN SPECTRAL DATA RECORD IINPTDB ROUTINE - STATEMENT 2402

2024 CALL ERRORA

CALL EXIT

C DIP ERROR IN DATA FOR RECORD 1 OF CROSS SECTION DATA

C IBLOCK (INPTDB ROUTINE - STATEMENT 2403)

2025 CALL ERRORA

CALL EXIT

C DIP ERROR IN DATA FOP SIGSOO RECORD IINPTDB ROUTINE -

C ISTATEMENT 30311

2226 CALL ERRORA

CALL EXIT

c. DIP ERROR IN DATA FOR SIGSI RECORD (INPTDB ROUTINE -

C ISTATEMENT 30711

2027 CALL ERRORA

CALL EXIT

C. .... DIP ERROR IN RESONANCE DATA (INPTOE ROUTINE STATEMENT 31120 )

2028 CALL ERRORA

CALL EXIT

C DIP ERROR IN DATA FOR SIGNG RECORD IINPTDE ROUTINE -

C ISTATEIAENT 31511

2029 CALL ERRORA

CALL. EXIT

C ITAPE COULD NOT BE READ

?. 330 CALL ERRORA

CALL EXIT

KTAPE COULD NOT RE READ

2031 CALL ERRORA 
CALL EXIT

BOTH MTAPE AND NTAPE FAILED .. TO COMPILE

2032 CALL ERRORA

CALL EXIT

C KTAPE FAILED TO COMPILE

2033 CALL ERRORA

CALL EXIT

C. DIMENSIONS IN MOMIN TABLE TOO LARGE.

2034 CALL ERRORA

CALL EXIT.

C TOO MANY LEVELS

2035 CALL ERRORA

CALL EXIT

C. ATIEMPTED TO DELETE NON-EXISTENT DATA BLOCK

CALL EXIT

c. ATTEMPTED TO RELOCATE DELETED BLOCK

2037 CALL ERRORA

CALL EXIT

C 2038 CALL ERRORA

CALL EXIT

2039 CALL ERRORA

... CALL EXIT

2040 RETURN

END $10,0.01$ 


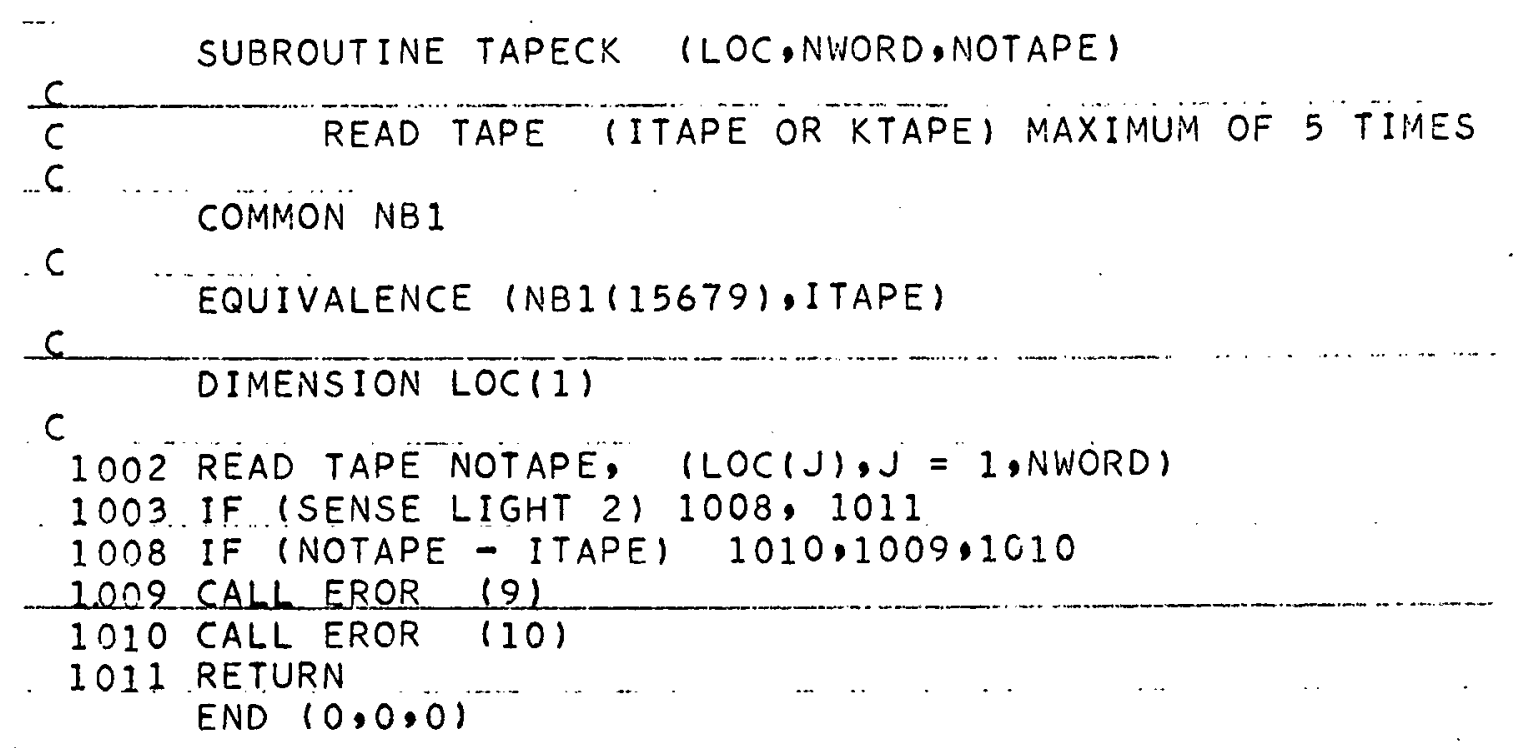




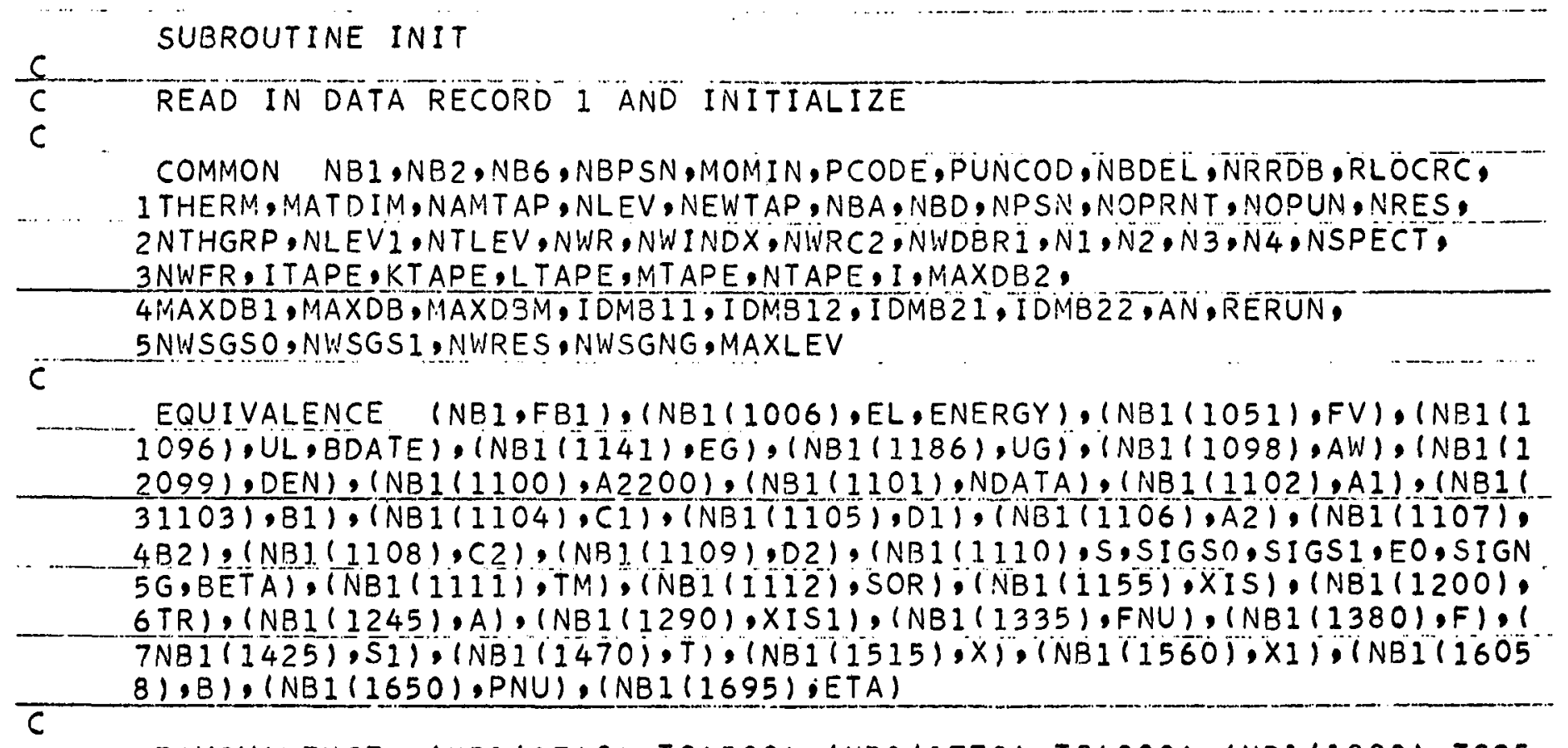

EQUIVALENCE (NBI (1740),TS4500), (NBI(1770),TS4000), (NBI(1800),TS35 $100),(N B I(1830), T S 3000)$, (NBI (1860), TS2500), (NB1(1890), TS2000), (NBI) $21920), T S 1500),(N B 1(1950), T S 1000),(N B 1(1980), T S 500),(N B 1(2010)$, TS68 $3),(N B 1(1310), R I 0),(N B I(1510), G N),(N B 1(1710), G G),(N B 2(1006), G F),(N B$ $42(1206), G),(N B 2(1406), P 0),(N B 2(1606), R),(N B 2(1806), E T A R),(N B 2, F B 2)$ 5 . (NBPSN $(2), B C O D E),(N B P S N(3), B N A M),(N B 6, F B 6)$

C

DIMENSION NBI(2100), FBI(2100), NB2(2050),FB2 2050$), N B 6(2010)$ IFB6 (2010), NBPSN(1992), BCODE (1991), BNAM(1990), MOMIN(4980),PCODE (500 2), PUNCOD $(500)$, NBDEL (499), NRRDB (499), RLOCRC(499), THERM(15), MATDIM(I $30)$, EL (45), FV (45), UL (45), EG(45),UG(45), BDATE(2),S(45), SIGSO (925), SI 4GS1(925), EO(200), SIGNG $(925)$, SOR(45), XIS(45), TR(45),A $(45), X I S 1(45)$, 5FNU (45), $F(45), S 1(45), T(45), X(45), X 1(45), B(45), P N U(45), E T A(45), T S 45$ $600(30)$, TS $4000(30)$, TS $3500(30)$,TS $3000(30)$, TS2500(30), TS2000(30), TSI5 $700(30)$, TS $1000(30)$, TS $500(30), T S 63(30), R I O(200), G N(200), G G(200), G F(2$ $800), G(200), P O(200), R(200), E T A R(200)$, ENERGY (45), NAMTAP(3)

$c$

THERM(1) $=4500.0$

$\operatorname{THERM}(2)=4000.0$

THERM(3) $=3500.0$

$\operatorname{THERM}(4)=3000.0$

THERM (5) $=2500.0$

$\operatorname{THERM}(6)=2000.0$

$\operatorname{THERM}(7)=1500.0$

$\operatorname{THERM}(8)=1000.0$

$\operatorname{THERM}(9)=500.0$

$\operatorname{THERM}(10)=68.0$

$C^{-}$NO. THERMAL GROUPS, NÖ: WORDS ON INDEX AND FINAL RECORDS NTHGRP $=10$

NWINDX $=2000$

NWFR $=1000$

C SET UP CROSS SECTION DATA BLOCK MAXIMUM DIMENSIONS

MAXLEV $=45$

NWSGSO $=626$ 


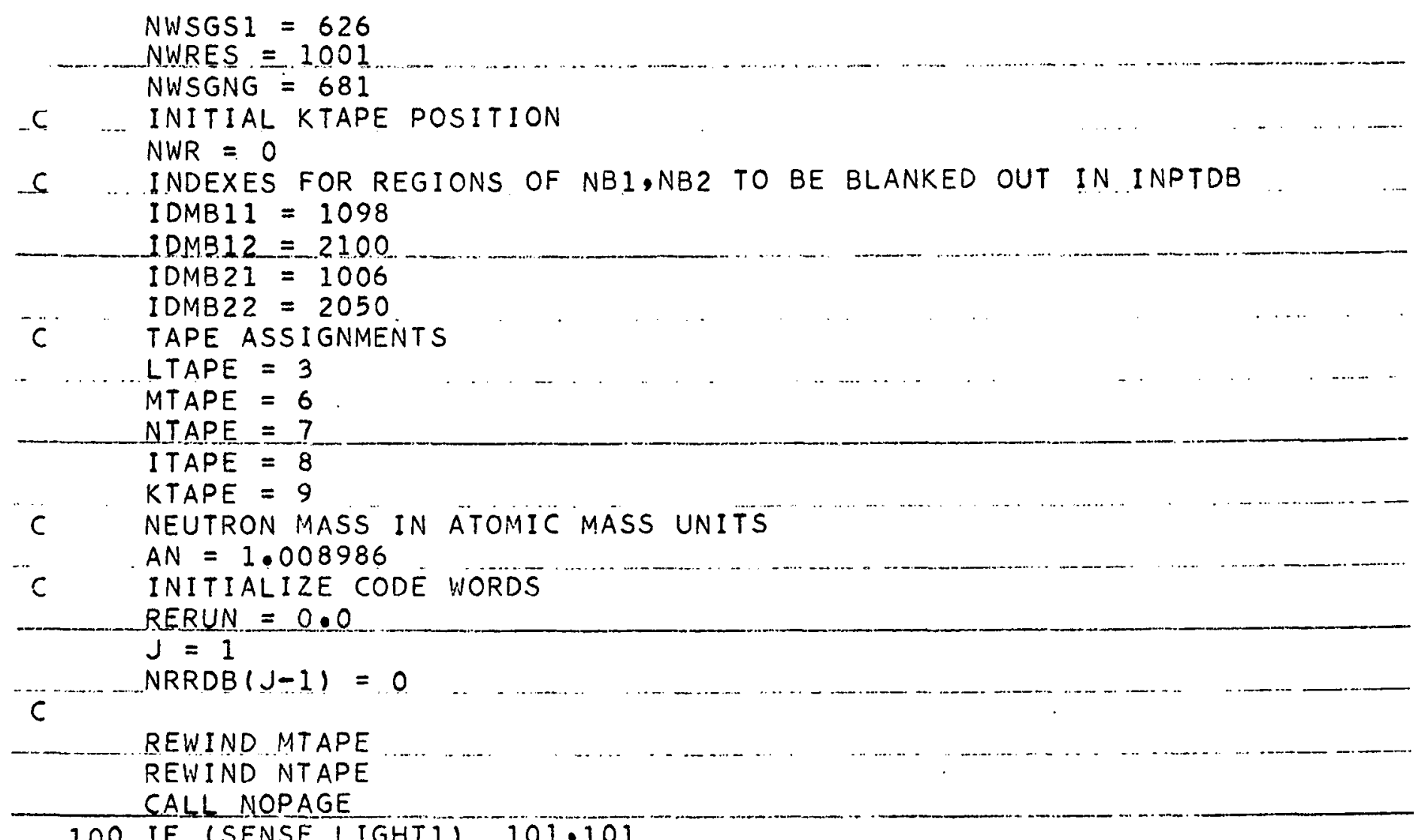

C READ IN FIRST RECORD OF DATA

101 READ DIP NAMTAP, NLEV, NEWTAP, NBA, NBD, NBDEL, NPSN, BNAM, BCODE,NBPSN, IMOMIN , NOPRNT, NOPUN, PCODE, PUNCOD, RERUN,BDATE, END IF (SENSE LIGHT I) 102,103

102 CALL EROR (1)

103 IF (RERUN) $1045,104,1045$

C SET UP HEADING FOR INDEX RECORD

104 NBI $(1)=$ NAMTAP $(1)$

$\ldots N B 1(2)=N A M T A P(2)$

NB $1(3)=$ NAMTAP $(3)$

NB1 $141=$ NLEV

C NLEVI $=$ NLEV +1

C TOTAL LEVELS $=$ NO. EPITHERMAL + NO. THERMAL̈ NTLEV = NLEV + NTHGRP

C NO WORDS - RECORD 2, CROSS-SECTION DATA BLOCK RECORD I, SPECTRAL IDATA ELOCK

NWRC2 $=2 * N T L E V+3 * N L E V+3$

NWDBR $1=6 *$ NTLEV + 30*NTHGRP + 16

NSPECT $=$ NTLEV 6

C MAXDB = MAX. NO. OF DATA BLOCKS, OTHERS ARE ASSOCIATED CONSTANTS MAXDB2 $=($ NWINDX) $/ 4$ $M A X D B 1=M A X D B 2-1$ $M A X D S=M A X D B 2 \quad 2$ MAXDBM = MAXDB2 - 3

GO TO 111

= CHECK DATA FOR ERRORS

$104500108 \mathrm{~J}=1$ N NBA 
IF ( (MOMIN $(10 * J-8)-M O M I N(10 * J-9)) *(M O M I N(10 * J-7)-M O M I N(10 * J-9))$ $1-N W S G S Q+11-105,105.109$ 105 IF ( MOMIN(10*J-5)-MOMIN $(10 * J-6)) *(M O M I N(10 * J-4)-M O M I N(10 * J-6))$ 1- NWSGS1 + 11 106,106,109

106 IF $(5 * M O M I N(10 * J-3)$ - NWRES +11$) 107,107,109$

-10.7 IF ( MOMIN (10*J-1) - MOMIN (10*J-2)) ) (MOMIN (10*J) -MOMIN(10*J-2))

1-NWSGNG + 1) $108,108,109$

108 CONTINUE IF (NLEV - MAXLEV) I11.111.110

109 CALL EROR (13)

110 CALL EROR (14)

111 RETURN

END $(0,0,0)$ 


\section{SUBROUTINE INDXNT}

\section{SET UP INDEX RECORD FOR NEW TAPE}

COMMON NB1,NB2, NB6, NBPSN,MOMIN,PCODE, PUNCOD, NBDEL, NRROB, RLOCRC, 1 THERM, MATDIM, NAMTAP, NLEV, NEWTAP, NBA, NBD, NPSN, NOPRNT, NOPUN, NRES, $2 N T H G R P, N L E V 1, N T L E V, N W R, N W I N D X, N W R C 2, N W D B R 1, N 1, N 2, N 3, N 4, N S P E C T$, 3NWFR, ITAPE, KT APE, L TAPE,MTAPE, NTAPE, I MAXDB2,

4 MAXDBI, MAXDB, MAXDBM, IDMBII, IDMBI2, IDMBZI, IDIVBZZ, AN

C....

EQUIVALENCE (NBI,FBI), (NB1(1006), EL,ENERGY), (NBI(1051),FV), (NBI(I $1096), U L, B D A T E),(N B 1(1141), E G),(N B 1(1186), U G),(N B 1(1098), A W),(N B 1$ (1) $2099), D E N),(N B 1(1100), A 2200)$, (NB1(1101), NDATA), (NB1(1102),A1), (NB1) $32103), 81),(N B 1(1104), C 1),(N B 1(1105), 01),(N B 1(1106), A 2),(N B 1(1107)$ $4 B 2),(N B I(1108),(2),(N B 1(1109), 02),(N B 1(1110), S, S I G S O, S I G S 1, E O, S I G N$ $5 G$.BETA), (NBI(1111), TM), (NBI(1112), SOR), (NB1(1155),XIS), (NBI(1200), $6 T R),(N B I(1245), A),(N B 1(1290), X I S 1),(N B 1(1335), F N U),(N B 1(1380), F), 1$ $7 N B 1(1425), S 1),(N B 1(1470), T),(N B 1(1515), X),(N B 1(1560), X 1),(N B 1(1605$. $8), B),(N B 1(1650), P N U),(N B 1(1695), E T A)$

$c$

EQUIVALENCE (NBI(1740),TS4500), (NB $1(1770), T S 4000),(N B 1(1800), T S 35$ $100)$, (NB1) 1830$)$, TS3000), (NB1 (1860), TS2500), (NB1(1890), TS2000), (NBI) $21920)$,TS1500), (NBI(1950), TS1000), (NBI(1980),TS500), (NBI(2010),TS68 3), (NB1 (1310),RI0), (NBI (1510),GN), (NB1 (1710),GG), (NE2(1006),GF), (NB $42(1206), G),(N B 2(1406), P 0),(N B 2(1606), R),(N B 2(1806), E T A R),(N B 2, F B 2)$ $5,(N B P S N(2), B C O D E),(N B P S N(3), B N A M),(N B 6, F B 6)$

C

DIMENSION NBI $(2100), F B I(2100), N B 2(2050), F B 2(2050), N B 6(2010)$,

IFB6(2010), NBPSN(1992), BCOOE(1991), BNAMI1990), MOMIN (4980), PCODE(500 2). PUNCOD (500), NBDEL (499), NRRDB (499), RLOCRC(499), THERM( 15 ), MATDIM(1 $30), E L(45)$, FV (45), UL (45), EG(45),UG(45),BDATE (2),S(45), SIGSO(925),SI 4GSI (925), EO (200), SIGNG (925), SOR (45),XIS(45),TR (45),A $(45), X I S I(45)$, $5 F N U(45), F(45), S 1(45), T(45), X(45), X 1(45), B(45), P N U(45), E T A(45), T S 45$ $600(30), T S 4000(30)$, TS3500(30),TS3000(30),TS2500(30),TS2000(30),TS15 $700(30), T S 1000(30)$, TS500(30),TS68(30),RIO(200), GN(200), GG(200), GF(2 $800), G(200), P 0(200), Q R(200)$,EIAR(200), ENERGY (45), NAMTAP(3)

C

120 NB1 $181=3$

C SET UP LOOP

DO $131 \quad I=1, M A X D B 1$

IF $(\bar{I}-\mathrm{NBA}) \quad 1201,1201,130$

C SEARCH FOR INFO FOR DATA BLOCK I

$1201001202 \mathrm{~J}=1, N B A$

IF $(I-\operatorname{NBPSN}(4 * J-3)) \quad 1202,1203,1202$

1202 CONT INUE

C CALCULATE NUMBER OF RECORDS IN DATA BLOCK I

$1203 K=1$

IF (MOMIN(10*J 9)) $122,123,122$

$122 \dot{K}=K+1$

123 IF (MOMIN(10*J 6)) 124,125:124

$124 K=K+1$

125 IF (MOMIN(10*J 3)) $126,127,126$

$126 \bar{K}=K+1$

127 IF (MOMIN(10*J 2)) $128,129,128$

$128 K=K+1$ 
C STORE INFO FOR DATA BLOCK I IN INDEX RECORD

129 EBI $(4 * 1+1)=$ BNAM $(4 * 1-3)$

$F B 1(4 * I+2)=\operatorname{BNAM}(4 * I-2)$

FB $1(4 * I+3)=B C O D E(4 * I-3)$

$N B 1(4 * I+8)=N B \cdot 1(4 * I+4)$

GO TO 131

C STORE REMAINDER ZEROS

$130 \mathrm{NB} 1(4 * I+1)=0$

NB $1(4 * I+2)=0$

$N B I(4 * I+3)=0$

NBI $(4 * I+8)=0$

131 CONTINUE

132 RETURN

END $(0,2,0)$ 
$-$

SUBROUTINE INDXRV

\section{REVISE THE OLD INDEX RECORD}

$c$

COMMON NB1,NB2,NB6,NBPSN,MOMIN, PCODE,PUNCOD,NBDEL,NRRDB,RLOCRC,

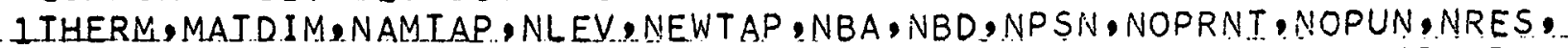
2 NTHGRP, NLEV I, NTLEV, NWR, NWINDX, NWFC 2 , NWDBR I, NI, N2, N3, N4, NSPECT,

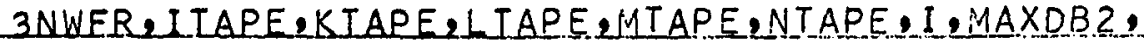
4MAXDB 1, MAXDB, MAXDBM, IDMB 11, IDMB I2, IDMB21, IDMB22, AN, RERUN,NCODOT, 5 FB $3 . N B$

C

EQUIVALENCE (NB1,FB1) (NB111006),EL,ENERGY),(NB1)(1051),FV), (NB1(1 1096), UL, BDATE), (NBI(1141),EG), (NBI(1186),UG), (NBI(1098),AW), (NBI(1

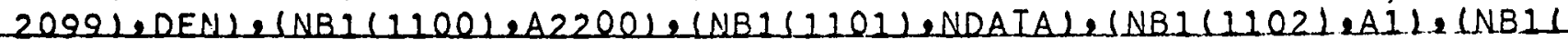
$31103), B 1),(N B 1(1104), C 1),(N B 1(1105), D 1),(N B 1(1106), A 2),(N B 1(1107)$, 4B2). (NBI (1108).C2), (NBI (1109),D2). (NBI11110),S,SIGSO,SIGSI,EO,SIGN $5 G, B E T A),(N B 1(1111), T M),(N B 1(1112)$, SOR), (NB1(1155),XIS), (NB1(1200),

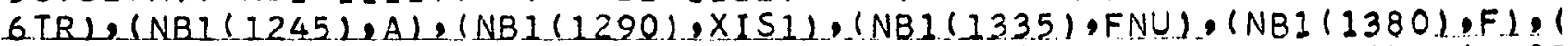
$7 N B 1(1425), S 1),(N B 1(1470), T),(N B 1(1515), X),(N B 1(1560), X 1),(N B 1(1605$ $8), B$ ) (NB1(1650), PNU), (NB1(1695), ETA)

C

EQUIVALENCE (NBI $(1740), T S 4500),(N B 1(1770), T S 4000)$, (NBI (1800),TS35 $100)$, (NB1 (1830), TS3000), (NB1(1860),TS2500), (NBI(2890),TS2000), (NBI)

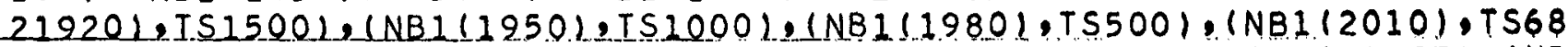
$3),(N B 1(1310), R I 0),(N B 1(1510), G N),(N B 1(1710), G G),(N B 2(1006), G F),(N B$ $42(1206) \cdot G),(N B 2(1406), P 0),(N B 2(1606), R),(N B 2(1806) \cdot E T(A R),(N B 2, E B 2)$ $5,(N B P S N(2), B C O D E),(N B P S N(3), B N A M),(N B 6, F B 6)$

$c$

DIMENSION NBI (2100), FBI $(2100)$ NB2 $(2050), F B 2(2050), N B 6(2010)$ IEB6 (2010),NBPSN(1992), BCODE (1991), BNAM(1990), MOMIN(4980),PCODE (500 2), PUNCOD( 500$)$, NBDEL (499), NRRDB (499), RLOCRC(499), THERM( 15$)$, MATDIMII $30), E L(45), F V(45), U L(45), E G(45), U G(45), B D A T E(2), S(45), S I G S 0(925), S I$ 4GS1 (925), EO(200), SIGNG (925), SOR (45), XIS(45), TR(45), A(45), XISI (45), SFNU (45), F $(45), S 1(45), T(45), \times(45), \times 1(45), B(45)$, PNU(45), ETA(45),TS45 $600(30)$, TS $4000(30)$, TS $3500(30)$, TS $3000(30)$, TS2500(30), TS2000(30), TS15 $70.0130)$, IS $10.00(30)$, TS500(30), TS68(30), RIO(200), GN(200), GG(200), GF(2 $800), G(200), P O(200), R(200)$, ETAR (200), ENERGY $(45)$, NAMTAP (3)

DIMENSION FB3(500) NB4 $(500)$

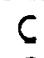

REWIND ITAPE

C.......READ IN OLD INDEX RECORD

CALL TAPECK (NBI, NWINDX, ITAPE)

DO $13999, L=1$ MAXDB

$F B 3(L)=F B 1(4 * L+3)$

$13999 N B 4(L)=N B I(4 * L+4)$

C CHECK FOR EXISTENCE OF BLOCKS TO BE DELETED, RELOCATED IF (NBD) . $14000,14007,14000$

$14000 K=1$

DO $14005 \quad I=1$ MAXDB

IF $(N B 1(4 * I+3)) 14001,14006,14001$

14001 DO $14004 \quad L=1$ NBD

IF (NBI(4*I+3) NBDFL(L)) $14004,14002,14004$

4002 IF $(K-N B D) \quad 14003,14007,14007$

$14003 K=K+1$ 
GO TO 14005

14004 CONIINUE

14005 CONTINUE

14006 CALL EROR (15)

14007 IF (NPSN) $14008,1410,14008$

$14.008 . K=1$

DO 14017 I $=1$ MAXOB

IF $(N B 1(4 * I+3)) \quad 14009,14018,24009$

14009 DO $14014 \quad L 1=1$,NPSN

$L=N B A+L 1$

IF (FBI(4*I+3) BCODE (4*L 31$)$ 14014,14010,14014

14010 IF $(\mathrm{NBD}) \quad 14011,14015914011$

14011 DO $14012 M=1, N B D$

If $(N B I(4 * I+3)$ NBDEL (M) 14012.14013 .14012

14012 CONTINUE

GO TO 14015

14013 CALL EROR (16)

14014 CONIINUE

GO TO 14017

14015 IF $(K-N P S N) \quad 14016,14100,14100$

$14016 K=K+1$

14017 CONIINUE

14018 CALL EROR (17.)

C RELOCATE DATE BLOCKS ON ITAPE BY FIRST PLACING ON KTAPE

IF (NPSN) $14100,1410,14100$

14100 READ TAPE ITAPE

REWIND KTAPE

$K=1$

DO $14116 \quad I=1, M A X D B$

$N=N B I(4 * I+8) \quad N B 1(4 * I 4)$

$J 1=N B A+1$

$12=N B A+N P S N$

DO $14101 \mathrm{~L}=\mathrm{J} 1 . \mathrm{J} 2$

IF (FBI $(4 * I+3) \quad B C O D E(4 * L-3)) \quad 14101,14102,14101$

14102 CONTINUE

GO TO 14113

C RELOCATE - CHECK TO SEE IF SPECTRAL OR CROSS SECTION DATA

14102 IF (FBI $(4 * I+3) \quad 500.0) \quad 14104.14103,14103$

C SPECTRAL

14103 CALL KTAPRC (NSPECT)

GO TO 14116

C CROSS-SECTION

14104 CALL KTAPRC (NWDBRI)

CALL DIMEN (NB2)

IF (N1) $14105,14106,14105$

14105 CALL KTAPRC (N1)

14106 IF (N2) $14107,14108,14107$

14107 CALL KTAPRC \{N2\}

14108 If (iN3) $14109+14110.14109$

14109 CALL KTAPRC (N3)

14110 IF (N4) 14111,14112,14111

14111 CALL KTAPRC (N4)

c

COPY INTO THE NRRDB REGION THE PHYSICAL RECORD NUMBER OF THE RELOCATED BLOCKSNOW ON THE KTAPE. THIS INFO WILL BE USED IN SUBROUTINE DATACT FOR TAPE POSITIONING. 
$14112 \operatorname{NRRDB}(K)=N+N R R D B(K-1)$

C STORE CODE NUMBER OF THIS QLOCK IN RLOCRC REGION $R L O C R C(K)=F B 1(4 * I+3)$

$K=K+1$

IF $(K-N P S N) \quad 14116,14116,1410$

14113 IF (N) $14116,14116,14114$

$141140014115 \mathrm{M}=1, \mathrm{~N}$

14115 READ TAPE ITAPE

14116 CONTINUE

C WRITE INDEX DATA IN NB2 BLOCK

$1410 \mathrm{~N}=\mathrm{NBA}+\mathrm{NBD}$

14117 IF... (N) $14120,14120,14118$

$141180014119 \quad I=1$, NWINOX

$14112 N B 2(1)=N B I(1)$

C SET UP RECORD NUMBER FOR THE FIRST DATA BLOCK

$N B 1(8)=3$

$C$ CHECK FOR DELETING

14120. IF (NBD) 1...111.15021411

$\frac{c}{C}$ DELEIION LOOP

$1411 K=1$

DO 144 I $=1, M A X D B$

C CHECK FOR DELETING THIS BLOCK

$J=I-K$

DO $142 \quad \mathrm{~L}=1, \mathrm{NBD}$

IF (NB2(4*I+3) NBDEL(L) $142,147,142$

142 CONTINUE

C DO NOT DELETE THIS BLOCK

$N B 1(4 * J+5)=N B 2(4 * I+1)$

$N B 1(4 * J+6)=N B 2(4 * I+2)$

$\frac{N B 1(4 * J+7)}{C}=\frac{N B 2(4 * I+3)}{\text { IS REST OF INDEX INFO ZEROS }}$

IF (NB2 $(4 * I+8) 1)$ I. 43.145 .143

$C$ UPDATE RECORD NUMBER

$143 N B 1(4 * J+12)=N B 1(4 * J+8) \ldots N B 2(4 * I 8)=N B 2(4 * I+4)$

144 CONTINUE GO IO 146

C SET INFO TO ZERO

$145 N B 1(4 * J+12)=0$.

146 NBI $(4 * J+9)=0$

$N B I(4 * J+10)=$ ?

$N B I(4 * J+12)=0$

C CHECK FOR IERMINATION OF LOOP

IF $(J-M A X D B M+N B A) \quad 1465,150,1465$

$1465 J=J+1$

GO TO 145

C DELETE THIS_BLOCK

$147 K=K+1$

CO TO 144
$C$ ADD TO INDEX RECORD
$C$ CHECK FOR ADDING
C.... IF (NBA) I5I,175,151 
C ADD IF DELETED SOME BLOCKS, RESTORE UPDATED INFO TO NB2

C...151.IF (NBD) .153,155,153

153 DO $154 \mathrm{~J}=1$,NWINDX

$\ldots 154 \operatorname{NB2}(J)=N B 1(J)$

C ADDITION LOOP

$155 K=1$

DO $168 \quad I=1, M A X D B$

C CHECK FOR ADDING AT THIS POSITION $J=I-K$

DO. $156 \ldots L=1, N B A$

1.56 CONTINUE

GO TO 166

c ADD AI THIS POSITION

157 FB1 $(4 * I+1)=\operatorname{BNAM}(4 * L-3)$

$F B I(4 * I+2)=\operatorname{BNAM}(4 * L-2)$

$F B 1(4 * I+3)=B C O D E(4 * L-3)$

C._CALCULATE. THE TOTAL NUMBER OF. RECORDS IN THE ADDED DATA BLOCK $M=O$

IF $(M O M I N(10 * 291) \quad 158,159,158$

$158 M=M+1$

- 159 IF (MOMIN(10*L 6)) . 160,161,160

$160 M=M+1$

- 161_IF (MOMIN(10*L 3)) $162,163,162 \ldots$

$162 M=M+1$

163 IE INOMIN (10*L 2)L $164,165.164$

$164 M=M+1$

C UPDATE RECORD NUMBER

$165 N B 1(4 * I+8)=N B I(4 * I+4) M+1$

$K=K+1$

GO TO 168

C COPY OLD INDEX INFO AND UPDATE THE RECORD NUMSER

$\begin{aligned} 166 \quad N B I(4 * I+1) & =N B 2(4 * J+5) \\ N B I(4 * I+2) & =N B 2(4 * J+6) \\ N B 1(4 * I+3) & =N B 2(4 * J+7) \\ \text { C. IS REST OF INDEX RECORD ZEROS } & \text { IF }(N B 2(4 * J+12)) \quad 167,169,167\end{aligned}$

$-167 N B 1(4 * I+8)=N B 1(4 * I+4) \quad N B 2(4 * J+12)-N B 2(4 * J+8)$

168 CONTINUE

6 SET INFO TO ZERO

$169 N B 1(4 * I+8)=0$

-...170. NBI $(4 * I+5)=0$

$N B 1(4 * I+6)=0$

$N B 1(4 * I+7)=0$

C CHECK FOR TERMINATION OF LOOP

IF $(I-M A X D B) \quad 171,175,175$

$171 \mathrm{I}=\mathrm{I}+1$

GO TO 169

$c$

C RELOCAIE INDEX RECORD INFO.

c

$c$

C CHECK FOR RELOCATION

c

175 IF (NPSN) $179,208,179$

RELOCATE 
C SET INDEX FOR STORING RELOCATION INFO IN LOWER NBG REGION $179 N G=M A X D B 2-N P S N$

C RELOCATION LOOP

$K=1$

DO $200 \quad I=1, M A X D B$

C CALCULATE THE NUMBER OF RECORDS FOR THIS DATA BLOCK

$N=N B I(4 * I+8) \quad N B I(4 * I 4)$

C CHECK FOR RELOCATING THIS DATA BLOCK

$J=N B A+1$

$J 1=N B A+N P S N$

DO $180 \quad L=J, J 1$

IF (FBI $(4 * I+3) \quad B C O D E(4 * L-3)) \quad 180,194,180$

180 CONTINUE

GO TO 125

C STORE INDEX INFO IN LOWER NBG REGION

$194 M=N G+K$

$N B 6(4 * M+1)=N B I(4 * I+1)$

$N B 6(4 * M+2)=N B 1(4 * I+2)$

$N B 6(4 * M+3)=N B I(4 * I+3)$

$N B 6(4 * M+4)=N$

$K=K+1$

GO TO 200

C DO NOT RELOCATE THIS BLOCK - COPY INDEX INFO INTO UPPER NB6

$195 L=I-K+1$

$N B 6(4 * L+1)=N B I(4 * 1+1)$

$N B 6(4 * L+2)=N B I(4 * 1+2)$

$N B 6(4 * L+3)=N B 1(4 * I+3)$

$N B 6(4 * L+4)=N$

200 CONTINUE

C INDEX INFO FOR RELOCATED RECORDS ARE NOW IN LOWER NB6 REGION

C AND FOR NON-RELOCATED BLOCKS IN UPPER NB6. RECORD NUMBERS

$C$ ARE REPLACED BY ACTUAL NUMBER OF OF RECORDS IN THE DATA BLOCK.

$C$ NEW INDEX RECORD IS COMPILED BY FIRST COMBINING THE INFO IN

$C$ THESE TWO REGIONS OF NB6 INTO NB2, KEEPING THE ACTUAL NUMBER

C OF RECORDS IN THE DATA BLOCK IN PLACE OF THE RECORD NUMBER.

$c$

$K=1$

$00204 \mathrm{I}=1$, MAXDS1

$D O 201 L=1, N P S N$

$N A=N B A+L$

IF $(I=N B P S N(4 * N A-3)) \quad 201.202 .201$

201 CONTINUE

GO TO 203

$2.02 .002021 \mathrm{~J}=1 . \mathrm{NPSN}$

$L=N G+J$

2021 CQNTINUE

$2022 N B 2(4 * I+I)=N B 6(4 * L+I)$

$N B 2(4 * I+2)=N B 6(4 * L+2)$

$N B 2(4 * I+3)=N B 6(4 * L+3)$

$N B 2(4 * I+4)=N B 6(4 * L+4)$

$K=K+1$

GO TO 204

$203 M=1-K+1$ 


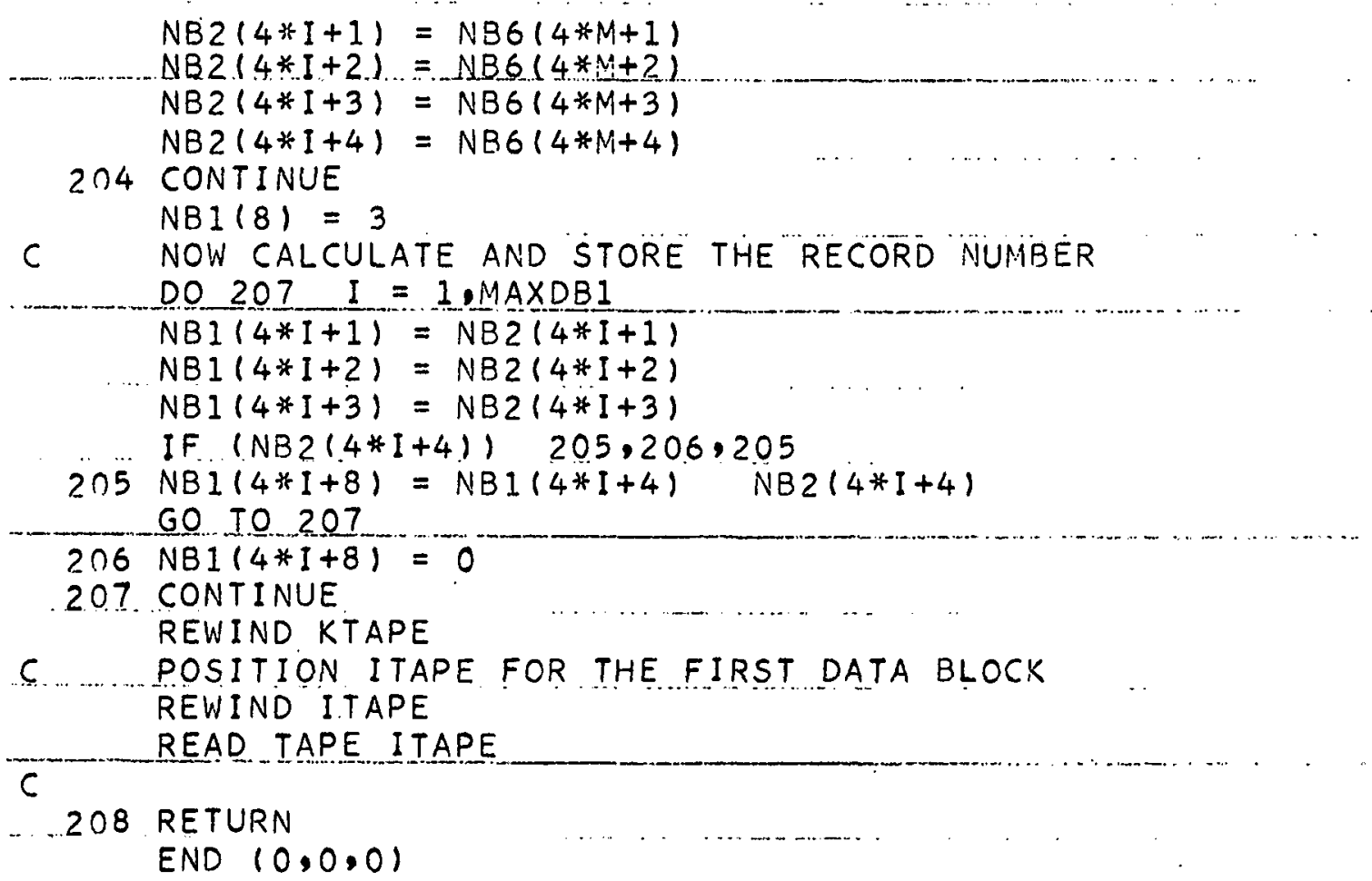


SUBROUTINE REC2

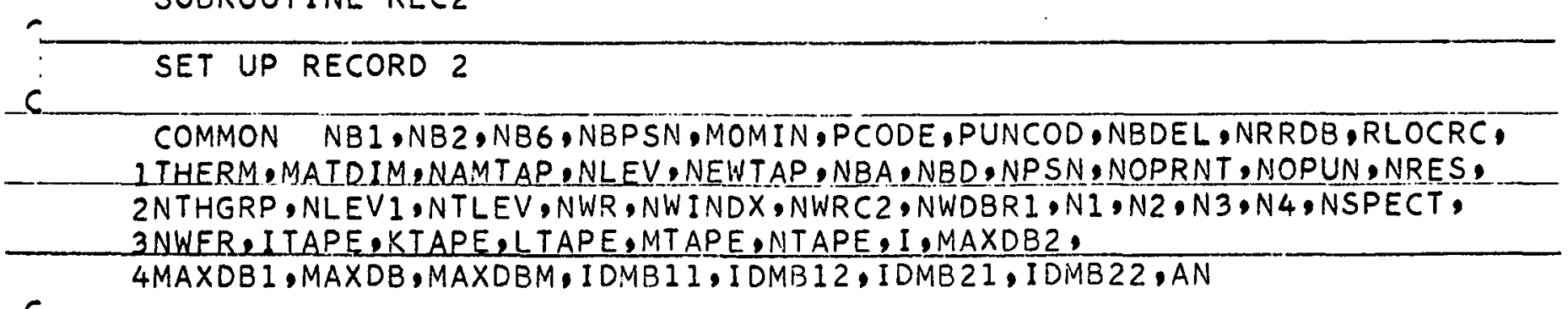

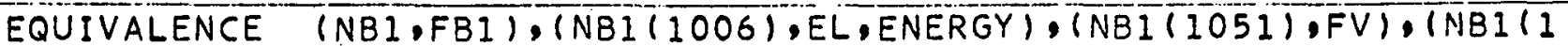

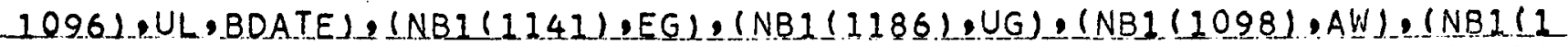
$2099), D E N),(N B 1(1100), A 2200),(N B 1(1101), N D A T A),(N B 1(1102), A 1),(N B 1)$ $31103), B 2) \cdot(N B 1(1104), C 1) \cdot(N B 1(1105), D 1),(N B 1(1106), A 2),(N B 1(1107)$, 4B2), (NBI ( 1108$), C 2),(N B 1(1109), D 2),(N B 1(1110), S, S I G S O, S I G S I, E 0, S I G N$ $5 G, B E T A),(N B 1(1111), T M),(N B 1(1112), S O R),(N B 1(1155), X I S),(N B 1(1200)$, $6 T R) \cdot(N B 1(1245), A),(N B 1(1290), X I S 1),(N B 1(1335), F N U),(N B 1(1380), F), 1$ $7 N B 1(1425), S 1),(N B)(1470), T),(N B 1(1515), X),(N B 1(1560), X 1),(N B 1(1605$ $8), B),(N B 1(1650), P N U),(N B 1(1695), E T A)$

$c$

EQUIVALENCE $($ NBI $(1740), T S 4500) \cdot(N B 1(1770), T S 4000),(N B 1(1800), T S 35$ $100),(N B)(1830), T S 3000),(N B 1(1860), T S 2500),(N B 1(1890), T S 2000),(N B 11$ $21920)$, TS1500), (NBI(1950), TS1000), (NB1(1980), TS500), (NB1(2010), TS68 3L, (NBI (1310),RIO), (NBI (1510),GN), (NBI(1710),GG), (NB2(1006),GF), (NB $42(1206), G),(N B 2(1406), P 0),(N B 2(1606), R),(N B 2(1806), E T A R),(N B 2, F B 2)$ $5,(N B P S N(2), B C O D E),(N B P S N(3), B N A M),(N B 6, F B 6)$

C

DIMENSION NB1 (2100), FB1 $(2100), N E 2(2050), F B 2(2050), N B 6(2010)$, 1FB6(2010), NBPSN (1992), BCODE (1991), BNAM(1990), MOMIN (4980),PCODE(500 2). PUNCOD (500), NBDEL (499), NRRDB (499), RLOCRC (499), THERM (15), MATDIM(1) $30), E L(45), F V(45), U L(45), E G(45), U G(45), B D A T E(2), S(45), S I G S O(925), S I$ $4 G S 1(225), E O(200)$, SIGNG $(225)$, SOR $(45), \times I S(45), T R(45), A(45), X 1 S 1(45)$, $5 F N U(45), F(45), S 1(45), T(45), X(45), X 1(45), B(45), P N U(45), E T A(45), T S 45$ $600(30)$, IS4000(30), TS3500(30), TS3000(30), TS2500 (30), TS2000 (30),TS15. $700(30)$, TS $1000(30)$, TS500(30), TS68(30), RIO(200), GN(200), GG(200), GF(2 $800), G(200), P O(200), R(200), E T A R(200), E N E R G Y(45)$, NAMTAP (3)

C CHECK TO SEE IF THIS IS A NEW OR A REVISED TAPE.

210 IF (NEWTAP) $212,211,212$

C. .... REVISED - READ DATA FROM OLD TAPE

211 CALL TAPECK (NBI,NWRC2,ITAPE)

GO IO 215

$C$ NEW READ CARD INPUT

212 READ DIP ELUULEEG UG 2FV,END

IF (SENSE LIGHT 1) 2121,2122

2121. CALL EROR (2)

2122 NBI(I) $=2$

C SET UP EL UL,EG,UG,FV INFO IN NBI REGION

$I 1=N T L E V+1$

DO $213 I=1$ NNTLEV

$F B I(I+I)=E L(I)$

$N A=I+11$

213 FBI $(\overline{N A})=U L(I)$

$I 1=2 * I 1-1$

$I 2=I I+N L E V I$ 


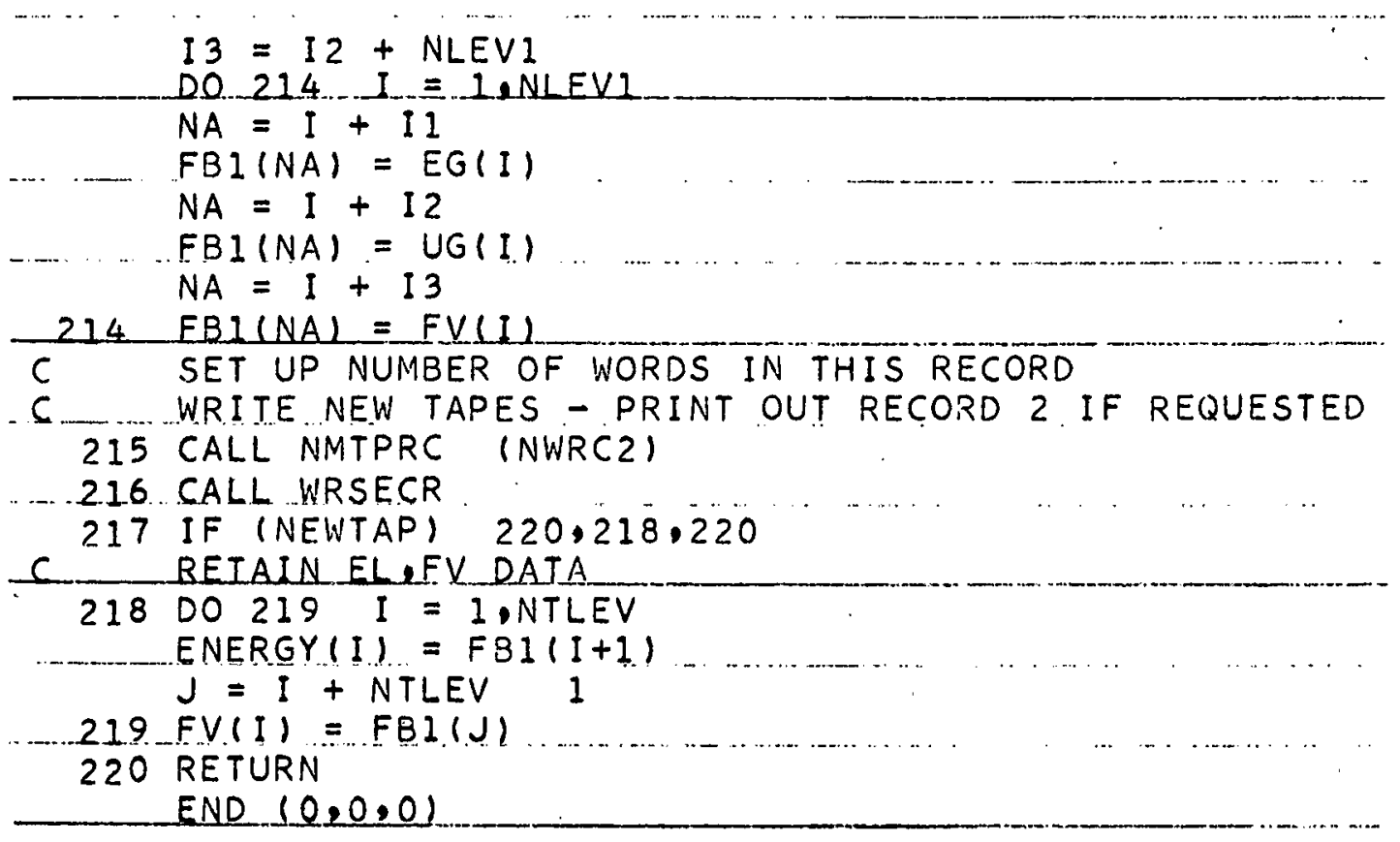




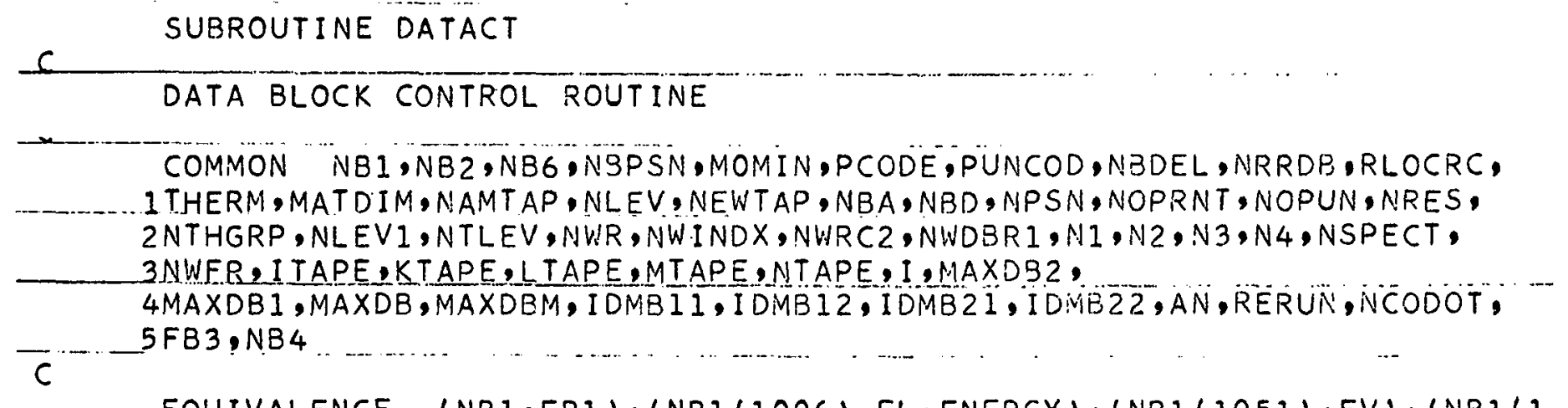

EQUIVALENCE (NB1, FBI), (NBI (1006), EL, ENERGY), (NBI(1051), FV), (NBI(1 1096), UL, BDATE), (NBI(1141),EG), (NBI(1186), UG), (NBI(1098),AW), (NBI(1 $2099), D E N),(N B)(1100), 42200),(N B 1(1101), N D A T A),(N B 1(1102), A 1),(N B 11$ $31103), B 1),(N B 1(1104), C 1),(N B 1(1105), 01),(N B 1(1106), A 2),(N B 1(1107)$, $4 B 2),(N B I(1108),(2),(N B I(1109), D 2),(N B I(1110), S, S I G S O, S I G S 1, E 0,5 I G N$ $5 G, B E T A),(N B I(1111), T M),(N B I(1112), S O R),(N B 1(1155), X I 5),(N B 1(1200)$, $6 T R),(N B 1(1245), A),(N B 1(1290), X I S 1),(N B 1(1335), F N U),(N B 1(1380), F), 1$ $7 N B 1(1425), S 1),(N B I(1470), T),(N B I(1515), X),(N B I(1560), X 1),(N B 1(1605$ $81, B),(N B 1(1650), P N U),(N B I(1695), E T A)$

$\mathrm{c}$

EQUIVALENCE (NBI $(1740), T S 4500),(N B 1(1770), T 54000),(N B 1(1800), T S 35$ $100),(N B 1(1830), T S 3000)$, (NBI(1860), TS2500), (NBI(1890), TS2000), (NBI) $21920), T S 1500),(N B 1(1950), T S 1000),(N B 1(1980), T S 500),(N B I(2010), T S 68$ $3),(N B 1(1310), R I 0),(N B 1(1510), G N),(N B 1(1710), G G),(N B 2(1006), G F),(N R$ $42(1206), G),(N B 2(1406), P 0),(N B 2(1606), R),(N B 2(1806), E T A R),(N B 2, F B 2)$ $5,(N B P S N(2), B C O D E),(N B P S N(3), B N A M),(N B 6, F B 6)$

$c$

DIMENSION NBI $(2100), F B 1(2100), N B 2(2050), F B 2(2050), N B 6(2010)$, IFB6(2010), NBPSN(1992), BCODE (1991), BNAM(1990), MOMIN(4980),PCODE(500 2), PUNCOD (500), NBDEL (499), NRRDB (499), RLOCRC(499), THERM(15), MATDIM(1 $30), E L(45), F V(45), 1 J E(45), E G(45), U G(45), B D A T E(2), S(45), S I G S O(925), S I$. 4GSI(925), EO(200), SIGNG (925), SOR(45), XIS(45), TR(45),A(45),XISI(45), $5 F N U(45), F(45), S 1(45), T(45), X(45), X 1(45), B(45)$, PNU(45), ETA(45), TS45 $600(30)$, TS4000(30), TS3500(30), TS3000(30), TS2500(30), TS2000(30), TS15 Z00(30), TSI000(30), TS500(30), TS68(30),RIO(200), GN(200), GG(200), GF(2 $800), G(200), P O(200), R(200)$, ETAR $(200)$, ENERGY (45), NAMT LP (3) DIMENSION FB3(500),NB4(500)

2 IF THIS IS A RERUN CASE, REESTAELISH INDEX I VALUE
220. IF (RERUN) 2202,2201,2202

$2201 \mathrm{NA}=1$

C. SET ITAPE INDEX

NCODOT $=0$

READ TAPE ITAPE

READ TAPE ITAPE

GO TO 2203

$2202 \mathrm{NA}=\mathrm{I}$

. 2203.DO 23985 I = NA,MAXDB2

C IF CODE NUMBER IS ZERO, THIS IS END" OF "DATA" BLUCKS IF $(N B 6(4 * I+3)) \quad 221.23997 .221$

c

C FIRST CHECK TO SEE WHERE DATA BLOCK I IS LOCATED -

C ITHAT IS, AS NEW CARD DATA,ON THE KTAPE, OR ON THE 2 ITAPE. 


\section{IF (NEWTAP) $240,222,240$}

222 IF (NBA) 223,225,223

C CHECK FOR ADDED DATA BLOCK

-223.DO $224-I I=1, N B A$

224 CONTINUE

C

225 IF (NPSN) 226.228 .226

C CHECK FOR RELOCATED DATA BLOCK

226 DO 227 II $1=1, N P S N$

$N A=I 1+N B A$

IF $(I-N B P S N(4 * N A-3)) \quad 227.2395 .227$

227 CONTINUE

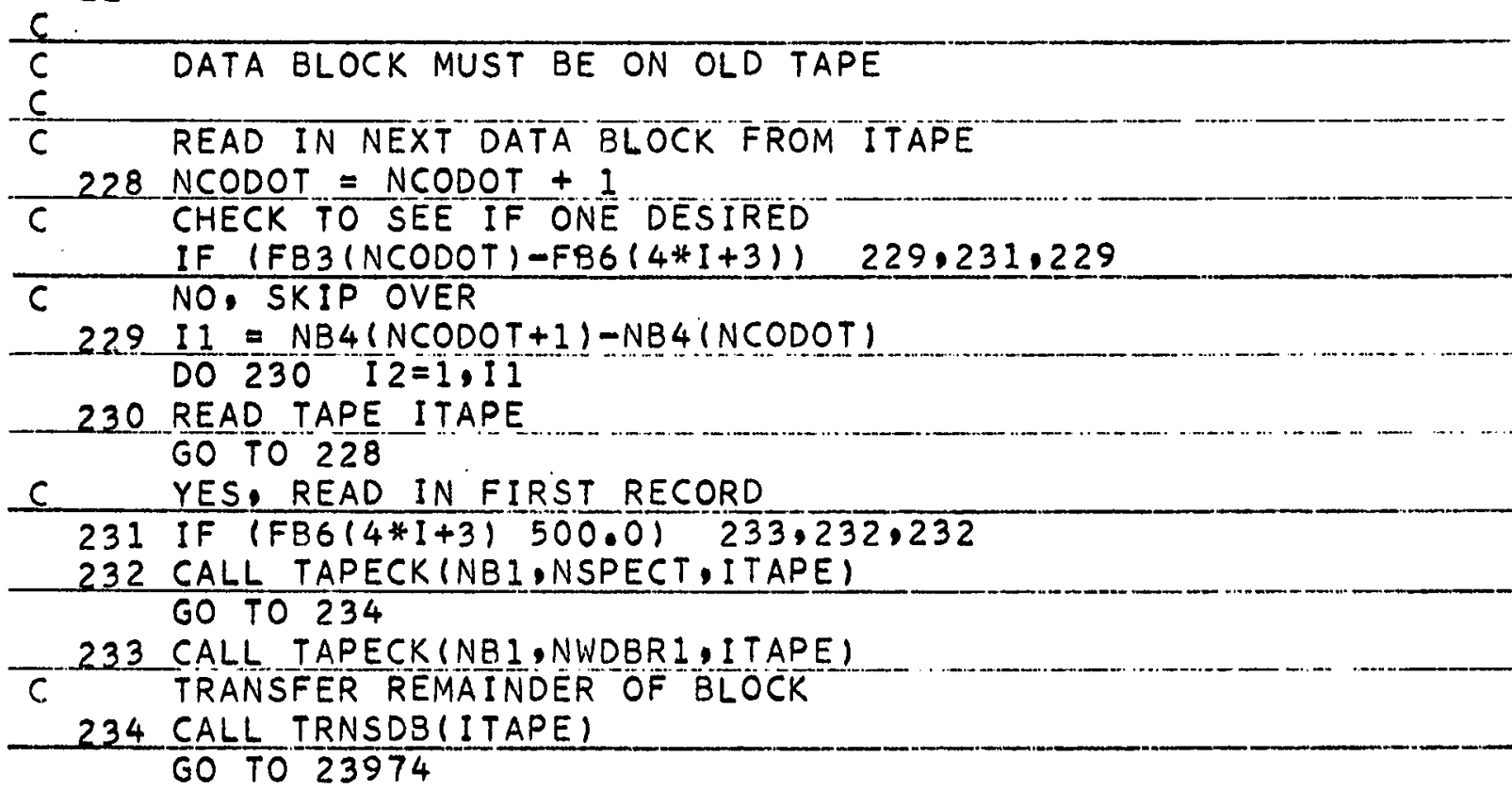

C READ DATA FROM INTERMEDIATE TAPE

2395 DO 23951 II 1 IONPSN

C FIND PHYSICAL RECORD NUMBER OF THIS DATA BLOCK ON KTAPE.

IF (FB6 (4*I+3) RLOCRC(II)) 23951,23952,23951

23951 CONTINUE

C CHECK RECORD NUMBER AGAINST CURRENT TAPE

C IPOSITION TO SEE IF WE NEED TO BACKSPACE OR FORWARD SPACE

23952 IF (NRRDB(II)-NWR) $23957,23957,23953$

C FORWARD SPACE OR DO NOT MOVE KTAPE

$23953 N=N R R D B(I I-1)-N W R$

IF (N) $23956,23956,23954$

23954 DO 23955 I2 $=1, N$

23955 READ TAPE KTAPE

23956 GO TO 23960

C $\quad$ BACKSPACE

$23957 \mathrm{~N}=N W R-N R R Q B(I 1-1)$

23958 DO 23959 I $2=1, N$

23959 BACKSPACE KTAPE

C UPDATE NWR FOR PRESENT KTAPE POSITION

$23960 \mathrm{NWR}=\mathrm{NRRDB}(\mathrm{I} 1)$ 
23971 CALL TAPECK (NBI,NWDBR1,KTAPE)

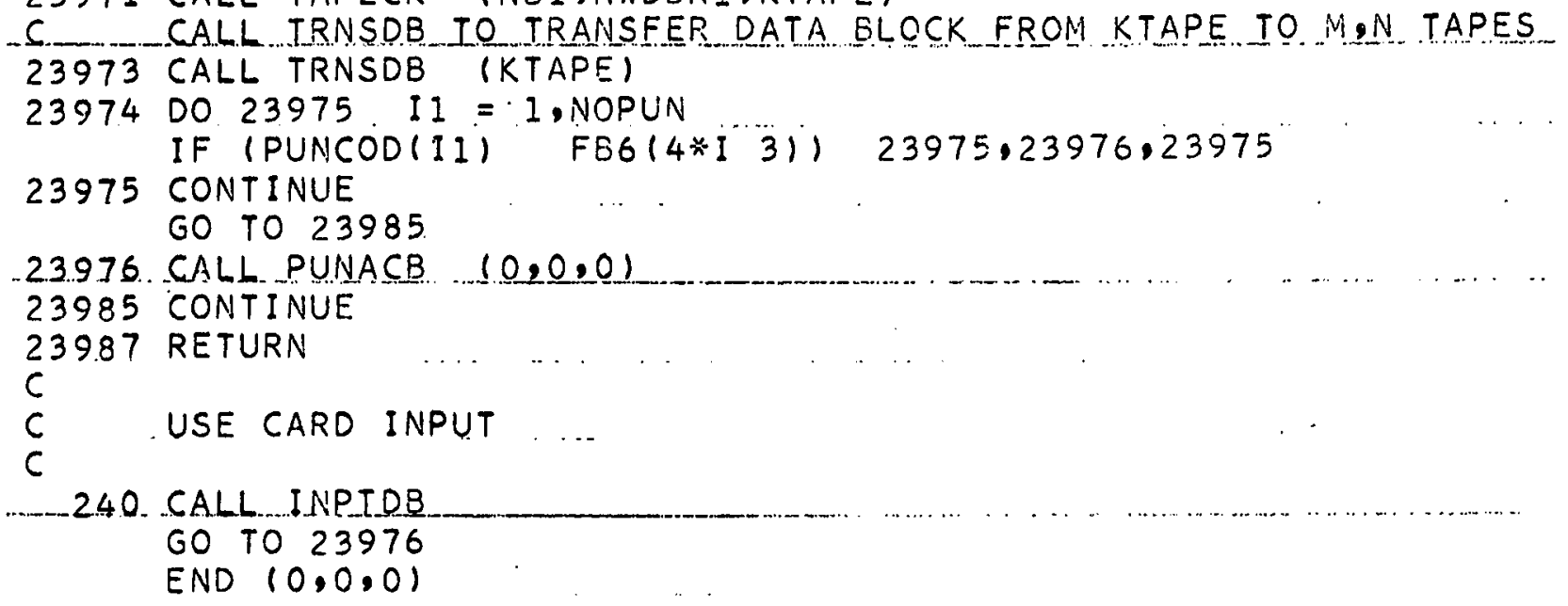




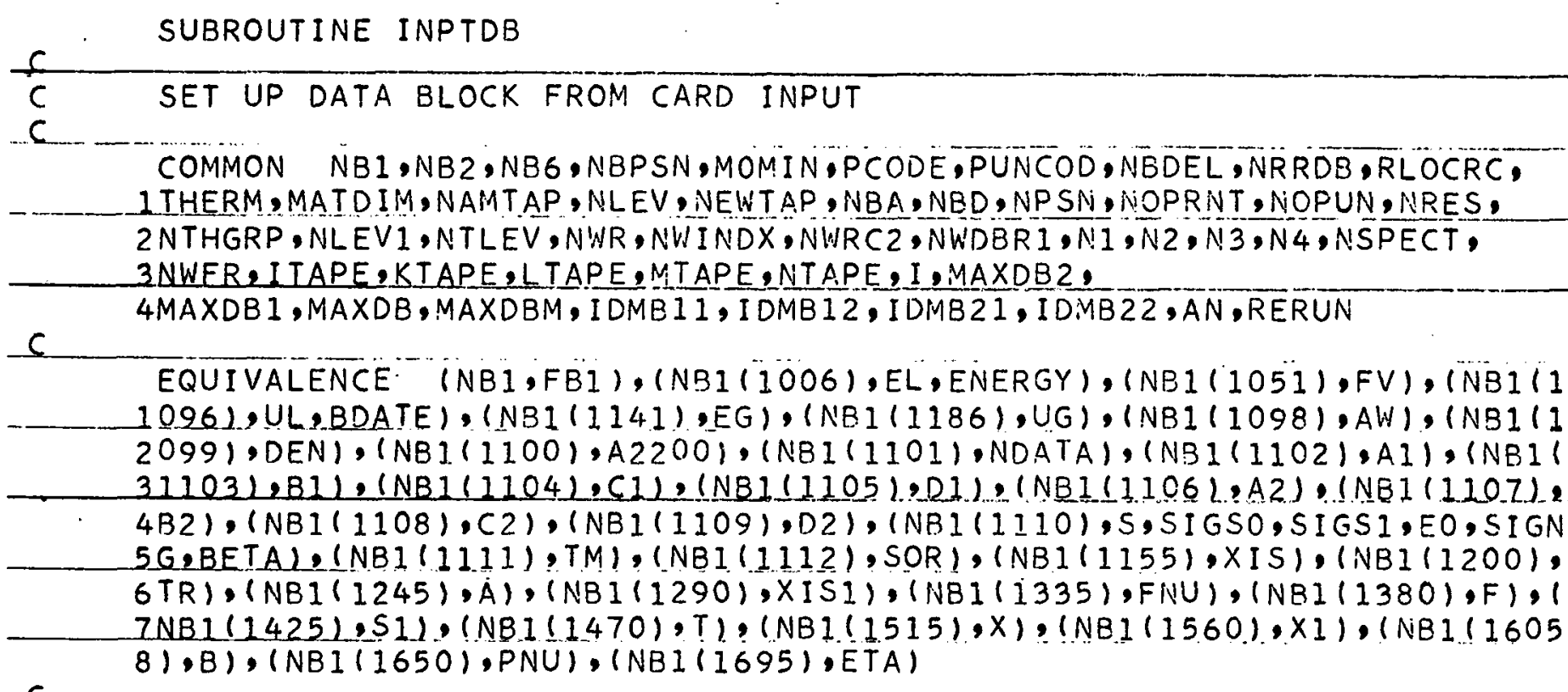

$C$

EQUIVALENCE (NB1(1740),TS4500),(NBI(1770),TS4000), (NB1(1800), TS35 $100),(N B 1(1830), T S 3000),(N B 1(1860), T S 2500),(N B 1(1890), T S 2000)$, (NBI) $21920), T S 1500),(N B 1(1950), T S 1000),(N B 1(1980), T S 500),(N B 1(2010)$, TS68 3). (NBI (13.10), RI0), (NBI (15.10), GN), (NBI $(1710), G G),(N B 2(1006), G F)$, (NA $42(1206), G),(N B 2(1406), P 0),(N B 2(1606), R),(N B 2(1806), E T A R),(N B 2, F B 2)$ $5,(N B P S N(2), B C O D E),(N B P S N(3), B N A M),(N B 6, F B 6)$

C

DIMENSION NBI(2100),FB1 $(2100), N B 2(2050), F B 2(2050), N B 6(2010)$, 1FB6 (2010), NBPSN(1992), BCODE(1991), BNAM(1990), MOMIN(4980),PCODE(500 2), PUNCOD (500), NBDEL (499) , NRRDB (499), RLOCRC (499), THERM(15), MATDIM(I 30), EL (45), FV (45), UL (45), EG (45), UG (45), BDATE( 2$), S(45), S I G S O(925), S I$ $4 G S I(925), E O(200), S I G N G(925), S O R(45), \times I S(45), T R(45), A(45), X I S 1(45)$. $5 F N U(45), F(45), S 1(45), T(45), X(45), X 1(45), B(45), P N U(45), E T A(45), T S 45$ $600(30)$, TS4000(30), TS3500(30), TS3000(30), TS2500(30), TS2000(30), TS15 $700(30)$, TS1000(30), TS500(30), TS68(30),RIO(200), GN(200), GG(200), GF(2 C - 8001.2.G $(200), P 0(200), R(200)$, ETAR $(200)$, ENERGY $(45)$, NAMTAP (3)

C BLANK OUT REGIONS OF NBI,NB2 DO $240 \mathrm{~J}=$ IDMBII, IDMBI2

$240 N B I(J)=0$ DO $2401 \mathrm{~J}=$ IDMB21.IDMB22

$2401 N B 2(J)=0$

C CHECK FOR SPECTRAL OR CROSS SECTION DATA IF (FB6(4*I+3) 500.0$) \quad 2403.2402 .2402$

C SPECTRAL - READ IN DATA

2402 READ DIP BDATE, BETA, TM, SOR, END IF (SENSE LIGHT I) 24021.24022

24021 CALL DIPERR (3)

24022 GO TO 320

C CROSS - SECTION - READ IN FIRST RECORD

2403 READ DIP BDATE,AW,DEN,A2200,NDATA,A1,B1,C1,D1,A2, B2,C2,D2,S,XIS, ITR, A, XISI, FNU, F, S1, T,X,X1, B, PNU, ETA, NCON, CONNO, WG T, TS4500,TS4000,T $2 S 3500$, TS 3000, TS2500,TS2000,TS1500,TS1000, TS500, TS 68 , END

IF (SENSE LIGHT 1 ) 24031.24032

24031 CALL DIPERR (4) 
c

-24032 IF (NDATA) 274.241 .274

241 DO $273 \mathrm{~J}=1$.NTLEV

IF $(F(J)) \quad 243.242,243$

242 IF (FNU(J)) $246,252,246$

243 IF (PNU(J)) $245,244,245$

244 PNU $(J)=A 1+B 1 * E N E R G Y(J)+C 1 *(E N E R G Y(J) * * 01$

$245 \mathrm{FNU}(\mathrm{J})=\operatorname{PNU}(\mathrm{J}) * \mathrm{~F}(\mathrm{~J})$

246 IF (AIJ) $257,247,257$

247 IF (TIJ)I 248.249 .248

248 IF (SIJ) $255,249,255$

249 IF (ETA (J)) 251.250 .251

250 ETA $(J)=A 2+(B 2+C 2 *(E N E R G Y(J)) * * D 2) * \operatorname{LOGF}(E N E R G Y(J))$

251A(J) = FNU(J)/ETA(J) GO TO 257

252 IF (A(J)) 257,253,257

253 IF (S(J)) $254,256,254$

254 IF $(T(J)) \quad 255,256,255$

$255 A(J)=T(J)-S(J)$

GO TO 260

$256 A(J)=F V(J) * A 2200$

GO TO 258

257 IF (S(J)) $260,258,260$

258 IF (T JU) $259,260.259$

$259 S(J)=T(J)-A(J)$

260 IF $(X I S(J)) \quad 264.261 .264$

261 IF $(X(J)) \quad 263,262,263$

$262 \times(J)=1.0+((1($ AW/AN $) \quad 1.0) * 2) /(2.0 *(A W / A N))) *$ LOGF

$1((1$ AW/AN $)-1.0) /((A W / A N)+1.0))$

$263 \times I S(J)=X(J) * S(J)$

264 IF (TR(J)) $268,265,268$

265 IF $(B(J)) \quad 267.266 .267$

$266 B(J)=1.0-(2.0 * A N) /(3.0 * A W)$

$-267 \quad T R(J)=B(J) * S(J)$

268 IF (XISI) J)) $274.269,274$

269 IF $(S I(J)) \quad 271.270 .271$

$270 S I(J)=S(J)-T R(J)$

271 IF $(X 1(\mathrm{~J})) \quad 273 \cdot 272.273$

272 IF $(A W-1.01) 2721.2725 .2725$

$-27.21 \times 1(J)=-0.6666667$

GO TO 273

$2725 \times 1(1)=1.6666667-(A W / A N) * 2+(((A W / A N)+2.0) *((A W / A N)-1.0) * 2$ $1 / 2.0) * \operatorname{LOGF}(((A W / A N)+1.0) /((A W / A N)-1.0))$

C

$273 \times I S I(J)=X I(J) * S 1 ! J)$

C ... SET UP FORMAT FOR RECORD I FROM THIS DATA IN NBI REGION

C

$274 N B 1(1)=N B 6(4 * I+4)$

$F B 1(2)=B D A T E(1)$

$F B 1(3)=B D A T E(2)$

$N B I(4)=N B 6(4 * I+3)$

$I 1=4+N T L E V$

$I 2=I 1+N T L E V$ 


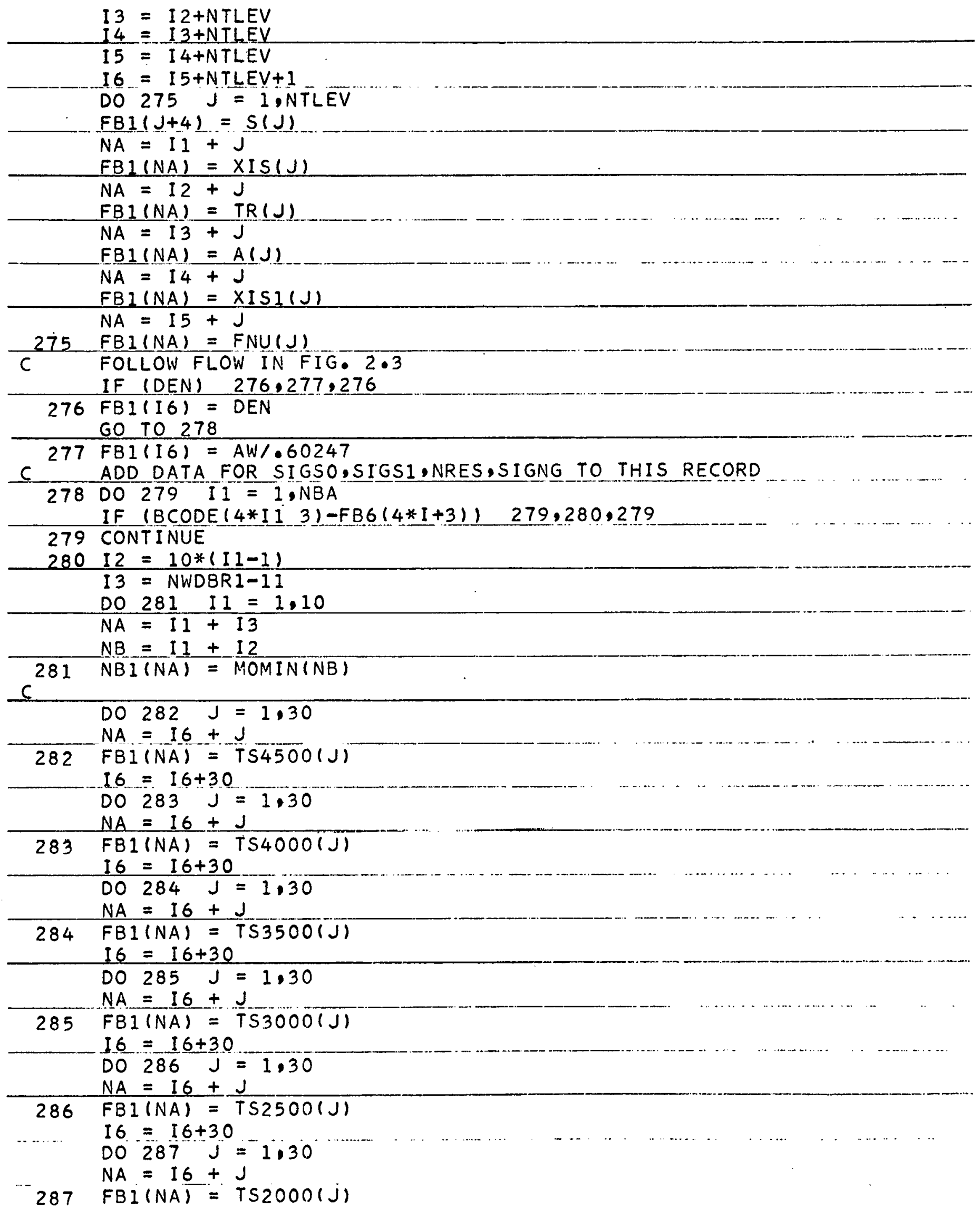




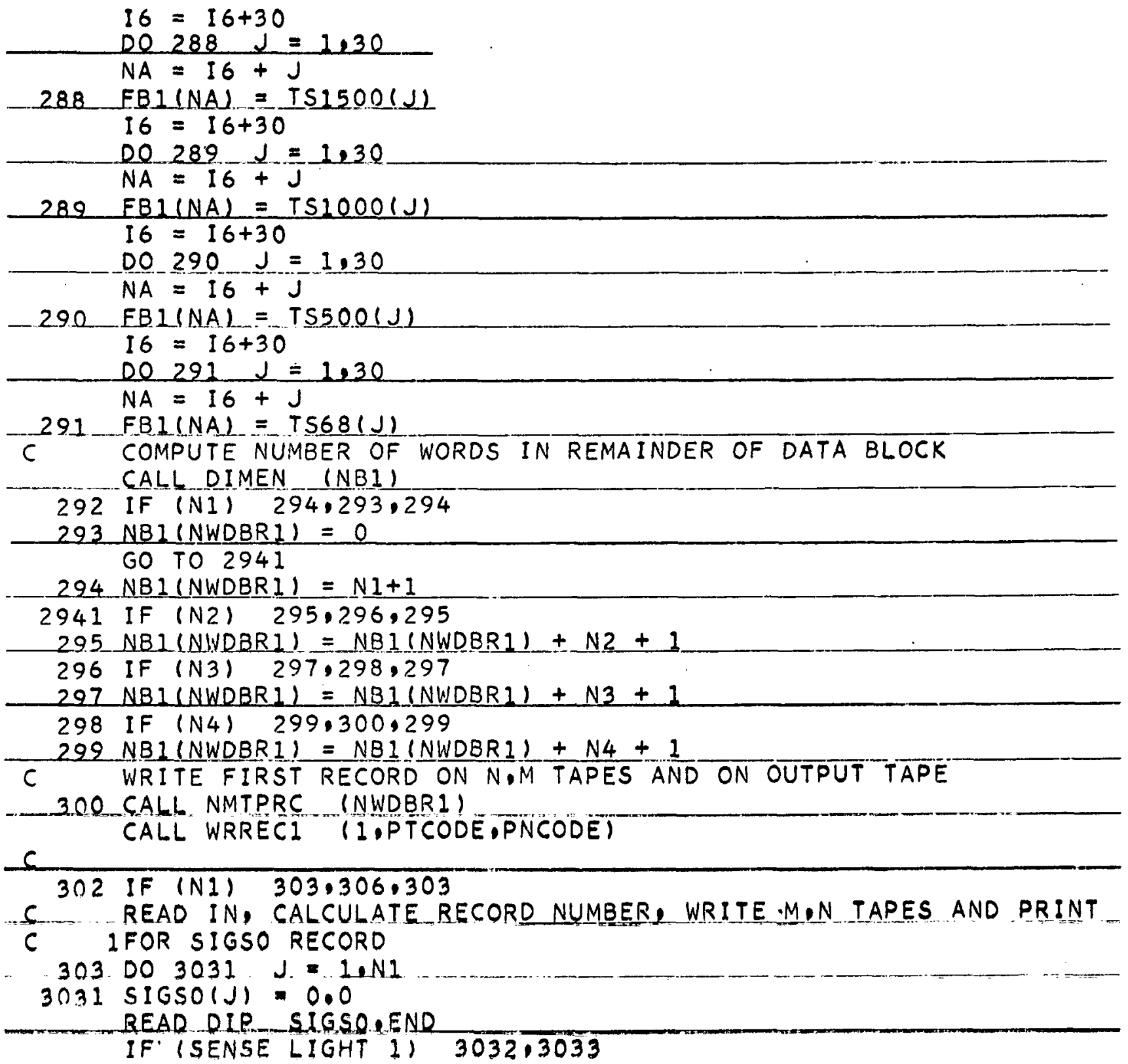

3032. CALL DIPERR (3)

$3033 N B 1(1) \cdot N B 1(1)+1$

$11-N 1-1$

DO $304 \mathrm{j}=1.11$

304 FBI $(\alpha+1)$ SIGSO $(\alpha)$

CALL NMTPRC (NI)

C 305 CALL WRREC2 ... (PTCODE PNCODE)

306 IF (N2) $\quad 307.310 .307$

C READ IN, CALCULATE RECORD NUMBER, WRITE MON TAPES AND PRINT

C IFOR SIGSI RECORD

$307003071 \mathrm{~J}=$ I.N2

- .307ISIGSI(J)=0.0

READ DIP SIGSI, END

IF (SENSE LIGHT 1 ) 3072,3073

3072 CALL DIPERR (6) 


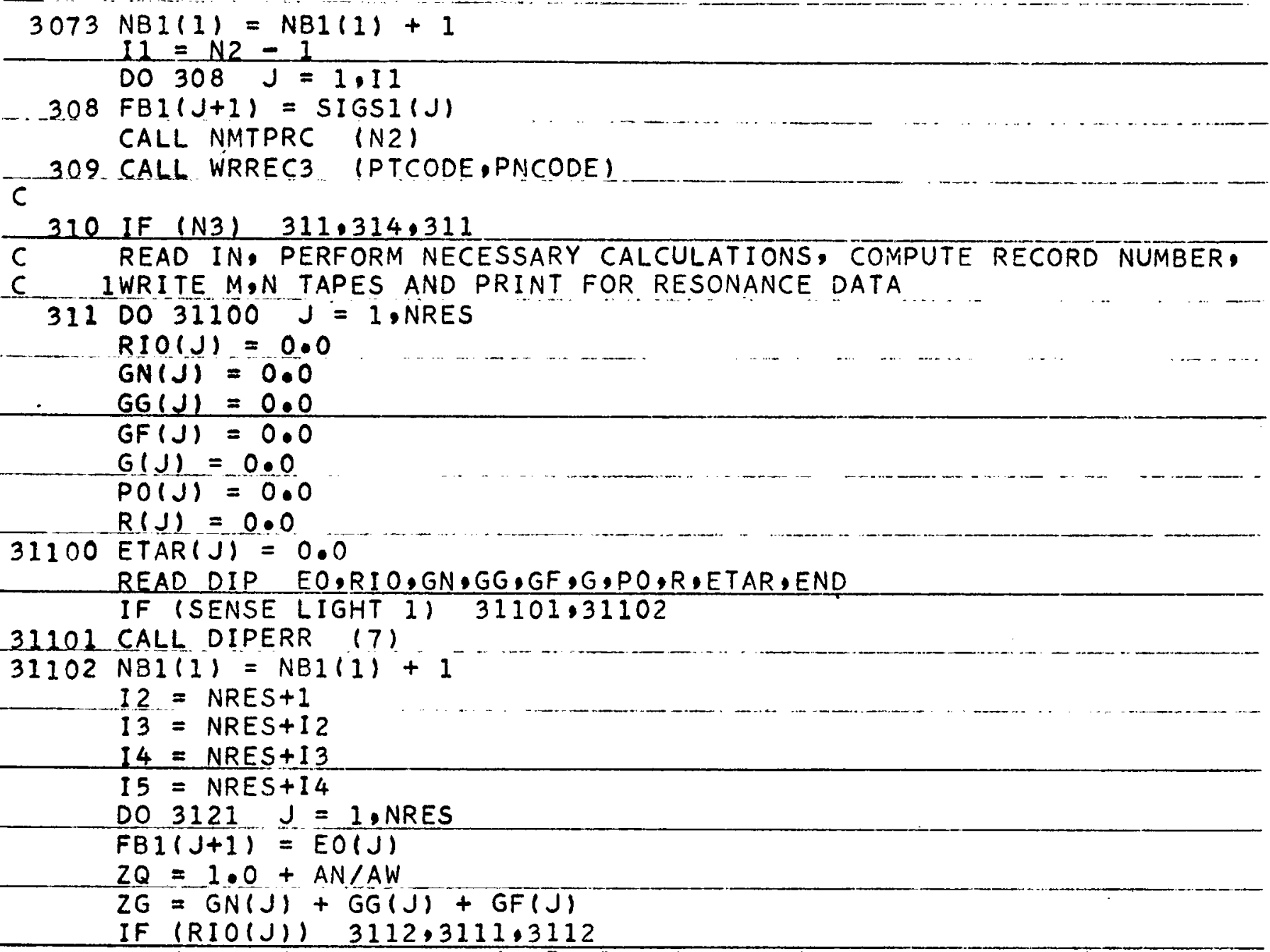

3111 RIO(J) $=(4.0896285 E+06 * 2 Q * Z Q * G(J) *(G G(J)+G F(J)) * G N(J) /$ $1(E O(J) * E O(J) * 2 G)$

$3112 \mathrm{NA}=J+12$

$F B I(N A)=R I O(J)$

IF $(P O(J)) \quad 3114,3113,3114$

$3113 P O(N)=(E O(J) * R I Q(J)) /(1.5707963 * 2 G)$

$3114 N A=J+13$

$F B I(N A)=P O(J)$

IF (R(J)) $3116,3115,3116$

$3115 R(J)=(3.44656 E-4 * A N * Z Q * E O(J)) /(A W * Z G * Z G)$

$3116 N A=J+I 4$

$F B I(N A)=R(J)$

IF IETAR(J) $312,3117,312$

3117 ETAR $(J)=((A 1-B 1 * E O(J) \quad C 1 * E O(J) * * D 1) * G F(J)) /(G G(J)$ GF $(J))$

$312 N A=J+I 5$

$3121 F B I(N A)=\operatorname{ETAR}(J)$

CALL NMTPRC (N3)

C

313 CALL WRREC4 (PTCODE,PNCODE)

314 IF (N4) $315,318,315$

C READ IN, CALCULATE RECORD NUMBER, WRITE M,N TAPES AND PRINT

C IFOR SIGNG RECORD

315 DO $3151 \mathrm{~J}=1, \mathrm{~N} 4$ 


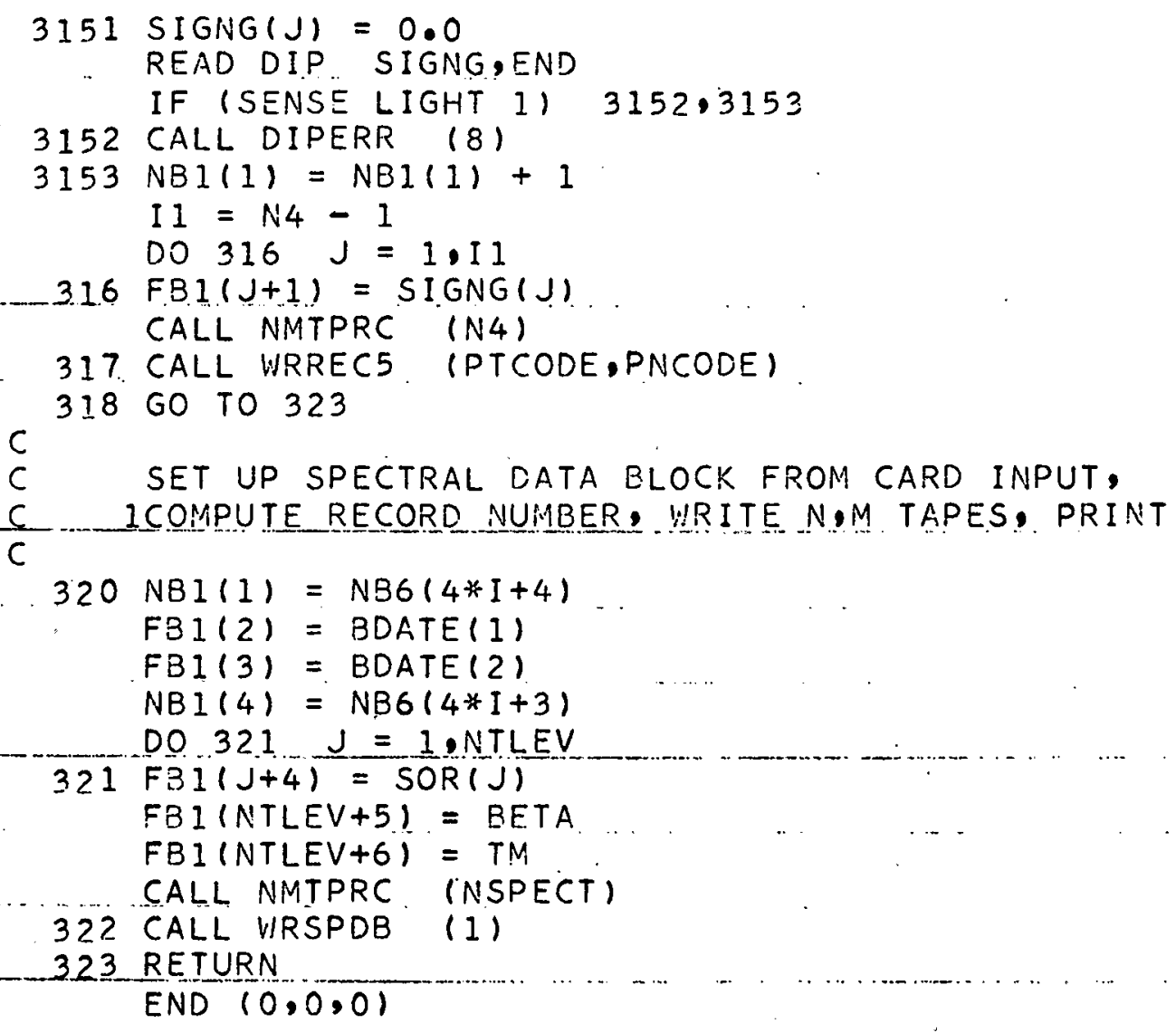




$\frac{\text { SUBROUTINE NMTPRC }}{C}$ (NW)

COMMON NBI, NB2, NB6, NBPSN,MOMIN,PCODE,PUNCOD,NBDEL, NRRDB, RLOCRC, 1 THERM, MATDIM,NAMT AP, NLEV, NEWTAP, NBA, NBD, NPSN, NOPRNT, NOPUN, NRES, 2NTHGRP, NLEVI, NTLEV, NWR, NWINDX,NWRC2,NWDBRI, NI, N2, N3, N4, NSPECT, 3NWFR, ITAPE, KTAPE, LTAPE, MTAPE, NTAPE, I, MAXDB2, 4MAXDB 1, MAXDB, MAXDBM, IDMB11, IDMB12, IDMB21, IDMB22, AN, RERUN

EQUIVALENCE (NBI, FBI), (NBI)(1006), EL, ENERGY), (NBI(1051), FV), TNBI(1 1096). UL, BDATE) , (NB1 (1141),EG), (NB1 $(1186), U G),(N B 1(1098), A W),(N B 1(1$ 2099 , DEN), (NBI(1100), A2200), (NBI(1101), NDATA), (NB1(1102), A1), (NBI1 $31103), B 1),(N B)(1104), C 1),(N B 1(1105), 01),(N B 1(1106), A 2),(N B 1(1) 107)$. $4 B 2),(N B 1(1108),(2),(N B 1(1109), 02),(N B 1(1110), 5, S 1 G S 0,5 I G S 1, E 0,51 G N$ 5G,BETA), (NBI! (1111), TM), (NB1 (1112), SOR), (NB1(1155),XIS), (NBI(1200), $6 T R),(N B 1(1245), A),(N B 1(1290), X I S 1),(N B I(1335), F N U),(N B 1(1380), F), i$ $7 N B 1(1425), S 1),(N B 1(1470), T),(N B 1(1515), X),(N B 1(1560), X 1),(N B 1(1605$ $8), B),(N B 1(1650), P N U),(N B 1(1695), E T A)$

C

EQUIVALENCE (NBI(1740),TS4500), (NB1(1770),TS4000), (NBI(1800),TS35 $100)$, (NB1 (1830), TS3000), (NB1 (1860), TS2500), (NB1(1890), TS2000), (NBI) $21920), T S 1500),(N B 1(1950), T S 1000),(N B 1(1980), T S 500),(N B 1(2010), T S 68$ 3), (NBI (1310),RI0), (NBI (1510), GN), (NB1(1710),GG), (NB2(1006),GF), (NB $42(1206), G),(N B 2(1406), P 0),(N B 2(1606), R),(N B 2(1806), E T A R),(N B 2, F B 2)$ 5 , (NBPSN $(2), B C O D E),(N B P S N(3), B N A M),(N B 6, F B 6)$

C

DIMENSION NB1 (2100),FB1 (2100), NB2(2050),FB2(2050),NB6(2010), 1FB6 (2010), NBPSN(1992), BCODE (199)), BNAM(1990), MOMIN (4980),PCODE (500 2), PUNCOD (500), NBDEL (499), NRRDB (499), RLOCRC (499), THERM (15), MATOIM(1 $30), E L(45)$, FV (45),UL(45), EG(45),UG(45), BDATE(2), S(45), SIGSO(925),SI 4GSI $(925)$, EO (200), SIGNG (925), SOR (45), XIS(45), TR (45), A $(45), X I S I(45)$, $5 F N U(45), F(45), S 1(45), T(45), X(45), X 1(45), B(45), P N U(45), E T A(45), T S 45$ $600(30)$,TS4000(30),TS3500(30), TS3000(30),TS2500(30),TS2000130), TS15. $700(30), T S 1000(30), T S 500(30), T S 68130), R I 0(200), G N(200), G G(200), G F(2$ $800), G(200), P O(200), R(200)$, ETAR (200), ENERGY (45), NAMTAP(3)

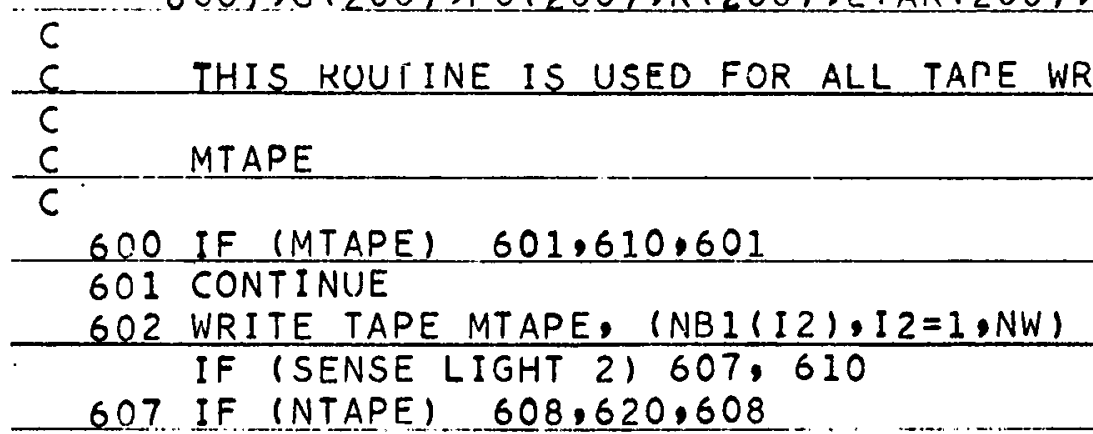

608 WRITE OUTPUT TAPE LTAPE, 609, MTAPE, NTAPE

609 FORMAT $11 \mathrm{H} 130 \mathrm{H} 2$ $1 S S / 37 \mathrm{H}$ $M T A P E=0$

DATA TAPEI $3,18 \mathrm{H}$ FAILED TO PROCE COMPILATION OF TAPEI $3,14 \mathrm{H}$ WILL CONTINUE)

$\stackrel{C}{C}$ NTAPE

5..610 IF (NTAPE)

$611,622,611$

611 CONTINUE 


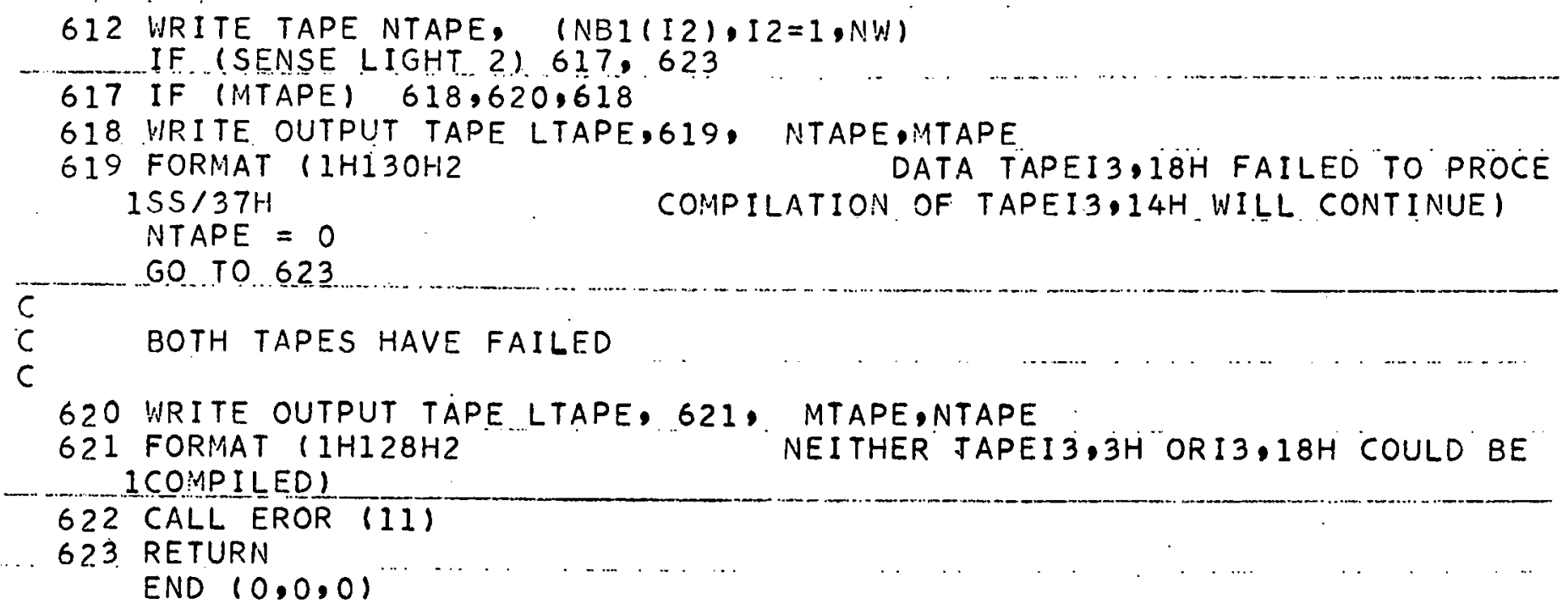




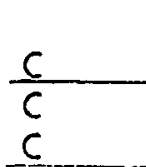

SUBROUTINE KTAPRC (NW)

WRITE RECORD ON INTERMEDIATE TAPE

COMMON NBI, NB2, NBG, NBPSN, MOMIN , PCODE, PUNCOD, NBDEL,NRRDB, RLOCRC, 1 THERM, MATDIM, NAMTAP, NLEV, NENTAP, NBA, NBD, NPSN, NOPRNT, NOPUN, NRES, 2NTHGRP, NLEVI, NTLEV, NWR, NWINDX, NWRC2, NWDBRI, NI, N2, N3, N4,NSPECT, 3NWFR, ITAPE, KTAPE, LTAPE, MTAPE, NTAPE, I, MAXDB2,

4MAXDB I, MAXDB,MAXD3M, IDMBII, IDMB12, IDMB2 I, IDMB $22, A N, R E R U N$

C

EQUIVALENCE (NBI,FBI), (NABI (1006), EL, ENERGY), (NBI(1051),FV), (NBI(I $1096), U L, B D A T E),(N B I(1141), E G),(N B 1(1186), U G),(N B 1(1098), A W),(N B I(1$ $2099), D E N),(N B 1(1100), A 2200),(N B 1(1101), N D A T A),(N B 1(1102), A 1),(N B I)$ $31103), B 1),(N B 1(1104), C 1),(N B 1(1105), D 1),(N B 1(1106), A 2),(N B 1(1107)$, $4 B 2),(N B I(1108), C 2),(N E 1(1109), D 2),(N B I(1110), 5, S I G S O, S I G S I, E O, S I G N$ $5 G, B E T A),(N B I(1111), T M),(N B I(1112), S O R),(N B I(1155), X I S),(N B I(1200)$, 6 TR), (NBI(1245), A), (NBI(1290),XISI), (NBI(1335), FNU), (NBI(I380i, F), $7 N B I(1425), S 1),(N B 1(1470), T),(N B I(1515), X),(N B 1(1560), X 1),(N B 1(1605$ $8), B),(N B I(1650), P N U T,(N B I(1695), E T A)$

$\underline{C}$

EQUIVALENCE (NB1(1740),TS4500),(NB1(1770),TS4000), (NBI) 11800),TS35 $100)$, (NB1 $(1830)$, TS3000), (NB1 (1860), TS2500), (NBI(1890), TS2000), (NBI) $21920)$, TS1500), (NB1(1950),TS1000), (NSI(1980),TS500), (NBI(2010),TS68 3), (NBI $(1310), R 10),(N B 1(1510), G N),(N B 1(1710), G G),(N B 2(1006), G F),(N B$ $42(1206), G),(N B 2(1406), P 0),(N B 2(1606), R),(N B 2(1806), E T A R),(N B 2, F B 2)$ $5,(N B P S N(2), B C O D E),(N B P S N(3), B N A M),(N B 6, F B 6)$

C

DIMENSION NB1 $(2100), F B 1(2100), N B 2(2050), F B 2(2050), N B 6(2010)$, IFB6(2010), NBPSN(1992), BCODE(1991), BNAM(1990), MOMIN(4980), PCODET500 2). PUNCOD (500), NBDEL(499), NRRDB (499), RLOCRC (499), THERM (15), MATDIM(1 30), EL(45), FV(45), UL (45), EG (45),UG(45), BDATE(2),S(45), SIGSO(925), SI

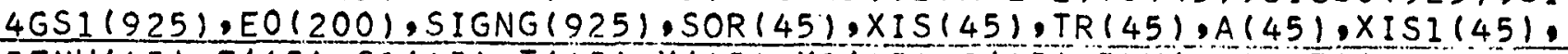
5FNU(45), $F(45), S 1(45), T(45), X(45), X 1(45), B(45)$, PNU $(45), E T A(45), T S 45$ $600(30), T S 4000(3)$, TS3500(30), TS3000(30), TS2500(30), TS2000(30), TS15 $700(30)$,TSI000(30), TS500(30), TS68(30), RIO(200), GN(200), GG(200), GF(2 $800.1, G(200)$, PO $(200)$, R (200), ETAR (200), ENERGY (45),NAMTAP (3)

$\bar{C}$

630 CALL TAPECK (NB2,NW, ITAPE)

631 WRITE TAPE KTAPE, (NB2) (I2), I $2=1$, NW)

IF (SENSE LIGHT 2) 636,638

636 CALL EROR (12)

638 RETURN

END $10,0,01$ 


\begin{tabular}{|c|c|}
\hline$c$ & SUBROUTINE DIMEN \\
\hline $\begin{array}{l}\mathrm{C} \\
\mathrm{c}\end{array}$ & COMPUTE DIMENSIONS \\
\hline & 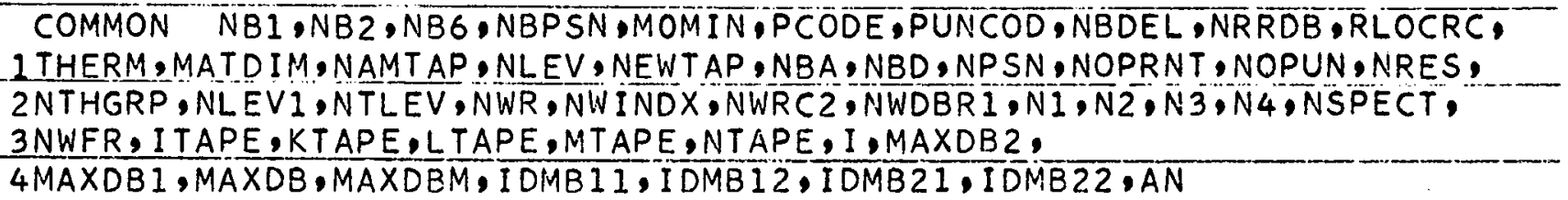 \\
\hline & 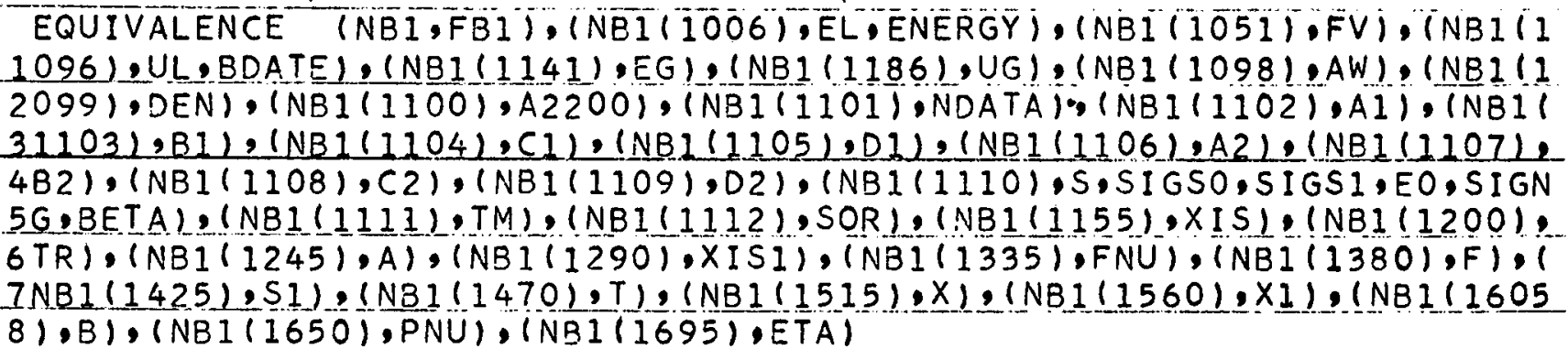 \\
\hline
\end{tabular}

$c$

EQUIVALENCE (NB1(1740),TS4500),(NBI(1770),TS4000),(NB1(1800),TS35 $100),(N B 1(1830), T S 3000)$, (NB1 (1860),TS2500), (NB1(1890), TS2000), (NB1) $21920)$, TS1500), (NB1(1950), TS1000), (NB1(1980), TS500), (NB1(2010),TS68 $3),(N B I(1310), R I 0),(N B 1(1510), G N),(N B I(1710), G G),(N B 2(1006), G F),(N B$ $42(1206), G),(N B 2(1406), P 0),(N B 2(1606), R),(N B 2(1806), E T A R),(N B 2, F B 2)$ $5,(N B P S N(2), B C O D E),(N B P S N(3), B N A M),(N B 6, F B 6)$

C

DIMENSION NB1(2100),FB1 $(2100), N B 2(2050), F B 2(2050), N B 6(2010)$, 1FB6 (2010), NBPSN(1992),BCODE (1991), BNAM(1990), MOMIN(4980),PCODE(500 2), PUNCOD (500), NBDEL (499), NRRDB (499), RLOCRC(499), THERM(15), MATDIM(I $30), E L(45), F V(45), U L(45), E G(45), U G(45), B D A T E(2), S(45), S I G S O(925)$, SI $4 G S I(925)$, EO $(200)$, SIGNG $(925)$, SOR $(45), X I S(45)$, TR $(45), A(45), X I S I(45)$. 5FNU(45), $F(45), S 1(45), T(45), X(45), X 1(45), B(45), P N U(45), E T A(45), T S 45$ $600(30)$. TS4000(30), TS35001301, TS3000(30), TS2500(30), TS20001301, TS15 $700(30)$, TS1000(30), TS500(30), TS68(30), RIO(200), GN(2,00), GG(200), GF(2 $800), G(200), P 0(200), R(200)$, ETAR (200), ENERGY $(45)$, NAMTAP (3)

$\bar{C}$ DIMENSION LOC(1)

$C$ THIS ROUTINE CALCULATES THE NUMBER OF WORDS IN THE

C ISIGSO, SIGSI, RESONANCE, AND SIGNG RECORDS AND STORES THIS

C 2INFO IN N1, N2, N3, N4 RESPECTIVELY. IT ALSO STORES THE

C 3 MOMIN,MOMAX,-- MPRIMEGAMMAMAX NUMBERS INTO REGION MATDIM

C $\quad 4$ TO BE USED IN THE WRREC SERIES ROUTINES.

640 .IF ILOCINWDBRI 10)) $642,641,642$

$641 N 1=0$

GO TO 643 .

$642 N 1=(L O C(N W D B R 1-9) 1-L O C(N W D B R 1-10) 1) *(L O C(N W D B R 1-8)$-LOC (NWDBR 1-10 $1)+1) 1$

643 IF (LOCINWOBRI 7 ) $\quad 645.6449645$

$644 N 2=0$

GO TO 646

$645 N 2=(L O C(N W D B R 1-6)-L O C(N W D B R I-7)+1) *(L O C(N W D B R I-5)-L O C(N W D B R I-7)+$ $111+1$

646 IF (LOCINWDBRI 4) $648,647,648$ 


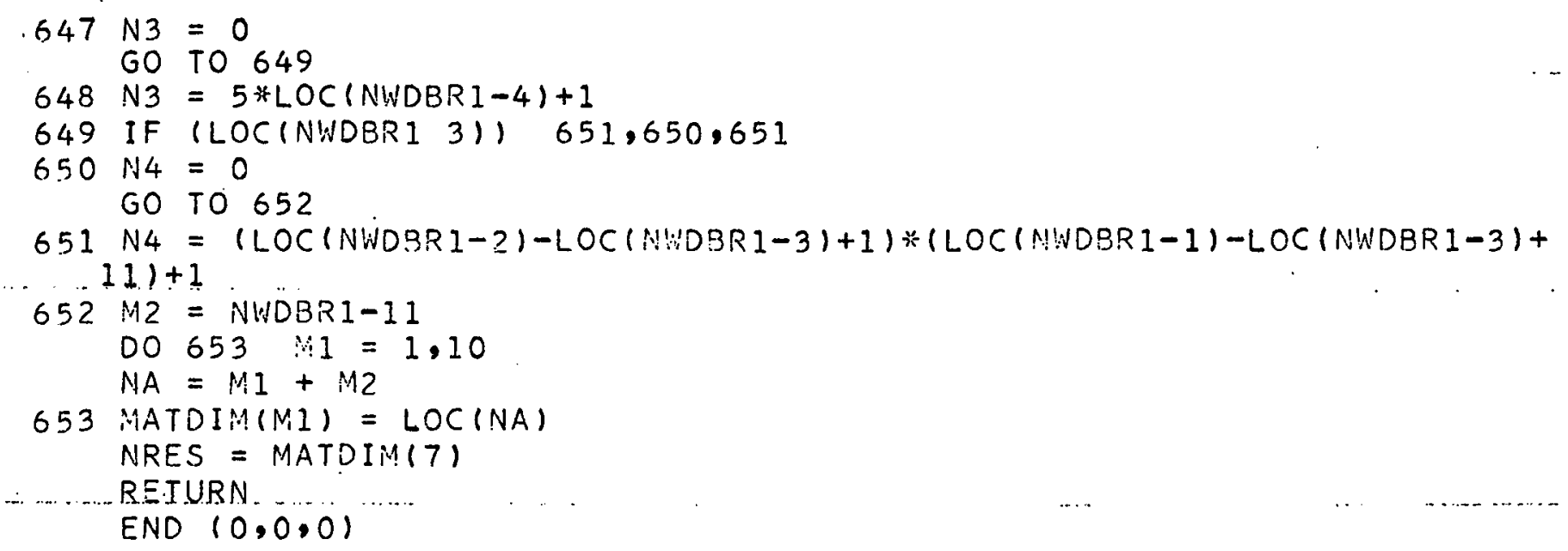




SUBROUTINE TRNSDB (ITAPI)
TRANSFER DATA BLOCK FROM INPUT OR INTERMEDIATE TAPE TO DATA TAPES

COMMON NEI, NB2, NB6, NBPSN, MOMIN,PCODE, PUNCOD, NBDEL, NRRDE, RLOCRC, 1 THERM , MATD IM, NAMTAP, NLEV , NEWTAP, NBA, NBD, NPSN, NOPRNT , NOPUN, NRES, 2NTHGRP, NLEVI, NTLEV, NWR, NWINDX, NWRC2, NWDBRI, NI, N2, N3,N4,NSPECT, 3 NUFR, I TAPE, KTAPE, LTAPE, MTAPE, NTAPE, I, MAXDB2,

4MAXDB1, MAXDS, MAXDBM, IDMB II, IDMS12, IDMB21, IDMB22,AN

C

EQUIVALENCE (NBI,FBI), (NBI (1006), EL, ENERGY), (NBI(1051), FV), (NBI(1 1096), UL, BDATE), (NB1 (1141),EG).,(NB1 (1186),UG), (NB1 (1098),AW), (NBI(1 $2099)$ DEN), (NB1(1100),A2200), (NB1(1101), NDATA), $(N B 1(1102), A 1),(N B 1)$ $31103), B 1),(N B 1(1104), C 1),(N B)(1105), 01),(N B I(1106), A 2) \cdot(N B 1(1107)$ ) $4 E 2),(N B 1(1108), C 2),(N B 1(1109), D 2),(N B 1(1110), S, S I G S O, S I G S 1, E 0, S I G N$ $5 G, B E T A),(N B 1(1111), T M),(N B 1(1112), S O R),(N B 1(1155), X I S),(N B 1(1200)$, $6 T R),(N B 1(1245), A),(N B 1(1290), X I S 1),(N B 1(1335), F N U),(N B 1(1380), F), 1$ INB $1(1425), S 1),(N B 1(1470), T),(N B 1(1515), X),(N B 1(1560), X 1),(N B 1(1605$ $8), B),(N B 1(1650), P N U),(N B 1(1695), E T A)$

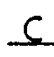

EQUIVALENCE (NB1(1740),TS4500),(NBI(1770),TS4000),(NB1(1800),TS35 $100)$, (NB) $(1830), T S 3000)$, (NB1 $(1860), T S 2500),(N B 1(1890), T S 2000),(N B 11$ $21920), T S 1500),(N B 1(1950), T S 1000),(N B 1(1980), T S 500),(N B 1(2010), T S 68$ $32,(N B 1(1310), R 10),(N B 1,(1510), G N),(N B 1(1710), G G),(N B 2(1006), G F),(N B$ $42(1206), G),(N B 2(1406), P 0),(N B 2(1606), R),(N B 2(1806), E T A R),(N B 2, F B 2)$ 5, (NBPSN (2), BCODE), (NBPSN (3), BNAM), (NB6,FB6)

C

DIMENSION NB1 $(2100), F B 1(2100), N B 2(2050), F B 2(2050), N B 6(2010)$, 1FB6(2010), NBPSN(1992), BCODE (1991), BNAM(1990), MOMIN 4980$), P C O D E(500$ 2). PUNCOD ( 500$)$, NBDEL (499), NRRDB (499), RLOCRC (499), THERM (15), MATDIM(I $30), E L(45), F V(45), U L(45), E G(45), U G(45), B D A T E(2), S(45), S I G S 0(925), S I$ 4GSI (225), EO (200), SIGNG (225), SOR (45),XIS(45), TR(45), A (45),XISI(45). $5 F N U(45), F(45), S 1(45), T(45), X(45), X 1(45), B(45), P N U(45), E T A(45), T S 45$ $600(30)$.TS4000(30), TS3500(30), TS3000(30), TS2500(30), TS2000(30), TS15 $700(30)$, TS $1000(30), T S 500(30), T S 68(30), R I O(200), G N(200), G G(200), G F(2$ $800), G(200), P 0(200), R(200)$, ETAR (200) ,ENERGY (45) , NAMTAP (3)

c

C SEI UP RECORD NUMBER

$N B I(1)=N B 6(4 * I+4)$

C $\ldots$ TEST.FOR SPECTRAL DATA

660 IF (FB6(4*I+3) 500.0$) 664.661 .661$

C SPECIRAL - WRITE MIN TAPES, PRINT IF DESIRED

661 CALL NMTPRC (NSPECT)

662 CALLWRSPDB (O)

663 GO TO 679

C CROSS-SECTION WRITE M,N TAPES, PRINT IF DESIRED

664 CALL NMTPRC (NWDBRI)

665 CALL DIMEN. (NBI)

666 CALL WRRECI (O,PTCODE,PNCODE)

C. DO SAME FOR REMAINING RECORDS OF DATA BLOCK AFTER READING THESE IN

IF (NI) $668,670,668$

$668 \mathrm{~J} 2=J 2+1$

CALL TAPECK (NBI,NI,ITAPI)

$N B I(1)=N B 6(4 * I+4)+J 2$ 
CALL NMTPRC (NI)

669 CALL WRREC2 (PTCODE,PNCODE)

670 IF (N2) $671,673,571$

$671 \mathrm{~J} 2=\mathrm{J} 2+1$

CALL TAPECK (NBI,N2,ITAPI)

$N B 1(1)=N B 6(4 * I+4)+J 2$

CALL NMTPRC (N2)

672 CALL WRREC3 (PTCODE,PNCODE)

673 IF (N3) $674,676,674$

$674 \mathrm{~J} 2=\mathrm{J} 2+1$

CALL TAPECK (NBI,N3, ITAPI)

$N B I(1)=N B 6(4 * I+4)+J 2$

CALL NMTPRC (N3)

675 CALL WRREC4 (PTCODE,PNCODE)

676 IF (N4) $677,679,677$

$677 \mathrm{~J} 2=\mathrm{J} 2+1$

CALL TAPECK (NBI,N4,ITAPI)

$N B 1(1)=N B 6(4 * 1+4)+J 2$

CALL NMTPRC (N4)

678 CALL WRREC5 (PTCODE, PNCODE)

679 RETURN

END $(0,0,0)$ 


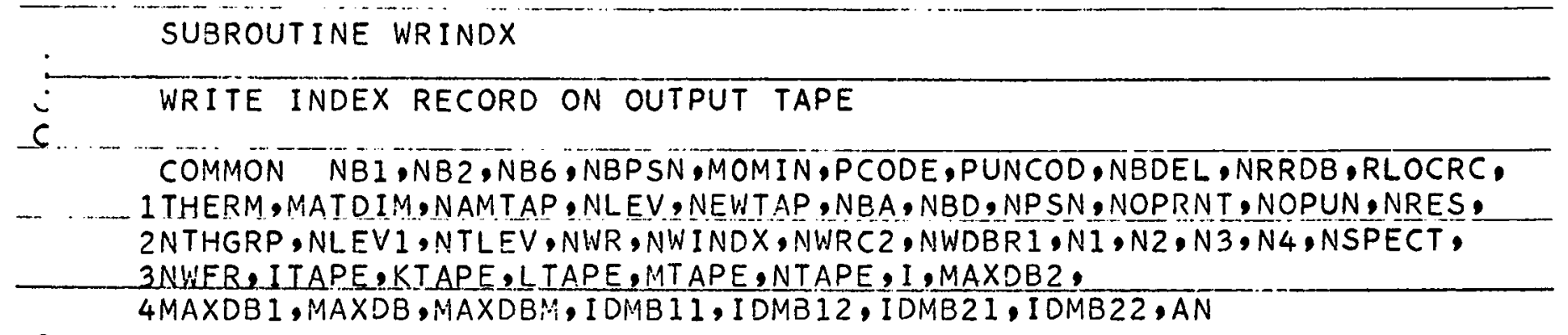

$C$

EQUIVALENCE (NBI,FBI), (NBI) 1006$), E L, E N E R G \bar{Y}),(N B I(1051), \overline{F V}),(N B I 11$ $.1096), U L, B D A T E),(N B 1(11.41), E G),(N B 1(1186), U G),(N B 1(1098), A W),(N B 111$ $2099)$, DEN), (NB1(1100), A2200), (NE111101), NDATA), (NB1(1102),A1), (NB11 $31103), B 1),(N B 1(1104), C 1),(N B 1(1105), D 1),(N B 1(1106), A 2),(N B I(1107)$ e 4B2), (NBI (1108), C2), (NE1(1109),D2), (NBI(1110),S,SIGSO,SIGSI,EO,SIGN $5 G, B E T A),(N B 1(1111), T M),(N B 1(1112), S O R),(N B 1(1155), X I S),(N B 1(1200)$. $6 T R),(N B 1(1245), A),(N B)(1290), X I S 1),(N B 1(1335), F N U),(N B 1(1380), F), 1$ $7 N B 1(1425), S 1),(N B 1(1470), T),(N E 1(1515), X),(N B 1(1560), X 1),(N B 1(1605$. $8), 8),(N B 1(1650), P N U),(N B 1(1695), E T A)$

$c$

EQUIVALENCE (NBI(1740),TS4500),(NB1(1770),TS4000),(NBI(1800),TS35 $100),(N B 1(1830), T S 3000)$, (NB) (1860),TS2500) , (NB I (1890), TS2000), (NB1) $21920)$, TS1500), (NB1(1950), TS1000), (NB1(1980),TS500), (NB1(2010), TS68 $31,(N B 1(1310), R I 0),(N B 1(1510), G N),(N B 1(1710), G G),(N B 2(1006), G F),(N B$ $42(1206), G),(N B 2(1406), P 0),(N B 2(1606), R),(N B 2(1806), E T A R),(N B 2, F B 2)$ $5,(N B P S N(2), B C O D E),(N B P S N(3), B N A M),(N B 6, F B 6)$

C

DIMENSION NB1 (2100),FB1 $(2100), N B 2(2050), F B 2(2050), N B 6(2010)$,

1 FB6 (2010), NBPSN(1992), BCODE (1991), BNAMI 1990$)$, MOMIN $(4980)$, PCODE $(500$ 2) . PUNCOD (500), NBDEL (499), NRRDE (499), RLOCRC(499), THERM (15), MATDIM(I $30), E L(45), F V(45), U L(45), E G(45), U G(45), B D A T E(2), S(45), S I G S O(925), S I$ $4 G S 1(925), E O(200), S I G N G(925), S O R(45), X I S(45), T R(45) \cdot A(45), X I S I(45)$, $5 F N U(45), F(45), S I(45), T(45), X(45), X 1(45), B(45), P N U(45), E T A(45), T S 45$ $600(30), T \$ 4000(30)$,TS3500(30),TS3000(30), TS2500(30), TS2000(30), TS15 $700(30)$, TS $1000(30)$, TS500(30), TS68(30),RIO(200), GN(200), GG(200), GF(2 $800), G(200), P O(200), R(200), E T A R(200)$, ENERGY $(45)$, NAMTAP ( 3$)$.

C

700 WRITE QUTPUT TAPE LTAPE, 701, (NAMTAP $(I), I=2,3)$, NLEV

701 FORMAT / 1 H157X,5HDATE $2 A 6 / / / 30 X, 12 \mathrm{HINDEX}$ RECORD///I33,7H LEVELS/10 1X,4HDATA/63H ELOCK NO. ... CODE NO. . .... DESIGNATION _... R

$2 E C O R D N O \cdot / / 1$

DO $705 \quad I=1, M A X D B 1$

WRITE OUTPUT TAPE LTAPE, 702 , I, NBI $(4 * I+3), N B 1(4 * 1+1)$, NBI $14 * 1+2)$; $1 N B 1(4 * I+4)$

702 FORMAT (I 14,F 18,4,4X,2A6,I12)

IF $(I-40 *(1 / 40)) 705,703,705$

703 WRITE CUTPUT TAPE LTAPE, 704 , NLEV

704. FORMAT ( 1 HI////30X,12HINDEX RECORD///133,7H LEVELS/10X,4HDATA/63H 1 BLOCK NO. CODE NO. DESIGNATION RECORD NO $/ 11$ C.T.5. CONTINUE

C...... PUNCH INDEX RECORD

CALL BRAND (NAMTAP $(2), 2)$

CALL PUNACB(NBI, NWINDX,O) 
CALL PUNACB $(0,0,0)$ DO $70 ? \quad I=1$, NWINDX

$7 \cap 7$ NB6(I) =NNE1(I)

RETURN

END $(0,0,0)$ 


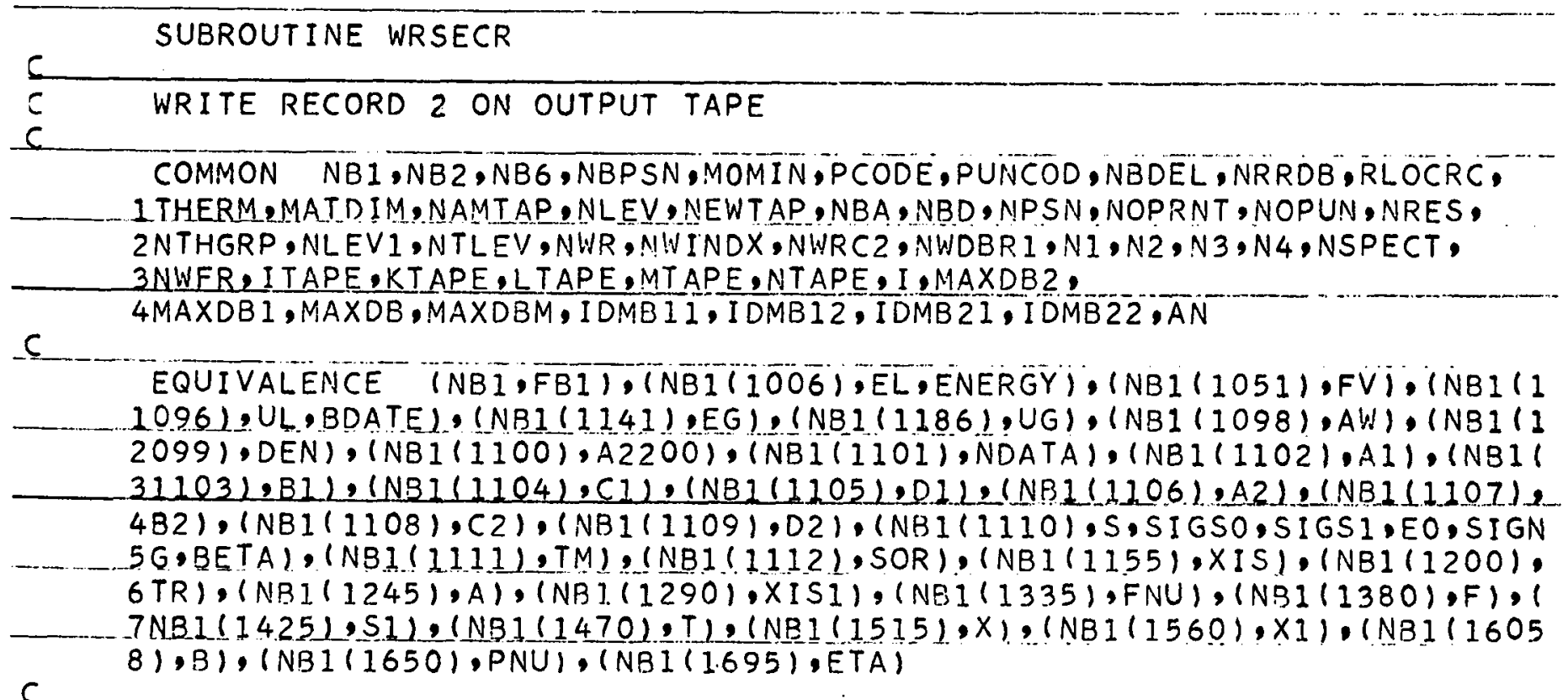

EQUIVALENCE (NB1(1740),TS4500), (NBI(1770):TS4000), (NBI(1800):TS35 $100),(N B I(1830)$, TS3000), (NB1 $(1860)$, TS2500), (NB1 $(1890)$, TS2000), (NBI1 $21920), T S 1500),(N B 1 .(1950)$, TS1000), (NB1 $(1980), T S 500),(N B 1(2010), T S 68$ $3) \cdot(N B I(1310), R I 0),(N B I(1510), G N),(N B 1(1710), G G),(N B 2(1006), G F)$, (NB $42(1206), G),(N B 2(1406), P 0),(N B 2(1606), R),(N B 2(1806), E T A R),(N B 2, F B 2)$ 5 . (NBPSN (2), BCODE), (NBPSN (3), BNAM), (NB6,FB6)

$\bar{C}$

DIMENSION NB1 (2100),FB1 $(2100), N B 2(2050), F B 2(2050), N B 6(2010)$, 1FB6(2010), NBPSN(1992),BCODE(1991), BNAM(1990), MONIN(4980),PCODE (500 2.).PUNCOD(500), NBDEL (499), NRRDB (499), RLOCRC(499), THERM(15), MATDIM(1 30), EL (45), FV (45), UL (45), EG(45),UG(45), BDATE(2), S(45), SIGSO(925), SI 4GSI (925), EO(200), SIGNG(925), SOR(45),XIS(45),TR(45),A(45),XISI(45), 5FNU(45), F(45),S1(45),T(45),X(45),XI(45),B(45),PNU(45),ETA(45), TS45 $600(30)$, TS4000(30), TS3500(30), TS3000(30), TS2500(30),TS2000(30), TS15 $700(30), T S 1000(30)$,TS500(30),TS68(30),RIO(200), GN(200), GG(200), GF(2 $C$ $800) .9(200), P O(200)$, R (200), ETAR (200), ENERGY (45), NAMTAP (3)

C CHECK FOR PRINTING SECOND RECORD

710 DO 711 II =1, NOPRNT

IF (PCOOE II I)-.000002) $711,712,711$

711 CONTINUE

C GO TO 730

PRINT

112 WRITE OUTPUT TAPE LTAPE. 713

713 FORMAT (52HI. LEVEL ENERGY (EV) "..... LETHARGY)

$12=N T L E V+1$

DO 718 I I $=1$, NLEV

$N A=I 1+I 2$

IF $((I 1-1)-5 *((I 1-1) / 5)) \quad 714,716,714$

714 WRITE OUTPUT TAPE LTAPE, $715,11, N B 1(11+1), N B I$ (NA)

715 FORMAT (I 19,1PE19.7,E17.7)

GO TO 718

716 WRITE OUTPUT TAPE LTAPE, 717, I1,NB1(I1+1), NB1(NA)

717 FORMAT (1HOI18,1PE19.7,E17.7)

718 CONTINUE 
$12=$ NLEV $1+N T L E V$

DO 723 II 1 , NTHGRP

$N A=N L E V 1+I 1$

$N B=11+12$

$N C=$ THERM(II)

IF $((I 1-1)-5 *((I)-1) / 5)) \quad 719,721,719$

719 WRITE OUTPUT TAPE LTAPE, 720, NC,NBI(NA), NB1 (NB)

720 FORMAT (I 20,1PE18.7,E17.7) GO TO 723

721. WRITE OUTPUT TAPE LTAPE, 722, NC,NB1 (NA), NBI (NB)

722 FORMAT (IHOI 19,IPEI8.7,E17.7)

723 CONTINUE

$I 2=2 * N T L E V+2$

$13=12+N$ LEV 1

$14=13+N L E V$

WRITE OUTPUT TAPE LTAPE, 724, NB1(I2),NB1(13)

724 FORMAT $162 \mathrm{H1}$ 1 FACTOR/63H $20 \mathrm{M} / \mathrm{SECI//1IH}$ LOWER GROUP ENERGY (EV) LETHARGY 1220 DO $729 \quad 11=1$, NLEV

$N A=I I+I 2$

$N B=I 1+13$

$N C=I 1+14$

IF $(I 1-5 *(I) / 5)) \quad 725,727,725$

725 WRITE OUTPUT TAPE LTAPE, 726, II,NBI (NA),NBI $(N B), 0 N B I(N C)$

726 FORMAT (I11.1PE19.7.1PE17.7.1PE17.7) GO TO 729

727 WRITE OUTPUT TAPE LTAPE, $728,11, N B 1$ (NA), NB1 (NB), NBI (NC)

728 FORMAT (IHOI 10,1PE19.7,1PE17.7,1PE17.7) c

729 CONTINUE

C PUNCH RECORD 2

C CHECK FOR PUNCHING

730 DO 731 I $1=1$, NOPUN

IF (PUNCOD(I I) $\quad .000002) \quad 731.732 .731$

731 CONTINUE

GO .TO ...734 _...

C PUNCH

. 73.2. CONT INUE

CALL BRAND(6HREC 2,1$)$

CALL_PUNACB (NBI, NWRC2.0)

CALL PUNACB $(0,0,0)$

734 RETURN

END $(0,0.0)$ 
SUBROUTINE WRSPDB (NG)

$r$ WRITE SPECTRAL DATA BLOCK ON OUTPUT TAPE

COMMON NBI, NB2, NB6, NBPSN,MOMIN,PCODE,PUNCOD, NBDEL, NRRDB, RLOCRC, 1. THERM , MATD IM, NAMTAP, NLEV_NEWTAP, NBA, NBD, NPSN, NOPRNT , NOPUN, NRES, 2 NTHGRP, NLEV 1 , NTLEV, NWR, NWINDX, NWRC2, NWDBR $1, N 1, N 2, N 3, N 4, N S P E C T$, $3 N W E R, I T A P F, K T A P E, L T A P E, M T A P E, N T A P E, I, M A X D 82$, 4MAXDB 1, MAXDB, MAXDBM, IDMB II, IDMB12, IDMB2I, IDMB22, AN

$C$

EQUIVALENCE (NBI, FB1), (NBI(1006), EL,ENERGY), (NBI(1051), FV), (NBI(1 1096), UL, BDATE), (NB1 (1141),EG), (NBI (1186),UG), (NBI(1098),AW), (NBI(1 $2099), D E N),(N B 1(1100), A 2200),(N B 1(1101), N D A T A),(N B 1(1102), A 1),(N B 1)$

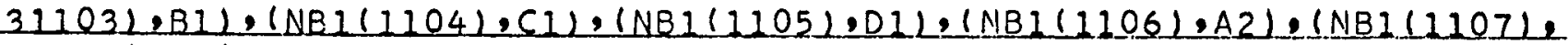
$4 B 2),(N B 1(1108), C 2),(N B I(1109), D 2),(N B 1(1110), S, S I G S O, S I G S 1, E 0, S I G N$ $5 G, B E T A),(N B 1(1111), T M),(N B I(1112), S O R),(N B 1(1155), X I S),(N B 1(1200)$. $6 T R),(N B 1(1245), A),(N B 1(1290), X I S 1),(N B 1(1335), F N U),(N B 1(1380), F), 1$ $7 N B 1(1425), S 1) \cdot(N B 1(1470), T),(N B 1(1515), X) \cdot(N B 1(1560), X 1),(N B 1(1605$. $8), B),(N B 1(1650), P N U),(N B 1(1695), E T A)$

$c$

EQUIVALENCE (NB1(1740),TS4500),(NB1(1770),TS4000), (NB1(1800),TS35 $100),(N B)(1830), T S 3000),(N B 1(1860), T S 2500),(N B 1(1890), T S 2000),(N B 1)$ $21920)$, TS1500), (NB1(1950), TS1000), (NB1(1980), TS500), (NE1(2010), TS68 $3),(N B 1(1310), R I 0),(N B 1(1510), G N),(N B 1(1110), G G),(N B 2(1006), G F),(N B$. $42(1206), G),(N B 2(1406), P 0),(N B 2(1606), R),(N B 2(1806), E T A R),(N B 2, F B 2)$ $5,(N B P S N(2), B C O D E),(N B P S N(3), B N A M),(N B 6, F B 6)$

C

DIMENSION NB1(2100),FB1 $(2100), N B 2(2050), F B 2(2050), N B 6(2010)$, 1FB6(2010), NBPSN(1992),BCODE(1991), BNAM(1990), MOMIN(4980),PCODE(500 2), PUNCOD (500), NBDEL (499), NRRDB (499), RLOCRC (499), THERM ( 15), MATOIM(1. $30), E L(45), F V(45), U L(45), E G(45), U G(45), B D A T E(2), S(45), S I G S O(925), S I$ $4 G S I(225), E O(200), S I G N G(925), \operatorname{SOR}(45), X I S(45), T R(45), A(45), X I S 1(45)$. $5 F N U(45), F(45), S 1(45), T(45), X(45), X 1(45), B(45), P N U(45), E T A(45), T 545$ $600(30)$, TS4000(30), TS3500(30),TS3000(30), TS2500(30),TS2000(30), TS15 $700(30)$, TS1000(30), TS500(30), TS68(30),RIO(200), GN(200), GG (200), GF(2 $800), G(200), P O(200), R(200), E T A R(200), E N E R G Y(45), N A M T A P(3)$

C

c. IF NG $=1$, THEN THIS IS AN ADDED DATA BLOCK AND IS TO BE

C IPRINTED AUTOMATICALLY, IF NG $=0$, THEN THIS BLOCK IS FROM EITHER

C 2ITAPE OR KTAPE AND A CHECK IS MADE FOR PRINTING.

739 IF (NG) $742,740,742$

740 DO 741 II $1=1$ NOPRNT

IF (PCODE (II)-FB6 $(4 * I+3)) 741,742,741$

741 CONTINUE

GO TO 754

C PRINT

742 WRITE OUTPUT TAPE LTAPE, $743,(N B I(I 1), I 1=2,3), N B 6(4 * I+1)$, $1 N B 6(4 * I+2), N B 1$ (NTLEV+5), NBI (NTLEV+6)

743 FORMAT 11 H156X,5HDATE $2 A 6 / / / 35 X, 25 H D E L A Y \quad$ PRECURSOR/6OH 1 DESIGNATION FRACTION

$2,1 P 2 E 18.4 / / 117 \mathrm{X}, 34 \mathrm{HLEVEL}$ ENERGY MEAN LIFE//13X,2A6 DO 748 I $I=1$, NLEV

IF $(1$ II-1)-5*((II-1)/5)) $744,746,744$

744 WRITE OUTPUT TAPE LTAPE, 745, I I, ENERGY (I 1$)$, NBI (I 1+4)

745 FORMAT (I20.1PE16.3.E18.7) 
GO TO 748

746 WRIIE OUTPUI IAPE LTAPE, 747 ULENERGYU11) NBI $(12+4)$

747 FORMAT (1HOI19,1PE16.3,E18.7)

7.48_CONTINUE

12 - NLEV+4

DO $753 \ldots 11 \equiv$.NTHGRP

$N A=11+N L E V$

$N B=11+12$

NC $=\operatorname{THERM}(11)$

IF $(11-1)-5 *(1,1-1) / 5) 1 \quad 749,751,749$

749 WRITE OUTPUT TAPE LTAPE, 750, NC,ENERGY (NA), NBI (NB)

750 FORMAT (121,2PE $15.3, E 18.7)$

60 TO 753

751 WRITE QUTPUT IAPE LTAPE, 7529

752 FORMAT (1HOI 20,1PE 15.3,E18.7)

753 CONTINUE

c

$C \quad$ PUNCH SPECTRAL DATA BLOCK

C CHECK FOR PUNCHING

75400755 II 1 .NOPUN

IF (PUNCOD(11) FB6 (4*l+3)) $755 \cdot 756175$

755 CONTINUE

GO TO 758

C PUNCH

736 CONIINUE

CALL BRAND $(N B 6(4 * I+I) \cdot 2)$

CALL PUNACBINBIENSPECT,O)

758 RETURN

END 10.0 .01

SUBROUTINE WRRECI (NG,PTCODE,PNCODE)

$\frac{c}{c}$

WRITE RECORD I OF CROSS SECTION DATA BLOCK ON OUTPUT TAPE

COMMON NBI, NB2, NB6, NBPSNOMOMIN,OCOOE,PUNCOD,NBDEL NRRDB,RLOCRC, ITHERM,$M A T D$ IM N NAMTAP NLEV NEWTAP N $3 A, N B D, N P S N, N O P R N T$ NOPUN NRES 2NTHGRP, NLEVI, NTLEV, NWR , NWI NDX, NWRC2, NWDBRI, NI, N2, N3,N4 NSPECT, 3 NWFB IIAPE IKIAPE, LIAPE IMTAPE INIAPE, I MAXDB2,

4MAXDB I.MAXDB,MAXDAM, IDME II, IDMB I2, IDMB I I IDMB 22, AN

$c$

EQUIVALENCE (NBI,FBIT, (NEIT1006), ELENERGYTINGITIOSI),FVT:INBITI

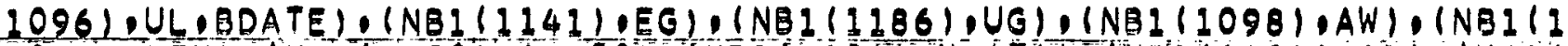

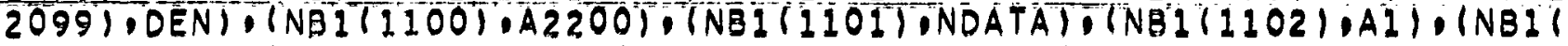
$3,1103), B 1) \cdot(N B 1(1104) \cdot C 1) \cdot(N B 1(1105), 01) \cdot(N B 1(1106), A 2),(N B 1(1107)$.

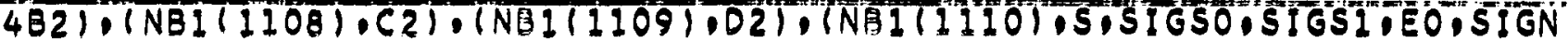
SG BETA), (NEI (1111),TM), (NBI(1112),SOR), (NBI(1155),XIS), (NBI(1200).

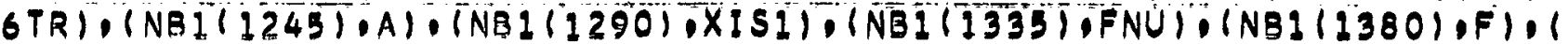

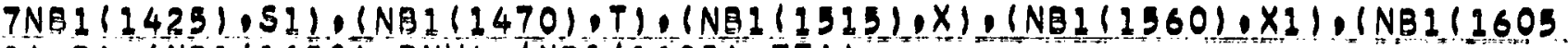
8 ) B IO (NBI(1650) OPNU), (NBI(1695),ETA)

c

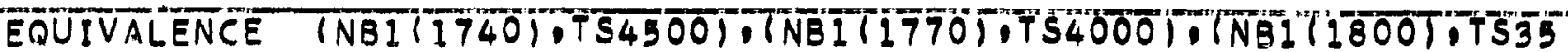

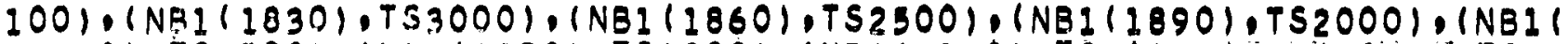
$21920) \cdot T S 1500) \cdot(N B 1(1950) \cdot T 51000) \cdot(N B 1(1980) \cdot T 5300) \cdot(N B 1(2010), T 568$ $3),(N B 1(1310), R 10)$, (NB1 (1510), GN), (NB1(1710),GG), (NB2)(1006),GF), (NB $42(1206) \cdot G),(N B 2(1406), 00),(N B 2(1606), R),(N B 2(1806), E T A R),(N B 2, F 82)$ 
$5,(N B P S N(2), B C O D E),(N B P S N(3), B N A M),(N B 6, F B 6)$

DIMENSION NBI(2100),FB1(2100),NB2(2050),FB2(2050),NB6(2010), IFB6 (2010), NBPSN (1992), BCODE (1991), BNAM(1990),MOMIN(4980),PCODE(500 2), PUNCOD (500), NBDEL (499), NRRDB (499), RLOCRC(499), THERM (15), MATDIM(I $30), E L(45), F V(45), U L(45), E G(45), U G(45), B D A T E(2), S(45), 51 G S O(925), S I$ 4GSI(925), EO(200),SIGNG (925), SOR(45),XIS(45), TR (45),A $(4.5), X I S I(45)$, $5 F N U(45), F(45), S 1(45), T(45), X(45), X 1(45), B(45), P N U(45), E T A(45), T S 45$ $600(30)$, TS4000(30), TS $3500(30), T S 3000(30)$, TS2500(30), TS2000(30), TS15 $700(30)$, TS $1000(30)$, TS500(30),TS68(30),RIO(200), GN(200), GG(200), GF(2 $800), G(200), P O(200), R(200)$, ETAR(200), ENERGY(45), NAMTAP(3)

$c$

IF NG $=1$, PRINTING IS AUTOMATIC, IF NG $=0$, PRINT IF REOUESTED.
759 IF (NG) $762,760,762$

760 DO 761 I $1=1$, NOPRNT

IF (PCODE (II)-FB6 $(4 * I+3)) \quad 761.762 .761$

761 CONTINUE

GO TO 808

C PRINT

762 WRITE OUTPUT TAPE LTAPE, 764, NB1(2), NB1(3),NB6(4*I+1),

$1 N B 6(4 * I+2) \cdot N B 6(4 * I+3) \cdot N B 1(6 * N T L E V+5)$

764 FORMAT (IHI57X,5HDATE 2A6//) 9X,53HDESIGNATION 1 DENSITY FACTOR//9X,2A6,F17.4,1PE22.5/1/70H

2 SIGMA XI SIGMA SIGMA SIGMA XII SIGMA/7OH LEVEL 3 ENERGY SCATTER SCATTER TRANSPORT ABSORPTION SCATTER II

$13=N T L E V+4$

$I 4=I 3+N T L E V$

$I 5=I 4+N T L E V$

$16=15+N T L E V$

$I 7=I 6+N T L E V$

DO 769 I $I=1$. NLEV

$N A=11+13$

$N B=11+14$

$N C=11+15$

$N D=I 1+16$

IF $((11-1)-5 *((1)-1) / 5)) \quad 765,767,765$

765 WRITE OUTPUT TAPE LTAPE, 766, II, ENERGY(II), NBI(I I+4), NBI (NA), NBII INB), NBI (NC), NBI (ND)

766 FORMAT $(14.1 \mathrm{PE} 11.3,4 E 11.4, E 12.4)$

GO TO 769

767 WRITE OUTPUT TAPE LTAPE, 768, II, ENERGY(II), NBI(II+4), NBI (NA), NBII $1 N B), N B 1(N C), N B 1$ (NO)

768 FORMAT (IHOI3,1PEII.3,4E11.4,E12.4)

769 CONTINUE

$12=N L E V+4$

$13=13+N L E V$

$14=14+N L E V$

$15=15+N L E V$

$16=16+N L E V$

$17=17+$ NLEV

DO 774 I I =1,NTHGRP

$J I=\operatorname{THERM}(I I)$

$N A=I 1+12$

$N B=11+13$

$N C=11+14$ 
$N D=11+15$

$N E=I 1+16$

$N F=I I+N L E V$

IF $((I 1-1)-5 *((I 1-1) / 5)) \quad 770,772,770$

770 WRITE OUTPUT TAPE LTAPE, 771, J1, ENERGY(NF), NBI (NA), NBI (NB), NBI (NC

1), NBI (ND), NBI (NE)

771 FORMAT (I5,1PE10.3,4E11.4,EI2.4) GO TO 774

772 WRITE OUTPUT TAPE LTAPE, 773, J1, ENERGY(NF), NBI (NA), NBI (NB), NBI (NC 1), NBI (ND), NBI (NE)

773 FORMAT $(1$ HOI 4,1 PE10.3,4E11.4,E12.4)

c

774 CONTINUE

$\frac{c}{c}$

77.5 WRITE OUTPUT TAPE LTAPE, 776, NB6 $(4 * I+1), N B 6(4 * I+2), N B 6(4 * I+3)$, 1 NB $1(6 * N T L E V+5)$

776 FORMAT $(63 \mathrm{H} 1$ DESIGNATION CODE NO.

IY FACTOR//10X,2A6,F17.4,1PE22.5///43X,8HNU SIGMA/21X,3OHLEVEL 2ENERGY

$13=16+$ NTHGRP

DO 781 I $1=1$, NLEV

$N A=11+13$

IF. $((I 1-1)-5 *(11-1) / 5)) \quad 777,779,777$

777 WRITE OUTPUT TAPE LTAPE, 778, I I,ENERGY(II), NBI (NA)

778 FORMAT (I24,1PE14.3.E14.4)

GO TO 781

779 WRITE OUTPUT TAPE LTAPE, 780, 11, ENERGY (I 1), NBI (NA)

780 FORMAT (IHOI23,1PE14.3,E14.4)

781. CONTINUE

$13=13+N L E V$

DO 786 I $1=1, N$,

$N A=11+N L E V$

$N B=11+13$

$N C=\operatorname{THERM}(11)$

IF $((I 1-1)-5 *(11-1) / 51) \quad 782,784,782$

782 WRITE OUTPUT TAPE LTAPE, 783 , NC,ENERGY (NA), NBI (NB)

283 FORMAT $(125,1 P E 13,3, E 14.4)$

GO TO 786

784 WRITE OUTPUT TAPE LTAPE, 785, NC, ENERGY (NA), NB I (NB)

785 FORMAT (IHOI24,1PE13.3,E14.4)

7.8.6 CONTINUE

C

C (PAGES $4-8$ )

$788 I 2=6 * N T L E V+6$

DO $793 \mathrm{~J}=1, \mathrm{NTHGRP}$

IF $((J-1)-2 *((J-1) / 2)) 791,789,791$

789 WRITE OUTPUT TAPE LTAPE, 790, NB6(4*I+1), NB6 $(4 * I+2)$

720 EORMAT (1H129X.2A6/./23X,25HTHERMAL TRANSFER MATRICES)

$79113=J+$ NLEV.

WRITE OUTPUT TAPE LTAPE, 792, NBI(I2),NB1(I2 1),NBI(12 2),NBI(I 3 1), NBI $(12+4), I 3, N B 1(I 2+5)$, NBI $1(2+6), N B 1(12+7), N B 1(I 2+8), N B I(12+9)$, $213, N B I(12+10), N B I(I 2+11)$, NBI $12+12)$, NBI $(12+13)$, NBI $(12+14), I 3$, $3 N B 1(I 2+15)$, NBI $(I 2+16)$, NBI I $2+17)$, N B I $(12+18), N B I(I 2+19), I 3$, 
$4 N B 1(12+20), N B 1(I 2+21), N B 1(I 2+22), N B 1(I 2+23)$, NBI $1(2+24)$. $513,13,13,13,13,13, N B 1(1225), N B 1(12+26), N 31(12+27), N B 1(12+28)$, $6 N B I(12+29)$

7.92 FORMAT. . $1 / 1 / 1 / 68 \mathrm{H}$ TEMPERATURE IGMA NU SIGMA/67H DEG.

SIGMA

F. SCATTER

FISSION//IPEI3.4.4EI4.4///3IX,9HNI

2... ABSORPT I ON

3SIGMA S $(\mathrm{NI/N} 2) / / 63 \mathrm{H}$

2121

2122

$21 I 4 / 1 \mathrm{E} 13.4,4 \mathrm{E} 14.4 / 63 \mathrm{H}$

$22 \mathrm{I} 4 / 1 \mathrm{E} 13.4,4 \mathrm{EI} \cdot \mathrm{H}$

$5-\frac{24}{22}-\frac{4}{23}$

6

$74 / 63 \mathrm{H} \quad 24 \quad 21$

$22 \quad 24$

2323.

2422
2324 $24 \quad 23$

$824 \mathrm{I} / 1 \mathrm{EE} 13.4,4 \mathrm{E} 14.4 / \mathrm{I}, 4 \mathrm{H} \quad 21 \mathrm{IIO}, 4 \mathrm{H} \quad 22 \mathrm{I} 10,4 \mathrm{H}$

$9 E 13.4,4 E 14.4)$

$.72312=12+30$

GO TO 794

$-c$

C (PAGE 9 )

c.

794 WRITE OUTPUT TAPE LTAPE, $795, N B 6(4 * 1+1), N 86(4 * I+2)$

795 FORMAT (1H130X,2A6////////1) IF (NI) 796,798,796

726 WRITE OUTPUT TAPE LTAPE, 797, MATDIM(1),MATDIM(2), MATDIM( I), MATDIM I(3)

797 FORMAI $132 \mathrm{X}, 8 \mathrm{HSIGMA} 50 / 127 \mathrm{H}$

TRANSFERS FROM GROUPS $13.8 \mathrm{H}$ THROUG 1HI $3,10 \mathrm{H}$ TO GROUPSI $3,8 \mathrm{H}$ THROUGH $3 / / / / / 1$

798 IF $(N 2) \quad 799.801,799$

799 WRITE OUTPUT TAPE LTAPE, 800, MATDIM(4), MATDIM(5), MATDIM(4), MATDIM $1(6)$

800 FORMAT $132 \times, 8 \mathrm{HSIGMA} 51 / / 27 \mathrm{H}$

TRANSFERS FROM GROUPSI 3 , 8 H THROUUG $1 \mathrm{H} 13,10 \mathrm{H}$ TO GROUPS $13,8 \mathrm{H}$ THROUGHI $3 / 1 / 1)$

801 IF (N3) $802,804,802$

802 WRIIE OUTPUT TAPE LTAPE, 803, MATDIM(7)

803 FORMAT $(29 \mathrm{X}, 14 \mathrm{HNO}$. RESONANCES//137////)

804 IF $(N 4) \quad 805,807.805$

805 WRITE OUTPUT TAPE LTAPE, 806, MATDIM(8), MATDIM(9), MATDIM(8), MATDIM $1(10)$

806 FORMAT (28X,15HSIGMA (N, GAMMA)//27H

IH IHROUGH I3, IOH IO GRQUPS I3,8H THROUGHI3I TRANSF̈ERS FROM GROUPSI 3.8

C SET PTCODE = 1,0 ACCORDINGLY AS THIS RECORD IS OR IS NOT

C. IPRINTED THUS AVOIDING A CHECK OF THE PCODE BLOCK IN

$C$ 2SUBSEQUENT RECORDS OF THIS DATA BLOCK

807 PTCODE $=1.0$ GO TO 809

808 RTCODE $=0.0$

c

$c$

$c$

c

$80900810 \ldots 11=1$ NOPUN

IF (PUNCOD(I1) FB6(4*I+3)) 810.811 .810

810. CONT INUE

GO TO 813

PUNCH

811 CONTINUE 
CALL 3RAND $(N 36(4 * I+1), 2)$

CALL PUNACB (NBI, N!:DBR I,O)

- SET PNCODE $=1,0$ ACCORDINGLY AS THIS RECORD IS OR IS NOT

C IPUNCHED THUS AVOIDING A CHECK OF THE PUNCOD BLOCK IN

C 2SUBSEQUENT RECORDS OF THIS DATA BLOCK.

PNCODE $=1 \cdot 0$

GO TO 814

813 PNCODE $=0.0$

814 RETURN

END $(0,0,0)$ 
SUBROUTINE WRREC2 (II3, II 4 )

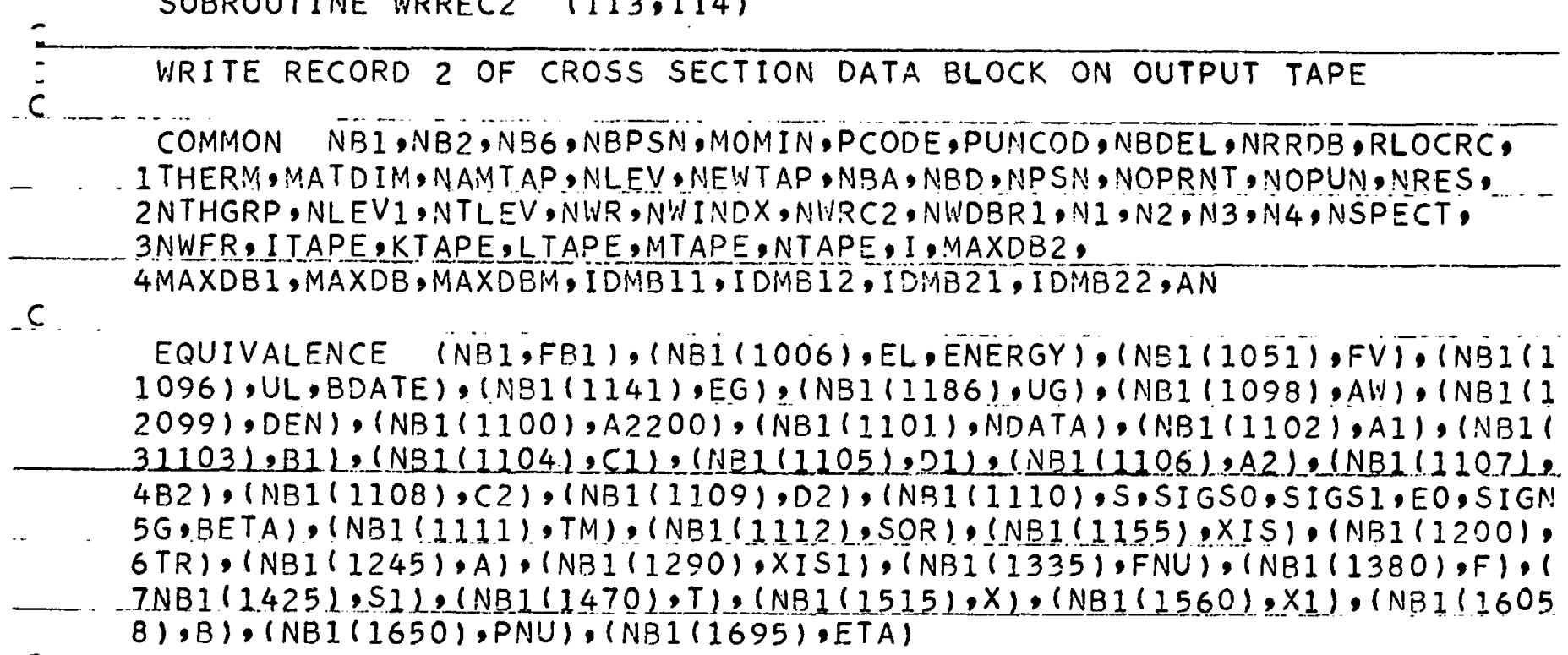

c

EQUIVALENCE (NBI(1740),TS4500), (NB1) 1770),TS4000), (NBIT1800),TS35 $100)$ (NBI $(1830)$, TS3000), (NB1 (1860), TS2500), (NB1(1890), TS2000), (NB1) $21920)$, TS1500), (NB1(1950), TS1000), (NB1(1980), TS500), (NBI(2010),TS68 $3) \cdot(N B 1(1310), R I 0),(N B 1(1510), G N),(N B 1(1710), G G),(N B 2(1006), G F),(N B$ $42(1206), G),(N B 2(1406), P O),(N B 2(1606), R),(N B 2(1.806), E T A R),(N B 2, F B 2)$ $5,(N B P S N(2), B C O D E),(N B P S N(3), B N A M),(N B 6, F B 6)$

C

DIMENSION NBI (2100),FBI $(2100), N B 2(2050), F B 2(2050), N B 6(2010)$,

IFB̈6(2010), NBPSN(1992), BCODE 1991$), B N A M(1990), M 0 M I N(4980), P C O D E T 500$ 2) . PUNCOD (500), NBDEL (499), NRRDB (499), RLOCRC(499), THERM (15), MATDIM(1 $30), E L(45)$, FV (45), UL (45), EG(45),UG(45), SDATE (2), S(45), SIGSO (925), SI $4 G S 1(925), E O(200)$, SIGNG $(925)$, SOR $(45), \times I S(45), T R(45), A(45), X I S I(45)$, 5FNU(45), F(45), SI (45), T(45),X(45),XI(45),B(45),PNU(45), ETA(45), TS45 $600(30), T S 4000(30)$, TS $3500(30), T S 3000(30), T S 2500(30)$, TS2000(30), TS15 $700(30)$, TS $1000(30), T S 500(30), T S 68(30), R I 0(200), G N(200), G G(200), G F(2$ $800) . G(200)$,PO(200),9R(200), ETAR(200), ENERGY(45), NAMTAP(3)

c

C SUBROUTINES WRREC2,URREC3 WRREC 5 EACH USE ROUTINE RC235

C ITO SET UP THE PRINT FORMAT. AS ALL RECORDS OF A DATA BLOCK

C ... 2WILL BE PRINTED IF THE FIRST IS, THE CODE WORDS 133,I44 WERE

C 3SET TO 1.0 IN WRRECI ACCORDINGLY AS THIS

C. $\quad 4 B L O C K$ IS OR IS NOT TO BE PRINTED, PUNCHED. THE VALUES OF

C SMATDIM(J) ARE MOMIN,MOMAX,... NG IS

C GA CODE WORD INDICATING THE PAGE HEADING TO BE PRINTED.

816 IF (II3) $817,822,817$

$817 M I=$ MATDIM(I)

$M 2=$ MATDIM(2)

820 CALL RC235 (MI,M2,NI,O)

c

C PUNCH RECORD 2 OF CROSS SECTION DATA BLOCK

822 IF (II 4 ) $823.824,823$

$823 N=$ NIVDBRI

CALL PUNACB $(N B 1, N 1, N)$ 
824 RETURN

END $(0,0,0)$ 
SUBROUTINE WRREC3 (II3,II4)

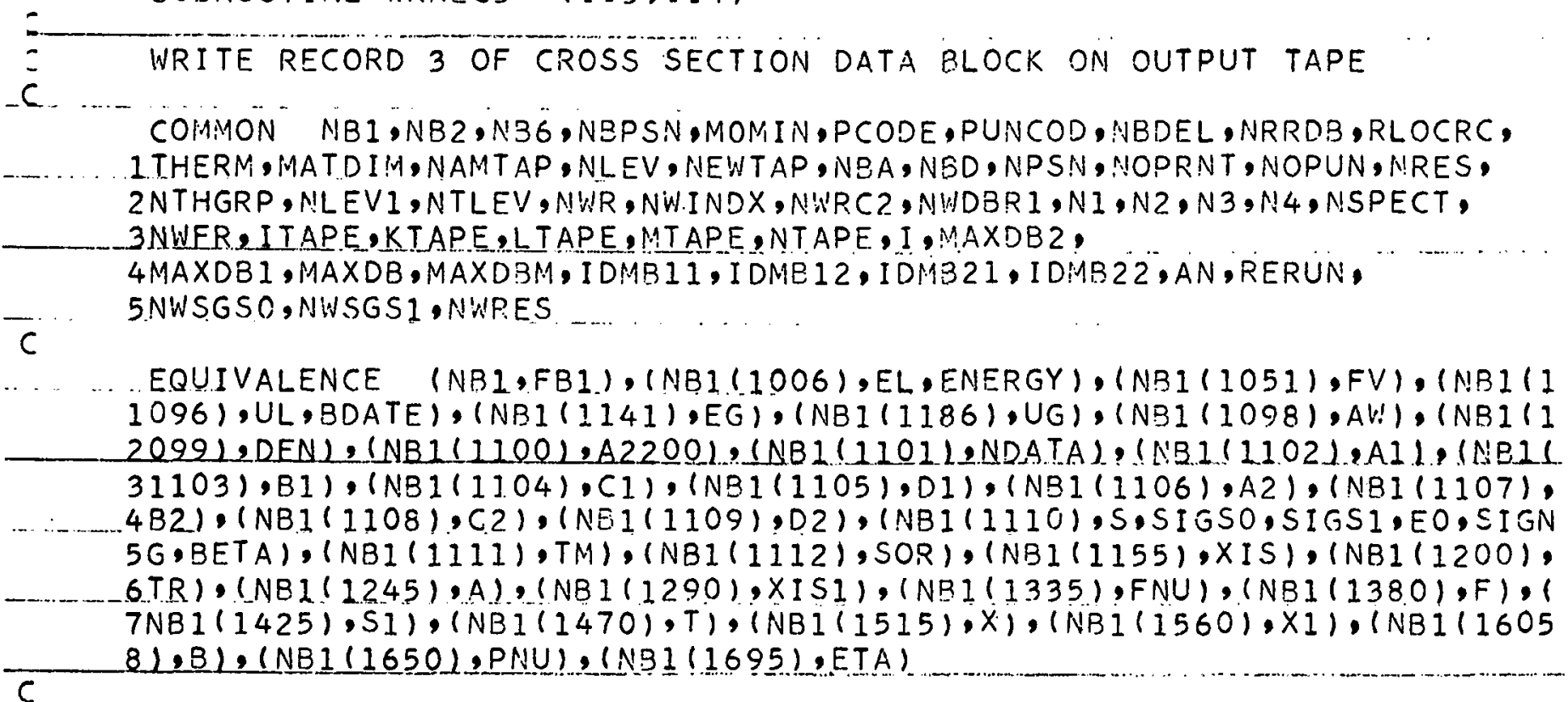

EQUIVALENCE (NBI $(17.40), T S 4500),(N B 1(1770), T S 4000),(N B 1(1800), T S 35$ $100),(N B 1(1830), T S 3000)$, (NS1 (1860), TS2500), (NB 1 (1890), TS2000), (NBI) $21920), T S 1500),(N B 1(1950), T S 1000),(N B I(1980), T S 500),(N B 1(2010), T S 68$ $3),(N B 1(1310), R I 0),(N B I(1510), G N),(N B 1(1710), G G),(N B 2(1006), G F),(N B$ $42(1206), G),(N B 2(1406), P 0),(N B 2(1605), R),(N B 2(1806), E T A R),(N B 2, F B 2)$ $5,(N B P S N(2), B C O D E),(N E P S N(3), B N A M),(N B 6, F 36)$

C

DIMENSION NBI $(2100), F B 1(2100), N B 2(2050), F B 2(2050), N B 6(2010)$; 1FB6 (2010), NBPSN(1992), BCODE (1991),BNAN(1990), MONIN(4980),PCODE (500 2). PUNCOD (500), NBDEL (499), NRRDB (499), RLOCRC(499), THERM(15), MATDIM(1 $30), E L(45), F V(45),(U L(45), E G(45), U G(45), B D A T E(2), S(45), S I G S O(925), S I$ 4GSI(925), EO(200),SIGNG(925), SOR(45),XIS(45), TR(45), A(45),XISI(45): 5ENU (45), F(45),S] (45),T(45), X(45), X $(45), 8(45), P N U(45), E T A(45)$, TS45 $600(30)$, TS4000(30), TS3500(30), TS3000(30), TS2500(30), TS2000(30), TS15 $700(30)$, TS $1000(30)$, TS500(30),TS68(30),RIO(200), GN(200),GG(200), GF(2 $800), G(200), P O(200), R(200)$, ETAR $(200)$, ENERGY (45), NAMTAP (3)

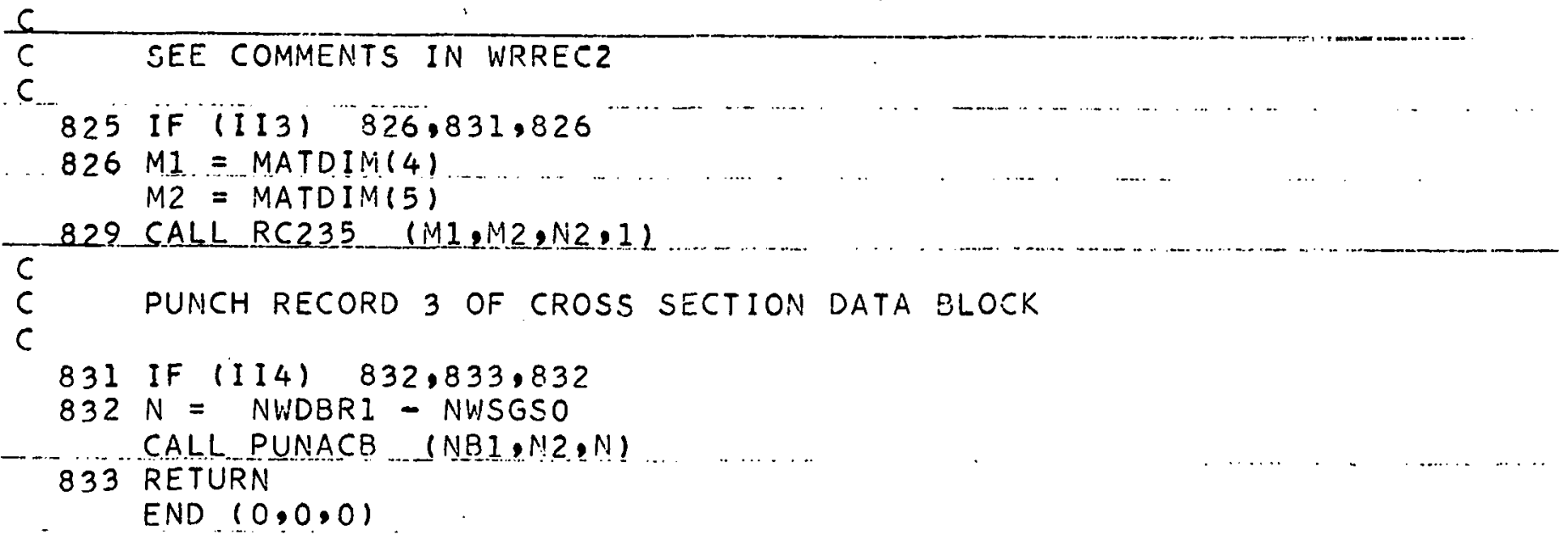




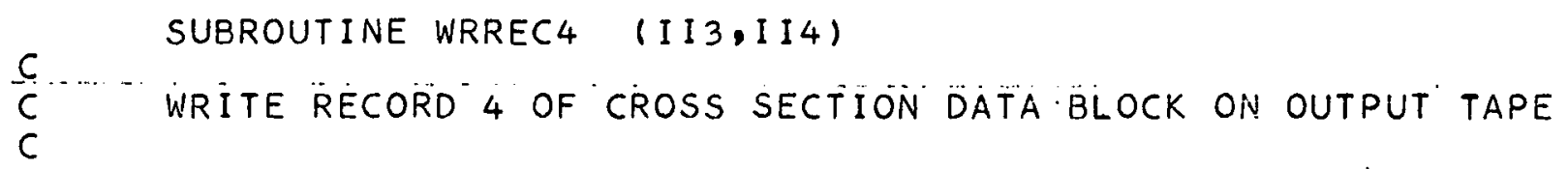

COMMON NBI, NB2, NB6, NBPSN, MOMIN,PCODE, PUNCOD, NBDEL, NRRDB, RLOCRC, 1 THERM, MATDIM, NAMTAP, NLEV, NEWTAP, NBA, NBD, NPSN, NOPRNT, NOPUN, NRES, $2 N T H G R P, N L E V 1, N T L E V, N W R, N$ INIDX, NIVRC2, NWDBR I, NI, N!2, N3, N4, NSPECT, $3 N W F R, I T A P E, K T A P E, L T A P E, M T A P E, N T A P E, I, M A X D B 2$,

4MAXDB I, MIAXDB, MAXXDBM, IDMB 11, IDHE12, IDMB21, IDMB22, AN, RERUN, 5 NWSGSO, NWSGS1, NWRES

$\bar{C}$

EQUIVALENCE (NBI, FB1), (NBI (1006), EL,ENERGY), (NB1(1051), FV), (NSI(1 $1096), U L, B D A T E),(N B I(1141), E G),(N B 1(1186), U G),(N B 1(1098), A W),(N B 1(1$ 2099), DEN), (NB1(1100), A2200), (NB1(1101), NDATA), (NEI(1102),AI), (NBI) $31103), B 1),(N B 1(1104), C 1),(N B 1(1105), 01),(N B 1(1106), A 2),(N B 1(1107)$, $4 B 2),(N B I(1108),(2),(N B I(1109), 02),(N B I(1110), S, S I G S O, S I G S I, E O, S I G N$ $5 G, B E T A),(N B 1(1111), T M),(N E 1(1112), S O R),(N B 1(1155), X I S),(N B I(1200)$, $6 T R),(N B 1(1245), A),(N B 1(1200), X I S 1),(N B 1(1335), F N U),(N B I(1380), F), 1$ $7 N B 1(1425), S 1),(N B I(1470), T),(N B 1(1515), X),(N B 1(1560), X 1),(N B 1(1605$ $8), B),(N B I(1650), P N U),(N B 1(1695), E T A)$

$\mathrm{C}$

EQUIVALENCE (NBI(1740),TS4500), (NBI(1770),TS4000),(NBI(18C0),TS35 $100),(N B 1(1830), T S 3000)$, (NB1 $(1860), T S 2500),(N B 1(1890), T S 2000),(N B 11$ $21920), T S 1500),(N B I(1950), T S 1000),(N B 1(1980), T S 500),(N B 1(2010), T S 68$ 3), (NBI (1310),RI0), (NBI(1510), GN), (NB1(1710),GG), (NB2(1006), GF), (NB $42(1206), G),(N B 2(1406), P 0),(N B 2(1606), R),(N B 2(1806), E T A R),(N B 2, F B 2)$ $5,(N B P S N(2), B C O D E),(N B P S N(3), B N A M),(N B 6, F B 6)$

C

DIMENSION NBI(2100), FBI(2100), NB2(2050), FB2 $(2050)$, NB6(2010), 1FB6 (2010), NBPSN (1992), BCODE (1991), BNAM(1990), MOMIN(4980), PCODE 500 2), PUNCOD (500), NBDEL (499), NRRDB (499), RLOCRC(499), THERM(15), MATDIM(I $30), E L(45), F V(45), U L(45), E G(45), U G(45), B D A T E(2), S(45), S I G S O(925), S I$ 4GSI(925), EO(200), SIGNG (925), SOR(45), XIS(45), TR (45),A(45),XISI(45): 5FNU (45), $F(45), S I(45), T(45), X(45), X 1(45) ; B(45), P N U(45), E T A(45), T S 45$ $600(30)$, TS $4000(30)$, TS3500(30), TS3000(30), TS2500(30), TS2000(30), TS15 $700(30), T S 1000(30), T S 500(30), T S 68(30), R I 0(200), G N(200), G G(200), G F(2$ $800), G(200), P O(200), R(200)$, ETAR(200), ENERGY (45), NAMTAP(3)

C SEE COMMENTS IN WRREC2

834 IF (II 3 ) $835,845,835$

835 WRITE OUTPUT TAPE LTAPE, $836,(N B 6(4 * I+1), N B 6(4 * 1+2))$

836 FORMAT TIHI30X,2A6///38H RESONANCE RESCNANCE 1
$2 I 12=$ ENERGY CROSS
$I 3=N R E S+I 2$
$I 4=N R E S+I 3$
$I 5=N R E S+I 4$
$D O 844 I 1=1, N R E S$
$N A=I 1+I 2$
$N B=I 1+13$
$N C=I 1+I 4$
$N D=I 1+I 5$
$I F(I I-1) 840,840,837$

PEAK $/ 65 \mathrm{H}$ ETA!

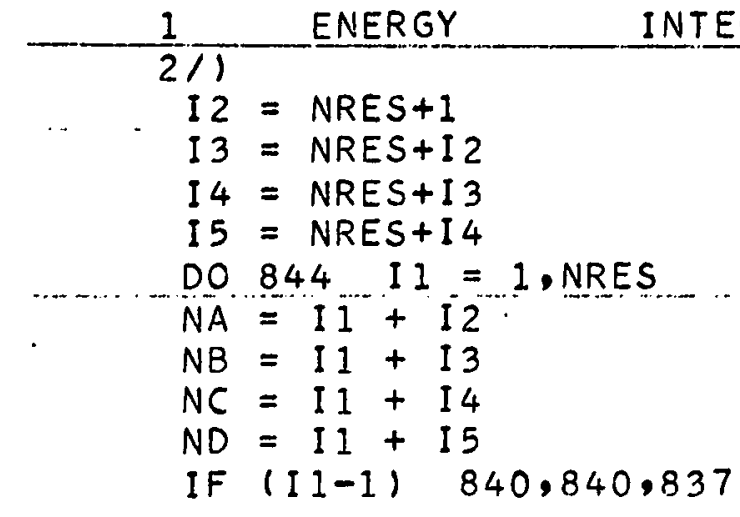


837 IF $((11-1)-4 C *((I I-1) / 40)) \quad 839,838,839$

838 URITE CUTPUT TA.PE LTAPE, $836,(N 96(4 * I+1)$, NBS $(4 * I+2))$

839 IF $((I 1-1)-5 *((I)-1) / 5)) \quad 840,842,840$

840 WRITE OUTPUT TAPE LTAPE,841, NBI(II+1), NEI(NA), NBI(NB), NBI(NC), YBI 1 (ND)

841 FORINAT (IPE13.4,4E14.4)

GO TO 844

. 842 WRITE OUTPUT TAPE LTAPE, 843, NSI(II+1),NGI(NA), NBI (NB),NBI(NC), MB1 1 (ND)

843 FORMAT (IHOIPEI2.4,4E14.4)

c

844 CONTINUE

C PUNCH RECORD 4 OF CROSS SECTION DATA BLOCK

C

845 IF (II 4 ) $846,847,846$

$846 \mathrm{~N}=$ NWDBRI - NWSGSO - NWSGSI

CALL PUNACB $\quad(N B 1, N 3, N)$

847 RETURN

END $(0,0,0)$ 


\begin{tabular}{|c|c|}
\hline$c$ & SUBROUTINE WRREC5 (II3,II4) \\
\hline $\begin{array}{l}C \\
C\end{array}$ & WRITE RECORD 5 OF CROSS SECTION DATA BLOCK ON OUTPUT TAPE \\
\hline & 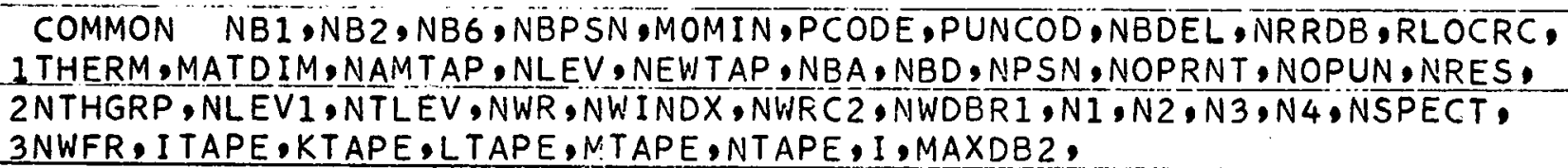 \\
\hline & $\begin{array}{l}\text { 4MAXDB 1, MAXDB, MAXDEM, IDMB } 11, I D M B 12, I D M B 21, I D M B 22, \bar{N}, \overline{R E R U N} \\
\text { 5NWSGSO,NWSGS } 1 \text { NWRES }\end{array}$ \\
\hline
\end{tabular}

EQUIVALENCE (NB1,FB1), (NB1 (1006), EL,ENERGY), (NB1 (1051), FV), (NBI ( 1 $1096), U L, B D A T E),(N B 1(1141), E G),(N B 1(1186), U G), 6 N B I(1008), A W),(N B I(1$ 2099), DEN), (NB1 $(1100), A 2200),(N B 1(1101), N D A T A),(N B 1(1102), A 1),(N B)($ $31103), B 1),(N B 1(1104), C 1),(N B 1(1105), D 1),(N B 1(1106), A 2),(N B 1(1107)$, $4 B 2),(N B 1(1108),(2),(N B 1(1109), D 2),(N B 1(1110), S, S I G S 0, S I G S 1, E 0, S I G N$ $5 G, B E T A),(N B 1(1111), T M),(N B 1(1112), S O R),(N B 1(1155), X I S),(N B 1(1200)$, $6 T R),(N B 1(1245), A),(N B 1(1290), X I S 1),(N B 1(1335), F N U),(N B 1(1380), F), 1$ 7NB1 $(1425), 51),(N B 1(1470), T),(N B 1(1515), X),(N B 1(1560), \times 1),(N B 1(1605$ $8), 8),(N B 1(1650), P N U),(N B 1(1695), E T A)$

$\mathrm{C}$

EQUIVALENCE $(N B 1(1740), T S 4500),(N B 1(1770), T S 4000),(N B 1(1800), T S 35$ $100),(N B 1(1830)$, TS3000), (NB1) 1860$)$, TS2500), (NBI(1890), TS2000), (NB1) $21920), T S 1500),(N B 1(1950), T S 1000),(N B 1(1980), T S 500),(N B 1(2010), T S 68$ $3),(N B 1(1310), R 10),(N B 1(1510), G N),(N B 1(1710), G G),(N B 2(1006), G F),(N B$ $42(1206), G),(N B 2(1406), P 0),(N B 2(1606), R),(N B 2(1 B 06), E T A R),(N B 2, F B 2)$ $5,(N B P S N(2), B C O D E),(N B P S N(3), B N A M),(N B 6, F 36)$

$C$

DIMENSION NBI(2100),FBI(2100),NB2(2050), FB2(2050), NB6(2010), 1FB6 (2010), NBPSN(1992), BCODE (1991), BNAM(1990), MOHIN(4980), PCODE(500 2), PUNCOD (500), NBDEL (499), NRRD9 (499), RLOCRC(499), THERM(15), MATDIM(1 $30), E L(45), F V(45), U L(45), E G(45), U G(45), B D A T E(2), S(45), S I G S O(925), S I$ $4 G S I(925), E O(200)$, SIGNG $(925)$, SOR $(45), X I S(45), T R(45), A(45), X I S I(45)$, 5FNU(45), $F(45), S I(45), T(45), X(45), X 1(45), E(45), P N U(45), E T A(45), T S 45$ $600(30)$, TS4000(30),TS3500(30), TS3000(30), TS2500(30), TS2000(30), TS15 $700(30), T S 1000(30)$, TS500(30), TS68(30),RIO(200), GN(200),GG(200),GF(2 $800), G(200), P O(200), R(200)$, ETAR(200), ENERGY (45), NAMTAP(3)

$\frac{c}{c}$

$\frac{c}{c}$

SEE COMMENTS IN WRREC2

848 IF (II 3 ) $849,854,849$

$8.49 M 1=$ MATDIM(8)

$M 2=M A T D I M(9)$

852 CALL RC235 (M1,M2,N4,2)

C

C PUNCH RECORD 5 OF CROSS SECTION CATA BLOCK

854 IF $(114) \quad 855.8551 .855$

$855 \mathrm{~N}=$ NWDBRI-NWSGSO-NWSGSI-NWRES CALL PUNACB $\quad(N B 1, N 4, N)$

8551 RETURN

END $(0,0,0)$ 


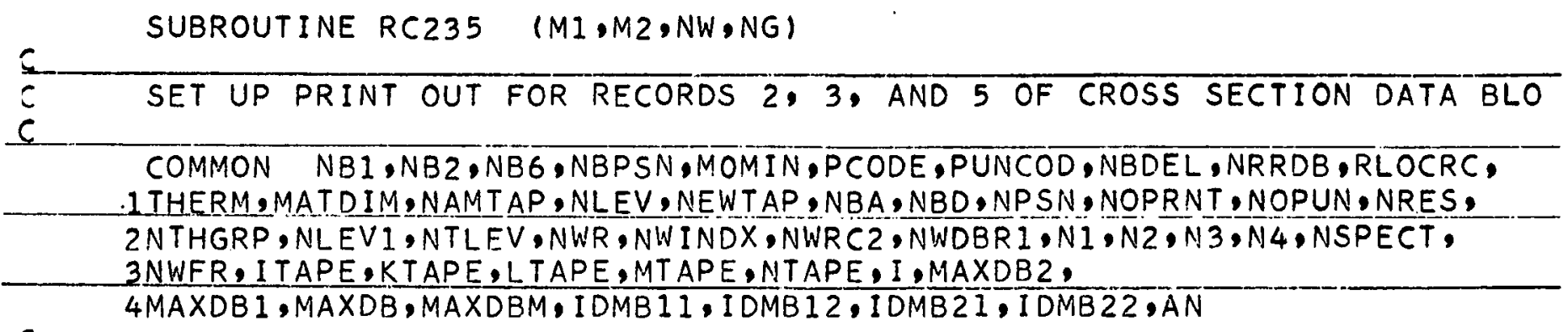

C

EQUIVALENCE (NBI, FBI), (NBI $(1006), E L, E N E R G \bar{Y}),(N B I(1051), F V),(N B I(1$ $1096), U L, B D A T E),(N B 1(1141), E G),(N B 1(1186), U G),(N B 1(1098), A W),(N B I(1)$ 2099), DEN), (NBI(1100),A2200), (NB1(1101), NDATA), (NB1(1102),A1), (NBI) $31103), B 1),(N B)(1104), C 1),(N B 1(1105), 01),(N B 1(1106), A 2),(N B 1(1107)$, $4 B 2),(N B I(1108), C 2),(N B I(1109), 02),(N B 1(1110), S, S I G S 0, S I G S I, E O, S I G N$ $5 G, B E T A),(N B 1(1111), T M),(N B 1(1112), S O R),(N B I(1155), X I S),(N B 1(1200)$. $6 T R),(N B I(1245), A),(N B 1(1290), X I S I),(N B 1(1335), F N U),(N B I(1380), F), 1$ ZNB $1(1425), S 1),(N B 1(1470), T),(N B 1(1515), X),(N B 1(1560), X 1),(N B 1(1605$ $8), B),(N B I(1650), P N U),(N B I(1695), E T A)$

C

EQUIVALENCE (NBI(1740), TS4500), (NBI(1770), TS4000), (NBI(1800), TS35 $100),(N B 1(1830), T S 3000),(N B 1(1860), T S 2500),(N B 1(1390), T S 2000)$, (NB1) $21920), T S 1500),(\overline{N B I}(1950), T S 1000),(N B 1)(1980), T S 500),(N B 1(2010), T S 68$ 3) . (NBI (1310),RI0), (NBI (1510),GN), (NBI(1710),GG),(NB2(1006),GF), (NB $42(1206), G),(N B 2(1406), P O),(N B 2(1606), R),(N B 2(1806), E T A R),(N B 2, F B 2)$ 5, (NBPSN (2), BCODE), (NBPSN (3),BNAM), (NB6,FB6)

$\mathrm{C}$

DIMENSION NBI (2100), FB1 (2100),NB2(2050),FB2(2050),NE6(2010),

IFB6(2010), NBP SN(1992), BCODE (1991), BNAM(1990), MOMIN( 4980$)$, PCODE(50O 2) .PUNCOD(500), NBDEL (499), NRRDB (499), RLOCRC (499), THERM( 15), MATDIM(I $30), E L(45)$, FV(45), UL (45), EG(45),UG(45), BDATE(2), S(45), SIGSO (925), SI 4GS (1925), EO(200), SIGNG(925), SOR (45),XIS(45), TR(45),A(45),XISI(45), SFNU(45), F(45),S1(45),T(45),X(45), XI(45), B(45), PNU(45), ETA(45), TS45 $600(30)$, TS4000(30), TS3500(30), TS3000(30),TS2500(30), TS2000(30), TS15 $700(30), T S 1000(30), T S 500(30), T S 68(30), R I O(200), G N(200), G G(200), G F(2$ $800), G(200), P O(200), R(200), E T A R(200)$ ? ENERGY (45),NAMTAP(3)

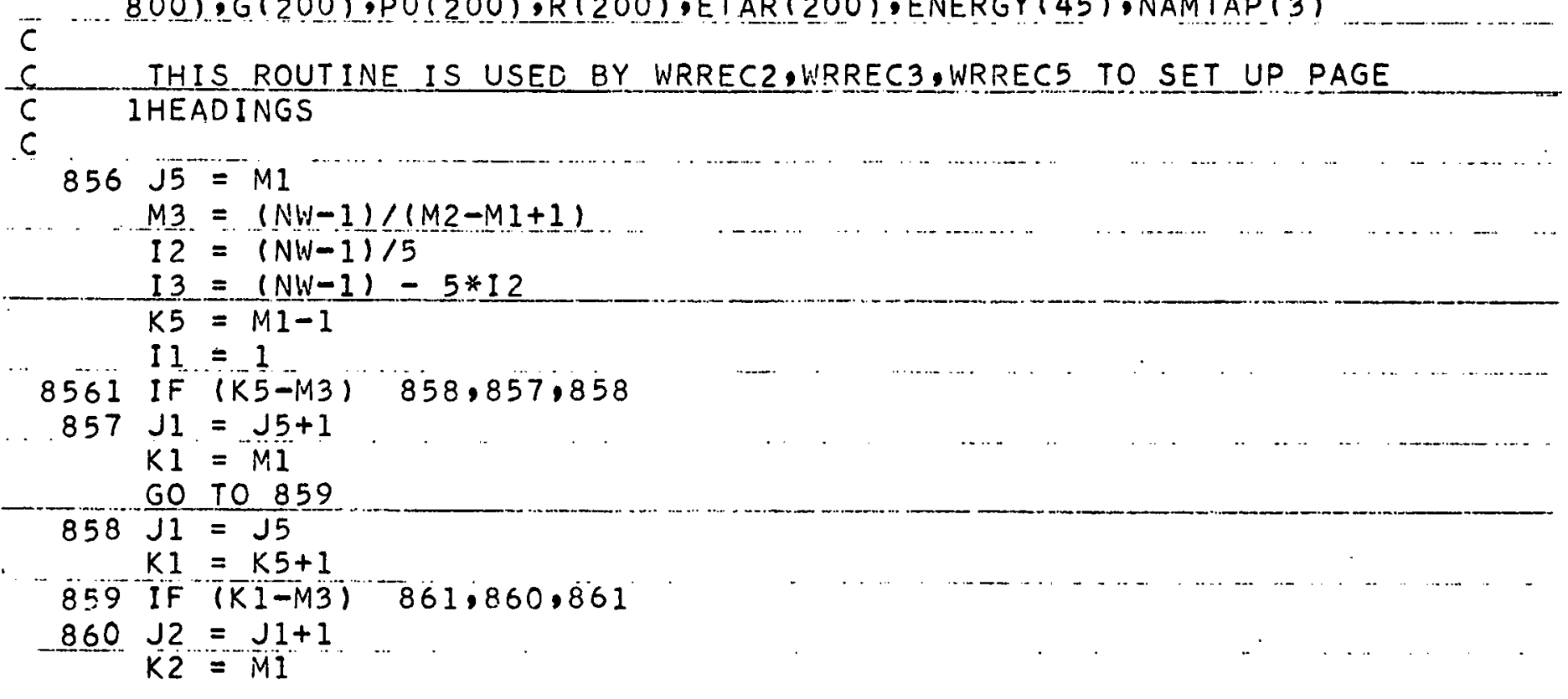




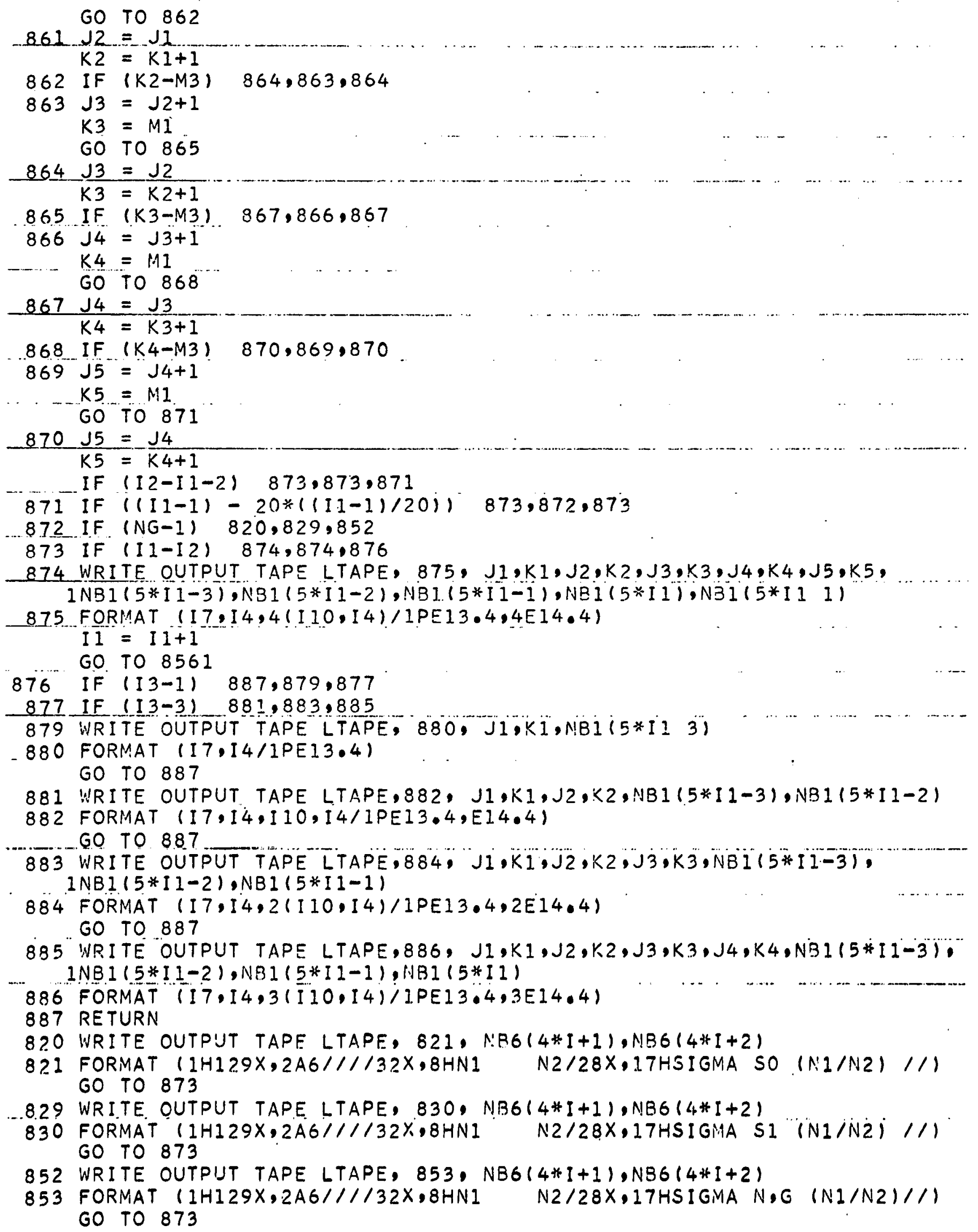




\section{END $(0,0,0)$}


SUBROUTINE FINREC

\begin{tabular}{|c|c|}
\hline $\begin{array}{l}C \\
C\end{array}$ & WR ITE FINAL RECORD ON OUTPUT TAPE \\
\hline - & 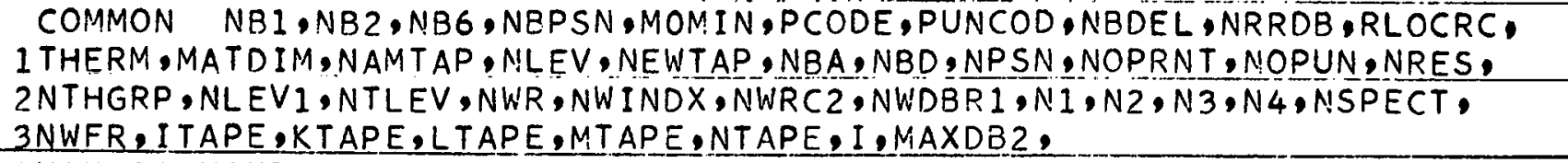 \\
\hline & $4 M A X D B 1, M A X D B, M A X D B M, I D M B 11, I D M B 12, I D M B 21, I D M B 22, A N$ \\
\hline & 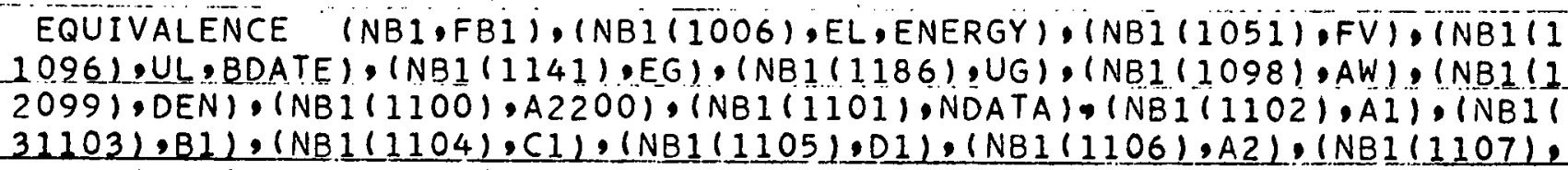 \\
\hline & 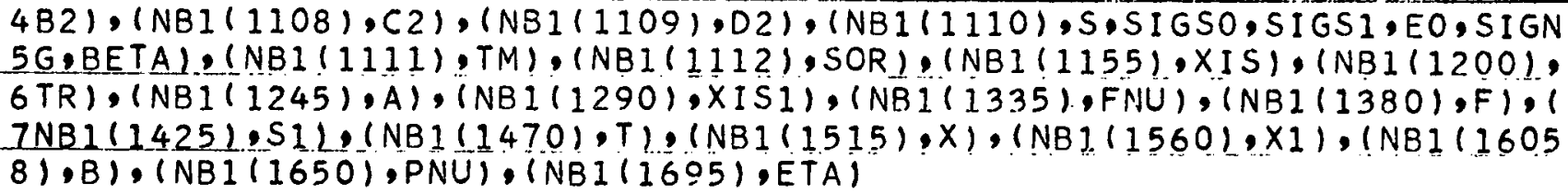 \\
\hline
\end{tabular}

$C$

EQUIVALENCE (NBI(1740),TS4500), (NB1)(1770),TS4000), (NB1)(1800),TS35 $100)$, (NB $1(1830)$, TS3000), (NB1(1860),TS2500), (NB1(1890), TS2000), (NB1) $21920)$, TS1500), (NB1(1950), TS1000), (NB1(1980), TS500), (NB1(2010), TS68. 3), (NB $1(1310), R ! 0),(N B 1(1510), G N),(N B 1(1710), G G),(N B 2(1006), G F),(N B$ $42(1206), G),(N B 2(1406), P 0),(N B 2(1606), R),(N B 2(1806), E T A R),(N B 2, F B 2)$ $5,(N B P S N(2), B C O D E),(N B P S N(3), B N A M),(N B 6, F B 6)$

C

DIMENSION NBI(2100),FB1 $(2100), N B 2(2050), F B 2(2050), N B 6(2010)$,

$1 F B 6(2010)$, NBPSN $(1992), B C O D E(1991), B N A M(1090), M O M I N(4980), P C O D E(500$ 2), PUNCOD (500), NBDEL (499), NRRDB (499), RLOCRC(499), THERM (15) ,MATDIM(1 $30), E L(45), F V(45), U L(45), E G(45), U G(45), B D A T E(2), S(45), S I G S O(925), S I$ $4 G S I(925), E O(200)$, SIGNG (925), SOR (45),XIS(45), TR (45), A (45), XISI (45), SFNU(45), $F(45), S I(45), T(45), X(45), X 1(45), B(45), P N U(45), E T A(45), T S 45$ $600(30)$, TS4000(30), TS3500(30), TS3000(30),TS2500(30), TS2000(30), TSI5 $700(30), T S 1000(30), T S 500(30), T S 68(30), R I O(200), G N(200), G G(200), G F(2$ $800), G(200), P O(200), R(200), E T A R(200)$,ENERGY (45), NAMTAP (3)

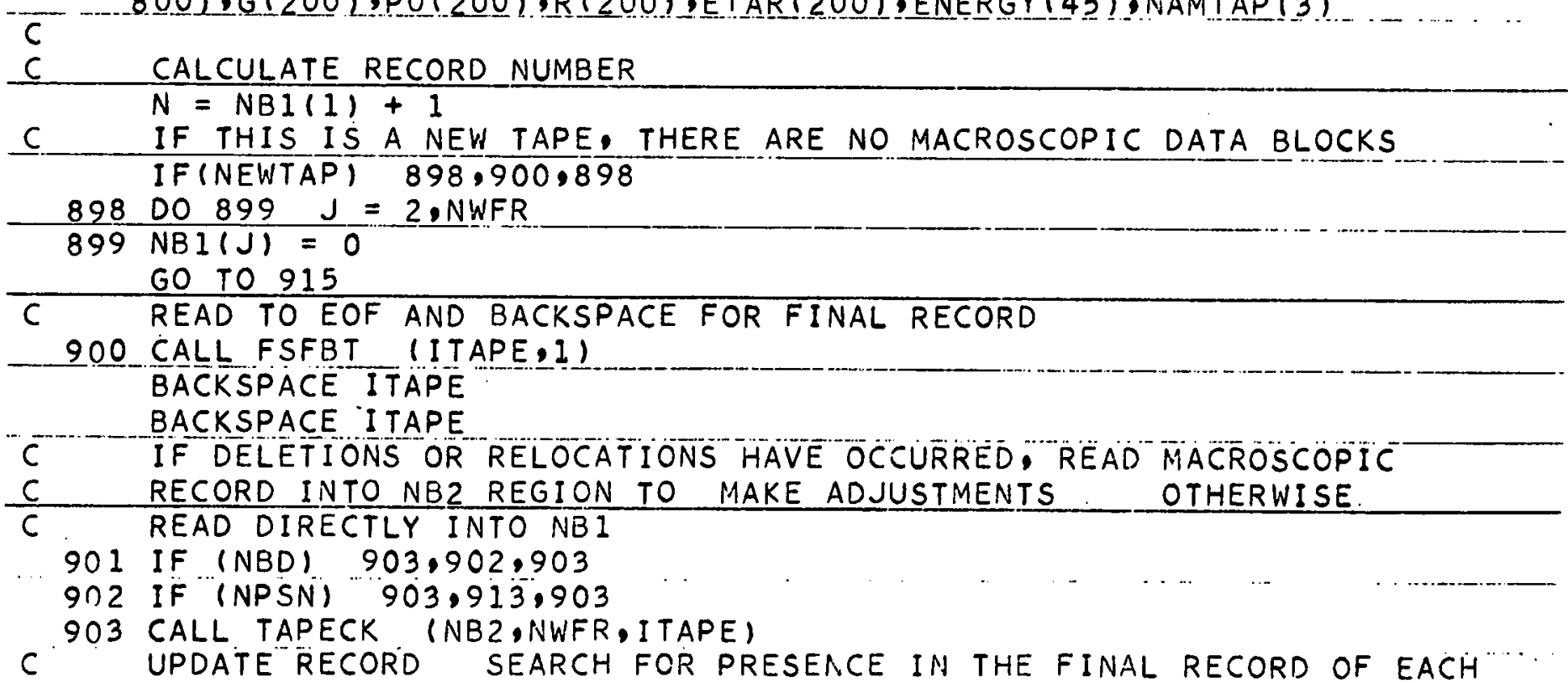


C ICODE NUMBER OF INDEX RECORD

$N E=1$

NB2 $(N W F R+1)=0$

DO $910 \quad I=1$, MAXDB

IF $(N B 6(4 * I+3)) 904.911 .904$

$904 N F=2$

905 IF (NB2(NF)) $906,910,906$

206 IF (NB2(NF) - NB6(4*I+3)) $907,908,907$

$907 \mathrm{NF}=\mathrm{NF}+2 *(\mathrm{NB} 2(\mathrm{NF}+1)+1)$

GO TO 905

C CODE NUMBER HAS BEEN LOCATED - COPY INFO TO NBI REGION

$908 N G=(N B 2(N F+1)+1) * 2$

DO $909 \mathrm{~J}=1 . \mathrm{NG}$

$N E=N E+1$

$N A=N F+J-1$

$909 N B 1(N E)=N B 2(N A)$

910 CONTINUE

C STORE ZEROS IN REMAINDER OF TABLE (INCLUDING NWFR+1)

911 DO $912 \quad K=N E$, NWFR

$912 N B 1(K+1)=0$ GO TO 914

$c$
$c$
$c$

913 CALL TAPECK (NB1,NWFR, ITAPE) $N B 1(N W F R+1)=0$

$\frac{c}{c}$ PRINT FINAL RECORD

914 IF (NBI(2)) $917,915,917$

915 WRITE OUTPUT TAPE LTAPE, 916

216 FORMAT (28H1 NO MACROSCOPIC DATA BLOCKS) GO TO 933

$917 \mathrm{NF}=2$

$\mathrm{NE}=0$

WRITE OUTPUT TAPE LTAPE, 918

918 FORMAT $149 \mathrm{HI}$

118 HCONSIITUENT TABLES//11

919 IF (NBI(NF)) 920.933 .920

$9.20 N G=N B 1(N F+1)$

IF (NE) $921,922,921$

9.21 CALL RESTO

$92200923 \quad I=1, M A X D B$

IF $(N B I(N F)-N B 6(4 * I+3)) \quad 923,924,923$

923 CONTINUE

224. WRITE OUTPUT TAPE LTAPE, 925, NBI (NF), NB6 $(4 * I+1), N B 1(4 * I+2)$

925 FORMAT $113 \mathrm{X}, 2$ OHMACROSCOPIC CODE NO 15X,11HDESIGNATION/F $28,4,20 \mathrm{X}, 2 \mathrm{~A}$ $16 / 158 \mathrm{H} \quad$ CONSTITUENT D DESIGNATION $29 \mathrm{H}$ CODE NO. WEI GHT/5

926 DO $932 \mathrm{~J}=1, \mathrm{NG}$

$N A=N F+1+2 * J$

$N B=N A-1$

DO 928 I $=1, M A X D B$

IF $(N B 6(4 * I+3)) \quad 927,9281,927$

927 IF (NB6(4*I+3) NBI (NB)i $928,930,928$ 
928 CONTINUE

2281 WRIIE QUTPUT IAPE LTAPE, 229, NBI (NB) N NBI(NA)

929 FORMAT (F21.4,1PE39.4)

GO TO 932

930 WRITE OUTPUT TAPE LTAPE, 931, NBI $(N B), N B 6(4 * I+1), N B 6(4 * I+2), N B 1(N$ 1AI

931 FORMAT (F21.4,9X,2A6,1PE18.4)

932 CONTINUE

$N E=1$

$N F=N F+2 *(N B 1(N F+1)+1)$

$c$

GO TO 919

C SEARCH FOR DELETED CONSTITUENTS

c

933 IF (NBD) $934,942,934$

934 CALL RESTO

$N F=2$

935 IF_(NBI (NF)) 9369942.936

$936 N A=N B 1(N F+1)$

DO $940 \quad J=N F+2 * J$

$D O 939, K=1, N B D$

IF (NBI(NB)-NBDELIK) $939,937,939$

237. WRITE OUTPUT TAPE LTAPE, 938, NBDEL (K),NBI (NF)

938 FORMAT (1OH4 MATERIALF $12.4,35 \mathrm{H}$ WHICH IS A CONSTITUENT OF MATERIALE

$112.4 / 21 \mathrm{X}, 29 H \mathrm{H}$ AS BEEN MODIFIEO OR DELETED. 1

GO TO 940

239 CONT I NUE

940 CONTINUE

$-941 \mathrm{NF}=\mathrm{NF}+2 *(\mathrm{NB} 1(\mathrm{NF}+1)+1)$

GO TO 935

C SET UP RECORD NUMBER

$942 N B 1(1)=N$

C.... WRITE N,M TAPES

CALL NMTPRC (NWFR)

C.- PUNCH FINAL RECORD

C CHECK FOR PUNCHING

943 IF (NOPUN) 9442948.944

944 DO $945 \mathrm{~J}=1, N O P U N$

IF (PUNCOD(J)) 945.946,945

945 CONTINUE

GO TO 948

946 CONTINUE

CALL BRAND (6HFINAL II)

CALL PUNACBINB1, NWFR,OI

CALL PUNACB $(0,0,0)$

948 CONTINUE

c END FILES

950 END FILE MTAPE

END FILE NTAPE

C REWIND TAPES

REWIND MTAPE

REWIND NTAPE 
IF (NEWTAP) 954,951,954

951 RE!! IND ITAPE

952 IF (NPSN) $953,954,953$

953 RENIND KTAPE

954 RETURN

END $(0,0,0)$ 


\section{SUBROUTINE DIPERR (N)}

\begin{tabular}{|c|}
\hline 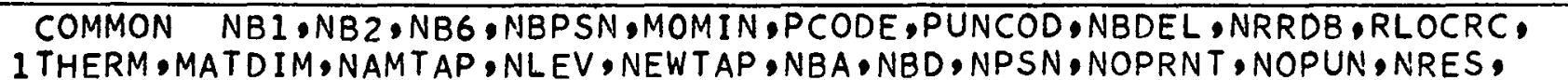 \\
\hline 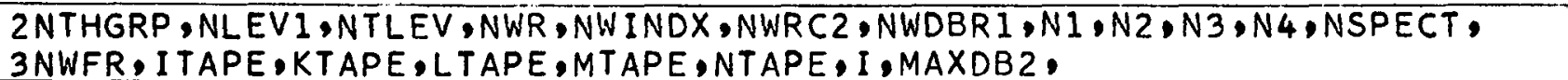 \\
\hline $\begin{array}{l}\text { 4MAXDB 1, MAXDB, MAXDBM, IDMB 11, IDMB 12, IDMB 21, IDMB } 22, A N, R E R U N, N C O D O T \text {, } \\
5 F B 3, N B 4\end{array}$ \\
\hline
\end{tabular}

EQUIVALENCE (NB1,FB1), (NBI(1006), EL,ENERGY), (NB1(1051),FV), (NB1) 1 $1096), U L, B D A T E),(N B I(1141), E G),(N B 1(1186), U G),(N B 1(1098), A W),(N B I 11$ $2099), D E N),(N B 1(1100), A 2200),(N B 1(1101), N O A T A),(N B 1(1) 02), A 1),(N B 11$ $31103), B 1),(N B 1(1104), C 1),(N B 1(1105) \cdot D 1),(N B 1(1106), A 2),(N B 1(1107)$, $482),(N B 1(1108), C 2),(N B 1(1109), D 2),(N B 1(1110), S, S I G S 0, S I G S 1, E 0, S I G N$ $5 G, B E T A),(N B 1(1111), T M),(N B 1(1112), S O R),(N B 1(1155), X I S),(N B 1(1200)$, $6 T R),(N B 1(1245), A),(N B 1(1290), X I S 1),(N B 1(1335), F N U),(N B I(1380), F), 1$ $7 N B 1(1425), S 1),(N B 1(1470), T),(N B 1(1515), X),(N B 1(1560), X 1),(N B 1(1605$ $8), B),(N B 1(1650), P N U),(N B 1(1695), E T A)$

$\mathrm{C}$

EQUIVALENCE (NBI(1740),TS4500),(NB1(1770),TS4000),(NB1(1800),TS35 $100),(N B 1(1830), T S 3000),(N B 1(1860), T S 2500),(N B 1(1890), T 52000),(N B 11$ $21920), T S 1500),(N B 1(1950), T S 1000),(N B 1(1980), T S 500),(N B 1(2010), T S 68$ $3),(N B 1(1310), R I 0),(N B 1(1510), G N),(N B 1(1710), G G),(N B 2(1006), G F),(N B$ $42(1206), 6),(N B 2(1406), P 0),(N B 2(1606), R),(N B 2(1806), E T A R),(N B 2, F B 2)$

$5,(N B P S N(2), B C O D E),(N B P S N(3), B N A M),(N B 6, F B 6)$

C

DIMENSION NB1(2100),FBI(2100), NB2(2050), FB2(2050),NB6(2010),

1FB6(2010), NBP SN (1992), BCODE (1991), BNAM(1990), MOMIN(4980), PCODE (500 2), PUNCOD (500), NBDEL (499), NRRDB (499), RLOCRC (499), THERM(15), MATDIM(I $30), E L(45), F V(45), U L(45), E G(45), U G(45), B D A T E(2), S(45), S I G S O(925), S I$ 4GSI(925), EO(200), SIGNG(925), SOR(45),XIS(45), TR(45),A(45), XISI (45), SFNU (45), F(45),S1(45),T(45),X(45),X1(45),B(45),PNU(45), ETA(45), TS45 $600(30)$, TS4000(30), TS3500(30), TS3000(30), TS2500(30), TS2000(30), TS15 $700(30), T S 1000(30), T S 500(30), T S 68(30), R I 0(200), G N(200), G G(200), G F(2$

$800), G(200), P 0(200), R(200), E T A R(200)$, ENERGY $(45)$,NAMTAP (3)

DIMENSION FB3(500),NB4(500)

$\mathrm{C}$

400 WRITE OUTPUT TAPE LTAPE, 401, (NB6 $(4 * I+1), N B 6(4 * 1+2), N B 6(4 * I+3)$, IMTAPE , NTAPE, ITAPE, KTAPE)

401 FORMAT 14 IHIA DIP ERROR HAS BEEN ENCOUNTERED IN DATA/7H BLOCK 2 A6, $111 \mathrm{H}$ ICODE NO.F12.4.3H ///11OH TO RERUN,//5OH 10 REMOVE ALL 2DATA PRECEDING THIS DATA BLOCK/47H 2. INSERT PUNCHED CARD DAT 3 A LABELED RERUN/3OH 3. REMOUNT LOGICAL TAPES/12H $44,4 \mathrm{H}$ ANDI $4 / 12 \mathrm{H} \quad$ B.I $4,21 \mathrm{H}$ IF REVISING OLD TAPE/12H

5 C.I $1,14 \mathrm{H}$ IF RELOCATING) PUNCH OUT NECESSARY DATA FOR RERUNNING THIS CASE USING THE

\begin{tabular}{ll}
$C$ & PUNCH OUT NECESSARY DATA \\
\hline$C$ & IPARTIALLY COMPILED TAPES
\end{tabular}

PUNCH 404, BDATE(1),BDATE(2)

404 FORMAT (9H5BDATE,2,2A6/9H=RERUN,1,)

CALL BRAND (6HRERUN ,1)

RERUN $=1.0$

$N C=N W I N D X / 4$

CALL PUNACB (FB, $, N C, 0)$

CALL PUNACB (NB $4, N C, 0)$

CALL PUNACB TEL;NTLEV,O) 
CALL PUNACB (FV,NTLEV,O)

$N A=4 *(N B A+N P S N)$

CALL PUNACB (NBPSN,NA,O)

- CALL PUNACB (NB6,NWINDX,0)

$N B=10 * N B A$.

CALL PUNACB (MOMIN,NB,O)

IF (NOPRNT) $4041,4042,4041$

-4041 CALL PUNACB (PCODE ONOPRNT,0)

4042 IF (NOPUN) $4043,4044,4043$

4043 CALL PUNACB (PUNCOD,NOPUN,0)

4044 IF (NBD) $4045,4046,4045$

4045 CALL PUNACB (NBDEL, NBD,0)

4046 IF (NPSN) . 4047,4048,4047

4047 CALL PUNACB (NRRDB NNPSNO)

CALL PUNACB (RLOCRC,NPSN,O)

4048 CALL PUNACB (THERM,68,0)

CALL PUNACB $(0,0,0)$

C PRINT ON-LINE COMMENT

PRINT 406, MTAPE, NTAPE

406 FORMAT (IH168H2A DIP ERROR HAS REEN ENCOUNTERED WITH RND TAPES PAR

ITIALLY COMPILED.//35H PLEASE SAVE TAPES ON LOGICAL UNITS//I $20 /$ I 201 IF (NEWTAP) $412,407,412$

407 PRINT 408 . ITAPE

... 408 FORMAT (I 20 )

409 IF (NPSN) $410,412,410$

410 PRINT 411 , KTAPE

411 FORMAT (120)

412 PRINT 413

413 FORMAT (IHI)

C. EXIT TO STOP

414 CALL EROR (N)

RETURN

END $(0,0,0)$ 
SUBROUTINE TRYOVR

C RERUN A CASE IN WHICH A DIP DATA ERRCR WAS ENCOUNTERED

COMMON NB1, NB2, NB6, NBPSN,MOMIN, PCODE, PUNCOD, NBDEL, NRRDB, RLOCRC, 1 THERM, MATDIM, NAMTAP, NLEV, NEWT AP, NBA, NBD, NPSN, NOPRNT, NOPUN, NRES, 2 NTHGRP, NLEV1, NTLEV, NWR, NWINDX, NWRC2, NWDBR I, N1, NI, N3,N4,NSPECT, 3 NWFR , I TAPE , KTAPE, LTAPE, MTAPE, NTAPE, I MAXDB2, 4MAXDB I, MAXDB, MAXDBM, IDMBII, IDMB 12, IDMB2I, IDMB22, AN, RERUN,NCODOT, $5 F B 3, N B 4$

C

EQUIVALENCE (NB1,FBI), (NB1(1006), EL, ENERGY), (NB.1(1051),FV), (NBI(1 $1096), U L, B D A T E),(N B I(1141), E G),(N B 1(1186), U G),(N B 1(1098), A W),(N B 1(3)$

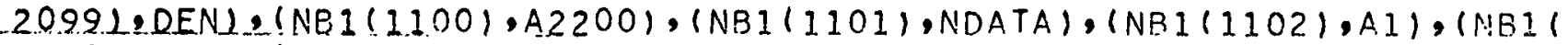
$31103), B 1),(N B 1(1104), C 1),(N B 1(1105), D 1),(N B 1(1106), A 2),(N B 1(1107)$, 4B2), (NBI (1108), C2), (NB1(1109),D2), (NBI(1110),S,SIGSO,SIGSI,EO,SIGN 5G,BETA), (NBI(1111), TM), (NBI(1112),SOR), (NB1(1155),XIS), (NBI(1200), $6 T R),(N B 1(1245), A),(N B I(1290), X I S 1),(N B 1(1335), F N U),(N B 1(1380), F), 1$ 7NBI $(1425), S 1),(N B I(1470), T),(N B I(1515), X),(N B 1(1560), X 1),(N B I(1605$ 8 8.B $),(N B !(1650), P N U),(N B I(1695), E T A)$

C

EQUIVALENCE (NB1(1740),TS4500),(NBI(1770),TS4000),(NB1(1800),TS35 $100)$, (NBI $(1830), T S 300 C),(N B I(1860), T S 2500),(N B I(1890), T S 2000),(N B I($ $21920)$,TS1500), (NE1(1950),TS1000), (NB1(1980),TS500), (NB1(?010),TS68 3), (NB1(1310),RI0), (NBI (1510),GN), (NB1(1710),GG), (NE2(1005), GF), (NB $42(1206), 0),(N B 2(1406), P 0),(N B 2(1606), R),(N B 2(1806), E T A R),(N B 2, F B 2)$

$5,(N B P S N(2), B C O D E),(N B P S N(3), B N A N),(N B 6, F B 5)$

C.

DIMENSION NE1(2100),FDI(2100), NB2(2050), FE2(2050), NB6(2010), $1 F B 6(2010)$, NEPSN (1972), ECODE (1991), BNAN(1990), MOMIN(4980),PCODE $(500$ 2), PUNCOD(500), NBDEL (499), NRRDB (499), RLOCRC(499), THERM(15), MATDIM( 1 $30), E L(45), F V(45), U L(45), E G(45), U G(45), E D A T E(2), S(45)$, SIGSO $(925), S I$ 4GSI(925), EO (200), SIGNG(925), SOR(45),XIS(45), TR(45), A(45),XISI(45), SFNU(45), F(45),SI(45),T(45),X(45),XI(45),B(45),PNU(45), ETA(45), TS45 $600(30)$, TS4000(30), TS3500(30), TS3000(30), TS2500(30), TS2000(30), TS15 $700(30), T \leq 1000(30), T S 500(30), T S 68(30), R I 0(200), G N(200), G G(200), G F(2$ $800), G(200), P 0(200), R(200), E T A R(200)$, ENERGY(45), NAMTAAP(3)

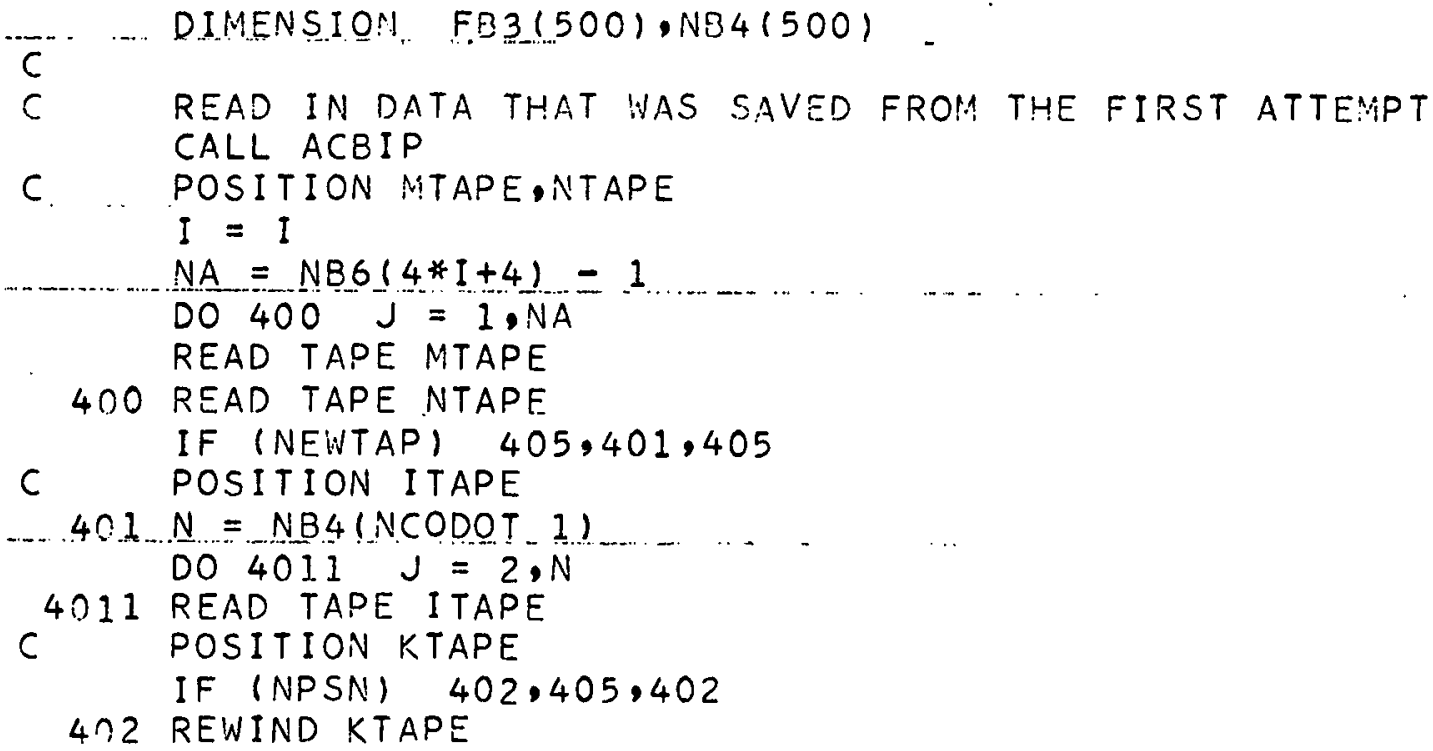


IF (NWR) $403,405,403$

$40300 \quad 404 \mathrm{~J}=1$. NWR

404 READ TAPE KTAPE

405 RETURN

END $(0,0,0)$ 


\subsection{Program Mnemonic Glossary}

Program

Mnemonic

Math Symbol

Description

$A_{1}$

$\sigma_{a i}$ or $\Sigma_{a i}$

Absorption cross section

AN

$m_{n}$

Mass of neutron in atomic mass units (1.008986 A.M.U.)

AW

A

Atomic weight

Al

$A_{1}$

Constant for calculation of $v$ (see

Eq. 2.19)

A2

$A_{2}$

Constant for calculation of $\eta$ (see Eq. 2.14)

A2200

$\sigma_{a(2200 \mathrm{~m} / \mathrm{sec})}$

$B_{1}$

$\left(1-\bar{\mu}_{0}\right)_{i}$

Absorption cross section at 2200

$\mathrm{m} / \mathrm{sec}$

$\bar{\mu}_{0}$ is the average scattering cosine in the laboratory system for isotropic scattering in the center-of-mass Bystem

$\mathrm{BC}_{\mathrm{DDE}}$

BDATE,

BETA

B

Data-block code number

Date of data-block addition to tape

Spectral delay fraction

BNAM $_{j}$

Bl

$\mathbf{B}_{\mathbf{1}}$

12-letter BCI data-block identification

Constant for calculation of $v$ (see Eq. 2.19)

B2

$\mathrm{B}_{2}$

Constant for calculation of $\eta$ (see Eq. 2.14)

$\mathrm{Cl}$

$\mathrm{C}_{1}$

Constant for calculation of $v$ (see Eq. 2.19)

C2

$\mathrm{C}_{2}$

Constant for calculation of $\eta$ (see Eq. 2.14)

DEN

$$
\rho
$$

Density $\left(\mathrm{gm} / \mathrm{cm}^{3}\right)$

$\mathrm{DF}$

$A / N_{0}$ or $\rho$

Density factor

$\mathrm{EG}_{\mathrm{m}}$

$\mathrm{EL}_{1}$

END

Group boundary energies (ev)

Level energies (ev)

Dumy location for end of record card 
Program

Mnemonic

Nath Symbol

Description

ENERGY

ETA $_{i}$

$\eta_{i}$

Same as $\mathrm{EL}_{1}$

ETAR

$\eta_{\ell}$

$v \sigma_{f} / \sigma_{a}$

EO

Eol

$v \Gamma_{f} /\left(\Gamma_{\gamma}+\Gamma_{f}\right)$

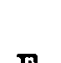

$\sigma_{f 1}$ or $\Sigma_{f i}$

Resonance energies (ev)

$F_{1}$

F'Bl

FB2

FB6

Fission cross section

Temporary storage array (same as $\mathrm{NBl}$ )

Temporary storage array (same as NB2)

Temporary storage array (same as NB6)

FNU $U_{i} \quad\left(v \sigma_{f}\right)_{i}$ or $\left(v \Sigma_{f}\right)_{i}$

FV

Flssion product cross section

Group $I / v$ factors (value in a $1 / v$ cross section in group $m$ with a value of 1.0 at a neutron velocity, $v$, of $2200 \mathrm{~m} / \mathrm{sec}$ )

G

8

Statistical weighting factor

GF

$r_{f}$

Resonance parameter (ev)

GG

$\Gamma$

Resonance parameter (ev)

GN

$\Gamma_{n}$

Resonance parameter (ev)

$\left.\begin{array}{l}\text { IDMBII } \\ \text { IDMBI2 } \\ \text { IDMB2I } \\ \text { IDMB22 }\end{array}\right\}$

$\left\{\begin{array}{l}\text { Limits of } D \phi \text { loops used for clearing } \\ \text { regions of memory }\end{array}\right.$

ITAPE

Old data tape being revised

KTAPE

Intermediate tape used for storage of data blocks being relocated

LPAPE

Output tape

MATDIM $_{n} \quad(n=1,2, \ldots, 10)$

Temporary storage location for $\mathrm{MO}_{\mathrm{MIN}}$, MOMAX, M'O $_{\text {MAX, }}$ M $_{\text {MIN }}, M_{M A X}, M^{\prime} I_{M A X}$,

NRES, MMIN, MIMAX, M' $\Gamma_{\text {MAX In that }}$ order for the material data block belng considered

MAXDB

Maximum tolerable number of data blocks 
Program

Mnemonic

MAXDBM

MAXDBI

MAXDB2

MAXIEV

MTAPE

$$
\begin{aligned}
& M \Gamma_{\text {MAX }} \\
& M \Gamma_{\text {MIN }} \\
& M^{\prime} \Gamma_{\text {MAX }} \\
& { }^{M O_{M A X}} \\
& { }^{M O_{M I N}}
\end{aligned}
$$$$
\mathrm{M}^{\prime} \mathrm{O}_{\mathrm{MAX}}
$$$$
\text { MI }
$$<smiles></smiles><smiles></smiles>

MOMIN

NAMTAP

NBA
Description

$\mathrm{MAXDB}-1$

$\mathrm{MAXDB}+1$

$\operatorname{MAXDB}+2$

Maximum permissible number of energy levels

One of the new data tapes

Last group from which $\sigma_{\mathfrak{n}, \gamma}\left(\mathrm{m} / \mathrm{m}^{\prime}\right)$ transfers occur (see MOMIN, MATDIM)

First group from which and into which $\sigma_{\mathrm{n}, \gamma}\left(\mathrm{m} / \mathrm{m}^{\prime}\right)$ transfers occur (see MOMIN, MATDIM)

Last group into which $\sigma_{\mathfrak{n}, \gamma}\left(\mathrm{m} / \mathrm{m}^{\prime}\right)$ transfers occur (see MOMIN, MATDIM)

Last group from which $\sigma_{\mathrm{so}}\left(\mathrm{m} / \mathrm{m}^{2}\right)$ transfers occur (see MOMIN, MATDIM)

First group from which and into which $\sigma_{\mathrm{so}}\left(\mathrm{m} / \mathrm{m}^{\prime}\right)$ transfers occur (see MOMIN, MATDTM)

Last group into which $\sigma_{\mathrm{so}}\left(\mathrm{m} / \mathrm{m}^{\prime}\right)$ transfers occur (see MOMIN, MATDIM)

Last groug from whlch $\sigma_{51}\left(\mathrm{~m} / \mathrm{m}^{\prime}\right)$ transfers occur (see MOMIN, MATDIM)

First group from which and into which $\sigma_{\mathrm{BI}}\left(\mathrm{m} / \mathrm{m}^{2}\right)$ transfers occur (see MOMIN, MATDIM)

Last group into which $\sigma_{81}\left(\mathrm{~m} / \mathrm{m}^{\prime}\right)$ transfers occur (see MOMIN, MATDIM)

Block for storling the 10 pleces of index data MOMIN,...., M' IMAX for date blocks added and relocated. The order is the same as in MATDIM, each data block occupying 10 consecutive locations

12-letter BCI for tape name and date Number of data blocks to be added to tape 
Program

Mnemonic

NBD

NBDEL $_{j}$

NBPSN $_{j}$

NBl

NB2

NB6

NDATA

NEWTAP

NIEV

NLEVI

INOPRNT

NøPUN

NPSN

NRES

$\mathrm{NRRDB}_{\mathrm{J}}$

NSPECT

NTAPE

NTHGRP

NTLEV

$\mathrm{NW}_{\boldsymbol{j}}$
Math Symbol

\section{Description}

Number of data blocks to be deleted from tape

Code numbers of blocks to be deleted

Data block position for blocks to be added and relocated

Temporary storage array (same as FBl)

Temporary storage array (same as FB2)

Temporary storage array (same as FB6)

Data code ( $=0$ to perform calculations to complete data, = 1 to use data as entered only)

Code for tape manufacture (= 0 to revise old tape, $=1$ to make data tape solely from card data)

Number of levels

$\mathrm{NLEV}+1$

Number of blocks to be printed

Number of blocks to be punched

Number of blocks to be relocated

Number of resonances for particular

block under consideration

An array containing the physical record number of the first record of each data block on the intermediate tape (see RIDCRC)

Number of words on a spectral data block (= NTLEV + 6)

One of the new data tapes

Number of thermal groups

Total number of levels ( $\Rightarrow$ NLEV + NTHGRP)

Number of words in data block following the first record 
Program Mnemonic

NWDBRI

NWFR

NWINDX

NWR

NWRC2

NWSGSO

NWSGSI

NWRES

NWSGNG

NI

N2
Math Symbol

\section{Description}

Number of words in first record of a material data block ( $=6 \times \mathrm{NTLEV}+$ $30 \times$ NTHGRP +16 )

Number of words in final record

Number of words in index record

Current KTAPE position--used in subroutine DATACT

Number of words in the second record $(=2 \times \mathrm{NTLEV}+3 \times \mathrm{NLEV}+3)$

Amount by which $\sigma_{51}\left(\mathrm{MI}_{\mathrm{MIN}} / \mathrm{M}^{\prime} \mathrm{l}_{\mathrm{MIN}}\right)$ is to be relocated downward from $\sigma_{\mathrm{BO}}\left(\mathrm{MO}_{\mathrm{MIN}} / \mathrm{M}^{\prime} \mathrm{O}_{\mathrm{MIN}}\right)$ when punching the SIGSI record of a material data block--depends on progrem RNDC requirements. It is independent of whether or not a SIGSO record exists

Relates the location of the initial word of a resonance record to the initial word of the SIGSI record as NWSGSO relates the initial word of the SIGSI record to the inftial word of a SIGSO record--1ndependent of whether or not SIGSI block exists

Relates the location of the Initial word of a SIGNG record to the initial word of a resonance record as NWSGSI relates the initial word of the resonance record to the initial word of the SIGSI record-aindependent of whether or not a resonance block exists

Number of words reserved for SIGNG record to meet program RNDC requirements

Number of words in SIGSO record-calculated by subroutine DIMEN

Number of words in SIGSI record-calculated by subroutine DIMEN

Number of words in resonance record-calculated by subroutine DIMEN 
Program Memonto

N4

$\mathrm{PC}_{\mathrm{DDE}}$

$\mathrm{PNU}_{1} \quad v_{i}$

PUINCodD

$\begin{array}{ll}\mathrm{PO}_{\ell} & \sigma_{\mathrm{o} \ell} \\ \mathrm{R}_{\ell} & \mathrm{R}_{\ell}\end{array}$

RERUN

$\mathrm{RI}_{\mathrm{O} \ell}$

$I_{\text {ol }}$

$\mathrm{RL}_{\mathrm{CRC}}$

$s_{1} \quad \sigma_{81}$ or $\Sigma_{81}$

SIGNG

$$
\begin{aligned}
& \sigma_{n, \gamma}\left(m / m^{\prime}\right) \text { or } \\
& \Sigma_{n, \gamma}\left(m / m^{\prime}\right)
\end{aligned}
$$

SIGSO

SIGSI

$\mathrm{SOR}_{1}$

SI

$T_{1}$

THERM $_{\mathbf{n}}$

TM

$\operatorname{TR}_{1}$

Math Symbol
Description

Number of words in SIGNG record-calculated by subroutine DIMEN

Code numbers of blocks to be printed

Number of neutrons emitted per fission

Code numbers of blocks to be punched

Peak cross section (barns)

Doppler temperature coefficient

$\left(\mathrm{K}^{-1}\right)$

Code word-mot ordinarily at user's option-to signify a rerun case. It is normally set to 0 . In case of a DIP error when reading data blocks from cards, It is punched together with other data by subroutine DIPERR.

Resonance integral (barns)

Code numbers of data blocks on intermediate tape (see NRRDB)

Scattering cross section (barns or $\mathrm{cm}^{-1}$ )

$n, \gamma$ transfer matrix

Scattering transfer matrix

FIrst-order scattering transfer matrix

Spectral level values

First-moment scattering cross section

Total cross section

Thermal levels--fixed in subroutine INIT

Mean life of neutron group percursor

Transport cross section 
Program

Mnemonic

TS...

$\mathrm{UG}_{\mathrm{m}}$

UL,

$x_{1}$

XIS

$X I S I_{1}$

$\mathrm{XI}_{1}$

m

${ }_{1}$
Math Symbol

$$
\begin{aligned}
& \sigma_{s}\left(k / k^{\prime}\right) \text { or } \\
& \Sigma_{s}\left(k / k^{\prime}\right)
\end{aligned}
$$

\section{Description}

Thermal scattering transfer matrix for $\cdots 0 \mathrm{~F}$

Group boundary lethargies

Level lethargies

Average logarithmic energy decrement

Slowing-down cross section

First-moment slowing-down cross section

First-moment average logarithmic energy decrement 


\subsection{REACTOR NUCLEAR DATA MACROSCOPIC PROGRAM}

\subsection{General Information}

The Reactor Muclear Data Macroscopic Program (RIDM) prepares macroscopic material cross-section data from microscopic cross section data present on the Reactor Nuclear Data Tape. Two new FND tapes, including these calculated macroscopic data blocks, are written and the Index and final records updated. If requested, the program will print out and punch on binary cards the new macroscopic blocks as well as any other data blocks appearling on the tape. All program output, whether writing the new binary tapes, printing binary-coded decimal output, or punching binary cards, is performed in accordance with the same specifications and formats as described in section 2.0 , for program RNDP.

\subsection{Equations and Dats Requirements}

The macroscopic cross sections are calculated from the microscopic cross sections with the following equation:

$$
\Sigma=\rho \sum_{i=1}^{N}\left[w_{1} \frac{N_{0}}{A_{1}} \sigma_{1}\right]
$$

where

p = material density in gm per cc,

$W_{1}$ = weight fraction of the ith constituent,

$\mathrm{N}_{0} / \mathrm{A}_{1}=$ density factor of the 1 th constituent,

$\sigma_{1}=$ microscoplc cross section of the ith constituent,

$\mathrm{N}=$ total number of constituents.

Several words in the data block are not calculated in the manner described above. The methods for treating these words are described below.

DEN - materlal density, supplied as imput

MOMIN - minimum MOMIN of the microscopic constituents, calculated

MOMAX - maximum MOMAX of the microscopic constituents, calculated

MOPMAX - maximum $M^{\prime} O_{\text {MAX }}$ of the microscopic constituents, calculated

MIMIN - minimum MIMIN of the microscoplc constituents, calculated

MIMAX - maximum MI MAX of the microscopic constituents, calculated

MIPMAX - maximum $M^{\prime} l_{\text {MAX }}$ of the microscopic constituents, calculated

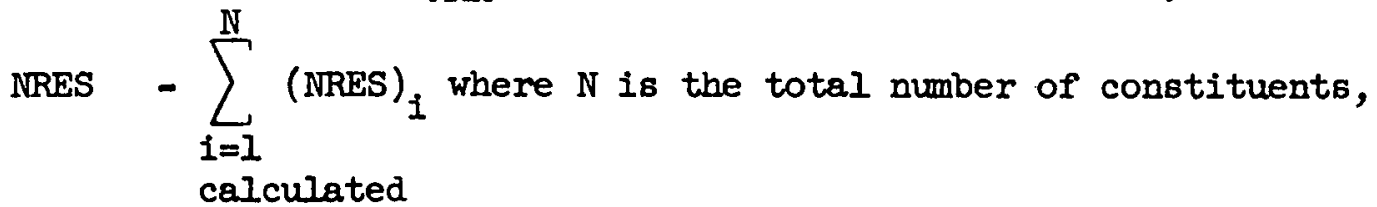

MGMIN - minimum $\mathrm{MT}_{\mathrm{MIN}}$ of the microscopic constituents, calculated 


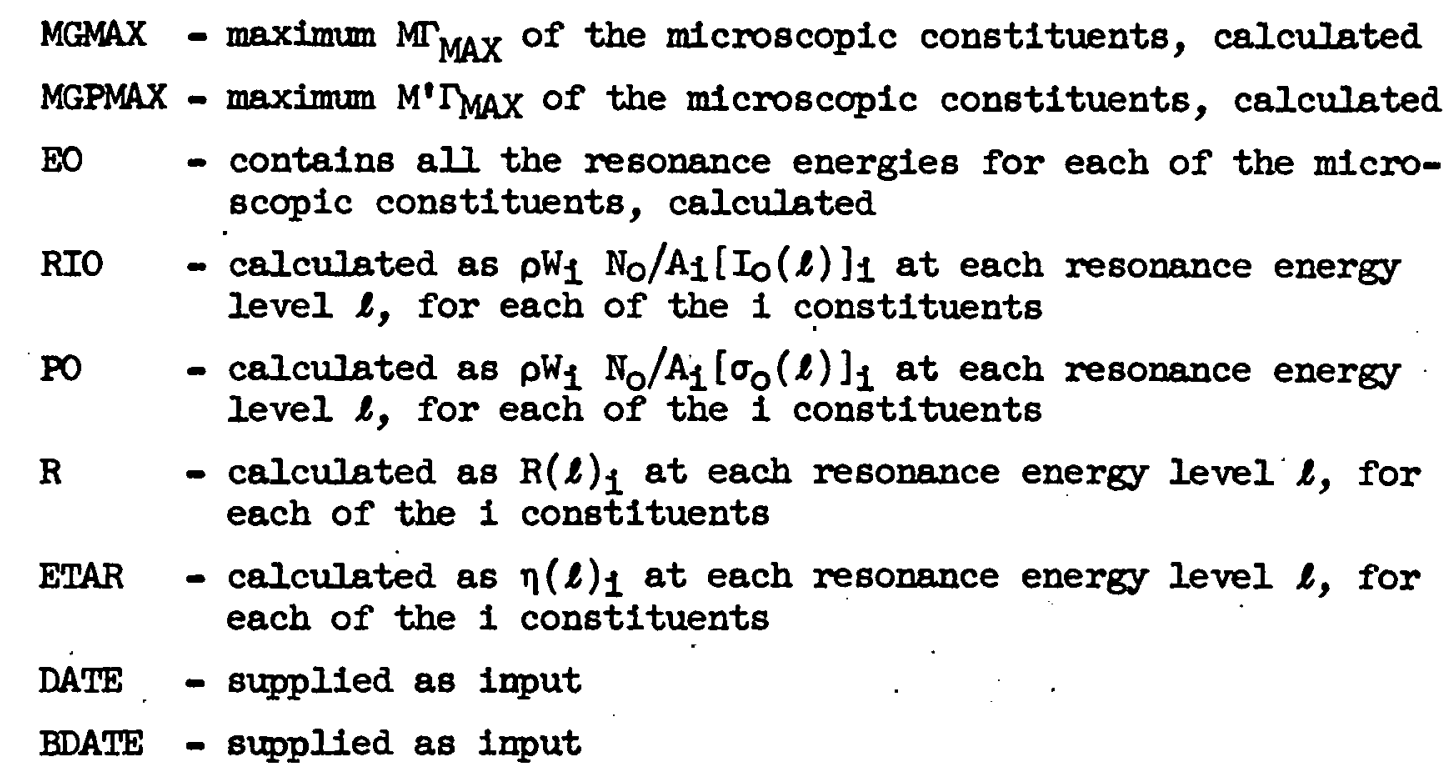

\subsection{Description of Subroutines Unique to this Program}

\subsubsection{Subroutine MACl}

This subroutine sets tape assignments and program constants, the temperature levels of the thermal groups, and the maximum possible number of data blocks. The first record of card Imput data and the index record from the master data tape are read in and stored in NB6. The tape Indices that depend on the number of energy levels are calculated, and the second record on the master tape is brought into core memory.

\subsubsection{Subroutine MAC2}

This subroutine collects the record location information from the master tape, 1dentifies the next macroscopic data block to be processed, and calls subroutine MAC3 to calculate the macroscoplc cross sections. The completed macroscopic data block is then written on an intermediate tape, KTAPE, and the index record is revised to reflect the addition of this data block. When all of the imput-specified number of macroscopic data blocks have thus been calculated, control is returned to the main program.

\subsubsection{Subroutine MAC3}

Thus subroutine brings into memory the necessary input data for the next macroscopic data block to be processed. The microscopic constituents are ordered as they appear on the master tape, and subroutine MAC4 is called to process in turn each constituent, computing the macroscopic cross section data. When all the constituents have been combined, the final record is up-dated to include this macroscopic data block and control is returned to MAC2.

\subsubsection{Subroutine MAC4}

This subroutine reads into memory each of the records within the microscopic constituent data block. Each piece of data is multiplied by 
the calculated weighting factor and added into the macroscopic cross section block. The matrix indices and number of resonances for the macroscopic data block are computed and stored in the appropriate locations. Control is then returned to subroutine MAC3.

\subsubsection{Subroutine MAC5}

This subroutine computes from information contained in record 1 , the number of words in records $2,3,4$, and 5 of the microscopic constituent data block.

\subsubsection{Subroutine MAC6}

This subroutine is used to position the master tape and read in the final record. The information for the new macroscopic data blocks is added, and the new revised final record is stored in core memory.

\subsubsection{Subroutine MAC7}

This subroutine is used to initlate the transfer of the revised index and final records, the second record, and all data blocks to the two new data tapes. As each data block is written on the new binary tapes, the program will check for possible printout of the data block. Only those data blocks, requested in the input, will be written on the output tape. The only exceptions are the index and final records which are automatically printed. The program will always punch on binary cards the index and final records, and all calculated macroscopic cross section data blocks. When all output operations have been completed, control is returned to the main program.

\subsubsection{Subroutine MAC8}

The purpose of this subroutine is to test for failures in reading or writing binsry tapes. If tape fallure does occur, sense light 2 1s turned on, and the program will stop. If the subroutine finds the sense light off, control is returned to the calling subroutine.

\subsubsection{Subroutine MAC9}

The purpose of this subroutine is two-fold. Flrst is serves to transfer a completed macroscopic data record from one portion of core storage to another, prior to writing this record on the intermediate tape KTAPE. Secondly, it computes the macroscoplc cross sections from the microscopic constituent data for the fifth record in the macroscopic data block.

\subsubsection{Subroutine MAClO}

This is an auxiliary subroutine used to transfer a particular set of data from one portion of core storage to another.

\subsection{Program Gross Flow Chart}

The program gross flow chart is shown in Flgure 3.1. 


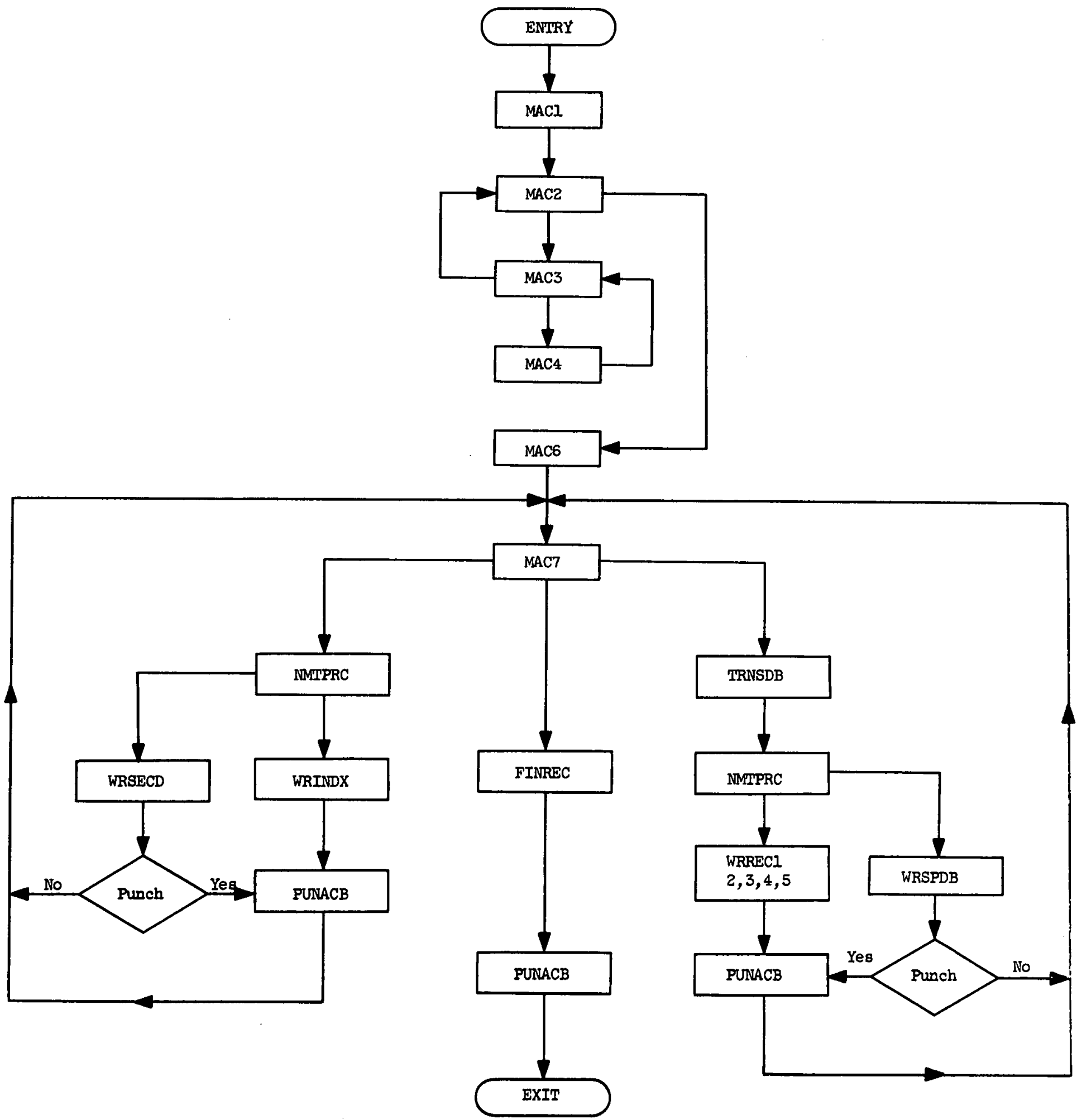

F1B. 3.1 Program Gross Flow Chart 


\subsection{Program Diagnost1cs}

External Formula Number

\section{$\underline{\text { Error }}$}

Subroutine MACl 900,902

901

Fifth consecutive failure in attempting to read the master tape

Sense light 1 after reading first DIP imput data

Subroutine MAC2

914

Negative value of $N$

915

Negative value of $\mathrm{N} 2$

916

Negative value of $\mathrm{N3}$

917

Negative value of $\mathrm{N} 4$

Subroutine MAC3

921

Sense light 1 after reading DIP Imput data

Subroutine MAC4

930

Negative value of $N I$

931

Negative value of $\mathrm{NZ}$

932

Negative value of $\mathrm{N3}$

933

Negative value of $\mathrm{N} 4$

934

Number of resonances equals or exceeds NRMAX originally set as 200

935

9351

Negative value of MOMIN in FBI

936

9361

Negative value of MOMIN in FB2

Negative value of MIMIN in FBI

Negative value of MMIN in FB2

937

Negat1ve value of MGMIN in FBI

9371

Negative value of MGMIN in FB2

Subroutine MAC5

940

Negative value of MOMIN in FBI

941

Negative value of MIMIN in FBI

942

Negative value of NRES in FBI

943

Negative value of MGMIN in FBI

Subroutine MAC8

980

Five consecutive failures in tryling to read or write a tape record and check its accuracy

against a second reading or writing 
External Formula

Number

Subroutine NMTPRC

624

Both data tapes have failed to check against a written record five consecutive times 
3.6 Program Input Forms

RNDM

PROGRAM RNDM

INDEX DATA

\begin{tabular}{|c|ccc|}
\hline 5DATE, 2, & \multicolumn{2}{|c|}{$/$} \\
\hline & MONTH & DAY & YEAR \\
& (2 Characters) & (2 Characters) & (2 Characters)
\end{tabular}

\begin{tabular}{|l|l|l|l|}
\hline 4N $\phi \mathrm{MAC}$, & N申PRNT, & \\
\hline & $\begin{array}{l}\text { Number of macroscopic } \\
\text { cross section blocks to b } \\
\text { calculated. }\end{array}$ & $\begin{array}{l}\text { Number of blocks to } \\
\text { be printed out. }\end{array}$
\end{tabular}

* The Index and Final records are always printed out.

Code Numbers of Blocks To Be Printed

$$
\left(2 \times 10^{-6}=\text { Record } 2\right)
$$

\begin{tabular}{|l|l|l|l|l|}
\hline $3 P C \phi D E$ &, & &, & \\
\hline
\end{tabular}

\begin{tabular}{|l|l|l|l|l|}
\hline (1) &, &, &, & \\
\hline
\end{tabular}
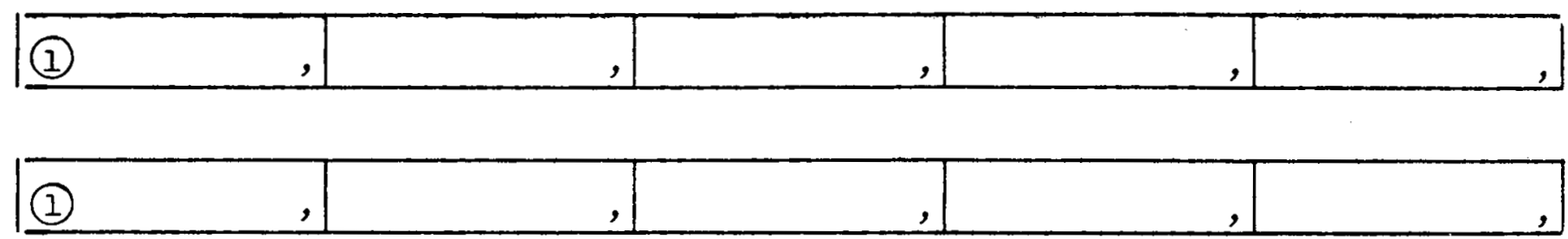

NOTE: The Index Record, all calculated macroscopic cross section blocks, and the final record are always punched out in binary. 
MACROSCOPIC CROSS SECTION BLOCKS

Index Data

\begin{tabular}{|c|c|} 
& $\begin{array}{c}\text { Data-Block Designation } \\
\text { (12 Characters) }\end{array}$ \\
\hline 5BNAM, 2, & \\
\hline$N_{1}^{*}$ & \\
\hline El7510,2, $^{*}{ }^{*}$ & \\
\hline El7508,2, & \\
\hline$N_{3}$ & \\
\hline El7506,2, & \\
\hline$N_{4}$ & \\
\hline$E 17504,2$, & \\
\hline$N_{5}$ & \\
\hline$E 17502,2$, & \\
\hline$N_{6}$ & \\
\hline$E 17500,2$, & \\
\hline
\end{tabular}
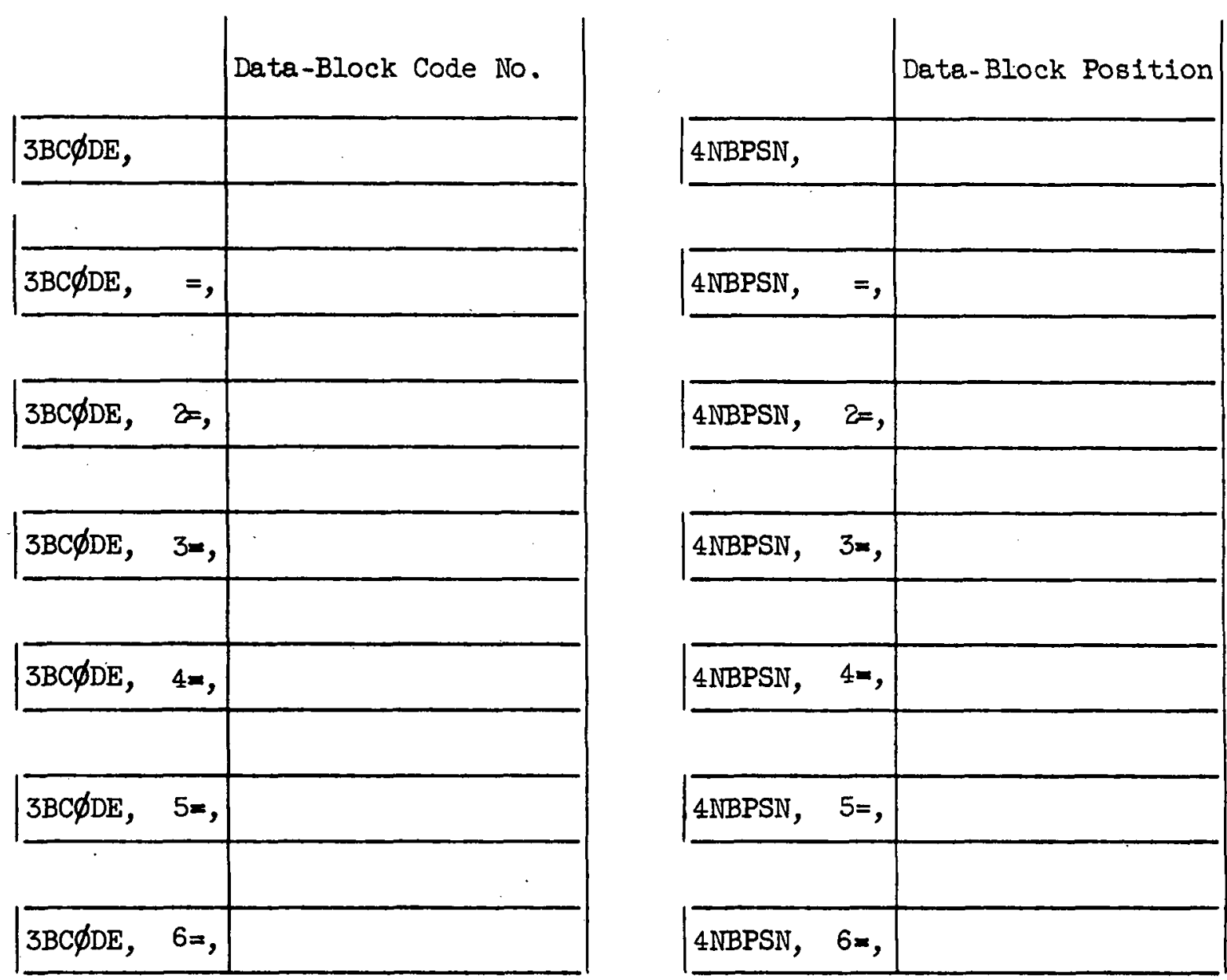

${ }^{*} N_{j}=$ (Location of BNAM) - $2 J$

\begin{tabular}{|l|l|}
\hline$=\mathrm{END}$, & 0, \\
\hline
\end{tabular}


MACROSCOPIC CROSS SECTIONS

RNDM

Constituent Data

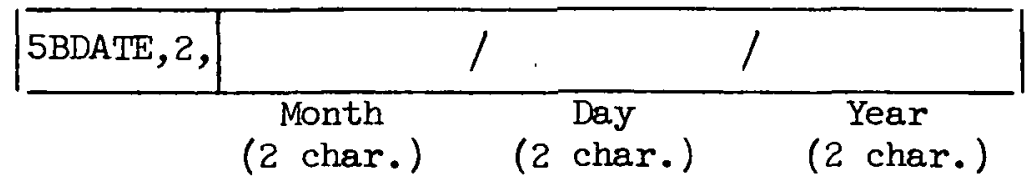

\begin{tabular}{|c|c|c|c|}
\hline 3DEN, & , & $4 \mathrm{NC} \phi \mathrm{N}$, & \\
\hline & Density & & $\begin{array}{c}\text { Number of Macroscopic } \\
\text { Constituents }\end{array}$ \\
\hline
\end{tabular}

- Microscopic Constituent Code Numbers

\begin{tabular}{|l|l|l|l|}
\hline 3 C $\varnothing \mathrm{NN} \phi$, &, & \multicolumn{3}{|c}{ Weight,} & \\
\hline
\end{tabular}

Microscopic Constituent Weight Fractions

\begin{tabular}{|c|c|c|c|}
\hline $3 c \phi N N \phi, \quad=$ & , & WGT, & , \\
\hline $3 c \phi \operatorname{NN} \phi, \quad 2=$ & , & WGT, $\quad 2=$, & , \\
\hline
\end{tabular}

\begin{tabular}{|l|l|l|l|l|l|}
\hline $3 C \phi \mathrm{NN} \phi$, & $3=$, & & WGT, $3=$, & \\
\hline $3 C \varnothing \mathrm{NN} \phi$, & $4=$, &, & WGT, $4=$, & \\
\hline
\end{tabular}

\begin{tabular}{|l|l|l|l|}
\hline $3 C \phi \mathrm{NN} \phi, 5=, 5$ &, & WGT, $5=$, & \\
\hline
\end{tabular}

\begin{tabular}{|l|l|l|l|l|}
\hline $3 C \phi \operatorname{NN} \phi, 6=$, & & WGT, $6=$, & \\
\hline $3 C \phi N N \phi, 7=$, &, & WGT, $7=$, & \\
\hline
\end{tabular}

\begin{tabular}{|l|l|}
\hline$=E N D$, & 0 \\
\hline
\end{tabular}




\subsection{Proprem Source Dook Iisting}

C PROGRAM RNDM

C

COMMON NB 1, NB2, NB6, NLOC, NF I NR, WGT, CONNO, BNAM, PCODE, BCODE, NCONN, INBP SN, PUNCOD, ENERGY, THERM, DATE, BDATE, NTHGRP, NWINDX, I TAPE, KTAPE, $2 L T A P E, M T A P E, N T A P E, M A X D B 2, M A X D B 1, M A X D B, I, N A, N B, N C, N D, N E, N F, N R, N X$, $3 M A 2, M A 3, M A 5, M B 2, M B 3, M B 5, M C 2, M C 3, M C 5, N 1, N 2, N 3, N 4, N R E S, N T R, M C O N$, 4 NCON, NOMAC, NOPRNT, NTLEV, NLEV, NWDBR1, NWRC2, NSPECT, NRMAX, MAXWD2, $5 N T W D S, D E N, N W F$ I NR, NOPUN, I I $3, J, M, N, N W S G S O, N W S G S 1$, NWRES

EQUIVALENCE (NBI, FB1), (NB2,FB2), (NB6, FB6), (NFINR, FINR), (NLOC,FLOC) DIMENSION FB1 $(2010)$, FB2 $(5010), F B 6(3010)$, NFINR(1010), FINR(1010) DIMENSION NB1 $(2010)$, NE2 $(5010)$, NB6(3010), THERM(10), NLOCC(2010), 1ENERGY (45),PCODE (124),BNAM(248), BCODE (124),NCONNTI24),FLOCT2OIO), 2 NBPSN (124), DATE (2), IVGT $(1000)$, CONNO $(1000)$, PUNCOD $(124)$, BDATE(2)

$c$
$C$
$C$
$c$
$C$
$C$
$c$
$c$
$c$
$c$
$c$
$c$ SET INDICES

1 CALL MACI

COMPILE MACROSCOPIC DATA BLOCKS AND PLACE ON INTERMEDIATE TAPE, REVISE INDEX RECORD, TABULATE FOR FINAL RECORD

2 CALL MAC2

COMPILE FINAL RECORD

3 CALL MAC6

OUTPUT

4 CALL MACT

CALL EXIT

END 
C. . SUBROUTINE TO SET RNDM INDICES SUBROUTINE MACI

C

COMMON NB I,NB 2, NB6, NLOC, NF INR, WG , CONNO, BNAM, PCODE, BCODE, NCONN, INBPSN, PUNCOD, ENERGY, THERM, DATE, BDATE, NTHGRP, NWI NDX, I TAPE, KTAPE, 2LTAPE , MT APE, NTAPE , MAXDB2, MAXDB1, MAXDB, I , NA, NB, NC, ND, NE INF, NRR , NX,$3 M A 2, M A 3, M A 5, M B 2, M B 3, M B 5, M C 2, M C 3, M C 5, N 1, N 2, N 3, N 4, N R E S, N T R, M C O N$, $4 N C O N, N O M A C$, NOPRNT, NTLEV, NLEV, NWDBR 1, NWRC2, NSPECT, NRMAX, MAXND2: $5 N T W D S, D E N, N W F$ I NR, NOPUN, I I $3, J, M, N, N W S G S O, N W S G S I$, NWRES

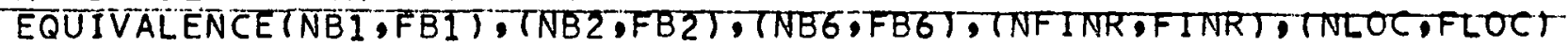
DIMENSION F8I (2010), FB2 (5010), FB6(3010), NFINR(1010), FINR(1010) DIMENSION NBI (2010), NB2 $(5010) ; N B 6(3010)$, THERM(10), NLOC $(2010)$, IENERGY (45), PCODE (124), BNAM(248),BCODE(124), NCONN(124), FLOC(2010), 2NBPSN $(124)$, DATE $(2)$, WGT $(1000)$, CONNO $(1000)$, PUNCOD $(124)$, BDATE $(2)$

100 NTHGRP $=10$ NWSGSO $=626$

NWSGS $1=626$

NRMAX $=200$

NWRES $=1001$

NWINDX $=2000$

NWF INR $=1000$

$N T R=20$

$\operatorname{THERM}(1)=4500$.

THERM $(2)=4000$

$\operatorname{THERM}(3)=3500$.

THERM $(4)=3000$.

$\operatorname{THERM}(5)=2500$.

THERM $(6)=2000$.

$\operatorname{THERM}(7)=1500$.

THERM $(8)=1000$.

$\operatorname{THERM}(9)=500$.

THERM $(10)=68$.

MAXDB $1=N W I N D X / 4-1$

$M A X D B=M A X D B 1=1$

I TAPE $=8$

RTAPE $=9$

LTAPE $=3$

$M T A P E=7$

NTAPE $=6$

REWIND ITAPE

REWIND KTAPE

REWIND MTAPE

REWIND NTAPE

CALL NOPAGE

C READ IN FIRST INPUT DATA RECORD

101 READ DIP DATE, NOMAC,NOPRNT,PCODE,BNAM,BCODE,

INBPSN, END

IF(SENSE LIGHTI)901,102

C READ INDEX RECORD

C 102 READ TAPE ITAPE, (FB6(II), II=1,NWINDX)

$\circ$ IF (SENSE LIGHT 2$)$ ) 900,1021
$C$ SET INDICES THAT DEPEND ON NLEV

1021 NLEV $=$ NB6 $(4)$ 
NTLEV $=N L E V+N T H G R P$

NWDBR $1=6$ *NTLEV $30 *$ NTHGRP 16

$N W R C 2=2 * N T L E V+3 * N L E V+3$

NSPECT $=$ NTLEV+6

MAXWD2 $=(N L E V+1) * 2+1$

NTWDS $=2 * M A X W D 2$ NWDBR $1+5 * N R M A X+20 * N T L E V+3$

103 READ TAPE I TAPE, $(F B 1(I 1), I 1=1, N W R C 2)$

IFISENSE LIGHT 2) 902,1031

$1031 M 4=N L E V+1$

DO $1032 \mathrm{~J} 1=1, M 4$

$J 2=2 * N T L E V+1+J 1$

1032 ENERGY $(J 1)=F B I(J 2)$

$N G=2 * M A X W D 2+2 * N L E V$

DO $1033 \mathrm{~J} 1=1$, NLEV

$J 2=N G+J 1$

$E R A=E N E R G Y(J 1) / E N E R G Y$ (J1 I)

$1033 \mathrm{FLOC}(\mathrm{J} 2)=\operatorname{LOGF}$ (ERA)

104 RETURN

900 CALL ERRORA STOP

901 CALL ERRORA

STOP

902 CALL ERRORA

STTOP

END 


\section{$C$ SUBROUTINE TO SET RNDM INDTCES}

C

COMMON NB 1,NB2, NB6, NLOC,NF INR,WG , CONNO, BNAM, PCODE, BCODE, NCONN, INBP SN, PUNCOD, ENERGY, THERM, DATE, BDATE, NTHGRP, NW I NDX , I T APE , KTAPE • 2LTAPE, MTAPE, NTAPE, MAXDBZ, MAXDBI, MAXXOB, I NA , NB, NC, ND, NE , NF, NR , NX, $3 M A 2, M A 3, M A 5, M B 2, M B 3, M B 5, M C 2, M C 3, M C 5, N 1, N 2, N 3, N 4, N R E S, N T R, M C O N$, $4 N C O N, N O M A C$, NOPRNT, NTLEV, NLEV, NWDBRI, NWRC $2, N \bar{S} \overline{E C}$ T, NRMAX, MAXWD2, $5 N T W D S, D E N, N W F$ I NR, NOPUN, I I $3, J, M, N, N W S G S O, N W S G S I, N W R E S$

EQUIVALENCE (NB1, FBI), (NB2,FB2), (NB6,FB6), (NFINR, FINR), (NLOC ,FLOC) DIMENSION FBI (2010), FB2 (5010), FB6(3010), NFINR(1010), FINR(1010)

DIMENSION NBI'(2010), NB2 $(5010)$, NB6 (3010), THERMTIO), NLOC(20IO). IENERGY (45), PCODE (124), BNAM (248), BCODE (124), NCONN(124), FLOC (2010),

2NBPSN $(124)$, DATE $(2)$, WGT (1000), CONNÖ (1000),PUNCODT124T.BDATE (2)

100 NTHGRP $=10$

NWSGSO $=626$

NWSGS $1=626$

NRMAX $=200$

NWRES $=1001$

NWI NDX $=2000$

NWF I NR $=1000$

$N T R=20$

$\operatorname{THERM}(1)=4500$.

THERM $(2)=4000$.

$\operatorname{THERM}(3)=3500$.

THERM $(4)=3000$.

$\operatorname{THERM}(5)=2500$.

THERM(6) $=2000$.

$\operatorname{THERM}(7)=1500$.

THERM $(8)=1000$.

$\operatorname{THERM}(9)=500$.

THERMP $10 \%=68$.

MAXDB I =NWI NOX/4-1

MAXDB $=M A X D B 1-1$

I TAPE $=8$

$K T A P E=9$

LTAPE $=3$

$M T A P E=7$

NTAPE $=6$

REWTND ITAPE

REWIND KTAPE

REWIND MTAPE

REWIND NTAPE

CALL NOPAGE

C READ IN FIRST INPUT DATA RECORD

101 REAO DIP DATE, NOMAC, NOPRNT, PCODE, BNAM, BCODE, INBPSN, END

IFISENSE LIGHTII901,102

C READ INDEX RECORD

C

102 READ TAPE ITAPE, (F B6 III) II=1,NWINDX)

IF(SENSE LIGHT 2) 900,1021

C $S E T$ INDICES THAT DEPEND ON NLEV

$1021 \mathrm{NLEV}=\mathrm{NB} 6(4)$ 
NTLEV $=N L E V+N T H G R P$

NWDBR $1=6 *$ NTLEV $30 *$ NTHGRP 16

$N W R C 2=2 * N T L E V+3 * N L E V+3$

NSPECT $=$ NTLEV+6

MAXWO2 $=(N L E V+1) * * 2+1$

NTWDS $=2 * M A X W D 2$ NWDBR $1+5 * N R M A X+20 * N T L E V+3$

103 READ TAPE ITAPE, (FBI (I 1 ), II=1, NWRC2)

IF(SENSE [IGHT 2 ) $902 ; 1031$

$1031 M 4=N L E V+1$

DO $1032 \mathrm{~J} 1=1, \mathrm{M4}$

$J 2=2 * N T L E V+1+J 1$

1032 ENERGYTJi)=FBI $(J 2)$

$N G=2 * M A X W D 2+2 * N L E V$

$D O-1033 \mathrm{JI}=1, N L E V$

$J 2=N G+J 1$

ERA $=E N E R G Y(J 1) / E N E R G Y T J 111$

$1033 F L O C(J 2)=\operatorname{LOGF}(E R A)$

104 RETURN

900 CALL ERRORA

STOP

901 CALL ERRORA

STOP

902 CALL ERRORA

STOP

END 
SUBROUTINE MAC2

\begin{tabular}{l}
\hline $\mathrm{C}$ \\
$\mathrm{C}$ \\
$\mathrm{C}$
\end{tabular}

SUBROUTINE TO ADD MACROSCOPIC DATA BLOCKS TO

C INTERMEDIATE TAPE

COMMON NB 1, NB2, NB6, NLOC,NFINR, WG T, CONNO, BNAM, PCODE, BCODE NCONN, INBPSN, PUNCOD, ENERGY, THERM, DATE, BDATE, NTHGRP, NWINDX, I TAPE, KTAPE, 2LTAPE, MTAPE, NTAPE, MAXDB2, MAXDBI, MAXOB,I, NA, NB, NC,ND, NE, NF ONR , NX, $3 M A 2, M A 3, M A 5, M B 2, M B 3, M B 5, M C 2, M C 3, M C 5, N 1, N 2, N 3, N 4, N R E S, N T R, M C O N$, $4 N C O N$, NOMAC,$N O P R N T$, NTLEV, NLEV $, N W D B R I, N W R C 2$ NSPEC I NRMAX OMAXWDZ? $5 N T W D S, D E N, N W F I N R$, NOPUN, I I $3, J, M, N, N W S G S O, N W S G S I, N W R E S$

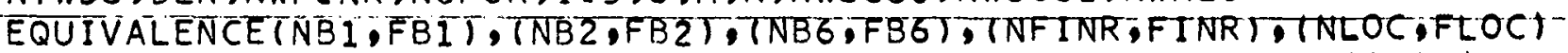
DIMENSION FBI (2010), FB2 $(5010)$, FB6 (3010), NFINR $(1010)$, FINR(1010)

DIMENSION NBI 2010 ) ONB2(5010), NB6 (3010),THERMT10), NLOCT2010T\%

IENERGY (45), PCODE (124), BNAM(248), BCODE (124), NCONN(124), FLOC (2010),

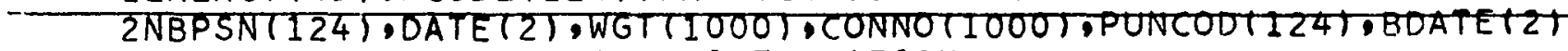

C COLLECT RECORD LOCATING INFORMATION

103 DO 104 T1 $=$ I, MAXOB

$I F(N B 6(4 * 11+8)-N B 6(4 * 11+4)) 1035,1035,104$

$1035 \mathrm{II}^{-}=\mathrm{II}^{-}$

GO TO 1041

104 CONTINUE

$113=M A \times D B$

$1041001045 \cdot \mathrm{J} 1=1.113$

FINR $(2 * J 1-1)=F B 6(4 * J 1+3)$

1045 CONT INUE

$N A=1$

$N R=0$

105 DO $1150 \quad I=1$ MAXDB

$105500106 \mathrm{~J} 1=1$, NOMAC

$N F=J 1$

IF(I NBPSN(J1)) 106,112,106

106 CONTINUE

111 GO TO 1150

$112001125-I 1=I$.NTWDS

$1125 \mathrm{NB2}(11)=0$

CALL MAC 3

DO 1126 I I = I.NWDBR I

I1.76 FBITII $=F B$ ?III

$N D=6 * N T L E V+6$

DO $1127 \mathrm{~J}=I$ INTHGRP

$F B 1(N D)=\operatorname{THERM}(J)$

ND $=N D+30$

1127 CONTINUE

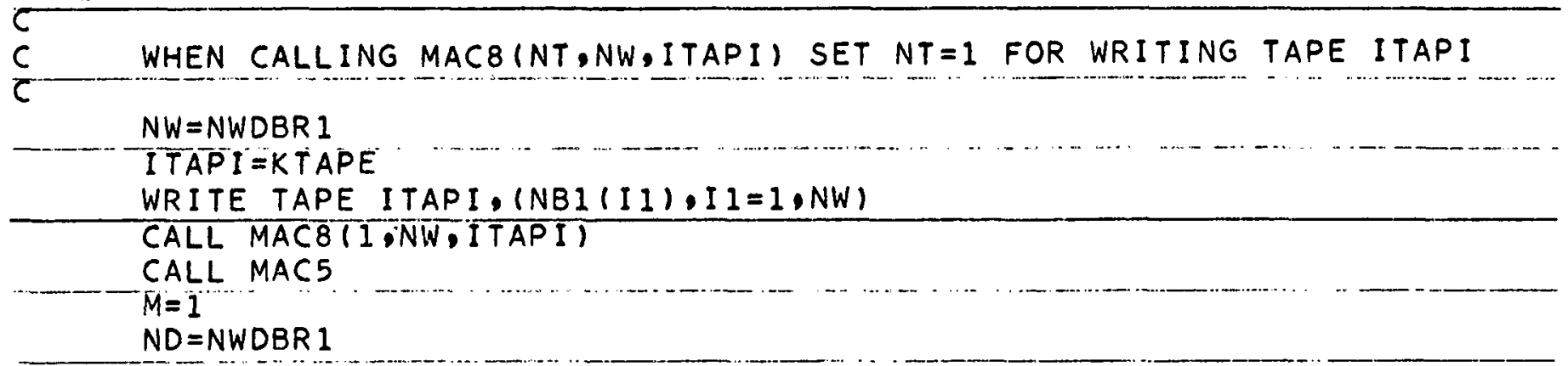


IF (N1) $914,1132,113$

$113 \quad M 4=N L E V+1$

CALL MAC9 (MA2,MB2,MC2,M4,0.0)

$\bar{M}=\bar{M}+1$

WRITE TAPE ITAPI, (NBI II),II=1,NI)

CALL MAC8 (1,NI, ITAPI)

$1132 N D=N D+M A X W D 2$

$\bar{I} F(N 2) 9 \overline{15}, 1135,1133$

$1133 M=M+1$

$M 4=N L E V+1$

CALL MAC9 (MA3,MB $3, M C 3, M 4,0.0)$

WRITE TAPE ITAPI, (NBITII),II=I,N2)

CALL MAC8 $11, N 2$, ITAP I)

$1 \longdiv { 3 5 } N \overline { N D } = N D + M A \overline { X } W \overline { D } 2$

I $F(N 3) 916,1142,1136$

$1136 \mathrm{M}=\mathrm{M} \quad 1$

$N W=$ NRES +1

DO II $37 \mathrm{JI}=1, \mathrm{NW}$

$J 2=N D+J 1$

$1137 \mathrm{FBI}(\mathrm{J} 1)=F B 2(\mathrm{~J} 2)$

$N D=N D+N R M A X+1$

DO $1138 \mathrm{JI}=1$, NRES

$J 2=N D+J 1$

$J_{3}=J i+N W$

$1138 F B 1(J 3)=F B 2(J 2)$

$N D=N D+N R M A X$

$N W=N W+N R E S$

DO $1139 \mathrm{~J} 1=1$.NRES

$J 2=N D+J 1$

$J_{3}=N W+J 1$

$1139 F B 1(J 3)=F B 2(J 2)$

$N D=N D+N \bar{M} A \bar{X}$

$N W=N W+N R E S$

DO $1140 \mathrm{JI}=$ I.NRES

$J 2=N D+J 1$

$J 3=N W+j i$

$1140 \mathrm{FBI}(\mathrm{J} 3)=\mathrm{FB} 2(\mathrm{~J} 2)$

$N D=N D+N \bar{R} M A$

$N W=N W+N R E S$

DO 1141 J1 $=1$.NRES

$J 2=N D+J 1$

$J 3=N W^{-}+J 1$

1141.FBI $(J 3)=F B 2(J 2)$

WRITE TAPE ITAPI, (NBITII),II $=1, N 3)$

CALL MAC8 $(1, N 3, I T A P I)$

$1142 \mathrm{ND}=$ NWDBRI + 2 *MAXWD2 + 5*NRMAX + 1 IF (N4) $917,1145,1143$

$1143 \mathrm{CALL}$ MACS (MAS, MBS, MC5,NTR,0.0) $M=M+1$

WRITE TAPE ITAPI, (NBITII),IIII,N4)

CALL MAC8 (I,N4, ITAPI)

C CORRECT INDEX RECORD

${ }_{6} 1145$ DO $1146 \mathrm{~J} 1=1$, NWINDX

$1146 \mathrm{FB1}(\mathrm{J1})=\mathrm{FB} 6(\mathrm{J1})$

$F B 6(4 * I+1)=B N A M(2 * N F-1)$ 
164

F.B $6(4 * I+2)=B N A M(2 * N F)$

FB $6(4 * I+3)=B C O D E(N F)$

$\operatorname{NB} 6(4 * I+8)=N B 6(4 * I+4)+M$

$N B=11$

DO 1149 I $1=N B, M A X D B$

$F B 6(4 * 11+1)=F B 1(4 * 11-3)$

$F B 6(4 * 11+2)=F B 1(4 * 11-2)$

$\bar{F} 6(4 * I I+3)=F B(14 * I I-1)$

IF (NBI $4 * I 1+4) \quad N B I(4 * I 1)) 1147,1147,1148$

$1147 \mathrm{NB} 6(4 * I 1+8)=0$

NB6 (NWINDX+1) $=N B 6(4 * I 1+4)$

60 TO 1150

$1148 \mathrm{NB} 6(4 * I 1+8)=N B 6(4 * I 1+4)+N B 1(4 * I 1+4)-N B 1(4 * I 1)$

1149 CONTINUE

1150 CONTINUE

1153 RETURN

9.14 CALL ERRORA

STOP

915. CALL ERRORA STOP

916 CALL ERRORA STOP

917 CALL ERRORA STOP

END 
SUBROUTINE MAC3

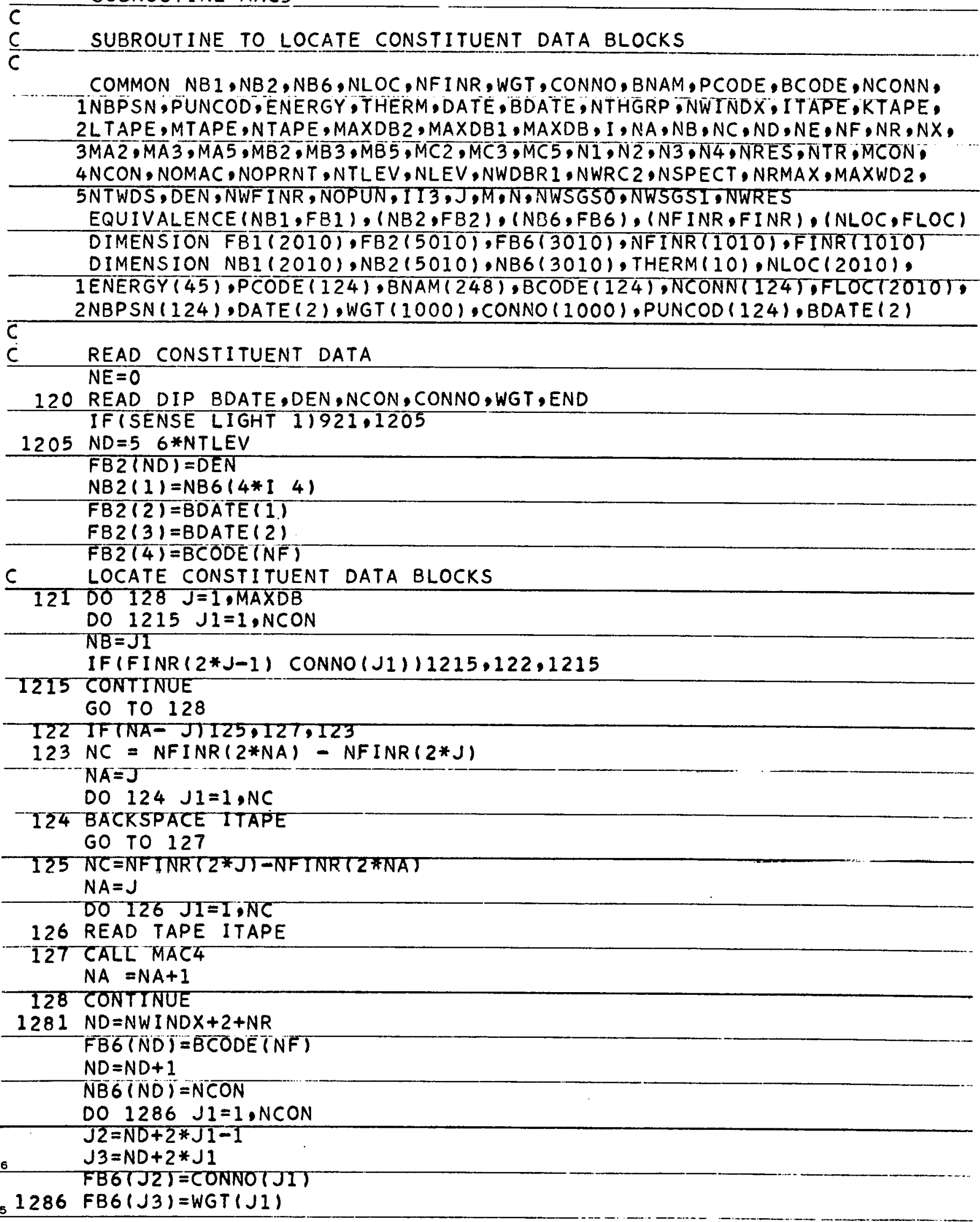


$N R=N R+2 * N C O N+2$

129 RETURN

921 CALL ERRORA STOP

END 
SUBROUTINE MAC4

$c$

$\mathrm{C}$

SUBROUTINE TO COMPILE MACROSCOPIC DATA BLOCKS

COMMON NB1, NB 2, NB6, NLOC, NFINR, WG , CONNO, BNAM, PCODE, BCODE, NCONN, INBPSN, PUNCOD, ENERGY, THERM, DATE, BDATE, NTHGRP, NW I NDX, I TAPE, KTAPE, $2 L T A P E, M T A P E, N T A P E, M A X D B 2, M A X D B 1, M A X D B, I, N A, N B, N C, N D, N E, N F, N R, N X$, $3 M A 2, M A 3, M A 5, M B 2, M B 3, M B 5, M C 2, M C 3, M C 5, N 1, N 2, N 3, N 4, N R E S, N T R, M C O N$, $4 N C O N, N O M A C, N O P R N T, N T L E V, N L E V, N W D B R 1, N W R C 2, N S P E C T$, NRMAX , MAXWD2,

5NTWDS, DEN, NWF INR, NOPUN, I I 3,J,M N, NWSGSO,NWSGS I, NWRES

EQUIVALENCE (NBI, FBI ), (NB, ,FB2), (NB6,FB6), (NFINR, FINR), (NLOC,FLOC)

DIMENSION FBI $(2010)$, FB2(5010), FB6(3010), NFINR(1010), FINR(1010)

DIMENSION NBI (2010), NB2 (5010), NB6(3010), THERM(10), NLOC $(2010)$,

1ENERGY (45), PCODE (124), BNAM (248),BCODE (124), NCONN7 124), FLOC (2010T," 2NBPSN (124), DATE(2),WGT (1000), CONNO(1000), PUNCOD (124), BDATE(2)

$\dot{C}$

$c$

C

C

C

WHEN CALLING MACB (NT, NW, ITAPI), SET NT=Ö FOR REAOING NWW WORDS

FROM ITAPI INTO FBI

C COMPUTE WEIGHTING FACTOR

READ TAPE ITAPE, $(N B I(J 1), J 1=1, N W D B R 1)$

CALL MACB (Ö,NWDBRI, I TAPE)

$N D=5 \quad 6 * N T L E V$

WGHTFR =DEN*WG T (NB) /FB I (ND)

C CALCULATE AND STORE MACROSCOPIC CONSTITUENTS

$N D=N D-1$

DO $13.1 J 1=5, N D$

$131 F B 2(J 1)=W G H T F R * F B 1(J 1)+F B 2(J 1)$

$N D=N D+2$

DO 132 I $2=1$, NTHGRP

DO $1316 \mathrm{Jl}=1,29$

$\mathrm{J} 2=\mathrm{ND}+\mathrm{J} 1$

$-1316-F B 2(J 2)=W G H T F R * F B I(J 2)+F B 2(J 2)$

$N D=N D+30$

C

132 CONTINUE

LOCATE AND STORE MINIMUM MOMIN, MAX MOIMAX, MAX MOOMAX

IF (NBI (NWDBRI-10)1935,135,1321

1321 IF.(NB2 (NWDBRI-10)) 9351,133,134

133 NB2 (NWDBR 1-10) =NS1 (NWDBR $1-105$

NB2 $($ NWDBR $1-9)=$ NB $1($ NWDBR 19$)$

NB2 $($ NWDBR $1-8)=$ NBI $($ NWDBR 1 8)

GO TO 135

134 NB2 (NWDBR 1-10) =XMINOF (NB2 (NWDBR1-10), NB1(NWDBR 1-10))

NE2 (NWDBR 1-9) = XMAXOF (NB2 (NWDBR 1-9), NBI (NWDBR 1-9))

N... NB2 (NWDBR 1-8) = XNAXOF (NB2 (NWDBR1-8), NBI (NWDBRI-8)

C LOCATE AND STORE MINIMUM MIMIN, MAXIMUM MIIMAX, MAXIMUM MIOMAX

135 IF (NEI(NWDBRI-7)) 936,138,1355

1355 IF(NB2(N:IDBR1-7))9361,136,137

136 NB2 (N!DBR $1-7)=$ NBI (NWDERI 7)

NB2 (NW:DBR $1-6)=$ NP1 (NWDBR 1 6)

NB2 (NWDBR 1 -5$)=$ NB1 (NWDBR1 5 )

GO TO 138

137 NB2 (NWDBR 1-7) $=X M I N O F(N B 2(N W D B R 1-7), N B 1(N W D B R 1-7))$

NB2 $(N W D B R 1-6)=X M A X O F(N B 2(N W D B R 1-6)$, NB1 $(N W D B R 1-6)$ ) 
NB2 (NWDBR 1-5) = XMAXOF (NB2 (NWDBR1-5), NB1 (NWDBR 1-5))

C ADD SUMS OF NRES TERMS

138 NB2 (NWDBR 1-4) =NB2 (NWDBR 14 ) +NB1 (NWDBR 1-4)

IF (NB 2 (NWDBRI-4)-NRMAX) $1381,934.934$

$C$ LOCATE AND STORE MINIMUM MGMIN, MAXIMUM MGIMAX, MAXIMUM MGOMAX

1381 IF (NB I (NWDBR1-3)) $937,1406,139$

139 IF (NB2 (NWDBR1-3)) $9371,140,1405$

140 NB2 (NWDBR $1-3)=$ NBI (NWDBRI 3 ) NB2 (NWDBR $1-2)=$ NB1 (NWDBR 12 2)

NB2 (NWDBR1-1) $=$ NBI (NWDBRI I)

GO TO 1406

1405 NB2 (NWDBR $1-3)=$ XMINOF (NB2 (NWDBR1-3),NB1 (NWDBR1-3)

NB2 (NWDBR 1-2) = XMAXOF (NB2 (NWDBR1-2), NBI (NWDBR 1-2))

NB2 (NWDBR1-1) = XMAXOF (NB2 (NWDBRI-1), NBI (NWDBRI-1)

C STORE SCATTERING CROSS-SECTIONS IN RECORD 2,3 MATRICES

$1406 \mathrm{NG}=2 * M A X W D 2$

DO $700 \quad J 1=1, N G$

700 FLOC $(J 1)=0.0$

$N G=2 * M A X W D 2+2 * N L E V$

$M 4=N L E V+1$

$\mathrm{NH}=1$

$N J=\overline{N T L E V+4}$

DO $1407 \mathrm{~J} 1=1, \mathrm{NLEV}$

$J \overline{2}=N H+J 1$

$J 3=N G+J 1$

$\mathrm{J} 4=\mathrm{N} \mathrm{J}+\mathrm{J} \mathrm{I}$

FLOC $(J 2+1)=F B 1(J 4) / F L O C(J 3)$

$F L O C(J 2)=F B 1(J 1+4)-F L O C(J 2+1)$

$\mathrm{NH}=\mathrm{NH}+\mathrm{M} 4$

$140 \overline{7}$ CONTINUE

$\mathrm{NH}=\mathrm{MAXWD2}$

$N J=4 * N T L E V+4$

DO $1408 \quad J 1=1, N L E V$

$J 2=N H+J 1$

$J 3=N G+J 1$

$J 4=N J+J 1$

$J 5=2 * N T L E V+4+J 1$

FLOC $(J 2+1)=F B 1(J 4) / F L O C(J 3)$

$F L O C(J 2)=F B 1(J 1+4)-F B I(J 5)-F L O C(J 21)$

$\mathrm{NH}=\mathrm{NH}+\mathrm{M} 4$

1408 CONTINUE

C COPY RECORDS $2,3,4,5$

141 CALL MAC5

ND $=$ NWDBR 1

IF (N1) $930,1425,1415$

1415 READ TAPE ITAPE, (FBITJI:JI:T:NI)

CALL MAC8 $(0, N 1$, ITAPE)

CALL MAC1O(MA2,MB2,MC2,M4,0)

DO 1416 J1 $=1$, MAXWD2

$J 2=N D+J 1$

$1416 F B 2(J 2)=W G H T F R * F L O C(J 1)+F B 2(J 2)$

$1425 N D=N D+M A X W D 2$

IF (N2) $931,145,143$

143 READ TAPE ITAPE, (FBI (J1),JI=1,N2)

CALL MAC8 $(0, N 2$, ITAPE) 
CALL MAC10 (MA3,MB3,MC3,M4,1)

DO $144 \mathrm{~J} 1=1, \mathrm{MAXWD} 2$

$J 2=N D+J 1$

$J 3=M A X W D 2+J I$

$144 F B 2(J 2)=W G H T F R * F L O C(J 3)+F B 2(J 2)$

$145 N D=N D+M A X W D 2$

IF (N3) $932,1485,1455$

1455 READ TAPE I TAPE, (FBI (J1);J) $=1 ; N \overline{3})$

CALL MAC8 $(0, N 3$, ITAPE)

NRES $1=$ NRES +1

DO $146 \mathrm{~J} 1=2, N R E S 1$

$\mathrm{J} 2=N D+N E+J 1$

$146 F B 2(J 2)=F B 1(J 1)$

$N D=N D+N R M A X+1$

DO $1465 \quad J 1=1$, NRES

$\sqrt{2}=N R E S 1+J 1$

$J 3=N D+N E+J 1$

1465 FB2 $(J 3)=W G H T F R * F B i(j 2)$

$N D=N D+N R M A X$

DO $147 \mathrm{~J} 1=1$, NRES

$\mathrm{J} 2=2 * N R E S+1+\mathrm{J} 1$

$\mathrm{J} 3=\mathrm{ND}+\mathrm{NE}+\mathrm{J} \mathrm{I}$

$147 F B 2(J 3)=W G H T F R * F B 1(J 2)$

$N D=N D+N R M A X$

DO $1475 \mathrm{~J} 1=1$, NRES

$J 2=3 * N R E S+1+J 1$

$J 3=N D+N E+J 1$

$1475 F B 2 T J 3)=F B I T J 2 T$

ND $=N D+N R M A X$

DO $148 \quad J 1=1$,NRES

$J 2=4 * N R E S+1+J 1$

$\therefore J 3=N D+N E+J 1$

$148 F B 2(J 3)=F B 1(J 2)$

NE $=$ NETNRES

1485 ND $=$ NWDBR1 + 2*MAXWD2 + 5*NRMAX + 1 IF (N4) $933,149,1486$

1486 READ TAPE ITAPE, (FBI (JI),JI $=1, N 4)$

CALL MACB (O.N4, ITAPE)

CALL MAC9 (MAS, MB5, MC5,NTR ,WGHTFR)

T49 RETURN

930 CALL ERRORA

STOP

931 CALL ERRORA STOP

932 CALL ERRORA STOP

933 CALL ERRORA STOP

934 CALL ERRORA STOP

935 CALL ERRORA

STOP

9351 CALL ERRORA STOP

936 CALL ERRORA 
170

\begin{tabular}{l} 
STOP \\
9361 CALL ERRORA \\
STOP \\
\hline 937 CALL ERRORA \\
STOP \\
9371 CALL ERRORA \\
STOP \\
END
\end{tabular}


SUBROUTINE MAC5

$c$
$C$
$C$

SUBROUTINE TO DETERMINE RECORD $2,3,4,5$ INDICES

COMMON NB1, NB2, NB6, NLOC, NF INR, WG , CONNO, BNAM, PCODE, BCODE, NCONN, INBPSN, PUNCOD, ENERGY, THERM, DATE, BDATE, NTHGRP, NWINDX, I TAPE, KTAPE, $2 L T A P E, M T A P E, N T A P E, M A X D B 2, M A X D B 1, M A X D B, I, N A, N B, N C, N D, N E, N F, N R, N X$, $3 M A 2, M A 3, M A 5, M B 2, M B 3, M B 5, M C 2, M C 3, M C 5, N 1, N 2, N 3, N 4, N R E S, N T R, M C O N$, $4 N C O N, N O M A C$, NOPRNT, NTLEV, NLEV, NWDBR 1 , NWRC2, NSPECT , NRMAX, MAXWD 2 ,.

$5 N T$ TOS, OEN, NWF INR, NOPUN, I 13 ,J,MON,NWSGSO , NWSGSI DNWRES

EQUIVALENCE (NB1, FB1), (NB2,FB2), $(N B 6, F B 6),(N F I N R, F I N R),(N L O C, F L O C)$

DIMENSION FBI $(2010), F B 2(5010), F B 6(3010)$, NFINRI 1010$)$, FINR(10I0)

DIMENSION NBI $(2010)$, NE2 $(5010), N B 6(3010)$, THERM(10), NLOC $(2010)$,

IENERGY (45), PCODE (124),BNAM(248), BCODE (124), NCONN(124), FLOC (2010), 2NBPSN (124), DATE (2), WGT (1000), CONNO (1000), PUNCOD(124), BDATE (2)

$\ddot{C}$

$150 \quad N 1=0$

$N 2=0$

N3 $=0$

$N 4=0$

1505 IF (NB I (NWDBR 1-10)) $940,152,151$

$151 M A 2=N B T(N W D B R 1 \cdots 10)$

$M B 2=N B 1$ (NWDBR 19 )

$M C 2=N B 1(N W D B R I 8)$

$\mathrm{Nl}=(M B 2-M A 2+1) *(M C 2-M A 2+1)+1$

152 IF (NBI (NWOBRI-7) /941,154,153

$153 M A 3=N B I$ (NWDBR 17 )

$M B 3=N B I T N W D B R 161$

$M C 3=N B I$ (NWDBR I 5 )

$N 2=(M B 3-M A 3+1) *(M C 3-M A 3+1)+1$

154 IF (NB I (NWDBR I-4) I942,156,155

$155 \mathrm{NRES}=$ NB 1 (NWDBR $1-4$ )

N3 $=5 * N R E S+1$

156 IF TNB 1 (NWDBR 1-3) $943,158,157$

157 MAS =NB I (NWDBR I 3 )

$M B 5=N B 1$ ( NWDBR 12 )

$M C 5=N B 1(N W D B R \& 1)$

$N 4=(M B 5-M A 5+1) *(M C 5-M A 5+1)+1$

158 RETURN

940 CALL ERRORA

STOP

941 CALL ERRORA STOP

942 CALL ERRORA STOP

943 CALL ERRORA

STOP

END 


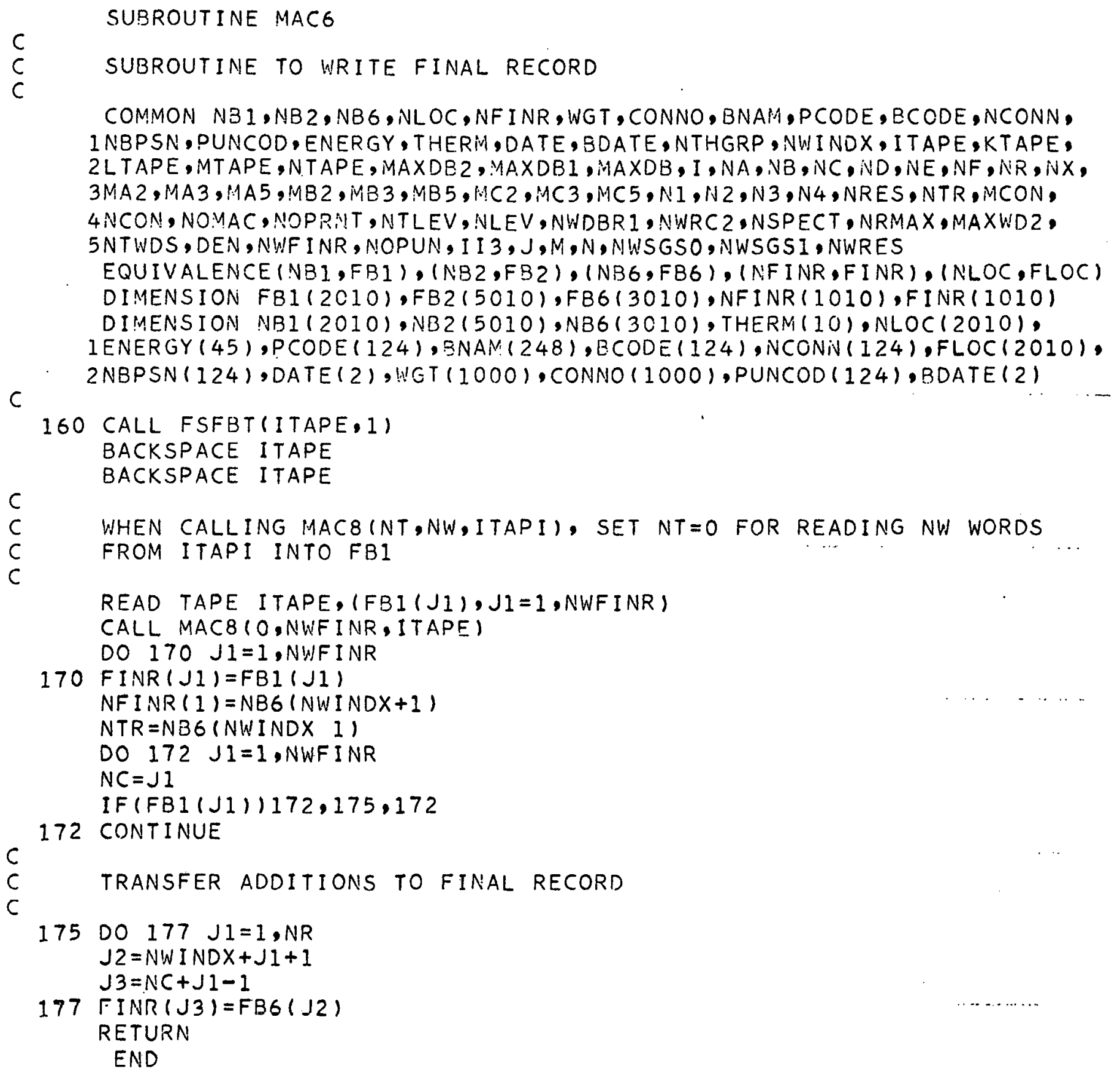


$c$ SUBROUTINE MAC7

\section{OUTPUT SUBROUTINE}

COMMON NB1, NB $2, N B 6, N L O C, N F I N R, W G T, C O N N O, B N A N$, PCODE, BCODE, NCONN, INBPSN, PUNCOD, ENERGY, THERM, DATE, BDATE, NTHGRP, NWINDX, ITAPE , KTAPE, 2LTAPE, MTAPE, NTAPE, MAXDB 2 , MAXDB1, MAXDB, I, NA, NB, NC, ND, NE, NF, NR , NX, $3 M A 2, M A 3, M A 5, M B 2, M B 3, M B 5, M C 2, M C 3, M C 5, N 1, N 2, N 3, N 4, N R E S, N T R, M C O N$, $4 N C O N, N O H A C$, NOPRNT , NTLEV, NLEV, NWDBR1, NWRC2, NSPECT, NRMAX, MAXWD2, $5 N T$ WDS, DEN, NWF INR, NCPUN, II $3, \mathrm{~J}, \mathrm{M}, \mathrm{N}, \mathrm{NWSGSO}, \mathrm{NWSGS} 1$, NWRES EQUIVALENCE (NBI, FB1), (NB2, FB2), (NB6, FB6), (NFINR, FINR), (NLOC,FLOC) DIMENSION FBI(2010), FB2(5010), FB6(3010), NFINR(1010), FINR(1010) DIMENSION NB1 $(2010)$, NB $2(5010)$, NB6 $(3010)$, THERM $(10)$, NLOC $(2010)$. IENERGY (45), PCODE (124), BNAN( 248$), B C O D E(124)$, NCONN $(124), F L O C(2010)$, 2NBPSN (124), DATE (2), ,GT $(1000)$, CONNO $(1000)$, PUNCOD $(124)$, BDATE ( 2$)$

$C$

$\mathrm{C}$

C

180 DO $181 \quad J I=1$,NWINDX

$181 F B 1(J 1)=F B 6(J 1)$

$N W_{j}=N W^{\prime}$ I NOX

CALL NMTPRC (NWINDX)

CALL WRINDX

C

c

C

182 REWIND ITAPE

REWIND KTAPE

READ TAPE ITAPE

$c$
$c$
$c$

WHEN CALLING MAC8 (NT,NW, ITAPI), SET NT=0 FOR READING NW WORDS FROM ITAPI INTO FBI

183 READ TAPE ITAPE, $(F B I(J I), J I=1, N W R C 2)$

CALL MAC8 (O,NWRC2, ITAPE)

1831 DO $1832 \mathrm{~J} 1=1$,NTLEV

$J 2=1 \mathrm{~J} I$

1832 ENERGY $(J 1)=F B 1(J 2)$

$N W=N W R C 2$

CALL NMTPRC(NWRC2)

c

1836 CALL WRSECR

$184 D O 1852 \quad I=1, M A X D B$

$N X=4 * I+3$

IF (NB6 (NX+1)-NTR ) 1841,186,1841

1841 DO $185 \mathrm{~J} 1=1$;NOMAC

IF (FB6(NX)-BCODE (J1)) 185,1851,185

185 CONTINUE

CALL TRNSDB(ITAPE, 0.0)

GO TO 1852

1851 CALL TRNSDB(KTAPE,1.0)

1852 CONTINUE

186 CALL FINREC RETURN

END 
C

TAPE CHECKING SUBROUTINE

COMMON NB 1, NB 2, NB6, NLOC, NF INR, WGT, CONNO, BNAM, PCODE, BCODE, NCONN, INBPSN, PUNCOD, ENERGY, THERM, OATE, BDATE, NTHGRP, NWINDX, ITAPE, KT APE, 2LTAPE, MTAPE, NTAPE, MAXDB2, MAXDB1 , MAXDB, I, NA, NB, NC, ND , NE , NF , NR , NX, $3 M A 2, M A 3, M A 5, M B 2, M B 3, M B 5, M C 2, M C 3, M C 5, N 1, N 2, N 3, N 4, N R E S, N T R, M C O N$, 4 NCON, NOMAC, NOPRNT , NTLEV , NLEV , NWDBR I , NWRC 2, NSPECT , NRMAX , MAXWD 2 * 5NTWDS, DEN, NWF INR, NOPUN, II $3, J, M, N, N W S G S O, N W S G S I, N W R E S$ EQUIVALENCE (NB1, FB1), (NB2, FB2), (NB6, FB6), (NF INR , FINR), (NLOC,FLOC) DIMENSION FBI(2010), FB2(5010), FB.613010), NFINR(1010), FINR(1010) DIMENSION NBI (2010), NB2(5010), NB6(3010), THERM(10), NLOC (2010), IENERGY (45), PCODE (124), BNAM (248), BCODE (124), NCONN(124), FLOC (2010), 2NBPSN (124), DATE (2), WGT (1000), CONNO (1000), PUNCOD (124), BDATE (2)

$\bar{C}$

$N T=N T$

$N W=N W$

ITAPI $=$ I TAPI

IFISENSE LIGHT 21980,198

198 RETURN

980 CALL ERRORA STOP

END 


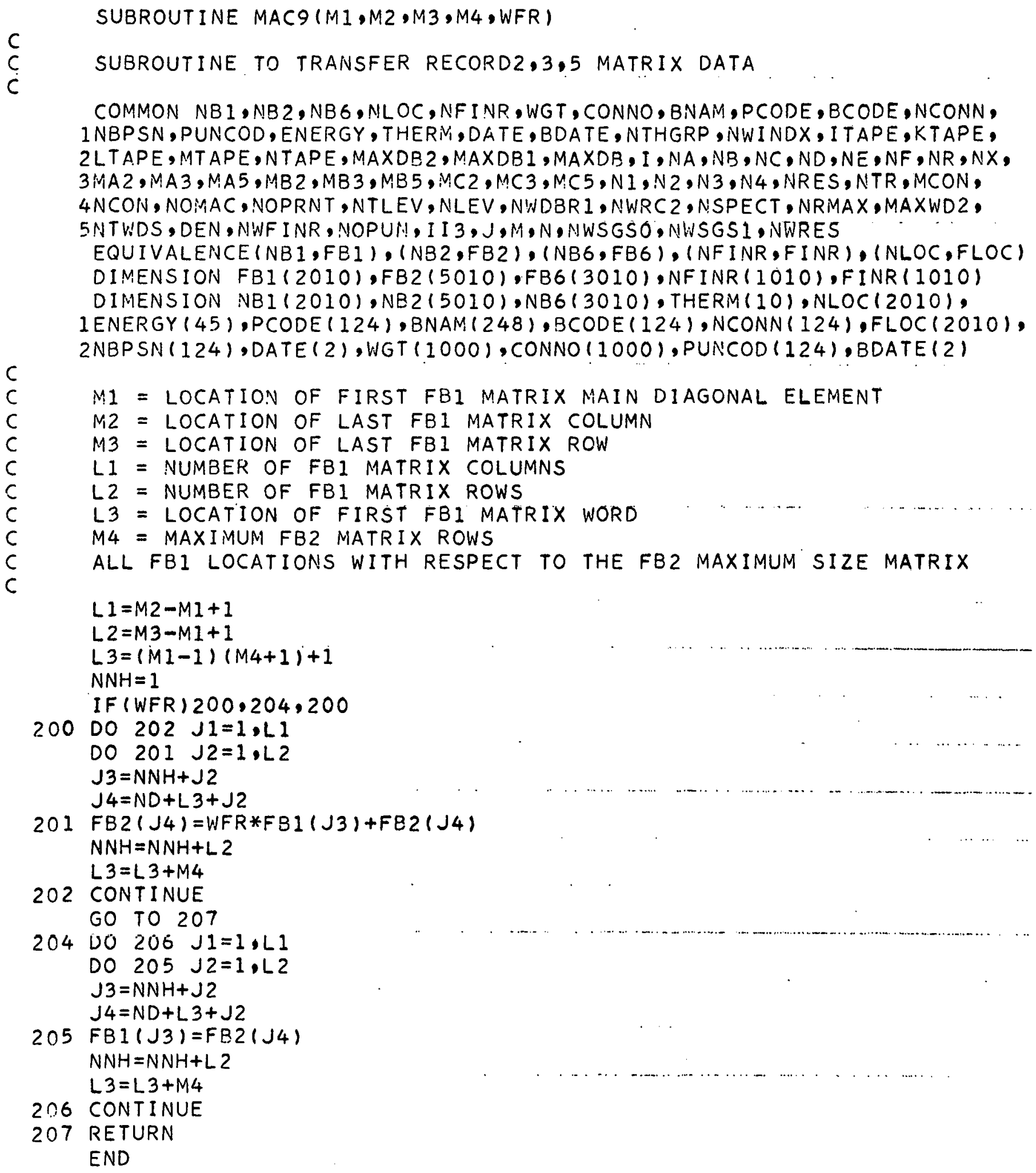


c SUBROUTINE TO TRANSFER RECORDS 2 AND 3 MATRIX DATA

COMMON NB 1,NB2, NB6, NLOC, NFINR, WGT, CONNO, BNAM, PCODE, BCODE, NCONN, INBP SN, PUNCOD, ENERGY, THERM, DATE, BDATE , NTHGRP, NWI NDX, ITAPE, KTA PE, 2LT APE , MT APE, NTAPE, MAXDB 2, MAXDB I, MAXDB, I , NA, NB, NC, ND, NE, NF, NR , NX, $3 M A 2, M A 3, M A 5, M B 2, M B 3, M B 5, M C 2, M C 3, M C 5, N 1, N 2, N 3, N 4, N R E S, N T R, M C O N$, $4 N C O N, N O M A C, N O P R N T, N T L E V, N L E V, N W D B R 1, N W R C 2, N S P E C T, N R M A X, M A X W D 2$, $5 N T W D S, D E N, N W F$ INR, NOPUN, I I $3, J, M, N, N W S G S O, N W S G S I$, NWRES EQUIVALENCE (NB1, FB1), (NB2, FB2), (NB6,FB6), (NFINR, FINR), (NLOC,FLOC)

DIMENSION FBI(2010), FB2 (5010), FB6(3010), NFINR(1010), FINR(1010) DIMENSION NBI (2010), NB2 (5010), NB6(3010), THERM(10), NLOC (2010), IENERGY (45), PCODE (124), BNAM(248), BCODE (124), NCONN(124), FLOC(2010), 2NBPSN (124), DATE (2), WGT (1000), CONNO (1000), PUNCOD (124), BDATE (2)

$\bar{C}$ $L 1=M 2-M 1+1$

$L 2=M 3-M 1+1$

$13=(M 1-1)(M 4+1)+1+N T * M A X W D 2$

$\mathrm{NNH}=1$

DO $212 \quad J 1=1, L 1$

DO 211 J2 $=1, L 2$

$\mathrm{J} 3=\mathrm{NNH}+\mathrm{J} 2$

$J 4=L 3+J 2$

$211 F L O C\left(J_{4}\right)=F B 1\left(J_{3}\right)$

$\mathrm{NNH}=\mathrm{NNH}+\mathrm{L} 2$

$\mathrm{L} 3=\mathrm{L} 3+\mathrm{M} 4$

212 CONTINUE

RETURN

END 


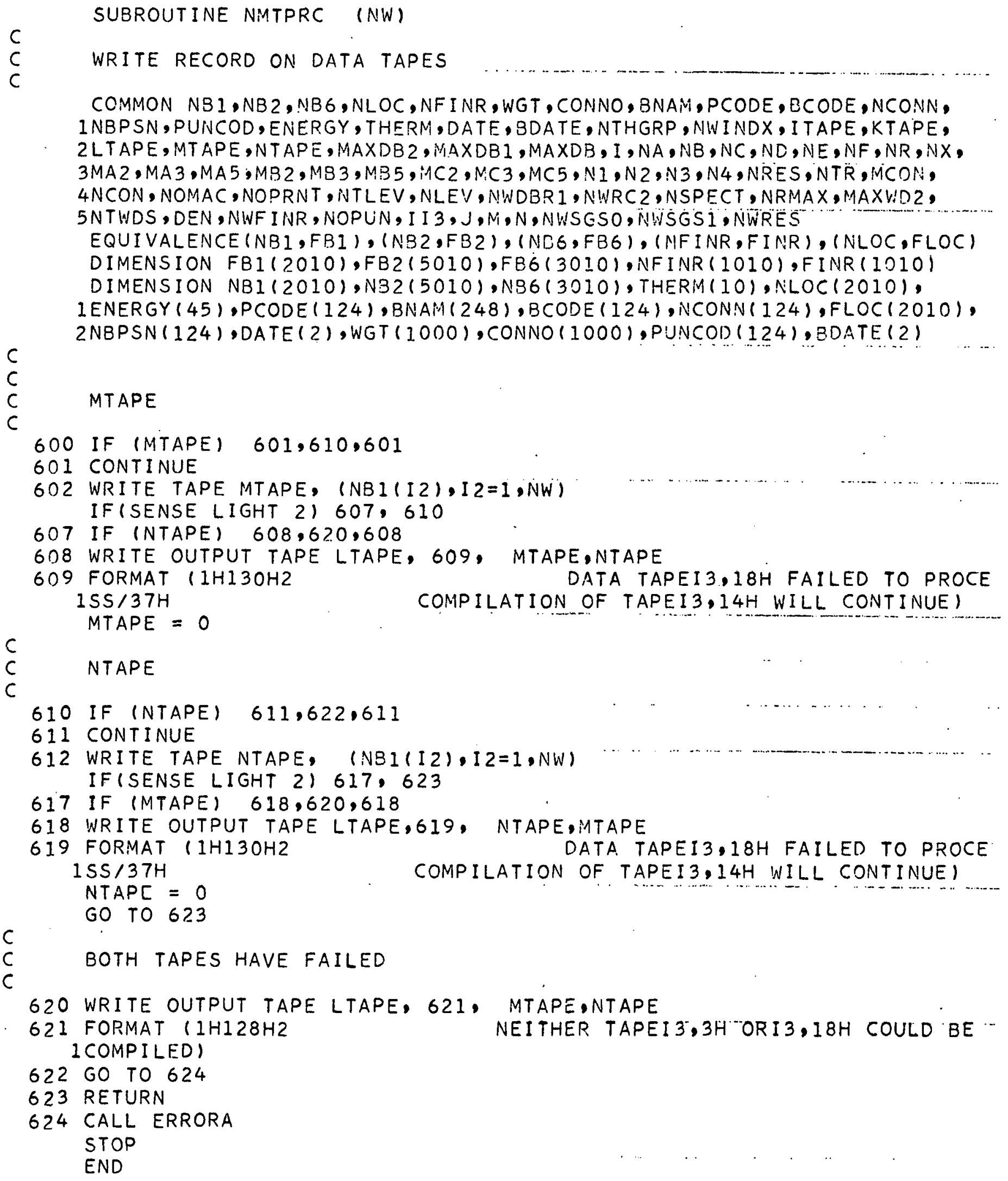




\section{SUBROUTINE TRNSDB(ITAPI, PNCODE)}

TRANSFER DATA BLOCK FROM INPUT OR INTERMEDIATE TAPE TO DATA TAPES

COMMON NB1, NB2, NB6, NLOC, NFINR, IVGT, CONNO, BNAIY, PCODE, BCODE,NCONN, INBPSN, PUNCOD, ENERGY, THERM, DATE, BDATE, NTHGRP, NHINDX, I TAPE , KTAPE, 2LTAPE, MTAPE, NTAPE, MAXDB $2, M A X D B 1, M A X D B, I, N A, N B, N C, N D, N E, N F, N R, N X$, $3 M A 2, M A 3, M A 5, M B 2, M B 3, M B 5, M C 2, M C 3, M C 5, N 1, N 2, N 3$, N4, NRES, NTR,MCON, $4 N C O N, N O M A C, N O P R N T$, NTLEV, NLEV , N!VDBR 1 , N!WRC 2 , NISPECT, NRMAX, MAXWD2, $5 N T W D S, D E N, N W F$ INR, NOPUN, I I $3, J, M, N$, NWSGSO, NWSGSI, NWRES

EQUIVALENCE (NB1, F91), $(N B 2, F B 2),(N B 6, F B 6),(N F I N R, F I N R),(N L O C, F L O C)$

DIMENSION FBI $(2010)$, FB2 $(5010)$, FB6 $(3010)$, NFINR(1010), FINR(1010)

DIMENSION NBI $(2010)$, NB2 $(5010)$, NB6(3010), THERII(10), NLOC $(2010)$, IENERGY (45), PCODE (124), BNAM(248), BCODE(124), NCONN(124), FLOC(2010), 2 NBPSN (124), DATE (2),WGT $(1000)$, CONNO (1000), PUNCOD (124), BDATE(2)

$C$

C TEST FOR SPECTRAL DATA

C SET UP RECORD NUMBER

$c$

C

C

660 IF (FB6 (4*I+3) 500.0$) \quad 664,661,66 I$

WHEN CALLING MACB (NT, NW, ITAPI) SET NT=0 FOR READING TAPE ITAPI

661 REAO TAPE ITAPI, (NBI (JI),JI=1,NSPECT)

CALL MAC8 (O,NSPECT, ITAPI)

NBI $(1)=N B 6(4 * I \quad 4)$

CALL NMTPRC(NSPECT)

662 CALL WRSPDB $(0)$

663 GO TO 679

664 READ TAPE ITAPI, (NB I (JI),JI=1,NWDBR I)

CALL MAC8 (O,NWDER 1, ITAPI)

C

$N B 1(1)=N B 6(4 * I 4)$

C

C

CROSS-SECTION WRITE M,N TAPES, PRINT IF DESIRED

CALL NMTPRC(NWDBR1)

665 CALL MAC5

666 CALL WRRECI (O,PTCODE,PNCODE) DO SAME FOR REMAINING RECORDS OF DATA BLOCK AFTER READING THESE IN

$667 \mathrm{~J} 2=0$

IF (NI) $668,670,668$

$668 \mathrm{~J} 2=\mathrm{J} 2+1$

READ TAPE ITAPI, $(N B 1(J 1), J 1=1, N 1)$

CALL MAC8 $(0, N 1$, ITAPI)

$N B 1(1)=N B 6(4 * I+4)+J 2$

CALL NMTPRC (NI)

669 CALL WRREC2 (PTCODE,PNCODE)

670 IF (N2) $671,673,671$

$671 \mathrm{~J} 2=\mathrm{J} 2+1$

READ TAPE ITAPI, $(N B I(J I), J 1=1, N 2)$

CALL MACB $(0, N 2$, ITAPI)

$N B I(1)=N B S(4 * I+4)+J 2$

CALL NMTPRC (N2)

672 CALL WRREC3 (PTCODE, PNCODE)

673 IF (N3) $674,676,674$

$674 \mathrm{~J} 2=\mathrm{J} 2+1$ 
READ IAPE ITAPI, (NBI $(J 1), J 1=1, N 3)$

CALL MAC8 $(0 . N 3$. ITAPI)

$N B I(I)=N B 6(4 * I+4)+J 2$

CALL NMTPRC (N3)

675 CALL WRREC4 (PTCODE,PNCODE)

676 IF (N4) $677,679,677$

$677 \mathrm{~J} 2=\mathrm{J} 2+1$

READ TAPE ITAPI, (NBIIJI),JI=I,N4)

CALL MAC8 $(0, N 4,1 T A P I)$

$N B I(1)=N B 6(4 * I+4)+J 2$

CALL NMTPRC (N4)

678 CALL WRREC5 (PTCODE,PNCODE)

679 IF (PNCODE) $680,681,680$

680 CALL PUNACB $(0,0,0)$

681 RETURN

END 


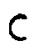

\section{SUBROUTINE WRINDX}

C WRITE INDEX RECORD ON OUTPUT TAPE

COMMON NB 1, NB $2, N B 6, N L O C, N F I N R, W G T$, CONNO, BNAN, PCODE, BCODE, NCONN, INBPSN, PUNCOD, ENERGY, THERM, DATE, EDATE, NTHGRP, NWINDX, I TAPE, KTAPE, 2LTAPE, MTAPE, NTAPE, MAXDB2, MAXDB1, MAXDB, I, NA, NB, NC, ND, NE, NF, NR, NX, $3 M A 2, M A 3, M A 5, M B 2, M B 3, M B 5, M C 2, M C 3, M C 5, N 1, N 2, N 3, N 4, N R E S, N T R, M C O N$, $4 N C O N, N O M A C, N O P R N T, N T L E V, N L E V, N W D B R 1$, NWRC $2, N S P E C T, N R M A X, N A X W D 2$, $5 N T W D S, D E N, N W F$ INR, NOPUN, I I $3, J, M, N, N W S G S O, N W S G S I, N I W R E S$

EQUIVALENCE (NB1, FBI), (NB2,FB2), (NB6, FB6), (NFINR, FINR), (NLOC,FLOC) DIMENSION FBI $(2010)$, FB2 $(5010)$, FB6 $(3010)$, NFINR(1010), FINR(1010) DIMENSION NB1 (2010), NB2 $(5010)$, NB6 (3010), THERM(10), NLOC $(2010)$, 1ENERGY (45), PCODE(124), BNAM(248), BCODE(124), NCONN(124), FLOC(2010),

700 WRITE OUTPUT TAPE.LTAPE, 701 , (DATE(I1), I1 $=1.2$ ), NLEV

701 FORMAT (1HI57X,5HDATE 2A6///30X,12HINDEX RECORD///I33,7H LEVELS/10 $1 X, 4 H D A T A / 63 H$ 2ECORD NO. //1 DO $705 \quad I=1, M A X D B I$

WRITE OUTPUT TAPE LTAPE, 702, I, NB1 $(4 * I+3)$,NBI $(4 * 1+1)$, NB1 $(4 * I+2), \cdots$ INB I $(4 * 1+4)$

702 FORMAT (I I $4, F 18,4,4 X, 2 A 6, I 12)$ IF $(I-40 *(I / 40)) 705,703,705$

703 WRITE OUTPUT TAPE LTAPE, 704 , NLEV

704 FORMAT (IHI////30X,12HINDEX RECORD///I33,7H LEVELS/10X,4HDATA/63H 1 BLOCK NO. CODE NO:

c 705 CONTINUE

PUNCH INDEX RECORD

CALL BRAND(DATE, 2 )

CALL PUNACB (NBI, NWINDX,0)

CALL PUNACB $(0,0,0)$

RETURN

END 
SUBROUTINE WRSECR

C WRITE RECORD 2 ON OUTPUT TAPE

COMMON NB1, NB2, NB6, NLOC, NFINR, WG , CONNO, BNAM, PCODE, BCODE, NCONN, 1 NBPSN, PUNCOD, ENERGY, THERM, DATE, BDATE, NTHGRP, NWINDX, I TAPE, KTAPE, 2LTAPE, MTAPE, NTAPE, MAXDB2, MAXDBI, MAXDB, I, NA, NB, NC,ND, NE, NF, NR, NX, $3 M A 2, M A 3, M A 5, M B 2, M B 3, M B 5, M C 2, M C 3, M C 5, N 1, N 2, N 3, N 4, N R E S, N T R, M C O N$, 4 NCON, NOMAC, NOPRNT, NTLEV, NLEV, NWIDBR1, NWRC2, NSPECT, NRMAX, MAXWD2,

5NTWDS, DEN, NWF INR, MOPUN, II $3, J, M, N, N W S G S O, N W S G S I, N W R E S$

EQUIVALENCE (NBI, FBI), $(N R 2, F B 2),(N B 6, F B 6),(N F I N R, F I N R),(N L O C, F L O C)$

DIMENSION FBI $(2010), F B 2(5010), F B 6(3010)$, NFINR(1010), FINR(1010)

DIMENSION NBI (2010), NB2(5010), NB6(3010), THERM(10), NLOC(2010),

IENERGY (45), PCODE (124), BNAM (248), BCODE(124), NCONN(124), FLOC (2010),

2 NBPSN (124), DATE (2),WGT $(1000)$, CONNO (1000), PUNCOD (124), BDATE(2)

CHECK FOR PRINTING SECOND RECORD

NLEVI = NLEV + 1

71000711 I $1=1$, NOPRNT

IF (PCODE (I 1)-.000002) 711,712,711

711 CONTINUE

GO TO 730

C

PRINT

712 WRITE OUTPUT TAPE LTAPE, 713

713 FORMAT $152 \mathrm{HI}$

LEVEL ENERGY (EV)

LETHARGY)

$12=N T L E V+1$

DO $718 \quad 11=1$, NLEV

$N A=11+12$

IF $((11-1)-5 *((11-1) / 5)) \quad 714,716,714$

714 WRITE OUTPUT TAPE LTAPE, 715, II,NBI(II+I),NBI(NA)-

715 FORMAT (I 19,1PE19.7,E17.7)

GO TO 718

716 WRITE OUTPUT TAPE LTAPE, 717, I1,NBI(11+1), NBI(NA)

717 FORMAT (IHOI18,1PE19:7,EI7.7)

718 CONTINUE

$I 2=N L E V I+N T L E V$

DO 723 I $1=1$, NTHGRP

$N A=N L E V 1+11$

$N B=I 1+12$

$N C=$ THERM(II)

IF $((11-1)-5 *((I 1-1) / 5)) 719,721,719$

719 WRITE OUTPUT TAPE LTAPE, 720, NC,NEI(NA), NBI(NB)

720 FORMAT (I 20,1 , PE $18,7, E 17,7$ )

GO TO 723

721 WRITE OUTPUT TAPE LTAPE, 722, NC,NBI(NA), NBI (NB)

722 FORMAT (IHOI19,IPEI8.7,E17.7)

723 CONTINUE

$12=2 * N T L E V+2$

$13=I 2+$ NLEV 1

$14=13+N L E V$

WRITE OUTPUT TAPE LTAPE, 724, NBI(12),NBI(13)

724 FORMAT $162 \mathrm{HI}$

1 FACTOR/63H

$20 \mathrm{M} / \mathrm{SEC} / / / 11 \mathrm{H}$

DO 729 II $1=1$, NLEV

LOWER

GROUP ENERGY (EV)

$0.1 P E 19.7, E 17.71$
UPPER

LETHARGY $1 / 7$ 1220 


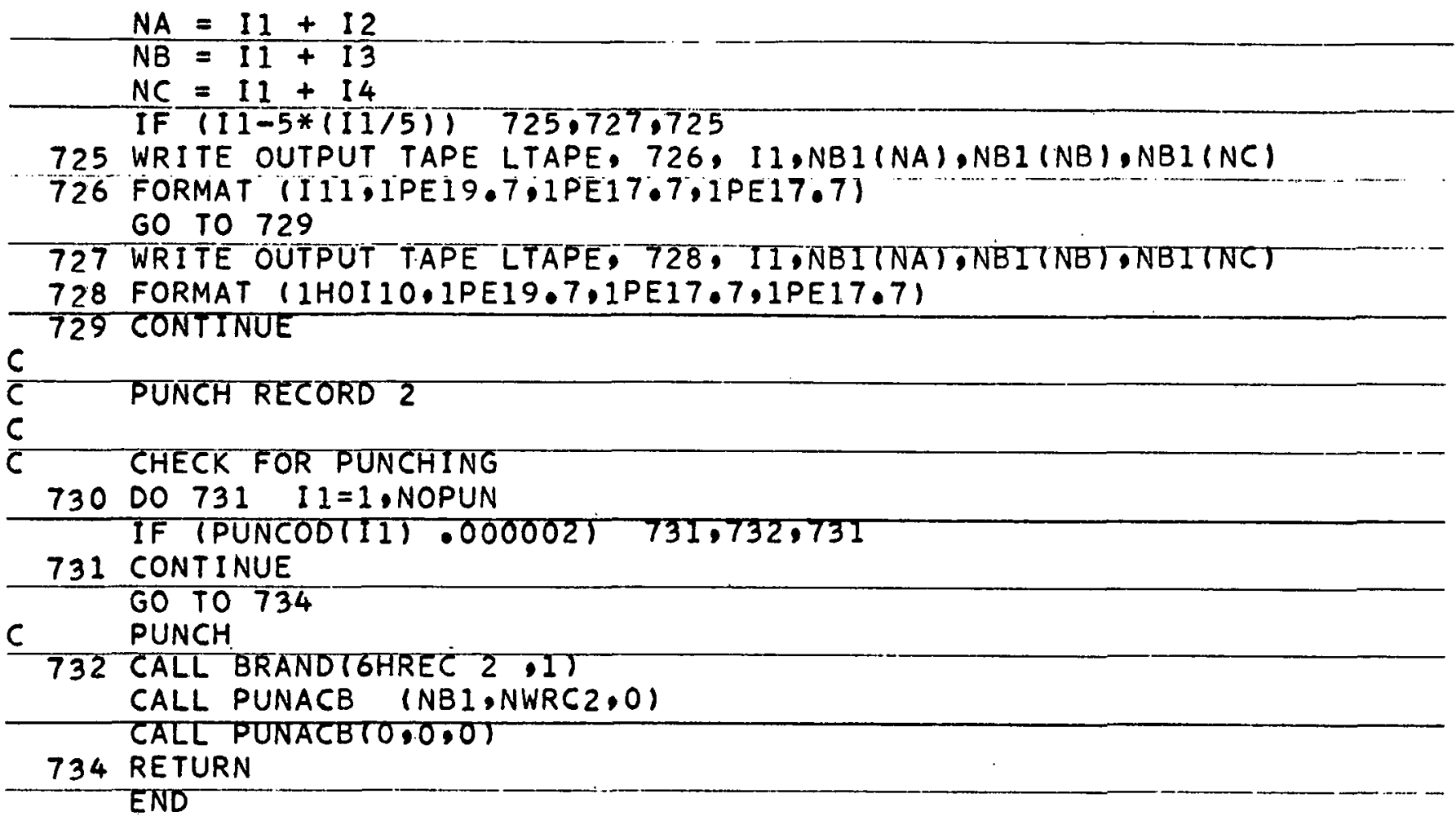




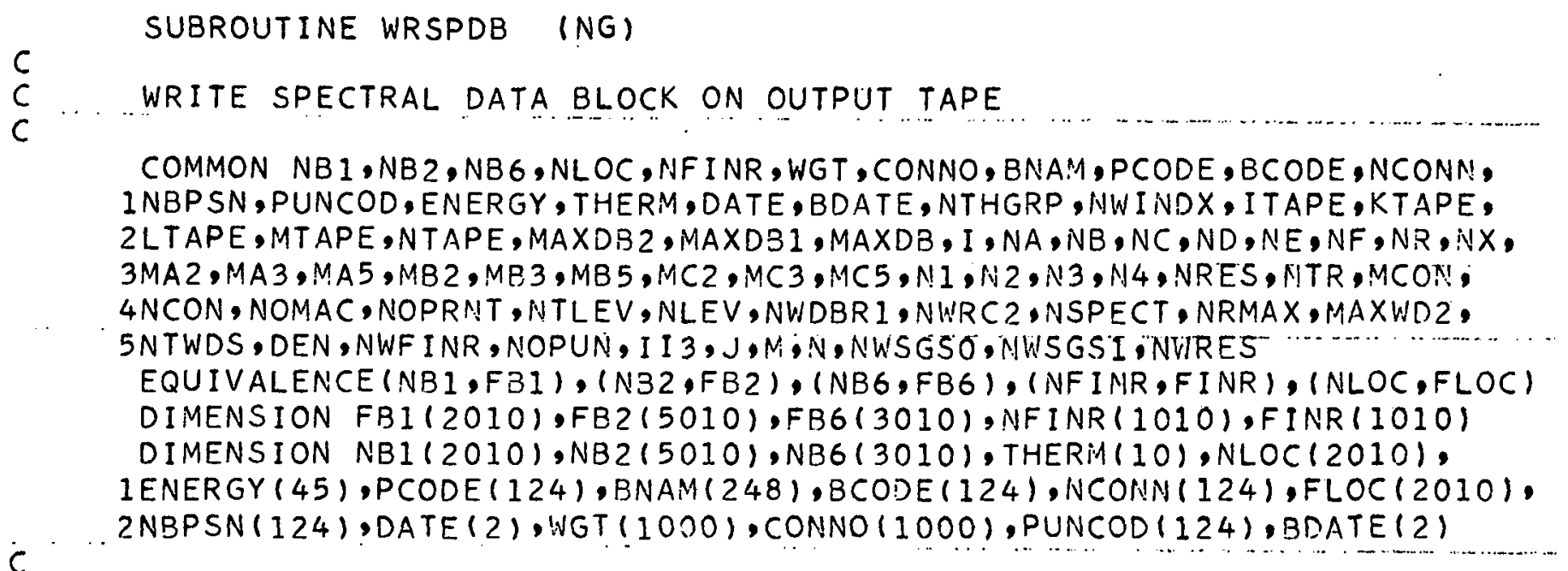

C

C

IF NG = 1, THEN THIS IS AN ADDED DATA BLOCK AND IS TO BE

1PRINTED AUTOMATICALLY, IF NG $=0$, THEN THIS BLOCK IS FROM EITHER

2ITAPE OR KTAPE AND A CHECK IS MADE FOR PRINTING.

739 IF (NG) $742,740,742$

740 DO 741 II $1=1$, NOPRNT

IF (PCODE (II)-FB6 $(4 * I+3)) \quad 741,742,741$

741 CONTINUE

C PRINT

GO TO 754

742 WRITE OUTPUT TAPE LTAPE, 743, (NBI(I 1$), 11=2,3$ ), NB6 $(4 * I+1)$, $1 N B 6(4 * I+2)$, NB 1 (NTLEV+5), NBI (NTLEV+6)

743 FORMATIIHIS6X,5HDATE 2 A $6 \% / 135 X, 25$ HDELAY 1 DESIGNATION FRACTION

$2,1 P 2 E 18.4 / / 117 X, 34 H L E V E L$ ENERGY

MEAN LIFE//13X,2A6

DO $748 \quad I 1=1$, NLEV

IF $((I 1-1)-5 *((I 1-1) / 5)) \quad 744,746,744$

744 WRITE OUTPUT TAPE LTAPE, 745, I I, ENERGY(11), NB $1(11+4)$

745 FORMAT $(120,1$ PE $16.3, E 18.7)$

GO TO 748

746 WRITE OUTPUT TAPE LTAPE, 747, 11, ENERGY(I1), NBI (1 1+4)

747 FORMAT (IHOI19,1PE16.3,E18.7)

748 CONTINUE

$12=N L E V+4$

DO 753 I $1=1$, NTHGRP

$N A=11+N L E V$

$N B=I 1+I 2$

$N C=$ THERM $(11)$

IF $((11-1)-5 *(1)-1) / 5)) \quad 749,751,749$

749 WRITE OUTPUT TAPE LTAPE, 750, NC,ENERGY (NA), NBI (NB)

750 FORMAT $(121,1$ PE15.3,E18.7) GO TO 753

751 WRITE OUTPUT TAPE LTAPE, 752, NC, ENERGY (NA), NBI (NB)

752 FORMAT (IHOI 20,1PE15.3,E18.7)

$c$

753 CONTINUE

c PUNCH SPECTRAL DATA BLOCK

$\varepsilon$

CHECK FOR PUNCHING

75400755 II $=1$, NOPUN 


\section{4}

IF (PUNCOD(II) FBG $(4 * I+3)$ ) $755,756 \cdot 755$

755 CONT INUE

GO TO 758

C PUNCH

$756 \quad M=4 * 1+1$

CALL BRAND (FB $6(M), 2)$

CALL PUNACB (NBI,NSPECT,O)

758 RETURN

END 


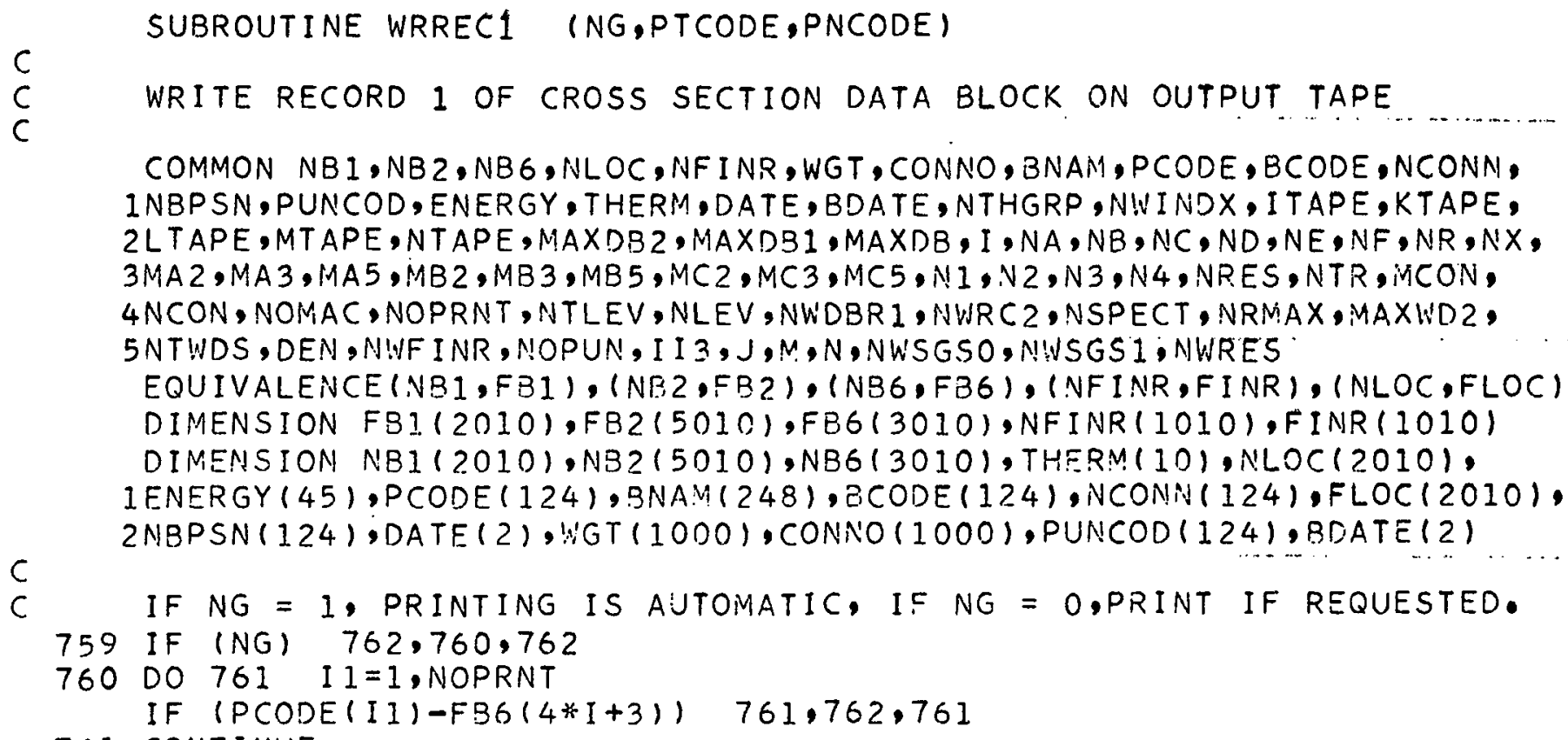

761 CONTINUE

C GO TO 808

762 WRITE OUTPUT TAPE LTAPE, 764, NBI(2), NB1(3), NB6 $(4 * I+1)$, $1 N B S(4 * I+2), N B 6(4 * I+3), N B 1(6 * N T L E V+5)$

764 FORMAT (IHI57X,5HDATE 2A6/// 9X,53HDESIGNATION CODE NO. 1 DENSITY FACTOR//9X,2A6,F17.4,1PE22.5/1/70H

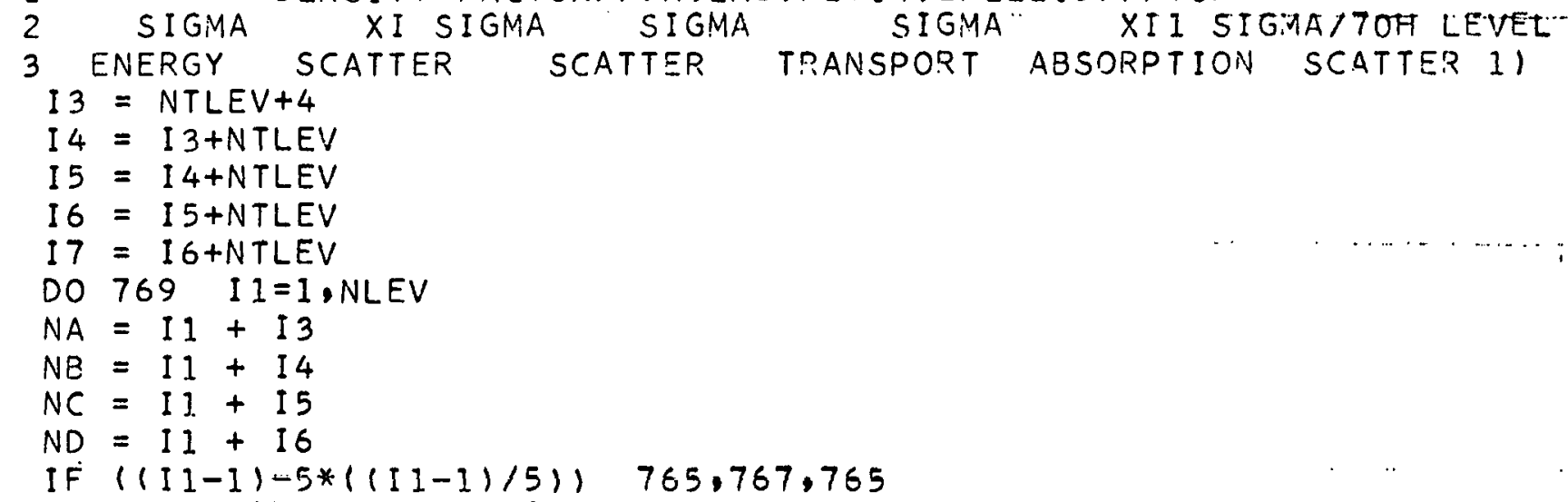

765 WRITE OUTPUT TAPE LTAPE, 766, I I, ENERGY(II), NEI(I 1+4), NBI(NA), NBI1 INB), NBI (NC),NBI (ND)

756 FORMAT (I4,IPEI1.3,4EI1.4,E12.4)

GO TO 769

767 IVITE OUTPUT TAPE LTAPE, 768, II,ENERGY(I I), NBI $11+4)$,NBI(NA), NBII INB), NBI (NC), NBI (ND)

768 FOR:MAT (1HOI3,IPE11.3,4E11.4,E12.4)

769 CONTINUE
$12=N L E V+4$
$13=13+N L E V$
$14=14+N L E V$
$I 5=15+N L E V$
$16=16+$ NLEV
$I 7=I 7+N L E V$
DO 774 II 1 , NTHGRP 


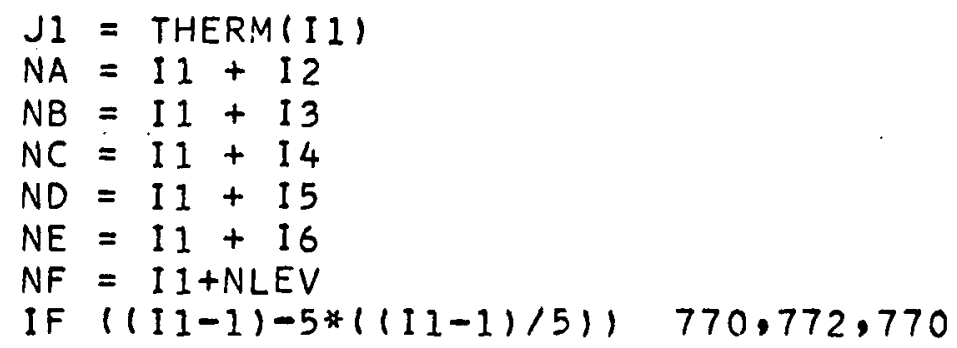

770 WRITE OUTPUT TAPE LTAPE, 771, J1, ENERGY(NF), NBI (NA), NBI (NB), NBI (NC $1)$, NBI (ND), NBI (NE)

771 FORMAT (I 5,IPE $10.3,4 E 11.4, E 12.4)$

GO TO 774

772 WRITE OUTPUT TAPE LTAPE, 773, J1, ENERGY(NF), NBI (NA), NBI (NB), NB1 (NC 1). NB1 (ND), NBI (NE)

773 FORMAT (IHOI4,1PE10.3,4E11.4,E12.4)

C

774 CONTINUE

C (PAGE 2)

C

775 WRITE OUTPUT TAPE LTAPE, 776, NB6(4*I+1),NB6(4*I+2),NB6(4*I+3), INBI (6*NTLEV+5)

776 FORMAT $163 \mathrm{HI}$ DESIGNATION CODE NO:

IY FACTOR//10X,2A6,F 17.4, IPE22.5///43X,8HNU SIGMA/21X,3OHLEVEL

2ENERGY FISSION)

$13=16+$ NTHGRP

DO 781 I $1=1$, NLEV

$N A=11+13$

IF $((11-1)-5 *(1) 1-1) / 5)\} \quad 777,779,777$

777 WRITE OUTPUT TAPE LTAPE, 778, I I, ENERGY (I 1),NBI (NA)

778 FORMAT (I24,1PE14.3,E14.4)

GO TO 781

779 WRITE OUTPUT TAPE LTAPE, 780, 11 , ENERGY (II) NBI (NA)

780 FORMAT (1HOI23,IPEI $4.3, E 14.4)$

781 "CONTINUE'

$13=13+N L E V$

DO $786 \quad 11=1$, NTHGRP

$N A=I 1+N L E V$

$N B=11+13$

$N C=$ THERM(II)

IF $((11-1)-5 *(1) 1-1) / 5)) 782 ; 784 ; 782$

782 WRITE OUTPUT TAPE LTAPE, 783, NC,ENERGY (NA), NBI (NB)

783 FORMAT $(125,1$ PE $13.3, E 14.4)$ GO TO 786

784 WRITE OUTPUT TAPE LTAPE, 785, NC, ENERGY (NA), NBI (NB)

785 FORMAT (IHOI24,1PE13.3.E14.4)

c

786 CONTINUE

$c$

$788 I 2=6 * N T L E V+6$

DO $793 J=1, N T H G R P$

IF $((J-1)-2 *((J-1) / 2)) \quad 791,789,791$

789 WRITE OUTPUT TAPE LTAPE, 790, NB6 $(4 * I+1), N B 6(4 * I+2)$

790 FORMAT (1H129X,2A6///23X,25HTHERMAL TRANSFER MATRICES)

$19113=J+N L E V$ 
WRITE OUTPUT TAPE LTAPE, 792, NB1(I2),NB1(I 21$)$, NBI(I 22$), N B 1(I 23$ 1) N NBI ( I $2+4), I 3, N B I(I 2+5), N B I(I 2+6), N B I(I 2+7), N B I(I 2+8), N B I(I 2+9)$, 213 , NBI $(12+10)$, NBI $(I 2+11)$, NBI $(12+12)$, NBI $(12+13)$, NBI $(12+14), I 3$, $3 N E 1(12+15), N B 1(I 2+16)$, NBI (I 2+17), NBI(I 2+18), NBITI $2+19) \cdot 13$ $4 N B I(12+20), N B I(I 2+21)$, NBI $(12+22), N B I(I 2+23), N B I(12+24)$, $513,13,13,13,13,13, N E 1(I 225), N E I(I 2+26), N B I(12+27), N B 1(I 2+28)$, $6 N B 1(12+29)$

792 FORMAT $1 / 1 / 1 / 68 \mathrm{H}$ TEMPERATURE SIGMA SIGMA NU SIGMA/67H

DEG. F. SCATTER

TRANSPORT SI 2 ABSORPTION

3SIGMA S (NI/N2) $/ 163 \mathrm{H}$ 2121 $21 \quad 22$ N27Z8X,I5H

$\begin{array}{lll}421 & 24\end{array}$ $21 \mathrm{I} 4 / 1 \mathrm{El} 3.4,4 \mathrm{E} 14.4 / 63 \mathrm{H} \quad 22 \quad 21$ $21 \quad 23$

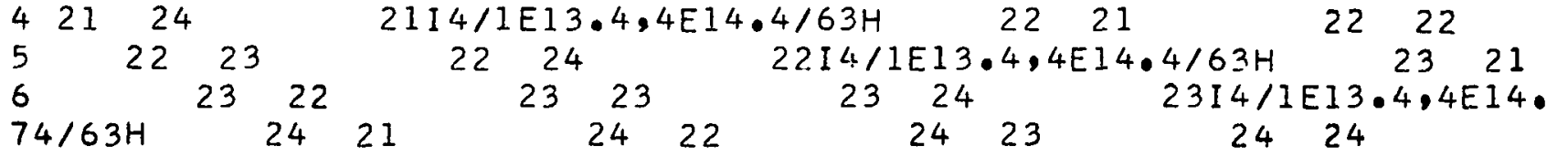
$82414 / 1 \mathrm{E} 13.4,4 \mathrm{E} 14.4 / 17,4 \mathrm{H} \quad 21110,4 \mathrm{H} \quad 22110,4 \mathrm{H} \quad 23110,4 \mathrm{H}$ 24II0,1471.. $9 E 13.4,4 E 14.41$

$79312=12+30$

GO TO 794

C

(PAGE 9 )

794 WRITE OUTPUT TAPE LTAPE, 795, NB6(4*I+1),NB6(4*I+2)

795 FORMAT (1H130X,2A6/////////)

IF (NI) $796,798,796$

796 WRITE OUTPUT TAPE LTAPE, 797,MA2, MB2,MA2,MC2

797 FORMAT $132 \mathrm{X}, 8 \mathrm{HSIGMA}$ SO//27H TRANSFERS FROM GROUPSI3,8H THROUG

$1 H I 3,10 H$ TO GROUPSI $3,8 H$ THROUGHI3/\%//)

798 IF (N2) 799,801,799

799 WRITE OUTPUT TAPE LTAPE, 800, MA3,MB3,MA3,MC3,

$8 \cap 0$ FORMAT $(32 \mathrm{X}, 8 \mathrm{HSIGMA} 51 / / 27 \mathrm{H}$

TRANSFERS FROM GROUPSI $3,8 \mathrm{H}$ THROUG

IHI $3,10 \mathrm{H}$ TO GROUPS I $3,8 \mathrm{H}$ THROUGH I $3 / / 1 / 1)$

801 IF (N3) $802,804,802$

802 WRITE OUTPUT TAPE LTAPE, 803 , NRES

803 FORMAT $(29 \mathrm{X}, 14 \mathrm{HNO}$. RESONANCES//I37////)

804 IF (N4) $805,807,805$

805 WRITE OUTPUT TAPE LTAPE, 806, MA5, MB 5,MA5,MC5

806 FORMAT $(28 X, 15 H S I G M A \quad(N, G A M M A) / / 27 H$

IH THROUGHI3,10H TO GROUPSI3,8H THROUGHI3)

C

C

c

c

C

8085 IF (PNCODE) $811,809,811$

809 DO $810 \quad 11=1$, NOPUN

IF (PUNCOD(II) FB6 $(4 * 1+3)$ ) $810,811,810$

810 CONTINUE

TRANSFERS FROM GROUPSI 3,8

SET PTCODE $=1,0$ AS THIS RECORD IS OR IS NOT PRINTED FOR REFERENCE IN OTHER RECORDS OF THIS DATA BLOCK

807 PTCODE $=1.0$

GO TO 8085

808 PTCODE $=0.0$

PUNCH RECORD 1 OF CROSS SECTION DATA BLOCK

CHECK FOR PUNCHING 
-_ GO TO 813

C PUNCH

- 811 $M=4 * I+1$

CALL BRAND(FB6(M).2)

CALL PUNACB (NBI, NWDBRI,0)

C

C SET PNCODE $=1,0$ AS THIS RECORD IS OR IS NOT PUNCHED FOR REFERENCE

C IN OTHER RECORDS OF THIS DATA BLOCK

P

GO TO 814

813 PNCODE $=0.0$

814 RETURN

END 


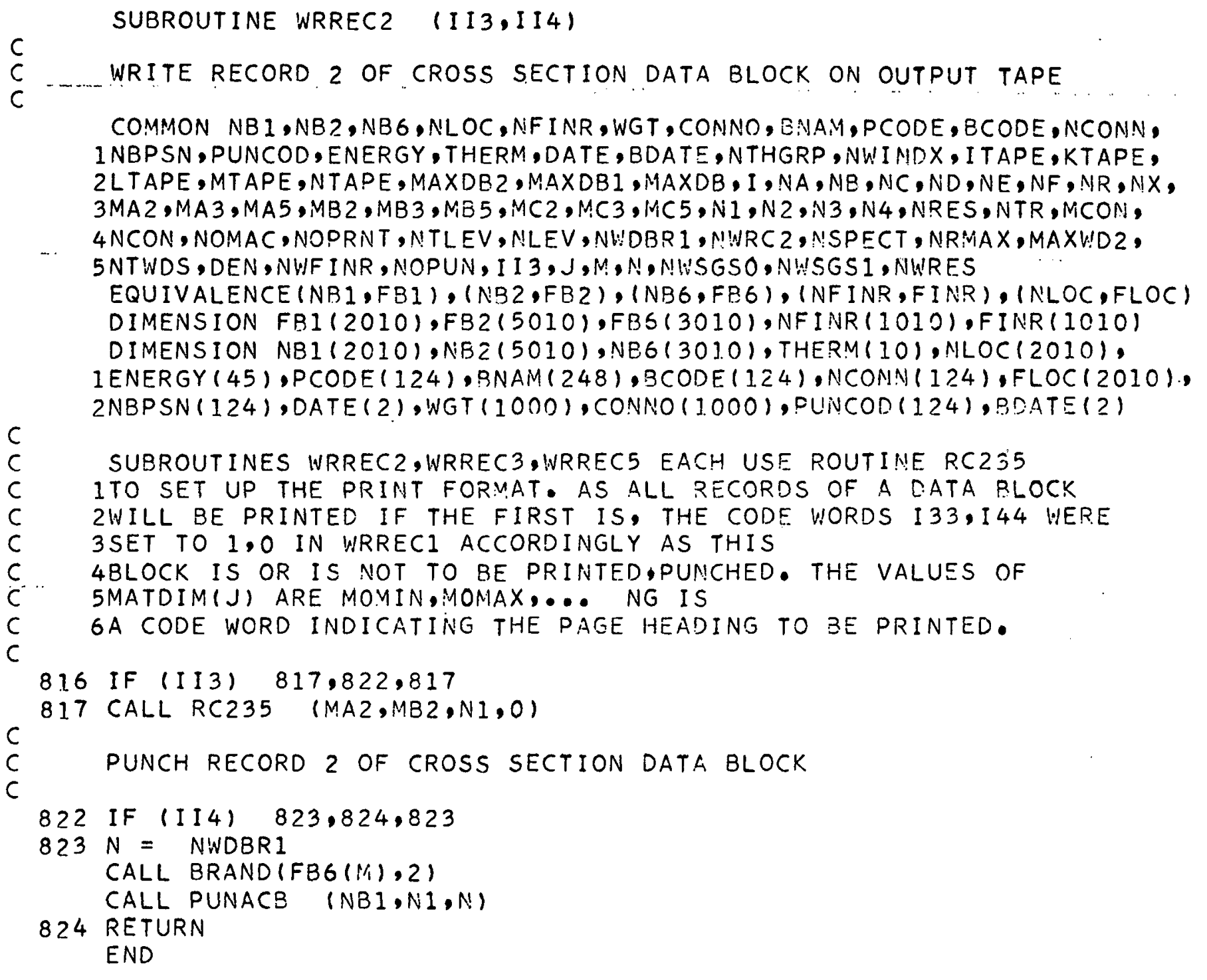


190

SUBROUTINE WRREC3 (II3,II4)

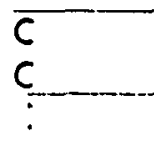

WRITE RECORD 3 OF CROSS SECTION DATA BLOCK ON OUTPUT TAPE

COMMON NB I, NB 2, NB6, NLOC, NF INR, WG T, CONNO, BNAM, PCODE, BCODE, NCONN, 1 INBP SN, PUNCOD, ENERGY, THERM, DATE, BDATE, NTHGRP, NWINDX, ITAPE, KTAPE, $2 L T A P E, M T A P E, N T A P E, M A X D B 2, M A X D B 1, M A X D B, I, N A, N B, N C, N D, N E, N F, N R, N X$, $3 M A 2, M A 3, M A 5, M B 2, M B 3, M B 5, M C 2, M C 3, M C 5, N 1, N 2, N 3, N 4, N R E S, N T R, M C O N$, $4 N C O N, N O M A C$, NOPRNT, NTLEV, NLEV, NWDBR 1, NWRC 2, NSPECT , NRMAX , MAXWD2, 5 NTWDS, DEN, NWF INR, NOPUN, II $3, J, M, N, N W S G S O, N W S G S I, N W R E S$ EQUIVALENCE (NBI, FB1), (NB2,FB2), (NB6, FB6), (NFINR, FINR), (NLOC, FLOC) DIMENSION FBI $(2010)$, FB2(5010), FB6(3010) INFINRI1010) , FINR (IOIO) DIMENSION NBI $(2010)$, NB2 $(5010)$,NB6(3010), THERM(10), NLOC $(2010)$, 1ENERGY (45), PCODE (124), BNAM (248), BCODE (124), NCONN $(124)$, FLOC (2010), 2NBPSN (124), DATE (2), WGT (1000), CONNO (1000), PUNCOD (124), BDATE (2)

c

c SEE COMMENTS IN WRREC2

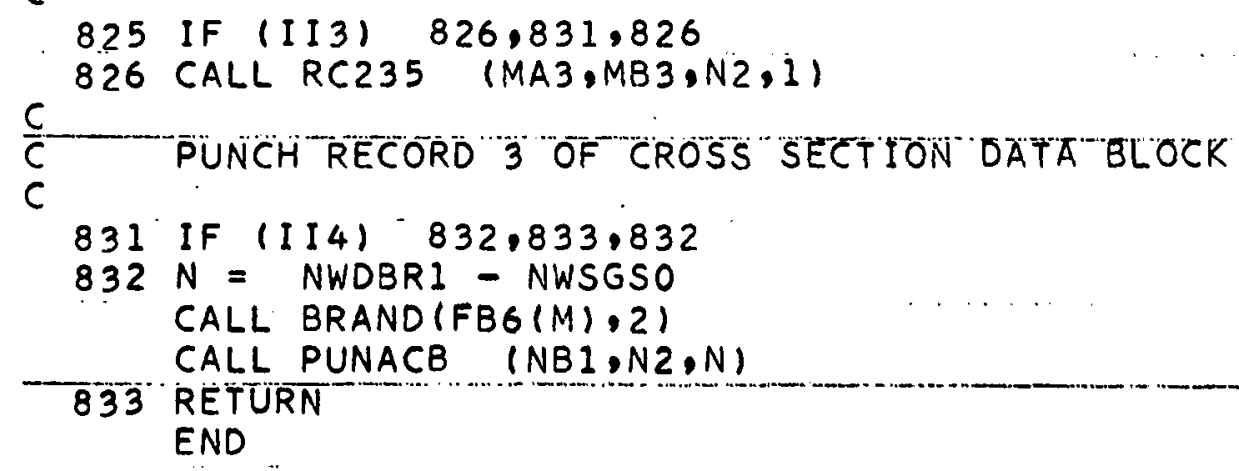


C

$C$

C

834 IF (II 3 ) $835,845,835$

835 WRITE OUTPUT TAPE LTAPE

836 FORMAT (1HI3OX,2A6///38H

1

$2 / 1$

ENERGY

$12=$ NRES +1

$13=N R E S+12$

$14=N R E S+13$

$I 5=N R E S+I 4$

DO $84411=1$, NRES

$N A=I 1+12$

$N B=11+13$

$N C=11+14$

$N D=11+15$

IF $(I 1-1) \quad 840,840,837$

837 IF $((I 1-1)-40 *((I 1-1) / 40)) 839,838,839$

838 WRITE OUTPUT TAPE LTAPE, 836, (NB6 $(4 * I+1), N B 6(4 * I+2))$

839 IF $((I 1-1)-5 *((I I-1) / 5)) \quad 840,842,840$

840 WRITE OUTPUT TAPE LTAPE,841, NBI(I1+1), NBI (NA), NBI(NB), NBI (NC), NBI

1 (ND)

841 FORMAT (IPE13.4,4E14.4)

GO TO 844

842 WRITE OUTPUT TAPE LTAPE, 843, NB1(I 1+1), NBI (NA), NBI (NB),NBI (NC), NBI 1 (ND)

843 FORMAT (1HOIPE12.4,4E14.4)

C

844 CONTINUE

C

C

\section{If (II 4 ) $\quad 846,847,846$}

$846 \mathrm{~N}=$ NWDBRI - NWSGSO - NWSGS 1

CALL BRAND(FB6(M),2)

CALL PUNACB (NBI,N3,N)

847 RETURN

END
36, (NB6 $(4 * I+1), N B 6(4 * I+2))$

RESONANCE RESONANCE

PEAK/65H

ETAY- 
C..... SUBROUTINE WRREC5 (II 3, I I 4 )

C.. WRITE RECORD 5 OF CROSS SECTION DATA BLOCK ON OUTPUT TAPE

COMMON NB 1, NB 2, NB6, NLOC, NF INR, WGT, CONNO, BNAM, PCODE, BCODE, NCONN, INBPSN, PUNCOD, ENERGY, THERM, DATE, BDATE, NTHGRP, NWINDX, I TAPE, KTAPE, 2LTAPE, MTAPE, NTAPE, MAXDB2, MAXDBI, MAXDB, I, NA, NB, NC,ND, NE, NF, NR , NX, $3 M A 2, M A 3, M A 5, M B 2, M B 3, M 35, M C 2, M C 3, M C 5, N 1, N 2, N 3, N 4, N R E S, N T R, M C O N$, $4 N C O N, N O M A C, N O P R N T$, NTLEV, NLEV, NWDBR 1 , NWRC2, NSPECT, NRMAX, MAXWD2,

$5 N T W D S, D E N$, NWF INR, NOPUN, II $3, \mathrm{~J}, M, N, N W S G S O, N W S G S 1$, NWRES EQUIVALENCE (NB1, FB1), (NB2, FB2), (NB6,FB6), (NFINR, FINR), (NLOC,FLOC) DIMENSION FBI (2010), FB2 (5010), FB6(3010), NFINR(1010), FINR(1010) DIMENSION NB1 (2010), NB2 (5010), NB6(3010), THERM(10), NLOC $(2010)$, IENERGY (45), PCODE (124), BNAM (248), BCODE (124), NCONN( 124$)$, FLOC $(2010)$, 2 NBPSN $(124)$, DATE $(2)$,WGT $(1000)$, CONNO $(1000)$, PUNCOD $(124)$, BDATE $(2)$

c

c SEE COMMENTS IN WRREC2

848 IF (II3) $849,854,849$

849 CALL RC235 (MA5,MB5,N4,2)

C PUNCH RECORD 5 OF CROSS SECTION DATA BLOCK

$c$

854 IF (II4) $855,8552,855$

$855 \mathrm{~N}=$ NWDBR 1 - NWSGSO - NWSGS1 - NWRES

CALL BRAND (FB6 $(M), 2)$

CALL PUNACB (NBI,N4,N)

8552 RETURN END 


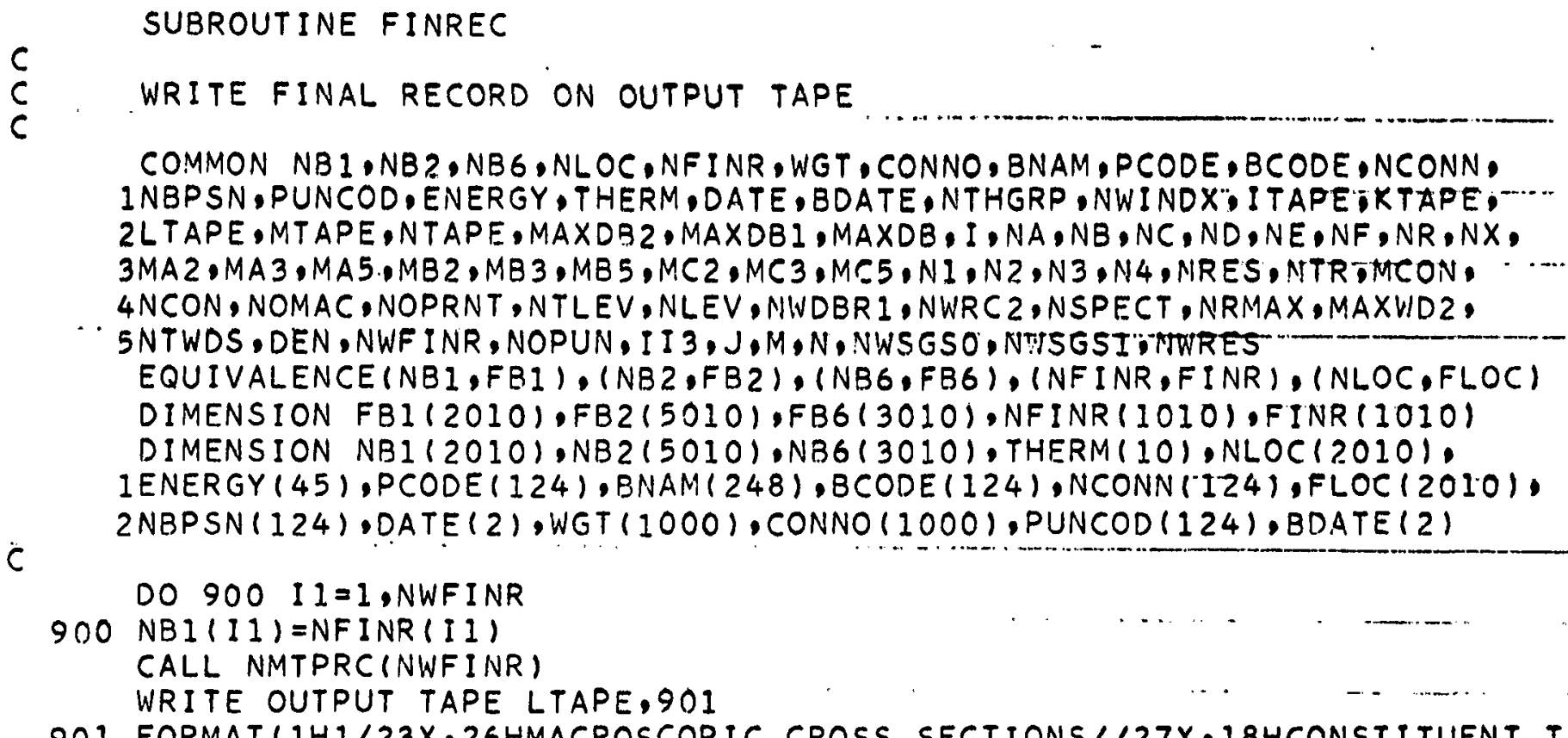

901 FORMAT (1H1/23X,26HMACROSCOPIC CROSS SECTIONS//27X,18HCONSTITUENT T

1 ABLES $/ / / / 1$

$N E=2$

$N C=0$

902 IF (NBI (NE) 1903,916,903

$903 N A=N B 1(N E+1)$

IF $(40-N C) 904,904,906$

904 WRITE OUTPUT TAPE LTAPE,905,NBIINET

905 FORMAT (1HI///26X,2OHMACROSCOPIC CODE NO./F $40.4 / / 1$

$N C=0$

GO TO 908

906 WRITE OUTPUT TAPE LTAPE,907, NBI (NE)

907 FORMAT (IHO/26X,2OHMACROSCOPIC CODE NO./F40.4//)

$908 N C=N C+B$

WR ITE OUTPUT .TAPE LTAPE 909

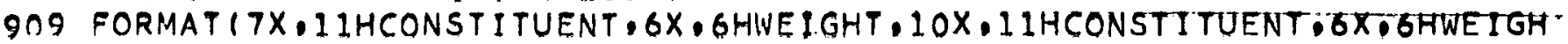

IT./8X,9HCODE NO.,6X,8HFRACTION,10X.9HCODE NO..6X,8HFRACTION//I

ND $(N A+1) / 2$

DO 915 II:1 ND

$J I=N E+4 * 11-2$

$N C=N C+1$

IF $(48-N C) 9091,9096,9096$

9091 WRITE OUTPUT TAPE LTAPE.9092

9092 FORMAT (1H1/////7X.11HCONSTITUENT .6X.6HWEIGHT.10X,IIHCONSTITUENT, 6X 1.6HWEIGHT/8X.9HCODE NO.,6X,8HFRACTION,10X.9HCODE NO..6X,8HFRACTI $20 N / 11$

$N C=3$

9096 IF(NA-2*(NA/2)) $910.913,910$

910 IF (NA-2HI1)911.911.913

911 WRITE OUTPUT TAPE LTAPE,912,NB1(J1),NB1(J1+1)

912 FORMAT (F18.4,IPE14.4) GO TO 915

913 WRITE OUTPUT TAPE LTAPE, 914 .NBI (J1),NBI(J1+1),NBI $(J 1+2) \cdot N B 1(J 1+3)$

914 FORMATIF18.4,1PE14.4,OPF19.4,1PE14.41

915 CONTINUE 


\section{4}

$N E=N E+2 * N A+2$

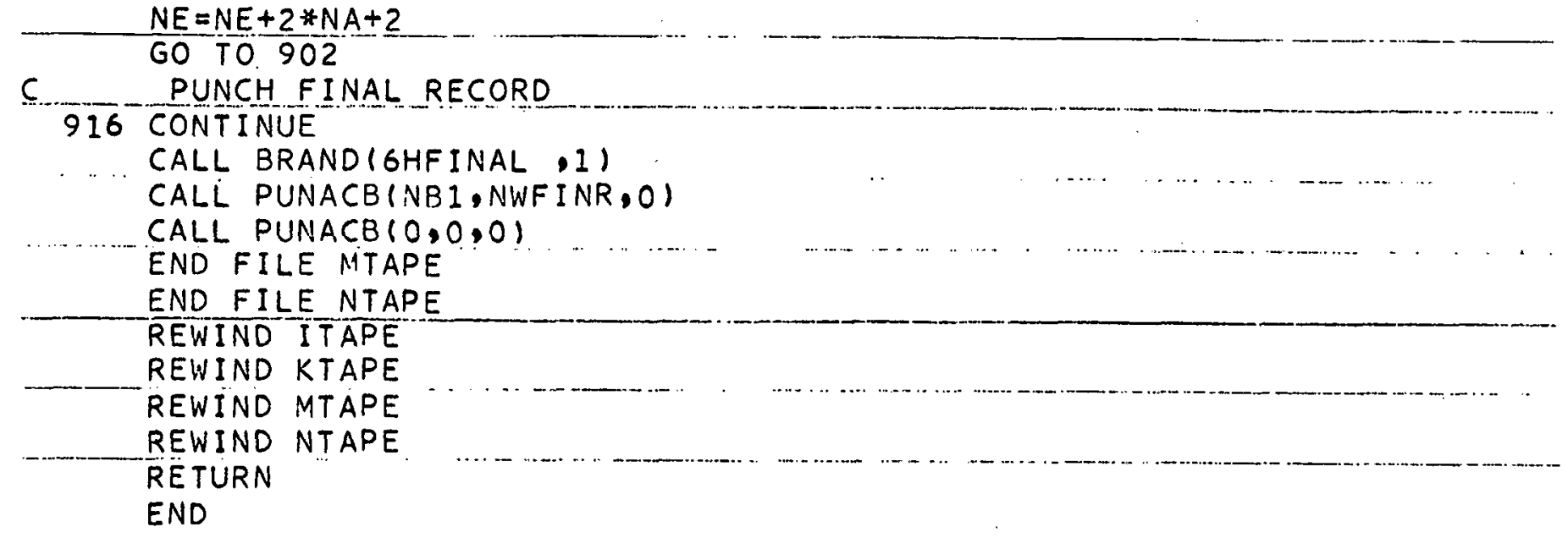




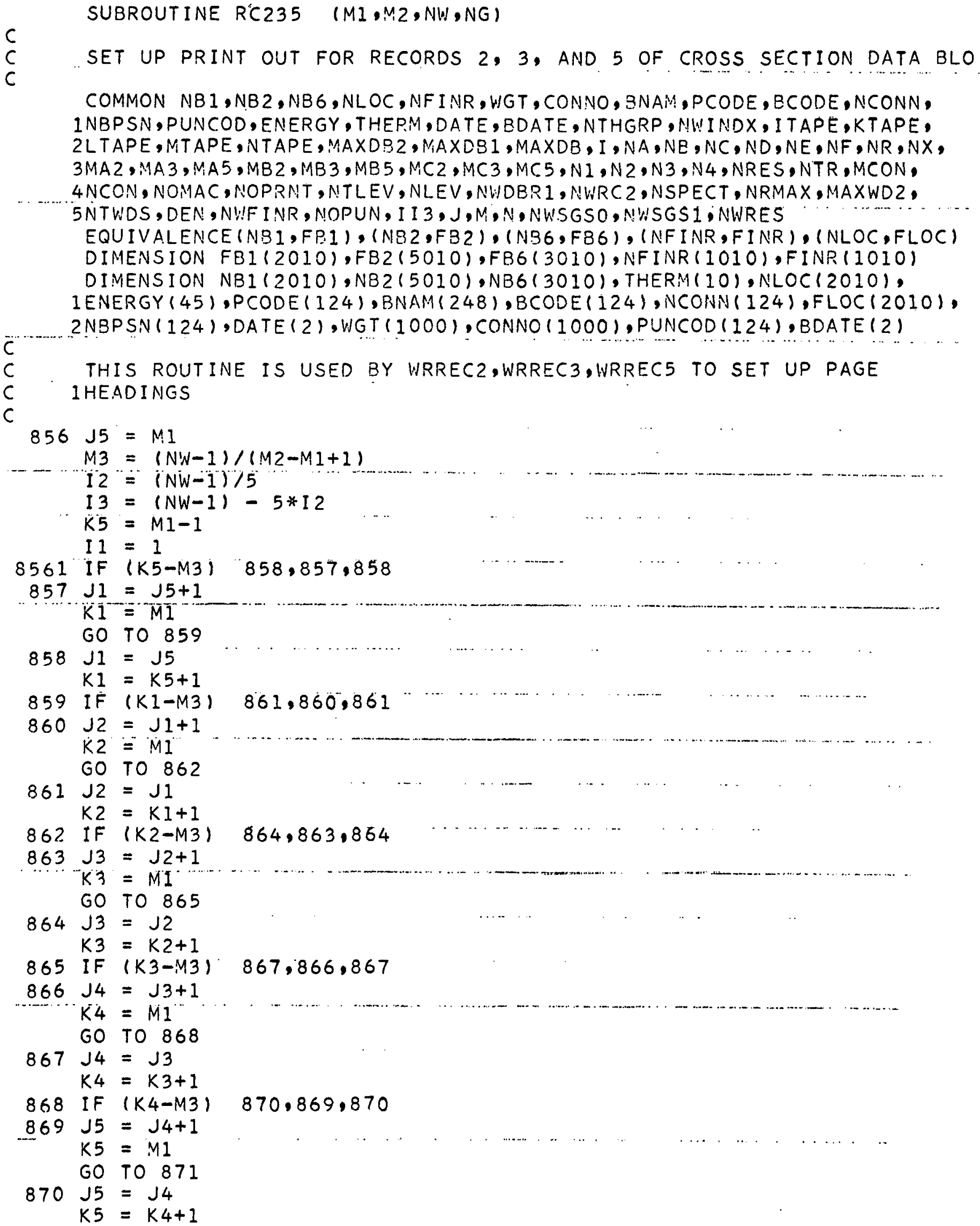




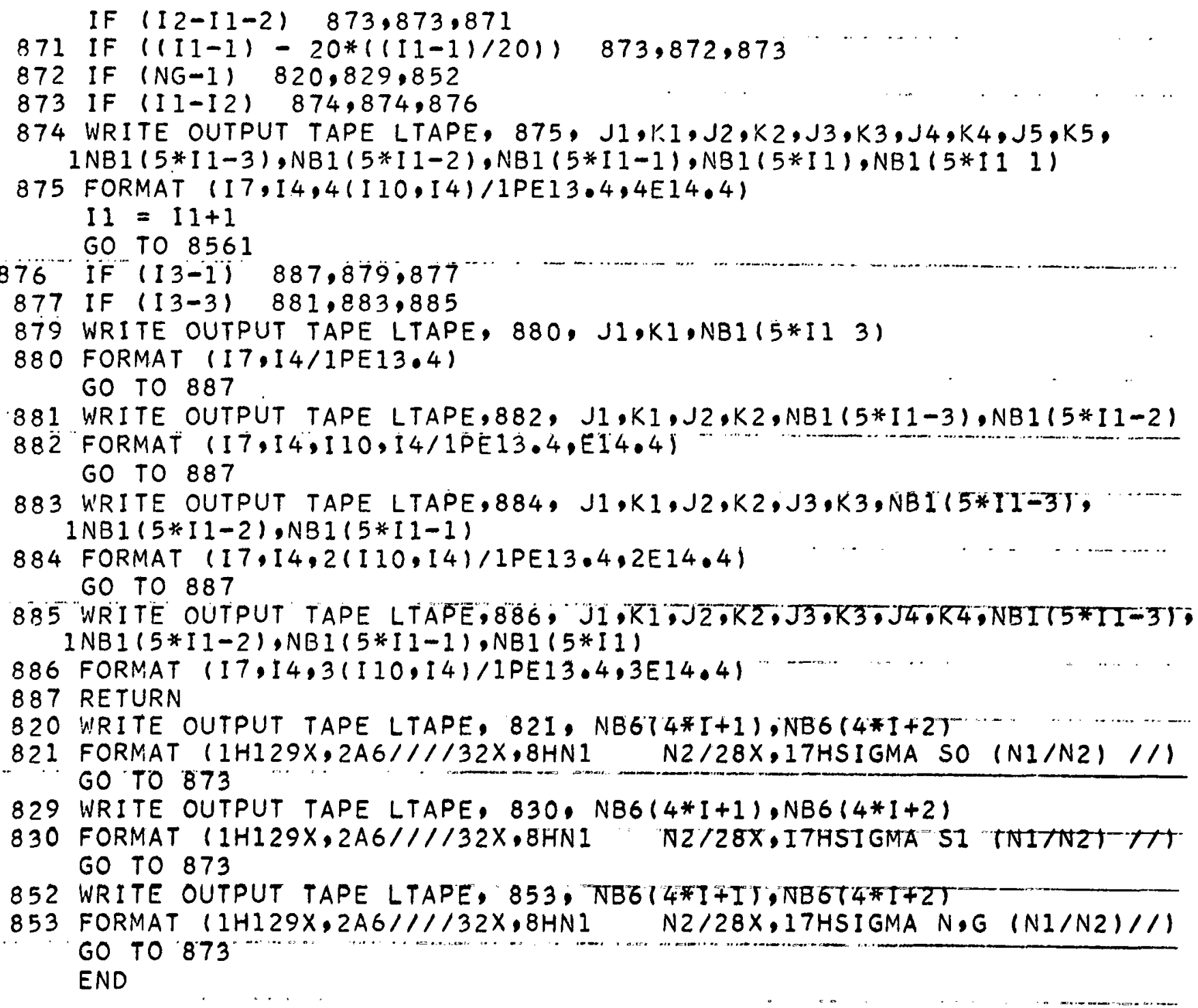




\subsection{Program Mnemonic Glossary}

Program

Mnemonic

$\mathrm{BC} \varnothing \mathrm{DE}$

BDATE

BNAM

cфNNф

DATE

DEN

ENERGY

FBI

FB2

FB6

FINR

FI $\phi C$

I

II3

ITAPE

ITAPI

$\mathrm{J}$

KTAPE

LTAPE

\section{Description}

Code number for a data block either microscopic or macroscopic

Date data block was added to Nuclear Data Tape, read in as card input in MAC3

2-word name of data block, read in as card imput in MACl

Code numbers (BCфDE) of microscopic constituents of a given macroscopic nuclear data block, read in as card input in MAC3

The date of a Nuclear Data Tape was colmiled, read in as card imput in MACl

Density factor of any data block, defined as (mass density)/(Avogadro's number) $\left(10^{24}\right)$, read in as card imput in MAC3

Energy of each of the energy levels expressed in ev, read from master tape record 2 in MAC7

Eq. NBl--core storage location of 2010 words used to store information read from and into tapes

Eq. NB2--core storage location of 5010 words used principally to store macroscopic data blocks while they are being calculated

Eq. NB6--core storage location of 3010 words used principally to store the index record and additions to the final record

Eq. NFINR--core storage location of 1010 words used to store old master tape indexing information while calculating macroscopic blocks and for storing corrected final record after all block calculations

Eq. NLфC--core storage location of 2010 words used

in MACl to store logarithmic decrements, and in MAC4 to store records 2 and 3

Index in comon used principally for locating index record information

Maximum number of data blocks on original tapes

Original master Nuclear Data Tape number, set in MACl

Tape number from which information is being read or into which information is being written

Index in common

Intermediate tape number, set in MACl

Output tape number, set in MACl 
Program

Mnemonic

M

MA2

MB2

MC2

MA3

MB3

MC3

MA5

MB5

MC5

MAXDB

MAXDBI

MAXWD2

METAPE

NTAPE

NI

N2

N3

N4

N

NA

NB

\section{Description}

The number of records in a given macroscopic data block used for correcting the index records

First group from which and into which $\sigma_{80}\left(\mathrm{~m} \mid \mathrm{m}^{\prime}\right)$ transfers occur--MOMIN

Last group from which $\sigma_{\text {so }}\left(\mathrm{m} / \mathrm{m}^{\prime}\right)$ transfers occur-MOMAX

Last group into which $\sigma_{\text {so }}\left(\mathrm{m} / \mathrm{m}^{\prime}\right)$ transfers occur-MOPMAX

Flrst group from which and into which $\sigma_{81}\left(\mathrm{~m} \mid \mathrm{m}^{\prime}\right)$ transfers occur--MIMIN

Last group from which $\sigma_{31}\left(m \mid m^{\prime}\right)$ transfers occur-MIMAX

Last group into which $\sigma_{51}\left(m \mid m^{\prime}\right)$ transfers occur-MIPMAX

First group from which and Into which $\sigma_{n, g}\left(\mathrm{~m} \mid \mathrm{m}^{\prime}\right)$ transfers occur--MGMIN Last group from which $\sigma_{n, g}\left(m / m^{\prime}\right)$ transfers occur--
MGMAX

Last group 1nto which $\sigma_{n, g}\left(m \mid m^{\prime}\right)$ transfers occur-MGPMAX

Maximum number of data blocks, (NWINDX/4) - 2, set In MACl

(NWINDX/4) - 1, set in MACl

Maximum number of words in data-block records 2 and 3, calculated in $\mathrm{MACl}$

New data tape number assigned in MACl

New data tape number assigned in MACl

Nimber of worda in data-block record 2 , set by MAC5 operating on data-block record 1 stored in FBI

Number of worda in data-block record 3, set by MAC5 Number of words in data-block record 4, set by MAC5 Number of words in date-block record 5, set by MAC5 Amount of relocation of records $2,3,4$, and 5 by PUNACB subroutine

Index record of data block on master tape in position to be read, also used in several writing subroutines

Used while calculating a macroscopic data block to indicate relative location of constituent date blocks on master tape, also used in several writing subroutines 


\footnotetext{
Program Mnemonic

Description

NC

Number of records okipped in positioning tape in MAC3, number of IInes in output printout in FINREC, also used in several writing subroutines

ND

Used extensively to Indicato information storage, locationa Benerally defined inmediately before use

NE

Used in MAC4 to Indicate number of words currently atored in the macroscopic data block record 4 belng calculated, also used in soveral writing subroutines

NF

Used wh1lo calculating macroscoplc date blocks to determine relative location of macroscoplc block data in cese Imput

NR

Number of words to be added to final record to describe celculated macroscoplc date blocks

$\mathbf{N X}$

Dummy Index used in MAC7

NBI

2010-word core storage locat1on, equivalent to FBl

NB2

NB6

NBPSN

5010-word core storage location, equivelent to FB2

3010-word core storage locat1on, equivalent to FB6

Index-recond position number of macroscoplc date blocks belng added to tape, read in as card Input data In MACI

$\operatorname{NC\phi N}$

Number of conot1tuent data blooks in a macroscoplc data block, road in as card laput for each macro. scoplc data block in MAC3

NFINR

1010-word core storage area used to store ITAPE Indexing Information and later on to store the revised final rocord, equivelent to FINR

NLEV

Number of oplthermal energy lovelo present read in MACl from orlginal tape index record

NOMAC

Number of macroscoplc blocks added to tape, read in as card Input in MACl

NФPRNI Number of data blocks to be printed, read in as card Input In MACl

N\PUN Mumber of date blocks to bo punchod by this program In addition to thone calculated by thi. program not now used, could bo added in MACl

NRE6 Number of rosonanco onerey lovols which oxiot in a given data block, sot by MAC5 oporating on datablock record 1 otored in NBI

NRMAX

Maximum number of resonanco energy lovels allowable In a macroscoplc data block, sot in MACl

NSPECT Number of words in opoctral data blocks computed in MACI uoIDg NLEV
} 
Program

Mnemonic

NTHGRP

NTLEV

NTR

NTWDS

NWDBRI

NWFINR

NWINDX

NWRC2

NWRES

IWWGSI

NWSGSO

PC $\varnothing D E$

PNCфDE

PTC $\phi D E$

PUNC $\varnothing D$

THETRM

WFR

WGHTFR

WGT
Description

Number of thermal energy levels, set in MACI

Total number of energy levels, computed in MACl as NLEV + NTLEV

Maximu number of rows in record-5 matrix set in MACl, final record number set in MAC6, used in MAC7

Maximum total number of words possible in a macroscoplc data block including all 5 records, computed in MACI

Number of words in data-block record 1, computed in MACI

Nrmber of words in final record, set in $\mathrm{MACl}$

Number of words in index record, set in MACl

Number of words in second record, computed in MAC2

Naximum number of words possible in data-block record 4 , set in $\mathrm{MACl}$

Maximum number of words possible in data-block record 3, set in MACI

Maximum number of words possible in data-block record 2, set in $\mathrm{MACl}$

Code numbers in date blocks to be printed, read in as card imput in $\mathrm{MACl}$

Control word for punchlng a given data block on binary cards

Control word for writing a given data block on the output tape

Code numbers of data blocks to be punched, in addition to those automatically punched, not now used, could be added as card Input to MACI

The temperature corresponding to each of the thermal energy levels, set in MACl

Welghting factor for compling the macroscopic-crosssection matrices

Welghting factor for converting microscoplc data to macroscople data

The weight fraction of a constituent in a macroscoplc data block, read-in as card 1nput in MAC3 


\subsection{REACTOR NUCLEAR DATA COPY AND CORRECT PROGRAM}

\section{I General Information}

The Reactor Nuclear Data Copy and Correct Program (RNDC) performs the following functions. First the program has the capability of correcting and/or copying a Reactor Nuclear Data Tape with a minimum of imput information. Corrections may be made in any record or data block on the tape. Two copies of the corrected tape may be requested. Second, the provision is made to manufacture a Reactor Muclear Data Tape directly from binary cards punched out by program RNDP. Third, the program will print out any record or data block on a Reactor Nuclear Data Tape, as the tape is copied, as the tape is made from binary cards, or as a separate function.

\subsection{Description of Subroutines Unique to this Program}

\subsubsection{Control Routine}

Four main functions are assigned to the control routine during an execution of program RNDC. The first is the initializing of various subroutines and the preassigning of values to certain variables. The next function is the clearing of the input data storage and the reading of the general imput data which also include any values for the program-preassigned variables that the user may wish to change. The third function the control routine performs is to make a logical consistency check on the general imput data, and, if an inconsistency is discovered, to halt the program. The last function is to direct the flow of the program by initializing and selecting the three first-order subroutines in the program, namely, the INDEX subroutine, ELlV subroutine, and the RAPRL subroutine; and, if the program has executed properly, denote the final stop.

\subsubsection{Subroutine INDEX}

The INDEX subroutine, as its name implies, processes all the information pertaining to the index record on the data tape. It is a first-order subroutine in the sense that it is entered from the control routine and its normal exit is to the control routine. This subroutine examines all the options connected with the control words NC $\phi \mathrm{DEI}$, N $\phi C \phi \mathrm{R}$, and NфTAPE and performs the necessary operations. The control word NQPRNT is not examined because the index record is always printed out whenever the program is used. In addition the INDEX subroutine performs one other major function and that is to determine the record number of the microscopic constituents record, the final record, as it was on the old data tape and as it should be on the new data tape. This is a safeguard designed in the program to make certain that the user has properly corrected the record numbers on the index record in the event of records being added to material data blocks through correction cards. It should be noted that error diagnostics have been programed into the INDEX subroutine so that only the normal return is to the control routine. 


\subsubsection{Subroutine ELIV}

The ELIV subroutine is another first-order subroutine which processes all the information pertaining to the energy-lethargy-l/v factor record on the data tape. It is also entered from the control routine, and its normal exit is to that same routine. This subroutine examines all the options connected with the control words NC $\phi \mathrm{DEl}$, N $\phi C \phi R$, and N $\phi \mathrm{TAPE}$ and performs the necessary operations. The control word N $\phi \mathrm{PRNT}$ is not examined because the energy-lethargy-1/v factor record is always printed out whenever the program is used. There are no special functions performed by this subroutine. There are error diagnostics in the ELIV subroutine.

\subsubsection{Subroutine RAPRL}

The RAPRL subroutine is the first-order subroutine which acts as a control routine for the second-order subroutines DBIKR, SPECT, and MICR $\phi$. The RAPRL subroutine establishes a reading and processing loop in which a record is read from the old binary data tape, the code number tested, and depending upon the value of the code number, control is transferred to one of the above-mentioned secondary subroutines.

- When control is transferred back to RAPRL from one of the secondary subroutines, the data tape will be in position for RAPRL to read the first record on the next data block. In the case in which the data block is on binary cards instead of tape, RAPRL performs in the same manner except for reading the cards rather than the tape. After the last record on tape has been processed, that is, the microscopic constituents record, and control has been transferred back to RAPRL from MICR $\varnothing$, RAPRL then transfers control to the program control routine. In the RAPRL subroutine there are no correction cards read, no binary tapes are written, no printout written, and only one record of a data block is read. There are error diagnostics in this subroutine, and only the normal return is to the control routine.

\subsubsection{Subroutine DBIKR}

If the code number examined by subroutine RAPRL is less than 500.0 in value, then RAPRL transfers control to the secondary subroutine DBLKR or the material-data-block subroutine. This subroutine is the largest and most complicated in the program due to the other options available within a material data block besides the normal NC $\phi D E l$, N $\phi C \phi R$, N $\phi P R N T$, and N $\phi \mathrm{TAPE}$ options. Since a material data block may have from one to five records on tape and since subroutine RAPRL only reads in the first record, subroutine DBLKR immediately must determine if any other records are in the data block, determine the type and length, and read them into their proper storage locations from the old data tape. It should be noted at this point that, if binary cards are being read rather than tape, subroutine RAPRL reads the complete set of cards representing the materiall data block, and hence no binary cards are read in DBLKR. The subroutine next determines if corrections are to be made to the data block, and, if so, the correction cards are read into their proper locations. Included in this reading of correction cards is, of course, the option of adding from one to four records to the material data block. Hence, the subroutine must recalculate the length of the records, 
determine the type of record, and calculate the proper record number of each of the records before the new tapes can be written. The last function performed by DBLKR is to determine if the material data block is to be printed out and, if it is, to do so. Control is then returned to subroutine RAPRL for the processing of the next record. There are error diagnostics in DBLKR, and only the normal return is to RAPRL.

\subsubsection{Subroutine SPECT}

If the code number examined by subroutine RAPRL is equal to or greater than 500.0, then RAPRL transfers control to the secondary subroutine SPECT or the spectral record subroutine. The purpose of this subroutine is to process each spectral record on the data tape. All the options connected with the control words NC $\phi D E l$, N $\phi C \phi R$, N $\phi \mathrm{TAPE}$, and NQPRNT are examined and the required operations performed. After the SPECT subroutine is completed, control is transferred to the RAPRL subroutine for the processing of the next record from the data tape. There are error diagnostics in SPECT, and only the normal return is to RAPRL.

\subsubsection{Subroutine MICR $\varnothing$}

If the code number examined by subroutine RAPRL is equal to zero, then RAPRL transfers control to the secondary subroutine MICR $\phi$ or the microscopic constituents record subroutine. This subroutine processes the last record of the data tape and, hence, is the last subroutine to be executed in the program. All the options connected with the control words NC $\phi D E J$, $N \phi C \phi R$, and N $\phi T A P E$ are examined and the required operations performed. The control word N $\phi$ PRNT is not examined because the microscopic constituents record is always printed out. If there are no macroscopic material data blocks on the tape and hence no microscopic constituents, a comment to this effect is printed out. Also if any of the microscopic constituents of a macroscopic material have been changed through correction cards, these changes are noted in the printout. The one special function the MICR $\phi$ subroutine performs is to check the logical record number of the microscoplc constituents record, to determine if the actual number of records present on the tape is identical to the record number of this last record. There are error diagnostics in MICR $\phi$, and only the normal return is to RAPRL.

\subsubsection{Subroutine CALDIM}

The CALDIM subroutine is a third-order subroutine which is used solely in conjunction with the DBLKR subroutine. It is entered from the DBIKR subroutine, and its only exit is to that same subroutine. The only function CAIDIM performs is to calculate the dimensions or length of the second, third, fourth, and fifth records of a material data block. The subroutine is entered twice, the first time being before the old data tape is read and the second time, after the corrections are read into the data block storage and before the new data tapes are written. There are no error diagnostics in CALDTM.

\subsubsection{Subroutine CLEAR}

The CLEAR subroutine is a third-order subroutine used in confunction with the control routine and the RAPRL subroutine. Its function is 
to set the two temporary data storage arrays to zero. There are no error diagnostics in CIEAR.

\subsubsection{Subroutine PSOLNG}

The PSOLNG subroutine is a third-order subroutine which is used solely in confunction with the DBLKR subroutine. It is entered from the DBLKR subroutine, and its only exit is to that same subroutine. The only function PSOING performs is to print out the isotropic scattering, first moment scattering, and $n-\gamma$ transfer matrices or records two, three, and five of a material data block. Hence, this subroutine can be entered a maximum of three times for each material data block. There are no error diagnostics in PSOLNG.

\subsubsection{Subroutine RBTIJK}

The RBTIJK subroutine is a third-order subroutine used in confunction with the ELIV, RAPRL, and DBIKR subroutines. Its purpose is to read a binary record from the old data tape of varlable length and test for a tape check. An attempt will be made to read the tape a total of ten times. There are no error diagnostics in the subroutine. However, sense light one is turned on if the tape cannot be read properly by the tenth attempt and subroutines ELIV, RAPRL, and DBIKR bave provision for monltoring sense light one.

\subsubsection{Subroutine RIJKT}

The RIJKT subroutine is a third-order subroutine used in conjunction with the INDEX, EIIV, RAPRL, DBIKR, SPECT, and MICR $\varnothing$ subroutines. Th1s subroutine simply rewinds ITAPE, JTAPE, and KTAPE. There are no error diagnostics in RIJKT.

\subsubsection{Subroutine SWTCH}

The SWTCH subroutine is a third-order subroutine which is used solely in conjunction with the DBLKR subroutine. It is entered from the DBIKR subroutine, and 1ts only exit is to that subroutine. The only function SWTCH performs is to "expand" the fourth record of the material data block, or resonance data array so that the corrections can be readin properly. On the binary data tape the resonance record is not written to its possible maximum length but only to the actual number of resonances given. However, when correcting the resonance array in storage, the maximum length must be allowed. The same logic holds for the binary card reading as for the blnary tape reading. There are no error diagnostics in SWTCH.

\subsubsection{Subroutine WBTIJ}

The WBTIJ subroutine is a third-order subroutine used in conjunct1on with the EIIV, DBIKR, SPECT, and MICR $\phi$ subroutines. Its purpose is to write a blnary record on a new data tape of varlable length. The subroutine will permit a maximum of five attempts at writing the new tape. There are no error diagnostics in the subroutine. However, sense light one is turned on if fallure occurs five times and subroutines ELIV, DBLKR, SPECT, and MICR $\varnothing$ have provisions for monitoring sense light one. 


\subsection{Program Flow Chart}

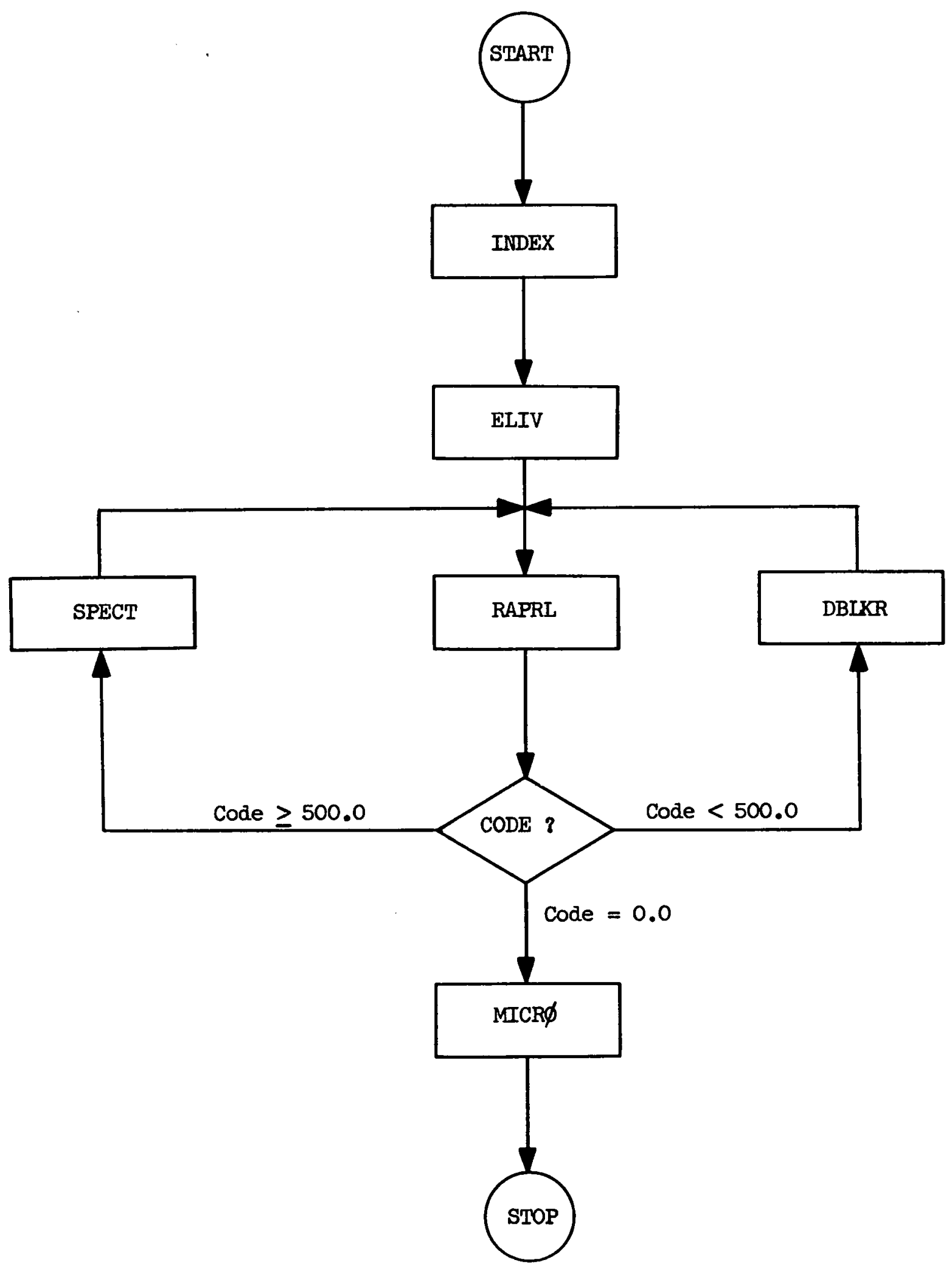

Fig. 4.1 Flow Chart of Control Routine 


\subsection{Program Diagnostics}

External Formula number

Control Routine

190

191

192

193

194

195

INDEX Subrout1ne

2905

2906

2907

2908

2909

2911

2913

EIlV Subroutine

3907

3908

3909

3910

3911

3912

3913

RAPRL Subroutine

8903
General input data error

NфTAPE control wond has minus or zero value

Inconsistency between $N \phi C \phi R$ control word and CC $\varnothing \mathrm{DE}$ table

Inconsistency between NфPRNT control word and PC $\phi \mathrm{DE}$ table

Tape number ITAPE has negat1ve value

Tape number JTAPE has negative value

Tape number KTAPE cannot be read properly'

Input data error in INDEX correction cards

Tape number ITAPE cannot be written properly

Ne1ther tape number ITAFE nor tape number JTAPE could be written properly

NФTAPE control word has negative value

More than 499 data blocks in index record of data tape

More than 499 data blocks in index record in core storage

Tape number KTAPE cannot be read properly

Input data error in ELIV correction cards

Tape number ITAPE cannot be written properly

Tape number ITAPE could not be written properly;

- tape number JTAPE had falled previously

Tape number JTAPE could not be written properly;

tape number ITAPE had falled previously

Tape number ITAPE has negative value

Tape number JTAPE has negative or zero value

Less than three records on the data tape. There must be present on the data tape at least the index, the energy-lethargy-l/v factor, and the microscopic constituents records. 
External Formula Number

8904

8905

DBLKR Subroutine

9938

9939

9940

9941

9942

.9943

9944

9945

9946

9947

9980

9981

9982

9983

9948

9949

9950

9951

9952

\section{Error}

Tape number KTAPE cannot be read properly

Calculated physical record number of microscopic constituents record does not agree with input final record number on the index record. This occurs when adding records to a material data block through correction cards.

MOMIN has negative value when read from KTAPE

Record 2, scattering transfer matrix, cannot be read properly from KTAPE

MIMIN has negative value when read from KTAPE

Record 3, first moment scattering transfer matrix, cannot be read properly from KTAPE

NRES has negative value when read from KTAPE

Record 4, resonance data, cannot be read properly from KTAPE

MGMIN has a negative value when read from KTAPE

Record 5, n-y transfer matrix, cannot be read properly from KTAPE

NC $\varnothing \mathrm{DEl}$ control word has negative value

Input data error in DBLKR correction cards

MOMIN has negative value after corrections read into storage

MIIN has negative value after corrections read into storage

NRES has negative value after corrections read into storage

MGMIN has negative value after corrections read. into storage

NфTAPE control word has zero value

A record of the material data block could not be written properly on tape number ITAPE

A record of the material data block could not be written properly on tape number ITAPE after tape number JTAPE had falled previously

MOMIN has negative value prior to writing tape ITAPE

MIMIN has negative value prior to writing tape ITAPE 
External Formula Number

9953

9954

9955

9956

9957

9958

9959

9960

9961

9962

9964

9965

9966

9967

9968

9969

9970

9971

\section{SPECT Subroutine}

9197

9198

9199
Error

NRES has negative value prior to writing tape ITAPE

MGMIN has negative value prior to writing tape ITAPE

Tape number JTAPE has negative value

A record of the material data block could not be written properly on tape number JTAPE after tape number ITAPE had failed previously

MOMIN has negative value prior to writing tape JTAPE

MIMIN has negative value prior to writing tape JTAPE

NRES has negative value prior to writing tape JTAPE

MGMIN has negative value prior to writing tape JTAPE

Tape number ITAPE has negative value

Code number of material data block not present in index record.

MOMIN has negative value prior to printout of scattering transfer matrix

MIMIN has negative value prior to printout of first moment scattering transfer matrix

NRES has negative value prior to printout of resonance data

MGMIN has negative value prior to printout of n-y transfer matrix

Record number of second record, scattering transter matrix, has' negative value

Record number of third record, first moment transfer matrix, has negative value

Record number of fourth record, resonance data, has negative value

Record number of fifth record, $n-\gamma$ transfer matrix, has negative value

Imput data error in SPECT correction cards

Tape number ITAPE cannot be written properly

Tape number ITAPE could not be written properly;

tape number JTAPE had failed previously 
External Formula Number

9131

9132

9133

9134

MICRф Subroutine 9062

9063

9064

9065

9066

9067

9068

9069

9070

9071

9072

9073

\section{Error}

Tape number JTAPE could not be written properly; tape number ITAPE had failed previously

Code number of spectrum not present in index record

Tape number ITAPE has negative value

Tape number JTAPE has negative value

$N \phi C \phi R$ control has negative value

Input data error in MICR $\phi$ correction cards

Calculated physical record number of microscoplc constituents record is less than final record number on the index record. This occurs when adding records to a materlal data block through correction cards.

Calculated physical record number of microscopic constituents record is greater than final record number on the index record

NфTAPE control has zero value

Tape number ITAPE cannot be written properly

Tape number ITAPE has negative value

Tape number ITAPE could not be written properly; tape number JTAPE had failed previously

Tape number JTAPE has negative value

Tape number JTAPE could not be written properly; tape number ITAPE had falled previously

Macroscoplc-materlal data-block code number cannot be found on index record

Microscopic-constituent material-data-block code number cannot be found on index record

\subsection{Program Input Forms}

All input data in Program RNDC are loaded by the DIP subroutine through READ DIP source statements followed by a list of variables representing those quantities to be loaded. All conventions, standards, and options noted in the DIP subroutine description are recognized by the program. Although end-of-record conditions are needed in the decimal-input-deck make-up, there is no end-of-file condition necessary since the logic of the program determines when it has exhausted its supply of input data. This, of course, imposes upon the user the necessity of assembling the decimal input deck correctly. As an added safeguard, the variables contained in the DIP Ilsts of the various subroutines for the most part are unique among themselves and hence the 
possibility of incorrect decimal-imput-deck assembly going undetected through a program execution is further reduced. The necessity for designing these safeguards into the program was occasioned by the fact that the identifying code number of a particular data block does not have to be given with the set of correction data for that data block, hence no cross-checking of code numbers could be effected.

\subsubsection{General Imput Data Sheets}

The general input data needed by Program RNDC consist of four control option words and two arrays of identifying data-block code numbers with an end-of-record card being the final card of the record.

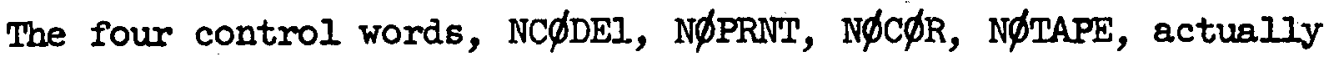
determine the type of program operation to perform and, except for one point, are explained fully on the data sheets. Under the NC $\phi$ DEI control word, if option minus one $(-1)$ is used, there must be a decimal identiflcation card preceeding each group of binary cards representing each of the varlous date blocks to be written on the tape. This decimal 1dentification card has only the first column read by the program and places the contents of the first column in a waste location. Hence any type of Hollerith information can be punched in the first column as well as the rest of the card.

The second general-imput data sheet contalns the two arrays of identifying data-block code numbers. The first array contains the code numbers of the data blocks to be printed, and the second the code numbers of the data blocks to be corrected. Again these arrays are fully explained on the data sheet. The code numbers can be given in any order in the print-code array, and, except when the final record is to be corrected, the same is true of the correction-code array. If the final record (microscoplc constituents record) is to be corrected, then its code (0) must be given as the last entry in the array.

\subsubsection{Correction Imput Data Sheet}

In general the correction imput data sheets are divided into five categories; index-record corrections, energy-lethargy record corrections, materlal-data-block corrections, spectral-record corrections, and microscoplc-constituents-record corrections. For the most part the varlous data sheets are self-explanatory when used in conjunction with the imput glossary. One point to be noted is that when correcting a value in an array, the equal sign $(\Rightarrow)$ convention in DIP to bypass storage locations is very helpful and eliminates the necessity of knowing or calculating the absolute memory location of a particular value. By preceeding the equal sign with an integer, $n$, the storage location of the variable is decreased by $n$.

With regard to index-record corrections, the two-word BCD Ident1fication of the first material or spectrum is assigned the mnemonic BIDENT. This card which has a loading address of (28100) 10 contains both words of the identification of the first material or spectrum. To arrive at the loading address of the second material or spectrum, simply subtract two from $(28100)_{10}$ or $(28098)_{10}$. This pattern 
continues for each succeeding identification until the last material or spectrum Identification loads at address (27104) 10 .

The remaining corrections on the index record should be selfexplanatory, as should be the case with the energy-lethargy record corrections.

A material data block on the Reactor Muclear Data Tape is composed of a minimum of one and a maximum of five separate binary records. However, when using Program RNDC to effect corrections to a material data block, all the corrections to all the blnary records of a block are read in with one record of decimal imput data. Thus, although the material data block correction sheets are divided into five parts each corresponding to its counterpart on the data tape, there is only one end-of-record card indicated and necessary for the whole material data block.

The material-data-block correction themselves should be selfexplanatory as should be the spectral-record corrections.

The fifth and last category of corrections to the data tape pertains to the microscopic-constituents record or last record of the tape. Since there is no set pattern to the contents of this record, the whole data array of 1000 words had to be indicated by one variable. This variable, NMICR $\phi$, is actually the physical record number of the microscopic-constituents record, a diagram of which appears in Figure 4.2 .

As is indicated on the diagram, there are only five "assigned" storage locations in the NMICR $\phi$ data array, and these are the first five locations. The remalnder of the array is varlable in content. In addition the array contains both fixed-point and floating-point variables. The fixed-point variables are the record number and the number of microscoplc constituents, whlle the floating-point variables are the various code numbers and the welght fractions. Thus the user, when correcting the microscopic-constituents record, must be careful not only to insert the corrections in the proper locations but also to have the proper control punch in column one of the correction card.

\subsubsection{Add1tion of Records to a Material Data Block Through Correction Cards}

It is possible, with Program RNDC, to add from one to four records to a material data block by reading the contents of the record or records into the proper locations through imput-data correction cards. This can only be done, however, with the last four records of the materlal data block, namely, the isotropic scattering transfer matrix, first moment scattering transfer matrix, resonance data, and the $n-\gamma$ transfer matrix. Additionally, the first record of the material data block containing the ordinary cross section data must already be present on the tape or on binary data cards for this option to work correctly. It should be noted that there is a considerable amount of work entailed in using this feature of the program. The logic of the program necessitates correcting, by decimal input, all of 


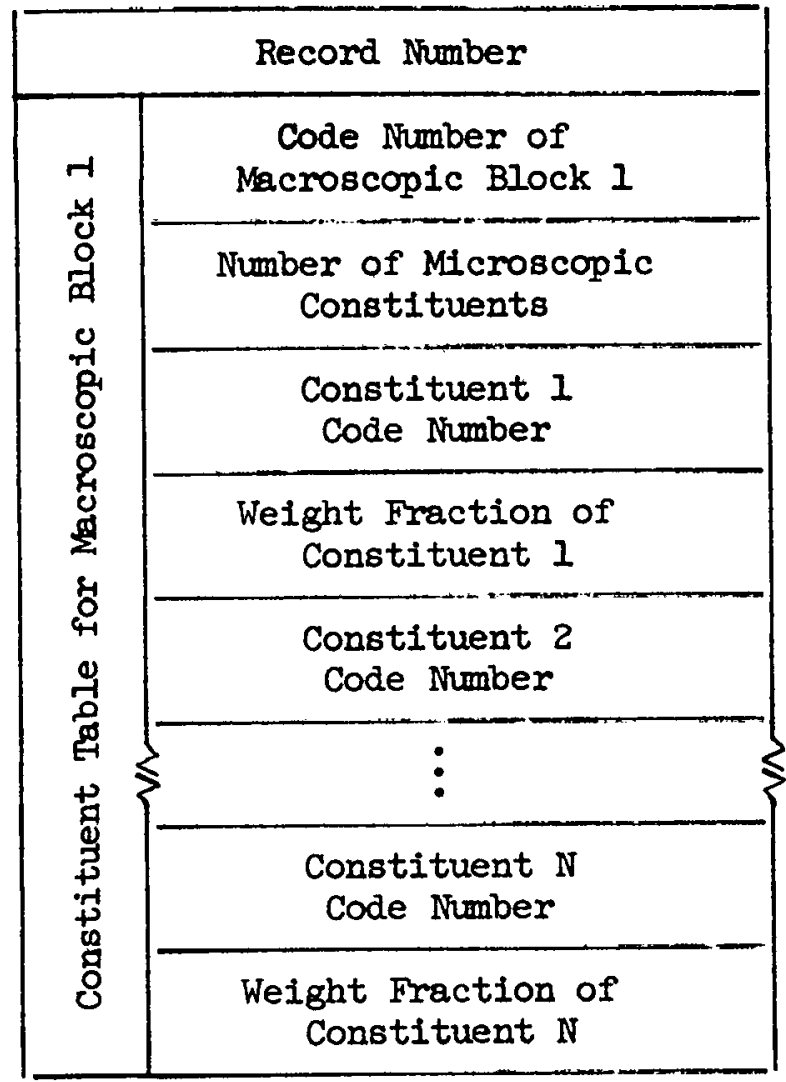

$:$

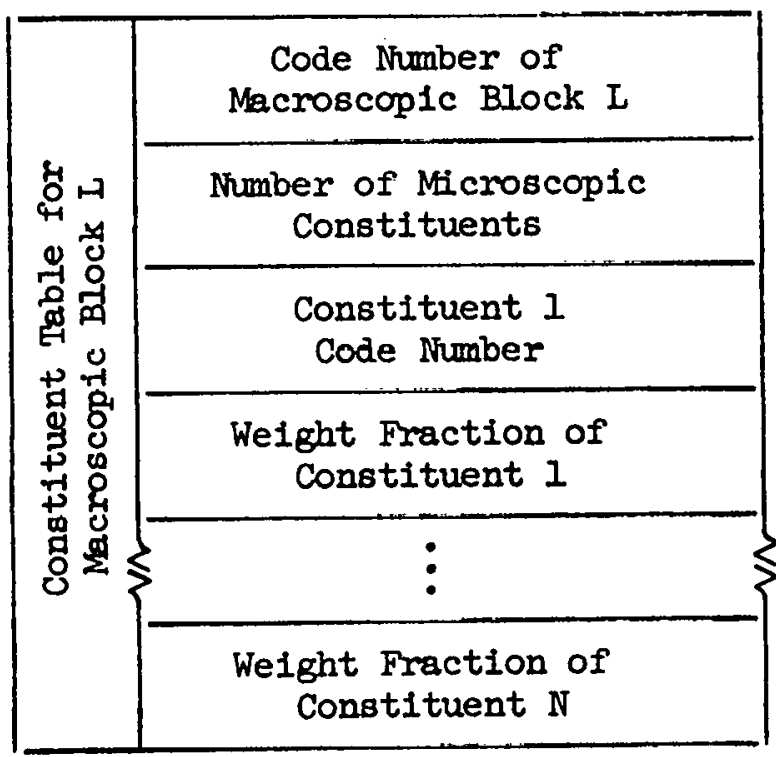

Fig. 4.2 - Nacroscopic-Constituent

Index (Final Record) 
the record numbers on the index record of those material data blocks and spectral records following that material data block which had a record or records added, in order to reflect the proper physical-position change on the data tape. Obviously, the record number of the microscopic-constituents record also must be corrected. Thus, if a material data block which is physically positioned near the beginning of the tape is to have records added to it, several-score index-record quantities might have to be corrected to effect this change. There is, however, no limit to the number of material data blocks that may have records added to them through correction cards, nor does this option in any way detract from the other options in the program.

4.5.4 Glossary for Imput Sheets

A An array containing absorption cross sections for a given material, cross sections are given in order from high to low energy, maximum of 34 , floating-point variables.

$\mathrm{BC} \varnothing \mathrm{DE}$ An array containing code numbers identifying the data blocks present on the tape, maximu of 499 , floatingpoint variables.

BETA A location containing the value of the spectral delay fraction of a given spectmm, floating-point variable.

$C C \varnothing D E$ An array containing the code numbers of data blocks on tape to be corrected, maximum of 499 , floating-point variables.

$C \varnothing D E$ A location containing the code number of a given data block on tape, floating-point varlable.

DATE Two locations containing 12 characters of $B C D$ information indicating the date of manufacture of the tape or the date of a data block.

DF A location containing the density factor of a given material, floating-point variable.

EG An array containing the group boundary energies of the data tape, energles are glven from high to low, maximum of 25 words, Iloating-point variables.

EL An array containing the level energies of the data tape, energies are given from high to low, maximum of 34 , floating-point variables.

END A location containing the value of the "end-of-record" card.

EO An array containing the resonance energies of a given material, maximum of 200 , floating-point variables.

ETAR An array containing the number of fission neutron produced per absorption for a material given in the order of resonance energies, maximum of 200, floating-point variables.

FNU An array containing fission production cross sections for a given material, cross sections are given in order from high to low energy, maximum of 34, floating-point variables. 
FV An array containing the group $I / v$ factors of the data tape, the $1 / v$ factors are given in order from high to low energy, maximum of 24 , floating-point variables.

MGIMAX A location containing the last group from which $n-\gamma$ transfers occur, fixed-point variable.

MGMIN A location containing the first group from which and first group into which $n-\gamma$ transfers occur, fixed-point variable.

MGळMAX A location containing the last group into which $n-\gamma$ transfers occur, fixed-point variable.

MOIMAX A location containing the last group from which scattering transfers occur, fixed-point variable.

MOMIN A location containing the first group from which and first group into which scattering transfers occur, fixed-point variable.

MO $\varnothing$ MAX A location containing the last group into whlch scattering transfers occur, fixed-point variable.

MIIMAX A location containing the last group from which first moment scattering transfers occur, flxed-point variable.

MUMIN A location containing the first group from whlch and first group into which first moment scattering transfers occur, fixed-point variable.

MØMAX A location containing the last group into which first moment scattering transfers occur, fixed-point variable.

NAME A location containing six characters of $B C D$ information identifying the tape by name.

NC $\phi$ DEI A location containing a control word having three options pertaining to the basic operation of Program RNDC, fixedpoint varlable.

NMICR $\varnothing$ An array containing the contents of the microscopic-const1tuents record or last record on tape, maximum of 1000 , the array contains both floating-point and positive fixedpoint variables.

NLEV A location containing the number of energy levels of the data tape, positive fixed-point variable.

$N \phi C \phi R$ A location containing a control word indicating the number of data blocks that are to be corrected, positive fixedpoint varlable.

NØPRNT A location contalning a control word having three options pertalning to the BCD output listing, fixed-point variable.

NфTAPE A location containing a control word having two options pertaining to the number of new binary tapes that are to be written, positive fixed-point variable.

NRES A location containing the number of resonances of a given material, positive fixed-point variable. 
NW A location containing a value indicating the total number of binary words contained in a material data block after the first record, positive fixed-point variable.

NIR A location containing the physical record number of the first record of a given data block, also pertains to the record number of the index record, second record, and final record, positive fixed-point variable.

When NLR is subscripted, it refers to an array containing the record numbers of the initial records of each data block, maximum of 499 , positive fixed-point variable.

N2R A location containing the physical record number of the second record of a given data block, positive fixedpoint variable.

N3R A location containing the physical record number of the third record of a given data block, positive fixed-point variable.

N4R A location containing the physical record number of the fourth record of a given data block, positive fixed-point variable.

N5R A location containing the physical record number of the fifth record of a given data block, positive fixed-point variable.

PC $\varnothing \mathrm{DE}$ An array containing the code numbers of data blocks on tape to be printed, maximm of 499 , floating-point variables.

PO An array containing the peak cross sections of a given material given in the order of the resonance energies, maximum of 200, floating-point variables.

$\mathrm{R} \quad$ An array conteining the Doppler temperature coefficlents of a given material given in the order of the resonance energles, maximum of 200, floating-point variables.

RIO An array containing the resonance integrals of a given material given in the order of the resonance energies, maximum of 200, floating-point variables.

$S \quad$ An array containing scattering cross sections for a given material, the cross sections are given in order from hlgh to low energy, maximum of 34, floating-point variables.

SIGNG An array containing the $n-\gamma$ transfer matrix of a material in which the elements are given by varying the column subscript (exit group) to its maximum for each row subscript (incident group) variation, also given in high to low energy, maximum of 680 , floating-point variables.

SIGSO An array containing the scattering transfer matrix of a material given in the order as described for the $n-\gamma$ transfer matrix, maximum of 625, floating-point variables.

SIGSI An array containing the first moment scattering transfer matrix for a material given in the order as described for the $n-\gamma$ transfer matrix, maximum of 625 , floating-point variables. 
$S \phi R$ An array containing the level spectral values of a spectrum given in order from high to low energy, maximm of 34, floating-point variables.

TM A location containing the mean life of the delayed neutron group precursor, floating-point variable.

TR An array containing transport cross sections for a given material, the cross sections are given in order from high to low energy, maximum of 34, floating-point variables.

TS4500 An array contalning the thermal scattering matrix for 4500 degrees F. for a given material, the values in the matrix are given by varying the column subscript (exit group) to its maximum for each row subscript (incident group) variation, also given in high to low enersy, maximum of 30, floating-point variables.

TS4000 An array containing the thermal scattering matrix for 4000 degrees F. for a given materlal as described for the 4500 degree matrix, maximum of 30, floating-point variables.

TS3500 An array containing the thermal scattering matrix for 3500 degrees $F$. for a given material as described for the 4500 degree matrix, maximu of 30 , floating-point variables.

TS3000 An array containing the thermal scattering matrix for 3000 degrees $F$. for a glven material as described for the 4500 degree matrix, maximu of 30, flosting-polnt variables.

TS2500 An array containing the thermal scattering matrix for 2500 degrees $F$. for a glven material as described for the 4500 degree matrix, maximum of 30 , floating-point variables.

TS2000 An erray containing the thermal scattering matrix for 2000 degrees F. for a given materlal as described for the 4500degree matrix, maximu of 30, floating-point variables.

TS1500 An array containing the thermal scattering matrix for 1500 degrees F. for a given materlal as described for the 4500 degree matrix, maximum of 30 , floating-point variables.

TS1000 An array containing the thermal scattering matrix for 1000 degrees F. for a given material as desoribed for the 4600 degree matrix, maximum of 30 , floating-polnt variables.

TS500 An array contalning the thermal scattering matrix for 500 degrees F. for a given material as described for the 4500 degree matrix, maximum of 30 , floating-point variables.

TS68 An array containing the thermal scattering matrix for 68 degrees F. for a given material as described for the 4500 degree matrix, maximum of 30 , floating-point variables.

UG An array containing the group boundary lethargies of the data tape, lethargies are given from low to high, maximum of 25, floating-polnt variables.

UL An array contalning the level lethargles of the data tape, lethargles are given from low to high, maximum of 34 , floating-polnt varlables. 
XIS An array containing slowing-down cross sections for a Given material, cross sections are given in order from high to low energy, maximu of 34 , floating-point variables.

XISI An array containing first-moment slowing-down cross sections for a given material, cross sections are given in order from high to low energy, maximum of 34, floatingpoint variables. 
GENERAL INPUT DATA

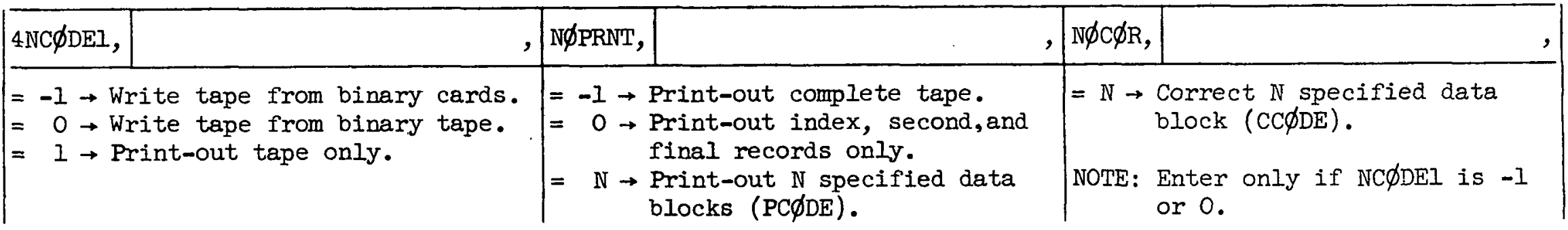

NOTE: Under the NфPRNT control, if $\mathrm{N}$ is specified, the index, second, and final records are not to be totaled in $\mathrm{N}$ as they are always printed out.

Under the $N \phi C \phi R$ control, if the index, second, and/or final records are to be corrected, they must be totaled in $\mathbb{N}$.

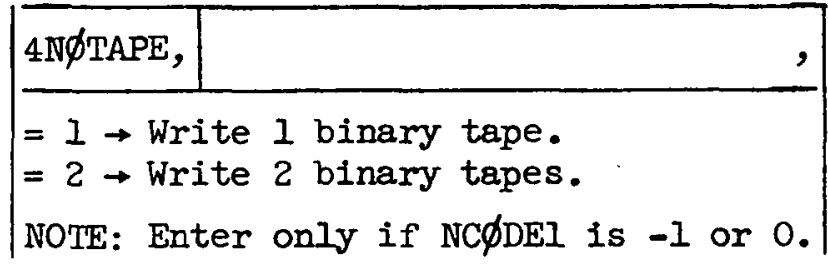


GENERAI INPUT DATA

PCфDE - Code Numbers of Blocks to be Printed 499 Code Numbers Maximum

\begin{tabular}{|l|l|l|l|l|}
\hline $3 P C \phi D E$ &, &, &, &, \\
\hline
\end{tabular}

\begin{tabular}{|l|l|l|l|l}
\hline (1), &, &, &, \\
\hline
\end{tabular}

\begin{tabular}{|l|l|l|l|l|l|}
\hline (1) & & & & & \\
\hline
\end{tabular}
CC $\varnothing \mathrm{DE}$ - Code Numbers of Blocks to be Corrected
499 Code Numbers Maximum

$\left[\begin{array}{rl}1 \times 10^{-6} & =\text { Code Index Record } \\ 2 \times 10^{-6} & =\text { Code Record } 2 \\ 0 & =\text { Code FInal Record }\end{array}\right]$

\begin{tabular}{|l|l|l|l|l|}
\hline $3 \mathrm{CC} \phi \mathrm{DE}$, &, &, &, \\
\hline
\end{tabular}

\begin{tabular}{|l|l|l|l|l|}
\hline$(1)$, &, &, &, \\
\hline
\end{tabular}
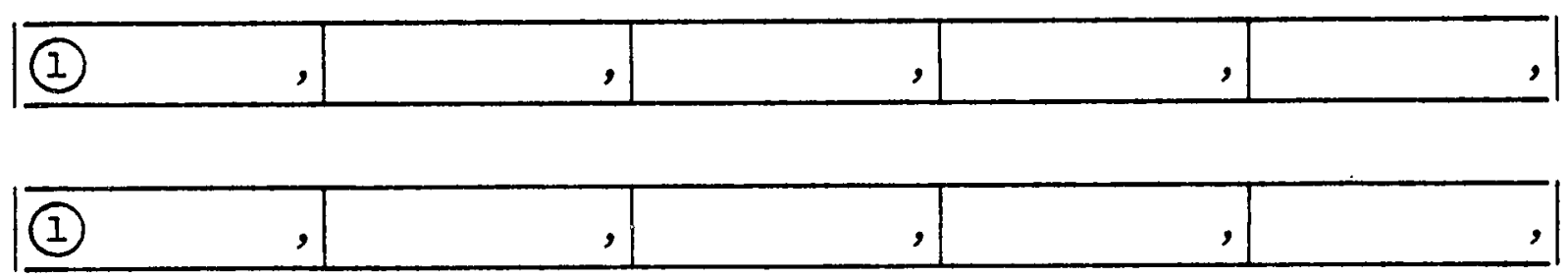

NOTE: If final record is to be corrected code number 0 must be given last.

$=\mathrm{END}$,


Tape Name

5NAME,1,

Date of Manufacture

5DATE, 2,

Number of Levels

4ILEV,

Identification of First Material

5BIDENT, 2 ,

E28100,2,

Code Numbers of Materials

\begin{tabular}{|r|r|r|r|r|r|}
\hline $3 \mathrm{BC} \phi \mathrm{DE}$, &, &, &, &, &, \\
\hline
\end{tabular}

Record Numbers of First Recorc of Each Material

\begin{tabular}{|r|r|r|r|r|r|}
\hline ANIR, &, &, &, & & \\
\hline
\end{tabular}

End of Record

\begin{tabular}{|l|l|}
\hline IEND, & 0, \\
\hline
\end{tabular}


Record Number

\begin{tabular}{|l|l|}
\hline $4 \mathrm{NIR}$, & \\
\hline
\end{tabular}

Ievel Energies

\begin{tabular}{|l|l|l|l|l|l|}
\hline 3EL, &, &, &, &, \\
\hline
\end{tabular}

Level Lethargies

\begin{tabular}{|l|l|l|l|l|l|}
\hline 3UL, &, &, &, &, \\
\hline
\end{tabular}

\begin{tabular}{|l|l|l|l|l|l|} 
Group Energies &, &, &, & \\
\hline
\end{tabular}

Group Lethargies

\begin{tabular}{|l|l|l|l|l|l|}
\hline 3UG, &, &, &, &, \\
\hline
\end{tabular}

Group 1/v Factors

\begin{tabular}{|l|l|l|r|r|r|}
\hline $3 F V$, &, &, &, &, \\
\hline
\end{tabular}

End of Record

\begin{tabular}{|l|l|}
\hline$=E N D$, & 0 \\
\hline
\end{tabular}


Record Number

\begin{tabular}{|l|l|}
\hline 4NIR, & , \\
\hline
\end{tabular}

Date

5DATE, 2,

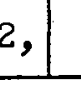

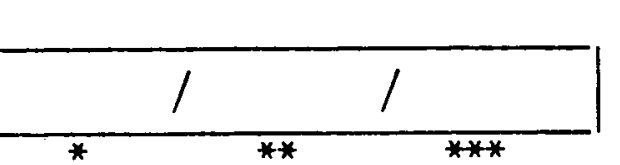

Code Number

\begin{tabular}{|l|l|}
\hline $3 \mathrm{C} \phi \mathrm{DE}$, & \\
\hline
\end{tabular}

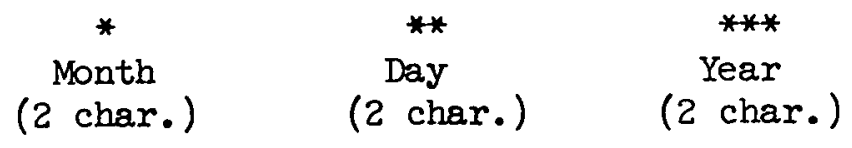

Scattering Cross Section

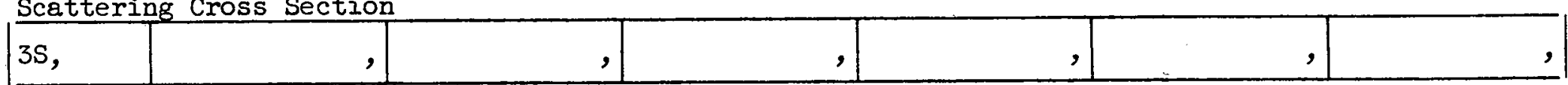

Slowing-Down Cross Section

\begin{tabular}{|l|r|r|r|r|r|}
\hline Slowing-Down Cross Section &, &, &, &, &, \\
\hline
\end{tabular}

Transport Cross Section

\begin{tabular}{|l|r|r|r|r|r|} 
Transport Cross Section &, &, &, &, \\
\hline $\mathrm{TR}$, & &, &, \\
\hline
\end{tabular}

Absorption Cross Section

\begin{tabular}{|l|r|r|r|r|r|}
\hline $3 \mathrm{~A}, \mathrm{n}$ &, &, &, &, \\
\hline
\end{tabular}

First-Moment Slowing-Down Cross Section \begin{tabular}{|l|l|l|}
\hline 3XISI, &,
\end{tabular}

,

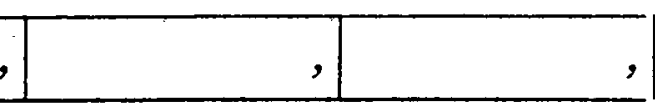

Fission Production Cross Section 3FNU, 
CORRECTIONS FOR MATERIAL DATA BLOCK RECORD ONE (continued)

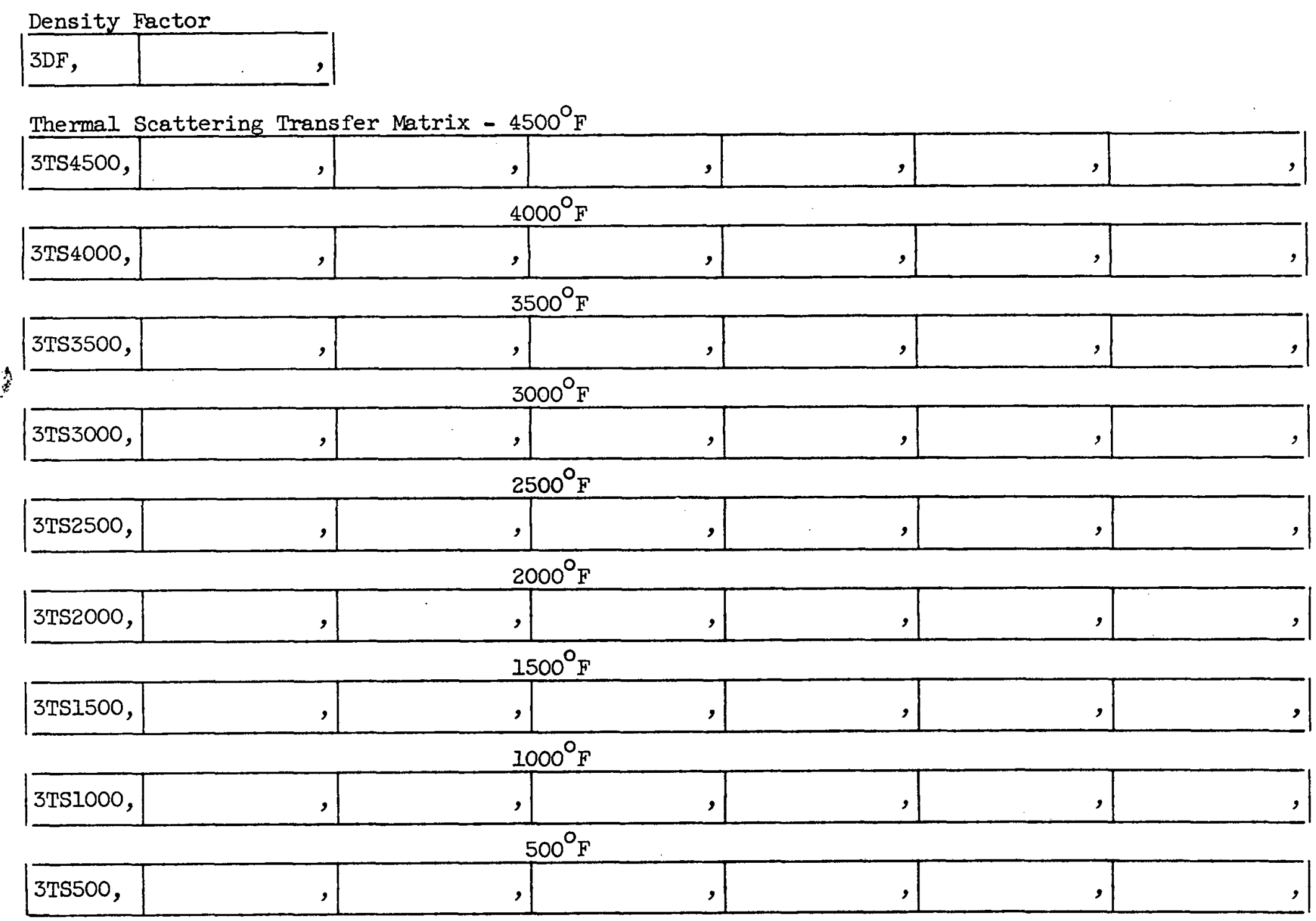


Thermal Scattering Transfer Matrix $-68^{\circ} \mathrm{F}$ \begin{tabular}{|l|r|r|}
\hline 3 TS68, &, &, \\
\hline
\end{tabular}

Flrst-Group Scatter Trans Zers

4MOMIN,

,

\begin{tabular}{|l|l|} 
Iast-Group Scatter From \\
\hline 4MOIMAX,
\end{tabular}

Last-Group Scatter Into 4MOфMAX,

First-Group Scatter One Transfers 4MIMIN,

Iast-Group Scatter One From 4MIIMAX,

Last-Group Scatter One Into 4MIфMAX,

,

Number of Resonances

4NRES, \begin{tabular}{l} 
First-Group N-Gamma Transfers \\
\hline 4MGMIN, \\
\hline
\end{tabular}

\begin{tabular}{l} 
Last-Group N-Garma From \\
\hline 4MGIMAX, \\
\hline
\end{tabular}

Last-Group N-Gamma Into

4MG $\varnothing \mathrm{MAX}$,

Number of Words in Rest of Data Block \begin{tabular}{|l|l|}
\hline $4 \mathrm{NW}$, & \\
\hline
\end{tabular} 
CCRRECTIONS FOR MATERIAL-DATA-BLOCK RECORD TWO

Record Number

\begin{tabular}{|l|l|}
\hline $4 \mathrm{~N} 2 \mathrm{R}$, & \\
\hline
\end{tabular}

Scattering Transfer Matrix

\begin{tabular}{|l|r|r|r|r|r|r|}
\hline 3SIGSO, &, &, &, & & &, \\
\hline
\end{tabular}

COFRECTIONS FOR MATERTAL-DATA-BLOCK RECORD THREE

Record Number

\begin{tabular}{|l|l|}
\hline $4 N 3 R$, & \\
\hline
\end{tabular}

First-Moment Scattering Transfer Matrix

\begin{tabular}{|l|l|l|l|l|l|l|}
\hline 3SIGSI, &, &, &, &, & &, \\
\hline
\end{tabular}


Record Number

\begin{tabular}{|l|l}
\hline $4 \mathrm{~N} 4 \mathrm{R}$, & \\
\hline
\end{tabular}

Resonance Energies

\begin{tabular}{|l|l|l|l|l|l|l|}
\hline Resonance Energies &, &, &, &, & & \\
\hline
\end{tabular}

\section{Resonance Integrals}

\begin{tabular}{|l|l|l|l|l|l|l|}
\hline Resonance Integrals &, &, &, &, & & \\
\hline
\end{tabular}

\section{Peak Cross Sections}

3PO,

,

,

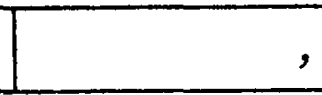

,

$|+|$

Doppler Temperature Coefficients

\begin{tabular}{|l|l|l|l|l|l|l|l|}
\hline 3R, & & & & & & & \\
\hline Eta Ratios & & & & & & \\
\hline 3ETAR, &, &, &, & & & & \\
\hline
\end{tabular}

CORRECTIONS FOR MATERTAL-DATA-BLOCK RECORD FIVE

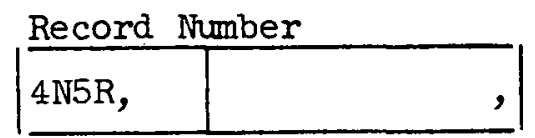

N-Gamma Transfer Matrix

\begin{tabular}{|l|r|r|r|r|r|r|}
\hline N-Gamma Transfer Matrix &, &, &, & &, \\
\hline
\end{tabular}

\begin{tabular}{|l|l|}
\hline \multicolumn{2}{|l}{ End of Record } \\
\hline END, & 0 \\
\hline
\end{tabular}


CORRECTIONS FOR SPECTRAL-DATA RECORD

Record Number
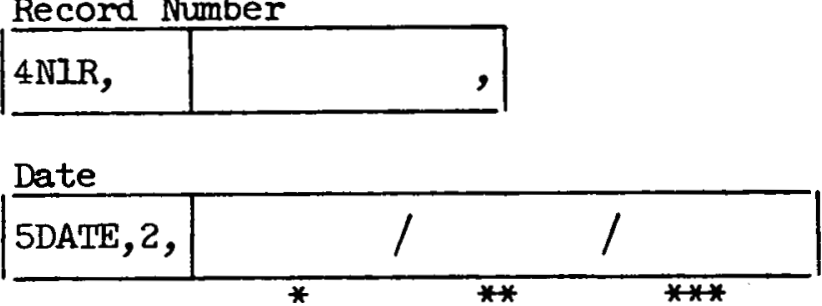

$\begin{array}{ccc}* * & * * & * * * \\ \text { Month } & \text { Day } & \text { Year } \\ (2 \text { char. }) & (2 \text { char. }) & (2 \text { char. })\end{array}$

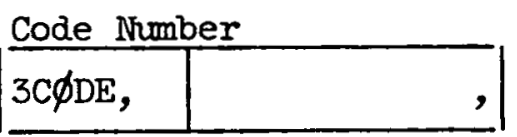

Level Spectral Values

\begin{tabular}{|l|r|r|r|r|l|l|}
\hline Level Spectral values $\phi \mathrm{R}$, &, &, &, &, &, \\
\hline
\end{tabular}

\begin{tabular}{|l|r|} 
Spectral Delay Fraction \\
\hline 3BETA,
\end{tabular}

\begin{tabular}{|l|l|} 
Mean Life \\
\hline $3 T M$, & \\
\hline
\end{tabular}

End of Record

\begin{tabular}{|l|l|}
\hline END, & 0 \\
\hline
\end{tabular}

CORRECTIONS FOR MICROSCOPIC-CONSTITUEIVTS RECORD

First Word - Record Number

4MMICR $\phi$,

\begin{tabular}{|l|l|} 
End of Record \\
\hline E END, & 0 \\
\hline
\end{tabular}




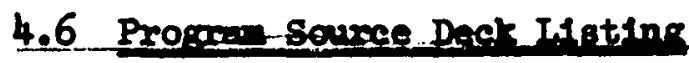

C THOMAS A HOFFMAN PROGRAM RNDC ANP NUMBER 599

C THIS IS THE RNDC CONTROL ROUTINE

c "THE CONTROL ROUTINES PREASSIGNS VALUES TO VARIOUS"

c VARIABLES, SETS THE TEMPORARY DATA ARRAYS TO ZERO,

C CLEARS THE INPUTT DATA READS THE GENERAL INPUT

C PLUS ANY PREASSIGNED VARIASLES THE USER IS DESIROUS

C OF CHANGING MAKES AN INPUT CONSISTENCY CHECK ON

C THE INPUT DATA, TRANSFERS CONTROL IN ORDER TO

c SUBROUTINES INDEX, ELIV,RAPRL, AND HAS THE FINAL STOP:

C COMMON FOR CONTROL BLOCK

COMMON DATA,PCODE, CCODE, NCODE I NOPRNT, NOCOR, NOTAPE, I TAPE,

1 ITAPE, KTAPE, NWOT, NOBLKS, NOTWDS, TEMP, THERM, NOTHGP, NLLEVS, EL, 2 NRNMCR,BNAMI, BNAM2,BCODE, NROMCR, NRSAVE

$\stackrel{c}{c}$

DIMENSION FOR CONTROL BLOCK

DIMEN SION DATA $(3454)$, PCODE (499), CCODET499), TEMP (2000, THERMT 115), EL (34), BNAM1 (499), BNAM2 (499), BCODE(499), NDATA (3454)

$c$

EQUIVALENCE FOR CONTROL BLOCK EQUIVALENCE (DATA,NDATA)

C- CONTROL INITIALIZATION ANO CONSISTENCY ROUTINE

C BEGIN EXECUTABLE "PART' OF ROUTINE

1 CONWOT $=2$

CALL WOT (NWOT)

IT $\overline{A P E}=5$

REWIND ITAPE

JTAPE $=6$

REWIND JTAPE

KTAPE $=7$

REWIND KTAPE

C CLLL NOPAGE

CALL CLEAR IDATA, TEMPI

C INITIALIZE QUASI INPUT

NOBLKS $=499$

NOTHGP $=10$

THERM $(1)=4500.0$

$\operatorname{THERM}(2)=4000.0$

$\operatorname{THERM}(3)=3500.0$

$\operatorname{THERM}(4)=3000.0$

$\operatorname{THERM}(5)=2500 \cdot 0$

$\operatorname{THERM}(6)=2000.0$

THERM $(7)=1500.0$

$\operatorname{THERM}(8)=1000 \cdot 0$

$\operatorname{THERM}(9)=500.0$

$\operatorname{THERM}(10)=68.0$ 
C... CLEAR DATA INPUT

NCODE $1=0$

NOCOR $=0$

NOTAPE $=0$

NOPRNT $=0$

DO $101 \quad I=1$, NOBLKS

CCODE (I ) $=0.0$

$P C O D E(I)=0.0$

101 CONTINUE

SENSE LIGHT 0

C... READ GENERAL INPUT

READ DIP PCODE, CCODE, NCODËI, NOCOR, NOTAPE, NOPRNT, I TAPE, JTAPE,

IKTAPE, NWOT, NOBLKS, NOTHGP, THERM, END

IF (SENSE LIGHT 1 ) 190,102

C MAKE CONSISTENCY CHECK

102 IF (NCODEI) I03,103,104

103 IF (NOTAPE) $191,191,105$

$105 \mathrm{~J}=0$

DO $106 \quad 1=1$, NOBLKS

IF (CCODE(I)) $107,108,107$

$107 \mathrm{~J}=\mathrm{J}+1$

106 CONTINUE

108 IF (NOCOR-J) $192,109,110$

$110 \mathrm{~J}=\mathrm{J}+1$

IF (NOCOR-J) $192,109,192$

104 NOCOR $=0$

NOTAPE $=0$

C TEST NOPRNT

109 IF (NOPRNT) $111,111,112$

$-112 \quad J=0$

DO $1131=1$, NOBLKS

IF (PCODE(I)) $114,115,114$

$114 \mathrm{~J}=\mathrm{J}+1$

II3 CONTINUE

115 IF (NOPRNT-J) $193,111,193$

C

PROCESS INDEX RECORD

111 NOTWDS $=$ NOBLKS $* 4+4$

CALL INDEX INCODE I, ITAPE, JTAPE,KTAPE, NOCOR, NOTAPE ONOBLKS :

INOTWDS, CCODE, TEMP, NRNMCR , NROMCR , DATA)

C DETERMINE NUMBER OF ZEVELS

NLLEVS $=$ NDATA $(4)$

C .... TRANSFER DESIGNATIONS AND CODE NUMBER

DO $118 \quad I=1$, NOBLKS

BNAMI (I ) =DATA $(I+4)$

BNAM $2(1)=$ DATA $(1+503)$

$B C O D E(I)=D A T A(I+1002)$

118 CONTINUE

PROCESS ELI/V RECORD

NOTWDS $=5 *$ NLLEVS $+2 *$ NOTHGP 3

C CLEAR STORAGE

CALL CLEAR (DATA,TEMP)

SENSE LIGHT O

CALL ELIV INCODEI, ITAPE, JTAPE,KTAPE, NOCOR, NOTAPE, NOTWDS,

1CCODE, TEMP, DATA, NLLEVS NOTHGP, THERMI

5 TRANSFER LEVEL ENERGIES 
$J=N L L E V S+N O T H G P$

DO II9 $I=1, J$

$E L(I)=D A T A(I+1)$

119 CONTINUE

C SET UP LOOP TO READ AND PROCESS THE REMAINING RECORDS

C OF BINARY DATA AND CORRECTIONS IF. ANY SENSE LIGHT O

CALL RAPRL REWIND KTAPE

IF (NOTAPE-I) 120.120 .121

121 IF (ITAPE) $194,122,123$

122 I TAPE $=5$

PRINT 124, ITAPE

124 FORMAT (1HII9X,5HTAPE I $1,23 \mathrm{H}$ COULD NOT BE PROCESSED) GO TO 120

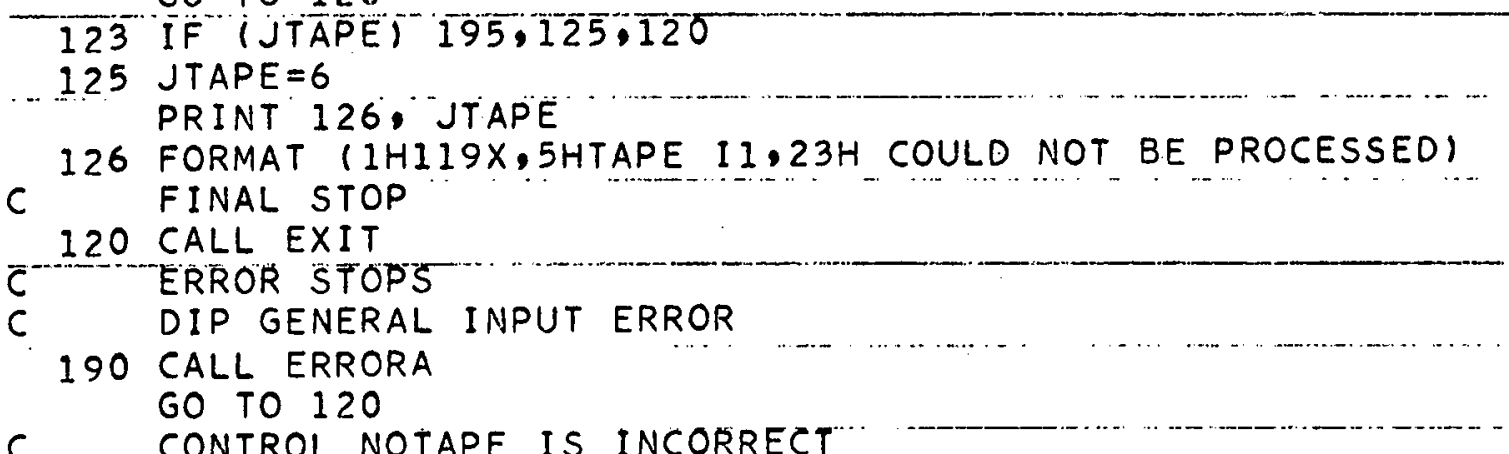

C CONTROL NOTAPE IS INCORRECT

191 CALL ERRORA

GO TOO 120

C NUMBER OF CORRECTIONS INCORRECT

192 CALL ERRORA GO TO 120

C NUMBER OF BLOCKS PRINTED INCORRECT

193 CALL ERRORA GO TO"120

C MACHINE ERROR TAPE NUMBER ITAPE IS NEGATIVE

194 CALL ERRORA GO TO 120

C MACHINE ERROR TAPE NUMBER JTAPE IS NEGATIVE

195 CALL ERRORA GO TO 120 END 
C S SUBROUTINE INDEX

C THE INDEX SUBROUTINE IS A FIRST ORDER SUBROUTINE

UNDER THE CONTROL OF THE CONTROL ROUTTINE ? THE INDEX

$\therefore \quad$ SUBROUTINE READS THE INDEX RECORD FROM TAPE ,CORRECTS

C THE INDEX RECORD IF NECESSARY - WRITES THE NEW

C INDEX RECORD ON THE NEW TAPES, AND PRINTS OUT

C THE INDEX RECORD - IF THE INDEX RECORD IS ON

C BINARY CARDS THESE ALSO ARE READ IN THIS ROUTINE.

C CONTROL IS TRANSFERRED BACK TO THE CONTROL ROUTINE.

$C$

SUBROUTINE INDEX (NCODEI, ITAPE, JTAPE, KTAPE, NOCOR,NOTAPE,NOBLKS, INOTWDS, CCODE, BIDENT, NRNMCR, NROMCR, DATA)

$\ddot{c}$

C COMMON FOR INDEX SUBROUTINE

COMMON NAME, DATE, NLEV,BNAM1, BNAM2,BCODE, NIR

$C$

C DIMENSION FOR INDEX SUBROUTINE

DIMENSION CCODE (499), BIDENT (2000),DATE(2), BNAMI (499),

IBNAM2 (499), BCODE (499), NIR(499), DATA(3454)

C - EQUIVALENCE FOR INDEXX SÜBROUUTINE

EQUIVALENCE (NAME, TNAME)

C BEGIN EXECUTABLE PART OF SUBROUTINE INDEX

C

2000 IF (NCODE1) $2100,2001,2001$

C READ BINARY TAPE

2001 READ TAPE KTAPE, NAME,DATE(1),DATE(2),NLEV, (BNAMI(I),BNAM2 (I),

$1 B C O D E(I), N I R(I), I=1$, NOBLKS)

IF (SENSE LIGHT 2) 2900,2002

C FIND OLD RECORD NUMBER OF MICROSCOPIC CONSTITUENT RECORD

2002 DO $2050 \quad I=1$, NOBLKS

IF (BCODE II) $2050,2051,2050^{\circ}$

2050 CONTINUE

GO TO 2910

2051 NROMCR $=$ NIR (I)

$C$ DETERMINE IF CORRECTIONS

DO $2006 \quad I=1, N O C O R$

If (CCODE (I) $-1.0 E-6) 2006,2008,2006$

2006 CONTINUE

NRNMCR $=$ NROMCR

GO TO 2007

C READ CORRECTIONS

$2008 \mathrm{~J}=1$

DO $2003 \mathrm{I}=1$, NOBLKS

$B I D E N T(J)=B N A M I(I)$

$B I D E N T(J+1)=B N A M 2(I)$

$J=J+2$

2003 CONTINUE

2004 READ DIP NAME, DATE, NLEV, BIDENT, BCODE, NIR, END

IF TSENSE LIGHT I) 2901,2052

C FIND NEW RECORD NUMBER OF MICROSCOPIC CONSTITUENT RECORD

$2052002053 \quad I=1$, NOBLKS

IF (BCODE (I)) $2053,2054,2053$ 
2053 CONTINUE

GO TO 2912

2054 NRNMCR $=N 1 R$ (I)

DETERMINE IF BINARY TAPES ARE TO BE WRITTEN

$J=1$

DO $2005 \quad I=1$, NOBLKS

BNAMI (I) $=$ BIDENT (J)

BNAM2 $(I)=B I D E N T(J+1)$

$J=J+2$

2005 CONTINUE

2007 IF (NOTAPE) $2904,2010,2009$

$C$ WRITE ITAPE AND CHECK

2009 WRITE TAPE ITAPE, NAME,DATE(1),DATE(2),NLEV, (BNAMI(I),BNAM2),

IBCODE (I), NIR (I), I = I, NOBLKS)

IF (SENSE LIGHT 2) 2014,2020

C ITAPE CANNOT BE WRITTEN PROPERLY

2014 IF (NOTAPE-1) 2902,2902,2019

2019 REWIND ITAPE

I TAPE $=0$

C DETERMINE IF JTAPE IS TO BE WRITTEN

2020 IF (NOTAPE-1) 2010.2010 .2022

C WRTTE JTAPE AND CHECR

2022 WRITE TAPE JTAPE,NAME,DATE(1),DATE(2), NLEV, (BNAMI I I),BNAM2 (I),

IBCODE (I),NIR (I), I = I, NOBLKS)

IF (SENSE LIGHT 2) 2026,2010

C PRINT OUT INDEX RECORD

2010 PRINT 2041,DATE(1),DATE(2), NLEV

2041 FORMAT (IH156X,5HDATE 2A677730X,I 2HINDEX RECORDTTII 33.7H LEVELSH $1 X, 4$ HDATA $/ 8 X, 9 H B L O C K$ NO.5X,8HCODE NO.6X, IIHDESIGNATION6X,1OHRECORD

$2 \mathrm{NO} \cdot 111$

DO $2032 \quad I=1$, NOBLKS

PRINT 2033,I, BCODE(I), BNAMI (I), BNAM2 (I) ONIRTI)

2033 FORMAT $(114, F 18,4,4 \times, 2 A 6,112)$

IF TT-40*(IT40)T $2032,2035,2032$

2035 PRINT 2036,NLEV

2036 FORMAT (1HI////30X,12HINDEX RECORD///133,7H LEVELSTIOX, 4RDATA78X,9 1HBLOCK NO.5X,8HCODE NO.6X,11HDESIGNAT ION $6 \mathrm{X}, 1$ OHRECORD NO $/ 1 / 1$

2032 CONTINUE

GO TO 2034

C JTAPE CANNOT BE WRTTTEN PROPERLY

2026 REWIND JTAPE

JTAPE $=0$

C DETERMINE IF ITAPE HAS BEEN WRITTEN PROPERLY

IF (ITAPE) $2903,2903,2010$

C READ BINARY CARDS

REAO HOLLERITH IDENTIFICATION CARD

2100 READ INPUT TAPE 2.2101

2 IOIFORMAT $(1 \mathrm{H})$

CALL ACBIP

$\bar{C}^{\cdots}$ STORE INOEX DATA PROPERLY

DO $2055 I=1$, NOBLKS

BIDENT $(I+4)=D A T A T 4 * I+1)$

BIDENT $(I+503)=$ DATA $(4 * I+2)$

BIDENT $(I+1002)=$ DATA $(4 * I+3)$

BIDENT $(I+150 I)=D A T A(4 * I+4)$ 
2055 CONT I NUE $J=4 * N O B L \overline{K S}$

_. DO $2056 I=1, J$

2056 DATA $(I+4)=$ BIDENT $(I+4)$

2056 CONTINUE

GO TO 2002

C ERROR STOPS

C KTAPE CANNOTT BE READ PROPERLY

2900 CALL RIJKT (ITAPE,JTAPE,KTAPE)

2905 CALL ERRORA

GO TO 2914

C DIP ERROR IN CORRECTIONS

2901 CALL RIJKT (ITAPE,JTAPE,KTAPE)

2906 CALL ERRORA

GO TO 2914

C ITAPE CANNNOT BE WRITTEN PROPERLY

2902 CALL RIJKT (ITAPE, JTAPE, KTAPE)

2907 CALL ERRORA

GO TO 2914

C NEITHER ITAPE NOR JTAPE CAN BE WRITTEN PRDPERLY

2903 CALL RIJKT (ITAPE, JTAPE,KTAPE)

2908 CALL ERRORA

GO TO 2914

C MACHINE ERROR CONTROL NOTAPE IS NEGATIVE

2904 CALL RIJKT (ITAPE,JTAPE,KTAPE)

2909 CALL ERRORA

GO TO 2914

C MORE THAN 499 BLOCKS ON INDEX RECORD

2910 CALL RIJKT (ITAPE, JTAPE,KTAPE)

2911 CALL ERRORA

GO TO 2914

C ….... MORE THAN 499 BLOCKS ON INDEX RECORD

2912 CALL RIJKT (ITAPE, JTAPE,KTAPE)

2913 CALL ERRORA

GO TO 2914

2914 CALL EXIT

2034 RETURN

END 


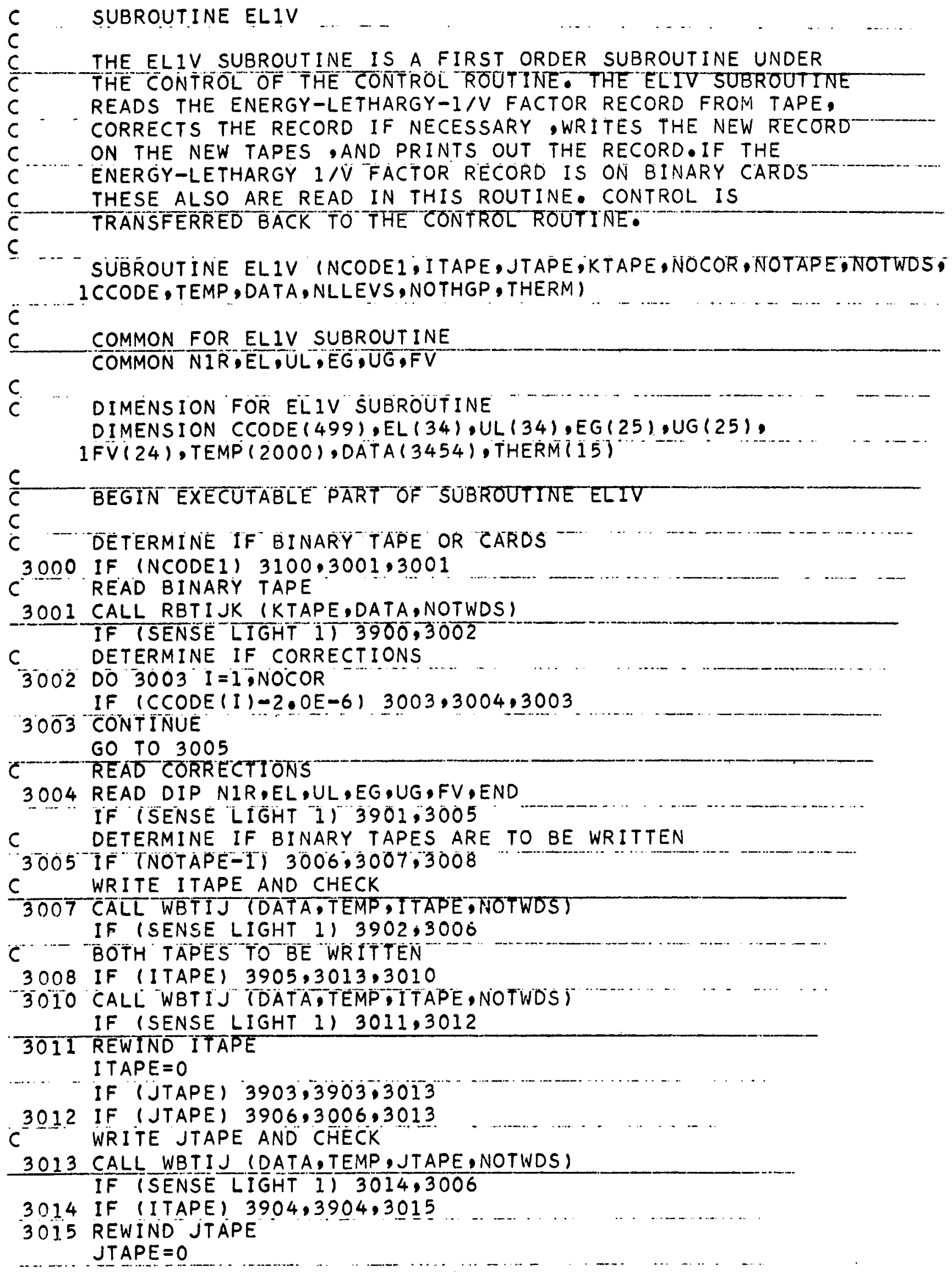


C PRINT OUT ELIV RECORD

3006 PRINT 3020

3020 FORMAT (IH115X,5HLEVEL $5 X$, 11HENERGY (EV) 7X,8HLETHARGY $/ 1$ )

DO $3025 I=1$,NLLEVS

IF $((1-1)-5 *((1-1) / 5)) 3022,3023,3022$

3022 PRINT 3024, I,EL(I),ULII)

3024 FORMAT (119,1PE19.7,E17.7)

GO TO 3025

3023 PRINT 3026, I,EL(I),ULII)

3026 FORMAT (1HO118,1PE19.7,E17.7)

3025 CONTINUE

$J=N L L E V S+1$

DO $3027 \quad I=1$, NOTHGP

$K=$ THERM(I)

If $(1 I-1)-5 *((I-1) / 5)) 3028,3029,3028$

BO28 PRINT $3030 . \mathrm{K}, E L(J), U L(J)$

3030 FORMAT (I 20,1PE18.7.E17.7)

$J=J+1$

GO TO 3027

3029 PRINT 3031 , K.ELIJ).UL(J)

3031 FORMAT (1HO119,IPE18.7.E17.7)

$J=J+1$

3027 CONTINUE

C PRINT SECOND PAGE ELIV RECORD

PRINT 3032, EG(1),UG (1)

3032 FORMAT (1H120X,5HLOWER12X,5HUPPER9X, 1OHIV FACTOR78X.5HGROUP5X.1IR

1ENERGY (EV) 7X,8HLETHARGY7X,12H(2200 M/SEC)//10X,1HO,1PE19.7,E17.7)

DO $30331=1$ NLLEVS

IF $(I-5 *(1 / 5)) \quad 3034,3035,3034$

3034 PRINT 3036 , IDEG(I+1), UG I +1) OFV(I)

3036 FORMAT (111,1PE19.7,1PE17.7,1PE17.7)

GO TO 3033

3035 PRINT 3038 , I,EG $(I+1)$,UG $(I+1)$,FV(I)

3038 FORMAT T1HOI10.1PE19.7.1PEI7.7.1PEIT.7)

3033 CONTINUE

60703037

c READ BINARY CARDS

C READ HOLLERITH IDENTIFICATION CARD

3100 READ INPUT TAPE 2,3101

3101 FORMAT (1H)

CALL ACBIP

GO TO 3002

C ERROR STOPS

C KTAPE CANNOT BE READ PROPERLY

3900 CALL RI JKT (ITAPE, JTAPE, KTAPE)

3907 CALL ERRORA

GO TO 3914

C DIP ERROR IN CORRECTIONS

3901 CALL RI JKT (ITAPE,JTAPE,KTAPE)

3908 CALL ERRORA

GO TO 3914

C ITAPE CANNOT BE WRITTEN PROPERLY

3902 CALL RIJKT (ITAPE, JTAPE, KTAPE)

3909 CALL ERRORA

GO TO 3914 
C ITAPE CANNOT BE WRITTEN PROPERLY LTARE ALREADY FAILED 3903 CALL RIJKT (ITAPE, JTAPE, KTAPE)

3910 CALL ERRORA

GO TO 3914

C

JTAPE CANNOT BE WRITTEN PROPERLY ITAPE ALREADY FAILED

3904 CALL RIJKT (ITAPE, JTAPE,KTAPE)

3911 CALL ERRORA

GO TO 3914

C MACHINE ERROR TAPE NUMBER ITAPE IS NEGATIVE

3905 CALL RI JKT IITAPE, JTAPE, RTAPE)

3912 CALL ERRORA

GO TO 3914

C MACHINE ERROR TAPE NUMBER JTAPE IS NEGATIVE

3906 CALL RIJKT (ITAPE, JTAPE,KTAPE)

3913 CALL ERRORA

GO TO 3914

3914 CALL EXIT

3037 RETURN

END 
SUBROUTINE RAPRL

$c$
$C$

$\vdots$
$C$
$C$
$C$
$C$
$C$
$C$
$C$
$C$
$C$
$C$
$C$
$C$

THE RAPRL SUBROUTINE IS A FIRST ORDER SUBROUTINE UNDER

THE CONTROL OF THE CONTROL ROUTINE. THE RAPRL

SUBROUTINE DOES NOT PROCESS ANY RECORS BUT ACTS AS A

$C$ SECONDARY CONTROL ROUTINE FOR SUBROUTINES DBLKR,

C SPECT AND MICRO. RAPRL. WILL READ ONE BINARY

C RECORD FROM TAPE OR ONE BLOCK OF BINARY CARDS,

C TEST THE CODE NUMBER OF THE RECORD OR BLOCK

C AND TRANASER CONTROL TO THE PROPER SUBROUTINE: THIS

C SUBROUTINE TRANSFERS CONTROL BACK TO RAPRL WHICH THEN

C PROCESSES THE NEXT CODE NUMBER. THIS PROCESS CONTINUES

$C$ UNTIL THE MICROSCOPIC CONSTITUENTS RECORD IS PROCESSED.

C AT WHICH TIME RAPRL TRANSFERS CONTROL TO THE

C CONTROL ROUTINE

C COMMON FOR SUBROUTINE RAPRL

COMMON DATA,PCODE, CCODE, NCODE I, NOPRNT, NOCOR, NOTAPE, I TAPE,

1 JTAPE, KTAPE, NWOT , NOBLKS, NOTWDS, TEMP, THERM, NOTHGP, NLLEVS, EL,

2 NRNMCR, BNAM1, BNAM $2, B C O D E$, NROMCR, NRSAVE

C -.... DIMENSION FOR SUBROUTINE" RAPRL

DIMENSION DATA (3454), PCODE (499), CCODE (499), TEMP (2000), THERM(15),

IEL (34), BNAM1 (499), BNAM2 (499), BCODE (499), NDATA (3454)

$C$ EQUIVALENCE FOR SUBROUTINE RAPRL

EQUIVALENCE (DATA,NDATA)

c

C BEGIN EXECUTABLE PART OF SUBROUTINE RAPRL

8000 NRSAVE $=3$

C TEST IF LESS THAN THREE RECORDS

IF (NROMCR-3) 8900,8001,8001

8001 DO $8011 \mathrm{~N}=1$,NOBLKS

CALL CLEAR (DATA,TEMP)

IF (NCODEI) $8002,8003,8003$

C READ BINARY CARDS

C READ HOLLERITH IDENTIFICATION CARD

8002 READ INPUT TAPE 2,8004

8004 FORMAT TIH )

CALL ACBIP

GO TO 8005

C READ BINARY TAPE KTAPE

8003 SENSE LIGHT 0

IF (BCODE (N)) $8016,8013,8016$

$8016^{\circ}$ IF IBCODE (N)-500.0) $8012,8014,8014$

8012 NOTWDS $=6 *$ (NLLEVS+NOTHGP) $(30 *$ NOTHGP $)+16$

GO TO 8015 .

8013 NOTWOS $=1000$

GO TO 8015

8014 NOTWDS =NLLEVS+NOTHGP+6

8015 CALL RBTI JK (KTAPE, DATA, NOTWDS)

IF (SENSE LIGHT 1) 8901,8005

6. DETERMINE WHAT RECORD

8005 IF (NROMCR-NDATA) $8902,8006,8007$ 
C MICROSCOPIC CONSTITUENT RECORD

8006 SENSE LIGHT 0

NOTWDS $=1000$

CALL MICRO INCODEI, NOCOOR,NOTAPE,ITAPE,JTAPE, KTAPE,NOTWOS,

I TEMP, DATA, CCODE, NRNMCR, NRSAVE, NROMCR, BCODE, BNAMI, BNAM 2 , NOBLKS) GO TO 8008

C DETERMINE IF SPECTRAL OR DATA BLOCK

8007 IF (DATA $(4)-500.0) 8009,8010.8010$

C SPECTRAL

$8010 "$ SENSE LIGHT"

NOTWDS $=$ NLLEVS+NOTHGP +6

CALL SPECT IDATA,PCODE, CCODE, NCODE I, NOPRNT, NOCOR, NOTAPE, ITAPE:

1 JTAPE , KTAPE, NOTWDS, TEMP, THERM, NOTHGP, NLLEVS, EL, BNAMI, BNAM2,

$2 B C O D E, N O B L K S, N R S A V E)$

GO TO 8011

C DATA BLOCK

8009 SENSE LIGHT 0

SENSE LIGHT O

NOTWDS $=6 *($ NL.LEVS+NOTHGP) $(30 *$ NOTHGP $)+16$

CALL DBLKR IDATA,PCODE, CCODE, NCODFI, NOPRNT ; NOCOR, NOT APE,

1 I TAPE, JTAPE, KTAPE, NOTWDS, TEMP, THERM, NOTHGP, NLLEVS, EL , BNAMI,

ZBNAM2, BCOODE, NOBLKS,NRSAVET

8011 CONTINUE

GO TO 8902

C ERROR STOPS

C LESS THAN THREE RECORDS ON KTAPE

8900 CALL RIJKT (ITAPE, JTAPE,KTAPE)

8903 CALL ERRORA

GO TO 8906

C KTAPE CANNOT BE PROCESSED CORRECTLY

8901 CALL RIJKT (ITAPE, JTAPE,KTAPE)

8904 CALL ERRORA

GO TO 8906

C … RECORD NUMBER OF MICROSCOPIC CONST ITUENT RECORD DOES NOT

C AGREE WITH FINAL RECORD NUMBER ON INDEX RECORD

8902 CALL RIJKT (ITAPE,JTAPE,KTAPE)

8905 CALL ERRORA

GO TO 8906

8906 CALL EXIT

8008 RETURN

END 


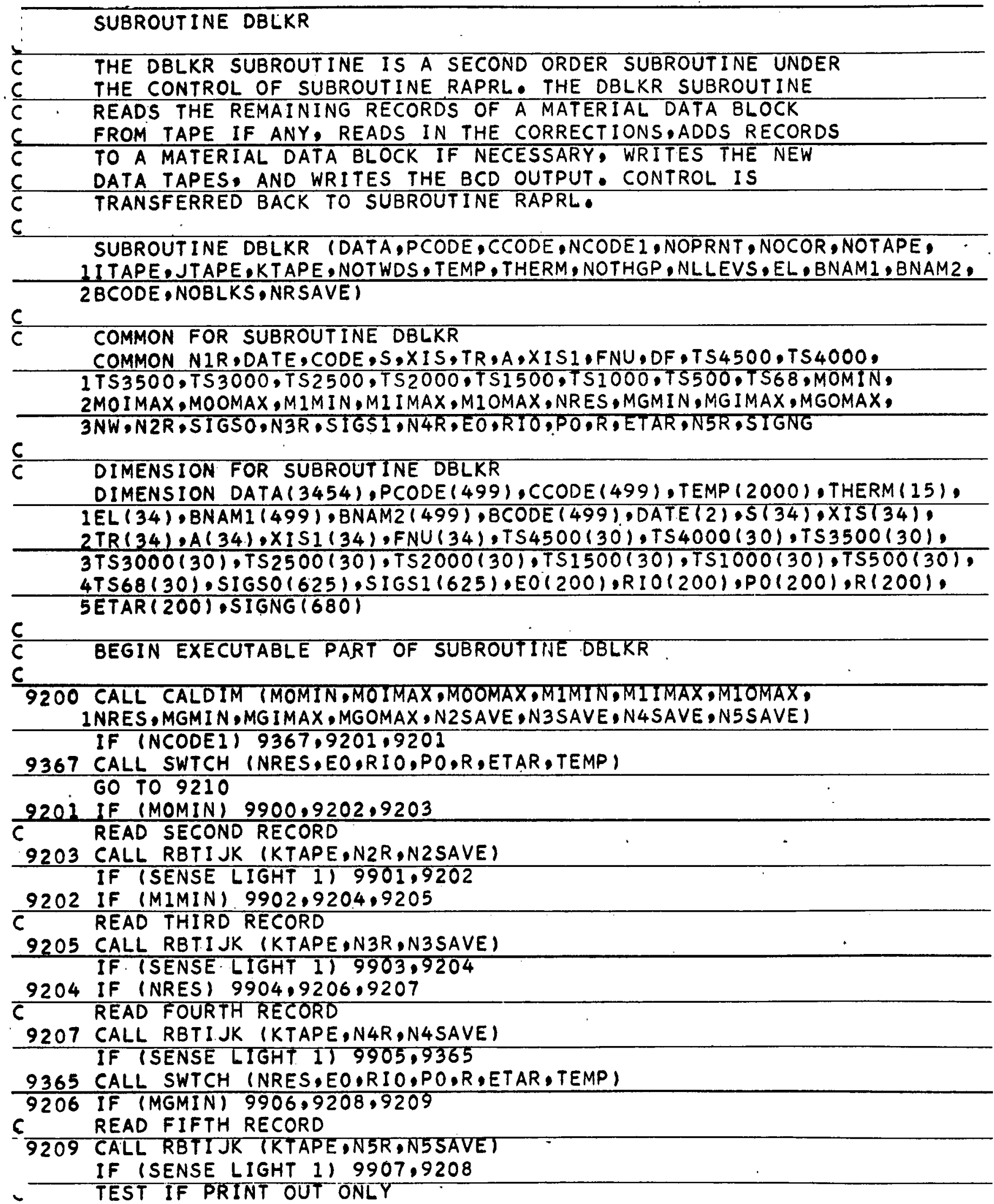


9208 IF (NCODEI) $9908,9210,9211$

6 TEST IF CORRECTIONS

$9210009212 \quad I=1, N O C O R$

IF (CCODE (I)-CODE) $9212,9213,9212$

9212 CONTINUE GO TO 9214

C READ CORRECTIONS

9213 READ DIP NIR,DATE,CODE, S,XIS,TR,A,XISI,FNU,DF,TS4500,TS4000, ITS3500,TS3000,TS2500,TS2000,TS1500,TS1000, TS500, TS68,MOMIN,MOIMAX. 2 MOOMAX, MIMIN, MI IMAX, MIOMAX, NRES, MGMIN, MG I MAX, MGOMAX,

$3 N W, N 2 R, S I G S O, N 3 R, S I G S 1, N 4 R, E O, R I O, P O, R$, ETAR,N5R, SI GNG,END IF (SENSE LIGHT 1) 9909.9214

9214 CALL CALDIM IMOMIN,MOIMAX,MOOMAX, MIMINOMIIMAX, MIOMAX,NRES, IMGMIN, MGIMAX , MGOMAX, N2SAVE, N3SAVE, N4SAVE, N5SAVE) DO 9368 I I I.NRES

$J=I+N R E S$

-

9368 CONTINUE DO 9369 I $=1, \bar{N} R \bar{R} \bar{S}$ $J=I+2 * N R E S$ EO(J) $=$ POII)

9369 CONTINUE DO 9370 I $=1$ INRES $J=I+3 *$ NRES $E O(J)=R(I)$

9370 CONTINUE DO 9371 I $=1$, NRES

$J=I+4 * N R E S$ EO(J) $=E$ TAR(I)

9371 CONTINUE

C SET UP PROPER RECORD NUMBERS

NIR = NRSAVE NRSAVE $=$ NRSAVE +1

IF (MOMIN) $9976,9330,9331$

$9331 N 2 R=N R \overline{S A V E}$ NRSAVE = NR SAVE +1

9330 IF TMIMINI $9977,9332,9333$

$9333 N 3 R=N R S A V E$ NRSAVE = NR SAVE +1

9332 IF (NRES) $9978,9334,9335$

$9335 N 4 R=N R S A V E$ NRSAVE $=$ NR SAVE +1

9334 IF (MGMIN) $9979,9336,9337$

9337 NSR =NRSAVE

NRSAVE $=$ NRS SAVE +1

C WRITE HOW MANY 3 INARY TAPES

9336 IF (NOTAPE-1) 9910.9215 .9216

C ITAPE ONLY

$9215^{\circ}$ CALL WBTIJ (N.IR,TEMP, ITAPE,NOTWDS)

IF (SENSE LIGHT 1 ) 9217.9218

C CHECK ERROR

9217 REWIND ITAPE

I TAPE $=0$

IF (NOTAPE-1) $9911,9911,9219$

9219 IF TJTAPET $9912,9912,9372$ 


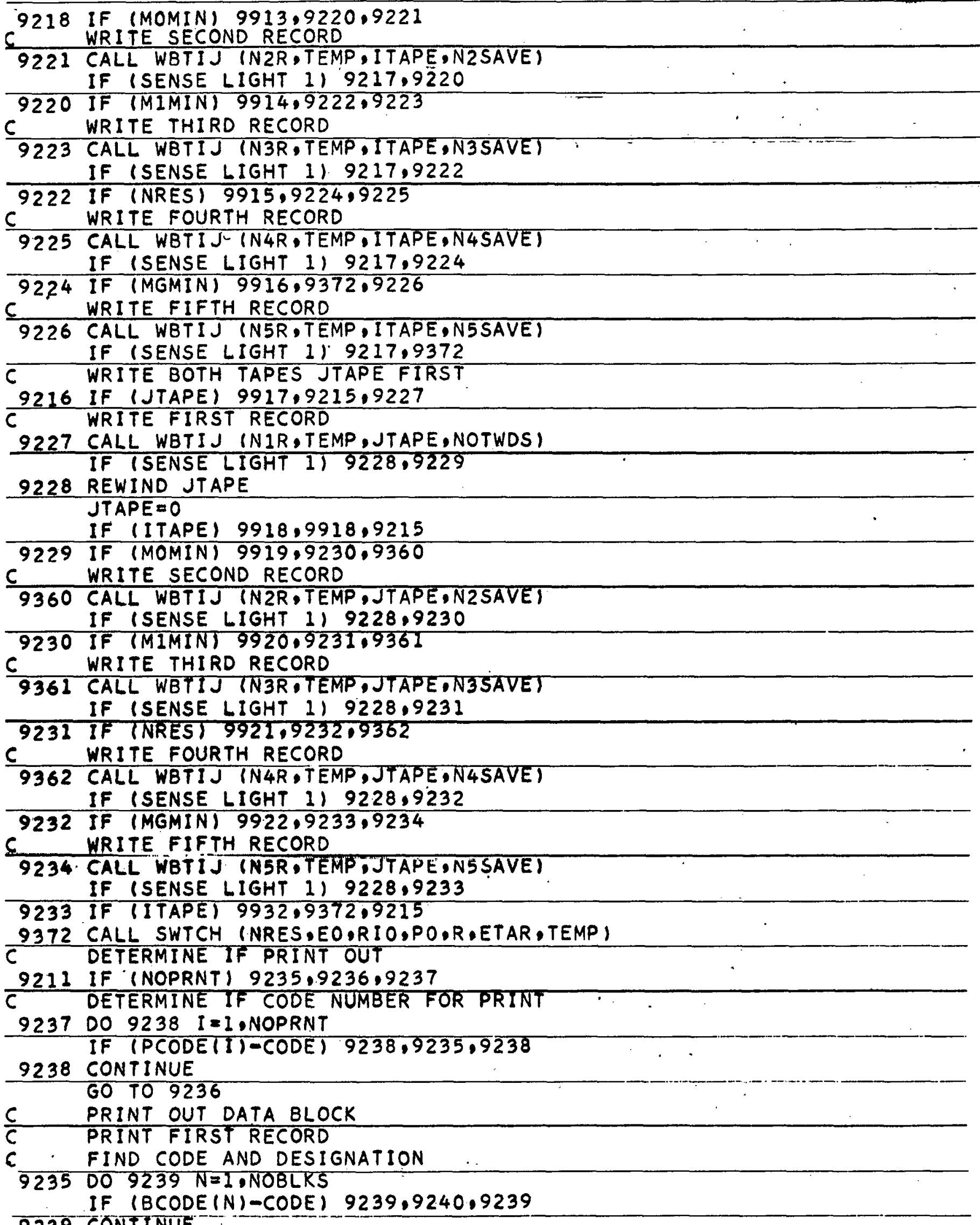

9239 CONTINUE 
GO TO 9923

9240 PRINT 9241, DATE (1),DATE (2), BNAM1 (N), BNAM2 (N),BCODE (N),DF

9241 FORMAT (IH157X,5HDATE 2A6///9X,11HDESIGNATION10X,8HCODE NO,10X,14H IDENSITY FACTOR//9X,2A6,F17.4, IPE22,5,///18X,5HSIGMA5X,8HXI SIGMA4X 2.5HSIGMA6X.5HSIGMA5X.9HXII SIGMA/7OH LEVEL ENERGY SCATTER 'SC 3ATTER TRANSPORT ABSORPTION SCATTER 11

DO $9242 I=1$,NLLEVS

IF $((I-1)-5 *((I-1) / 5)) 9243,9244,9243$

9243 PRINT 9245, I.EL(I),S(I),XIS(I),TR(I),AII).XISITI

9245 FORMAT (I4,IPEII.3,4E11.4,E12.4)

GO TO 9242

9244 PRINT 9246, I,EL(I),S(I),XIS(I),TR(I),A(I),XISI(I)

9246 FORMAT (1HO13,1PEI1.3,4E11.4.E12.4)

9242 CONTINUE

$J=N L L E V S+1$

DO $9247 \quad I=1$, NOTHGP

$K=$ THERM II)

IF $((1-1)-5 *((I-1) / 5) 19248,9249,9248$

9248 PRINT $9250, \mathrm{~K}, E L(J), S(J), \overline{X I S(J), T R(J), A T J), X I S I T J) ~}$

9250 FORMAT (I5.IPE 10.3.4E11.4,E12.4)

$J=J+1$

GO TO 9247

9249 PRINT $9251, \bar{K}, E L(J), S(J), \bar{X} \bar{S}(J), T R(J), A(J), \overline{X I S I(J) ~}$

9251 FORMAT (1HOI4,1PE10.3,4E11.4,E12.4)

$J=J+1$

9247 CONTINUE

C

C PAGE 2 RECORD 1 DATA BLOCK PRINT OUT

C

PRINT 9252, BNAMI (N),BNAM2 $(N), B C O D E(N), D F$

9252 FORMAT (IHI8X,IIHDESIGNATIONIOX.8HCODE NO.1OX.14HDENSITY FACTOR//9

$1 X, 2 A 6, F 17,4,1$ PE $22,5, / / / 43 X, 8 H N U$ SIGMA/21X,5HLEVEL $5 X, 6 H E N E R G Y 7 X, 7 H F$

2ISSION)

DO $9253 I=1$,NLLEVS

IF $(I I-1)-5 *((I-I) / 5)) 9254,9255.9254$

9254 PRINT 9256 , I,EL(I), FNU(1)

9256 FORMAT (I24,IPE 14.3, E 14.4$)$

GO TO 9253

9255 PRINT 9257 . I.EL(I).FNU(I)

9257 FORMAT (IHOI23,IPE14.3,E14.4)

9253 CONTINUE

$J=N L L E V S+1$

DO 9258 I $=1$ INOTHGOP

$K=\operatorname{THERM(I)}$

IF $((I-1)-5 *((I-1) / 5)) 9259,9260.9259$

9259 PRINT $9261, K, E L(J), F N U(J)$

9261 FORMAT (I25.IPE 13.3, E I4.4)

$J=J+1$

GO TO $9258^{\circ}$

9260 PRINT 9262, K,EL(J),FNU(J)

9262 FORMAT (IHOI24,1PE13.3,E14.4)

$J=J+1$

9258 CONTINUE

c

PAGES 3 THROUGH 7 RECORD I DATA BLOCK PRINT OUT 
$9264 K=1$

DO $9275 \quad I=1$,NOTHGP

IF $((I-1)-2 *((I-1) / 2)) 9276.9277 .9276$

9277 PRINT 9278 , BNAM1 (N),BNAM2(N)

9278 FORMAT 1 IH129X,2A6///23X,25HTHERMAL TRANSFER MATRICES I

$9276 \mathrm{~J}=I+N L L E V S$

PRINT 9279, TS4500(K),TS4500(K+1),TS4500(K+2), TS4500(K 3), TS4500 $1(K+4) \cdot J \cdot T S 4500(K+5) \cdot T S 4500(K+6) \cdot T S 4500(K+7) \cdot T S 4500(K+8), T S 4500(K$ $2+9), J, T S 4500(K 10), T S 4500(K+11), T S 4500(K+12) \cdot T S 4500(K+13), T S 4500$

$3(K+14), J, T S 4500(K+15), T S 4500(K+16), T S 4500(K+17), T S 4500(K+18)$.

$4 T S 4500(K+19), J, T S 4500(K+20) \cdot T S 4500(K+21) \cdot T S 4500(K+22), T S 4500(K+$ $523) \cdot T S 4500(K+24), J, J, J, J, J, J, T S 4500(K+25), T S 4500(K+26) \cdot T S 4500$

$6(K+27), T 54500(K+28), T S 4500(K+29)$

9279 FORMAT $1 / / / / / 3 X, 11$ HTEMPERATURE $5 X, 5 H S I G M A 9 X, 5 H S I G M A 9 X, 5 H S I G M A 8 X, 8 H N$

IU SIGMA/ $4 X, 8$ HDEG F.6X,7HSCATTER $6 X, 9$ HTRANSPOR $5 X, 1$ IHABSORPTION5X, 27HFISSION//1PE13.4,4E14.4///31X,2HN15X,2HN2/28X,15HSIGMA S (N1/N2)

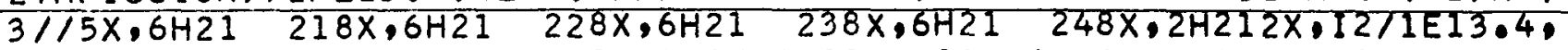

$44 E 14,4 / 5 X, 6 H 22 \quad 218 X, 6 H 22 \quad 228 X, 6 H 22 \quad 238 X, 6 H 22 \quad 248 X, 2 H 222 X, 12 / 1 E$

$513.4,4 \mathrm{E} 14.415 \mathrm{X}, 6 \mathrm{H} 23 \quad 218 \mathrm{X}, 6 \mathrm{H} 23 \quad 228 \mathrm{X}, 6 \mathrm{H} 23 \quad 238 \mathrm{x}, 6 \mathrm{H} 23 \quad 248 \mathrm{X}, 2 \mathrm{~Hz} 32 \mathrm{X}$.

$6 I 2 / 1 \mathrm{E} 13.4,4 \mathrm{E} 14.4 / 5 \mathrm{X}, 6 \mathrm{H} 24 \quad 218 \mathrm{x}, 6 \mathrm{H} 24 \quad 228 \mathrm{X}, 6 \mathrm{H} 24 \quad 238 \mathrm{X}, 6 \mathrm{H} 24 \quad 248 \mathrm{X}, 2 \mathrm{H}$

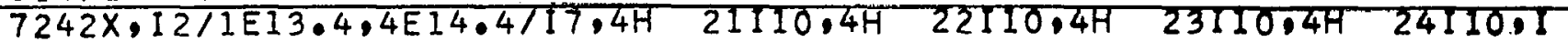

$84 / 1 E 13.4,4 E 14.41$

$K=K+30$

9275 CONTINUE

$\bar{C}$

C PAGE 8 RECORD 1 DATA BLOCK PRINT OUT

C

PRINT 9280, BNAMI (N), BNAM2 (N)

9280 FORMAT (IH130X.2A6/7/7/T/T/

IF (MOMIN) $9925,9281,9282$

9282 PRINT 9283, MOMIN,MOIMAX ,MOMIN,MOOMAX

9283 FORMAT $132 \mathrm{X}, 8 \mathrm{HSIGMA}$ SO/16X,21HTRANSFERS FROM GROUPSI3,8H THROUGHI 3

$1,10 \mathrm{H}$ TO GROUPSI $3,8 \mathrm{H}$ THROUGHI37777

9281 IF (MIMIN) $9926,9284,9285$

9285 PRINT 9286 , MIMIN,MIIMAX,MIMIN,MIOMAX

9286 FORMAT $(32 X, 8$ HSIGMA S1/16X,21HTRANSFERS FROM GROUPSI $3,8 H$ THROUGHI 3

$1,10 \mathrm{H}$ TO GROUPSI $3,8 \mathrm{H}$ THROUGH $13 / 17 / 1$

9284 IF (NRES) $9927,9287,9288$

9288 PRINT 9289, NRES

9289 FORMAT $(29 \times, 14 H N O$. RESONANCES//137////)

9287 IF (MGMIN) 9928.9290.9291

9291 PRINT 9292. MGMIN,MGIMAX,MGMIN,MGOMAX

9292 FORMAT $(28 X, 15 H S I G M A$ IN.GAMMA) $/ 76$, 21HTRANSFERS FROM GROUPSI 3.8H T IHROUGHI3, 1OH TO GROUPS $13,8 \mathrm{H}$ THROUGHI $3 / / / / 1$

$\bar{C}$

9290 BNAMX1 $=B N A M 1(N)$

$B N A M \times 2=B N A M 2(N)$

$c$

C DETERMINE IF SECOND RECORD IS PRESENT

IF (N2R) $9933,9293,9294$

C PRINT SECOND RECORD OF DATA BLOCK

9294 NFORK $=0$

CALL PSOING (MOMIN,MOIMAX,N2SAVE BNAMXI, BNAMX Z NFORK STGSOI

DETERMINE IF THIRD RECORO IS PRESENT 
C

9293 IF (N3R) $9929,9296,9297$

PRINT THIRD RECORD DATA BLOCK

9297 NFORK $=1$

CALL PSOING (MIMIN, MIIMAX,N3SAVE, BNAMX I, BNAMX 2,NFORK, SIGSI)

$\ddot{C}$

$\ddot{C}$

9296 IF $^{-1 N 4 R)} 9930.9299,9300$

C PRINT FOURTH RECORD

9300 PRINT 9301 , BNAMI (N), BNAM2 $(N)$

9301 FORMAT (1H130X,2A6///3X, 9HRESONANCE5X,9HRESONANCE $8 X, 4 H P E A K / 5 X, 6 H E N$ IERGY 7X,8HINTEGRAL 4X; 13 HCROSS SECTION6X,1HR 12X,3HETA//) DO $9302 \quad 1=1$, NRES

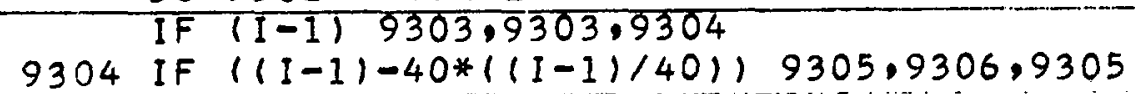

9306 PRINT 9307 , BNAMI (N), BNAM2TNS

9307 FORMAT (1H130X,2A6///3X, 9HRESONANCE $5 X, 9 H R E S O N A N C E 8 X, 4 H P E A K / 5 X, 6 H E N$ 1ERGY $7 X, 8 H I N T E G R A L 4 \bar{X}, 13$ HCROSS SECTION6X,1HR12X,3HETA//1)

9305 IF $((I-1)-5 *((I-1) / 5)) 9303,9308,9303$

9303 PRINT 9309 , EOIII.RIOTII.POTII.RIIT,ETARTIT

9309 FORMAT (IPE13.4,4E14.4)

GO TO 9302

9308 PRINT 9310, EO(I),RIO(I),PO(I),R(I),ETAR(I)

9310 FORMAT (1HOIPEI2.4,4E14.4)

9302 CONTINUE

C

C DETERMINE IF FIFTH RECORD IS PRESENT

C 299 IF (NSR) $9931,9236,9311$

C PRINT FIFTH RECORD

9311 NFORK $=2$

CALL PSOING TMGMIN MG IMAX ,N5SAVE, BNAMXI, BNAMX2,NFORK, SIGNG

GO TO 9236

¿ ERROR STOPS

C MACHINE ERROR MOMIN IS NEGATIVE

9900 CALL RIJKT (ITAPE, JTAPE,KTAPE)

9938 CALL ERRORA

GO TO 9984

C RECORD 2 DATA BLOCK KTAPE COULD NOT BE READ PROPERLY

9901\% CALL RIJKT' (ITAPE, JTAPE,KTAPE)

9939 CALL ERRORA

GO TO 9984

C MACHINE ERROR MIMIN IS NEGATIVE

9902 CALL RIJKT (ITAPE, JTAPE, RTAPE)

9940 CALL ERRORA

GO TO 9984

C. RECORD 3 DATA BLOCK KTAPE COULD NOT BE READ PROPERLY

9903 CALL RIJKT (ITAPE, JTAPE,KTAPE)

9941 CALL ERRORA

GO TO 9984

f. MACHINE ERROR NRES IS NEGATIVE

$990^{\circ}$ CALL RIJRT' (ITAPE, JTAPE,RTAPE)

9942 CALL ERRORA

GO TO 9984 
C RECORD 4 DATA BLOCK KTAPE COULD NOT BE READ PROPERLY

9905 CALL RI JKT (ITAPE,JTAPE,KTAPE)

9943 CALL ERRORA

GO TO 9984

C MACHINE ERROR MGMIN IS NEGATIVE

9906 CALL RIJKT (ITAPE, JTAPE,KTAPE)

9944 CALL ERRORA

GO TO 9984

C RECORD 5 DATA BLOCK KTAPE COULD NOT BE READ PROPERLY

9907 CALL RIJKT (ITAPE,JTAPE,KTAPE)

9945 CALL ERRORA

GO TO 9984

C MACHINE ERROR NCODEI MINUS AT ILLOGICAL POINT

9908 CALL RIJKT (ITAPE,JTAPE,KTAPE)

9946 CALL ERRORA

GO TO 9984

C ERROR IN READING DIP CORRECTIONS

9909 CALL RIJKT (ITAPE,JTAPE,KTAPE)

997 CALL ERRORA

GO TO 9984

C MACHINE ERROR MOMIN IS NEGATIVE

9976 CALL RIJKT (ITAPE, JTAPE,KTAPE)

9980 CALL ERRORA

GO TO 9984

C MACHINE ERROR MIMIN IS NEGATIVE

9977 CALL RIJKT (ITAPE,JTAPE,KTAPE)

9981 CALL ERRORA

GO TO 9984

C MACHINE ERROR NRES IS NEGATIVE

9978 CALL RIJKT (ITAPE, JTAPE,KTAPE)

9982 CALL ERRORA

GO TO 9984

C MACHINE ERROR MGMIN IS NEGATIVE

9979 CALL RI JKT (ITAPE, JTAPE,KTAPE)

9983 CALL ERRORA

GO TO 9984

C MACHINE ERROR NOTAPE ZERO AT ILLOGICAL POINT

9910 CALL RI JKT (ITAPE, JTAPE,KTAPE)

9948 CALL ERRORA

GO TO 9984

C A RECORD OF DATA BLOCK ITAPE COULD NOT BE WRITTEN PROPERLY

9911 CALL RIJKT (ITAPE, JTAPE,KTAPE)

9949 CALL ERRORA

GO TO 9984

C A RECORD OF DATA BLOCK ITAPE COULD NOT BE WRITTEN

C PROPERLY JTAPE ALREADY FAILED

9912 CALL RIJKT (ITAPE, JTAPE, RTAPE)

9950 CALL ERRORA

GO TO 9984

C MACHINE ERROR MOMIN IS NEGATIVE

9913 CALL RIJKT (ITAPE, JTAPE,KTAPE)

9951 CALL ERRORA

GO TO 9984

C MACHINE ERROR MIMIN IS NEGATIVE

9914 CALL RIJKT TITAPE,JTAPE,KTAPE) 
9952 CALL ERRORA

GO TO 9984

C MACHINE ERROR NRES IS NEGATIVE

9915 CALL RIJKT (ITAPE, JTAPE, KTAPE)

9953 CALL ERRORA

GO TO 9984

$C$ MACHINE ERROR MGMIN IS NEGATIVE

9916 CALL RIJKT (ITAPE, JTAPE, KTAPE)

9954 CALL ERRORA

GO TO 9984

C MACHINE ERROR TAPE NUMBER. JTAPE IS NEGATIVE

9917 CALL RIJKT (ITAPE, JTAPE, KTAPE)

9955 CALL ERRORA

GO TO 9984

C A RECORD OF DATA BLOCK JTAPE COULD NOT BE WRITTEN PROPERLY

C ITAPE ALREADY FAILED

9918 CALL RI JKT (ITAPE, JTAPE, KTAPE)

9956 CALL ERRORA

GO TO 9984

C MACHINE ERROR MOMIN IS NEGATIVE

9919 CALL RIJKT (ITAPE, JTAPE OKTAPE)

9957 CALL. ERRORA

GO TO 9984

C MACHINE ERROR MIMIN IS NEGATIVE

9920 CALL RI JKT (ITAPE, JTAPE, KTAPE)

9958 CALL ERRORA

GO TO 9984

C MACHINE ERROR NRES IS NEGATIVE

9921 CALL RIJKT (ITAPE, JTAPE, KTAPE)

9959 CALL ERRORA

GO TO 9984

C MACHINE ERROR MGMIN IS NEGATIVE

9922 CALL RIJKT (ITAPE.JTAPE, RTAPE)

9960 CALL ERRORA

GO TO 9984

C MACHINE ERROR TAPE NUMBER ITAPE IS NEGATIVE

9932 CALL RIJKT (ITAPE, JTAPE. KTAPE)

9961 CALL ERRORA

GO TO 9984

C CODE NUMBER OF DATA BLOCK NOT PRESENT IN INOEX RECORD

9923 CALL RIJKT (ITAPE, JTAPE, OKTAPE)

9962 CALL ERRORA

GO TO 9984

C MACHINE ERROR MOMIN IS NEGATIVE

9925 CALL RIJKT (ITAPE, JTAPE, RTAPE)

9964 CALL ERRORA

GO TO 9984

C MACHINE ERROR MIMIN IS NEGATIVE

9926 CALL RIJKT (ITAPE, JTAPE, KTAPE)

9965 CALL ERRORA

GO TO 9984

6 MACHINE ERROR NRES IS NEGATIVE

9927 CALL RIJKT (ITAPE OJTAPE ,KTAPE)

9966 CALL ERRORA

GO TO 9984 
C MACHINE ERROR MGMIN IS NEGATIVE

9928 CALL RIJKT (ITAPE IJTAPE,KTAPE)

9967 CALL ERRORA

GO TO 9984

C MACHINE ERROR N2R IS NEGATIVE

9933 CALL RI JKT (ITAPE, JTAPE,KTAPE)

9968 CALL ERRORA GO TO 9984

C MACHINE ERROR N3R IS NEGATIVE

9.929 CALL RI JKT (ITAPE, JTAPE,KTAPE)

9969 CALL ERRORA GO TO 9984

C MACHINE ERROR N4R IS NEGATIVE

'9930 CALL RIJKT (ITAPE, JTAPE,KTAPE)

9970 CALL ERRORA GO TO 9984

C MACHINE ERROR N5R IS NEGATIVE

9931 CALL RIJKT (ITAPE, JTAPE,KTAPE)

9971 CALL ERRORA GO TO 9984

9984 CALL EXIT

c.

9236 RETURN END 
C SUBROUTINE SPECT

$C$

C THE SPECT SUBROUTINE IS A SECOND ORDER SUBROUTINE UNDER

C THE CONTROL OF SUBROUTINE RAPRL. THE SPECT SUBROUTINE

C READS IN THE CORRECTIONS, WRITES THE NEW DATA TAPES, AND

C W....WRITES THE BCD OUTPUT FOR A SPECTRAL RECORD - CONTROL

C IS TRANSFERRED BACK TO SUBROUTINE RAPRL.

$\underline{C}$

SUBROUTINE SPECT IDATA,PCODE, CCODE,NCODEI,NOPRNT, NOCOR,NOTAPE, 1 I TAPE, JTAPE, KTAPE, NOTWDS, TEMP, THERM, NOTHGP, NLLEVS, EL , BNAMI, 2BNAM2, BCODE, NOBLKS, NIR SAVE)

C

C COMMON FOR SUBROUTINE SPECT

COMMON NIR, DATE, CODE, SOR, BETA, TM

$\bar{c}$

C DIMENSION FOR SUBROUTINE SPECT

DIMENS ION DATA $(3454)$, PCODE(499), CCODE $(499)$, TEMP(2000), THERMI

$115)$, EL (34),DATE(2), SOR (34), BNAMI (499), BNAM2 (499), BCODE (499)

C

C BEGIN EXECUTABLE PART OF SUBROUTINE SPECT

$\mathrm{C}$

9100 DO $9101 \quad I=1$, NOCOR

IF (CCODE (I)-CODE) 9101,9102,9101

9101 CONTINUE

GO TO 9103

C READ DIP CORRECTIONS

9102 READ DIP NIR,DATE, CODE, SOR, BETA,TM,END

IF (SENSE LIGHT I) 9190.9103

9103 NIR $=$ NRSAVE

NRSAVE = NRSAVE+1

C TEST NCODEI

IF (NCODE 1) $9104,9104,9105$

9104 IF (NOTAPE-1) $9105,9106,9107$

C WRITE ITAPE AND CHECK

9106 CALL WBTI J (DATA,TEMP, ITAPE,NOTWDS)

IF (SENSE LIGHT 1 ) 9191,9105

C WRITE AND CHECK BOTH TAPES

9107 IF (ITAPE) $9195,9108,9109$

9109 CALL WBTIJ (DATA,TEMP, ITAPE, NOTWDS)

IF (SENSE LIGHT 1 ) 9110.9111

9110 REWIND ITAPE

I TAPE $=0$

IF (JTAPE) $9192,9192,9108$

9111 IF (JTAPE) $9196,9105,9108$

9108 CALL WBTIJ (DATA, TEMP, JTAPE, NOTWDS)

IF (SENSE LIGHT 1 ) 9112,9105

9112 IF (ITAPE) $9193,9193.9113$

9113 REWIND JTAPE

JTAPE $=0$

C TEST FOR PRINT OUT

9105 IF (NOPRNT) 9114,9115.9116

\& DETERMINE IF THIS RECORD IS TO BE PRINTED

9116 DO $9117 \mathrm{I}=1$, NOPRNT

$5 \quad$ IF (PCODE (I)-CODE) 9117.9114 .9117

9117 CONTINUE 
GO TO 9115

LOCATE DESIGNATION

9114 DO $9118 \mathrm{~J}=1$,NOBLKS

IF $(B C O D E(J)-C O D E) \quad 9118,9119,9118$

9118 CONTINUE

GO TO 9194

C PRINT THIS RECORD OUT

9119 PRINT 9120, DATE (1),DATE (2),BNAMI (J),BNAM2 (J),BETA,TM

9120 FORMAT (1H156X,5HDATE 2A6///34X,5HDELAY11X,9HPRECURSOR/12X,1IHDESI IGNATIONIOX,8HFRACTION9X,9HMEAN LIFE/112X,1A6,1A6,1PE18,4,1PE18,4//

$2 / 17 X, 5 H L E V E L 6 X, 6$ HENERGY9X,8HSPE(TRUM)

DO $9121 \quad 1=1$,NLLEVS

IF $((1-1)-5 *(1)-1) / 5)) 9122,9123,9122$

9122 PRINT 9124 , I,EL(I),SOR(I)

9124 FORMAT (I21,1PE15.3.E18.7)

GO TO 9121

9123 PRINT 9125, I,EL(I),SOR(I)

9125 FORMAT (1HOI20.1PE15.3.E18.7)

9121 CONTINUE $J=N L L E V S+1$

DO $9126 \quad I=1$,NOTHGP

$K=T H E R M(I)$

IF $((I-1)-5 *((I-1) / 5)) 9127,9128,9127$

9127 PRINT $9129, K, E L(J), S O R(J)$

9129 FORMAT $(122,1$ PE1 $4,3, E 18.7)$

$J=J+1$

GO TO 9126

9128 PRINT 9130, K,EL(J),SOR (J)

9130 FORMAT (IHOI21,IPE14.3.E18.7)

$J=J+1$

$9 \overline{126}$ CONTINUE

GO TO 9115

C ERROR STOPS

C ERROR IN READING DIP CORRECTIONS

9190 CALL RIJKT (ITAPE, JTAPE,KTAPE)

9197 CALL ERRORA

GO TO 9135

C ERROR IN WRITING ITAPE

9191 CALL RIJKT (ITAPE OJTAPE,KTAPE)

9198 CALL ERRORA

GO TO 9135

C ERROR IN WRITING ITAPE JTAPE ALREADY FAILED

9192 CALL RIJKT (ITAPE,JTAPE,KTAPE)

9199 CALL ERRORA

GO TO 9135

C ERROR IN WRITING JTAPE ITAPE ALREADY FAILED

9193 CALL RIJKT (ITAPE, JTAPE,KTAPE)

9131 CALL ERRORA

GO TO 9135

C SPECTRAL CODE NUMBER NOT PRESENT IN INDEX RECORD

9194 CALL RIJKT (ITAPE, JTAPE,KTAPE)

69132 CALL ERRORA

GO TO 9135

f MACHINE ERROR TAPE NUMBER ITAPE IS NEGATIVE

9I95 CALL RIJKT (ITAPE, JTAPE,KTAPE) 
9133 CALL ERRORA GO TO 9135

C MACHINE ERROR TAPE NUMBER JTAPE IS NEGATIVE

9196 CALL RIJKT (ITAPE, JTAPE,KTAPE)

9134 CALL ERRORA

GO TO 9135

9135 CALL EXIT

9115 RETURN

END 


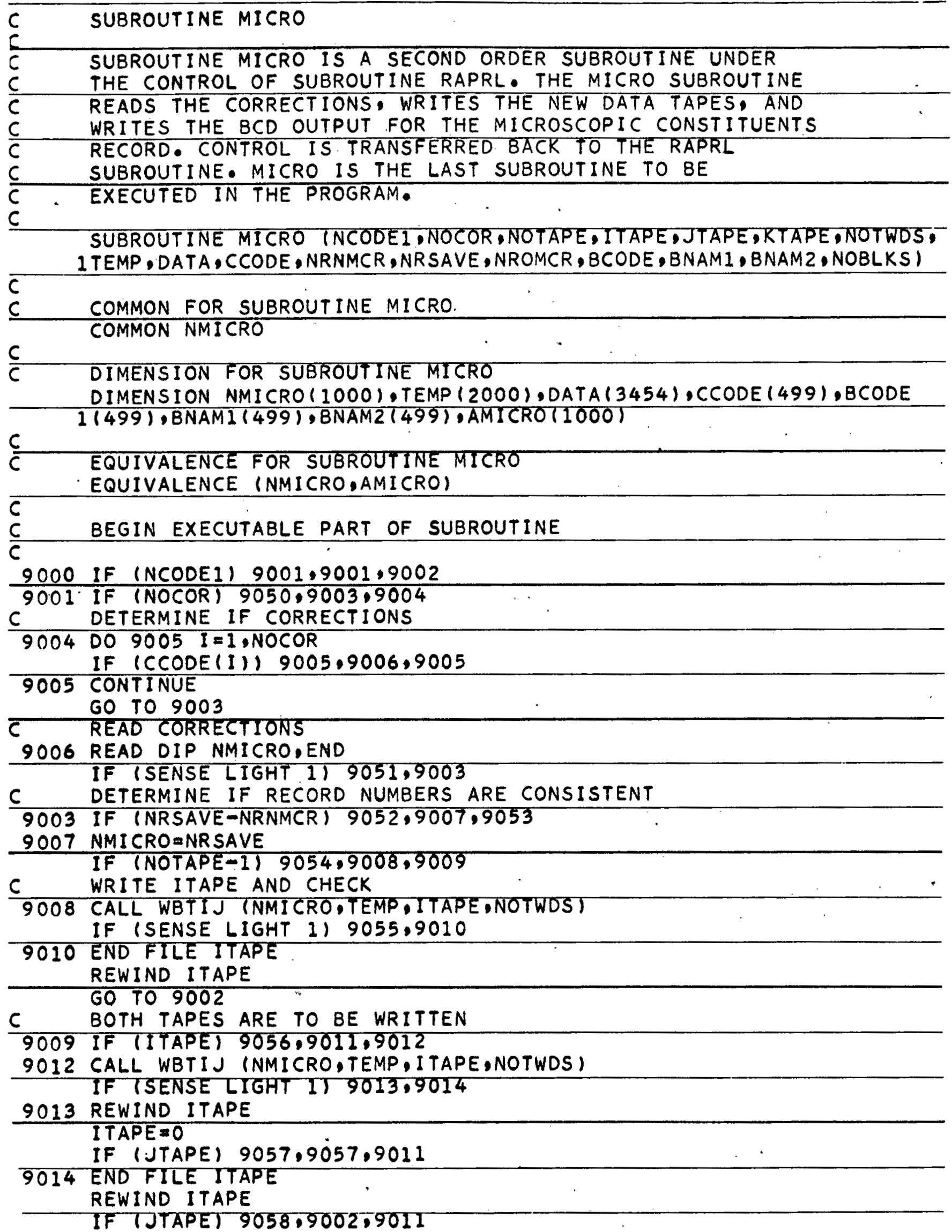


9011 CALL WBTIJ (NMICRO, TEMP, JTAPE, NOTWDS)

IF (SENSE LIGHT 1 ) 9015,9016

9016 END FILE JTAPE

REWIND JTAPE

GO TO 9002

9015 IF (ITAPE) $9059,9059,9017$

9017 REWIND JTAPE

JTAPE $=0$

C

C PRINT OUT MICROSCOPIC CONSTITUENT RECORD

$9002 \mathrm{~N}=2$

9029 IF (NMICRO(N)) $9020,9021,9020$

9020 DO $9022 I=1$, NOBLKS

IF (AMICROIN)-BCODE(I)) $9022,9023,9022$

9022 CONTINUE

GO TO 9060

C PRINT HEADING

9023 PRINT 9024, NMICRO (N), BNAMI (I), BNAM2 (I)

9024 FORMAT I IHI22X,26HMACROSCOPIC CROSS SECTIONS//27X,18HCONSTITUENT T 1 ABLES///13X,2OHMACROSCOPIC CODE NO.15X,11HDESIGNATION/F 28,4,20X,2A $26 / / 10 X, 11$ HCONST I TUENT $9 X, 1$ IHDESIGNATIONIIX,6HWEIGHT/11X,9HCODE NO. $331 \times, 8 H F R A C T I O N / / 1$

C SET UP PRINT LOOP

$I=N M I C R O(N+1)$

DO $9025 \mathrm{~J}=1, \mathrm{I}$

DO $9026 K=1$, NOBLKS

IF (BCODE $(K)$-AMICRO $(N+2)) \quad 9026.9027 .9026$

9026 CONTINUE

GO TO 9061

9027 PRINT 9028, NMICRO $(N+2), B N A M I(K), B N A M 2(K), N M I C R O(N+3)$

9028 FORMAT (F21.4.9X,2A6,IPE 18.4)

$N=N+2$

9025 CONTINUE

$\mathrm{N}=\mathrm{N}+2$

GO TO 9029

9021 IF (NMICRO(2)) 9030.9031 .9030

9031 PRINT 9032

9032 FORMAT (43HINO MACROSCOPIC DATA BLOCKS PRESENT ON TAPE)

GO TO 9033

C DETERMINE IF MICROSCOPICS HAVE BEEN CORRECTED

9030 PRINT 9034

9034 FORMAT (IHI)

$N=2$

9041 IF (NMICRO(N)) $9035,9033,9035$

$9035 M=N$

$I=N M I C R O(N+1)$

DO $9036 J=1 . N O C O R$

$N=M$

DO $9037 \mathrm{~K}=1, \mathrm{I}$

IF (CCODE (J)-AMICRO $(N+2)) 9038,9039,9038$

9039 PRINT 9040. CCODE (J),NMICRO(M)

9040 FORMAT $110 \mathrm{H}$ MATERIALIX,FII.4,35H WHICH IS A CONSTITUENT OF MATERI

IALIX,F11.4/30X, 18HHAS BEEN MODIFIED. I

GO TO 9036

$3038 \mathrm{~N}=\mathrm{N}+2$ 


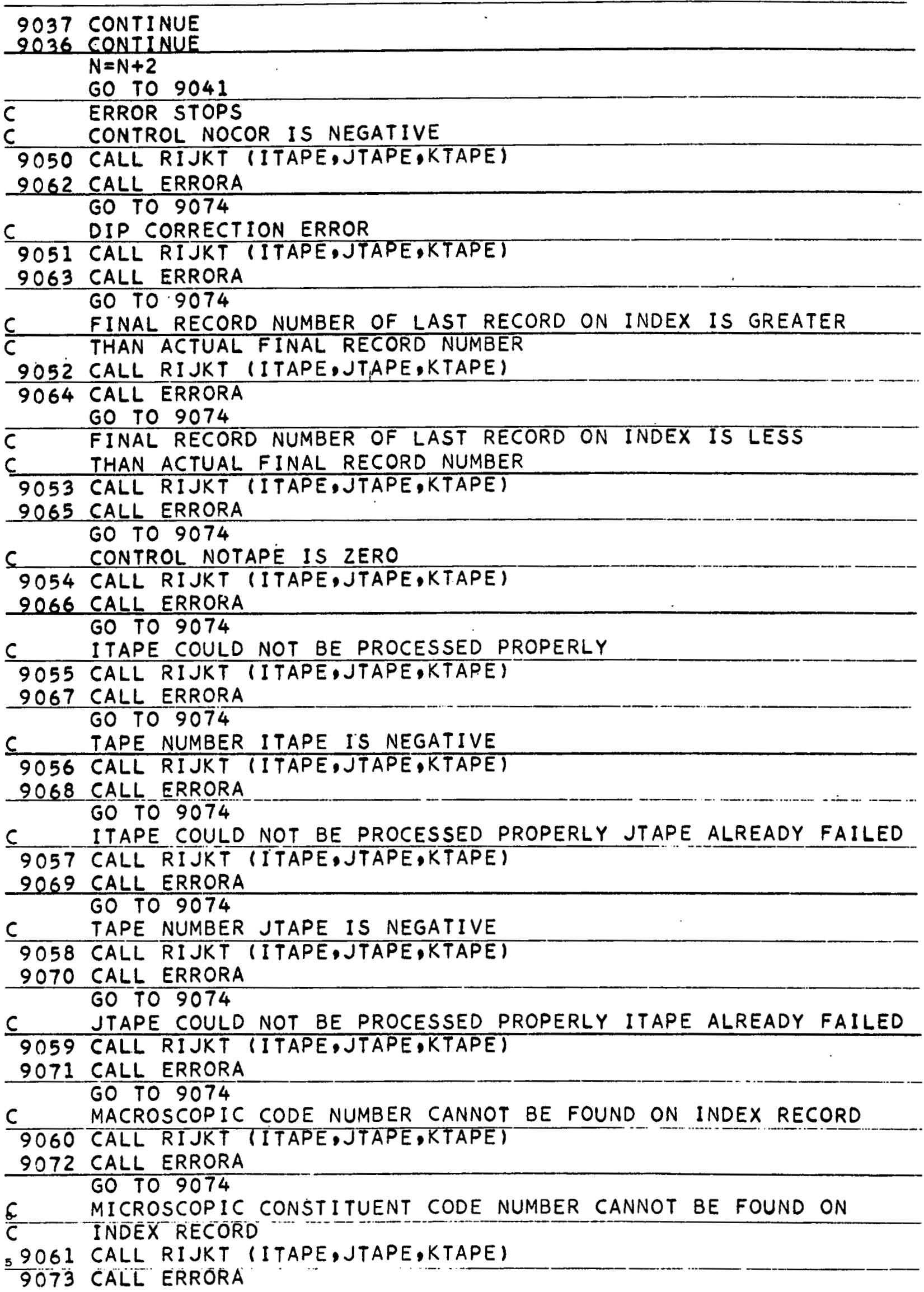




\section{GO TO 9074}

9074 CALL EXIT

C EXIT TO CONTROL BLOCK

9033 RETURN

END 
C SUBROUTINE CALDIM

$c$

C SUBROUTINE CALDIM

CALCULATE THE NUMBER OF WORDS IN THE SCATTERING:

C FIRST MOMENT SCATTERING, AND N-GAMMA TRANSFER MATRICES

$C$ AND THE RESONANCE DATA ARRAY OF A MATERIAL DATA BLOCK.

C SUBROUTINE CALDIM IS USED ONLY IN CONJUNCTION WITH

C SUBROUTINE DBLKR.

C

SUBROUTINE CALDIM (MOMIN,MOTMAX, MOOMAX,MIMINOMI IMAX OMIOMAXINRES, MGMIN, MG IMAX, MGOMAX, N2SAVE, N3SAVE, N4 SAVE, N5SAVEI

$C$

C BEGIN EXECUTABLE PART OF SUBROUTINE CALDIM

C

9800 N2SAVE $=($ MOIMAX MOMIN+1)*(MOOMAX-MOMIN+1) +1 N3SAVE $=(M I I M A X$ MIMIN+I)*(MIOMAX-MIMIN+I) II N4SAVE $=5 * N R E S+1$ N5SAVE $=($ MGIMAX MGMIN+1)*(MGOMAX-MGMIN+I) + I RETURN 
$c$ SUBROUTINE CLEAR

C

C SUBROUTINE CLEAR IS A THIRD ORDER SUBROUTINE USED TO

C SET THE TWO TEMPORARY STORAGE ARRAYS TO ZERO:

C SUBROUTINE CLEAR IS USED IN CONJUNCTION WITH THE

C CONTROL ROUTINE AND SUBROUTINE RAPRL.

$\mathrm{C}$

C DIMENSION FOR CLEAR SUBROUTINE

DIMENSION DATA $(3454)$, TEMP $(2000)$
C BEGIN EXECUTABLE PART OF SUBROUTINE CLEAR

1000 DO $1001 \quad I=1.3454$

$\operatorname{DATA}(I)=0.0$

1001 CONTINUE

DO $1002 \quad I=1 \cdot 2000$

TEMP $(i)=0.0$

1002 CONTINUE

RETURN

END 
c. ... SUBROUTINE PSOING

C THE PSOING SUBROUTINE IS A THIRD ORDER SUBROUTINE UNDER

C THE CONTROL OF SUBROUTINE DBLKR. THE PSOING SUBROUTINE

- PRINTS OUT THE SCATTERING, FIRST MOMENT SCATTERING, AND

C N-GAMMA TRANSFER MATRICES. CONTROL IS TRANSFERRED BACR

C TO SUBROUTINE DBLKR.

c

SUBROUTINE PSOING (N1,N2,NWDS, BNAMX1,BNAMX2,NFORK, ARRAY)

C DIMENSION FOR SUBROUTINE PSOING

DIMENSION ARRAY (681)

C BEGIN EXECUTABLE PART OF SUBROUTINE PSOING

C

$970011=1$

$12=(N W D S-1) / 5$

I $3=N W D S-5 * 12-1$

$N 3=(N W D S-1) /(N 2-N 1+1)$

$J 5=N 1$

$K 5=N 1-1$

9724 IF $(K 5-N 3) 9701,9702,9701$

$9702 J 1=J 5+1$

$K I=N I$

GO TO 9703

$9701 \mathrm{JI}=\mathrm{J} 5$

$K 1=K 5+1$

9703 IF TRI-N3) $9704,9705,9704$

$9705 \mathrm{~J} 2=\mathrm{J} 1+1$

$\mathrm{K} 2=N I$

GO TO 9706

$9704 J 2=J 1$

$K 2=K 1+1$

9706 IF TK2-N3T 9707.9708 .9707

$9708 \mathrm{~J} 3=\mathrm{J} 2+1$

K3 $=N I$

60 TO 9709

$9707 \sqrt{3}=\mathrm{J} 2$

$K 3=K 2+1$

9709 IF IR3-N3) 9710.9711 .9710

$9711 \quad \sqrt{4}=\mathrm{J} 3+1$

R4=NI

GO TO 9712

$9710-J 4=-\sqrt{3}$

$K 4=K 3+1$

9712 IF $(K 4-N 3) \quad 9713.9714 .9713$

$9714 \mathrm{~J} 5=\mathrm{J} 4+1$

$R 5=N 1$

GO TO 9715

$-9713-15=J 4$

$K 5=K 4+1$

9715 IF $(1-12) 9716.9717 .9718$

9716 If $((11-1)-20 *((11-1) / 20)) 9717,9719.9717$

9719 IF (NFORK-I) $9720,972 I ; 9722$

9717 PRINT $9723, J 1, K 1, J 2, K 2, J 3, K 3, J 4, K 4, J 5, K 5, A R R A Y(5 * I 1-4)$, 
258

IARRAY (5*I 1-3),ARRAY (5*11 2) ARRAY (5*11-1),ARRAY(5*I1)

9723 FORMAT $(17,14,41110,14) / 1$ PE13.4.4E14.4)

$I 1=I 1+1$

$60-109724$

9718 IF $(13-1) 9732,9726,9727$

9727 IF $(I 3-3) \quad 9728,9729,9730$

9726 PRINT 9731 , JI,KI,ARRAY (5*I1-4)

9731 FORMAT (IT,14/1PE13.4 GO TO 9732

9728 PRINT $9733, J 1, K 1, J 2, K 2$, ARRAYT5*I 41,ARRAYTS*II-3

9733 FORMAT $(17,14,110,14 / 1$ PE13.4,E14.4) GO TO 9732

9729 PRINT 9734, J1,K1,J2,K2,J3,K3,ARRAY (5*I1-4),ARRAY (5*I I 3),ARRAY $1(5 * I 1-2)$

9734 FORMAT $(17,14.2(110,14) / 1$ PE13.4,2E14.4) GO TO 9732

9730 PRINT $9735, J 1, K 1, J 2, K 2, J 3, K 3, J 4, K 4, A R R A Y(5 * I 1-4), A R R A Y(5 * 11$ 1-3),ARRAY (5*I1 2$)$,ARRAY $(5 * I 1-1)$

9735 FORMAT $(17,[4,3(I 10,14) / 1 P E 13.4,3 E 14.4)$ GO TO 9732

9720 PRINT 9736, BNAMX1,BNAMX2

9736 FORMAT IIHI29X,2A6/7T/32X,2HNI4X,2HN2/28X,16HSIGMA 5O INITIITI GO TO 9717

9721 PRINT 9737 , BNAMX1, BNAMX 2

9737 FORMAT (IHI29X,2A6////32X,2HN14X,2HN2/28X,16HSIGMA S1 (N1/N2)//) GO TO 9717

9722 PRINT 9738, BNAMX1,BNAMX2

9738 FORMAT IIHI29X,2A6/T/32X.2HNI4X.2HN2728X.I7HSIGMA N,G INI/N2TII GO TO 9717

9732 RETURN END 
C SUBROUTINE RBTIJK

C _... SUBROUTINE RBTIJK IS A THIRD ORDER SUBROUTINE USED TO READ THE OLD BINARY DATA TAPE AND TEST FOR TAPE CHECKS. IF A TAPE CHECK IS INDICATED BY THE IBM SUBROUTINE

C RER AFTER NINE ATTEMPTS AT READING, SENSE LIGHT 2

C IS TURNED ON. IBM SUBROUTINE RER HAS BEEN MODIFIED

C TO DO THIS. SUBROUTINE RBTIJK IS USED IN CONJUNCTION

C..... WITH SUBROUTINES ELIV,RAPRL, DBLKR.

SUBROUTINE RBTI JK(NTAPE, ARRAY,NWDS)

C DIMENSION FOR SUBROUTINE RBTIJK

DIMENSION ARRAY (2000)

C BEGIN EXECUTABLE PART OF SUBROUTINE RBTIJTR

4000 READ TAPE NTAPE, (ARRAY $(I), I=1$, NWOS)

IF ISENSE LIGHT 2T 4001,4002

4001 SENSE LIGHT 1

4002 RETURN

END 
260

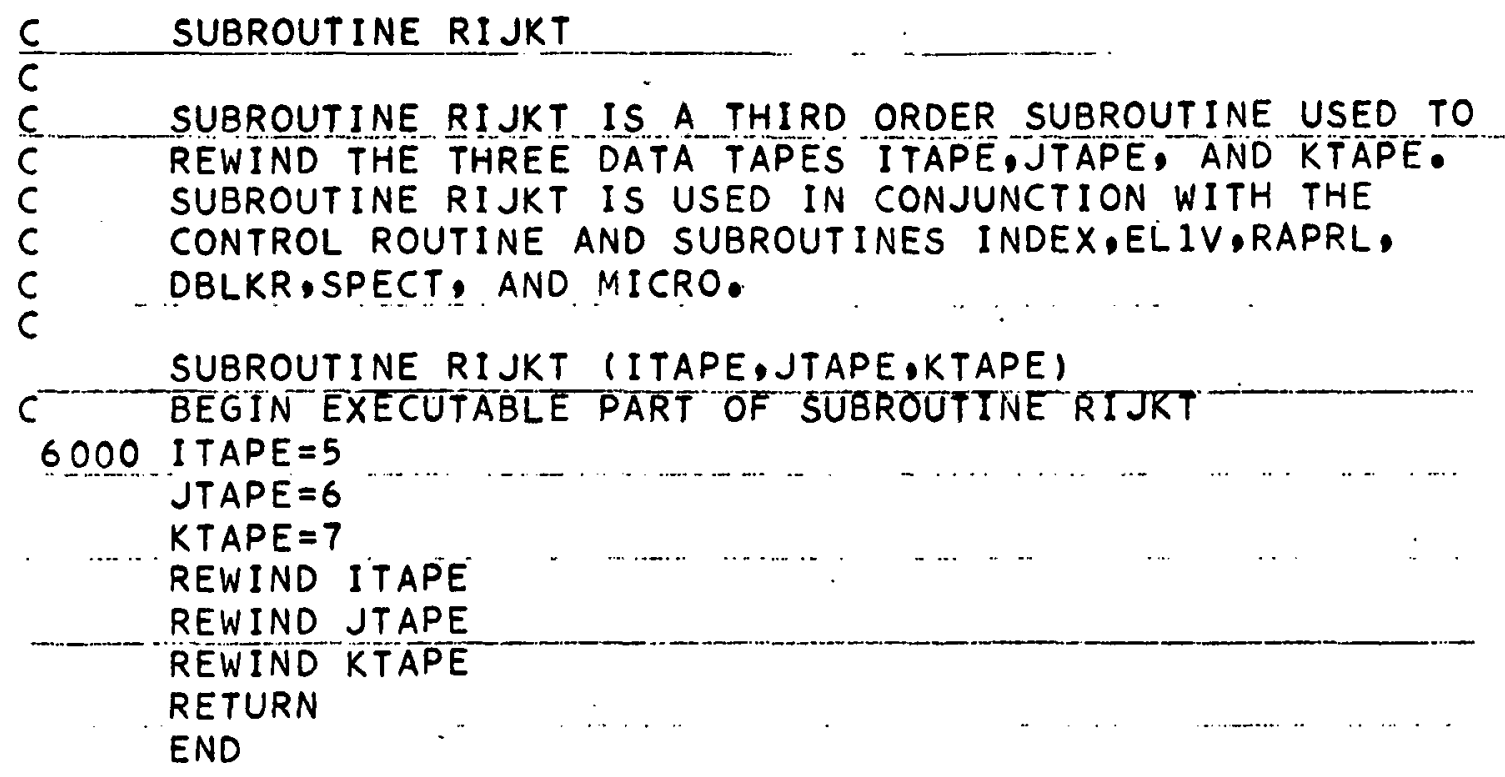


C SUBROUTINE SWITCH - C SUBROUTINE SWTCH IS A THIRD ORDER SUBROUTINE USED TO

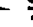

EXPAND THE RESONANCE DATA ARRAY TO ITS MAXIMUM AL
LENGTH IN STORAGE. ON BINARY TAPE ONLY THE ACTUAL

C NUMBER OF RESONANA CES GIVEN ARE WRITTEN. HOWEVER IN

C STORAGE FOR CORRECTION PURPOSES THE TAPE RECORD MUST

C BE EXPANDED: SUBROUTINE SWTCH IS USED ONLY IN CONJUNCTION

C WITH SUBROUTINE DBLKR •

SUBROUTINE SWTCH (NRES,EO,RIO,PO,R,ETAR, TEMP)

C DIMENSION FOR SUBROUTINE SWTCH

DIMENSION EO(200),RIO(200),PO(200),R(200),ETAR(200),TEMP(2000)

$\mathrm{C}$

$9505.009504 \quad I=I .2000$

$\operatorname{TEMP}(I)=0.0$

$9 \overline{504}$ CONT INUE

9500 DO $9501 \quad I=1$, NRES

$J=I+N R E S$

$\operatorname{TEMP}(I)=E O(J)$

$K=I+2 * N R E S$

$\operatorname{TEMP}(1+200)=E O(K)$

$L=I+3 * N R E S$

$\operatorname{TEMP}(I+400)=E O(L)$

$M=I+4 *$ NRES

$\operatorname{TEMP}(1+600)=E O(M)$

9501 CONTINUE

DO 9502 I =1, NRES

$R I O(I)=T E M P(I)$

$P O(I)=T E M P(I+200)$

$R(I)=T E M P(I+400)$

$E T A R(I)=T E M P(I 600)$

9502 CONTINUE

DO $9503 \quad I=1.2000$

9503 CONTINUE

$\operatorname{TEMP}(I)=0.0$

RETURN

END 


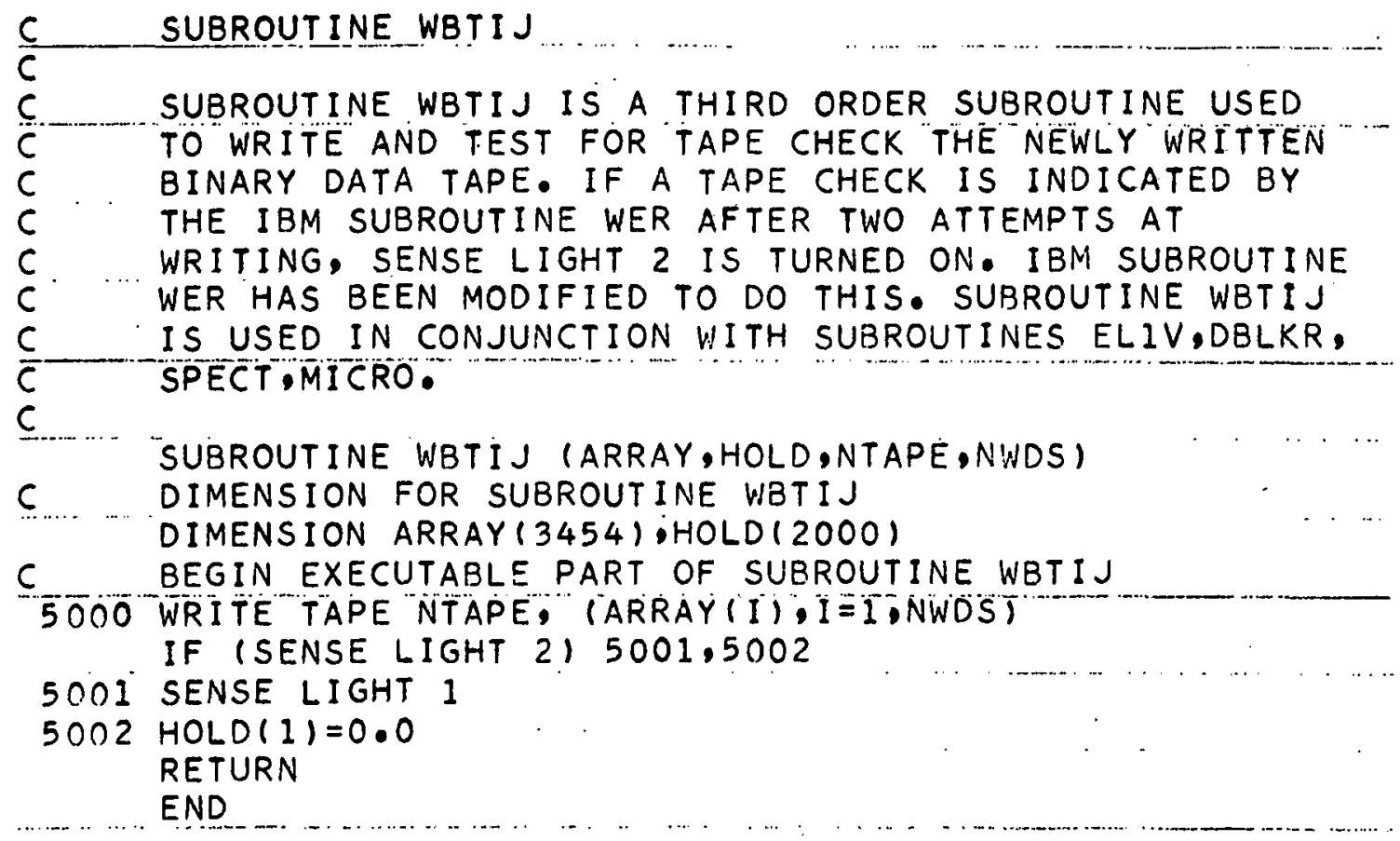




\subsection{Program Mnemonic Glossary}

This glossary contains all the variables used in Program RNDC which are not described in the input glossary with the exception of those fixed-point variables used simply as upper limits of $D \phi$ loops.

\begin{tabular}{|c|c|}
\hline $\begin{array}{l}\text { Program } \\
\text { Mnemonic }\end{array}$ & Description \\
\hline AMICR $\varnothing$ & Refer to NMICR $\phi$ in the input glossary \\
\hline ARRAY & $\begin{array}{l}\text { A temporary array identical to DATA below, used in } \\
\text { binary data tape reading and writing subroutines. }\end{array}$ \\
\hline BNAMI & $\begin{array}{l}\text { For the } 7090 \text { version refer to BIDENT in the imput } \\
\text { glossary }\end{array}$ \\
\hline BNAM2 & $\begin{array}{l}\text { For the } 7090 \text { version refer to BIDENT in the lnput } \\
\text { glossary }\end{array}$ \\
\hline BNAMXI & $\begin{array}{l}\text { A location containing the first word of the two-word } \\
\text { material-data-block identification or spectral-record } \\
\text { ldentification }\end{array}$ \\
\hline BNAMX2 & $\begin{array}{l}\text { A location containing the second word of the two-word } \\
\text { material-data-block Identification or spectral-record } \\
\text { identification }\end{array}$ \\
\hline DATA & $\begin{array}{l}\text { The main temporary storage array of the program into } \\
\text { which the contents of the original data tape records } \\
\text { and decimal correction cards are read, and from which } \\
\text { the new data tapes and BCD output are written }\end{array}$ \\
\hline EL & $\begin{array}{l}\text { A permanent storage array containing the level } \\
\text { energies of the data tape }\end{array}$ \\
\hline H $\phi$ D & $\begin{array}{l}\text { A temporary storage array used in checking the con- } \\
\text { tents of a record written on the new binary data tape } \\
\text { for accuracy. This feature is not used in the } 7090 \\
\text { version. }\end{array}$ \\
\hline ITAPE & $\begin{array}{l}\text { A location containing the logical tape unit number of } \\
\text { the primary new data tape to be written, preassigned } \\
\text { to } 5\end{array}$ \\
\hline JTAPE & $\begin{array}{l}\text { A location containing the logical tape unit number of } \\
\text { the secondary new data tape to be written, preassigned } \\
\text { to } 6\end{array}$ \\
\hline
\end{tabular}

KIAPE A location containing the logical tape unit number of the original data tape to be read, preassigned to 7

NDATA Refer to DATA in this glossary

NFORK A location containing varlous values used in determining which of the three transfer matrices are being used

NLLEVS Refer to NLEV in the imput glossary

$N \phi B L K S \quad A$ location containing the number of material data blocks and spectral records present on the data tape, preassigned to 499 
Program

Mnemonic

NØTHGP

NфTWDS

NRNMCR

NRфMCR

NRSAVE

NTAPE

NWDS

NI

N2

NZSAVE

N3SAVE

N4SAVE

N5SAVE

TEMP

THERM

TNAME
Description

A location containing the number of thermal groups on the data tape, preassigned to 10

A location containing the number of words contained in various data arrays

A location containing the new physical record number of the microscopic-constituents record; this will be different from the old record number only when records are added to material data blocks

A location containing the old physical record number of the microscopic-constituents record

A location containing the physical record number of the record being written on the new binary data tapes

Refer to ITAPE, JTAPE, and KTAPE in this glossary

Refer to NфTWDS in this glossary

A location equivalent to MOMIN, MIMIN, or MGMIN, refer to these in the imput glossary

A location equivalent to MOIMAX, MLIMAX, or MGIMAX, refer to these in the imput glossary

A location containing the number of words in the second record of a material data block

A location containing the number of words in the third record of a material data block

A location containing the number of words in the fourth record of a material data block

A location contalning the number of words in the fifth record of a material data block

A temporary storage array used in realigning the resonance data read from the old data tape for the purpose of corrections and print-out

A permanent storage array containing the Fahrenheit temperatures of the thermal transfer matrices

Refer to NAME in the imput glossary 
APPEIDIX A - Semple Problem

Al - Sample Problem Input

SNAMTAP, 3,RNDT $2505 / 15 / 61$

4 NLEV, 24,

4 NEWTAP, $1, N B A, 2, N B D, 0$,

4NPSN, 0 ,

5BNAM, 2, URANIUM 235

$3 B$ CODE, $92 \cdot 235$,

4 NBP SN, 1 ,

4 MOMIN $, 1,10,16,0,0,0,45,1,34,11$,

E28406,2,35 SPECTRUM

$3 B C O D E, 4=, 500.0$,

$4 N B P S N, 4=2$,

4 MOMIN, $10=, 0,0,0,0,0,0,0,0,0,0$,

4 NOPRNT, 3, NOPUN, 0 ,

3PCODE, $2.06,92 \cdot 235,500.0$

$=E N D, 0$,

$3 E L, 1.0+7,6.065307+6,3.67879446 .2 .2313016+6,1.3533528+6$,

$8.2084998 \quad 5,4.9787068+5,3.0197384+5,1.8315639+5,6 \cdot 737947+4$,

$2.47875224,9.1188197+3,3.3546263+3,1.2340980+3,453.99930$,

$87.190524,35.449023,13.040967,5.0434766,1.4449802$,

$.68256034, .25109992, .09237450, .025301182$,

$.302295, .271820, .241345, .210870, .180395$,

$.149920, .119445,8 \cdot 897017-2,5.849518-2,3.216477-2$,

$3 \cup L, 0, .5,1,1.5,2.0,2.5,3.0,3.5,4.0,5.0,6.0,7,8,9,10,11.65$,

$12 \cdot 55,13 \cdot 55,14 \cdot 5,15 \cdot 75,16 \cdot 5,17 \cdot 5,18 \cdot 5,19 \cdot 795$,

$17 \cdot 31433,17 \cdot 42061,17 \cdot 53950,17 \cdot 67451,17 \cdot 83061$

$18.01565,18 \cdot 24290,18.53745,18.95681,19.55488$, 
$3 E G, 1.2840254+7,7 \cdot 7880078+6,4 \cdot 7236655+6,2.8650479+6,1.7377394+6$, $1.05399226 .6 .3927861+5.3 .8774208+5,2.3517746+5.1 .1108997+5$, $4.08677144 .1 .5034392+4.5 .5308437+3,2.0346837+3.7 .4851831+2$, $1.98958392,55.595138,21.500919,8.1099823,2.6995785$, $.99311943, .41399378, .1522998,4 \cdot 8344436-2,2.5301182-2$, $3 \cup G,-.25,+.25, .75,1.25,1.75,2.25,2.75,3.25,3 \cdot 75,4 \cdot 50,5 \cdot 5,6.5$, $7.5,8.5,9.5,10.825,12 \cdot 1,13.05,14 \cdot 025,15 \cdot 125,16 \cdot 125,17 \cdot 0,18 \cdot 0$, $19 \cdot 1475,19 \cdot 795$,

$3 F V \cdot 5.0428002-5,6 \cdot 4750836-5,8 \cdot 3141724-5,1.0675608-4 \cdot 1.3707754-4$, $1.76010994,2.2600263-4,2 \cdot 901931-4,3.9793804-4,6 \cdot 1914462-4,1.0207969-3$, $1.6830096 \quad 3,2 \cdot 7748135-3,4 \cdot 5748942-3,8 \cdot 2454357-3,1.5773244-2$, $2.73050592,4 \cdot 4204265-2.7 .4459926-2,1.2559766-1, .20021685$, $.32072485, .55045328, .85441628$.

$=E N D, 0$,

5BDATE, 2, DEC 011960

$3 A W, 235 \cdot 117$

$3 A 2200,683.0$,

4NDATA, 0 ,

$35,4 \cdot 26738,5 \cdot 58365,6 \cdot 55996,6.00605,5 \cdot 27471$,

$3 S \cdot 5=, 5 \cdot 41810,6 \cdot 21584,7 \cdot 47755,9 \cdot 01324,10 \cdot 32709$,

$3 S, 10=, 10.461536,10.044369,10.034058,11.925448,13.353193$,

$35,15=, 16 \cdot 138427,25,994430,11 \cdot 276972,10 \cdot 183404,11.604643$,

$35,20=, 12 \cdot 826825,13.767262,14.351427,14.600489,13.336363$,

$3 S, 25=.13 .435080,13.539832,13.651001,13.769034,13.894640$,

$35.30=.14 \cdot 029776,14.179675,14.354820,14.531250$,

$3 \times 15,3.52337,4.03556,3.62979,2.82049,2.02572$,

$3 \times 1 S, 5=, 1.46414,1 \cdot 27568,1.08309,7.90604-1,2.84583-1$,

$3 \times 1 S, 10=, 8.9529824-2,8.59597032,8.5871466-2,1.0205798-1,1.1427661-1$, 
$3 \times 15,15=, 1.3811265-1,2.22460361,9.6508325-2,8.6379215-2,9.8434668-2$, $3 \times 15,20=, 1.0880165-1,1.16778761,1.2173385-1,1.2384648-1,1.1312374-1$, $3 \times 1 S, 25=1.1396109-1,1.14849531 .1 .1579260-1.1 .1679381-1,1.1785923-1$. $3 \times 15,30=, 1.1900542-1,1.20277001,1.2176258-1,1.2325917-1$,

$3 T R, 1.80654,2 \cdot 69551,3.44762,3.62976,3.54510$,

$3 T R, 5=.3 .80178,4 \cdot 59503,5 \cdot 89240,7 \cdot 79027,10.02487$.

$3 \mathrm{TR}, 10=, 10.431147,10.015641,10,005360,11 \cdot 891341,13 \cdot 315003$,

$3 T R, 15=, 16.092269,25 \cdot 920085,11 \cdot 244720,10 \cdot 154528,11 \cdot 571738$,

$3 T R, 20=, 12.790454,13 \cdot 728224,14 \cdot 310734,14 \cdot 559089,13 \cdot 298548$,

$3 T R \cdot 25=, 13.396985,13.501440,13 \cdot 612293,13 \cdot 729992,13.855241$,

$3 T R \cdot 30=, 13 \cdot 989996,14 \cdot 139469,14 \cdot 314117,14 \cdot 490047$.

$3 A \cdot 1.8753,1.4500,1.3100,1.4100,1 \cdot 4000$

$3 A, 5=, 1 \cdot 33782,1 \cdot 42387,1 \cdot 56988,1 \cdot 84390,2 \cdot 27496$,

$3 A, 10=, 3.6902647,5 \cdot 2241154,7.3007675,10.816236,23 \cdot 062310$,

$3 A, 15=, 36 \cdot 484936,72,662618,109 \cdot 86888,40 \cdot 863138,42 \cdot 582235$,

$3 A, 20=\cdot 79 \cdot 233875,208 \cdot 11720,330.04422,568 \cdot 15945,163 \cdot 56938$,

$3 A \cdot 25=, 175,65111 \cdot 189 \cdot 92243 \cdot 207 \cdot 03358,227 \cdot 89864,253 \cdot 92676$,

$3 A, 30=, 287,77645,336 \cdot 12386,420 \cdot 92899,591 \cdot 16768$,

$3 F N U, 6.9925210,4 \cdot 5403728,3 \cdot 6345988,3 \cdot 6462461,3.4146576$,

$3 F N U, 5=, 3 \cdot 1485105,3 \cdot 1571243,3 \cdot 4727328,4 \cdot 0150673,5 \cdot 2671745$,

$3 F N U, 10=, 7.1500528,9 \cdot 7836984,13 \cdot 226331,18.956019,38 \cdot 800426$,

$3 F N U, 15=, 58.754542,116.91770,168.16753,41.692175,78.811172$,

$3 F N U, 20=, 166 \cdot 50628,422 \cdot 16808,695 \cdot 97333,1 \cdot 1812683+3,335.85637$,

$3 F N U, 25=, 360 \cdot 91845,390 \cdot 49181,425 \cdot 96756,469 \cdot 29679,523.60643$,

$3 F N U \cdot 30=, 594 \cdot 71092,696 \cdot 80226,875 \cdot 02786,1 \cdot 2277724+3$,

$3 T S 4500,4500.0,15 \cdot 265845,15 \cdot 222557,943.88058,1.9530248+3$,

$3 T S 4500,5=, 12.029478,0.7973474 \quad 00000$

$3 T S 4500,10=, 0.7437936,12 \cdot 511322,0.5392118,3 \cdot 2044314-6,0$ 


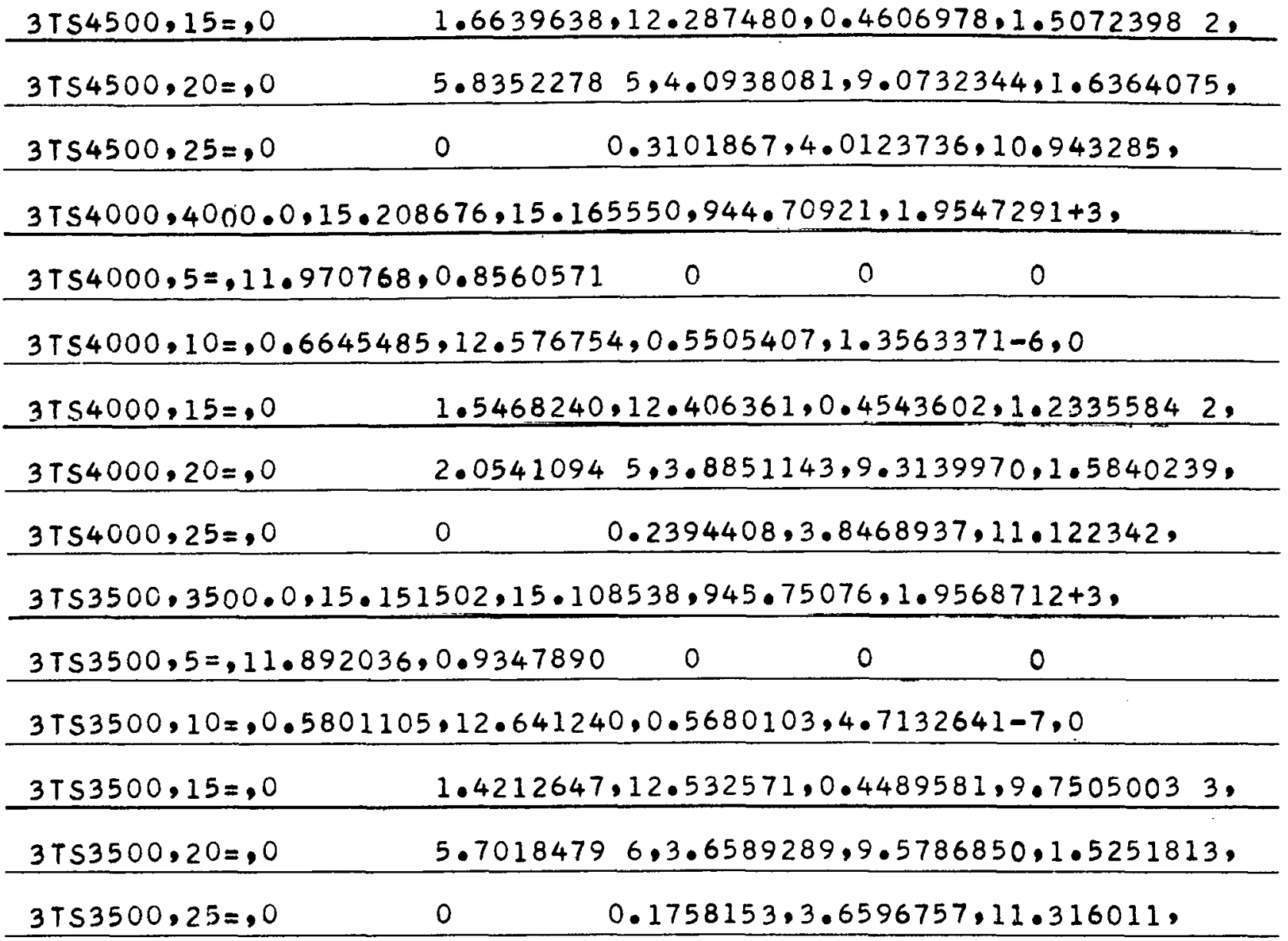

$3 T 53000,3000.0,15.094271,15.051470,947.09890,1.9596440+3$,

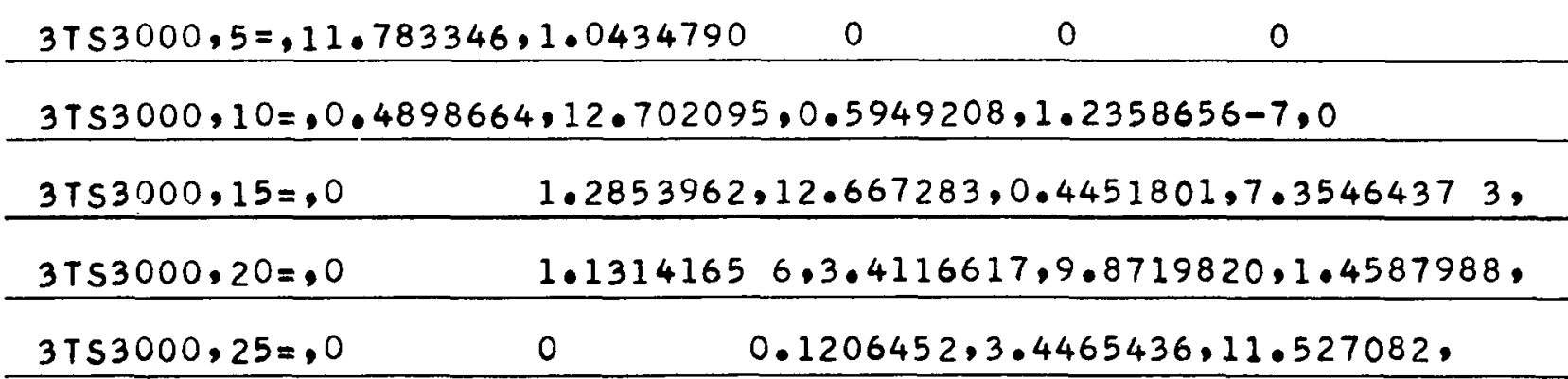

$3 T S 2500,2500 \cdot 0,15.037002,14 \cdot 994363,948 \cdot 91293,1.9633749+3$,

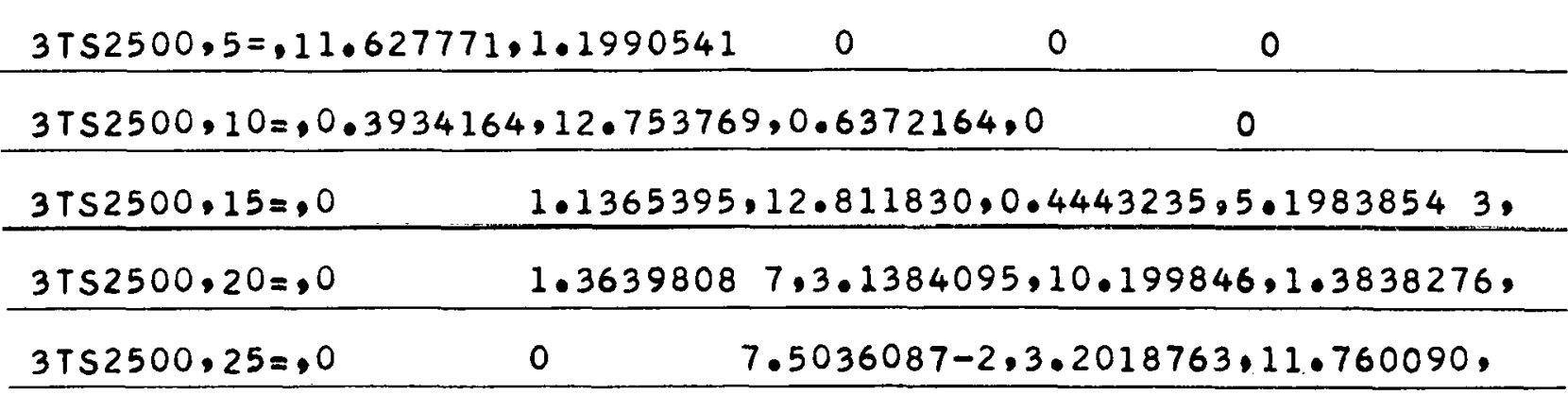

$3 T \$ 2000,2000.0,14.979667,14.937191,951.48435,1.9686636+3$, 
$3 T S 2000,5=, 11.405204,1.4216209$

3 TS2000,10=,0.2911094,12.784236,0.7065755,0

0

$3 T S 2000,15=, 0$

$0.9708546,12 \cdot 967380,0.4490104 \cdot 3 \cdot 32958653$,

$3 T S 2000,20=, 0$

0

$2.8319907,10.570816,1.2989155$,

$3 T 52000,25=, 0$

0

$4.0195439-2.2 .9182793 .12 .021192$,

$3 T S 1500,1500 \cdot 0,14 \cdot 922367,14 \cdot 880053,955 \cdot 41233 \cdot 1.9767426+3$,

$3 T S 1500,5=, 11.230525,1.5963000$

$3 T 51500,10=, 0.1856281,12 \cdot 765008,0.8287936,0$

0

\begin{tabular}{lll}
3 TS $1500,15=, 0$ & $0.7825674,13.133747,0.4651406,1.81455613$, \\
3 TS $1500,20=, 0$ & 0 & $2.4815050,10.996860,1.2029958$, \\
3 TS $1500,25=.0$ & 0 & $1.6769986-2,2.5848607,12.320736$, \\
\hline
\end{tabular}

$3 T S 1000,1000.0,14.865152,14.823000,962.15161,1.9906035+3$,

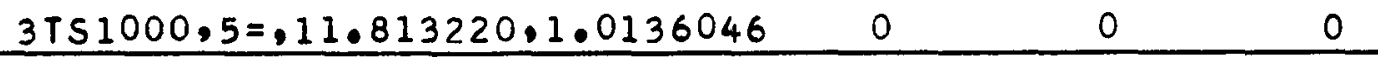

$3 T 51000,10=, 8,6243144-2,12.619881,1.0707746,0 \quad 0$

$3 T S 1000,15=.0 \quad 0.5630644,13.303380,0.5088123,7.25753794$,

\begin{tabular}{|c|c|c|}
\hline $3 T 51000,20=0$ & 0 & 2.0680512 .11 .496843 .1 .0961113$, \\
\hline $3 T 51000,25=.0$ & 0 & $4 \cdot 3602102-3,2.1839834,12 \cdot 676808$, \\
\hline \multicolumn{3}{|c|}{$3 T S 500,500.0,14 \cdot 808389,14 \cdot 766398,976.38466,2.0199+3$, } \\
\hline \multicolumn{2}{|c|}{$3 T S 500,5=.12 \cdot 241785,0.5850404$} & 0 \\
\hline \multicolumn{3}{|c|}{$3 T S 500,10=, 0.1134155,12.113705,1.5468854,0$} \\
\hline $3 T S 500,15=, 0$ & \multicolumn{2}{|c|}{$0.3031290,13.425721,0.6397316,1.3613176-4}$, \\
\hline $3 T S 500,20=, 0$ & 0 & $1.5529061 .12 .103530,0.9842148$, \\
\hline $3 T S 500,25=, 0$ & 0 & $3.6960635-4.1 .6796702,13.128349$, \\
\hline \multicolumn{3}{|c|}{$3 T S 68.68 .0,14.760995,14.719139,1.0129743 \quad 3,2.0951457+3}$, \\
\hline \multicolumn{2}{|c|}{$3 T S 68,5=.12 .222044,0.6047812$} & 0 \\
\hline \multicolumn{3}{|c|}{$3 \mathrm{TS} 68,10=, 0.2235824,13.094448,0.4521462,0 \quad 0$} \\
\hline $3 T 568,15=.0$ & \multicolumn{2}{|c|}{$7.0153109-2,13.196108,1.0960923,3.31003226}$, \\
\hline $3 T S 68,20=, 0$ & \multicolumn{2}{|c|}{$0 \quad 0.9460556,12.757702,0.9193123}$, \\
\hline
\end{tabular}


$=E N D, O$.

$351 G S 0,2.65229,5 \cdot 62880-2,1 \cdot 22036-1.3 \cdot 23729-1,4 \cdot 14813-1.3 \cdot 32078-1$,

$1.97235-1,9 \cdot 73160-2,5 \cdot 36222-2,1.53572-2,2 \cdot 25409-3,3 \cdot 12669-4$,

$4 \cdot 33862-5,5 \cdot 77496-6,8 \cdot 50695-7,6 \cdot 57875-8$,

$1=, 3.49580,1.18770-1,2.82907-1,5.07902-1,5.04908-1,3.43593-1$,

$1.84432-1,1.07617-1,3.21209-2,4.81681-3,6.73561-4,9.37369-5$,

$1.24911-5,1 \cdot 84079-6,1 \cdot 42377-7$

$2=, 4.46065,1.99935-1,4.28538-1,5.48782-1,4.38712-1.2 .60387-1$,

$1.62761-1.5 .10488-2.7 .85511-3,1.10915-3.1 .54901-4,2.06701-5$,

$3.04759-6,2 \cdot 35763-7$

$3=, 4 \cdot 13582 \cdot 3 \cdot 16898-1,4 \cdot 72050-1,4 \cdot 65211-1,3 \cdot 14944-1,2 \cdot 15564-1$,

$7.21696-2,1 \cdot 14891-2,1.64326-3,2 \cdot 30564-4,3.08226-5,4 \cdot 54743-6$,

$3.51878-7$,

$4=, 3.71480,4 \cdot 22449-1.4 \cdot 24908-1,3.39149-1,2.60360-1,9.46524-2$,

$1.57307-2 \cdot 2 \cdot 28696-3,3 \cdot 22790-4,4 \cdot 32515-5 \cdot 6 \cdot 38643-6,4 \cdot 94334-7$,

$5=, 4.18909,4.03646-1,3.54544-1,3 \cdot 16344-1,1.28069-1,2.25179-2$,

$3 \cdot 34474-3,4 \cdot 75790-4,6 \cdot 39470-5,9 \cdot 45261-6,7 \cdot 31972-7$,

$6=, 5.00225,5.06570-1,4.51323-1,2.09184-1,3.94914-2,6.02808-3$,

$8 \cdot 66062-4,1 \cdot 16853-4 \cdot 1 \cdot 72972-5,1 \cdot 34014-6$,

$7=, 6.39308,6.74630-1,3.29322-1,6.79689-2,1.07386-2,1.56239-3$,

$2 \cdot 11846-4,3 \cdot 14140-5,2 \cdot 43550-6$,

$8=, 8.10232,7.66825-1,1.20430-1,2 \cdot 01897-2,3.00238-3,4.10600-4$,

$6 \cdot 10740-5,4 \cdot 74057-6$,

$9=.9 .89183,4.15259-1,1.69811-2,2.60670-3,3.60959-4,5.39315-5$,

4.19332-6,

$=E N D, 0$,

$3 E 0,1 \cdot 1270,2 \cdot 040,2 \cdot 820,3 \cdot 140,3 \cdot 610,4 \cdot 840,5 \cdot 450,6 \cdot 120$, 
$6.40,7 \cdot 10,8 \cdot 820,9 \cdot 270,9 \cdot 740,10 \cdot 150,10 \cdot 640,11 \cdot 110,11 \cdot 660$, $12 \cdot 40,13 \cdot 40,13 \cdot 80,14 \cdot 110,14 \cdot 60,15 \cdot 50,16 \cdot 10,16 \cdot 80,18 \cdot 10$, $18 \cdot 70 \cdot 19 \cdot 30,20 \cdot 60,21 \cdot 10,22 \cdot 10,22 \cdot 90,23 \cdot 50,24 \cdot 40,25 \cdot 30$. $25 \cdot 80 \cdot 26 \cdot 50 \cdot 27 \cdot 30,27 \cdot 90,30 \cdot 70,31 \cdot 10,32 \cdot 10,33 \cdot 60$, $34 \cdot 60 \cdot 35 \cdot 30$,

$3 R 10,24 \cdot 130790,3 \cdot 878203,0.6532495,5 \cdot 929572,7 \cdot 213012,4 \cdot 643314$, $1.458542 \cdot 1.497982,15.206050,4.460899,31.124390,3.064682,0.7460382$, $1.210974,0.5940221,0.7792993,8.730255,17.601930,0.8402182,1.606561$, $2.057776,1.623623,2.324958,2.227811,2.089894,2.930858,1.272149$, $17.294380,1.757571,4 \cdot 212773,0.3966072,2.615329,6.567533$, $1.498573,1.226491,0.9410788,1.458386,0.3032624,2.080898$, $0.9532018,0.3679257,2 \cdot 239809,3 \cdot 927770,7 \cdot 703968,11 \cdot 773240$, $3 P 0,1.154095+2,1.144487+2,1.172729+1,7.182484+1,1.725944+2$, $3.040662+2.5 .6214931 .6 .482829+1.1 .062629+3.3 .593573+2$. $1.727114+3,2.006718 \quad 2.5 .137977+1.8 .688540+1.4 .469147+1$, $6.121121+1.9 .5892452 .2 .003966+3.7 .957589+1.1 .565661+2$. $2.049289+2.1 .6736532 .2 .541415+2.2 .529233+2.2 .475649+2$, $3.733007+2.1 .6787082,1.724675+3,2.550759+2,6.224115+2$, $6.193514+1.4 \cdot 2051232.1 .070379+3.2 \cdot 574026+2.2 .185657+2$, $1.711651+2.2 .7186492,5.849111+1.4 .070861+2.2 .059937+2$. $8.078390+1.5 .0225012 .9 .112174+2.1 .791821+3.2 .707388+3$, $3 R \cdot 7 \cdot 375385-5,1 \cdot 551314-3,4 \cdot 152949-4,1.698016-4,5 \cdot 763438-4,3 \cdot 219614-3$, $9.904626-4,1.112073 \quad 3.2 .772802-3,3.321382-3,1.268646-3,1.680702-3$. $1.769587-3,1.8430043,1.933175-3,2.017940-3,3.759977-3,3.798455-3$. $2.432444-3,2.5008673,2.554184-3,2.644698-3,2.801291-3,2.909134-3$. $3.035212-3,3.257003 \quad 3,3.383777-3,1.872474-3,3.715475-3,3.759287-3$, $4.009864-3,4 \cdot 1024003,4 \cdot 107448-3,4 \cdot 393889-3,4.561245-3,4 \cdot 659376-3$, $4.765236-3.4 \cdot 9516363,4 \cdot 984659-3,5.528006-3,5.632976-3$, 
$5.692221-3,5.8207843,5 \cdot 681367-3,5,443816-3$,

3ETAR, $0.80,0.3181818,0.70,0.8181818,0.68750,0.3617021$,

$0.6666667,0.6666667,0.4827586,0.4642857,0.70,0.6666667$.

$0.6666667,0.6666667,0.6666667,0.6666667,0.2686567,0.4411765$,

$0.6666667,0.6666667,0.6666667,0.6666667,0.6666667,0.6666667$,

$0.6666667,0.6666667,0.6666667 .0 .750,0.6666667,0.6666667$,

$0.6666667,0.6666667,0.6666667,0.6666667,0.6666667,0.6666667$,

$0.6666667,0.6666667,0.6666667,0.6666667,0.6666667,0.6666667$,

$0.6666667 .0 .6666667,0.6666667$

$=E N D, 0$,

3SIGNG,

$0 \quad 0$

$\begin{array}{lllll}2.6856-02 & 6.6246-02 & 2.7214-01 & 8.3971-01 & 1.9963+00 \\ 4.3131+00 & 8.0623+00 & 9.3979+00 & 7.1617-02 & \end{array}$

$0 \quad 0$

\begin{tabular}{lllll}
$1.9943-02$ & $4.9192-02$ & $2.0209-01$ & $6.2354-01$ & $1.4824+00$ \\
$3.2028+00$ & $5.9868+00$ & $6.9786+00$ & $5.3181-02$ & \\
\hline
\end{tabular}

$0 \quad 0$

$\begin{array}{lllll}1.8801-02 & 4.6375-02 & 1.9051-01 & 5.8783-01 & 1.3975+00 \\ 3.0194+00 & 5.6439+00 & 6.5790+00 & 5.0135-02\end{array}$

$0 \quad 0$

$\begin{array}{lllll}1.9936-02 & 4.9175-02 & 2.0202-01 & 6.2333-01 & 1.4819+00\end{array}$

$3.2017+00 \quad 5.9847+00 \quad 6.9762+00 \quad 5.3162-02$

00

\begin{tabular}{lllll}
$1.9638-02$ & $4.8441-02$ & $1.9900-01$ & $6.1402-01$ & $1.4598+00$ \\
$3.1539+00$ & $5.8954+00$ & $6.8721+00$ & $5.2369-02$ & \\
\hline
\end{tabular}

00

$\begin{array}{lllll}1.8955-02 & 4.6755-02 & 1.9207-01 & 5.9265-01 & 1.4090+00\end{array}$


$3.0441+00$

$5.6902+00$

$6.6328+00$

$5.0546-02$

00

$\begin{array}{llll}1.9790-02 & 4.8816-02 & 2.0054-01 & 6.1878-01 \\ 3.1783+00 & 5.9410+00 & 6.9253+00 & 5.2774-02\end{array}$

00

$\begin{array}{llll}2.1636-02 & 5.3491-02 & 2.1975-01 & 6.7803-01 \\ 3.4827+00 & 6.5100+00 & 7.5885+00 & 5.7828-02\end{array}$

$0 \quad 0$

\begin{tabular}{lllll}
$2.5496-02$ & $6.2889-02$ & $2.5835-01$ & $7.9716-01$ & $1.8952+00$ \\
$4.0946+00$ & $7.6538+00$ & $8.9217+00$ & $6.7988-02$ & \\
\hline
\end{tabular}

00

\begin{tabular}{lllll}
$3.3653-02$ & $8.3010-02$ & $3.4101-01$ & $1.0522+00$ & $2.5015+00$ \\
$5.4046+00$ & $1.0103+01$ & $1.1776+01$ & $8.9741-02$ & \\
\hline
\end{tabular}

00

$\begin{array}{lllll}4.6870-02 & 1.1561-01 & 4.7495-01 & 1.4655+00 & 3.4840+00 \\ 7.5273+00 & 1.4070+01 & 1.6401+01 & 1.2499-01 & \end{array}$

00

$\begin{array}{lllll}6.7991-02 & 1.6771-01 & 6.8898-01 & 2.1259+00 & 5.0540+00 \\ 1.0919+01 & 2.0411+01 & 2.3792+01 & 1.8131-01 & \\ & & & \end{array}$

$\begin{array}{lllll}9.3337-02 & 2.3023-01 & 9.4581-01 & 2.9183+00 & 6.9380+00\end{array}$

$1.4990+01 \quad 2.8020+01 \quad 3.2662+01 \quad 2.4890-01$

00

\begin{tabular}{lllll}
$1.3825-01$ & $3.4101-01$ & $1.4009+00$ & $4.3225+00$ & $1.0276+01$ \\
$2.2202+01$ & $4.1502+01$ & $4.8377+01$ & $3.6866-01$ & \\
\hline
\end{tabular}

00

\begin{tabular}{lllll}
$2.8010-01$ & $6.9092-01$ & $2.8384+00$ & $8.7579+00$ & $2.0821+01$ \\
$4.4985+01$ & $8.4087+01$ & $9.8018+01$ & $7.4694-01$ \\
\hline
\end{tabular}


0

$\begin{array}{lllll}4.4698-01 & 1.1026+00 & 4.5294+00 & 1.3976+01 & 3.3226+01 \\ 7.1786+01 & 1.3418+02 & 1.5641+02 & 1.1920+00\end{array}$

0

$\begin{array}{lllll}8.8428-01 & 2.1812+00 & 8.9607+00 & 2.7648+01 & 6.5731+01 \\ 1.4201+02 & 2.6546+02 & 3.0944+02 & 2.3581+00\end{array}$

0.0

$\left.\begin{array}{llll}1.3090+00 & 3.2288+00 & 1.3264+01 & 4.0927+01\end{array}\right] .9 .7300+01$

$0 \quad 0$

4.8909-01 $1.2064+00$

$7.8548+01 \quad 1.4682+02$

$4.9561+00 \quad 1.5292+01$

$3.6356+01$

$1.4682+02 \quad 1.7115+02 \quad 1.3042+00$

$0 \quad 0$

$\begin{array}{lllll}5.7386-01 & 1.4155+00 & 5.8151+00 & 1.7943+01 & 4.2657+01 \\ 9.2162+01 & 1.7227+02 & 2.0081+02 & 1.5303+00 & \end{array}$

00

$1.1411+00 \quad 2.8148+00 \quad 1.1563+01 \quad 3.5679+01 \quad 8.4823+01$

$1.8326+02 \quad 3.4256+02 \cdot 3.9931+02 \quad 3.0430+00$

00

\begin{tabular}{|c|c|c|c|c|c|}
\hline & $3.0167+00$ & $7 \cdot 4411+00$ & $3.0569+01$ & $9.4321+01$ & $2.2424+02$ \\
\hline & $4.8448+02$ & $9.0560+02$ & $1.0556+03$ & $8.0444+00$ & - \\
\hline \multicolumn{6}{|c|}{00} \\
\hline & $4.7394+00$ & $1.1690+01$ & $4.8026+01$ & $1.4818+0 ?$ & $3.5229+02$ \\
\hline & $7.6114+02$ & $1.4228+03$ & $1.6585+03$ & $1.2638+01$ & \\
\hline \multicolumn{6}{|c|}{$0 \quad 0$} \\
\hline & $7 \cdot 7934+00$ & $1.9224+01$ & $7.8973+01$ & $2.4367+02$ & $5.7931+02$ \\
\hline & $1.2516+03$ & $2.3396+03$ & $2.7272+03$ & $2.0782+01$ & \\
\hline 0 & & & . & & \\
\hline
\end{tabular}




\begin{tabular}{|c|c|c|c|c|}
\hline $1.2917+01$ & $3.1861+01$ & $1.3089+C 2$ & $4.0386+02$ & $9.6014+02$ \\
\hline $2.0744+03$ & $3.8776+03$ & $4.5200+03$ & $3.4445+01$ & \\
\hline \multicolumn{5}{|l|}{$0 \quad 0$} \\
\hline $1.2926+01$ & $3 \cdot 1885+01$ & $1.3099+02$ & $4.0416+02$ & $9.6086+02$ \\
\hline $2.0760+03$ & $3.8805+03$ & $4.5234+03$ & $3 \cdot 4470+01$ & \\
\hline \multicolumn{5}{|l|}{$0 \quad 0$} \\
\hline $1 \cdot 2941+01$ & $3.1922+01$ & $1.3114+02$ & $4.0463+02$ & $9.6197+02$ \\
\hline $2.0784+03$ & $3.8850+03$ & $4.5286+03$ & $3.4510+01$ & \\
\hline \multicolumn{5}{|l|}{0.0} \\
\hline $1.2959+01$ & $3 \cdot 1966+01$ & $1.3132+02$ & $4.0519+02$ & $9.6330+02$ \\
\hline $2.0812+03$ & $3.8903+03$ & $4.5348+03$ & $3.4558+01$ & \\
\hline 00 & & & . & \\
\hline $1.2984+01$ & $3.2026+01$ & $1.3157+02$ & $4.0596+02$ & $9.6512+02$ \\
\hline $2.0852+03$ & $3.8977+03$ & $4.5434+03$ & $3.4623+01$ & \\
\hline \multicolumn{5}{|l|}{$\begin{array}{ll}0 & 0 \\
\end{array}$} \\
\hline $1.3020+01$ & $3.2116+01$ & $1.3194+02$ & $4.0710+02$ & $9.6783+02$ \\
\hline $2.0910+03$ & $3.9086+03$ & $4.5562+03$ & $3.4720+01$ & \\
\hline $\begin{array}{ll}0 & 0\end{array}$ & & & & \\
\hline
\end{tabular}

\begin{tabular}{lllll}
$1.3074+01$ & $3.2249+01$ & $1.3248+02$ & $4.0877+02$ & $9.7182+02$ \\
\hline $2.0996+03$ & $3.9247+03$ & $4.5749+03$ & $3.4863+01$ \\
\hline
\end{tabular}

00

\begin{tabular}{|c|c|c|c|c|}
\hline $1.3166+01$ & $3 \cdot 2476+01$ & $1.3342+02$ & $4.1166+02$ & $9.7868+02$ \\
\hline $2.1145+03$ & $3.9524+03$ & $4.6072+03$ & $3.5109+01$ & \\
\hline \multicolumn{5}{|l|}{00} \\
\hline $1.3359+0.1$ & $3.2952+01$ & $1.3537+02$ & $4.1769+02$ & $9.9301+02$ \\
\hline $2.1454+03$ & $4.0103+03$ & $4.6747+03$ & $3.5624+01$ & $\therefore$ \\
\hline \multicolumn{5}{|l|}{$0 \quad 0$} \\
\hline $1.3858+01$ & $3 \cdot 4184+01$ & $1.4043+02$ & $4.3330+02$ & $1.0301+0.3$ \\
\hline
\end{tabular}


$=E N D, 0$,

5BDATE,2,12/07/60

3BETA, 1.0,TM,0.0,

\begin{tabular}{|c|c|c|c|c|c|}
\hline $3 S O R$ & 0.0120488 & 0.1122472 & 0.3278114 & 0.4631222 & 0.4218180 \\
\hline & 0.2953138 & 0.1762738 & 0.0955957 & 0.0421600 & 0.0125488 \\
\hline & 0.0028805 & 0.0006485 & 0.0001451 & 0.0000324 & 0.0000060 \\
\hline & 0.0000008 & 0.0000001 & 0.0000000 & 0. & 0. \\
\hline & 0. & $\underline{0}$. & 0. & c. & 0. \\
\hline & 0. & 0. & 0. & 0. & 0. \\
\hline & 0. & 0. & 0. & 0. & \\
\hline
\end{tabular}

$=E N D, 0$, 
A2 - Sample Problem Output

4-814

RGHERRMANN

RNOP

CHARGE 54323, SEQUENCE 4-814, CHANNELS TAPES

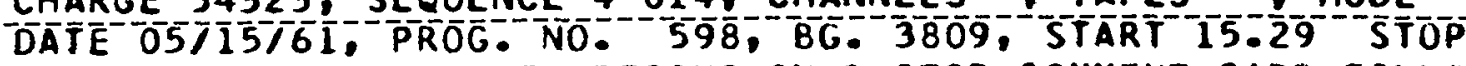
START TIME AFTER INSTRUCTIONS ON I STOP COMMENT CARD FOLLOHED 15.300 
5 XEE 
DATA

EX̄ECUUT'TONN

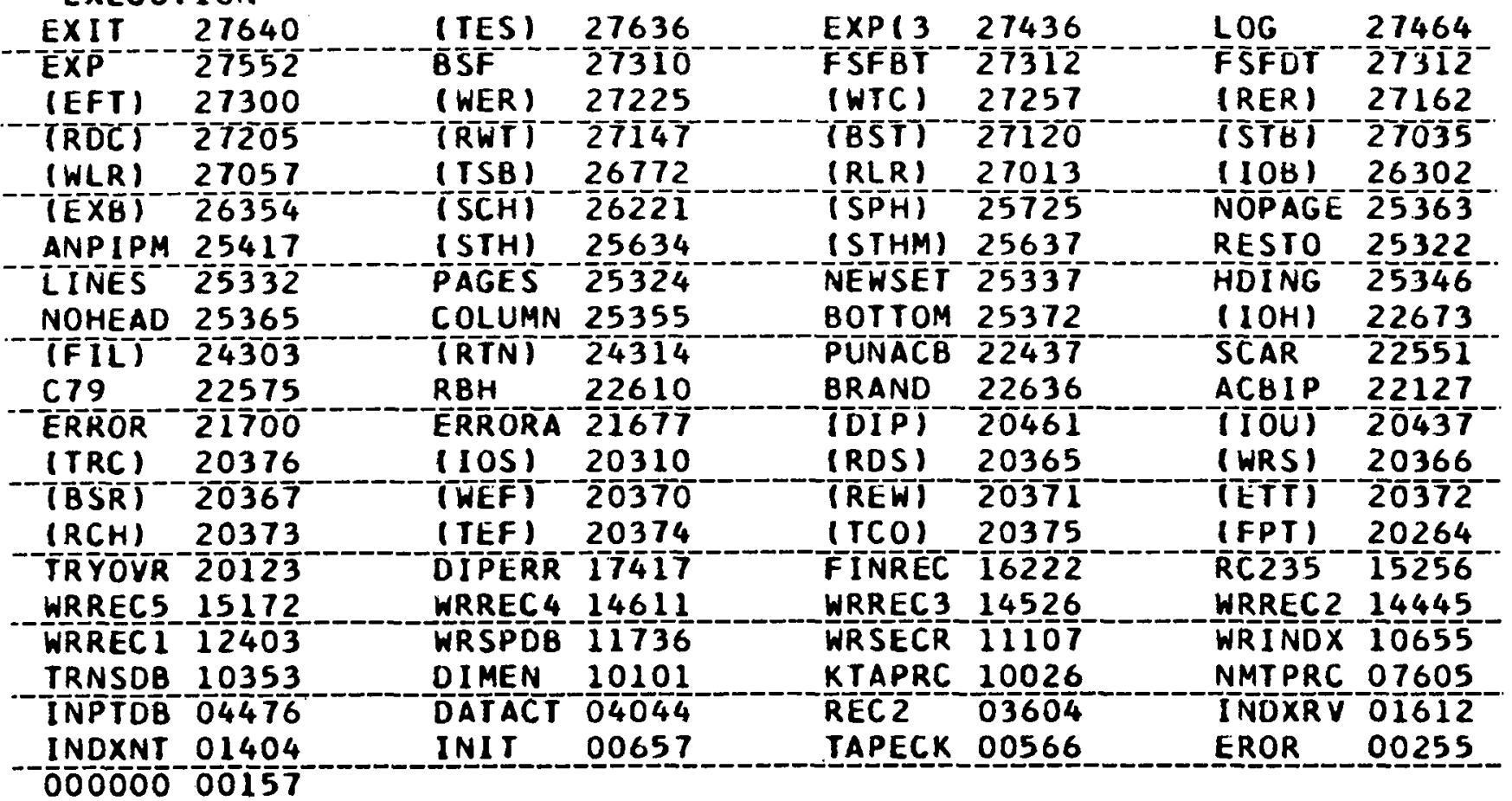


OATÉT OSTISTEI

INOEX RECORD

DATA

24 LEVELS

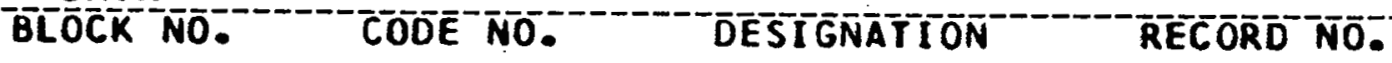

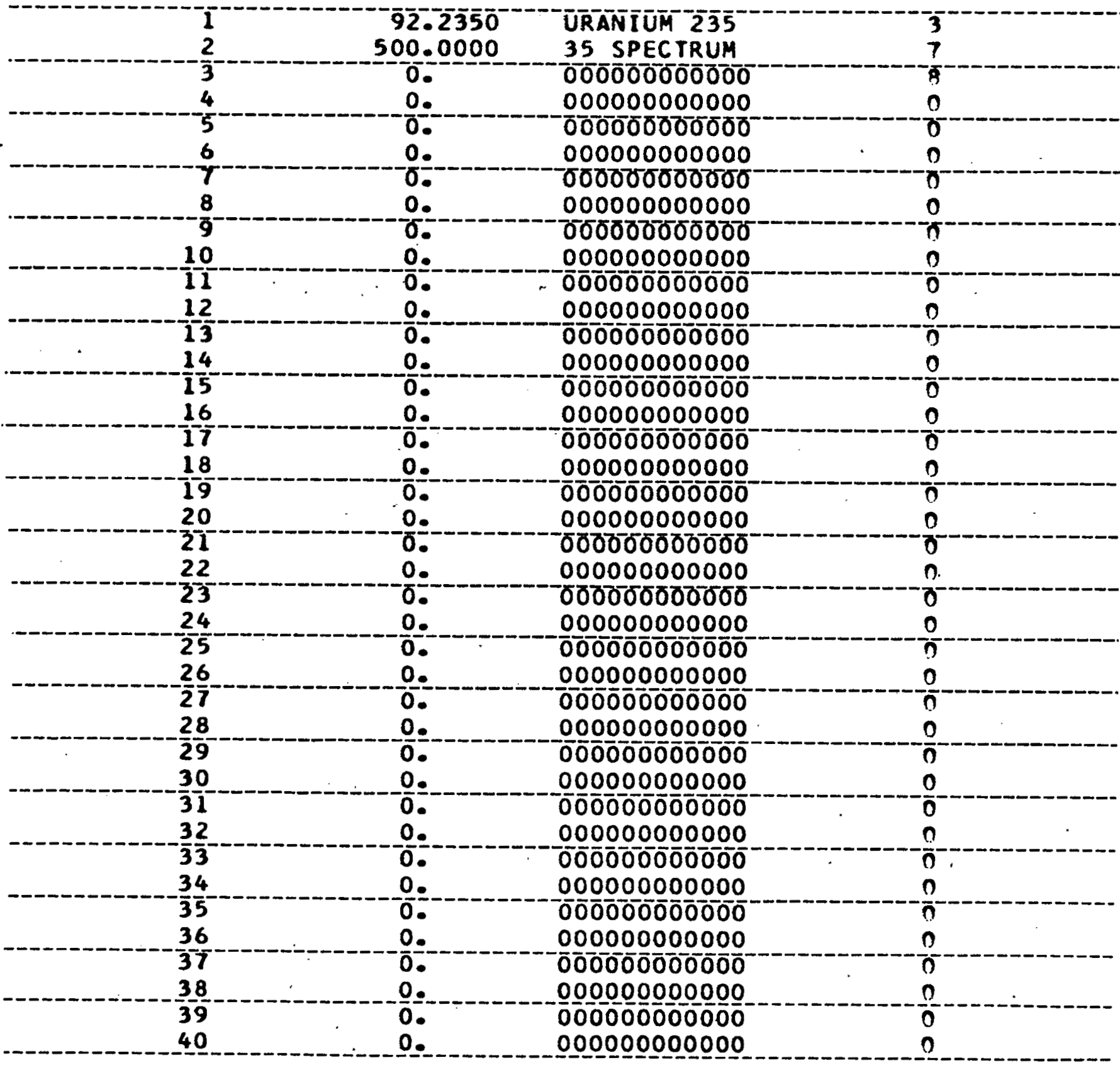

Twe Index record on the tape will always be a flxed length, containing 2000

words of Index information for the tape heading and 499 data blocks. 


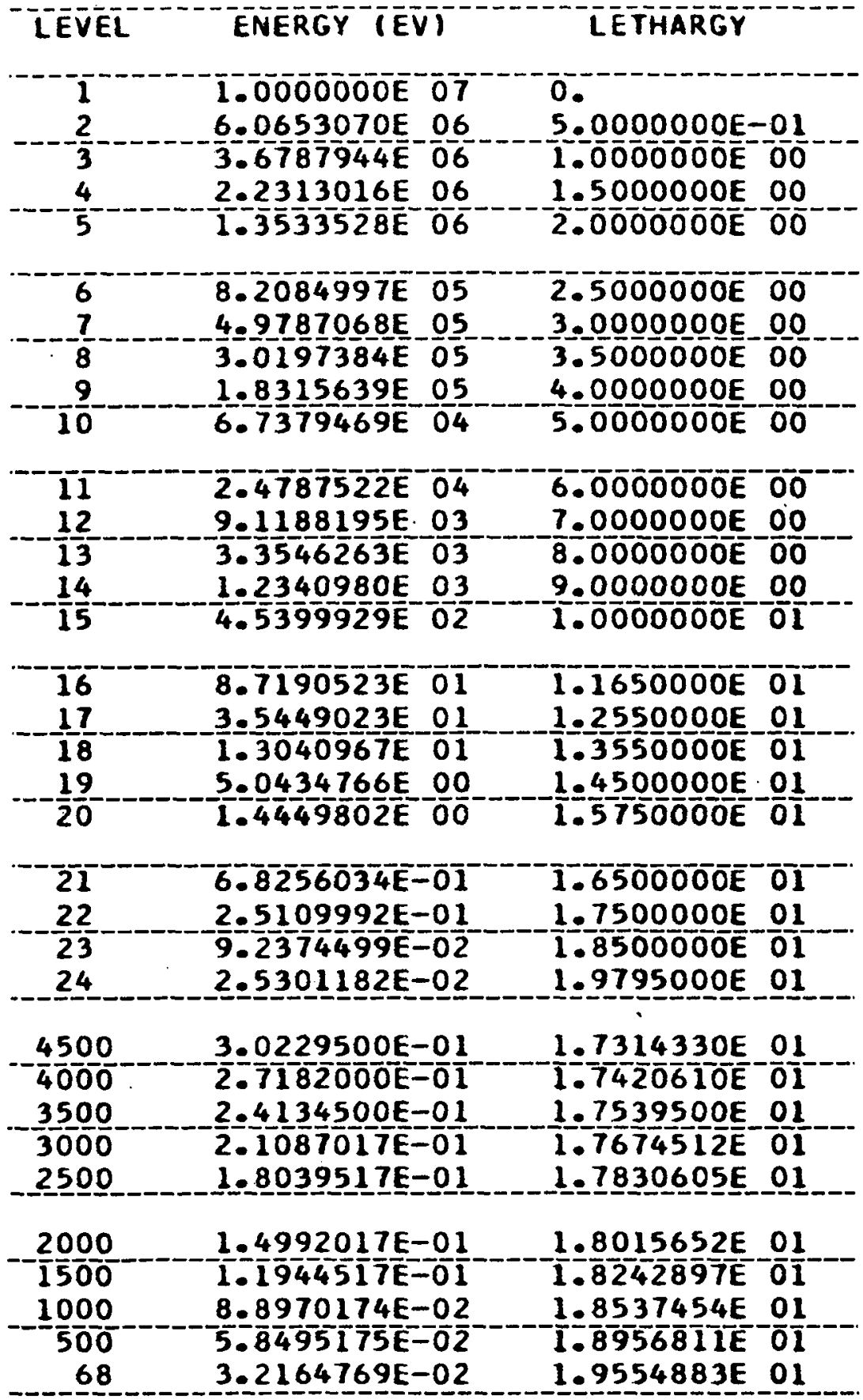




\begin{tabular}{|c|c|c|c|}
\hline GROUP & $\begin{array}{l}\text { LOWER } \\
\text { ENERGY (EV) }\end{array}$ & $\begin{array}{c}\text { UPPER } \\
\text { LEIHARGY }\end{array}$ & $\begin{array}{l}1 / V \text { FAC } \\
12200 \mathrm{M} / \mathrm{SEC}\end{array}$ \\
\hline $\begin{array}{l}-\frac{0}{1} \\
\frac{2}{3} \\
-\frac{4}{4}\end{array}$ & 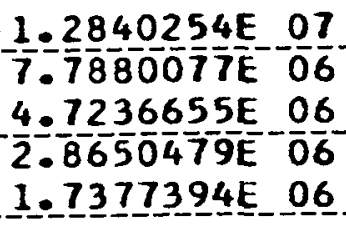 & $\begin{array}{r}-\frac{2}{2} \cdot \frac{5000000 E-01}{5000000 E-01} \\
7 \cdot 5000000 E-01 \\
1.2500000 E-00 \\
1.7500000 E-00\end{array}$ & $\begin{array}{l}5.0428001 \mathrm{E}=05 \\
6.4750835 \mathrm{E}-05 \\
8.3141723 \mathrm{E}=05 \\
1.0675608 \mathrm{E}-04\end{array}$ \\
\hline $\begin{array}{r}-\frac{5}{6} \\
7 \\
-8 \\
8\end{array}$ & 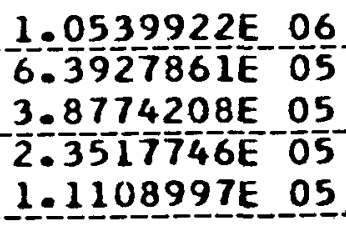 & $\begin{array}{l}\frac{2}{2} \cdot \frac{2500000 E}{7500000 E}-00 \\
\frac{3}{3} \cdot 2500000 E \\
3 \cdot 7500000 \mathrm{E}-\frac{00}{00} \\
4.5000000 E \text { OO }\end{array}$ & $\begin{array}{l}1.3707754 \mathrm{E}-04 \\
1.7601099 \mathrm{E}=04 \\
2.2600263 \mathrm{E}-04 \\
2.9019310 \mathrm{E}=04 \\
3.9793803 \mathrm{E}-04\end{array}$ \\
\hline $\begin{array}{r}10 \\
112 \\
12 \\
-\frac{1}{3} \\
14\end{array}$ & 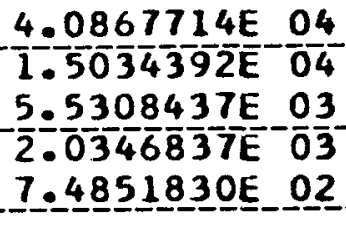 & 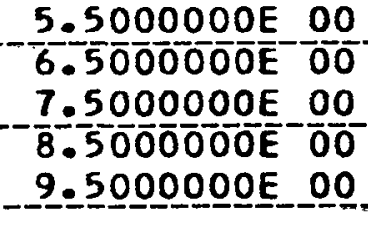 & $\begin{array}{l}6.1914461 \mathrm{E}-04 \\
1.0207969 \mathrm{E}-03 \\
1.6830096 \mathrm{E}-03 \\
2.7748135 \mathrm{E}=03 \\
4.5748941 \mathrm{E}-03\end{array}$ \\
\hline $\begin{array}{l}-\frac{15}{16} \\
17 \\
-18 \\
-19\end{array}$ & $\begin{array}{l}1.9895839 \mathrm{E}-02 \\
\frac{1}{5.5595137 \mathrm{E}} 01 \\
2.1500919 \mathrm{E}-01 \\
8.1099823 \mathrm{E}-00 \\
2.6995785 \mathrm{E} O 0\end{array}$ & $\begin{array}{l}1.0825000 E \\
1.2100000 E \\
1.3050000 E \\
-1.4025000 E \\
1.5125000 E \text { 01 }\end{array}$ & $\begin{array}{l}8.2454356 \mathrm{E}-03 \\
1.5773244 \mathrm{E}-0 \frac{2}{2} \\
\frac{2}{4} \cdot \frac{7305059 \mathrm{E}-02}{4204265 \mathrm{E}-02} \\
7.4459925 \mathrm{E}-02\end{array}$ \\
\hline $\begin{array}{r}20 \\
-\frac{1}{1} \\
22 \\
-\frac{2}{3} \\
24\end{array}$ & $\begin{array}{l}9.9311942 \mathrm{E}-01 \\
4.1399378 \mathrm{E}=01 \\
1.5229980 \mathrm{E}-01 \\
4.8344435 \mathrm{E}=02 \\
2.5301182 \mathrm{E}-02\end{array}$ & $\begin{array}{l}1.6125000 E \text { O1 } \\
1.7000000 E \\
1.8000000 E \\
1.91 \\
1.979501\end{array}$ & 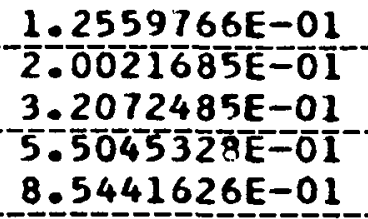 \\
\hline
\end{tabular}


283

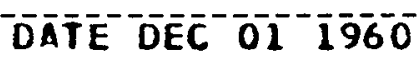

DESIGNATION

URANIUM 235
CODE NO.

92.2350
DENSIIY FACTOR

$3: 90255 E \quad 02$

LEVEL_ENERGY SCAIIER SI SIGMA SIGMA

- 1 1.000E $07 \quad 4.2674 \mathrm{E} \quad 00 \quad 3.5234 \mathrm{E} \quad 00 \quad 1.8065 \mathrm{E} \quad 00 \quad 1.8753 \mathrm{E} \quad 0.0-2.2758 \mathrm{E} \quad 00$

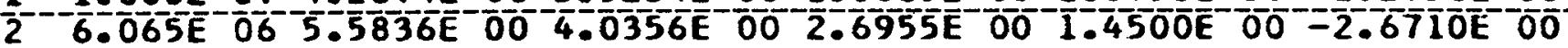

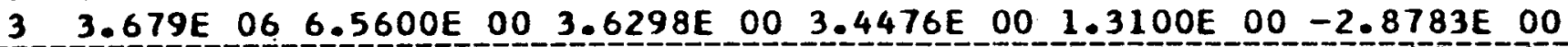

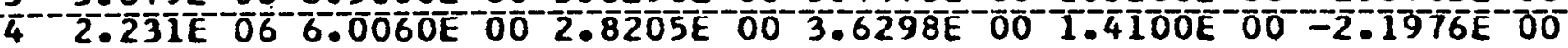

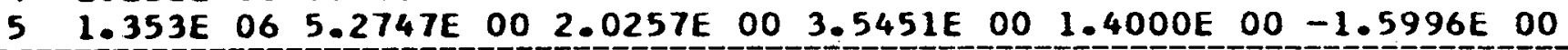

$6 \quad 8.208 E$ O5 $5.4181 E \quad 00 \quad 1.4641 E \quad 00 \quad 3.8018 E \quad 00 \quad 1.3378 E \quad 00-1.4948 E \quad 00$

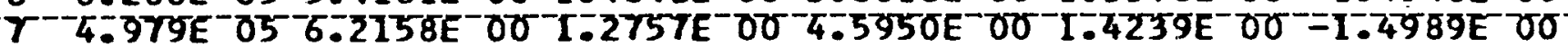
$8 \quad 3.020 E$ O5 $7.4775 E$ OO $1.0831 E$ O 00 5.8924E $00 \quad 1.5699 E \quad 00-1.4660 E \quad 00$

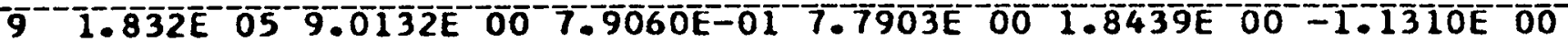
$106.738 \mathrm{E} \quad 04 \quad 1.0327 \mathrm{E} \quad 01 \quad 2.8458 \mathrm{E}-01 \quad 1.0025 \mathrm{E} \quad 01$ 2.2750E $00 \quad-2.7949 \mathrm{E}-01$

$11 \quad 2.479 E \quad 04 \quad 1.0462 E \quad 01 \quad 8.9530 E-02 \quad 1.0431 E \quad 01 \quad 3.6903 E \quad 00-2.8104 E-02$

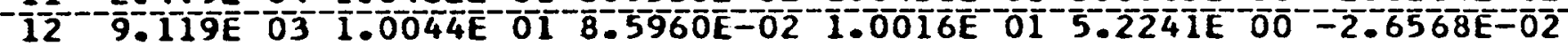
$13 \quad 3.355 E \quad 03 \quad 1.0034 E \quad 018.5871 E-02 \quad 1.0005 E \quad 01 \quad 7.3008 E \quad 00-2.6540 E-02$

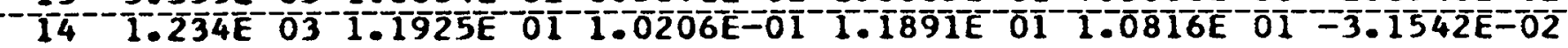
$154.540 \mathrm{E} \quad 02$ 1.3353E 01 1.1428E-01 1.3315E 01 2.3062E $01-3.5318 \mathrm{E}-02$

$\begin{array}{llllllllllll}16 & 8.719 E & 01 & 1.6138 E & 01 & 1.3811 E-01 & 1.6092 E & 01 & 3.6485 E & 01 & -4.2687 E-02\end{array}$

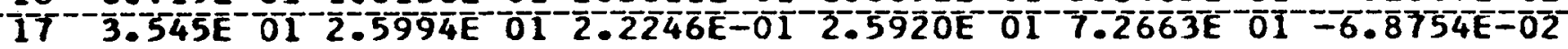
$18 \quad 1.304 E \quad 01 \quad 1.1277 E \quad 01 \quad 9.6508 E-02 \quad 1.1245 E \quad 01 \quad 1.0987 E \quad 02-2.9827 E-02$ $19-5.043 E^{-} 00-1.0183 E^{-01} 8.6379 E-02$ 1.0155E $01-4.0863 E-01-2.6705 E-02$ 20 1.445E 00 1.1605E 01 9.8435E-02 1.1572E 01 4.2582E $01-3.0431 \mathrm{E}-02$

$21 \quad 6.826 E-01 \quad 1.2827 E \quad 01 \quad 1.0880 E-01 \quad 1.2790 E \quad 01 \quad 7.9234 E \quad 01-3.3636 E-02$

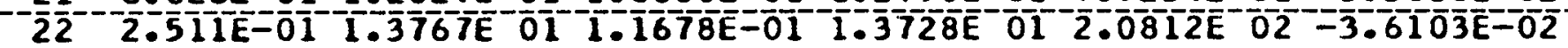
$239.237 E-021.4351 E \quad 01 \quad 1.2173 E-01 \quad 1.4311 E \quad 013.3004 E-02-3.7633 E-02$

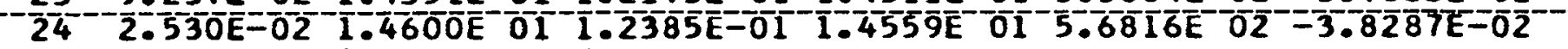

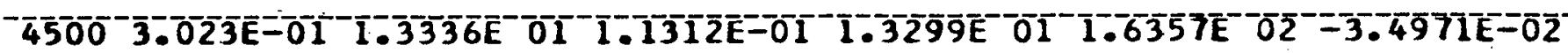
$40002.718 E-011.3435 E$ O 1 1.1396E-01 $1.3397 E \quad 01 \quad 1.7565 E \quad 02-3.5230 E-02$

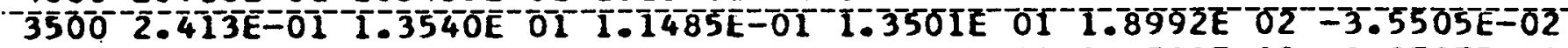
$30002.109 E-01 \quad 1.3651 E$ E $011.1579 E-01 \quad 1.3612 E \quad 012.0703 E \quad 02-3.5797 E-02$

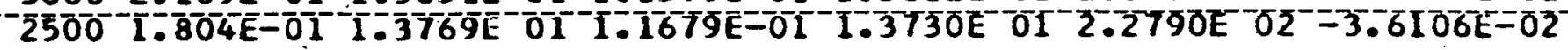

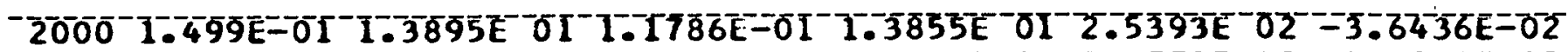
$15001.194 \mathrm{E}-01$ 1.4030E 01 1.1901E-01 1.3990E 01 2.8778E $02-3.6789 \mathrm{E}-02$

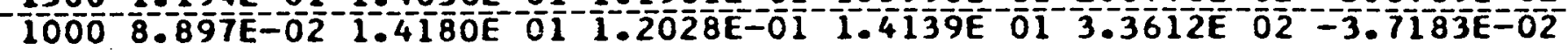
$5005.850 E-02 \quad 1.4355 E$ O 01 1.2176E-01 $1.4314 \mathrm{E} \quad 01 \quad 4.2093 \mathrm{E} \quad 02-3.7642 \mathrm{E}-02$

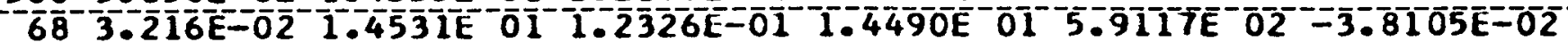




DESIGNATION

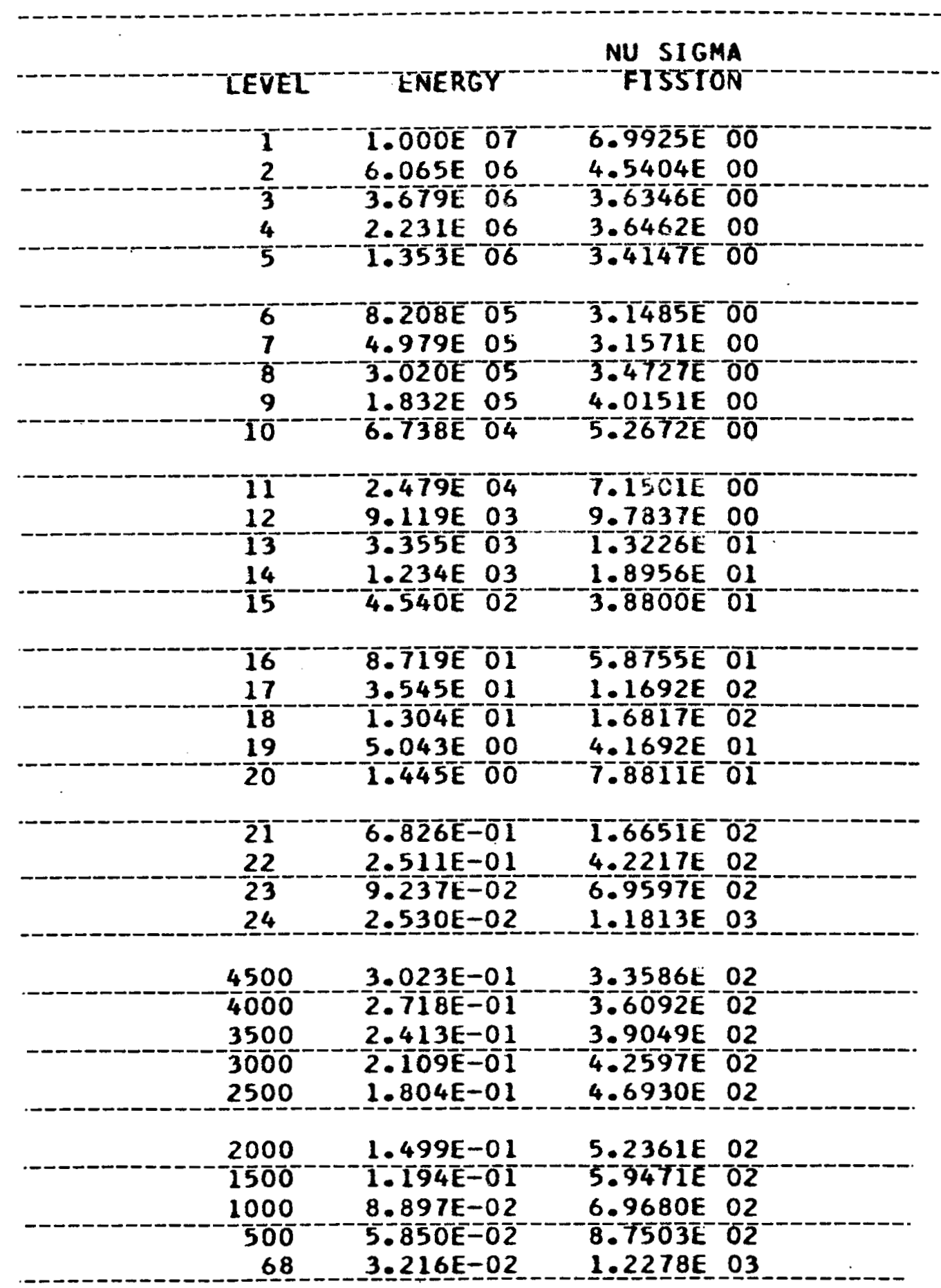




\section{URĀNITUM}

\section{THERMAL TRANSFER MATRICES}

TEMPERATURE DEG. $4 . \overline{5} 0 \overline{0} \overline{0 E} \overline{0} \overline{3}$

\section{SIGMA \\ SCATTER}

SIGMA TRANSPORT
ABSORPTION

$9.4 \overline{8} \overline{8} \overline{\mathrm{O}} \overline{2}$
NU SIGMA FISSION

$-3$

N1 N2

SIGAA S (NTINNT)

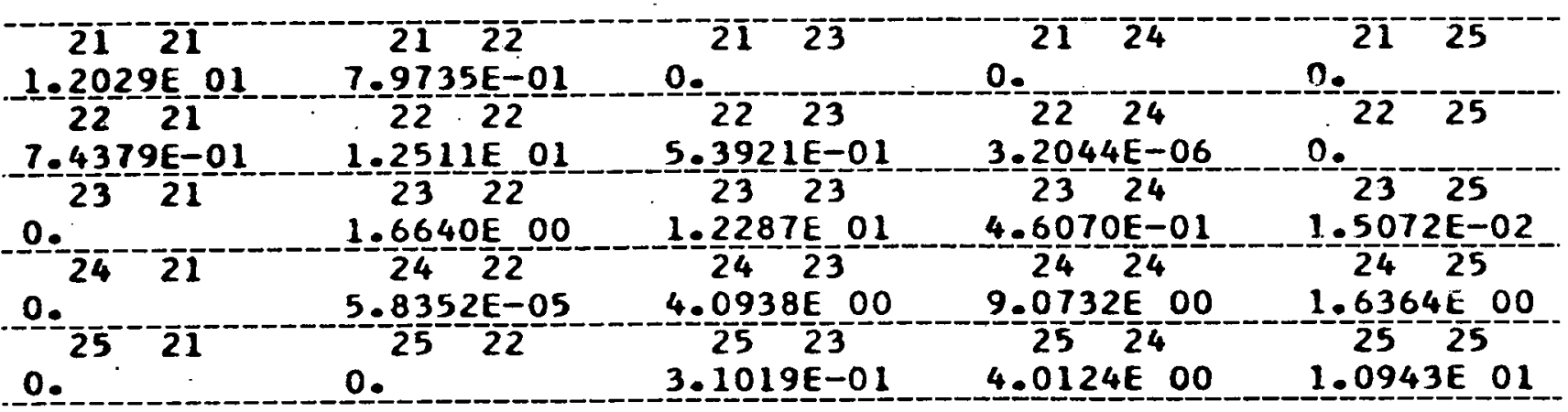

\begin{tabular}{|c|c|c|c|c|}
\hline IEMPERATURE & SCIGMA & $=\frac{S I G M A}{T R A N S P O R T}$ & SIGMA & $\frac{\text { NU SIGMA }}{\text { FISSION }}$ \\
\hline $4.0000 \mathrm{E} 03$ & $1.5209 \bar{E} \quad 01$ & $1.5 \overline{166 E} 0 \overline{1}$ & $9.4471 \mathrm{E} 02$ & $1.9547 \mathrm{E} 03$ \\
\hline
\end{tabular}

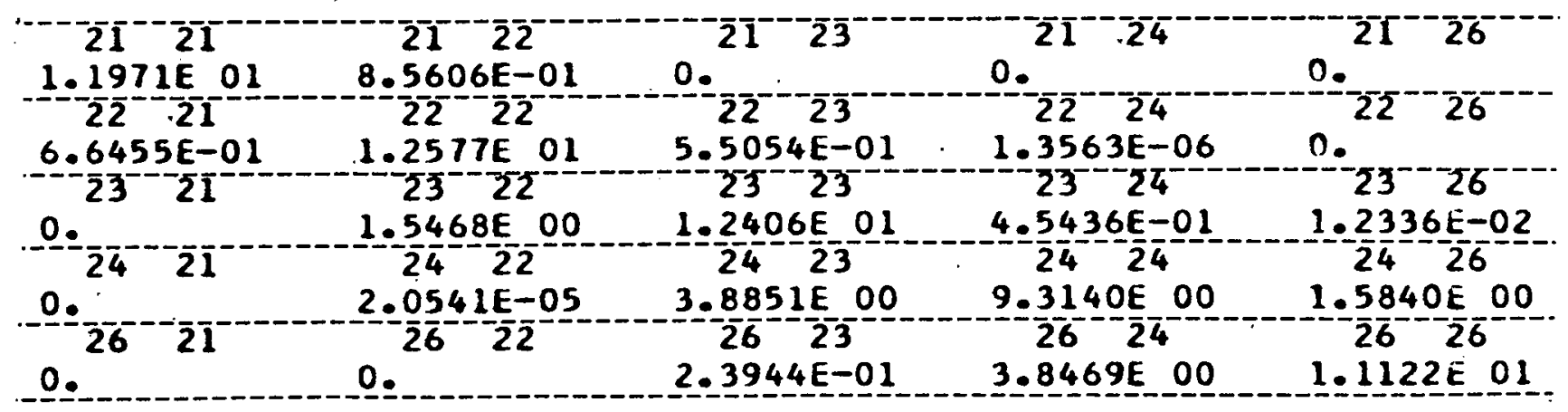


URAĀNITYM $\overline{2} \overline{3} \bar{S}$

THERMAL TRANSFER MAIRICES

TEMPERAIURE

SI GMA

SIGMA

SI GMA

NU SIGMA

DE G.

SCATTER

TRANSPORT

ABSORPTION

FISSION

$\overline{3} \cdot \overline{5} \overline{0} \bar{O} \overline{\mathrm{E}} \overline{0} \overline{3}$

$\overline{1} . \overline{1} \overline{1} \overline{2} \overline{\mathrm{O}}$

$\overline{1} \cdot \overline{5} \overline{1} 0 \overline{9} \overline{0} \bar{I}$

$\overline{9} . \overline{4} \overline{7} \overline{5} \overline{\mathrm{O}} \overline{\mathrm{O}}$

$\overline{1} . \overline{56} \overline{\mathbf{0}} \overline{0}$

\section{NI \\ N2 \\ STGMA S (NNTINZ)}

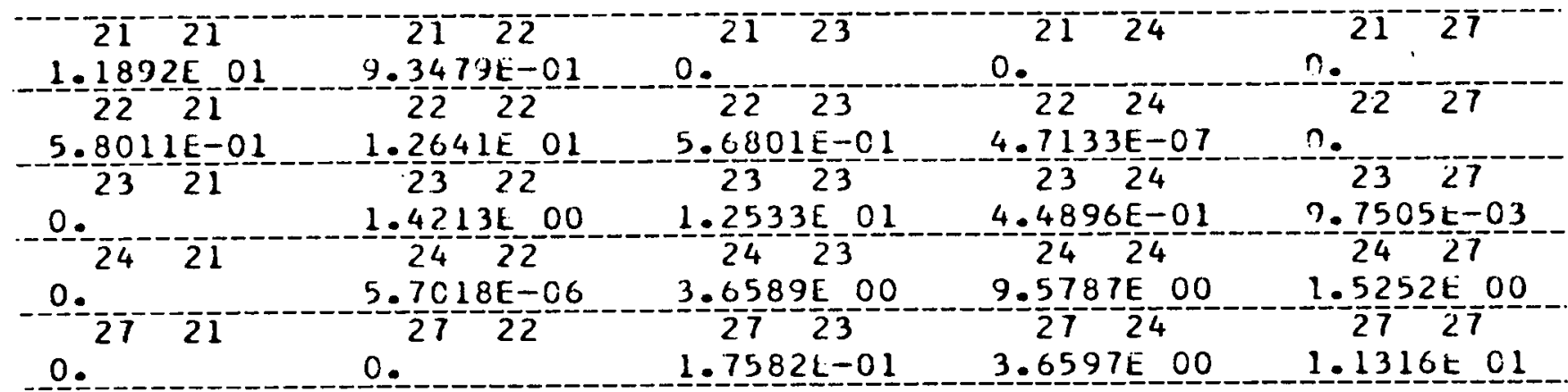

TEMPERATURE

$\overline{D E G}$. $\bar{F}$.

उ. OOOOOE
SIOMA

SCATTER

$1.5094 \bar{E} 01$
SIGMA

TRANSPORT

1. $50 \overline{5} \overline{1} \overline{0} \overline{1}$
SI GMA

ABSORPTIUN

$9.4 \overline{1} 0 \overline{\mathrm{E}} \mathrm{O} 2$
NU SICMA

FISSIONN

\section{$S\left(G M A-\frac{N}{S}\left(N \frac{N}{2} \frac{N}{N}\right)\right.$}

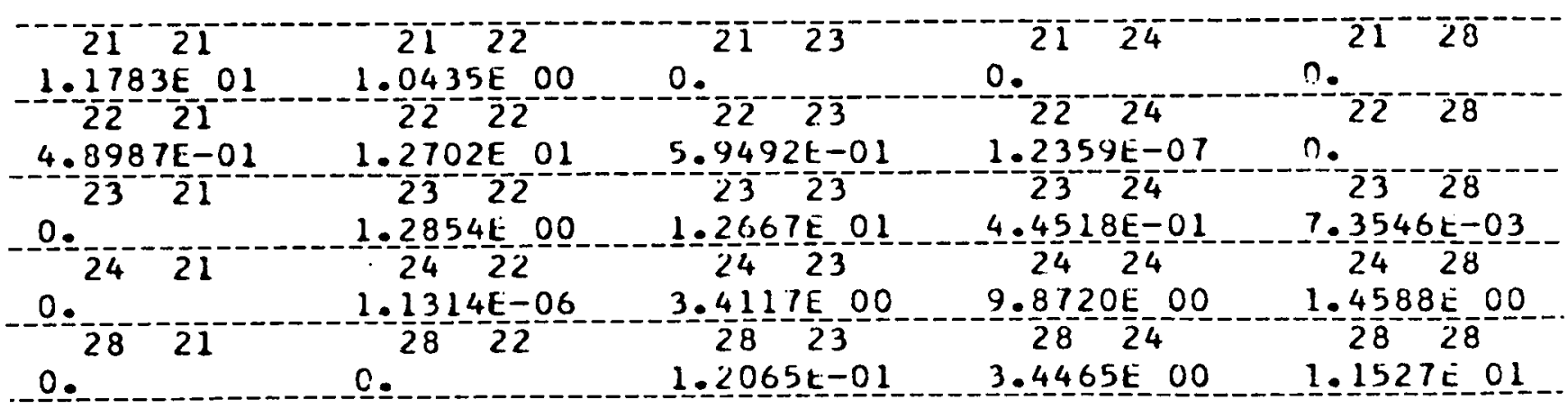


IEMPERATURE

DEG. F.

$2.5 \bar{O} 0 \overline{O E} \overline{0} \overline{3}$
SCATTER

$1.5037 \bar{E} 01$
TRANSPORT

$\overline{1.4} \overline{9} \overline{4} \overline{\mathrm{E}} \mathrm{O} \overline{\mathrm{I}}$
ABSORP TION

$9.4 \overline{9} \overline{\mathrm{IE}} 0 \overline{2}$
NU SI GMA

FISSION

$1 . \overline{9634} \overline{\mathrm{E}} \overline{\mathrm{O}}$

\section{SIGMA $\frac{N}{S}\left(\frac{N}{N} \frac{N}{T N}\right.$}

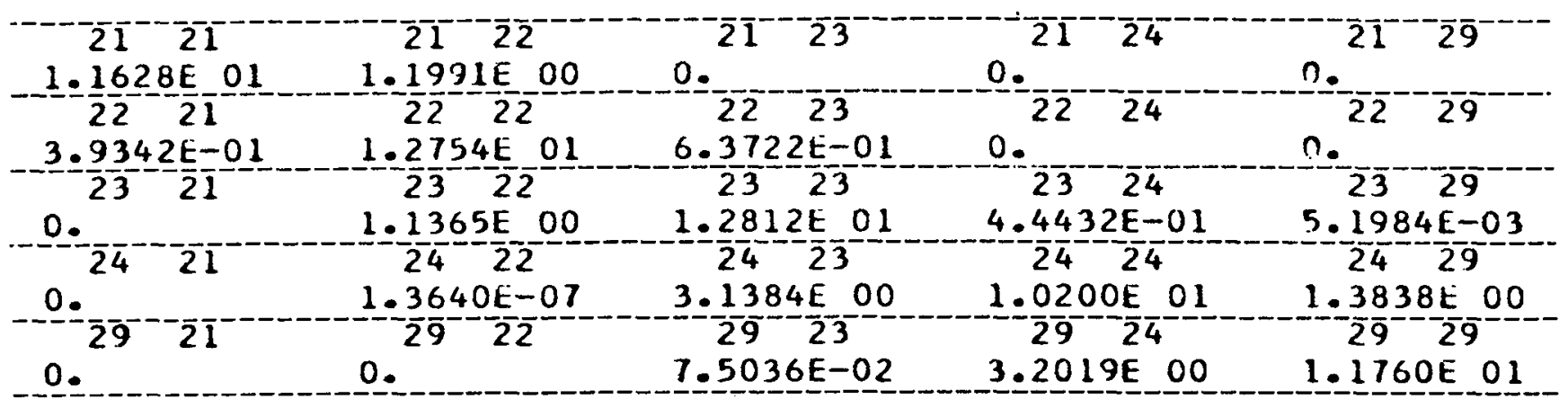

TEMPERATURE
SIGMA

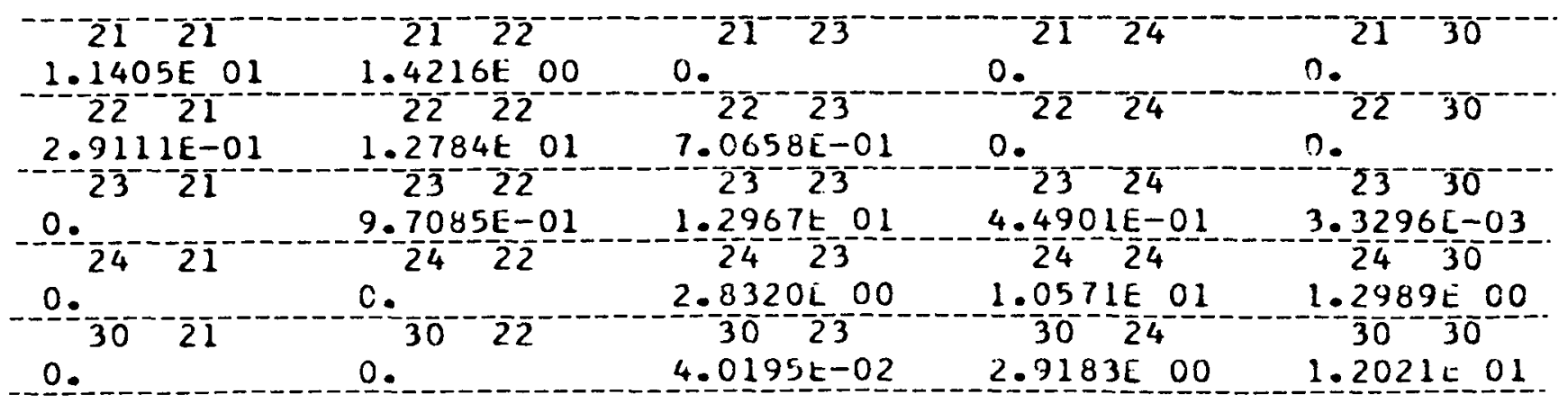


TEMPERATURE

$\bar{D} \bar{E} \bar{G}$.

1. $5000 \bar{E}-0 \overline{3}$
SIGMA

SC ATTTER

$1.4922 E^{-} 01$
SIGMA TRANATSTORT
SI GMA

$\bar{A} \bar{B} \bar{S} O \bar{R} \bar{P} \bar{T} \bar{I} O \bar{N}$

NU SIGMA

F I SSIOON

\section{N1 N2 \\ SIGMA- 5 (NTITN2)}

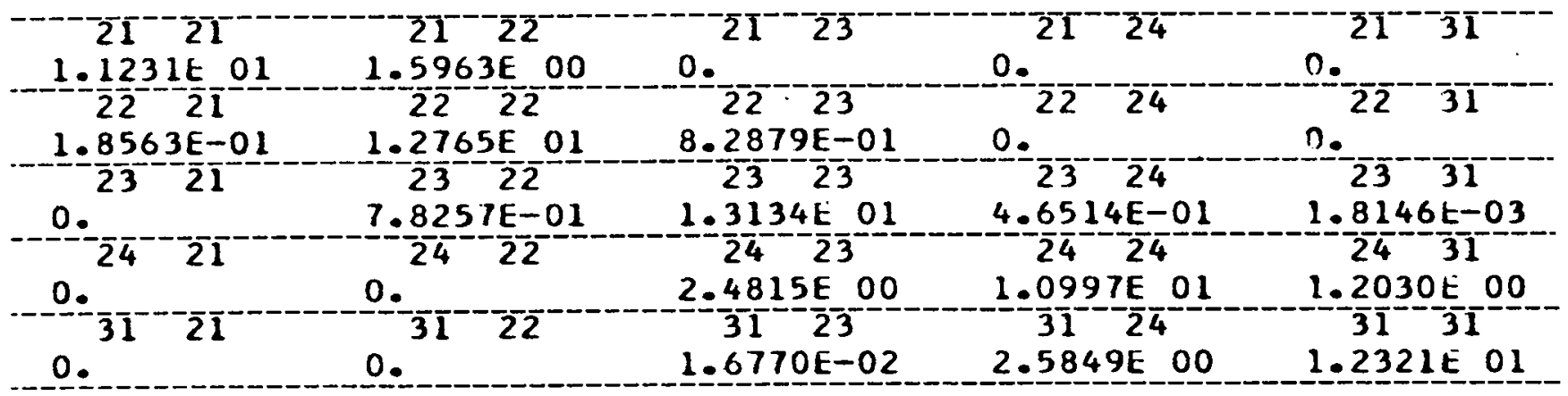

TEMPERATURE

DEG.

SIGMA

SCATTTER

$\overline{1} \cdot \overline{0} 0 \overline{0} 0 \bar{E}-\overline{0} \overline{3}$

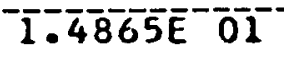

SIGMA

TRAN'SPORT
SIGMA

ABSORPTION

$\overline{9} . \overline{6} \overline{1} \overline{\mathrm{E}} \overline{\mathrm{O}} \overline{2}$

I.9767E $03^{-}$ 
TEMPERATURE

$\bar{D} \bar{E} \cdot-\bar{F}$.

$-\overline{5} \cdot \overline{0} \bar{O} \bar{O} \bar{O} \bar{E}^{-} \overline{0} \overline{2}$
SIGMA

STCATTER

$\overline{1} . \overline{4} \overline{80} \overline{8} \overline{\mathrm{E}}^{-} \mathrm{O} \mathrm{I}$
SIGMA

TRANSTORT

$\overline{1} \cdot \overline{4} \overline{7} \overline{6} \overline{[} \overline{0} \bar{l}$
SIGMA

$\bar{A} \bar{B} \bar{S} O \bar{P} \bar{P} \bar{T} \bar{I} \bar{O}$

$\overline{9} . \overline{6} \overline{3} \overline{8} \overline{0} \overline{2}$
NU SI CMA

$\bar{F} \overline{I S} \bar{S} \overline{I O N}$

NI N2

STGMA S-

\begin{tabular}{|c|c|c|c|c|}
\hline $1.2242 E$ OI & $5.8504 \mathrm{E}-01$ & 0.2123 & 0.2124 & 0.2133 \\
\hline $1.1342 \mathrm{E}-01$ & $1.2114 \mathrm{E} O 1$ & $1.5469 t 00$ & 0. & $0.22 \quad 33$ \\
\hline 0. & $\begin{array}{r}23 \\
3.0313 \mathrm{E}-01\end{array}$ & $\begin{array}{l}23 \\
1.3426 E \quad 01\end{array}$ & $\begin{array}{l}23 \\
6.3973 E-01\end{array}$ & $\begin{array}{l}233 \\
1.3613 z-04\end{array}$ \\
\hline 0. & $0.24-2$ & $\begin{array}{l}24 \\
1.5529 E 00\end{array}$ & $1.2104 E 01$ & $9.84211-01$ \\
\hline & 0. & $3.6961 \mathrm{E}-04$ & $\begin{array}{r}33 \\
1.6797 \mathrm{E} 00\end{array}$ & $\begin{array}{l}1.3128 E 01 \\
1.35\end{array}$ \\
\hline
\end{tabular}

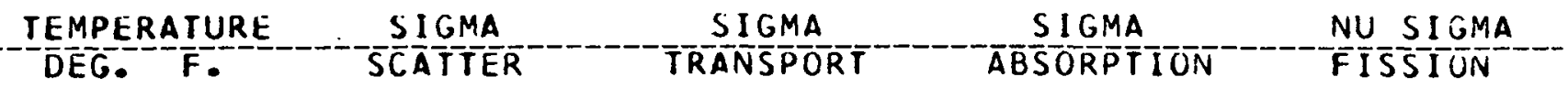

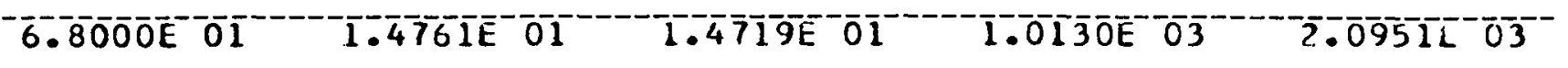

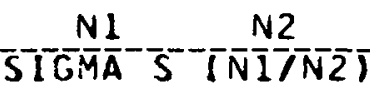

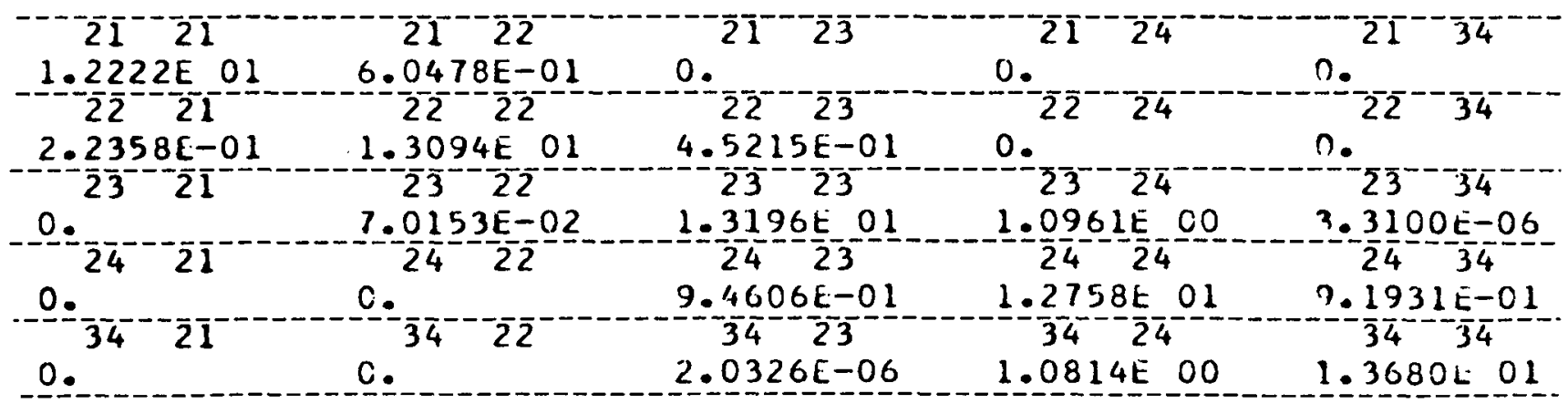




\section{SIGMA SO}

TRANSFERS FROM GROUPS 1 IHROUGH 10 TO GROUPS I THROUGH 16

NO. RESONANCES

45

SIGMA (N, GAMMA)

IRANSFERS FROM GROUPS 1 THROUGH 34 IO GROUPS 1 THROUGH 11 


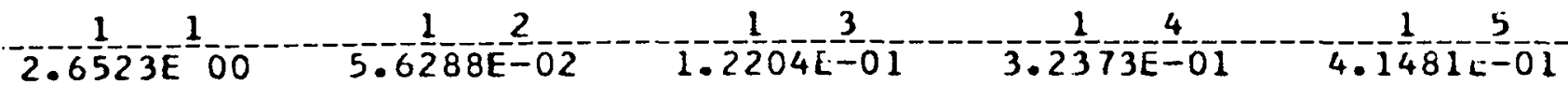

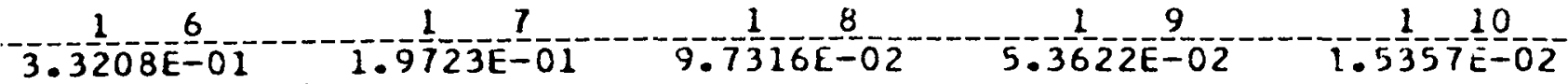

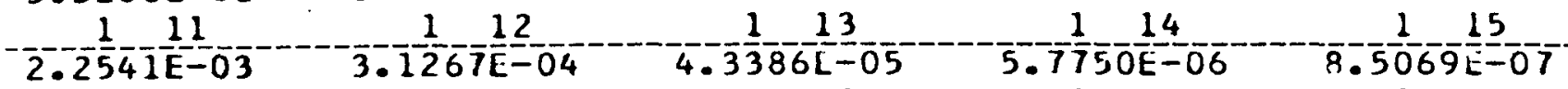

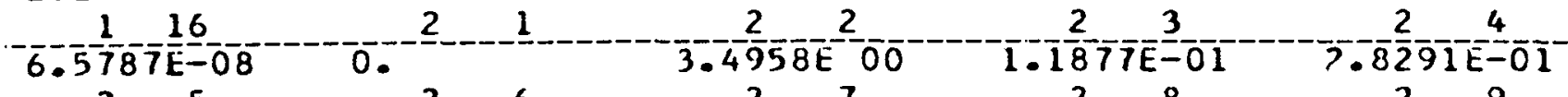

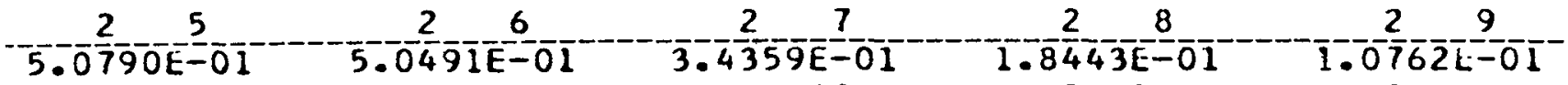

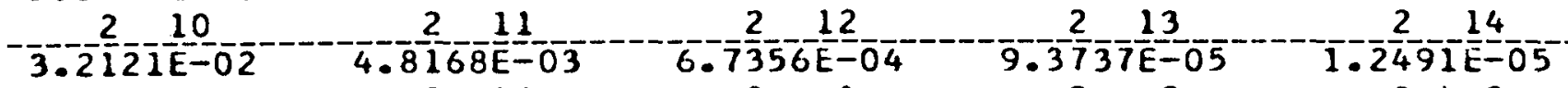

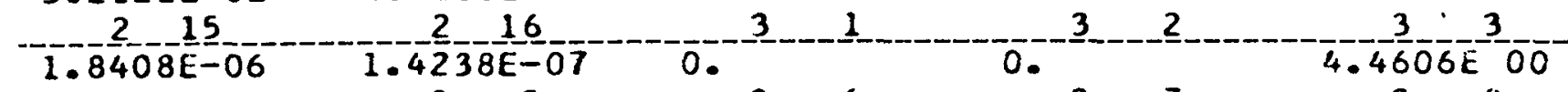

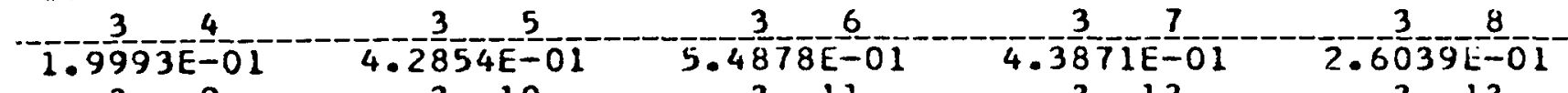

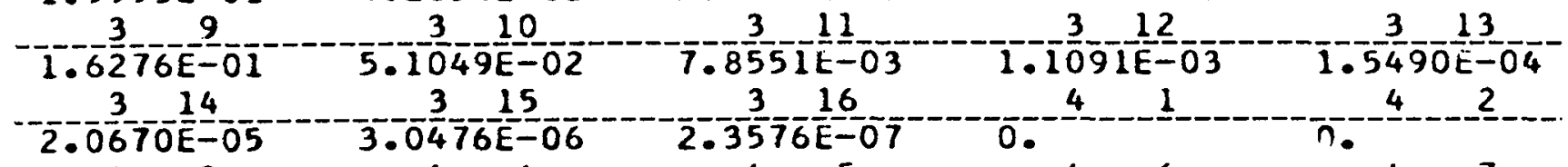

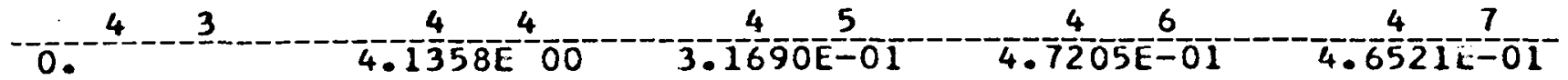

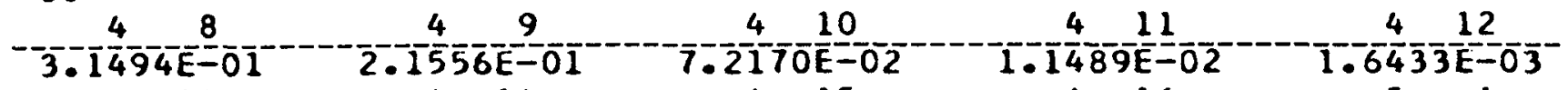

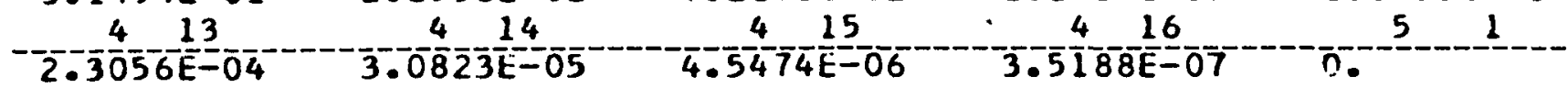

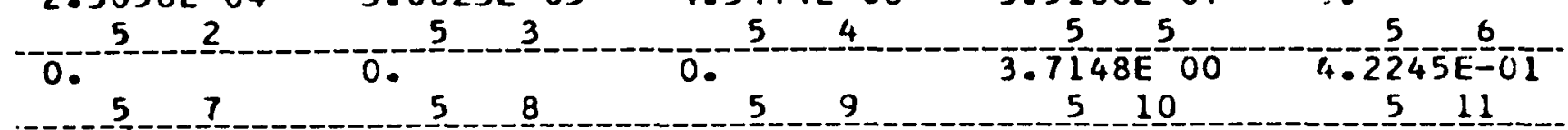

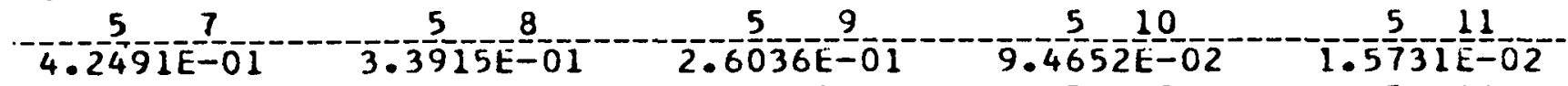

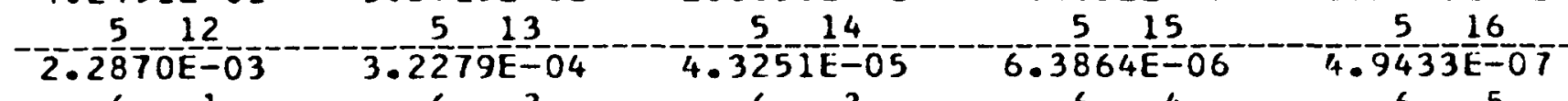
-0.

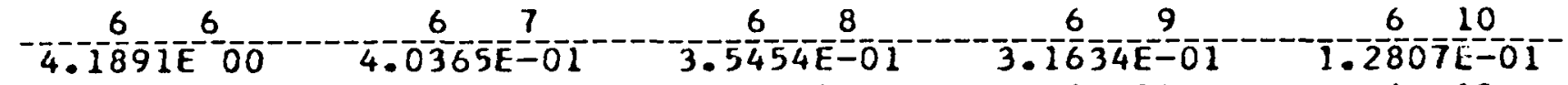

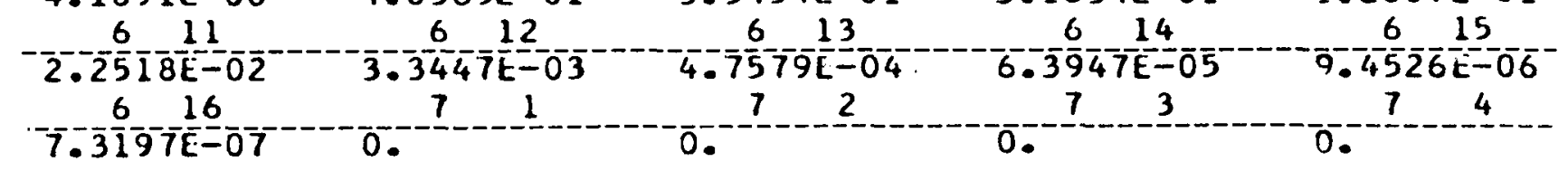




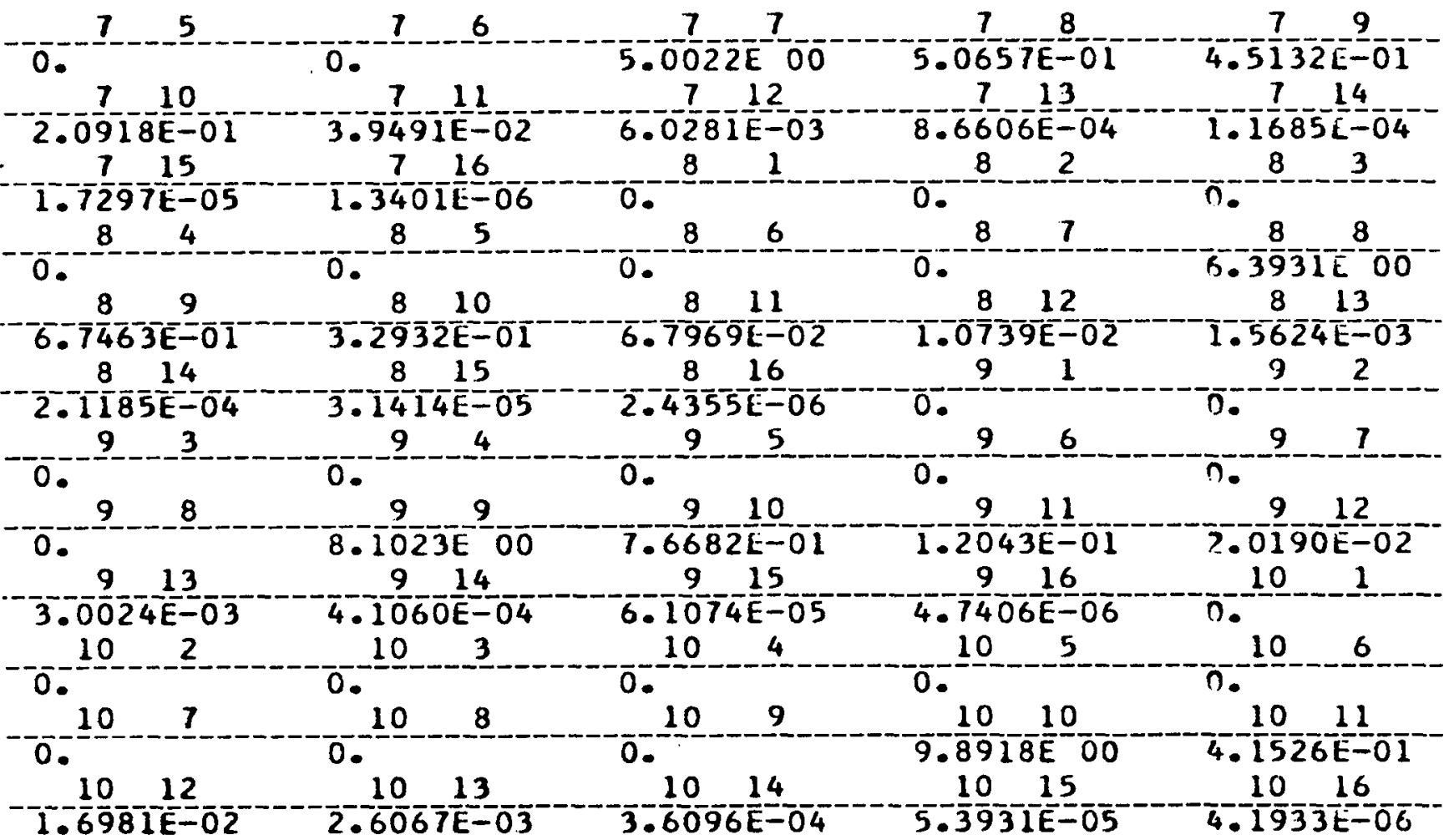


U'RĀNIUUM

RESONANCE
RESONANCE


URATNIUMM $2 \overline{3} \overline{5}$

$\bar{N} \overline{1}-\cdots$

SIGMA N,G (N1/N2)

0.

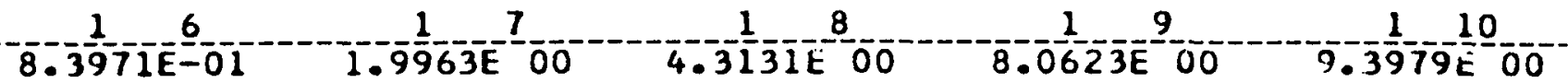

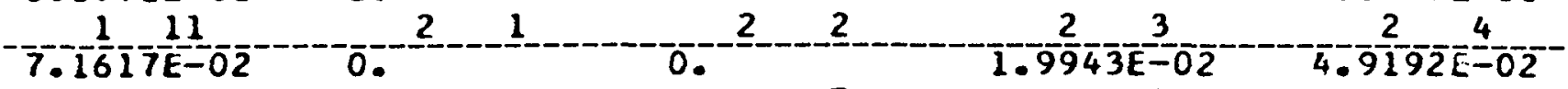

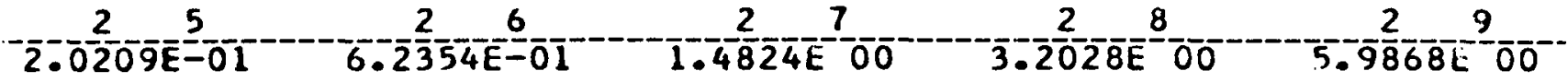

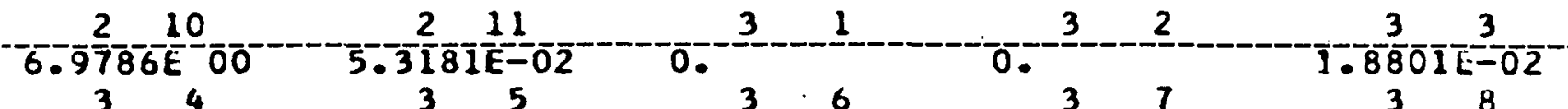

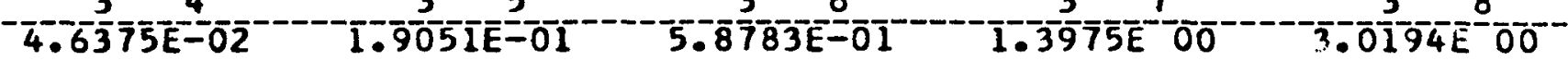

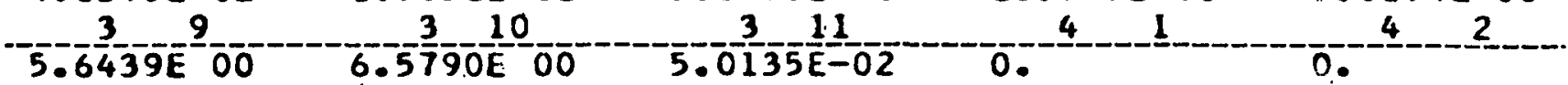

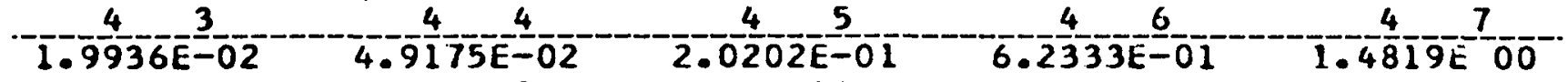

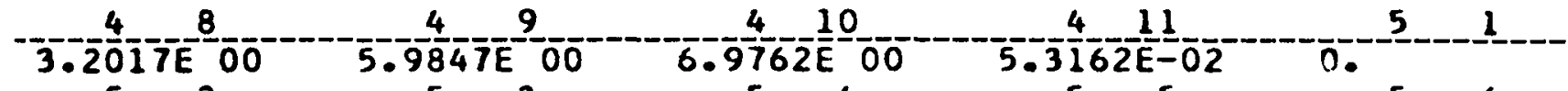

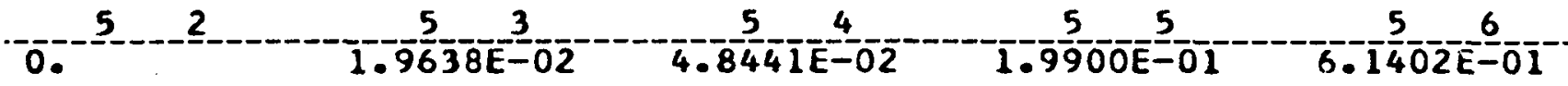

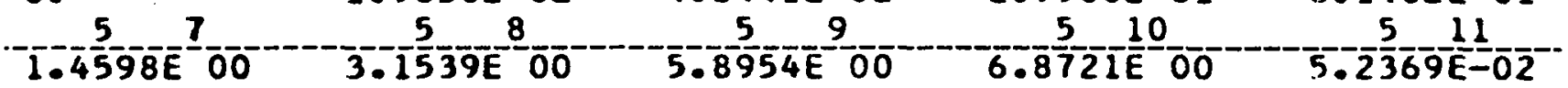

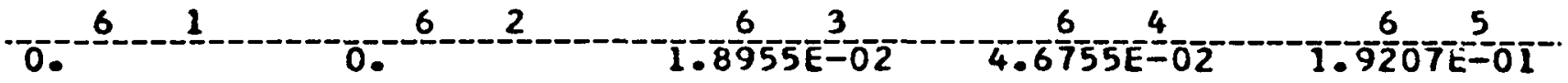

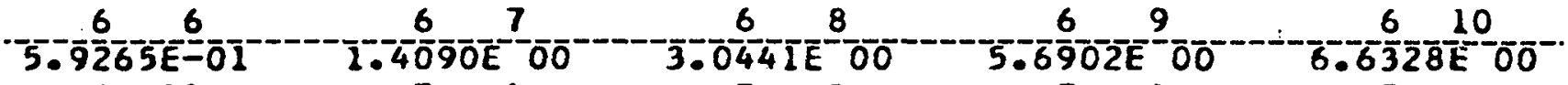

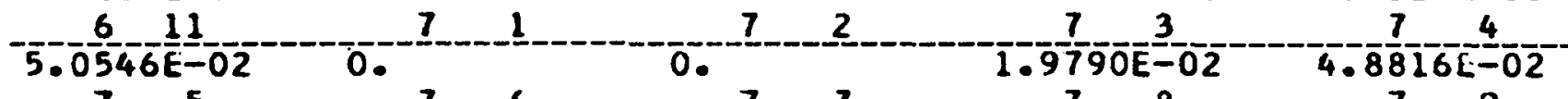

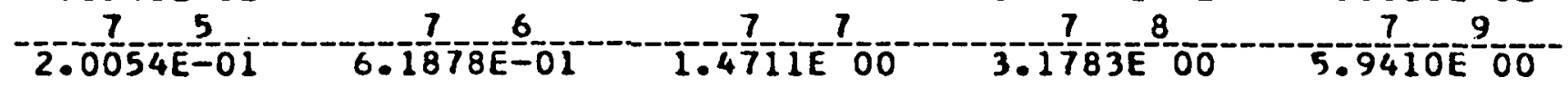

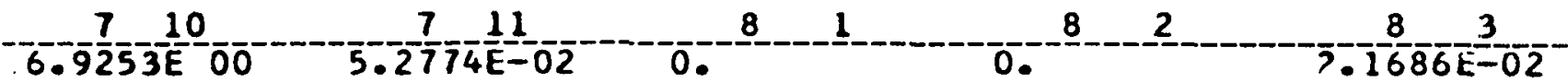

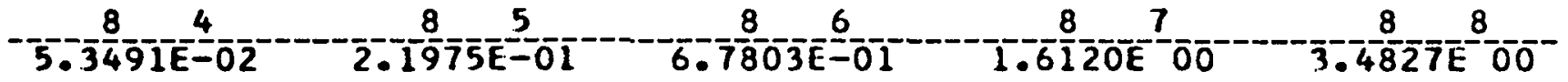

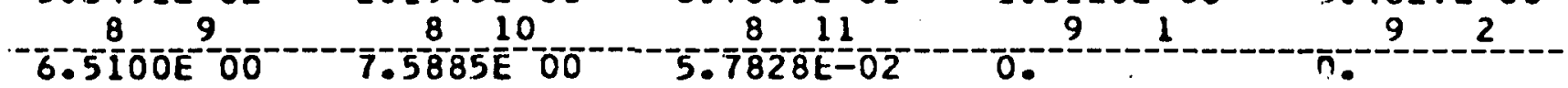

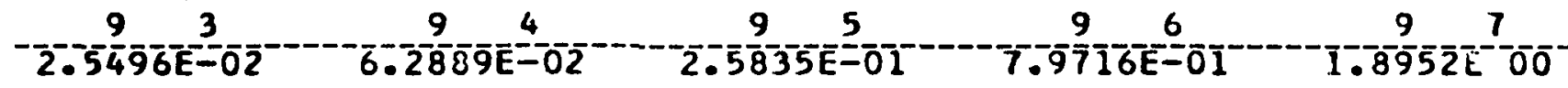

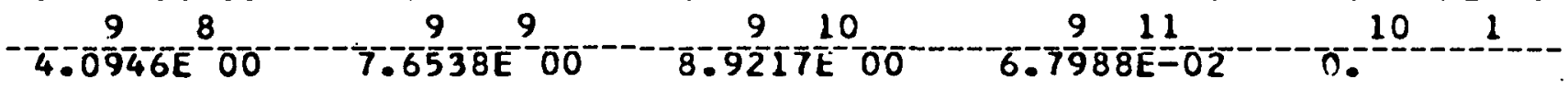


URANĀIUM $2 \overline{3} \overline{5}$

$\mathrm{N} 1$

SIGMA N.G (N1/N2)

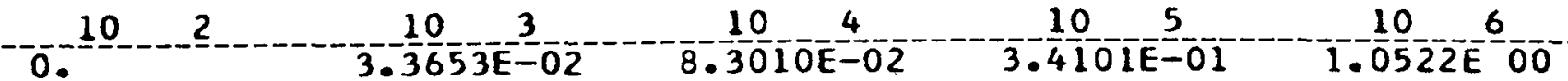

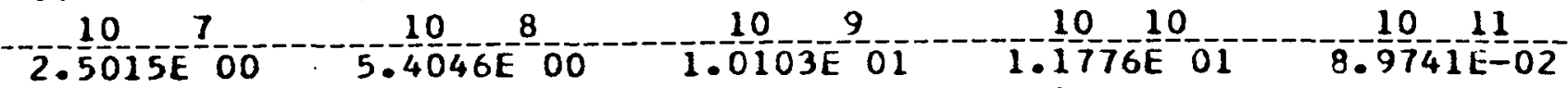

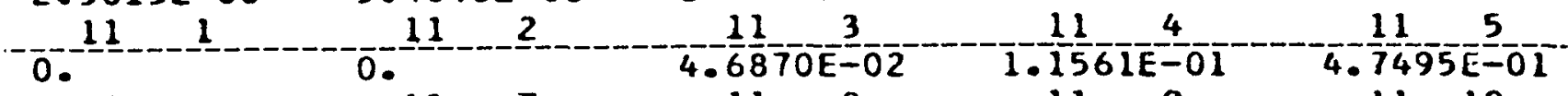

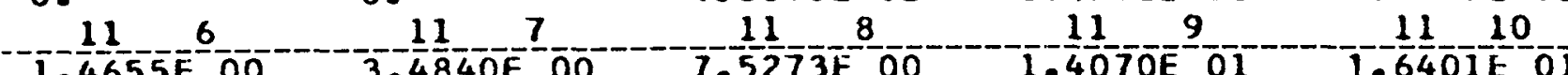

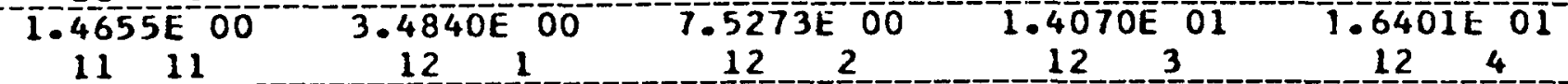
$1.2499 \mathrm{E}-01 \mathrm{I}^{-} 0_{0}$

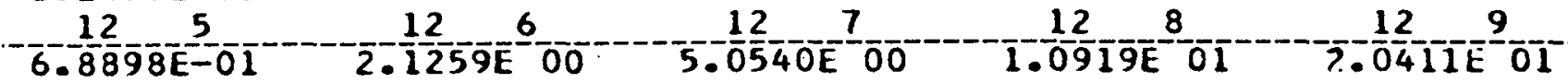
$-12-10$

$2.3792 \mathrm{E}$ OI $1.8131 \mathrm{E}-01$

$--\frac{13}{302}-4$

$9.4 \frac{13}{58}-\frac{5}{1 E-0} \overline{1}$ 0 .

139 $13 \quad 10$ $2.9183 \mathrm{E}-00$ $3.2662 \mathrm{E} 01$ 2.4890E-01 $-1 \frac{14}{3} 825 \mathrm{E}-\mathrm{O}$ 3. $-\frac{14}{4} \frac{4}{1} \bar{O} \overline{1} \frac{4}{E}-\bar{O} \overline{1}$ $\frac{14}{1 .} \frac{5}{4009 E} 00$ 0.13 $6 . \frac{1}{9} \frac{3}{380}-\frac{7}{0}-10$ $9.3337 E-02$ $14 \quad 8$
$-20 \quad 14$

$2.2202 \bar{O}$ 4. $\overline{1} \overline{1} \overline{0} \overline{2} \mathrm{E}$ Ō $4.8377 \mathrm{E}$ OI 0.1 $-15$ $2 . \frac{15}{80} \overline{1} \mathrm{O}^{3}-0 \mathrm{O}$

$--\frac{15}{2}-\frac{7}{8}-1 E^{-01}$ $4 . \frac{15}{4} \overline{8} \overline{5} \mathrm{E}^{8}-\overline{0}$ 0. 16

$-16-6$ 0. 162

(-)
$6-\frac{15}{909}-\frac{4}{2}$ $-\frac{14}{32} 25 E^{6}-0$ $-\frac{13}{1.4990 \mathrm{E}^{-1}}$ $16-11$ 1. $1920 \mathrm{E} 0 \overline{0}$ $3.3226 \mathrm{E}^{-1} \mathrm{01}$ 159 $--\frac{1}{0} \frac{7}{6}-5$ 0. $17-1$

1710

$3.0944 E-02$

$2.7648 \bar{E}-61$ $8.4087 E^{-} O 1$ $-3.6866 \bar{E}-11$ $\frac{16}{4.4698}-\frac{3}{16}-01$ $2.2 \frac{1}{8}$ 15 010 14. 2 $-\frac{18}{3 .} \frac{8}{2} \overline{8} \overline{8}-\overline{0}$ 2. $.358 \overline{1}-00$ 189 $-\frac{18}{32} 6 \frac{5}{10}$ 7. $\frac{16}{1786 E}-\frac{8}{01}$ 1510 $9 . \overline{8} \overline{1} \overline{8} \mathrm{E}^{-} \mathrm{O}$ $1 . \frac{14}{0} \overline{7} \frac{7}{6}-\overline{0}-$ 
URANIUMM 235

$N 1$

SIGMA N.G (NI/N2)

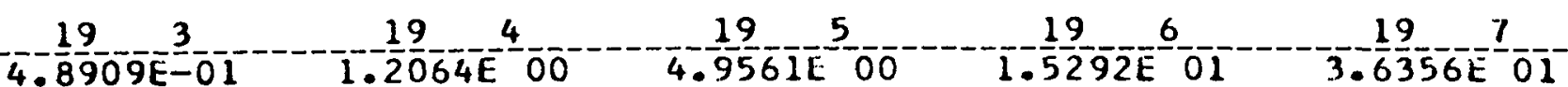

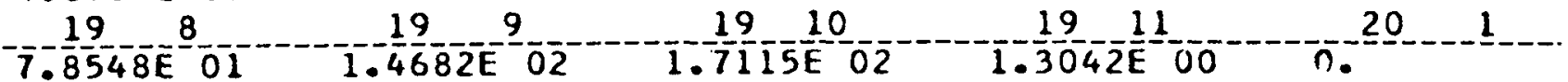

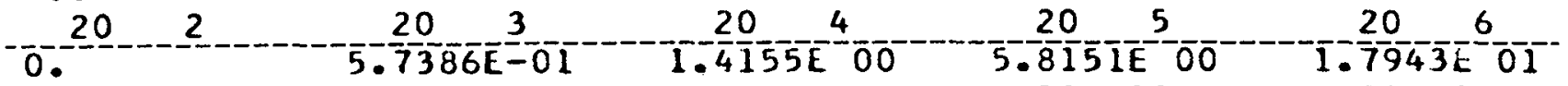

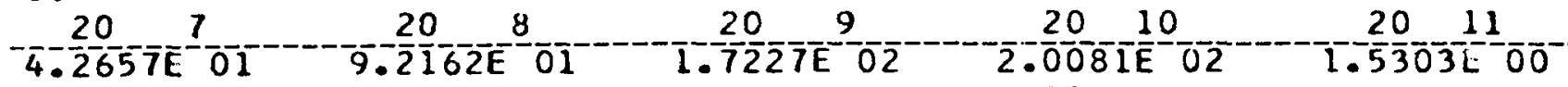

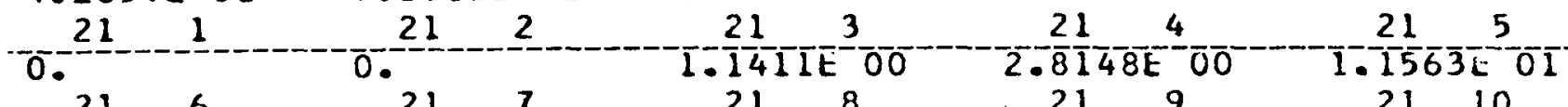

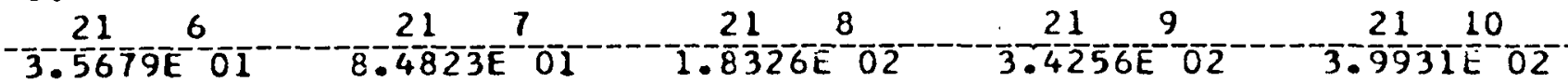

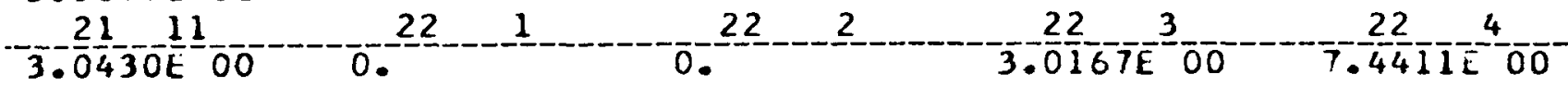

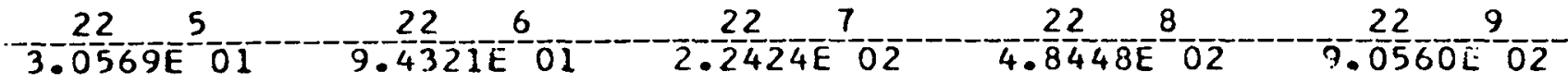

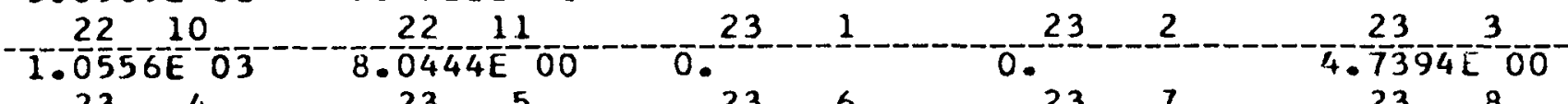

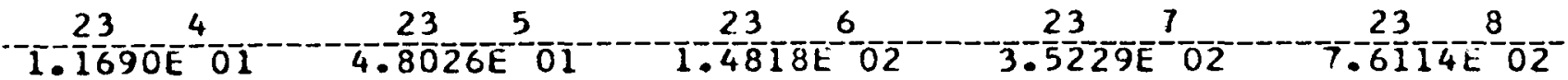

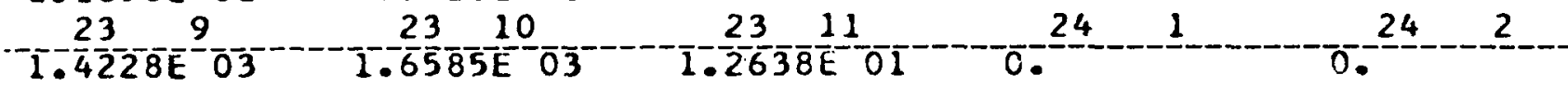

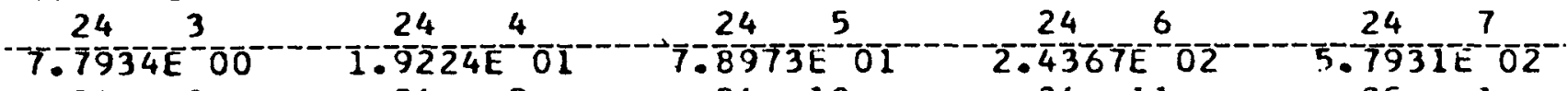

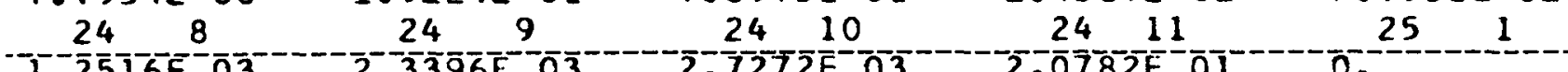

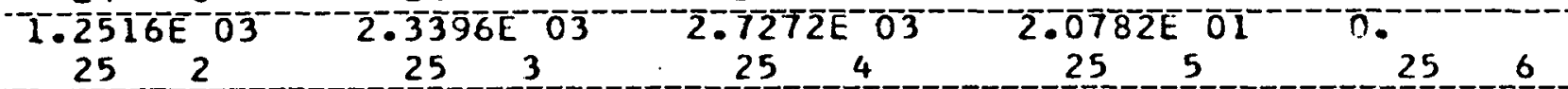

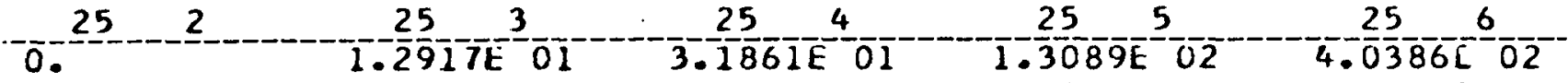

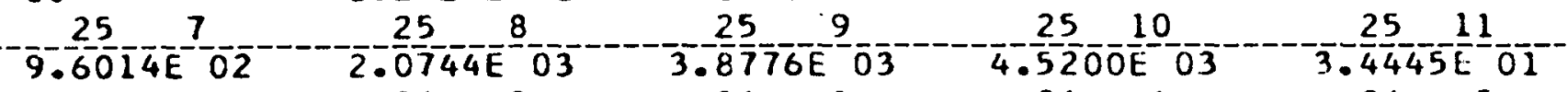

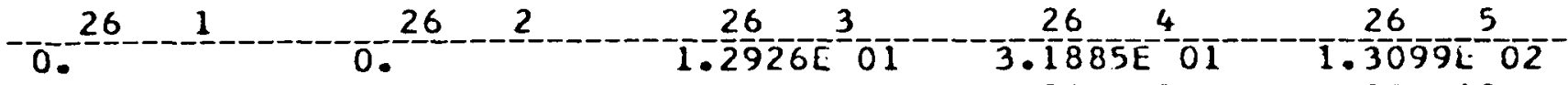

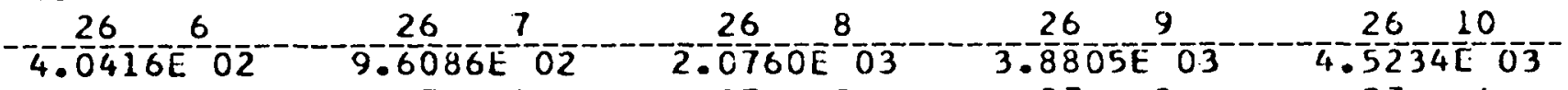

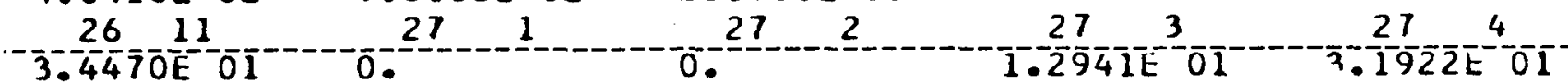

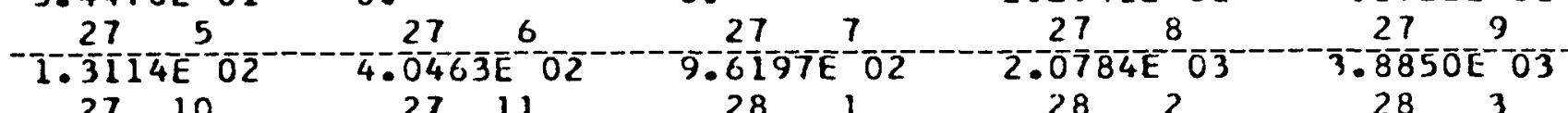

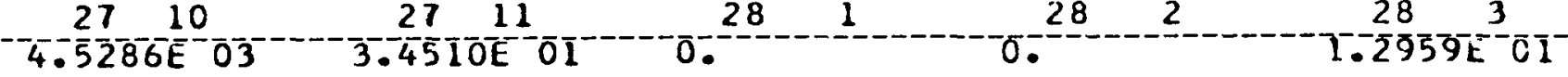


SIGMA N,G (NI/N2)

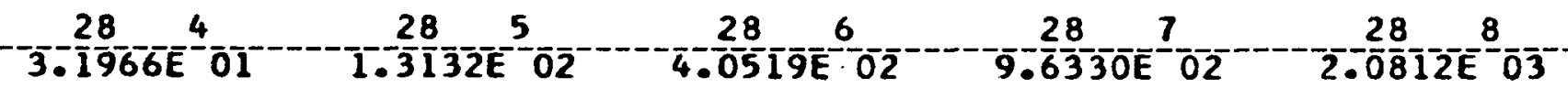

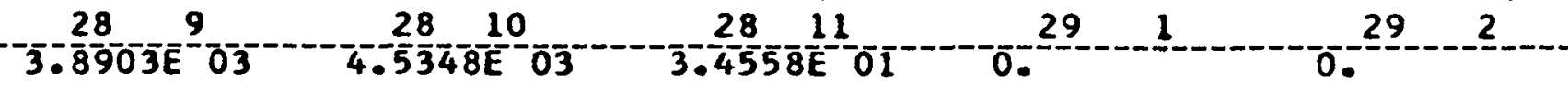

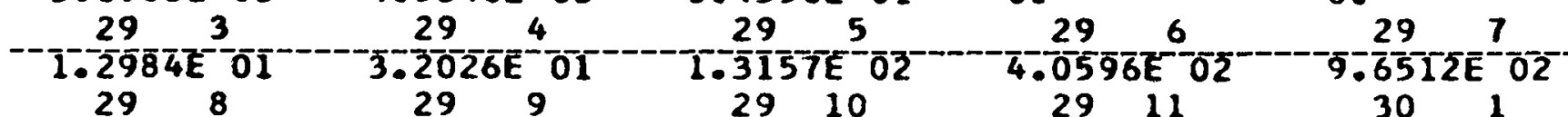

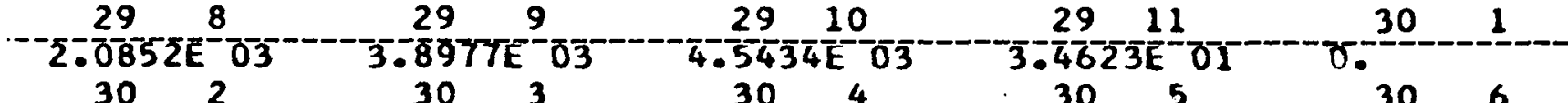

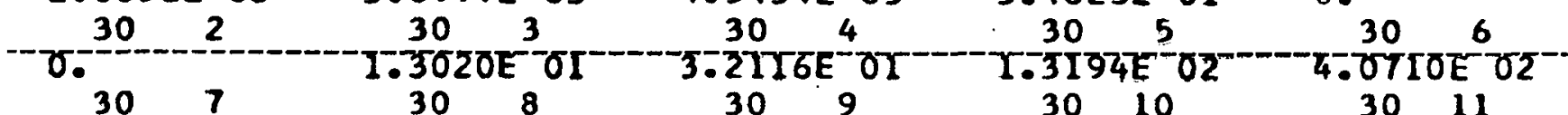

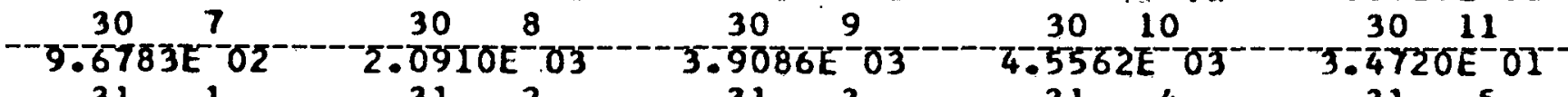

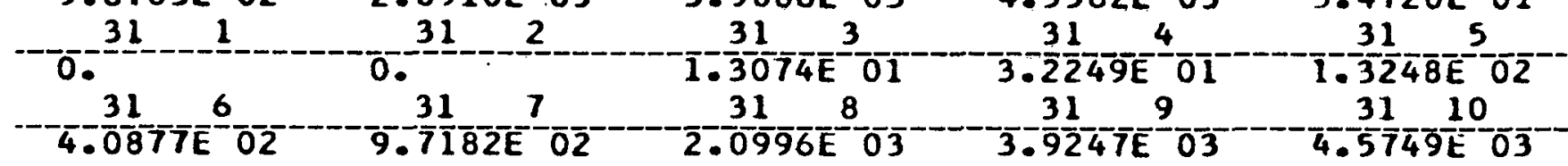

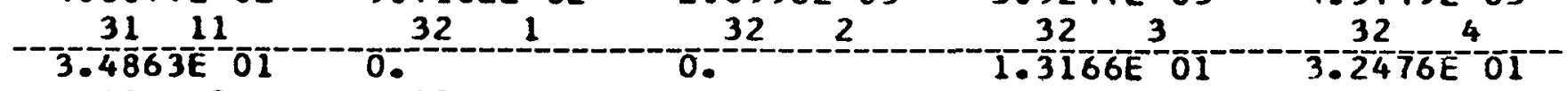

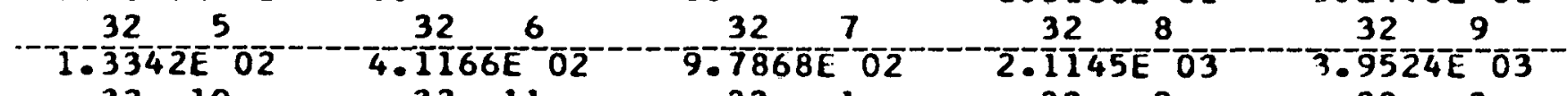

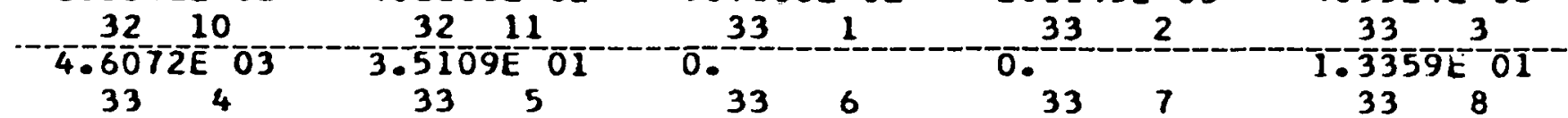

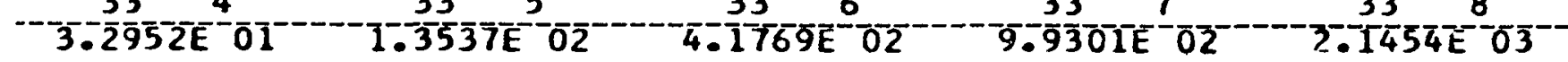

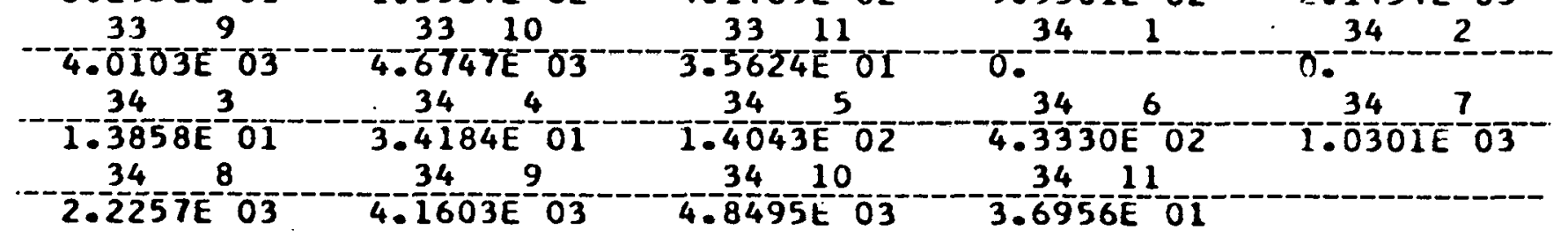




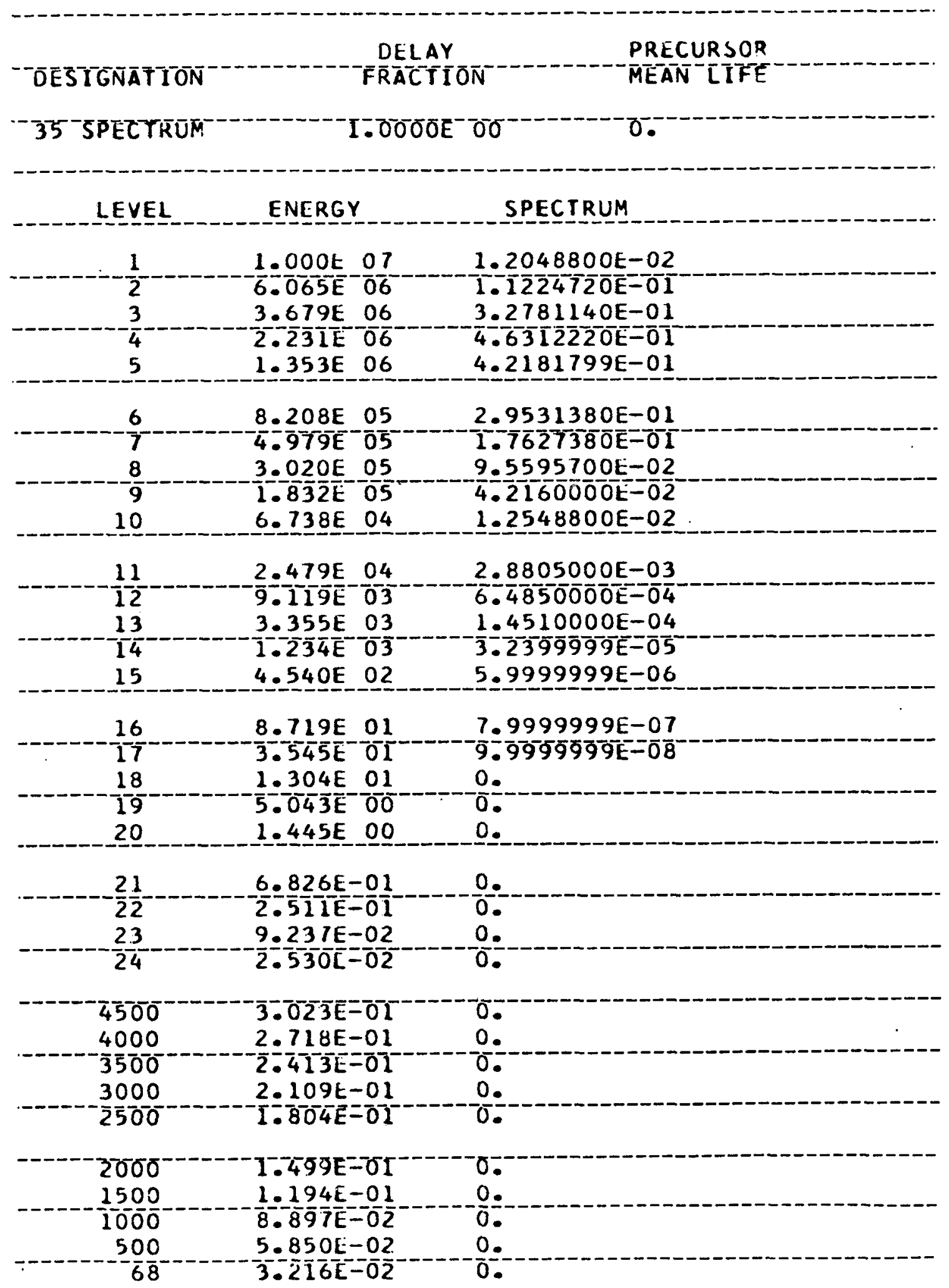




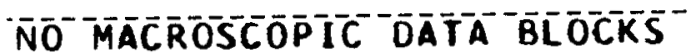


+ THIS PROGRAM PROCESSED ALL AVAILABLE CARDS

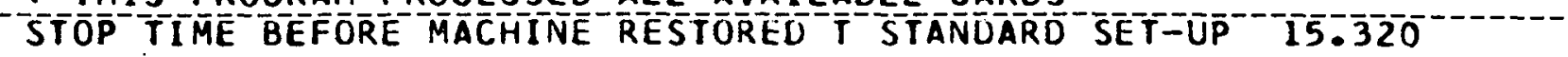
RESTORE MACHINE IO STANDARD SEI_UP_- - PUSH STARI. 
APPENDIX B - DIP Imput Subroutine

DIP

\section{SUBROUTINES}

\section{IDENTIFICATION:}

Title: DIP - Decimal Input

Source Language: FAP

Application: 7090 FORTRAN

Author: Dorothea S. Clarke

Unit: CTD

Installation: General Electric - Evendale

Date: April 1960

GENERAL INEORMATION:

The DIP input program loads from the peripheral input tape, converts and stores integers, floating-point data, alphabetic data or octal instructions. Column 1 of every data card is reserved for control information, which indicates whether the data is to be converted to integers, floating-point, six-bit binary-coded decimal, or an octal instruction. Other controls used are end-of-record, absolute location, and end-of-file. The data cards may be set up for any number of fields, limited only by the 72 columns on the card.

USAGE:

\section{Data Card Format}

Column 1

Column 1 is reserved for control punches. Below is a list of the various control punches that are permitted by DIP and their meaning:

\begin{tabular}{|c|c|c|}
\hline Control & Symbol & Designation \\
\hline $0(Z$ ero $)$ & 0 & Octal Instruction \\
\hline 2 & 2 & $\begin{array}{l}\text { Data is Binary-Coded Decimal stored } \\
\text { ascending order }\end{array}$ \\
\hline 3 & 3 & Data is Floating-Point \\
\hline 4 & 4 & Data is Fixed-Point \\
\hline 5 & 5 & $\begin{array}{l}\text { Data is Binary-Coded Decimal stored } \\
\text { descending order }\end{array}$ \\
\hline 8 & 8 & End-of-File when followed by 5 blanks \\
\hline $8-0$ & $\mathbf{Y}$ & Octal Instruction - End-of-Record \\
\hline $8-3$ & $=$ & Data is Floating-Point - End-of-Record \\
\hline
\end{tabular}




\section{DIP}

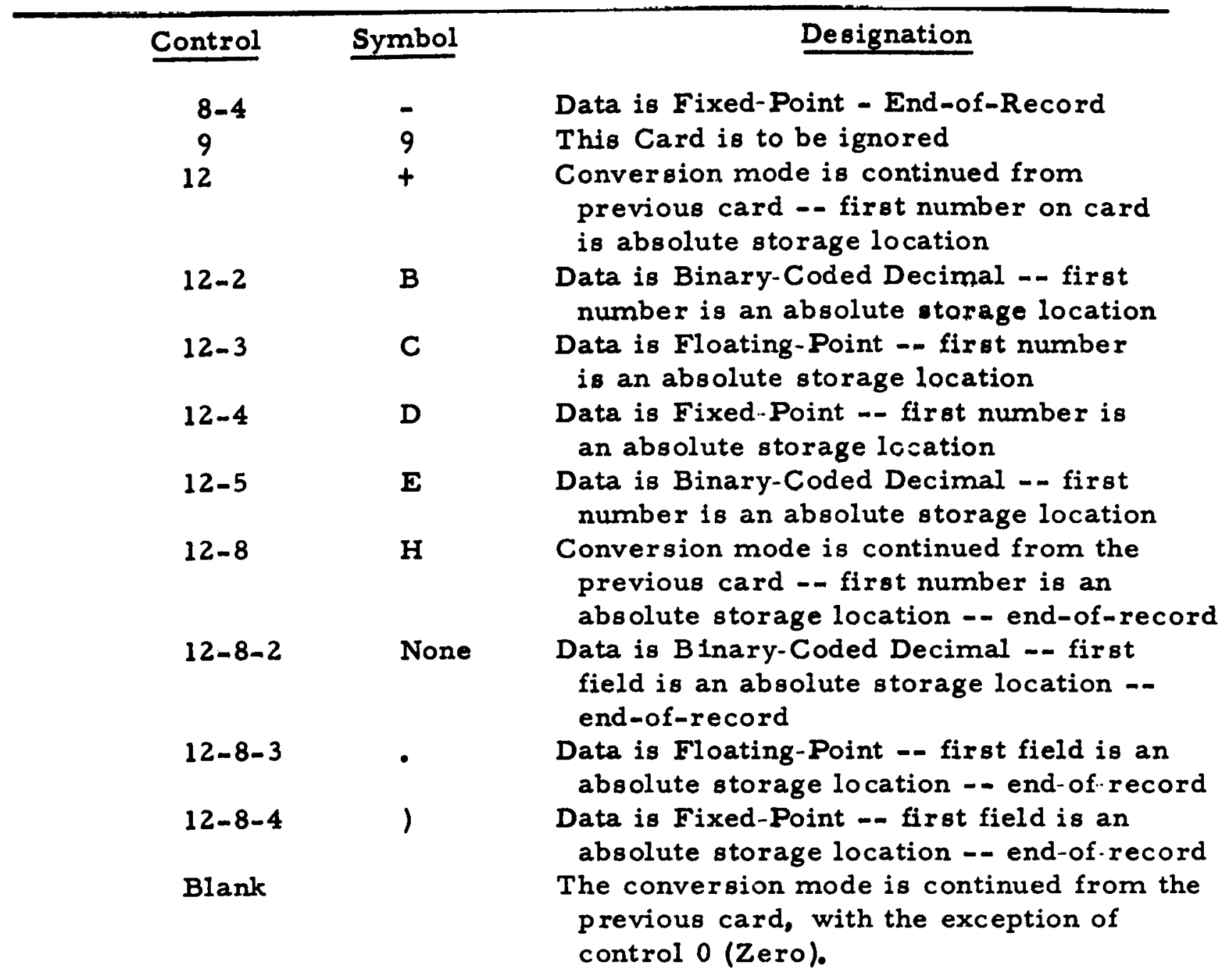

Absolute Locations

Whenever there is a 12 punch in column 1, the next number on the card must be an absolute location. DIP will then store all the data that follow in descending order with the first piece of data stored at this location.

Variable Storage Reference

Whenever a variable is found on the data card, DIP searches through the List of Variables and equates it to an absolute storage location. The variable may appear anywhere on the card with the 
exception of column 1 , and all data that follow are stored with reference to the absolute location.

Data

Floating Point: - A 3 punch in column 1 indicates that all tize data on this card are to be converted to 704 floating-point notation. There are a variety of acceptable ways to punch floating-point data, e. 8. :

$$
\begin{aligned}
& 8126 \\
& +8126.00 \\
& 8.126 / 3 \\
& +8.126 /+3 \\
& \\
& 8.126+3 \\
& +812600 /-2 \\
& 812600-2
\end{aligned}
$$

The plus sign on the data is optional. The exponent is separated from the mantissa by either a $/, t$, or - sign. A blank or a comma tells DIP that this is the end of the number.

Fixed Point: - A.4 punch in column 1 indicates that all data on this card are to be converted to a binary integer and stored in the decrement field of the current absolute storage location. The data on the card must be punched as an integer and may be preceded by a sign. The absolute value of the integer must not exceed 32767. The end of the integer is indicated by a blank or a comma.

Bypassing Storage Locations: - An equal sign immediately followed by a blank or a comma causes the storage location to be decreased by one. An integer ( $n$ ) immediately preceding the equal sign causes the storage location to be decreased by $n$. This feature enables the loading of data into scattered locations without continually defining the storage location.

Octal-Instruction Cards: - If column 1 contains a zero punch, this indicates to DIP that this is an octal-instruction card. Each octal-instruction card must have a zero punch in column 1 , an octal address in columns $7-11$, a sign and 12 octal numbers of the instruction in columns $18-30$. 
Binary-Coded Decimal Data: - If column 1 contains a 2 or a 5 punch, the data on the card are stored in six-bit binary-coded decimal form. If there is also a 12 punch in column 1, then the first information must be the absolute storage location. If the re is no 12 punch in column 1, the first character of the next field is examined to determine if it is numeric or alphabetic. If alphabetic, the field is interpreted to be the variable defining the storage location. Following the storage reference, and separated from it by either a blank or a comma, is the integer $\mathrm{N}$ where $\mathrm{N} \times 6$ is the number of BCD characters to be stored. If the first character of the first field is numeric, the field is interpreted as $\mathrm{N}$ and the storage location is sequential from the previous card. The integer $\mathrm{N}$ is followed by a blank or comma and the next character will be the first $B C D$ character to be stored. DIP will store BCD information in ascending locations if the column 1 control punch is $a 2$, or in descending order if the column I control punch is a 5. (When BCD information is being read for use as format by $I O H$, the control 5 must be used.)

When it is necessary to continue BCD information for more than one card, the following cards must have a blank or an 8 punch (EOR) in column 1 followed by the integer $\mathrm{N}$, a blank or comma, and the BCD information. Note: $N$ may not exceed the physical capacity of one card. Succeeding cards must each contain $\mathrm{N}$.

End-of-File: - When either a physical or control punch end-of-file is encountered the standard QUIT sequence is executed without comment.

Error Codes: - When DIP encounters an error, it reads in, but does not convert the remaining cards until an end-of-record control punch occurs in column 1. While bypassing the data cards, DIP will continue to search for errors, printing out each as it is found. After bypassing the data cards, DIP exits to the ERROR subroutine. The standard error procedure print-out is produced on the peripheral output tape as:

\section{AN ERROR HAS BEEN FOUND AT OR NEAR}

EXTERNAL FORMULA NO. $\alpha$, INTERNAL FORMULA NO. $\beta$, of (DIP)

where $\alpha$ is the error code and $\beta$ is $1000 \times$ card count + column number) 10 where the ror occurred. The error codes are: 


\section{DIP}

\begin{tabular}{lll}
\hline Code & 1 & Column l control not acceptable \\
& 5 & Error in data character \\
& 7 & Integer is too large \\
& 8 & Variable not given in list \\
& 9 & Tape failure
\end{tabular}

\section{CODING INFORMATION:}

\section{Restrictions}

1. The control punch zero must be on every octal-instruction card. In the octal flelds, blanks are equivalent to zero.

2. A data fleld is terminated by either a blank or a comma. Any number of blanks may separate the data fields.

3. Absolute storage locations, if specified, must be designated in the first field of the card.

4. An end-of-file error exit may be obtained by either an end-of-file control card or, if there is an attempt to read more data cards, when no more are aväilable.

5. A control character including a BCD 5 control and an EOR control is an illegal character unacceptable to the peripheral card reader. 
AIRCRAFT NUCLEAR PROPULSION DEPARTMENT

$$
\text { GENERAL ELECTRIC }
$$

